\title{
Efficient Access to Chiral Benzhydrols via Asymmetric Transfer Hydrogenation of Unsymmetrical Benzophenones with Bifunctional Oxo-tethered Ruthenium Catalysts
}

Taichiro Touge* ${ }^{[\mathrm{a}]}$ Hideki Nara, ${ }^{[\mathrm{a}]}$ Mitsuhiko Fujiwhara, ${ }^{[\mathrm{a}]}$ Yoshihito Kayaki* ${ }^{[\mathrm{b}]}$ and Takao Ikariya $^{[\mathrm{b}]}$

${ }^{[\mathrm{a}]}$ Corporate Research \& Development Division, Takasago International Corporation, 1-4-11 Nishi-yawata, Hiratsuka City, Kanagawa 254-0073, Japan

${ }^{[b]}$ Department of Chemical Science and Engineering, School of Materials and Chemical Technology, Tokyo Institute of Technology, 2-12-1-E4-1 O-okayama, Meguro-ku, Tokyo 152-8552, Japan

taichiro touge@takasago.com

ykayaki@o.cc.titech.ac.jp 


\section{Contents}

$\begin{array}{ll}\text { General Information } & \text { S2 }\end{array}$

$\begin{array}{ll}\text { A. Synthesis of Ketones. } & \text { S3 }\end{array}$

B. Asymmetric Transfer Hydrogenation of Unsymmetrical Benzophenones Using $(R, R)-3$ or $(R, R)-4$.

$\begin{array}{ll}\text { C. Characterization Data for Reduction Products. } & \text { S9 }\end{array}$

D. Synthesis of Chiral Benzo[c]chromene. $\quad$ S22

E. Determination of Absolute Configuration of Products. S23

F. References. $\quad$ S36

G. NMR Spectra for Ketones, Reduction Products and Derivatized Products. S37

H. HR-MS Spectra for Ketones, Reduction Products and Derivatized Products. S79

I. Chiral GC and HPLC Charts for Reduction Products. S101

\section{General Information}

All reactions and manipulations were conducted under a nitrogen atmosphere unless otherwise noted. Synthesis of ruthenium catalysts was performed in commercial anhydrous solvents. NMR Spectra were obtained on Agilent 400-MR DD2 and Bruker BioSpin Avance III 500 Systems. NMR chemical shifts are reported in ppm relative to $\mathrm{CHCl}_{3}$ (7.26 ppm for ${ }^{1} \mathrm{H}$, and $77.0 \mathrm{ppm}$ for $\left.{ }^{13} \mathrm{C}\right)$, or $\mathrm{CH}_{3} \mathrm{OH}\left(3.30 \mathrm{ppm}\right.$ for ${ }^{1} \mathrm{H}$, and $49.0 \mathrm{ppm}$ for ${ }^{13} \mathrm{C}$ ). The following abbreviations were used to designate peak splitting patterns: $\mathrm{s}=$ singlet, $\mathrm{d}=$ doublet, $\mathrm{t}=$ triplet, $\mathrm{q}=$ quartet, $\mathrm{m}=$ multiplet, $\mathrm{br}=$ broad. Optical rotations were obtained on a JASCO P-1020 Polarimeter. Mass spectra were recorded on SHIMADZU LCMS-IT-TOF and JEOL JMS-T100GCV. Elemental analyses were carried out using a PE2400 Series II CHNS/O Analyser (Perkin Elmer). High performance liquid chromatography (HPLC) analysis was performed using a system comprised of a GL-Science GL-7400 series; column oven: GL-7430, a gradient unit, a pump, degasser: GL-7430, a UV detector: GL-7450, an auto sampler: GL-7420. Recyclable preparative HPLC was performed on a Japan Analytical Industry LC-9225 NEXT system. IR Spectra were obtained on Thermo Fisher Scientific NICOLET iS10.

Ketones (5a, 5b, 5c, 5e, 5f, 5g, 5h, 5i, 5j, 5l, 5m, 5n, 10a, 10b, 10c, 10d, 10e, 10h, 10m) were purchased from TCI (Tokyo Chemical Industry Co., Ltd.). Ketones (6d, 6k) were purchased froma Sigma-Aldrich. Ketone (10i) was purchased from Combi blocks. Ketones (11j and 11k) were purchased from Wako Chemical Ltd. and Alfa Aesar, respectively. 


\section{A. Synthesis of Ketones.}

(a) Synthesis of Phenyl(3,4,5-trifluorophenyl)methanol ((rac)-11f)<smiles>O=C(F)c1cc(F)c(F)c(F)c1</smiles>

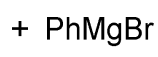

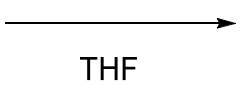<smiles>OC(c1ccccc1)c1cc(F)c(F)c(F)c1</smiles>

$11 f$

To a stirred mixture of 3,4,5-trifluorobenzaldehyde (4.00 g, $25.0 \mathrm{mmol})$ in dry THF (100 $\mathrm{mL}$ ), a solution of phenylmagnesium bromide (26.2 $\mathrm{mL} 26.23 \mathrm{mmol}, 1.0 \mathrm{M}$ in THF) was added dropwise at $0{ }^{\circ} \mathrm{C}$, and then the reaction temperature was raised to room temperature. After stirring for $2 \mathrm{~h}$, water $(50 \mathrm{~mL})$ and EtOAc $(50 \mathrm{~mL})$ was added, and $\mathrm{HCl}$ conc. (ca. 1 $\mathrm{mL}$ ) was slowly added to acidify the reaction mixture. The product was extracted with EtOAc $(2 \times 50 \mathrm{~mL})$ and the combined organic layers were washed with brine $(2 \times 100 \mathrm{~mL})$, dried over anhydrous $\mathrm{MgSO}_{4}$, and concentrated under reduced pressure to afford the crude product. Purification by silica gel column chromatography gave the product (11f) as a colorless oil ( $4.9 \mathrm{~g}, 83 \%$ yield).

${ }^{1} \mathrm{H}$ NMR (400 MHz, $\left.\mathrm{CDCl}_{3}\right) \delta$ 7.37-7.27 (m, 5H), 7.00-6.94 (m, 2H), $5.68(\mathrm{~d}, J=2.8 \mathrm{~Hz}$, $1 \mathrm{H}), 2.51(\mathrm{~d}, J=2.8 \mathrm{~Hz}, 1 \mathrm{H}) ;{ }^{13} \mathrm{C} \mathrm{NMR}\left(125 \mathrm{MHz}, \mathrm{CDCl}_{3}\right) \delta 151.1(\mathrm{dd}, J=10.0,3.8 \mathrm{~Hz})$, , $142.5,139.9(\mathrm{~m}), 137.8(\mathrm{~m}), 128.9,128.4,126.5,110.3(\mathrm{dd}, J=17.5,5.0 \mathrm{~Hz}), 74.9$.

HRMS (FI) calcd for $\mathrm{C}_{13} \mathrm{H}_{9} \mathrm{~F}_{3} \mathrm{O}[\mathrm{M}]^{+}: 238.0606$. Found: 238.0617.

IR (neat) 3376, 2978, 2876, 1622, 1528, 1447, 1343, 1234, 1036, 758, 704, $613 \mathrm{~cm}^{-1}$.

\section{(b) Synthesis of Phenyl(3,4,5-trifluorophenyl)methanone (10f) ${ }^{[1]}$}<smiles>OC(c1ccccc1)c1cc(F)c(F)c(F)c1</smiles>

$11 f$

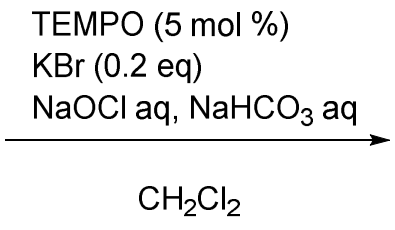

$\mathrm{CH}_{2} \mathrm{Cl}_{2}$<smiles>O=C(c1ccccc1)c1cc(F)c(F)c(F)c1</smiles>

$10 f$

To a solution of $11 \mathrm{f}(2.00 \mathrm{~g}, 8.39 \mathrm{mmol})$ in $\mathrm{CH}_{2} \mathrm{Cl}_{2}(73 \mathrm{~mL})$ were added $\mathrm{KBr}(0.204 \mathrm{~g}, 1.71$ mmol), 2,2,6,6-tetramethylpiperidine 1-oxyl (TEMPO) (65.5 $\mathrm{mg}, 0.419 \mathrm{mmol})$, and saturated aqueous $\mathrm{NaHCO}_{3}(50 \mathrm{~mL})$. The biphasic mixture was vigorously stirred, and aqueous $\mathrm{NaOCl}(36.7 \mathrm{~mL}, 0.7 \mathrm{M})$ was added. The resulting bright orange mixture was stirred for $2 \mathrm{~h}$, and the orange color dissipated. The colorless biphasic layers were separated, the aqueous phase was extracted with $\mathrm{CHCl}_{3}(2 \times 50 \mathrm{~mL})$, and the combined organic portions were dried over $\mathrm{MgSO}_{4}$, and concentrated to give a crude liquid. The mixture was filtered through a plug of silica gel and concentrated to give the product (10f) as a colorless liquid (1.9 g, 97\% yield). 
${ }^{1} \mathrm{H}$ NMR (400 MHz, $\left.\mathrm{CDCl}_{3}\right) \delta$ 7.79-7.75 (m, 2H), 7.65-7.60 (m, 1H), 7.55-7.40 (m, 4H); ${ }^{13} \mathrm{C}$ NMR $\left(125 \mathrm{MHz}, \mathrm{CDCl}_{3}\right) \delta 192.8,151.9(\mathrm{dd}, J=10.0,3.8 \mathrm{~Hz}), 149.9(\mathrm{dd}, J=10.0,3.8$ $\mathrm{Hz}), 142.7$ (m), 136.1, $133.0(\mathrm{~m}), 129.8,128.6,114.5$ (dd, $J=16.3,5.0 \mathrm{~Hz})$.

HRMS (FI) calcd for $\mathrm{C}_{13} \mathrm{H}_{7} \mathrm{~F}_{3} \mathrm{O}[\mathrm{M}]^{+}: 236.0449$. Found: 236.0448 .

IR (neat) 3391, 1662, 1596, 1526, 1434, 1344, 1232, 1046, 886, 763, 727, 700, $667 \mathrm{~cm}^{-1}$.

(c) Synthesis of (3,5-Dinitrophenyl)(phenyl)methanone (10g)<smiles>O=C(O)c1cc([N+](=O)[O-])cc([N+](=O)[O-])c1</smiles>

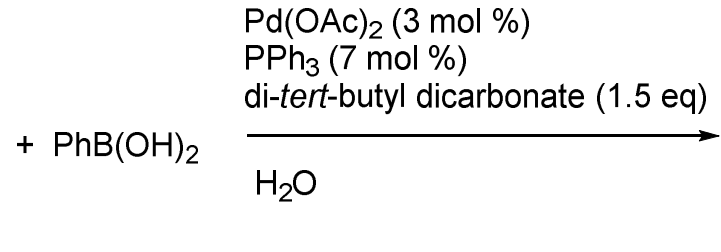<smiles>O=C(c1ccccc1)c1cc([N+](=O)[O-])cc([N+](=O)[O-])c1</smiles>

$10 \mathrm{~g}$

A THF solution $(60 \mathrm{~mL})$ containing $\mathrm{Pd}(\mathrm{OAc})_{2}(67.2 \mathrm{mg}, 0.30 \mathrm{mmol}), \mathrm{PPh}_{3}(184.0 \mathrm{mg}, 0.70$ $\mathrm{mmol})$, 3,5-dinitrobenzoic acid $(2.121 \mathrm{~g}, 10.0 \mathrm{mmol})$, phenylboronic acid $(1.463 \mathrm{~g}, 12.0$ mmol), di-tert-butyl dicarbonate $(2.764 \mathrm{~g}, 15.0 \mathrm{mmol})$, and $\mathrm{H}_{2} \mathrm{O}(0.450 \mathrm{~mL})$ was heated under Ar atmosphere at $60{ }^{\circ} \mathrm{C}$, for $15 \mathrm{~h}$. After cooling the reaction mixture, the insoluble materials were filtered off through a pad of Florisil. The Florisil was washed with $\mathrm{Et}_{2} \mathrm{O}(50$ $\mathrm{mL}$ ) and the combined filtrates were washed with a saturated aqueous solution of $\mathrm{NaHCO}_{3}$ $(3 \times 20 \mathrm{~mL})$, and brine $(10 \mathrm{~mL})$, and then dried over $\mathrm{MgSO}_{4}$. After removal of organic solvents, the residue was purified by silica gel column chromatography (hexane/AcOEt $=$ 6/1), followed by preparative HPLC equipped with JAIGEL-1H and $-2 \mathrm{H}$ columns using $\mathrm{CHCl}_{3}$ as an eluent at a flow rate of $14 \mathrm{~mL} \mathrm{~min}^{-1}$ gave $\mathbf{1 0 g}$ as a white solid in $24 \%$ yield $(0.650 \mathrm{~g}, 2.39 \mathrm{mmol})$.

${ }^{1} \mathrm{H}$ NMR (400 MHz, $\left.\mathrm{CDCl}_{3}\right) \delta 9.25(\mathrm{~s}, 1 \mathrm{H}), 8.93(\mathrm{~s}, 2 \mathrm{H}), 7.83-7.80(\mathrm{~m}, 2 \mathrm{H}), 7.78-7.72(\mathrm{~m}$, 1H), 7.62-7.57 (m, 2H); ${ }^{13} \mathrm{C}$ NMR (125 MHz, $\left.\mathrm{CDCl}_{3}\right) \delta 191.6,148.5,140.7,135.0,134.2$, 130.0, 129.4, 129.1, 121.5.

HRMS (FI) calcd for $\mathrm{C}_{13} \mathrm{H}_{8} \mathrm{~N}_{2} \mathrm{O}_{5}[\mathrm{M}]^{+}:$272.0433. Found: 272.0442 .

IR (neat) 3100, 1670, 1545, 1348, 1282, 1079, 916, 809, $710 \mathrm{~cm}^{-1}$.

\section{(d) Synthesis of (3,5-Dinitrophenyl)(4-methoxyphenyl)methanone (10I)}

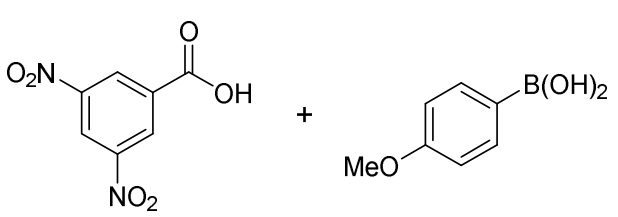

$\mathrm{Pd}(\mathrm{OAc})_{2}(3 \mathrm{~mol} \%)$

$\mathrm{PPh}_{3}(7 \mathrm{~mol} \%)$

di-tert-butyl dicarbonate $(1.5 \mathrm{eq})$

$\mathrm{H}_{2} \mathrm{O}$

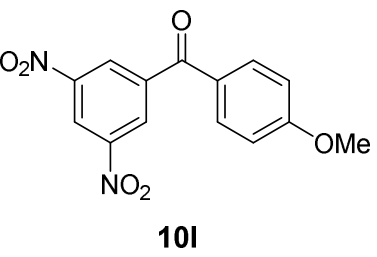

10I

A THF solution $(60 \mathrm{~mL})$ containing $\mathrm{Pd}(\mathrm{OAc})_{2}(67.2 \mathrm{mg}, 0.30 \mathrm{mmol}), \mathrm{PPh}_{3}(184.0 \mathrm{mg}, 0.70$ 
mmol), 3,5-dinitrobenzoic acid (2.121 g, $10.0 \mathrm{mmol})$, 4-methoxyphenylboronic acid (1.824 $\mathrm{g}, 12.0 \mathrm{mmol})$, di-tert-butyl dicarbonate $(2.765 \mathrm{~g}, 15.0 \mathrm{mmol})$, and $\mathrm{H}_{2} \mathrm{O}(0.450 \mathrm{~mL})$ was heated under Ar atmosphere at $60{ }^{\circ} \mathrm{C}$, for $18 \mathrm{~h}$. After cooling the reaction mixture, the insoluble materials were filtered off through a pad of Florisil. The Florisil was washed with $\mathrm{Et}_{2} \mathrm{O}(50 \mathrm{~mL})$ and the combined filtrates were washed with a saturated aqueous solution of $\mathrm{NaHCO}_{3}(3 \times 20 \mathrm{~mL})$, and brine $(10 \mathrm{~mL})$, and then dried over $\mathrm{MgSO}_{4}$. After removal of organic solvents, the residue was purified by silica gel column chromatography (hexane/AcOEt $=5 / 1$ ), followed by preparative HPLC equipped with JAIGEL-1H and $-2 \mathrm{H}$ columns using $\mathrm{CHCl}_{3}$ as an eluent at a flow rate of $14 \mathrm{~mL} \mathrm{~min}^{-1}$ gave $\mathbf{1 0 1}$ as a white solid in $33 \%$ yield (0.976 g, $3.23 \mathrm{mmol})$.

${ }^{1} \mathrm{H}$ NMR (400 MHz, $\left.\mathrm{CDCl}_{3}\right) \delta 9.22(\mathrm{~s}, 1 \mathrm{H}), 8.89(\mathrm{~s}, 2 \mathrm{H}), 7.82(\mathrm{~d}, J=8.8 \mathrm{~Hz}, 2 \mathrm{H}), 7.04(\mathrm{~d}$, $J=8.8 \mathrm{~Hz}, 2 \mathrm{H}), 3.94(\mathrm{~s}, 3 \mathrm{H}) ;{ }^{13} \mathrm{C} \mathrm{NMR}\left(125 \mathrm{MHz}, \mathrm{CDCl}_{3}\right) \delta 190.2,164.6,148.5,141.5$, 132.7, 129.1, 127.7, 121.1, 114.5, 55.70.

HRMS (ESI) calcd for $\mathrm{C}_{14} \mathrm{H}_{10} \mathrm{~N}_{2} \mathrm{O}_{6}[\mathrm{M}]^{+}: 302.0539$. Found: 302.0539 .

IR (neat) 3092, 1661, 1597, 1546, 1538, 1264, 1165, 1023, 846, 729, $608 \mathrm{~cm}^{-1}$.

\section{(e) Synthesis of (3-Nitrophenyl)(thiophen-2-yl)methanol ((rac)-11n)}<smiles>O=Cc1cccc([N+](=O)[O-])c1</smiles><smiles>O=[N+]([O-])c1cccc(C(O)c2cccs2)c1</smiles>

A solution of 3-nitrobenzaldehyde $(3.02 \mathrm{~g}, 20.0 \mathrm{mmol})$ in dry THF $(25 \mathrm{~mL})$ was cooled to $0{ }^{\circ} \mathrm{C}$, and commercially available 2-thienyl lithium (1.0 M in hexane/THF) was carefully added dropwise under Ar atmosphere. The resulting solution was allowed to warm to room temperature followed by stirring for $3 \mathrm{~h}$. After the reaction was quenched with aqueous $\mathrm{NH}_{4} \mathrm{Cl}(5 \mathrm{~mL})$, the resulting mixture was extracted with $\mathrm{Et}_{2} \mathrm{O}(3 \times 15 \mathrm{~mL})$ and then washed with brine $(5 \mathrm{~mL})$. The organic layer was dried over $\mathrm{MgSO}_{4}$ and the volatiles were removed under reduced pressure. The residue was purified by column chromatography on silica gel using hexane/AcOEt $=3 / 1$ as eluents. The following purification by preparative HPLC equipped with JAIGEL-1H and $-2 \mathrm{H}$ columns using $\mathrm{CHCl}_{3}$ as an eluent at a flow rate of 14 $\mathrm{mL} \mathrm{min}^{-1}$ gave (3-nitrophenyl)(thiophen-2-yl)methanol (11n) as a colorless oil in $48 \%$ yield $(2.25 \mathrm{~g}, 9.56 \mathrm{mmol})$.

${ }^{1} \mathrm{H}$ NMR $\left(400 \mathrm{MHz}, \mathrm{CDCl}_{3}\right) \delta 8.32-8.31(\mathrm{~m}, 1 \mathrm{H}), 8.15-8.12(\mathrm{~m}, 1 \mathrm{H}), 7.78-7.76(\mathrm{~m}, 1 \mathrm{H})$, 7.54-7.52 (m, 1H), 7.30-7.29 (m, 1H), 6.97-6.94 (m, 2H), $6.15(\mathrm{~s}, 1 \mathrm{H}), 2.83(\mathrm{br}, 1 \mathrm{H}) ;{ }^{13} \mathrm{C}$ NMR $\left(125 \mathrm{MHz}, \mathrm{CDCl}_{3}\right) \delta 148.3,146.6,145.1,132.2,129.4,126.9,126.2,125.5,122.8$, 121.2, 71.1 .

HRMS (FI) calcd for $\mathrm{C}_{11} \mathrm{H}_{9} \mathrm{NO}_{3} \mathrm{~S}[\mathrm{M}]^{+}: 235.0303$. Found: 235.0294 . IR (neat) 3392, 2917, 2848, 1529, 1350, 1094, 1022, 811, 760, $707 \mathrm{~cm}^{-1}$. 
(f) Synthesis of (3-Nitrophenyl)(thiophen-2-yl)methanone (10n)<smiles>O=[N+]([O-])c1cccc(C(O)c2cccs2)c1</smiles>

$11 \mathrm{n}$

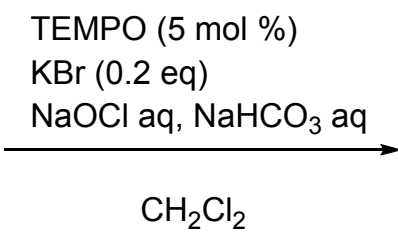

$\mathrm{CH}_{2} \mathrm{Cl}_{2}$<smiles>O=C(c1cccc([N+](=O)[O-])c1)c1cccs1</smiles>

$10 n$

To a solution of $11 \mathrm{n}(1.00 \mathrm{~g}, 4.25 \mathrm{mmol})$ in $\mathrm{CH}_{2} \mathrm{Cl}_{2}(36 \mathrm{~mL})$ were added $\mathrm{KBr}(0.102 \mathrm{~g}, 0.86$ mmol), 2,2,6,6-tetramethylpiperidine 1-oxyl (TEMPO) $(32.8 \mathrm{mg}, 0.210 \mathrm{mmol}$ ), and saturated aqueous $\mathrm{NaHCO}_{3}(25 \mathrm{~mL})$. The biphasic mixture was vigorously stirred, and aqueous $\mathrm{NaOCl}(18.3 \mathrm{~mL}, 0.7 \mathrm{M})$ was added. The resulting bright orange mixture was stirred for $3 \mathrm{~h}$, and the orange color dissipated. The colorless biphasic layers were separated, the aqueous phase was extracted with $\mathrm{CHCl}_{3}(2 \times 25 \mathrm{~mL})$, and the combined organic portions were dried over $\mathrm{MgSO}_{4}$, and concentrated to give a crude liquid. The mixture was filtered through a plug of silica gel and concentrated to give the product (10n) as a pale white solid (0.94 $\mathrm{g}, 95 \%$ yield).

${ }^{1} \mathrm{H}$ NMR $\left(400 \mathrm{MHz}, \mathrm{CDCl}_{3}\right) \delta 8.72-8.71(\mathrm{~m}, 1 \mathrm{H}), 8.46-8.43(\mathrm{~m}, 1 \mathrm{H}), 8.22-8.19(\mathrm{~m}, 1 \mathrm{H})$, 7.82-7.80 (m, 1H) , 7.78-7.75 (m, 1H), 7.65-7.63 (m, 1H), 7.22-7.20 (m, 1H); ${ }^{13} \mathrm{C} \mathrm{NMR}$ $\left(125 \mathrm{MHz} \mathrm{CDCl}_{3}\right) \delta 185.6,148.1,142.4,139.5,135.5,135.3,134.7,129.8,128.4,126.6$, 124.0.

HRMS (FI) calcd for $\mathrm{C}_{11} \mathrm{H}_{7} \mathrm{NO}_{3} \mathrm{~S}[\mathrm{M}]^{+}:$233.0147. Found: 233.0134.

\section{B. Asymmetric Transfer Hydrogenation of Unsymmetrical Benzophenones Using $(R, R)-3$ of $(R, R)-4$}

General procedures under the conditions of $\mathrm{S} / \mathrm{C}=100,60^{\circ} \mathrm{C}$, and $5 \mathrm{~h}$.

Under $\mathrm{N}_{2}$ atmosphere, a mixture of ketone $(1.0 \mathrm{mmol})$ and the $\mathrm{Ru}$ catalyst $(0.01 \mathrm{mmol})$ in an azeotrope of formic acid and triethylamine $(5: 2,0.5 \mathrm{~mL})$ was stirred at $60{ }^{\circ} \mathrm{C}$ for $5 \mathrm{~h}$. After the reaction completion, water $(3 \mathrm{~mL})$ and EtOAc $(5 \mathrm{~mL})$ was added. The biphasic layers were separated, the aqueous layer was extracted with EtOAc $(3 \times 5 \mathrm{~mL})$, and the combined organic portions were washed with brine $(3 \mathrm{~mL})$. After drying over $\mathrm{MgSO}_{4}$, filtration, and solvent removal under reduced pressure, the crude residue was purified by silica gel column chromatography to afford the desired product. The optical purity of product was determined by chiral HPLC analysis using a Daicel Chiralcel OD-H, OJ-H or Chiralpak AD-H, AS-H column $(4.6 \mathrm{~mm} \times 25 \mathrm{~cm})$ with hexane/2-propanol as the eluent where a clear base-line separation was obtained. 
Figure S1. Structure of non-tethered and tethered Ru-DPEN catalysts.
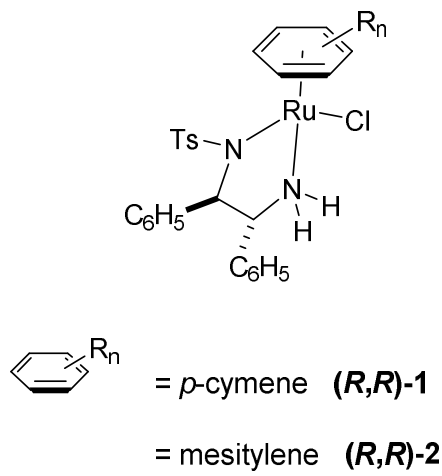

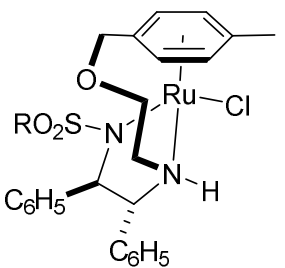

$\mathrm{SO}_{2} \mathrm{R}=\mathrm{Ts}(\boldsymbol{R}, \boldsymbol{R})-3 \quad$ (Ts-DENEB)

Ms $(R, R)-4$ (Ms-DENEB)

Table S1. Asymmetric transfer hydrogenation of 2-substituted benzophenones<smiles>[R][Z]1ccc(C(=O)c2ccccc2)c([R])c1</smiles><smiles>[R][R]1ccc([C@@H](O)c2ccccc2)c([R])c1</smiles>

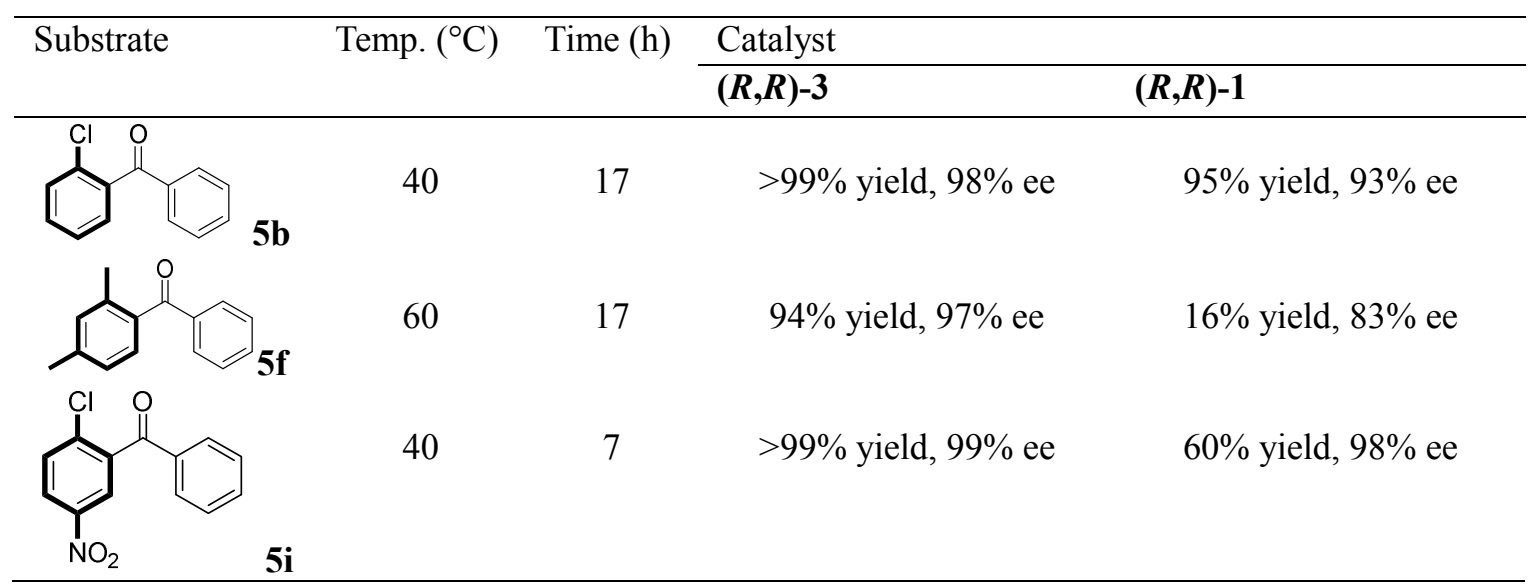


Table S2. Asymmetric transfer hydrogenation of non-ortho-substituted diaryl ketones<smiles>[R]c1ccc(C(=O)c2ccccc2)cc1[R]</smiles>

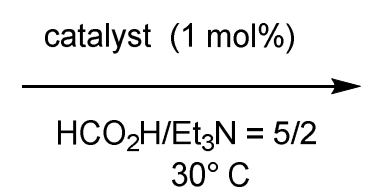<smiles>[R]c1ccc([C@@H](O)c2ccccc2)cc1[R]</smiles>

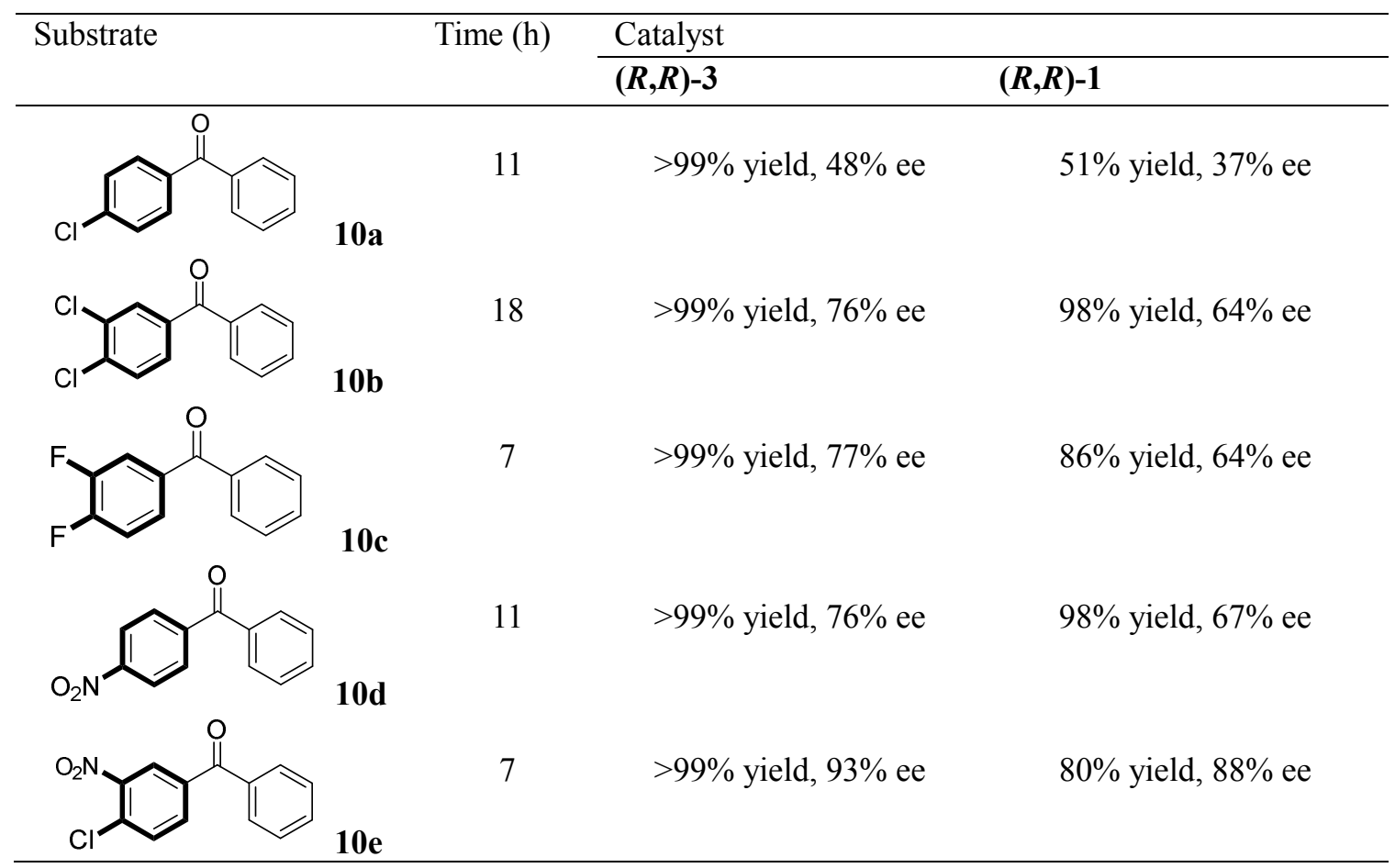




\section{Characterization Data for Reduction Products.}

\section{(S)-Phenyl(o-tolyl)methanol (6a)}<smiles>Cc1ccccc1[C@@H](O)c1ccccc1</smiles>

According to the general procedure (ketone: $0.196 \mathrm{~g}(1 \mathrm{mmol})$, cat. $(\boldsymbol{R}, \boldsymbol{R})-3), 0.194 \mathrm{~g}$ of $\mathbf{6 a}$ was obtained as a white solid ( $98 \%$ yield).

${ }^{1} \mathrm{H}$ NMR $\left(400 \mathrm{MHz}, \mathrm{CDCl}_{3}\right) \delta$ 7.52-7.50 (m, 1H), 7.33-7.14 (m, 8H), $6.01(\mathrm{~d}, J=3.2 \mathrm{~Hz}$, $1 \mathrm{H}), 2.25(\mathrm{~s}, 1 \mathrm{H}), 2.12(\mathrm{~d}, J=3.2 \mathrm{~Hz}, 1 \mathrm{H}) ;{ }^{13} \mathrm{C} \mathrm{NMR}\left(125 \mathrm{MHz}, \mathrm{CDCl}_{3}\right) \delta 142.9,141.4$, $135.4,130.5,128.5,127.6,127.5,127.1,126.3,126.1,73.4,19.4$. All characterization data are in agreement with the previously reported data ${ }^{[2]}$.

HRMS (FI) calcd for $\mathrm{C}_{14} \mathrm{H}_{14} \mathrm{O}[\mathrm{M}]^{+}:$198.10446. Found: 198.10457.

The enantiomeric excess was determined by HPLC analysis (Chiralcel OD-H, $250 \times 4.6$ $\mathrm{mm}$ column, hexane/2-propanol 97:3,1.0 mL/min, $220 \mathrm{~nm}, 30^{\circ} \mathrm{C},(R)$ isomer $15.8 \mathrm{~min},(S)$ isomer $17.3 \mathrm{~min}) ;[\alpha]_{\mathrm{D}}{ }^{20}+7.3\left(\mathrm{c} 0.735\right.$ in $\left.\mathrm{CHCl}_{3}\right) 98 \%$ ee $(S)\left(\right.$ lit. $^{[2]}[\alpha]_{\mathrm{D}}{ }^{22}+6.38(\mathrm{c} 0.906$ in $\left.\mathrm{CHCl}_{3}\right) 93 \%$ ee $\left.(S)\right)$.

IR (neat) 3375, 3064, 3027, 1492, 1454, 1017, 699, $667 \mathrm{~cm}^{-1}$.

\section{(S)-(2-Chlorophenyl)(phenyl)methanol (6b)}<smiles>OC(c1ccccc1)c1ccccc1Cl</smiles>

According to the general procedure (ketone: $0.216 \mathrm{~g}(1 \mathrm{mmol})$, cat. $(\boldsymbol{R}, \boldsymbol{R})-\mathbf{3}), 0.217 \mathrm{~g}$ of $\mathbf{6 b}$ was obtained as a clear oil ( $>99 \%$ yield).

${ }^{1} \mathrm{H}$ NMR (400 MHz, $\left.\mathrm{CDCl}_{3}\right) \delta$ 7.60-7.57 (m, 1H), 7.39-7.18 (m, 8H), $6.20(\mathrm{~s}, 1 \mathrm{H}), 2.44(\mathrm{br}$, $1 \mathrm{H}) ;{ }^{13} \mathrm{C}$ NMR $\left(125 \mathrm{MHz}, \mathrm{CDCl}_{3}\right) \delta 142.2,141.0,132.5,129.5,128.7,128.5,128.0,127.7$, $127.0,126.9,72.7$. All characterization data are in agreement with the previously reported data $^{[2]}$.

HRMS (FI) calcd for $\mathrm{C}_{12} \mathrm{H}_{11} \mathrm{ClO}[\mathrm{M}]^{+}:$218.04984. Found: 218.05035.

The enantiomeric excess was determined by HPLC analysis (Chiralcel OD-H, $250 \times 4.6$ $\mathrm{mm}$ column, hexane/2-propanol 97:3, $1.0 \mathrm{~mL} / \mathrm{min}, 220 \mathrm{~nm}, 30^{\circ} \mathrm{C},(R)$ isomer $14.6 \mathrm{~min},(S)$ isomer $18.6 \mathrm{~min}$ ); $[\alpha]_{\mathrm{D}}{ }^{20}-15.2$ (c 1.51 in $\left.\mathrm{CHCl}_{3}\right) 98 \%$ ee $(S)\left(\right.$ lit. $^{[2]}[\alpha]_{\mathrm{D}}{ }^{20}-21.51$ (c 1.136 in $\left.\mathrm{CHCl}_{3}\right) 97 \%$ ee $\left.(S)\right)$.

IR (neat) 3355, 3064, 3031, 1441, 1183, 1020, 699, $646 \mathrm{~cm}^{-1}$.

\section{(S)-(2-Bromophenyl)(phenyl)methanol (6c)}


<smiles>OC(c1ccccc1)c1ccccc1Br</smiles>

According to the general procedure (ketone: $3.0 \mathrm{~g}(11.5 \mathrm{mmol})$, cat. $(\boldsymbol{R}, \boldsymbol{R})-3), 0.301 \mathrm{~g}$ of $\mathbf{6 c}$ was obtained as a clear oil ( $>99 \%$ yield).

${ }^{1} \mathrm{H}$ NMR $\left(400 \mathrm{MHz}, \mathrm{CDCl}_{3}\right) \delta$ 7.60-7.53 (m, 2H), 7.42-7.28 (m, 6H), 7.18-7.13 (m, 1H), $6.19(\mathrm{~s}, 1 \mathrm{H}), 2.56(\mathrm{br}, 1 \mathrm{H}) ;{ }^{13} \mathrm{C} \mathrm{NMR}\left(125 \mathrm{MHz}, \mathrm{CDCl}_{3}\right) \delta 142.5,142.1,132.8,129.1$, $128.4,128.4,127.7,127.7,127.0,122.8,74.7$. All characterization data are in agreement with the previously reported data ${ }^{[2]}$.

HRMS (FD) calcd for $\mathrm{C}_{13} \mathrm{H}_{11} \mathrm{BrO}[\mathrm{M}]^{+}: 261.9993$. Found: 261.9996.

The enantiomeric excess was determined by HPLC analysis (Chiralcel OD-H, $250 \times 4.6$ $\mathrm{mm}$ column, hexane/2-propanol 97:3, $1.0 \mathrm{~mL} / \mathrm{min}, 220 \mathrm{~nm}, 30^{\circ} \mathrm{C},(R)$ isomer $15.8 \mathrm{~min},(S)$ isomer $22.3 \mathrm{~min}$ ); $[\alpha]_{\mathrm{D}}{ }^{20}-41.6$ (c 1.40 in $\left.\mathrm{CHCl}_{3}\right) 99 \%$ ee $(S)\left(\right.$ lit. $^{[2]}[\alpha]_{\mathrm{D}}{ }^{\mathrm{RT}}-41.9$ (c 1.19 in $\left.\mathrm{CHCl}_{3}\right) 96 \%$ ee $\left.(S)\right)$.

IR (neat) 3354, 3063, 3030, 1735, 1438, 1184, 1016, $699 \mathrm{~cm}^{-1}$.

\section{(S)-Phenyl[2-(trifluoromethyl)phenyl]methanol (6d)}<smiles>OC(c1ccccc1)c1ccccc1C(F)(F)F</smiles>

According to the general procedure (ketone: $0.250 \mathrm{~g}(1 \mathrm{mmol})$, cat. $(\boldsymbol{R}, \boldsymbol{R})-\mathbf{3}), 0.250 \mathrm{~g}$ of $\mathbf{6 d}$ was obtained as a clear oil (99\% yield).

${ }^{1} \mathrm{H}$ NMR $\left(400 \mathrm{MHz}, \mathrm{CDCl}_{3}\right) \delta$ 7.67-7.62 (m, 2H), 7.58-7.52 (m, 1H), 7.40-7.24 (m, 6H), $6.31(\mathrm{~d}, J=3.2 \mathrm{~Hz}, 1 \mathrm{H}), 2.34(\mathrm{~d}, J=3.2 \mathrm{~Hz}, 1 \mathrm{H}) ;{ }^{13} \mathrm{C} \mathrm{NMR}\left(125 \mathrm{MHz}, \mathrm{CDCl}_{3}\right) \delta 142.7$, $132.3,129.5,128.4,127.8,127.7,127.5,126.4,125.6,125.4,123.3,70.8$.

HRMS (FI) calcd for $\mathrm{C}_{14} \mathrm{H}_{11} \mathrm{~F}_{3} \mathrm{O}[\mathrm{M}]^{+}:$252.07620. Found: 252.07615.

The enantiomeric excess was determined by HPLC analysis (Chiralcel OD-H, $250 \times 4.6$ $\mathrm{mm}$ column, hexane/2-propanol 97:3,1.0 mL/min, $220 \mathrm{~nm}, 30{ }^{\circ} \mathrm{C},(R)$ isomer $9.1 \mathrm{~min},(S)$ isomer $13.4 \mathrm{~min})$; $[\alpha]_{\mathrm{D}}{ }^{20}-71.7$ (c 1.53 in $\left.\mathrm{CHCl}_{3}\right)>99 \%$ ee $(S)$; the stereochemistry was determined based on the reported literature ${ }^{[9]}$.

IR (neat) 3356, 3066, 3032, 1454, 1313, 1161, 1123, 1037, 767, 737, 700, $649 \mathrm{~cm}^{-1}$.

\section{(S)-2-(1'-Hydroxybenzyl)phenol (6e)}<smiles>Oc1ccccc1[C@@H](O)c1ccccc1</smiles>

According to the general procedure (ketone: $0.198 \mathrm{~g}(1 \mathrm{mmol})$, cat. $(\boldsymbol{R}, \boldsymbol{R})-4), 0.188 \mathrm{~g}$ of $\mathbf{6 e}$ 
was obtained as a clear oil ( $94 \%$ yield).

${ }^{1} \mathrm{H}$ NMR $\left(400 \mathrm{MHz}, \mathrm{CDCl}_{3}\right) \delta 7.96(\mathrm{br}, 1 \mathrm{H}), 7.35-7.26(\mathrm{~m}, 5 \mathrm{H}), 7.26-7.12(\mathrm{~m}, 1 \mathrm{H})$, 6.85-6.76 (m, 3H), $5.92(\mathrm{~s}, 1 \mathrm{H}) ;{ }^{13} \mathrm{C} \mathrm{NMR}\left(125 \mathrm{MHz}, \mathrm{CDCl}_{3}\right) \delta 155.2,141.8,129.2,128.6$, $128.2,128.1,126.8,126.7,120.0,117.1,76.7$. All characterization data are in agreement with the previously reported data ${ }^{[7]}$.

HRMS (ESI) calcd for $\mathrm{C}_{13} \mathrm{H}_{12} \mathrm{O}_{2}[\mathrm{M}-\mathrm{H}]^{-}:$199.0765. Found: 199.0766.

The enantiomeric excess was determined by HPLC analysis (Chiralcel OD-H, $250 \times 4.6$ $\mathrm{mm}$ column, hexane/2-propanol 80:20, $1.0 \mathrm{~mL} / \mathrm{min}, 220 \mathrm{~nm}, 30^{\circ} \mathrm{C},(R)$ isomer $6.0 \mathrm{~min},(S)$ isomer $8.0 \mathrm{~min}$ ); $[\alpha]_{\mathrm{D}}{ }^{20}-46.1\left(\mathrm{c} 1.40\right.$ in $\left.\mathrm{CHCl}_{3}\right) 77 \%$ ee $(R)\left(\right.$ lit. $^{[7]}[\alpha]_{\mathrm{D}}{ }^{25}-5.68$ (c 0.827 in $\left.\mathrm{CH}_{3} \mathrm{CN}\right) 99 \%$ ee).

IR (neat) 3347, 3062, 3032, 1587, 1489, 1456, 1014, $699 \mathrm{~cm}^{-1}$.

\section{(S)-(2,4-Dimethylphenyl)(phenyl)methanol (6f)}<smiles>Cc1ccc([C@@H](O)c2ccccc2)c(C)c1</smiles>

According to the general procedure (ketone: $0.210 \mathrm{~g}(1 \mathrm{mmol})$, cat. $(\boldsymbol{R}, \boldsymbol{R})-3), 0.199 \mathrm{~g}$ of $\mathbf{6 f}$ was obtained as a white solid (94\% yield).

${ }^{1} \mathrm{H}$ NMR (400 MHz, $\left.\mathrm{CDCl}_{3}\right) \delta$ 7.36-7.24 (m, 6H), 7.05-7.02 (m, 2H), 6.96 (s, 1H), 5.97 (s, $1 \mathrm{H}), 2.31(\mathrm{~s}, 3 \mathrm{H}), 2.22(\mathrm{~s}, 3 \mathrm{H}), 2.09(\mathrm{br}, 1 \mathrm{H}) ;{ }^{13} \mathrm{C} \mathrm{NMR}\left(125 \mathrm{MHz}, \mathrm{CDCl}_{3}\right) \delta 143.1,138.6$, $137.2,135.3,131.4,128.4,127.4,126.9,126.7,126.4,73.2,21.0,19.3$. All characterization data are in agreement with the previously reported data ${ }^{[3]}$.

HRMS (FI) calcd for $\mathrm{C}_{15} \mathrm{H}_{16} \mathrm{O}[\mathrm{M}]^{+}: 212.12011$. Found: 212.11955.

The enantiomeric excess was determined by HPLC analysis (Chiralcel OD-H, $250 \times 4.6$ $\mathrm{mm}$ column, hexane/2-propanol 97:3, $1.0 \mathrm{~mL} / \mathrm{min}, 220 \mathrm{~nm}, 30^{\circ} \mathrm{C},(R)$ isomer $13.3 \mathrm{~min},(S)$ isomer $15.9 \mathrm{~min}$ ); $[\alpha]_{\mathrm{D}}{ }^{20}-2.0$ (c 2.93 in $\left.\mathrm{CHCl}_{3}\right) 97 \%$ ee $(S)\left(\right.$ lit. $^{[3]}[\alpha]_{\mathrm{D}}{ }^{23}+8.9$ (c 0.80 in $\left.\mathrm{CHCl}_{3}\right) 82 \%$ ee $(R)$ ).

IR (neat) 3335, 3061, 3029, 2917, 1615, 1493, 1452, 1187, 1033, 1020, 800, 760, 699, 638 $\mathrm{cm}^{-1}$.

\section{(S)-Phenyl(2,4,5-trimethylphenyl)methanol (6g)}<smiles>Cc1cc(C)c([C@@H](O)c2ccccc2)cc1C</smiles>

According to the general procedure (ketone: $0.224 \mathrm{~g}(1 \mathrm{mmol})$, cat. $(\boldsymbol{R}, \boldsymbol{R})-\mathbf{2}), 0.222 \mathrm{~g}$ of $\mathbf{6 g}$ was obtained as a white solid ( $98 \%$ yield).

${ }^{1} \mathrm{H}$ NMR $\left(400 \mathrm{MHz}, \mathrm{CDCl}_{3}\right) \delta 7.35-7.20(\mathrm{~m}, 6 \mathrm{H}), 6.92(\mathrm{~s}, 1 \mathrm{H}), 5.96(\mathrm{~d}, J=2.8 \mathrm{~Hz}, 1 \mathrm{H})$, $2.23(\mathrm{~s}, 3 \mathrm{H}), 2.22(\mathrm{~s}, 3 \mathrm{H}), 2.19(\mathrm{~s}, 3 \mathrm{H}), 2.07(\mathrm{~d}, J=2.8 \mathrm{~Hz}, 1 \mathrm{H}) ;{ }^{13} \mathrm{C}$ NMR $(125 \mathrm{MHz}$, 
$\left.\mathrm{CDCl}_{3}\right) \delta 143.2,138.8,135.7,134.1,132.5,132.0,128.4,127.6,127.4,126.9,73.2,19.4$, 19.2, 18.7 .

HRMS (FI) calcd for $\mathrm{C}_{16} \mathrm{H}_{18} \mathrm{O}[\mathrm{M}]^{+}: 226.13576$. Found: 226.13621.

The enantiomeric excess was determined by HPLC analysis (Chiralcel OD-H, $250 \times 4.6$ $\mathrm{mm}$ column, hexane/2-propanol 97:3, $1.0 \mathrm{~mL} / \mathrm{min}, 220 \mathrm{~nm}, 30{ }^{\circ} \mathrm{C},(R)$ isomer $14.4 \mathrm{~min},(S)$ isomer $21.3 \mathrm{~min}$ ); $[\alpha]_{\mathrm{D}}{ }^{20}+24.7$ (c 1.02 in $\left.\mathrm{CHCl}_{3}\right) 98 \%$ ee $(S)$.

IR (neat) 3362, 3032, 2968, 2892, 1504, 1452, 1264, 1069, 1012, 869, 746, 703, $687 \mathrm{~cm}^{-1}$.

\section{$(S)-(2,4-D i c h l o r o p h e n y l)(p h e n y l) m e t h a n o l ~(6 h)$}<smiles>O[C@H](c1ccccc1)c1ccc(Cl)cc1Cl</smiles>

According to the general procedure (ketone: $0.251 \mathrm{~g}(1 \mathrm{mmol})$, cat. $(\boldsymbol{R}, \boldsymbol{R})-3), 0.246 \mathrm{~g}$ of $\mathbf{6 h}$ was obtained as a clear oil (97\% yield).

${ }^{1} \mathrm{H}$ NMR (400 MHz, $\left.\mathrm{CDCl}_{3}\right) \delta 7.57(\mathrm{~d}, J=8.4 \mathrm{~Hz}, 1 \mathrm{H}), 7.35-7.26(\mathrm{~m}, 7 \mathrm{H}), 6.15(\mathrm{~d}, J=3.2$ $\mathrm{Hz}, 1 \mathrm{H}), 2.37(\mathrm{~d}, J=3.2 \mathrm{~Hz}, 1 \mathrm{H}) ;{ }^{13} \mathrm{C} \mathrm{NMR}\left(125 \mathrm{MHz}, \mathrm{CDCl}_{3}\right) \delta 141.8,139.6,133.8$, $133.1,129.3,128.9,128.6,128.0,127.4,126.9,72.3$. All characterization data are in agreement with the previously reported data ${ }^{[6]}$.

HRMS (FI) calcd for $\mathrm{C}_{13} \mathrm{H}_{10} \mathrm{Cl}_{2} \mathrm{O}[\mathrm{M}]^{+}:$252.01087. Found: 252.01169.

The enantiomeric excess was determined by HPLC analysis (Chiralcel OD-H, $250 \times 4.6$ $\mathrm{mm}$ column, hexane/2-propanol 97:3, $1.0 \mathrm{~mL} / \mathrm{min}, 220 \mathrm{~nm}, 30^{\circ} \mathrm{C},(R)$ isomer $14.2 \mathrm{~min},(S)$ isomer $16.0 \mathrm{~min}$ ); $[\alpha]_{\mathrm{D}}{ }^{20}-2.82\left(\right.$ c $^{2} .75$ in $\left.\mathrm{CHCl}_{3}\right) 99 \%$ ee $(S)\left(\right.$ lit. $^{[6]}[\alpha]_{\mathrm{D}}{ }^{22}-15.4$ (c 0.17 in $\left.\mathrm{CHCl}_{3}\right) 93 \%$ ee $\left.(S)\right)$.

IR (neat) 3336, 3064, 3031, 1589, 1470, 1454, 1381, 1183, 1103, 1033, 1021, 865, 697, 668, $626 \mathrm{~cm}^{-1}$.

\section{(S)-(2-Chloro-5-nitrophenyl)(phenyl)methanol (6i)}<smiles>O=[N+]([O-])c1ccc(Cl)c([C@H](O)c2ccccc2)c1</smiles>

According to the general procedure (ketone: $0.262 \mathrm{~g}(1 \mathrm{mmol})$, cat. $(\boldsymbol{R}, \boldsymbol{R})-\mathbf{3}), 0.262 \mathrm{~g}$ of $\mathbf{6 i}$ was obtained as a clear oil $(>99 \%$ yield $)$.

${ }^{1} \mathrm{H} \mathrm{NMR}\left(400 \mathrm{MHz}, \mathrm{CDCl}_{3}\right) \delta 8.66(\mathrm{~d}, J=2.4 \mathrm{~Hz}, 1 \mathrm{H}), 8.10-8.07(\mathrm{~m}, 1 \mathrm{H}), 7.48(\mathrm{~d}, J=8.8$ $\mathrm{Hz}, 1 \mathrm{H}), 7.40-7.28(\mathrm{~m}, 5 \mathrm{H}), 6.19(\mathrm{~d}, J=3.2 \mathrm{~Hz}, 1 \mathrm{H}), 2.49(\mathrm{~d}, J=3.2 \mathrm{~Hz}, 1 \mathrm{H}) ;{ }^{13} \mathrm{C} \mathrm{NMR}$ $\left(125 \mathrm{MHz}, \mathrm{CDCl}_{3}\right) \delta 147.0,143.0,140.9,138.9,130.5,128.9,128.5,127.1,123.4,123.0$ 72.5 .

HRMS (FI) calcd for $\mathrm{C}_{13} \mathrm{H}_{10} \mathrm{NClNO}_{3}[\mathrm{M}]^{+}:$263.03492. Found: 263.03539. 
The enantiomeric excess was determined by HPLC analysis (Chiralcel OD-H, $250 \times 4.6$ $\mathrm{mm}$ column, hexane/2-propanol 97:3, $1.0 \mathrm{~mL} / \mathrm{min}, 220 \mathrm{~nm}, 30^{\circ} \mathrm{C},(R)$ isomer $23.3 \mathrm{~min},(S)$ isomer $25.9 \mathrm{~min}) ;[\alpha]_{\mathrm{D}}{ }^{20}+169.9\left(\mathrm{c} 1.53\right.$ in $\left.\mathrm{CHCl}_{3}\right)>99 \%$ ee $(S)$.

IR (neat) 3385, 3101, 1609, 1576, 1525, 1456, 1346, 1183, 1023, 918, 836, 768, 743, 699 $\mathrm{cm}^{-1}$.

\section{(S)-(2,5-Difluorophenyl)(phenyl)methanol (6j)}<smiles>OC(c1ccccc1)c1cc(F)ccc1F</smiles>

According to the general procedure (ketone: $0.218 \mathrm{~g}(1 \mathrm{mmol})$, cat. $(\boldsymbol{R}, \boldsymbol{R})-\mathbf{3}), 0.200 \mathrm{~g}$ of $\mathbf{6 j}$ was obtained as a clear oil (91\% yield).

${ }^{1} \mathrm{H}$ NMR $\left(400 \mathrm{MHz}, \mathrm{CDCl}_{3}\right) \delta$ 7.40-7.23 (m, 6H), 7.00-6.86 (m, 2H), $6.08(\mathrm{~d}, J=3.6 \mathrm{~Hz}$, $1 \mathrm{H}), 2.37(\mathrm{~d}, J=3.6 \mathrm{~Hz}, 1 \mathrm{H}) ;{ }^{13} \mathrm{C} \mathrm{NMR}\left(125 \mathrm{MHz}, \mathrm{CDCl}_{3}\right) \delta 158.9(\mathrm{~d}, J=240 \mathrm{~Hz}), 155.6$ $(\mathrm{d}, J=240 \mathrm{~Hz}), 142.2,132.7(\mathrm{dd}, J=15.6,8.0 \mathrm{~Hz}), 128.7,128.1,126.4,116.4(\mathrm{dd}, J=25.0$, $8.0 \mathrm{~Hz}), 115.3(\mathrm{dd}, J=25.0,8.0 \mathrm{~Hz}), 114.1(\mathrm{dd}, J=25.0,4.0 \mathrm{~Hz}), 69.8(\mathrm{~d}, J=2.0 \mathrm{~Hz})$. HRMS (FI) calcd for $\mathrm{C}_{13} \mathrm{H}_{10} \mathrm{~F}_{2} \mathrm{O}[\mathrm{M}]^{+}: 220.06997$. Found: 220.07077 .

The enantiomeric excess was determined by HPLC analysis (Chiralcel OJ-H, $250 \times 4.6 \mathrm{~mm}$ column, hexane/2-propanol 97:3, $1.0 \mathrm{~mL} / \mathrm{min}, 220 \mathrm{~nm}, 30^{\circ} \mathrm{C},(R)$ isomer $26.7 \mathrm{~min},(S)$ isomer $29.3 \mathrm{~min}) ;[\alpha]_{\mathrm{D}}{ }^{20}+29.6\left(\mathrm{c} 2.1\right.$ in $\left.\mathrm{CHCl}_{3}\right) 91 \%$ ee $(S)$.

IR (neat) 3354, 3065, 3032, 2916, 2848, 1491, 1429, 1241, 1181, 1134, 1035, 1022, 884, $834,818,769,699 \mathrm{~cm}^{-1}$.

\section{(S)-[2-Fluoro-3-(trifluoromethyl)phenyl](phenyl)methanol (6k)}<smiles>OC(c1ccccc1)c1cccc(C(F)(F)F)c1F</smiles>

According to the general procedure (ketone: $0.268 \mathrm{~g}(1 \mathrm{mmol})$, cat. $(\boldsymbol{R}, \boldsymbol{R})-\mathbf{3}), 0.268 \mathrm{~g}$ of $\mathbf{6 k}$ was obtained as a white solid ( $>99 \%$ ee).

${ }^{1} \mathrm{H}$ NMR $\left(400 \mathrm{MHz}, \mathrm{CDCl}_{3}\right) \delta 7.82-7.78(\mathrm{~m}, 1 \mathrm{H}), 7.58-7.50(\mathrm{~m}, 1 \mathrm{H}), 7.45-7.22(\mathrm{~m}, 6 \mathrm{H})$, $6.21(\mathrm{~d}, J=4.0 \mathrm{~Hz}, 1 \mathrm{H}), 2.36(\mathrm{~d}, J=4.0 \mathrm{~Hz}, 1 \mathrm{H}) ;{ }^{13} \mathrm{C} \mathrm{NMR}\left(125 \mathrm{MHz}, \mathrm{CDCl}_{3}\right) \delta 156.8(\mathrm{qd}$, $J=260.0,2.5 \mathrm{~Hz}) 142.0,132.6(\mathrm{~d}, J=12.5 \mathrm{~Hz}), 131.6(\mathrm{~d}, J=5.0 \mathrm{~Hz}), 128.8,128.2,126.3$, $126.3(\mathrm{q}, J=5.0 \mathrm{~Hz}), 124.1(\mathrm{~d}, J=3.8 \mathrm{~Hz}), 122.6(\mathrm{q}, J=270 \mathrm{~Hz}), 118.3(\mathrm{qd}, J=32.5,12.5$ $\mathrm{Hz}), 69.5$.

HRMS (FI) calcd for $\mathrm{C}_{14} \mathrm{H}_{10} \mathrm{~F}_{4} \mathrm{O}[\mathrm{M}]^{+}: 270.06678$. Found: 270.06633 .

The enantiomeric excess was determined by HPLC analysis (Chiralcel OD-H, $250 \times 4.6$ $\mathrm{mm}$ column, hexane/2-propanol 97:3, $1.0 \mathrm{~mL} / \mathrm{min}, 220 \mathrm{~nm}, 30^{\circ} \mathrm{C},(R)$ isomer $15.0 \mathrm{~min},(S)$ 
isomer $16.3 \mathrm{~min}) ;[\alpha]_{\mathrm{D}}^{20}+1.90$ (c 2.1 in $\left.\mathrm{CHCl}_{3}\right) 90 \%$ ee $(S)$.

IR (neat) 3236, 1623, 1594, 1467, 1326, 1227, 1144, 1110, 1023, 831, 793, 746, $696 \mathrm{~cm}^{-1}$.

\section{(S)-(Perfluorophenyl)(phenyl)methanol (61)}<smiles>O[C@H](c1ccccc1)c1c(F)c(F)c(F)c(F)c1F</smiles>

According to the general procedure (ketone: $0.272 \mathrm{~g}(1 \mathrm{mmol})$, cat. $(\boldsymbol{R}, \boldsymbol{R})-3), 0.272 \mathrm{~g}$ of $\mathbf{6} \mathbf{I}$ was obtained as a white solid ( $>99 \%$ yield).

${ }^{1} \mathrm{H}$ NMR $\left(400 \mathrm{MHz}, \mathrm{CDCl}_{3}\right) \delta 7.42-7.30(\mathrm{~m}, 5 \mathrm{H}), 6.24(\mathrm{~d}, J=7.2 \mathrm{~Hz}, 1 \mathrm{H}), 2.65(\mathrm{~d}, J=7.2$ $\mathrm{Hz}, 1 \mathrm{H}) ;{ }^{13} \mathrm{C} \mathrm{NMR}\left(125 \mathrm{MHz}, \mathrm{CDCl}_{3}\right) \delta 144.6(\mathrm{~m}), 140.8(\mathrm{~m}), 140.6,137.7(\mathrm{~m}), 128.8$, $128.3,125.4(\mathrm{~m}), 117.0(\mathrm{~m}), 67.6$. All characterization data are in agreement with the previously reported data ${ }^{[7]}$.

HRMS (FI) calcd for $\mathrm{C}_{13} \mathrm{H}_{7} \mathrm{~F}_{5} \mathrm{O}[\mathrm{M}]^{+}:$274.04171. Found: 274.04238 .

The enantiomeric excess was determined by HPLC analysis (Chiralcel OD-H, $250 \times 4.6$ $\mathrm{mm}$ column, hexane/2-propanol 97:3, $1.0 \mathrm{~mL} / \mathrm{min}, 254 \mathrm{~nm}, 30{ }^{\circ} \mathrm{C},(R)$ isomer $9.8 \mathrm{~min},(S)$ isomer $12.1 \mathrm{~min}$ ); $[\alpha]_{\mathrm{D}}{ }^{20}-45.0\left(\mathrm{c} 1.90\right.$ in $\left.\mathrm{CHCl}_{3}\right)>99 \%$ ee $(S)\left(\right.$ lit. $^{[7]}[\alpha]_{\mathrm{D}}{ }^{20}+42.0$ (c 1.224 in $\left.\mathrm{CHCl}_{3}\right) 70 \%$ ee $\left.(R)\right)$.

IR (neat) 3275, 1654, 1522, 1505, 1304, 1121, 995, 948, 699, $644 \mathrm{~cm}^{-1}$.

\section{(S)-(2-Chlorophenyl)(4-chlorophenyl)methanol (6m)}<smiles>O[C@H](c1ccc(Cl)cc1)c1ccccc1Cl</smiles>

According to the general procedure (ketone: $0.251 \mathrm{~g}(1 \mathrm{mmol})$, cat. $(\boldsymbol{R}, \boldsymbol{R})-\mathbf{3}), 0.251 \mathrm{~g}$ of $\mathbf{6 m}$ was obtained as a clear oil ( $>99 \%$ yield).

${ }^{1} \mathrm{H}$ NMR $\left(400 \mathrm{MHz}, \mathrm{CDCl}_{3}\right) \delta$ 7.56-7.54 (m, 1H), 7.36-7.22 (m, 7H), $6.19(\mathrm{~d}, J=3.6 \mathrm{~Hz}$, $1 \mathrm{H}), 2.40(\mathrm{~d}, J=3.6 \mathrm{~Hz}, 1 \mathrm{H}) ;{ }^{13} \mathrm{C}$ NMR $\left(125 \mathrm{MHz}, \mathrm{CDCl}_{3}\right) \delta 140.7,140.6,133.5,132.4$, 129.6, 129.0, 128.6, 128.3, 127.9, 127.2, 72.0; All characterization data are in agreement with the previously reported data ${ }^{[4]}$.

HRMS (FI) calcd for $\mathrm{C}_{13} \mathrm{H}_{10} \mathrm{NCl}_{2} \mathrm{O}[\mathrm{M}]^{+}: 252.01087$. Found: 252.01037.

The enantiomeric excess was determined by HPLC analysis (Chiralcel OD-H, $250 \times 4.6$ $\mathrm{mm}$ column, hexane/2-propanol 97:3, $1.0 \mathrm{~mL} / \mathrm{min}, 220 \mathrm{~nm}, 30^{\circ} \mathrm{C},(R)$ isomer $15.6 \mathrm{~min},(S)$ isomer $23.6 \mathrm{~min})$; $[\alpha]_{\mathrm{D}}{ }^{20}-42.1\left(\mathrm{c} 1.55\right.$ in $\left.\mathrm{CHCl}_{3}\right) 97 \%$ ee $(S)\left(\right.$ lit. $^{[4]}[\alpha]_{\mathrm{D}}{ }^{23}+40.0$ (c 1.04 in $\left.\mathrm{CHCl}_{3}\right) 96 \%$ ee $\left.(R)\right)$.

IR (neat) 3370, 1489, 1438, 1183, 1091, 1056, 1014, 798, $668 \mathrm{~cm}^{-1}$. 


\section{(S)-(2-Chlorophenyl)(4-fluorophenyl)methanol (6n)}<smiles>O[C@H](c1ccc(F)cc1)c1ccccc1Cl</smiles>

According to the general procedure (ketone: $0.235 \mathrm{~g}(1 \mathrm{mmol})$, cat. $(\boldsymbol{R}, \boldsymbol{R})-3), 0.233 \mathrm{~g}$ of $\mathbf{6 n}$ was obtained as a clear oil (99\% yield).

${ }^{1} \mathrm{H}$ NMR $\left(400 \mathrm{MHz}, \mathrm{CDCl}_{3}\right) \delta$ 7.60-7.57 (m, 1H), 7.40-7.20 (m, 5H), 7.03-6.95 (m, 2H), $6.19(\mathrm{~d}, J=3.2 \mathrm{~Hz}, 1 \mathrm{H}), 2.39(\mathrm{~d}, J=3.2 \mathrm{~Hz}, 1 \mathrm{H}) ;{ }^{13} \mathrm{C} \mathrm{NMR}\left(125 \mathrm{MHz}, \mathrm{CDCl}_{3}\right) \delta 162.2(\mathrm{~d}$, $J=245.0 \mathrm{~Hz}), 140.8,138.0(\mathrm{~d}, J=3.8 \mathrm{~Hz}), 132.4,129.6,128.9,128.6(\mathrm{~d}, J=8.8 \mathrm{~Hz})$, $127.8,127.2,115.3(\mathrm{~d}, J=21.3 \mathrm{~Hz}), 72.1$. All characterization data are in agreement with the previously reported data ${ }^{[5]}$.

HRMS (FI) calcd for $\mathrm{C}_{13} \mathrm{H}_{10} \mathrm{NClFO}[\mathrm{M}]^{+}:$236.04042. Found: 1236.04013.

The enantiomeric excess was determined by HPLC analysis (Chiralcel OD-H, $250 \times 4.6$ $\mathrm{mm}$ column, hexane/2-propanol 97:3, $1.0 \mathrm{~mL} / \mathrm{min}, 220 \mathrm{~nm}, 30^{\circ} \mathrm{C},(R)$ isomer $12.7 \mathrm{~min},(S)$ isomer $18.3 \mathrm{~min}$ ); $[\alpha]_{\mathrm{D}}{ }^{20}-15.2$ (c 1.51 in $\left.\mathrm{CHCl}_{3}\right) 97 \%$ ee $(S)\left(\right.$ lit. $^{[5]}[\alpha]_{\mathrm{D}}{ }^{20}+9.9$ (c 0.82 in $\left.\mathrm{CHCl}_{3}\right) 83 \%$ ee $\left.(R)\right)$.

IR (neat) 3351, 1604, 1509, 1471, 1441, 1158, 1056, 1023, 842, 813, $668 \mathrm{~cm}^{-1}$.

\section{(S)-(4-Chlorophenyl)(phenyl)methanol (11a)}<smiles>OC(c1ccccc1)c1ccc(Cl)cc1</smiles>

According to the general procedure (ketone: $0.217 \mathrm{~g}(1 \mathrm{mmol})$, cat. $(\boldsymbol{R}, \boldsymbol{R})-\mathbf{3}), 0.217 \mathrm{~g}$ of $\mathbf{1 1 a}$ was obtained as a white solid ( $>99 \%$ yield).

${ }^{1} \mathrm{H}$ NMR (400 MHz, $\left.\mathrm{CDCl}_{3}\right) \delta 7.34-7.27(\mathrm{~m}, 9 \mathrm{H}), 5.79(\mathrm{~d}, J=3.2 \mathrm{~Hz}, 1 \mathrm{H}), 2.28(\mathrm{~d}, J=3.2$ $\mathrm{Hz}, 1 \mathrm{H}) ;{ }^{13} \mathrm{C} \mathrm{NMR}\left(125 \mathrm{MHz}, \mathrm{CDCl}_{3}\right) \delta 143.4,142.2,133.3,128.6,128.6,127.9,126.5$, 75.6. All characterization data are in agreement with the previously reported data ${ }^{[2]}$.

HRMS (FI) calcd for $\mathrm{C}_{13} \mathrm{H}_{11} \mathrm{ClO}[\mathrm{M}]^{+}: 218.04984$. Found: 218.05004 .

The enantiomeric excess was determined by HPLC analysis (Chiralpak AD-H, $250 \times 4.6$ $\mathrm{mm}$ column, hexane/2-propanol 97:3, $1.0 \mathrm{~mL} / \mathrm{min}, 220 \mathrm{~nm}, 30^{\circ} \mathrm{C},(R)$ isomer $18.5 \mathrm{~min},(S)$ isomer $20.5 \mathrm{~min})$; $[\alpha]_{\mathrm{D}}{ }^{20}+8.0\left(\mathrm{c} 1.51\right.$ in $\left.\mathrm{CHCl}_{3}\right) 48 \%$ ee $(S)\left(\right.$ lit. $^{[2]}[\alpha]_{\mathrm{D}}{ }^{\mathrm{RT}}+2.77$ (c 0.932 in $\left.\mathrm{CHCl}_{3}\right) 9 \%$ ee $\left.(S)\right)$.

IR (neat) 3370, 3030, 1488, 1454, 1407, 1185, 1090, 1013, 795, 701, $668 \mathrm{~cm}^{-1}$.

\section{(S)-(3,4-Dichlorophenyl)(phenyl)methanol (11b)}


<smiles>O[C@H](c1ccccc1)c1ccc(Cl)c(Cl)c1</smiles>

According to the general procedure (ketone: $0.251 \mathrm{~g}(1 \mathrm{mmol})$, cat. $(\boldsymbol{R}, \boldsymbol{R})-3), 0.251 \mathrm{~g}$ of 11 b was obtained as a clear oil ( $>99 \%$ yield).

${ }^{1} \mathrm{H}$ NMR $\left(400 \mathrm{MHz}, \mathrm{CDCl}_{3}\right) \delta$ 7.48-7.47 (m, 1H), 7.40-7.13 (m, 7H), $5.72(\mathrm{~d}, J=3.2 \mathrm{~Hz}$, $1 \mathrm{H}), 2.46(\mathrm{~d}, J=3.2 \mathrm{~Hz}, 1 \mathrm{H}) ;{ }^{13} \mathrm{C} \mathrm{NMR}\left(125 \mathrm{MHz}, \mathrm{CDCl}_{3}\right) \delta 143.8,142.8,132.5,131.4$, $130.3,128.8,128.3,128.1,126.5,125.8,75.1$.

HRMS (FI) calcd for $\mathrm{C}_{13} \mathrm{H}_{10} \mathrm{Cl}_{2} \mathrm{O}[\mathrm{M}]^{+}: 252.01087$. Found: 252.00984 .

The enantiomeric excess was determined by HPLC analysis (Chiralcel OJ-H, $250 \times 4.6 \mathrm{~mm}$ column, hexane/2-propanol 90:10, $1.0 \mathrm{~mL} / \mathrm{min}, 220 \mathrm{~nm}, 30{ }^{\circ} \mathrm{C},(R)$ isomer $10.1 \mathrm{~min},(S)$ isomer $11.2 \mathrm{~min}) ;[\alpha]_{\mathrm{D}}{ }^{20}+31.2\left(\mathrm{c} 1.70\right.$ in $\left.\mathrm{CHCl}_{3}\right) 76 \%$ ee $(S)$.

IR (neat) 3311, 3220, 1495, 1458, 1398, 1269, 1029, 896, 812, 704, $636 \mathrm{~cm}^{-1}$.

\section{(S)-(3,4-Difluorophenyl)(phenyl)methanol (11c)}<smiles>O[C@H](c1ccccc1)c1ccc(F)c(F)c1</smiles>

According to the general procedure (ketone: $0.218 \mathrm{~g}(1 \mathrm{mmol})$, cat. $(\boldsymbol{R}, \boldsymbol{R})-3), 0.218 \mathrm{~g}$ of $11 \mathrm{c}$ was obtained as a clear oil ( $>99 \%$ yield).

${ }^{1} \mathrm{H}$ NMR (400 MHz, $\left.\mathrm{CDCl}_{3}\right) \delta$ 7.40-7.05 (m, 8H), $5.77(\mathrm{~d}, J=3.2 \mathrm{~Hz}, 1 \mathrm{H}), 2.33(\mathrm{~d}, J=3.2$ $\mathrm{Hz}, 1 \mathrm{H}) ;{ }^{13} \mathrm{C} \mathrm{NMR}\left(125 \mathrm{MHz}, \mathrm{CDCl}_{3}\right) \delta 150.9(\mathrm{dd}, J=12.5,8.5 \mathrm{~Hz}), 149.0(\mathrm{dd}, J=12.5$, $8.5 \mathrm{~Hz}), 143.1,140.7$ (t, $J=3.8 \mathrm{~Hz}), 128.7,128.1,126.5,122.3(\mathrm{dd}, J=6.2,3.8 \mathrm{~Hz}), 117.1$ $(\mathrm{d}, J=17.5 \mathrm{~Hz}), 115.5(\mathrm{~d}, J=17.5 \mathrm{~Hz}), 75.2$.

HRMS (FI) calcd for $\mathrm{C}_{13} \mathrm{H}_{10} \mathrm{~F}_{2} \mathrm{O}[\mathrm{M}]^{+}: 220.06997$. Found: 220.07028 .

The enantiomeric excess was determined by HPLC analysis (Chiralcel OD-H, $250 \times 4.6$ $\mathrm{mm}$ column, hexane/2-propanol 97:3, $1.0 \mathrm{~mL} / \mathrm{min}, 220 \mathrm{~nm}, 30^{\circ} \mathrm{C},(R)$ isomer $22.8 \mathrm{~min},(S)$ isomer $26.7 \mathrm{~min}) ;[\alpha]_{\mathrm{D}}^{20}+20.7\left(\mathrm{c} 1.29\right.$ in $\left.\mathrm{CHCl}_{3}\right) 77 \%$ ee $(S)$.

IR (neat) 3514, 2961, 2886, 1724, 1287, 1186, 1069, 1036, 957, 931,851, $754 \mathrm{~cm}^{-1}$.

\section{(S)-(4-Nitrophenyl)(phenyl)methanol (11d)}<smiles>O=[N+]([O-])c1ccc([C@H](O)c2ccccc2)cc1</smiles>

According to the general procedure (ketone: $0.227 \mathrm{~g}(1 \mathrm{mmol})$, cat. $(\boldsymbol{R}, \boldsymbol{R})-3), 0.227 \mathrm{~g}$ of 11d was obtained as a pale yellow solid ( $>99 \%$ yield). 
${ }^{1} \mathrm{H}$ NMR $\left(400 \mathrm{MHz}, \mathrm{CDCl}_{3}\right) \delta 8.19-8.16(\mathrm{~m}, 2 \mathrm{H}), 7.58-7.55(\mathrm{~m}, 2 \mathrm{H}), 7.40-7.30(\mathrm{~m}, 5 \mathrm{H})$, $5.91(\mathrm{~d}, J=2.8 \mathrm{~Hz}, 1 \mathrm{H}), 2.47(\mathrm{~d}, J=2.8 \mathrm{~Hz}, 1 \mathrm{H}) ;{ }^{13} \mathrm{C} \mathrm{NMR}\left(125 \mathrm{MHz}, \mathrm{CDCl}_{3}\right) \delta 150.7$, $147.2,142.7,128.9,128.4,127.0,126.7,123.7,75.5$. All characterization data are in agreement with the previously reported data ${ }^{[6]}$.

HRMS (FI) calcd for $\mathrm{C}_{13} \mathrm{H}_{11} \mathrm{NO}_{3}[\mathrm{M}]^{+}:$229.07389. Found: 229.07343.

The enantiomeric excess was determined by HPLC analysis (Chiralpak AD-H, $250 \times 4.6$ $\mathrm{mm}$ column, hexane/2-propanol $90: 10,1.0 \mathrm{~mL} / \mathrm{min}, 220 \mathrm{~nm}, 30^{\circ} \mathrm{C},(R)$ isomer $12.3 \mathrm{~min}$, $(S)$ isomer $15.4 \mathrm{~min}) ;[\alpha]_{\mathrm{D}}{ }^{20}+51.5\left(\right.$ c 1.57 in $\left.\mathrm{CHCl}_{3}\right) 76 \%$ ee $(S)\left(\right.$ lit. $^{[6]}[\alpha]_{\mathrm{D}}{ }^{22}+71.0($ c 0.27 in $\left.\mathrm{CHCl}_{3}\right) 92 \%$ ee $\left.(S)\right)$.

IR (neat) 3466, 1595, 1515, 1450, 1345, 1190, 1055, 867, 813, 754, 745, 708, $692 \mathrm{~cm}^{-1}$.

\section{(S)-(4-Chloro-3-nitrophenyl)(phenyl)methanol (11e)}<smiles>O=[N+]([O-])c1cc([C@@H](O)c2ccccc2)ccc1Cl</smiles>

According to the general procedure (ketone: $0.261 \mathrm{~g}(1 \mathrm{mmol})$, cat. $(\boldsymbol{R}, \boldsymbol{R})-3), 0.262 \mathrm{~g}$ of $11 \mathrm{e}$ was obtained as a pale yellow solid ( $>99 \%$ yield).

${ }^{1} \mathrm{H}$ NMR $\left(400 \mathrm{MHz}, \mathrm{CDCl}_{3}\right) \delta 7.94(\mathrm{~d}, J=1.6 \mathrm{~Hz}, 1 \mathrm{H}), 7.53-7.48(\mathrm{~m}, 2 \mathrm{H}), 7.40-7.30(\mathrm{~m}$, 5H), $5.86(\mathrm{~s}, 1 \mathrm{H}), 2.37(\mathrm{br}, 1 \mathrm{H}) ;{ }^{13} \mathrm{C} \mathrm{NMR}\left(125 \mathrm{MHz}, \mathrm{CDCl}_{3}\right) \delta 147.5,144.1,142.3,131.8$, 131.0, 129.1, 128.6, 126.7, 125.7, 123.3, 74.8.

HRMS (FI) calcd for $\mathrm{C}_{13} \mathrm{H}_{10} \mathrm{ClNO}_{3}[\mathrm{M}]^{+}:$263.03492. Found: 263.03518 .

The enantiomeric excess was determined by HPLC analysis (Chiralcel OJ-H, $250 \times 4.6 \mathrm{~mm}$ column, hexane/2-propanol 90:10, $1.0 \mathrm{~mL} / \mathrm{min}, 220 \mathrm{~nm}, 30{ }^{\circ} \mathrm{C},(R)$ isomer $22.7 \mathrm{~min},(S)$ isomer $27.1 \mathrm{~min}) ;[\alpha]_{\mathrm{D}}{ }^{20}+52.5\left(\mathrm{c} 1.91\right.$ in $\left.\mathrm{CHCl}_{3}\right) 96 \%$ ee $(S)$.

IR (neat) 3578, 3428, 1530, 1454, 1350, 1191, 1048, 1024, 827, 768, $715 \mathrm{~cm}^{-1}$.

\section{(S)-Phenyl(3,4,5-trifluorophenyl)methanol (11f)}<smiles>O[C@H](c1ccccc1)c1cc(F)c(F)c(F)c1</smiles>

According to the general procedure (ketone: $0.236 \mathrm{~g}(1 \mathrm{mmol})$, cat. $(\boldsymbol{R}, \boldsymbol{R})-\mathbf{3}), 0.236 \mathrm{~g}$ of $\mathbf{1 1 f}$ was obtained as a clear oil $(>99 \%$ yield).

${ }^{1} \mathrm{H}$ NMR (400 MHz, $\left.\mathrm{CDCl}_{3}\right) \delta$ 7.37-7.27 (m, 5H), 7.00-6.94 (m, 2H), $5.68(\mathrm{~d}, J=2.8 \mathrm{~Hz}$, $1 \mathrm{H}), 2.51(\mathrm{~d}, J=2.8 \mathrm{~Hz}, 1 \mathrm{H}) ;{ }^{13} \mathrm{C} \mathrm{NMR}\left(125 \mathrm{MHz}, \mathrm{CDCl}_{3}\right) \delta 151.1(\mathrm{dd}, J=10.0,3.8 \mathrm{~Hz})$, , 142.5, 139.9 (m), 137.8 (m), 128.9, 128.4, 126.5, 110.3 (dd, $J=17.5,5.0 \mathrm{~Hz}), 74.9$.

HRMS (FI) calcd for $\mathrm{C}_{13} \mathrm{H}_{9} \mathrm{~F}_{3} \mathrm{O}[\mathrm{M}]^{+}:$238.0606. Found: 238.0617 .

The enantiomeric excess was determined by HPLC analysis (Chiralcel OD-H, $250 \times 4.6$ 
$\mathrm{mm}$ column, hexane/2-propanol 95:5, $1.0 \mathrm{~mL} / \mathrm{min}, 220 \mathrm{~nm}, 30^{\circ} \mathrm{C},(R)$ isomer $16.1 \mathrm{~min},(S)$ isomer $21.2 \mathrm{~min})$; $[\alpha]_{\mathrm{D}}{ }^{20}+52.1\left(\mathrm{c} 1.46\right.$ in $\left.\mathrm{CHCl}_{3}\right) 95 \%$ ee $(S)$.

IR (neat) 3376, 2978, 2876, 1622, 1528, 1447, 1343, 1234, 1036, 758, 704, $613 \mathrm{~cm}^{-1}$.

\section{(S)-(3,5-Dinitrophenyl)(phenyl)methanol (11g)}<smiles>O=[N+]([O-])c1cc([C@H](O)c2ccccc2)cc([N+](=O)[O-])c1</smiles>

According to the general procedure (ketone: $0.136 \mathrm{~g}(0.5 \mathrm{mmol})$, cat. $(\boldsymbol{R}, \boldsymbol{R})-3), 0.123 \mathrm{~g}$ of $11 \mathrm{~g}$ was obtained as a yellow oil (90\% yield).

${ }^{1} \mathrm{H}$ NMR (400 MHz, $\left.\mathrm{CDCl}_{3}\right) \delta 8.94-8.92(\mathrm{~m}, 1 \mathrm{H}), 8.61-8.60(\mathrm{~m}, 1 \mathrm{H}), 7.43-7.35(\mathrm{~m}, 5 \mathrm{H})$, $6.00(\mathrm{~s}, 1 \mathrm{H}), 2.66(\mathrm{br}, 1 \mathrm{H}) ;{ }^{13} \mathrm{C}$ NMR $\left(125 \mathrm{MHz}, \mathrm{CDCl}_{3}\right) \delta 148.6,148.3,141.7,129.4$, 129.1, 126.7, 126.4, 117.7, 74.9.

HRMS (FI) calcd for $\mathrm{C}_{13} \mathrm{H}_{10} \mathrm{~N}_{2} \mathrm{O}_{5}[\mathrm{M}]^{+}:$274.0590. Found: 274.0596.

The enantiomeric excess was determined by HPLC analysis (Chiralpak AS-H, $250 \times 4.6$ $\mathrm{mm}$ column, hexane/2-propanol $90: 10,1.0 \mathrm{~mL} / \mathrm{min}, 220 \mathrm{~nm}, 30{ }^{\circ} \mathrm{C},(S)$ isomer $17.2 \mathrm{~min}$, $(R)$ isomer $18.6 \mathrm{~min}) ;[\alpha]_{\mathrm{D}}{ }^{20}+72.8$ (c 1.15 in $\left.\mathrm{CHCl}_{3}\right) 96 \%$ ee $(S)$.

IR (neat) 3351, 3106, 1597, 1560, 1541, 1450, 1348, 1266, 1041, 912, 752, 728, 703, 679 $\mathrm{cm}^{-1}$.

\section{(R)-(4-Methoxyphenyl)(phenyl)methanol (11h)}<smiles>COc1ccc([C@@H](O)c2ccccc2)cc1</smiles>

According to the general procedure (ketone: $0.212 \mathrm{~g}(1 \mathrm{mmol})$, cat. $(\boldsymbol{R}, \boldsymbol{R})-3), 0.171 \mathrm{~g}$ of 11h was obtained as a white solid ( $80 \%$ yield).

${ }^{1} \mathrm{H}$ NMR (400 MHz, $\left.\mathrm{CDCl}_{3}\right) \delta$ 7.40-7.25 (m, 7H), 6.87-6.80 (m, 2H), $5.80(\mathrm{~s}, 1 \mathrm{H}), 3.78(\mathrm{~s}$, $3 \mathrm{H}), 2.18$ (br, 1H); ${ }^{13} \mathrm{C}$ NMR $\left(125 \mathrm{MHz}, \mathrm{CDCl}_{3}\right) \delta$ 159.1, 144.0, 136.2, 128.4, 127.9, 127.4, $126.4,113.9,55.3$. All characterization data are in agreement with the previously reported $\operatorname{data}^{[3]}$.

HRMS (FI) calcd for $\mathrm{C}_{14} \mathrm{H}_{14} \mathrm{O}_{2}[\mathrm{M}]^{+}:$214.09938. Found: 214.09995.

The enantiomeric excess was determined by HPLC analysis (Chiralpak AD-H, $250 \times 4.6$ $\mathrm{mm}$ column, hexane/2-propanol $90: 10,0.5 \mathrm{~mL} / \mathrm{min}, 220 \mathrm{~nm}, 30{ }^{\circ} \mathrm{C},(R)$ isomer $22.5 \mathrm{~min}$, $(S)$ isomer $24.3 \mathrm{~min}) ;[\alpha]_{\mathrm{D}}{ }^{20}+1.50\left(\mathrm{c} 1.08\right.$ in $\left.\mathrm{CHCl}_{3}\right) 5 \%$ ee $(R)\left(\right.$ lit. $^{[3]}[\alpha]_{\mathrm{D}}{ }^{29}+24.6(\mathrm{c} 0.80$ in $\left.\mathrm{CHCl}_{3}\right) 90 \%$ ee $(R)$ ).

IR (neat) 3403, 2952, 2837, 1611, 1588, 1516, 1495, 1446, 1305, 1252, 1178, 1034, 1019, $841,811,727,697,655,624 \mathrm{~cm}^{-1}$. 
(S)-(4-Chlorophenyl)(4-methoxyphenyl)methanol (11i)<smiles>COc1ccc([C@@H](O)c2ccc(Cl)cc2)cc1</smiles>

According to the general procedure (ketone: $0.123 \mathrm{~g}(0.5 \mathrm{mmol})$, cat. $(\boldsymbol{R}, \boldsymbol{R})-3), 0.073 \mathrm{~g}$ of $11 \mathrm{i}$ was obtained as a white solid (59\% yield).

${ }^{1} \mathrm{H}$ NMR (400 MHz, $\left.\mathrm{CDCl}_{3}\right) \delta$ 7.31-7.29 (m, 4H), 7.25-7.23 (m, 2H), 6.87-6.85 (m, 2H), $5.76(\mathrm{~d}, J=2.8 \mathrm{~Hz}, 1 \mathrm{H}), 3.79(\mathrm{~s}, 3 \mathrm{H}), 2.20(\mathrm{~d}, J=2.8 \mathrm{~Hz}, 1 \mathrm{H}) ;{ }^{13} \mathrm{C}$ NMR $(125 \mathrm{MHz}$, $\left.\mathrm{CDCl}_{3}\right) \delta 159.2,142.4,135.8,133.1,128.5,127.9,127.7,114.0,75.2,55.3$. All characterization data are in agreement with the previously reported data $^{[3]}$.

HRMS (FI) calcd for $\mathrm{C}_{14} \mathrm{H}_{13} \mathrm{ClO}_{2}[\mathrm{M}]^{+}:$248.06041. Found: 248.06039 .

The enantiomeric excess was determined by HPLC analysis (Chiralcel OD-H, $250 \times 4.6$ $\mathrm{mm}$ column, hexane/2-propanol 95:5, $0.5 \mathrm{~mL} / \mathrm{min}, 220 \mathrm{~nm}, 30^{\circ} \mathrm{C},(S)$ isomer $34.0 \mathrm{~min},(R)$ isomer $36.3 \mathrm{~min}$ ); $[\alpha]_{\mathrm{D}}{ }^{20}+16.6\left(\mathrm{c} 0.73\right.$ in $\left.\mathrm{CHCl}_{3}\right) 53 \%$ ee $(S)\left(\right.$ lit. $^{[3]}[\alpha]_{\mathrm{D}}{ }^{27}+36.6$ (c 0.80 in $\left.\mathrm{CHCl}_{3}\right) 89 \%$ ee $\left.(S)\right)$.

IR (neat) 3315, 1611, 1513, 1488, 1253, 1174, 1091, 1035, 1008, 859, 806, $773 \mathrm{~cm}^{-1}$.

\section{(S)-4-[(4-Chlorophenyl)hydroxymethyl]phenol (11j)}<smiles>Oc1ccc([C@@H](O)c2ccc(Cl)cc2)cc1</smiles>

According to the general procedure (ketone: $0.116 \mathrm{~g}(0.5 \mathrm{mmol})$, cat. $(\boldsymbol{R}, \boldsymbol{R})-4), 0.116 \mathrm{~g}$ of 11j was obtained as a white solid ( $>99 \%$ yield).

${ }^{1} \mathrm{H}$ NMR (400 MHz, CD $\left.3 \mathrm{OD}\right) \delta$ 7.33-7.27 (m, 4H), 7.15-7.12 (m, 2H), 6.74-6.72 (m, 2H), $5.67(\mathrm{~S}, 1 \mathrm{H}), 4.86(\mathrm{br}, 1 \mathrm{H}) ;{ }^{13} \mathrm{C} \mathrm{NMR}\left(125 \mathrm{MHz}, \mathrm{CDCl}_{3}\right) \delta 157.9,145.1,136.5,133.6$, 129.2, 129.1, 116.1, 75.9, 49.0 .

HRMS (FI) calcd for $\mathrm{C}_{13} \mathrm{H}_{11} \mathrm{NClO}_{2}[\mathrm{M}]^{+}:$234.0448. Found: 234.0454 .

The enantiomeric excess was determined by HPLC analysis (Chiralcel OD-H, $250 \times 4.6$ $\mathrm{mm}$ column, hexane/2-propanol $90: 10,1.0 \mathrm{~mL} / \mathrm{min}, 220 \mathrm{~nm}, 30{ }^{\circ} \mathrm{C},(S)$ isomer $16.3 \mathrm{~min}$, $(R)$ isomer $18.8 \mathrm{~min})$; $[\alpha]_{\mathrm{D}}{ }^{20}+23.2(\mathrm{c} 0.97$ in $\mathrm{MeOH}) 55 \%$ ee $(S)$. Absolute configuration was determined by HPLC analysis of a demethylated compound derived from $(S)$-11i.

IR (neat) 3384, 3142, 1614, 1598, 1513, 1489, 1455, 1372, 1242, 1172, 1093, 1004, 832, $817 \mathrm{~cm}^{-1}$.

\section{(R)-(4-Methoxyphenyl)(4-nitrophenyl)methanol (11k)}


<smiles>COc1ccc([C@@H](O)c2ccc([N+](=O)[O-])cc2)cc1</smiles>

According to the general procedure (ketone: $0.257 \mathrm{~g}(1 \mathrm{mmol})$, cat. $(\boldsymbol{R}, \boldsymbol{R})-3), 0.257 \mathrm{~g}$ of $11 \mathbf{k}$ was obtained as a pale yellow oil ( $>99 \%$ yield).

${ }^{1} \mathrm{H}$ NMR $\left(400 \mathrm{MHz}, \mathrm{CDCl}_{3}\right) \delta 8.15(\mathrm{~d}, J=8.8 \mathrm{~Hz}, 2 \mathrm{H}), 7.54(\mathrm{~d}, J=8.8 \mathrm{~Hz}, 2 \mathrm{H}), 7.23(\mathrm{~d}, J$ $=8.4 \mathrm{~Hz}, 2 \mathrm{H}), 6.86(\mathrm{~d}, J=8.4 \mathrm{~Hz}, 2 \mathrm{H}), 5.85(\mathrm{~s}, 1 \mathrm{H}), 3.78(\mathrm{~s}, 3 \mathrm{H}), 2.50(\mathrm{br}, 1 \mathrm{H}) ;{ }^{13} \mathrm{C} \mathrm{NMR}$ $\left(125 \mathrm{MHz} \mathrm{CDCl}_{3}\right) \delta 159.5,151.1,147.0,135.0,128.1,126.9,123.6,114.2,75.0,55.3$.

HRMS (FI) calcd for $\mathrm{C}_{14} \mathrm{H}_{13} \mathrm{NO}_{4}[\mathrm{M}]^{+}: 259.08446$. Found: 259.0853 .

The enantiomeric excess was determined by HPLC analysis (Chiralpak AD-H, $250 \times 4.6$ $\mathrm{mm}$ column, hexane/2-propanol $90: 10,1.0 \mathrm{~mL} / \mathrm{min}, 220 \mathrm{~nm}, 30{ }^{\circ} \mathrm{C},(S)$ isomer $19.5 \mathrm{~min}$, $(R)$ isomer $23.9 \mathrm{~min}) ;[\alpha]_{\mathrm{D}}{ }^{20}+43.1\left(\mathrm{c} 1.14\right.$ in $\left.\mathrm{CHCl}_{3}\right) 79 \%$ ee $(R)$.

IR (neat) 3454, 1068, 1513, 1463, 1347, 1249, 1173, 1109, 1032, 834, 804, $739 \mathrm{~cm}^{-1}$.

\section{(S)-(3,5-Dinitrophenyl)(4-methoxyphenyl)methanol (111)}<smiles>COc1ccc([C@H](O)c2cc([N+](=O)[O-])cc([N+](=O)[O-])c2)cc1</smiles>

According to the general procedure (ketone: $0.152 \mathrm{~g}(0.5 \mathrm{mmol})$, cat. $(\boldsymbol{R}, \boldsymbol{R})-3), 0.141 \mathrm{~g}$ of 111 was obtained as a yellow liquid (92\% yield).

${ }^{1} \mathrm{H}$ NMR $\left(400 \mathrm{MHz}, \mathrm{CDCl}_{3}\right) \delta 8.91-8.89(\mathrm{~m}, 1 \mathrm{H}), 8.58-8.57(\mathrm{~m}, 1 \mathrm{H}), 7.26(\mathrm{~d}, J=8.4 \mathrm{~Hz}$, $2 \mathrm{H}), 6.90(\mathrm{~d}, J=8.4 \mathrm{~Hz}, 1 \mathrm{H}), 5.94(\mathrm{~s}, 1 \mathrm{H}), 3.80(\mathrm{~s}, 3 \mathrm{H}), 2.80(\mathrm{br}, 1 \mathrm{H}) ;{ }^{13} \mathrm{C} \mathrm{NMR}(125 \mathrm{MHz}$, $\left.\mathrm{CDCl}_{3}\right) \delta 160.1,148.5,133.9,132.6,128.2,126.4,117.5,114.7,74.4,55.4$.

HRMS (ESI) calcd for $\mathrm{C}_{14} \mathrm{H}_{12} \mathrm{~N}_{2} \mathrm{O}_{6}[\mathrm{M}+\mathrm{Cl}]^{-:}$339.0389. Found: 339.0382 .

The enantiomeric excess was determined by HPLC analysis (Chiralcel OJ-H, $250 \times 4.6 \mathrm{~mm}$ column, hexane/2-propanol 85:15, $1.0 \mathrm{~mL} / \mathrm{min}, 220 \mathrm{~nm}, 30^{\circ} \mathrm{C},(S)$ isomer $68.9 \mathrm{~min},(R)$ isomer $85.4 \mathrm{~min}) ;[\alpha]_{\mathrm{D}}{ }^{20}+73.9\left(\mathrm{c} 0.9\right.$ in $\left.\mathrm{CHCl}_{3}\right) 99 \%$ ee $(S)$.

IR (neat) 3421, 3107, 2917, 2849, 1598, 1541, 1254, 1174, 1113, 1031, 840, $730 \mathrm{~cm}^{-1}$.

\section{(S)-Phenyl(ferrocenyl)methanol (11m)}

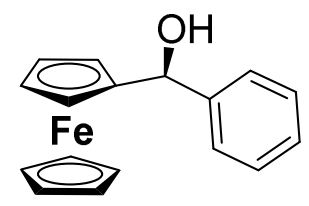

According to the general procedure (ketone: $0.290 \mathrm{~g}(1 \mathrm{mmol})$, cat. $(\boldsymbol{R}, \boldsymbol{R})-3), 0.154 \mathrm{~g}$ of $11 \mathrm{~m}$ was obtained as a red solid (53\% yield). 
${ }^{1} \mathrm{H}$ NMR (400 MHz, $\left.\mathrm{CDCl}_{3}\right) \delta 7.45-7.23(\mathrm{~m}, 5 \mathrm{H}), 5.47(\mathrm{~d}, J=3.2 \mathrm{~Hz}, 1 \mathrm{H}), 4.23(\mathrm{~s}, 9 \mathrm{H})$, $2.43(\mathrm{~d}, J=3.2 \mathrm{~Hz}, 1 \mathrm{H}) ;{ }^{13} \mathrm{C} \mathrm{NMR}\left(125 \mathrm{MHz}, \mathrm{CDCl}_{3}\right) \delta 143.2,128.2,127.4,126.2,94.3$, $72.0,68.5,68.2,68.1,67.5,66.0$. All characterization data are in agreement with the previously reported data ${ }^{[8]}$.

HRMS (ESI) calcd for $\mathrm{C}_{17} \mathrm{H}_{16} \mathrm{OFe}[\mathrm{M}]^{+}:$292.0545. Found: 292.0537.

The enantiomeric excess was determined by HPLC analysis (Chiralpak AS-H, $250 \times 4.6$ $\mathrm{mm}$ column, hexane/2-propanol 95:5, $1.0 \mathrm{~mL} / \mathrm{min}, 220 \mathrm{~nm}, 30^{\circ} \mathrm{C},(R)$ isomer $9.8 \mathrm{~min},(S)$ isomer $10.8 \mathrm{~min}$ ); $[\alpha]_{\mathrm{D}}{ }^{20}+80.8$ (c 0.05 in $\left.\mathrm{CHCl}_{3}\right) 90 \%$ ee $(S)\left(\right.$ lit. $^{[8]}[\alpha]_{\mathrm{D}}-94.4$ (c 0.016 in $\left.\mathrm{CHCl}_{3}\right) 98 \%$ ee $\left.(R)\right)$.

IR (neat) 3566, 3415, 3083, 3027, 2957, 2919, 2859, 1731, 1494, 1453, 1409, 1372, 1320, $1182,1048,1017,1000,823,720,700 \mathrm{~cm}^{-1}$.

\section{(R)-(3-Nitrophenyl)(thiophen-2-yl)methanol (11n)}<smiles>O=[N+]([O-])c1cccc([C@H](O)c2cccs2)c1</smiles>

According to the general procedure (ketone: $0.233 \mathrm{~g}(1 \mathrm{mmol})$, cat. $(\boldsymbol{R}, \boldsymbol{R})-3), 0.226 \mathrm{~g}$ of 11n was obtained as a clear oil (96\% yield).

${ }^{1} \mathrm{H}$ NMR $\left(400 \mathrm{MHz}, \mathrm{CDCl}_{3}\right) \delta 8.32-8.31(\mathrm{~m}, 1 \mathrm{H}), 8.15-8.12(\mathrm{~m}, 1 \mathrm{H}), 7.78-7.76(\mathrm{~m}, 1 \mathrm{H})$, 7.54-7.52 (m, 1H), 7.30-7.29 (m, 1H), 6.97-6.94 (m, 2H), $6.15(\mathrm{~s}, 1 \mathrm{H}), 2.83(\mathrm{br}, 1 \mathrm{H}) ;{ }^{13} \mathrm{C}$ NMR $\left(125 \mathrm{MHz}, \mathrm{CDCl}_{3}\right) \delta 148.3,146.6,145.1,132.2,129.4,126.9,126.2,125.5,122.8$, 121.2, 71.1 .

HRMS (FI) calcd for $\mathrm{C}_{11} \mathrm{H}_{9} \mathrm{NO}_{3} \mathrm{~S}[\mathrm{M}]^{+}:$235.0303. Found: 235.0294 .

The enantiomeric excess was determined by HPLC analysis (Chiralcel OJ-H, $250 \times 4.6 \mathrm{~mm}$ column, hexane/2-propanol 90:10, $1.0 \mathrm{~mL} / \mathrm{min}, 220 \mathrm{~nm}, 30{ }^{\circ} \mathrm{C},(S)$ isomer $34.9 \mathrm{~min},(R)$ isomer $38.9 \mathrm{~min})$; $[\alpha]_{\mathrm{D}}{ }^{20}+19.4\left(\mathrm{c} 1.43\right.$ in $\left.\mathrm{CHCl}_{3}\right) 98 \%$ ee $(R)$.

IR (neat) 3392, 2917, 2848, 1529, 1350, 1094, 1022, 811, 760, $707 \mathrm{~cm}^{-1}$. 


\section{Synthesis of Chiral Benzo[c]chromene Compound.}<smiles>OC(c1ccccc1)c1ccccc1Br</smiles>

$(99 \%$ ee)
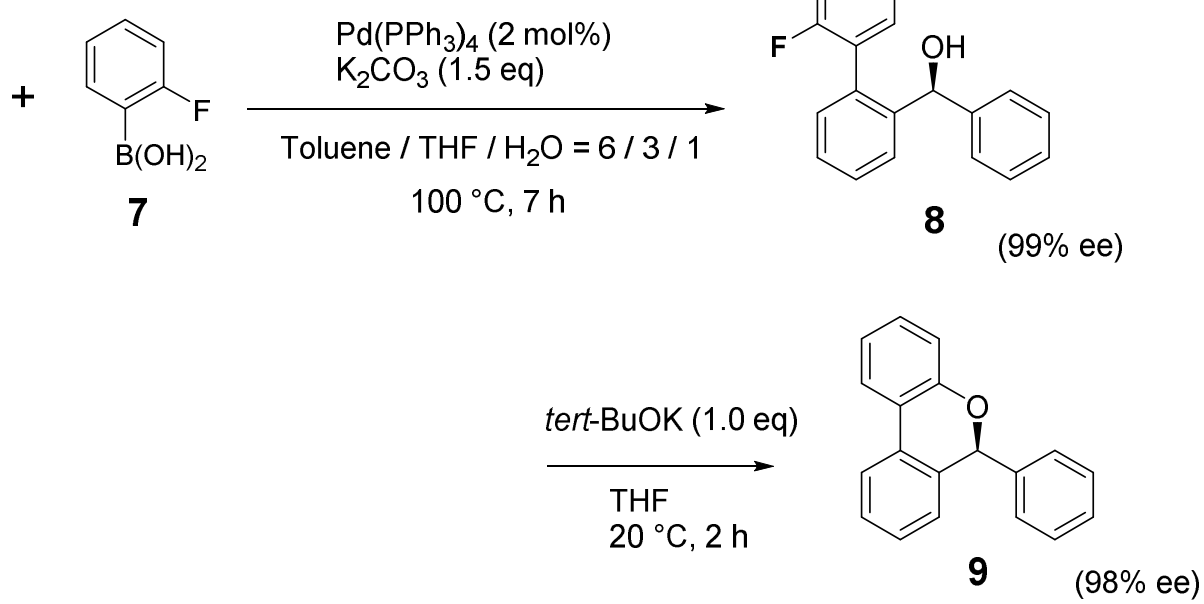

To a solution of $\mathbf{6 c}(0.5 \mathrm{~g}, 1.9 \mathrm{mmol})$ in toluene $(6 \mathrm{~mL})$ and THF $(3 \mathrm{~mL})$ were added (2-fluorophenyl)boronic acid (7) (0.399 g, $2.85 \mathrm{mmol}), \mathrm{Pd}\left(\mathrm{PPh}_{3}\right)_{4}(43.9 \mathrm{mg}, 0.038 \mathrm{mmol}$ ), $\mathrm{K}_{2} \mathrm{CO}_{3}(0.394 \mathrm{~g}, 2.85 \mathrm{mmol})$, and $\mathrm{H}_{2} \mathrm{O}(10 \mathrm{~mL})$. The biphasic mixture was vigorously stirred at $100{ }^{\circ} \mathrm{C}$ for $7 \mathrm{~h}$. The biphasic layers were separated, the aqueous phase was extracted with EtOAc $(2 \times 10 \mathrm{~mL})$, and the combined organic portions were dried over $\mathrm{MgSO}_{4}$, and concentrated to give a crude liquid of $\mathbf{8}$. The crude product was used for the following cyclization reaction without further purification (90\% yield).

The enantiomeric excess of $\mathbf{8}$ was determined by HPLC analysis (Chiralcel OD-H, $250 \times$ $4.6 \mathrm{~mm}$ column, hexane/2-propanol 97:3, $1.0 \mathrm{~mL} / \mathrm{min}, 254 \mathrm{~nm}, 30^{\circ} \mathrm{C},(S)$ isomer $10.4 \mathrm{~min}$, $(R)$ isomer $11.7 \mathrm{~min})$.

To a solution of crude liquid of $8(0.05 \mathrm{~g}, 0.18 \mathrm{mmol})$ in toluene $(1 \mathrm{~mL})$ were added tert-BuOK $(20.2 \mathrm{mg}, 0.18 \mathrm{mmol})$. After the reaction mixture was stirred at $20{ }^{\circ} \mathrm{C}$ for $2 \mathrm{~h}$, aqueous $\mathrm{NH}_{4} \mathrm{Cl}(5 \mathrm{~mL})$ was added to acidify the solution. The biphasic layers were separated, the aqueous phase was extracted with EtOAc $(3 \times 5 \mathrm{~mL})$, and the combined organic portions were washed with brine $(2 \times 3 \mathrm{~mL})$, dried over $\mathrm{MgSO}_{4}$, and concentrated under reduced pressure to afford the crude product which was purified by silica gel column chromatography to afford the product (9) as a white solid (28.9 mg, 70\% yield).

${ }^{1} \mathrm{H}$ NMR (400 MHz, $\left.\mathrm{CDCl}_{3}\right) \delta 7.78-7.74(\mathrm{~m}, 2 \mathrm{H}), 7.42-7.28(\mathrm{~m}, 6 \mathrm{H}), 7.26-7.18(\mathrm{~m}, 2 \mathrm{H})$, 7.08-6.95 (m, 2H), $6.84(\mathrm{~d}, J=7.6 \mathrm{~Hz}, 1 \mathrm{H}), 6.16(\mathrm{~s}, 1 \mathrm{H}) ;{ }^{13} \mathrm{C} \mathrm{NMR}\left(125 \mathrm{MHz}, \mathrm{CDCl}_{3}\right) \delta$ $153.6,139.6,134.0,130.0,129.6,128.5,128.5,128.4,128.1,127.6,126.2,123.1,122.8$, 122.1, 117.9, 79.6.

HRMS (APCI) calcd for $\mathrm{C}_{19} \mathrm{H}_{14} \mathrm{O}[\mathrm{M}]^{+}: 258.1039$. Found: 258.1019.

The enantiomeric excess was determined by HPLC analysis (Chiralcel OJ-H, $250 \times 4.6 \mathrm{~mm}$ column, hexane/2-propanol 98:2, $1.0 \mathrm{~mL} / \mathrm{min}, 220 \mathrm{~nm}, 30^{\circ} \mathrm{C},(R)$ isomer $17.4 \mathrm{~min},(S)$ isomer $20.7 \mathrm{~min}$ ); $[\alpha]_{\mathrm{D}}{ }^{20}-80.7$ (c 0.07 in $\left.\mathrm{CHCl}_{3}\right) 98 \%$ ee $(S)$.

IR (neat) 3065, 3033, 2960, 2922, 2852, 1726, 1593, 1486, 1439, 1245, 1010, 722, 699, $612 \mathrm{~cm}^{-1}$. 


\section{E. Determination of Absolute Configuration of Products}

a) Synthesis of $(S)$-(2-Chloro-5-nitrophenyl)(phenyl)methyl 4-nitrobenzoate (13i)<smiles>O=[N+]([O-])c1ccc(Cl)c([C@H](O)c2ccccc2)c1</smiles>

$6 \mathbf{i}$<smiles>CC(F)(F)[C@@H](C(=O)O[C@H](c1ccccc1)c1cc([N+](=O)[O-])ccc1Cl)C(F)(F)F</smiles>

12

$13 \mathbf{i}$

To a mixture of 4-nitrobenzoylchloride (12) (400 $\mathrm{mg}, 2.17 \mathrm{mmol})$ and $N, N$-dimethyl-4-aminopyridine $(256 \mathrm{mg}, 2.17 \mathrm{mmol})$ in THF $(10 \mathrm{~mL})$ was added alcohol $\mathbf{6 i}$ $(0.57 \mathrm{mg}, 2.17 \mathrm{mmol})$ in THF $(10 \mathrm{~mL})$. The reaction mixture was stirred for $2 \mathrm{~h}$ at room temperature and then quenched by water. The aqueous layer was extracted with $\mathrm{CHCl}_{3}(\times 3)$. The combined organic portions were dried over $\mathrm{Mg}_{2} \mathrm{SO}_{4}$ and evaporated in vacuo. The residue was purified by column chromatography to give ester $13 \mathbf{i}$ in $90 \%$ yield as a white solid. Single crystals were obtained by recrystallization from a slow diffusion of hexane into a THF solution.

${ }^{1} \mathrm{H}$ NMR $\left(400 \mathrm{MHz}, \mathrm{CDCl}_{3}\right) \delta 8.46(\mathrm{~d}, J=2.8 \mathrm{~Hz}, 2 \mathrm{H}), 8.35-8.29(\mathrm{~m}, 4 \mathrm{H}), 8.16(\mathrm{dd}, J=$ $2.8,8.7 \mathrm{~Hz}, 1 \mathrm{H}), 7.61(\mathrm{~d}, J=8.7 \mathrm{~Hz}, 1 \mathrm{H}), 7.48-7.38(\mathrm{~m}, 6 \mathrm{H}) ;{ }^{13} \mathrm{C} \mathrm{NMR}\left(125 \mathrm{MHz}, \mathrm{CDCl}_{3}\right)$ $\delta 163.4,150.9,147.0,139.7,139.4,136.6,134.7,131.2,131.0,129.2,129.1,127.5,124.3$, 123.8, 123.0, 74.6. HRMS (APCI) calcd for $\mathrm{C}_{20} \mathrm{H}_{13} \mathrm{~N}_{2} \mathrm{O}_{6} \mathrm{Cl}$ [M-H] : 411.0389. Found: 411.0405. Anal. calcd for $\mathrm{C}_{20} \mathrm{H}_{13} \mathrm{ClN}_{2} \mathrm{O}_{6}$ : C, 58.19; H, 3.17; N, 6.79. Found: C, 58.48; H, $3.23 ; \mathrm{N}, 6.62$.

IR (neat) 1728, 1522, 1346, 1264, 1249, 1095, 1054, 852, 742, $717 \mathrm{~cm}^{-1}$.

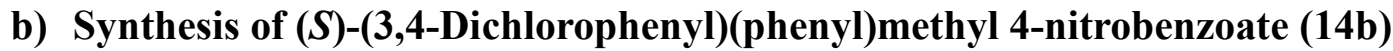<smiles>CC(C)CC(C)(C)C(=O)OC(c1ccccc1)c1ccc(Cl)c(Cl)c1</smiles>

To a mixture of 4-nitrobenzoylchloride (12) (352 $\mathrm{mg}, 1.90 \mathrm{mmol})$ and $\mathrm{N}, \mathrm{N}$-dimethyl-4-aminopyridine $(232 \mathrm{mg}, 1.90 \mathrm{mmol})$ in THF $(10 \mathrm{~mL})$ was added alcohol $11 \mathrm{~b}(0.50 \mathrm{mg}, 1.90 \mathrm{mmol})$ in THF $(10 \mathrm{~mL})$. The reaction mixture was stirred for $2 \mathrm{~h}$ at room temperature and then quenched by water. The aqueous layer was extracted with $\mathrm{CHCl}_{3}(\times 3)$. The combined organic portions were dried over $\mathrm{Mg}_{2} \mathrm{SO}_{4}$ and evaporated in vacuo. The residue was purified by column chromatography to give ester $\mathbf{1 4 b}$ in $90 \%$ yield as a white solid. Single crystals were obtained by recrystallization from an $\mathrm{Et}_{2} \mathrm{O}$-hexane solution. 
${ }^{1} \mathrm{H}$ NMR $\left(400 \mathrm{MHz}, \mathrm{CDCl}_{3}\right) \delta 8.33-8.27(\mathrm{~m}, 4 \mathrm{H}), 7.51-7.50(\mathrm{~m}, 1 \mathrm{H}), 7.45-7.35(\mathrm{~m}, 6 \mathrm{H})$, 7.27-7.25 (m, 1H), $7.06(\mathrm{~s}, 1 \mathrm{H}) ;{ }^{13} \mathrm{C} \mathrm{NMR}\left(125 \mathrm{MHz}, \mathrm{CDCl}_{3}\right) \delta 163.5,150.8,139.7,138.4$, $135.1,132.9,132.5,130.9,130.7,129.0,128.9,128.7,127.0,126.5,123.7,76.8$. HRMS (APCI) calcd for $\mathrm{C}_{20} \mathrm{H}_{13} \mathrm{NO}_{4} \mathrm{Cl}_{2}[\mathrm{M}-\mathrm{H}]^{-}$: 400.0149. Found: 400.0144. Anal. calcd for $\mathrm{C}_{20} \mathrm{H}_{13} \mathrm{Cl}_{2} \mathrm{NO}_{4}$ : C, 59.72; H, 3.26; N, 3.48. Found: C, 59.84; H, 3.35; N, 3.43.

IR (neat) 1724, 1523, 1493, 1469, 1342, 1323, 1302, 1269, 1115, 1030, 1015, 983, 873, 856, $717,695 \mathrm{~cm}^{-1}$.

c) Synthesis of (S)-(4-Chloro-3-nitrophenyl)(phenyl)methyl 4-nitrobenzoate (14e)

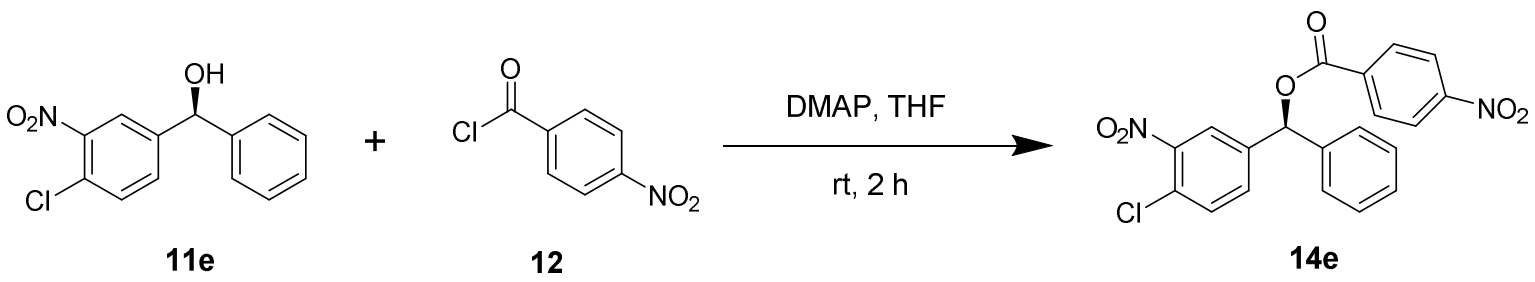

To a mixture of 4-nitrobenzoylchloride (12) (352 $\mathrm{mg}, 1.90 \mathrm{mmol})$ and $N, N$-dimethyl-4-aminopyridine $(232 \mathrm{mg}, 1.90 \mathrm{mmol})$ in THF $(10 \mathrm{~mL})$ was added alcohol $11 \mathrm{e}(0.50 \mathrm{mg}, 1.90 \mathrm{mmol})$ in THF $(10 \mathrm{~mL})$. The reaction mixture was stirred for $2 \mathrm{~h}$ at room temperature and then quenched by water. The aqueous layer was extracted with $\mathrm{CHCl}_{3}(\times 3)$. The combined organic portions were dried over $\mathrm{Mg}_{2} \mathrm{SO}_{4}$ and evaporated in vacuo. The residue was purified by column chromatography to give ester $14 \mathrm{e}$ in $90 \%$ yield as a white solid. Single crystals were obtained by recrystallization from a heptane solution.

${ }^{1} \mathrm{H}$ NMR (400 MHz, $\left.\mathrm{CDCl}_{3}\right) \delta 8.34-8.28(\mathrm{~m}, 4 \mathrm{H}), 7.93-7.92(\mathrm{~m}, 1 \mathrm{H}), 7.57-7.56(\mathrm{~m}, 2 \mathrm{H})$, 7.43-7.40 (m, 5H), $7.13(\mathrm{~s}, 1 \mathrm{H}) ;{ }^{13} \mathrm{C} \mathrm{NMR}\left(125 \mathrm{MHz}, \mathrm{CDCl}_{3}\right) \delta 163.5,150.9,148.0,140.1$, 137.7, 134.7, 132.3, 131.7, 130.9, 129.2, 129.1, 127.1, 127.0, 124.1, 123.8, 78.0. HRMS (APCI) calcd for $\mathrm{C}_{20} \mathrm{H}_{13} \mathrm{~N}_{2} \mathrm{O}_{6} \mathrm{Cl}[\mathrm{M}-\mathrm{H}]^{-}:$411.0389. Found: 411.0401. Anal. calcd for $\mathrm{C}_{20} \mathrm{H}_{13} \mathrm{ClN}_{2} \mathrm{O}_{6}$ : C, 58.19; H, 3.17; N, 6.79. Found: C, 58.16; H, 2.98; N, 6.60.

IR (neat) 1729, 1535, 1337, 1278, 1117, 1106, 732, 720, $702 \mathrm{~cm}^{-1}$.

\section{d) Synthesis of $(S)$-Phenyl(2,4,5-trimethylphenyl)methyl 4,5-dichloro -2-((3aR,6S)-8,8-dimethyl-2,2-dioxidohexahydro-3H-3a,6-methanobenzo[c]isothi azole-1-carbonyl)benzoate ${ }^{[10]}(13 g)$}




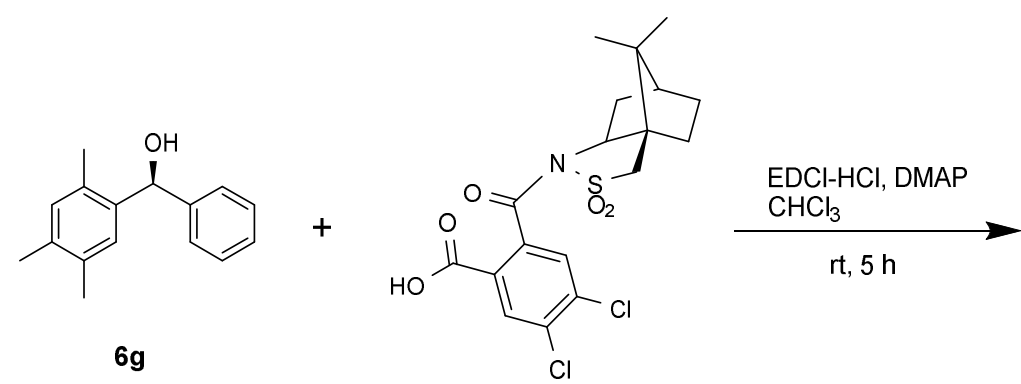

$(+)-15$

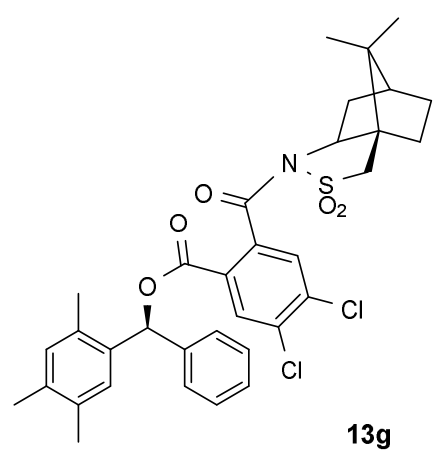

To a mixture of $N$-(2-carboxy-4,5-dichlorobenzoyl)-(+)-10,2-camphorsultam ((+)-15) (500 mg, $1.16 \mathrm{mmol})$, 1-ethyl-3-(3-dimethylaminopropyl) carbodiimide hydrochloride (220 $\mathrm{mg}, 1.16 \mathrm{mmol}$ ), and $\mathrm{N}, N$-dimethyl-4-aminopyridine (142 $\mathrm{mg}, 1.16 \mathrm{mmol}$ ) in $\mathrm{CHCl}_{3}(3$ $\mathrm{mL})$ was added alcohol $\mathbf{6 g}(201 \mathrm{mg}, 0.89 \mathrm{mmol})$ in $\mathrm{CHCl}_{3}(2 \mathrm{~mL})$. The reaction mixture was stirred for $5 \mathrm{~h}$ at room temperature and then quenched by a saturated aqueous $\mathrm{NH}_{4} \mathrm{Cl}$ solution. The aqueous layer was extracted with $\mathrm{CHCl}_{3}(\times 3)$. The combined organic portions were dried over $\mathrm{Mg}_{2} \mathrm{SO}_{4}$ and evaporated in vacuo. The residue was purified by column chromatography to give ester $\mathbf{1 3 g}$ in $95 \%$ yield as a white solid. Single crystals were obtained by recrystallization from a methanol solution.

${ }^{1} \mathrm{H}$ NMR $\left(500 \mathrm{MHz}, \mathrm{CDCl}_{3}\right) \delta 8.15(\mathrm{~s}, 1 \mathrm{H}), 7.49(\mathrm{~s}, 1 \mathrm{H}), 7.35-7.27(\mathrm{~m}, 5 \mathrm{H}), 7.12-7.11(\mathrm{~m}$, $2 \mathrm{H}), 6.93(\mathrm{~s}, 1 \mathrm{H}), 3.62-3.58(\mathrm{~m}, 1 \mathrm{H}), 3.31-3.21(\mathrm{~m}, 2 \mathrm{H}), 2.42-2.37(\mathrm{~m}, 1 \mathrm{H}), 2.24(\mathrm{~s}, 3 \mathrm{H})$, $2.21(\mathrm{~s}, 3 \mathrm{H}), 2.20(\mathrm{~s}, 3 \mathrm{H}), 2.03-1.98(\mathrm{~m}, 1 \mathrm{H}), 1.84-1.82(\mathrm{~m}, 3 \mathrm{H}), 1.30-1.24(\mathrm{~m}, 2 \mathrm{H}), 0.94$ $(\mathrm{s}, 3 \mathrm{H}), 0.89(\mathrm{~s}, 3 \mathrm{H}) ;{ }^{13} \mathrm{C}$ NMR $\left(125 \mathrm{MHz}, \mathrm{CDCl}_{3}\right) \delta 165.0,162.6,139.0,136.8,136.3$, $134.9,134.8,134.5,134.1,133.2,131.9,131.9,131.0,128.9,128.5,128.5,127.9,127.6$, 76.3, 65.4, 52.8, 48.3, 47.6, 44.7, 37.6, 32.8, 26.4, 20.4, 19.9, 19.4, 19.3, 18.8. HRMS (ESI) calcd for $\mathrm{C}_{34} \mathrm{H}_{35} \mathrm{NO}_{5} \mathrm{SCl}_{2}[\mathrm{M}+\mathrm{Na}]^{+}:$662.1505. Found: 662.1504. Anal. calcd for $\mathrm{C}_{34} \mathrm{H}_{35} \mathrm{Cl}_{2} \mathrm{NO}_{5} \mathrm{~S}: \mathrm{C}, 63.74 ; \mathrm{H}, 5.51 ; \mathrm{N}, 2.19$. Found: $\mathrm{C}, 63.84 \mathrm{H}, 5.38 ; \mathrm{N}, 2.38$.

IR (neat) 2960, 1727, 1674, 1552, 1461, 1331, 1316, 1301, 1242, 1167, 1139, 1117, 1091, $1067,753,703 \mathrm{~cm}^{-1}$.

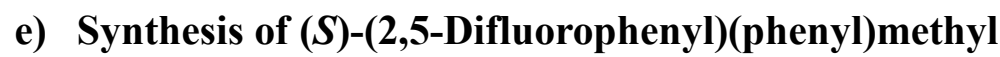
4,5-dichloro-2-((3aS,6R)-8,8-dimethyl-2,2-dioxidohexahydro-3H-3a,6-methanobenz o[c]isothiazole-1-carbonyl)benzoate ${ }^{[10]}(13 \mathbf{j})$

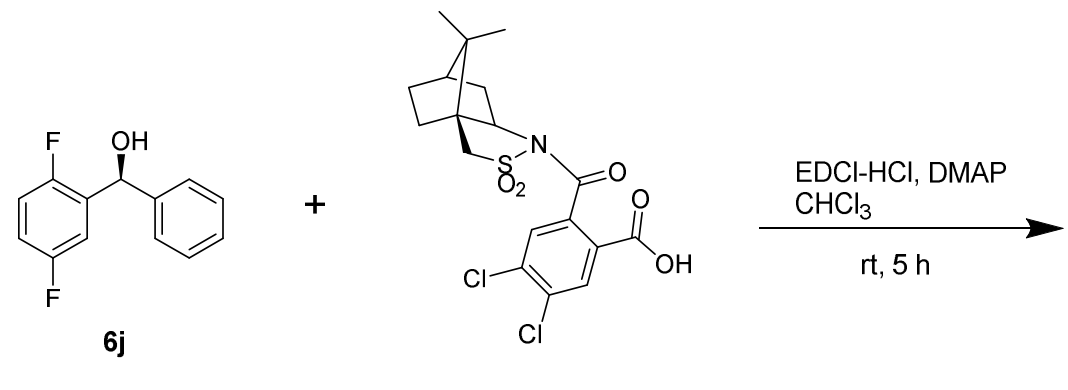

$(-)-15$

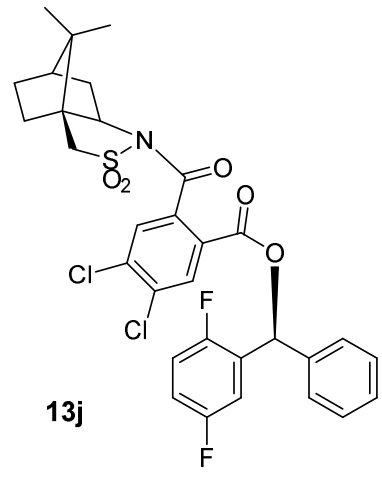


To a mixture of $N$-(2-carboxy-4,5-dichlorobenzoyl)-(-)-10,2-camphorsultam ((-)-15) (406 $\mathrm{mg}, 0.94 \mathrm{mmol}), 1$-ethyl-3-(3-dimethylaminopropyl) carbodiimide hydrochloride (180 $\mathrm{mg}$, $0.94 \mathrm{mmol})$, and $\mathrm{N}, \mathrm{N}$-dimethyl-4-aminopyridine $(115 \mathrm{mg}, 0.94 \mathrm{mmol})$ in $\mathrm{CHCl}_{3}(2 \mathrm{~mL})$ was added alcohol $\mathbf{6 j}(138 \mathrm{mg}, 0.63 \mathrm{mmol})$ in $\mathrm{CHCl}_{3}(2 \mathrm{~mL})$. The reaction mixture was stirred for $5 \mathrm{~h}$ at room temperature and then quenched by a saturated aqueous $\mathrm{NH}_{4} \mathrm{Cl}$ solution. The aqueous layer was extracted with $\mathrm{CHCl}_{3}(\times 3)$. The combined organic portions were dried over $\mathrm{Mg}_{2} \mathrm{SO}_{4}$ and evaporated in vacuo. The residue was purified by column chromatography to give ester $\mathbf{1 3}$ j in $94 \%$ yield as a white solid. Single crystals were obtained by recrystallization from a methanol solution.

${ }^{1} \mathrm{H}$ NMR $\left(500 \mathrm{MHz}, \mathrm{CDCl}_{3}\right) \delta 8.12(\mathrm{~s}, 1 \mathrm{H}), 7.53(\mathrm{~s}, 1 \mathrm{H}), 7.39-7.32(\mathrm{~m}, 5 \mathrm{H}), 7.18(\mathrm{~s}, 1 \mathrm{H})$, 7.18-7.15 (m, 1H), 7.06-6.96 (m, 2H), 3.71-3.68 (m, 1H), 3.39-3.27 (m, 2H), 2.40-2.35 $(\mathrm{m}, 1 \mathrm{H}), 2.80-2.20(\mathrm{~m}, 1 \mathrm{H}), 1.89-1.85(\mathrm{~m}, 3 \mathrm{H}), 1.33-1.28(\mathrm{~m}, 2 \mathrm{H}), 1.03(\mathrm{~s}, 3 \mathrm{H}), 0.92(\mathrm{~s}$, $3 \mathrm{H}) ;{ }^{13} \mathrm{C} \mathrm{NMR}\left(125 \mathrm{MHz}, \mathrm{CDCl}_{3}\right) \delta 164.9,162.5,157.8,156.8,155.0,137.7,137.1,135.1$, $134.7,131.8,131.3,128.7,128.5,128.2,127.2,116.9(\mathrm{dd}, J=23.8,8.8 \mathrm{~Hz}), 116.2(\mathrm{dd}, J=$ 23.8, 8.8 Hz), $114.8(\mathrm{~d}, J=28.8 \mathrm{~Hz}), 72.5,65.5,53.0,48.4,47.7$, 44.7, 37.6, 32.9, 26.4, 20.5, 19.9. HRMS (ESI) calcd for $\mathrm{C}_{31} \mathrm{H}_{27} \mathrm{NO}_{5} \mathrm{~F}_{2} \mathrm{SCl}_{2}[\mathrm{M}+\mathrm{Na}]^{+}: 656.0847$. Found: 656.0831. Anal. calcd for $\mathrm{C}_{31} \mathrm{H}_{27} \mathrm{Cl}_{2} \mathrm{~F}_{2} \mathrm{NO}_{5} \mathrm{~S}$ : C, 58.68; H, 4.29; N, 2.21. Found: C, 59.00; H, 4.31; N, 2.21 .

IR (neat) 2959, 1734, 1686, 1496, 1337, 1299, 1243, 1169, 1141, 1116, 1092, 1063, 764 $\mathrm{cm}^{-1}$.

\section{f) Synthesis of (S)-(2-Fluoro-3-(trifluoromethyl)phenyl)(phenyl)methyl 4,5-dichloro-2-((3aS,6R)-8,8-dimethyl-2,2-dioxidohexahydro-3H-3a,6-methanobenz $0[c]$ isothiazole-1-carbonyl)benzoate ${ }^{[10]}(13 \mathrm{k})$}
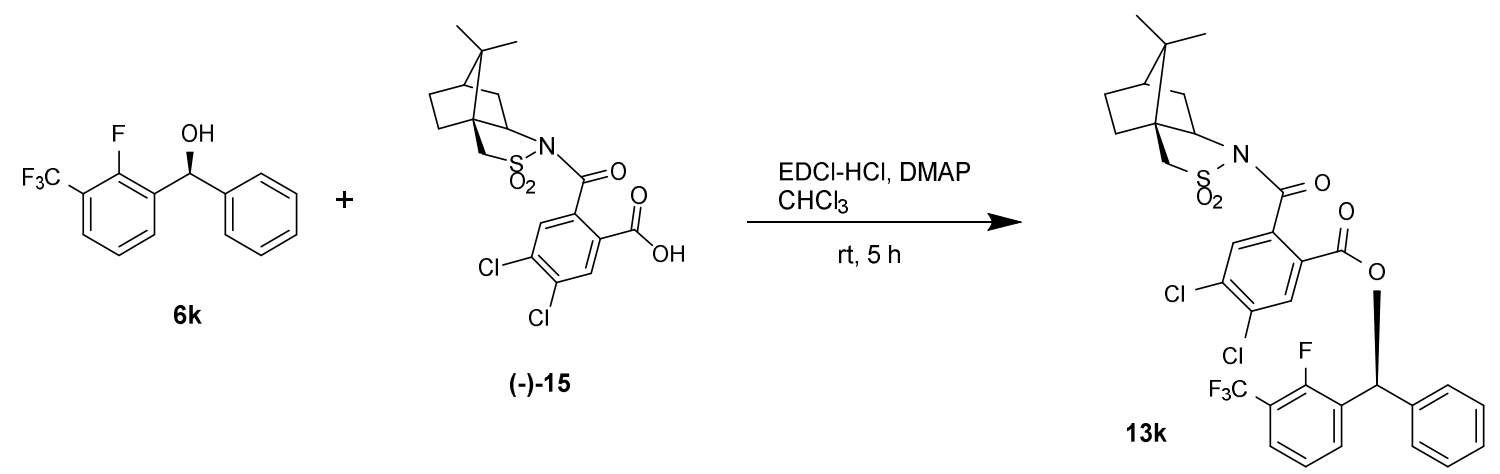

To a mixture of $N$-(2-carboxy-4,5-dichlorobenzoyl)-(-)-10,2-camphorsultam ((-)-15) (500 $\mathrm{mg}, 1.16 \mathrm{mmol})$, 1-ethyl-3-(3-dimethylaminopropyl) carbodiimide hydrochloride $(220 \mathrm{mg}$, $1.16 \mathrm{mmol})$, and $N, N$-dimethyl-4-aminopyridine $(142 \mathrm{mg}, 1.16 \mathrm{mmol})$ in $\mathrm{CHCl}_{3}(3 \mathrm{~mL})$ was added alcohol $\mathbf{6 k}(240 \mathrm{mg}, 0.89 \mathrm{mmol})$ in $\mathrm{CHCl}_{3}(2 \mathrm{~mL})$. The reaction mixture was stirred for $5 \mathrm{~h}$ at room temperature and then quenched by a saturated aqueous $\mathrm{NH}_{4} \mathrm{Cl}$ solution. The aqueous layer was extracted with $\mathrm{CHCl}_{3}(\times 3)$. The combined organic portions were dried over $\mathrm{Mg}_{2} \mathrm{SO}_{4}$ and evaporated in vacuo. The residue was purified by column chromatography to give ester $\mathbf{1 3 k}$ in $95 \%$ yield as a white solid. Single crystals were obtained by recrystallization from a 2-propanol solution. 
${ }^{1} \mathrm{H}$ NMR $\left(500 \mathrm{MHz}, \mathrm{CDCl}_{3}\right) \delta 8.13(\mathrm{~s}, 1 \mathrm{H}), 7.68-7.65(\mathrm{~m}, 1 \mathrm{H}), 7.60-7.56(\mathrm{~m}, 1 \mathrm{H}), 7.51(\mathrm{~s}$, $1 \mathrm{H})$, 7.39-7.13 (m, 5H), 7.29-7.25 (m, 2H), 3.68-3.65 (m, 1H), 3.36-3.23 (m, 2H), 2.42-2.36 (m, 1H), 2.08-2.02 (m, 1H), 1.88-1.85 (m, 3H), 1.33-1.25 (m, 2H), $1.03(\mathrm{~s}, 3 \mathrm{H})$, $0.92(\mathrm{~s}, 3 \mathrm{H}) ;{ }^{13} \mathrm{C}$ NMR $\left(125 \mathrm{MHz}, \mathrm{CDCl}_{3}\right) \delta 164.9,162.3,158.0,155.9,137.5(\mathrm{~d}, J=27.5$ $\mathrm{Hz}), 135.0,134.9,132.0,131.7,131.2,128.8,128.7,128.6,127.9,127.3,127.0$ (d, $J=5.0$ $\mathrm{Hz}), 124.3(\mathrm{~d}, J=3.8 \mathrm{~Hz}), 122.4(\mathrm{q}, J=270.0 \mathrm{~Hz}), 118.7(\mathrm{qd}, J=32.5,12.5 \mathrm{~Hz}), 72.2,65.5$, 52.9, 48.4, 47.7, 44.7, 37.5, 32.9, 26.4, 20.5, 19.9. HRMS (ESI) calcd for $\mathrm{C}_{32} \mathrm{H}_{27} \mathrm{NO}_{5} \mathrm{~F}_{4} \mathrm{SCl}$ $[\mathrm{M}+\mathrm{Na}]^{+}:$706.0815. Found: 706.0804. Anal. calcd for $\mathrm{C}_{32} \mathrm{H}_{27} \mathrm{Cl}_{2} \mathrm{~F}_{4} \mathrm{NO}_{5} \mathrm{~S}: \mathrm{C}, 56.14 ; \mathrm{H}$, 3.98; N, 2.05. Found: C, 55.78; H, 4.00; N, 2.03.

IR (neat) 2962, 1736, 1685, 1474, 1335, 1296, 1265, 1244, 1165, 1129, 1110, 1094, 1061, $795,758,697 \mathrm{~cm}^{-1}$.

\section{g) Synthesis of (S)-(3,4-Difluorophenyl)(phenyl)methyl 4,5-dichloro-2-((3aR,6S)-8,8-dimethyl-2,2-dioxidohexahydro-3H-3a,6-methanobenz o[c]isothiazole-1-carbonyl)benzoate ${ }^{[10]}(14 c)$}

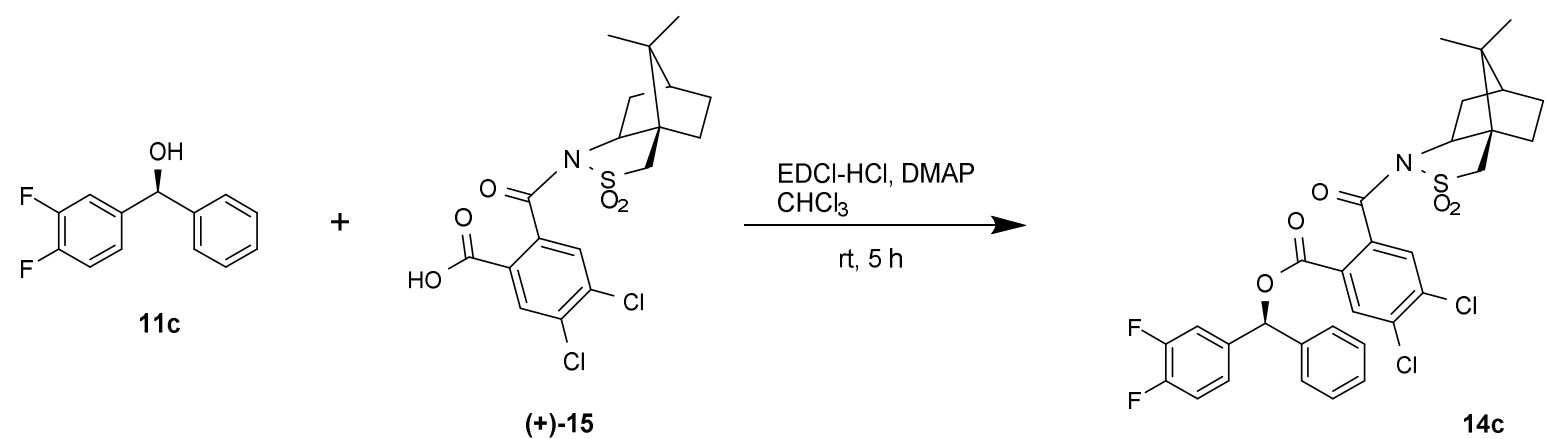

To a mixture of $N$-(2-carboxy-4,5-dichlorobenzoyl)-(+)-10,2-camphorsultam $((+)-15)(500$ $\mathrm{mg}, 1.16 \mathrm{mmol})$, 1-ethyl-3-(3-dimethylaminopropyl) carbodiimide hydrochloride (220 $\mathrm{mg}$, $1.16 \mathrm{mmol}$ ), and $N, N$-dimethyl-4-aminopyridine $(142 \mathrm{mg}, 1.16 \mathrm{mmol})$ in $\mathrm{CHCl}_{3}(3 \mathrm{~mL})$ was added alcohol 11c $(196 \mathrm{mg}, 0.89 \mathrm{mmol})$ in $\mathrm{CHCl}_{3}(2 \mathrm{~mL})$. The reaction mixture was stirred for $5 \mathrm{~h}$ at room temperature and then quenched by a saturated aqueous $\mathrm{NH}_{4} \mathrm{Cl}$ solution. The aqueous layer was extracted with $\mathrm{CHCl}_{3}(\times 3)$. The combined organic portions were dried over $\mathrm{Mg}_{2} \mathrm{SO}_{4}$ and evaporated in vacuo. The residue was purified by column chromatography to give ester $\mathbf{1 4 c}$ in $93 \%$ yield as a white solid. Single crystals were obtained by recrystallization from a methanol solution.

${ }^{1} \mathrm{H}$ NMR $\left(500 \mathrm{MHz}, \mathrm{CDCl}_{3}\right) \delta 8.10(\mathrm{~s}, 1 \mathrm{H}), 7.52(\mathrm{~s}, 1 \mathrm{H}), 7.41-7.33(\mathrm{~m}, 5 \mathrm{H}), 7.23-7.19(\mathrm{~m}$, 1H), 7.16-7.05 (m, 2H), $6.93(\mathrm{~s}, 1 \mathrm{H}), 3.80-3.76(\mathrm{~m}, 1 \mathrm{H}), 3.39-3.30(\mathrm{~m}, 2 \mathrm{H}), 2.42-2.38(\mathrm{~m}$, $1 \mathrm{H}), 2.10-2.06(\mathrm{~m}, 1 \mathrm{H}), 1.91-1.88(\mathrm{~m}, 3 \mathrm{H}), 1.34-1.32(\mathrm{~m}, 2 \mathrm{H}), 1.01(\mathrm{~s}, 3 \mathrm{H}), 0.93(\mathrm{~s}, 3 \mathrm{H})$; ${ }^{13} \mathrm{C}$ NMR $\left(125 \mathrm{MHz}, \mathrm{CDCl}_{3}\right) \delta 165.0,162.6,151.1(\mathrm{dd}, J=26.3,12.5 \mathrm{~Hz}), 149.1(\mathrm{~d}, J=$ 26.3, 12.5 Hz), 138.4, 137.1, $136.4(\mathrm{~m}), 135.0,134.8,131.4(\mathrm{~m}), 128.8,128.7,128.2,127.6$, $126.5,123.3(\mathrm{~m}), 117.2(\mathrm{~d}, J=18.0 \mathrm{~Hz}), 116.5(\mathrm{~d}, J=18.0 \mathrm{~Hz}), 77.5,65.5,53.0,48.4,47.6$, 44.7, 37.5, 33.0, 26.4, 20.4, 20.0. HRMS (ESI) calcd for $\mathrm{C}_{31} \mathrm{H}_{27} \mathrm{NO}_{5} \mathrm{~F}_{2} \mathrm{SCl}_{2}[\mathrm{M}+\mathrm{Na}]^{+}$: 656.0847. Found: 656.0831. Anal. calcd for $\mathrm{C}_{31} \mathrm{H}_{27} \mathrm{Cl}_{2} \mathrm{~F}_{2} \mathrm{NO}_{5} \mathrm{~S}$ : C, 58.68; H, 4.29; N, 2.21 . 
Found: C, 58.60; H, 4.15; N, 2.36.

IR (neat) 2969, 1732, 1673, 1515, 1328, 1299, 1264, 1244, 1169, 1141, 1114, 1093, 1068, 754, 738, $709 \mathrm{~cm}^{-1}$.

h) Synthesis of $\quad(S)$-Phenyl(3,4,5-trifluorophenyl)methyl 4,5-dichloro-2-((3aS,6R)-8,8-dimethyl-2,2-dioxidohexahydro-3H-3a,6-methanobenz o[c]isothiazole-1-carbonyl)benzoate ${ }^{[10]}(14 f)$

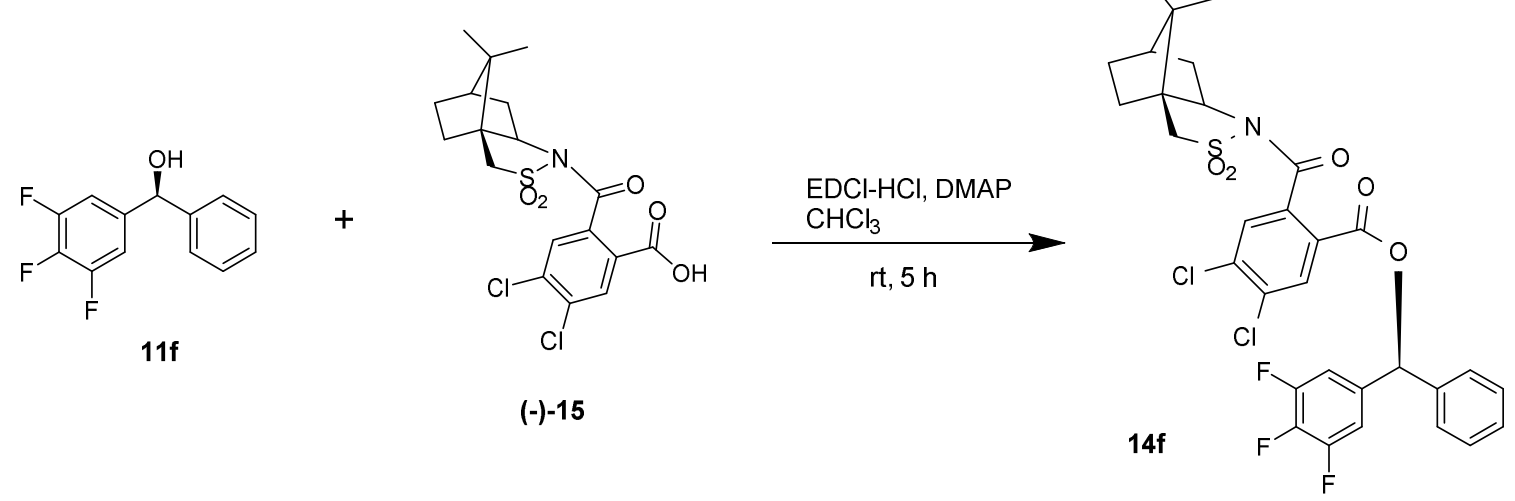

To a mixture of $N$-(2-carboxy-4,5-dichlorobenzoyl)-(-)-10,2-camphorsultam ((-)-15) (500 $\mathrm{mg}, 1.16 \mathrm{mmol})$, 1-ethyl-3-(3-dimethylaminopropyl) carbodiimide hydrochloride (220 $\mathrm{mg}$, $1.16 \mathrm{mmol})$, and $\mathrm{N}, \mathrm{N}$-dimethyl-4-aminopyridine (142 $\mathrm{mg}, 1.16 \mathrm{mmol})$ in $\mathrm{CHCl}_{3}(3 \mathrm{~mL})$ was added alcohol $11 \mathrm{f}(212 \mathrm{mg}, 0.89 \mathrm{mmol})$ in $\mathrm{CHCl}_{3}(2 \mathrm{~mL})$. The reaction mixture was stirred for $5 \mathrm{~h}$ at room temperature and then quenched by a saturated aqueous $\mathrm{NH}_{4} \mathrm{Cl}$ solution. The aqueous layer was extracted with $\mathrm{CHCl}_{3}(\times 3)$. The combined organic portions were dried over $\mathrm{Mg}_{2} \mathrm{SO}_{4}$ and evaporated in vacuo. The residue was purified by column chromatography to give ester $\mathbf{1 4 f}$ in $95 \%$ yield as a white solid. Single crystals were obtained by recrystallization from an ethanol solution.

${ }^{1} \mathrm{H}$ NMR $\left(500 \mathrm{MHz}, \mathrm{CDCl}_{3}\right) \delta 8.10(\mathrm{~d}, J=0.5 \mathrm{~Hz}, 1 \mathrm{H}), 7.52(\mathrm{~d}, J=0.5 \mathrm{~Hz}, 1 \mathrm{H}), 7.40-7.32$ (m, 5H), 7.03-6.99 (m, 2H), $6.88(\mathrm{~s}, 1 \mathrm{H}), 3.71-3.68(\mathrm{~m}, 1 \mathrm{H}), 3.39-3.26(\mathrm{~m}, 2 \mathrm{H}), 2.40-2.37$ $(\mathrm{m}, 1 \mathrm{H}), 2.10-2.04(\mathrm{~m}, 1 \mathrm{H}), 1.89-1.88(\mathrm{~m}, 3 \mathrm{H}), 1.33-1.26(\mathrm{~m}, 2 \mathrm{H}), 1.06(\mathrm{~s}, 3 \mathrm{H}), 0.93(\mathrm{~s}$, $3 \mathrm{H}) ;{ }^{13} \mathrm{C} \mathrm{NMR}\left(125 \mathrm{MHz}, \mathrm{CDCl}_{3}\right) \delta 164.9,162.6,152.2(\mathrm{~d}, J=10.0 \mathrm{~Hz}), 150.2(\mathrm{~d}, J=6.3$ $\mathrm{Hz}), 137.9$, 137.3, 135.7 (m), 135.2, 134.7, 131.8, 131.3, 128.8, 128.8, 128.0, 127.4, 111.7 (dd, $J=16.3,5.0 \mathrm{~Hz}), 76.8,65.5,53.0,48.4,47.7,44.7,37.7,32.9,26.4,20.6,19.9$. HRMS (ESI) calcd for $\mathrm{C}_{31} \mathrm{H}_{26} \mathrm{NO}_{5} \mathrm{~F}_{3} \mathrm{SCl}_{2}[\mathrm{M}+\mathrm{Na}]^{+}$: 674.0753. Found: 674.0748. Anal. calcd for $\mathrm{C}_{31} \mathrm{H}_{26} \mathrm{Cl}_{2} \mathrm{~F}_{3} \mathrm{NO}_{5} \mathrm{~S}$ : C, 57.06; H, 4.02; N, 2.15. Found: C, 57.32; H, 4.02; N, 2.19.

IR (neat) 2960, 1732, 1684, 1531, 1455, 1338, 1299, 1241, 1169, 1142, 1116, 1091, 1047, $701 \mathrm{~cm}^{-1}$.

i) Synthesis of $\quad(R)-(3-N i t r o p h e n y l)($ thiophen-2-yl)methyl 4,5-dichloro-2-((3aS,6R)-8,8-dimethyl-2,2-dioxidohexahydro-3H-3a,6-methanobenz o[c]isothiazole-1-carbonyl)benzoate ${ }^{[10]}(14 n)$ 

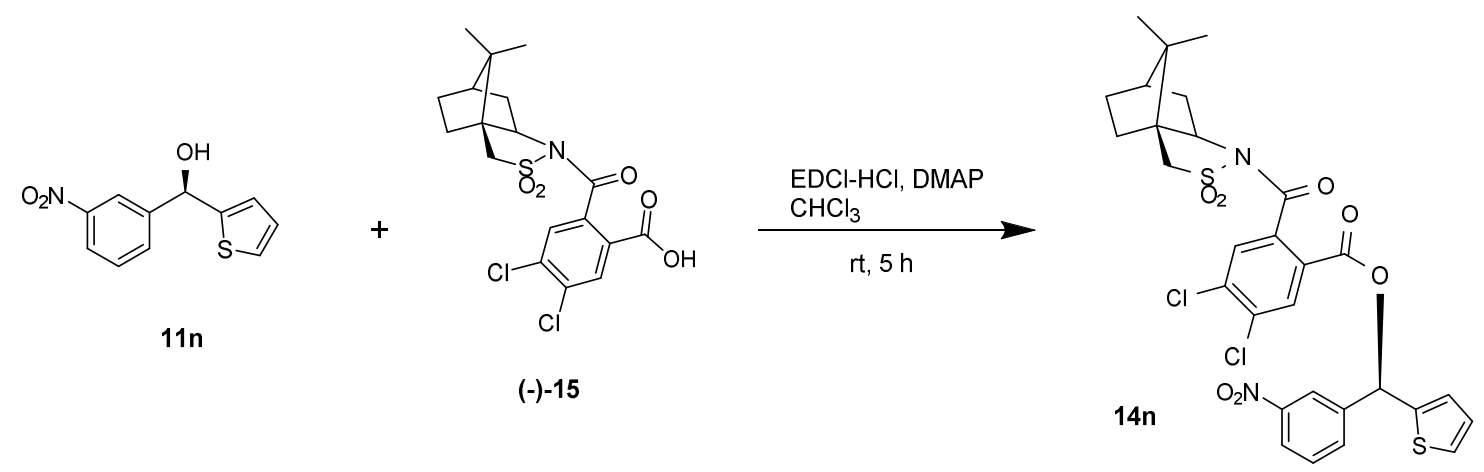

To a mixture of $N$-(2-carboxy-4,5-dichlorobenzoyl)-(-)-10,2-camphorsultam ((-)-15) (500 $\mathrm{mg}, 1.16 \mathrm{mmol}$ ), 1-ethyl-3-(3-dimethylaminopropyl) carbodiimide hydrochloride (220 mg, $1.16 \mathrm{mmol})$, and $N, N$-dimethyl-4-aminopyridine $(142 \mathrm{mg}, 1.16 \mathrm{mmol})$ in $\mathrm{CHCl}_{3}(3 \mathrm{~mL})$ was added alcohol $11 \mathrm{n}(136 \mathrm{mg}, 0.58 \mathrm{mmol})$ in $\mathrm{CHCl}_{3}(2 \mathrm{~mL})$. The reaction mixture was stirred for $5 \mathrm{~h}$ at room temperature and then quenched by a saturated aqueous $\mathrm{NH}_{4} \mathrm{Cl}$ solution. The aqueous layer was extracted with $\mathrm{CHCl}_{3}(\times 3)$. The combined organic portions were dried over $\mathrm{Mg}_{2} \mathrm{SO}_{4}$ and evaporated in vacuo. The residue was purified by column chromatography to give ester $\mathbf{1 4 n}$ in $92 \%$ yield as a white solid. Single crystals were obtained by recrystallization from a 2-propanol solution.

${ }^{1} \mathrm{H}$ NMR $\left(500 \mathrm{MHz}, \mathrm{CDCl}_{3}\right) \delta 8.33-8.32(\mathrm{~m}, 1 \mathrm{H}), 8.23-8.20(\mathrm{~m}, 1 \mathrm{H}), 8.14(\mathrm{~s}, 1 \mathrm{H})$, 7.84-7.82 (m, 1H), 7.61-7.57 (m, 1H), 7.16 (s, 1H), 7.37-7.36 (m, 1H), 7.27-7.26 (m, 1H), 7.04-7.03 (m, 1H), 7.02-7.00 (m, 1H), 3.83-3.80 (m, 1H), 3.42-3.29 (m, 2H), 2.44-2.42 (m, 1H), 2.14-2.09 (m, 1H), 1.92-1.86 (m, 3H), 1.36-1.34 (m, 2H), $1.18(\mathrm{~s}, 3 \mathrm{H}), 0.97(\mathrm{~s}$, $3 \mathrm{H}) ;{ }^{13} \mathrm{C}$ NMR $\left(125 \mathrm{MHz}, \mathrm{CDCl}_{3}\right) \delta 164.9,162.4,148.4,141.1,140.9,137.4,135.1,134.8$, $132.8,131.8,131.2,129.8,127.9,127.6,127.2,127.1,123.6,122.3,73.5,65.6,52.9,48.5$, 47.7, 44.8, 37.7, 33.0, 26.4, 20.8, 20.0. HRMS (ESI) calcd for $\mathrm{C}_{29} \mathrm{H}_{26} \mathrm{~N}_{2} \mathrm{O}_{7} \mathrm{~S}_{2} \mathrm{Cl}_{2}[\mathrm{M}+\mathrm{Na}]^{+}$: 671.0451. Found: 671.0457. Anal. calcd for $\mathrm{C}_{29} \mathrm{H}_{26} \mathrm{Cl}_{2} \mathrm{~N}_{2} \mathrm{O}_{7} \mathrm{~S}_{2}$ : C, 53.62; H, 4.03; N, 4.31 . Found: C, 53.78 H, 3.95; N, 4.23 .

IR (neat) 2959, 1732, 1685, 1532, 1339, 1300, 1243, 1169, 1141, 1116, 1090, $1064 \mathrm{~cm}^{-1}$. 
X-ray Structure Determination for $13 \mathbf{i}, 14 \mathbf{b}, 14 \mathbf{e}, 13 \mathrm{~g}, 13 \mathbf{j}, 13 \mathbf{k}, 14 \mathbf{c}, 14 \mathbf{f}$, and $14 \mathbf{n}$.

Measurements were made on a Rigaku Saturn CCD area detector equipped with graphite-monochromated Mo- $K \alpha$ radiation $(\lambda=0.71070 \AA)$ under nitrogen stream at $93 \mathrm{~K}$. Indexing was performed from eighteen images. The crystal-to-detector distance was $45.05 \mathrm{~mm}$. The data were collected to a maximum $2 \theta$ value of $55.0^{\circ}$. A total of 720 oscillation images were collected. A sweep of data was carried out using $\omega$ scans from -110.0 to $70.0^{\circ}$ in $0.5^{\circ}$ steps, at $\chi=45.0^{\circ}$ and $\phi=0.0^{\circ}$. A second sweep was performed using $\omega$ scans from -110.0 to $70.0^{\circ}$ in $0.5^{\circ}$ steps, at $\chi=45.0^{\circ}$ and $\phi=90.0^{\circ}$. Intensity data were collected for Lorentz-polarization effects as well as absorption. Structure solution and refinements were performed with the CrystalStructure program package. The heavy atom positions were determined by direct methods (SIR2002), and the remaining non-hydrogen atoms were found by subsequent Fourier techniques. An empirical absorption correction based on equivalent reflections was applied to all data. All non-hydrogen atoms other than solvent molecules were refined anisotropically by full-matrix least-square techniques based on $F^{2}$. All hydrogen atoms were constrained to ride on their parent atom. Relevant crystallographic data are compiled in Tables S3-S5.

Table S3. Crystallographic Data for 13i, 14b, 14e, and 13g

\begin{tabular}{|c|c|c|c|c|}
\hline & $13 \mathbf{i}$ & $14 \mathrm{~b}$ & $14 \mathrm{e}$ & $13 \mathrm{~g}$ \\
\hline empirical formula & $\mathrm{C}_{20} \mathrm{H}_{13} \mathrm{ClN}_{2} \mathrm{O}_{6}$ & $\mathrm{C}_{20} \mathrm{H}_{13} \mathrm{Cl}_{2} \mathrm{NO}_{4}$ & $\mathrm{C}_{20} \mathrm{H}_{13} \mathrm{ClN}_{2} \mathrm{O}_{6}$ & $\mathrm{C}_{34} \mathrm{H}_{35} \mathrm{Cl}_{2} \mathrm{NO}_{5} \mathrm{~S}$ \\
\hline formula weight & 412.79 & 402.23 & 412.79 & 640.62 \\
\hline crystal color & Colorless & Colorless & Colorless & Colorless \\
\hline crystal system & Monoclinic & Monoclinic & Orthorhombic & Orthorhombic \\
\hline space group & $P 2_{1}(\# 4)$ & $P 2_{1}(\# 4)$ & $P 2{ }_{1}{ }_{1} 2_{1}(\# 19)$ & $P 2{ }_{1}{ }_{1} 2_{1}(\# 19)$ \\
\hline$a, \AA$ & $13.373(4)$ & $5.902(2)$ & $7.210(2)$ & $10.5269(13)$ \\
\hline$b, \AA$ & $7.840(2)$ & $12.201(3)$ & $15.714(4)$ & $11.2332(14)$ \\
\hline$c, \AA$ & $18.471(6)$ & $12.309(3)$ & $15.938(4)$ & $26.231(3)$ \\
\hline$\beta, \operatorname{deg}$ & $109.497(4)$ & 101.322(4) & & \\
\hline$V, \AA^{3}$ & 1815.6(9) & $869.2(4)$ & $1805.7(7)$ & $3101.8(7)$ \\
\hline$Z$ & 4 & 2 & 4 & 4 \\
\hline$D_{\text {calcd, }} \mathrm{g} \mathrm{cm}^{-3}$ & 1.510 & 1.537 & 1.518 & 1.372 \\
\hline $\mathrm{F}_{000}$ & 848.00 & 412.00 & 848.00 & 1344.00 \\
\hline$\mu, \mathrm{cm}^{-1}(\mathrm{MoK} \alpha)$ & 2.531 & 4.006 & 2.544 & 3.198 \\
\hline Exposure rate & $16.0 \mathrm{sec} /{ }^{\circ}$ & $10.0 \mathrm{sec} /{ }^{\circ}$ & $16.0 \mathrm{sec} /{ }^{\circ}$ & $16.0 \mathrm{sec} /{ }^{\circ}$ \\
\hline no. of reflections measured & 15140 & 7112 & 15014 & 25817 \\
\hline no. of unique reflections & 7694 & 3893 & 4139 & 6996 \\
\hline no. of variables & 550 & 258 & 276 & 424 \\
\hline$R 1(I>2.00 \sigma(I))$ & 0.0604 & 0.0422 & 0.0393 & 0.0467 \\
\hline $\mathrm{w} R 2$ (All reflections) & 0.1009 & 0.0977 & 0.0883 & 0.1116 \\
\hline GOF on $F^{2}$ & 1.010 & 1.000 & 1.000 & 1.000 \\
\hline Flack parameter & $0.09(6)$ & $-0.00(5)$ & $-0.04(6)$ & $-0.06(5)$ \\
\hline
\end{tabular}
$R 1=\Sigma|| F_{\mathrm{o}}|-| F_{\mathrm{c}} \| / \Sigma\left|F_{\mathrm{o}}\right|, \mathrm{w} R 2=\left[\Sigma\left(w\left(F_{\mathrm{o}}{ }^{2}-F_{\mathrm{c}}{ }^{2}\right)^{2}\right) / \Sigma w\left(F_{\mathrm{o}}{ }^{2}\right)^{2}\right]^{1 / 2}$. 
Table S4. Crystallographic Data for $\mathbf{1 3 j}$, 13k, and $\mathbf{1 4 c}$

\begin{tabular}{|c|c|c|c|}
\hline & 13j & 13k & $14 \mathrm{c}$ \\
\hline empirical formula & $\mathrm{C}_{31} \mathrm{H}_{27} \mathrm{Cl}_{2} \mathrm{~F}_{2} \mathrm{NO}_{5} \mathrm{~S}$ & $\mathrm{C}_{32} \mathrm{H}_{27} \mathrm{Cl}_{2} \mathrm{~F}_{4} \mathrm{NO}_{5} \mathrm{~S}$ & $\mathrm{C}_{31} \mathrm{H}_{27} \mathrm{Cl}_{2} \mathrm{~F}_{2} \mathrm{NO}_{5} \mathrm{~S}$ \\
\hline formula weight & 634.52 & 684.53 & 634.52 \\
\hline crystal color & Colorless & Colorless & Colorless \\
\hline crystal system & Monoclinic & Monoclinic & Orthorhombic \\
\hline space group & $C 2(\# 5)$ & $C 2(\# 5)$ & $C 2(\# 5)$ \\
\hline$a, \AA$ & $33.429(9)$ & $24.814(7)$ & $31.304(13)$ \\
\hline$b, \AA$ & $7.746(2)$ & $7.731(2)$ & $7.830(3)$ \\
\hline$c, \AA$ & $12.033(3)$ & $19.580(5)$ & $12.515(6)$ \\
\hline$\beta, \operatorname{deg}$ & 111.194(4) & $126.078(3)$ & $110.626(6)$ \\
\hline$V, \AA^{3}$ & 2924.3(12) & $3035.7(13)$ & $2871(2)$ \\
\hline Z & 4 & 4 & 4 \\
\hline$D_{\text {calcd }}, \mathrm{g} \mathrm{cm}^{-3}$ & 1.441 & 1.498 & 1.468 \\
\hline$F_{000}$ & 1312.00 & 1408.00 & 1312.00 \\
\hline$\mu, \mathrm{cm}^{-1}(\mathrm{MoK} \alpha)$ & 3.479 & 3.502 & 3.544 \\
\hline Exposure rate & $6.0 \mathrm{sec} /{ }^{\circ}$ & $10.0 \mathrm{sec} /{ }^{\circ}$ & $4.0 \mathrm{sec} /{ }^{\circ}$ \\
\hline no. of reflections measured & 12127 & 12547 & 11834 \\
\hline no. of unique reflections & 5792 & 6422 & 6423 \\
\hline no. of variables & 407 & 434 & 407 \\
\hline$R 1(I>2.00 \sigma(I))$ & 0.0437 & 0.0385 & 0.0360 \\
\hline $\mathrm{w} R 2$ (All reflections) & 0.1023 & 0.0906 & 0.0854 \\
\hline GOF on $F^{2}$ & 1.000 & 1.000 & 1.000 \\
\hline Flack parameter & $-0.08(6)$ & $-0.03(5)$ & $-0.02(4)$ \\
\hline
\end{tabular}

$R 1=\Sigma|| F_{\mathrm{o}}|-| F_{\mathrm{c}} \| / \Sigma\left|F_{\mathrm{o}}\right|, \mathrm{w} R 2=\left[\Sigma\left(w\left(F_{\mathrm{o}}^{2}-F_{\mathrm{c}}^{2}\right)^{2}\right) / \Sigma w\left(F_{\mathrm{o}}{ }^{2}\right)^{2}\right]^{1 / 2}$.

Table S5. Crystallographic Data for $\mathbf{1 4 f}$ and $\mathbf{1 4 n}$

\begin{tabular}{|c|c|c|}
\hline & $14 f$ & $14 n$ \\
\hline empirical formula & $\mathrm{C}_{31} \mathrm{H}_{26} \mathrm{Cl}_{2} \mathrm{~F}_{3} \mathrm{NO}_{5} \mathrm{~S}$ & $\mathrm{C}_{29} \mathrm{H}_{26} \mathrm{Cl}_{2} \mathrm{~N}_{2} \mathrm{O}_{7} \mathrm{~S}_{2}$ \\
\hline formula weight & 652.51 & 649.56 \\
\hline crystal color & Colorless & Colorless \\
\hline crystal system & Monoclinic & Monoclinic \\
\hline space group & $P 2_{1}(\# 4)$ & $P 2{ }_{1} 2_{1}(\# 19)$ \\
\hline$a, \AA$ & $7.590(2)$ & $7.0524(10)$ \\
\hline$b, \AA$ & $33.801(6)$ & $15.8433(22)$ \\
\hline$c, \AA$ & $12.053(2)$ & $25.4267(31)$ \\
\hline$\beta$, deg & $107.278(3)$ & \\
\hline$V, \AA^{3}$ & $2952.9(10)$ & $2841.0088(0)$ \\
\hline$Z$ & 4 & 4 \\
\hline$D_{\text {calcd }}, \mathrm{g} \mathrm{cm}^{-3}$ & 1.468 & 1.519 \\
\hline $\mathrm{F}_{000}$ & 1344.00 & 1344.00 \\
\hline$\mu, \mathrm{cm}^{-1}(\mathrm{MoK} \alpha)$ & 3.514 & 4.270 \\
\hline Exposure rate & $10.0 \mathrm{sec} /{ }^{\circ}$ & $10.0 \mathrm{sec} /{ }^{\circ}$ \\
\hline no. of reflections measured & 24300 & 23544 \\
\hline no. of unique reflections & 11664 & 6486 \\
\hline no. of variables & 828 & 405 \\
\hline$R 1(I>2.00 \sigma(I))$ & 0.0402 & 0.0416 \\
\hline $\mathrm{w} R 2$ (All reflections) & 0.0961 & 0.0989 \\
\hline GOF on $F^{2}$ & 1.000 & 1.000 \\
\hline Flack parameter & $-0.03(4)$ & $-0.02(5)$ \\
\hline
\end{tabular}


Figure S2. X-ray crystallographic structure of 13i.
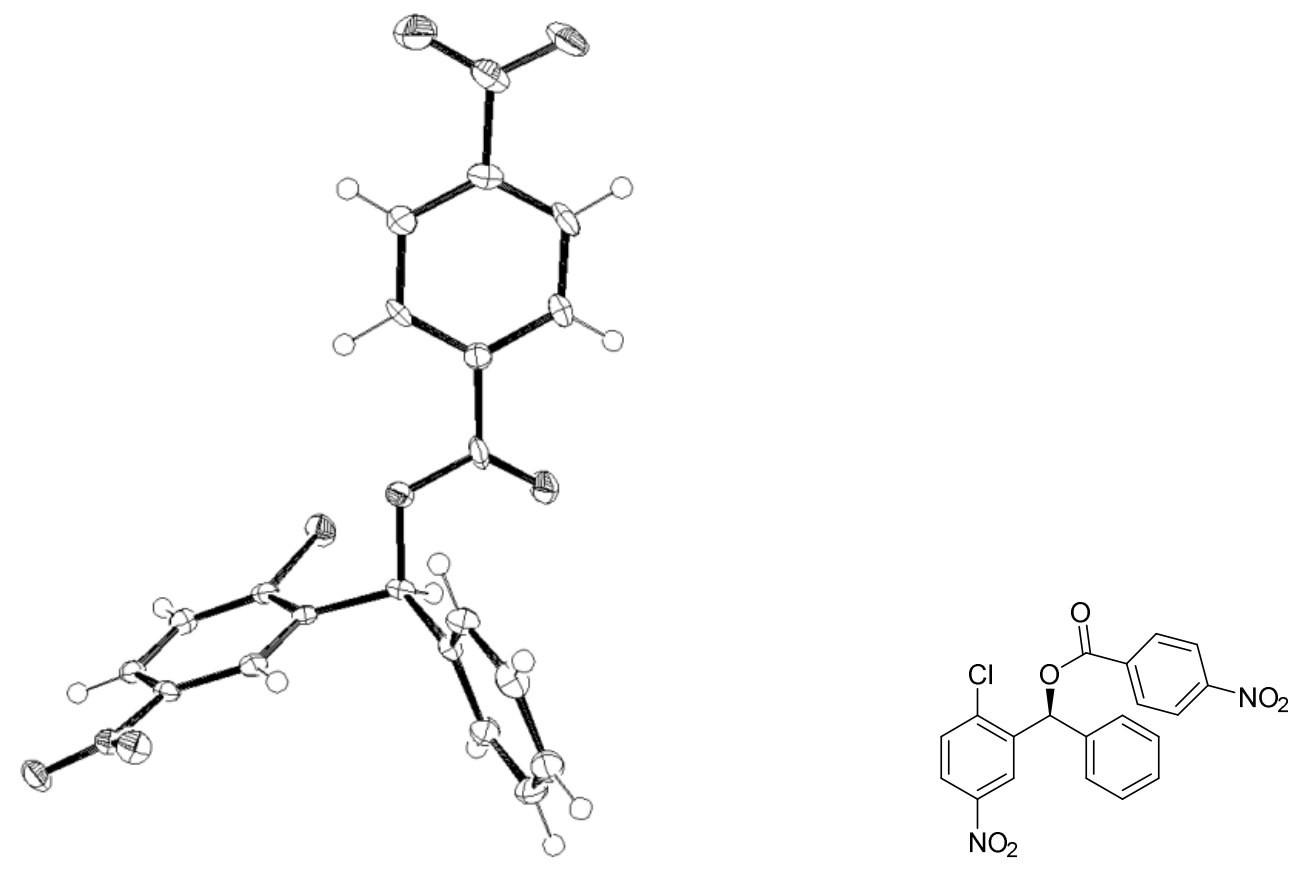

Figure S3. X-ray crystallographic structure of $\mathbf{1 4 b}$.

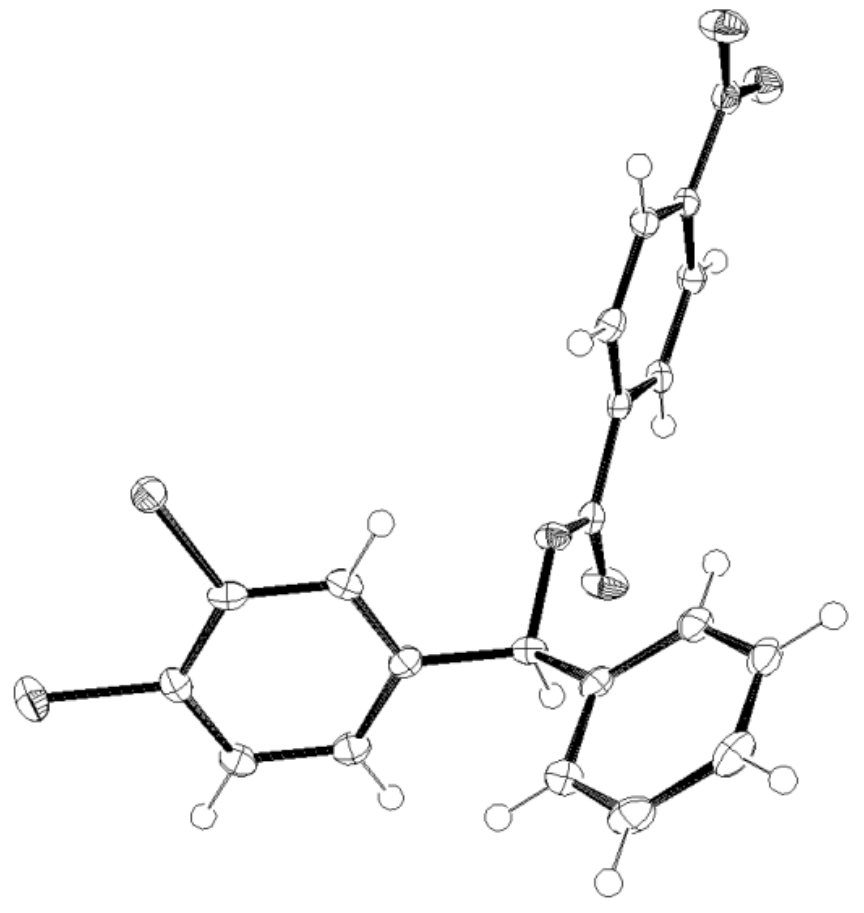<smiles>O=C(OC(c1ccccc1)c1ccc(Cl)c(Cl)c1)c1ccc([N+](=O)[O-])cc1</smiles> 
Figure S4. X-ray crystallographic structure of $14 \mathrm{e}$.
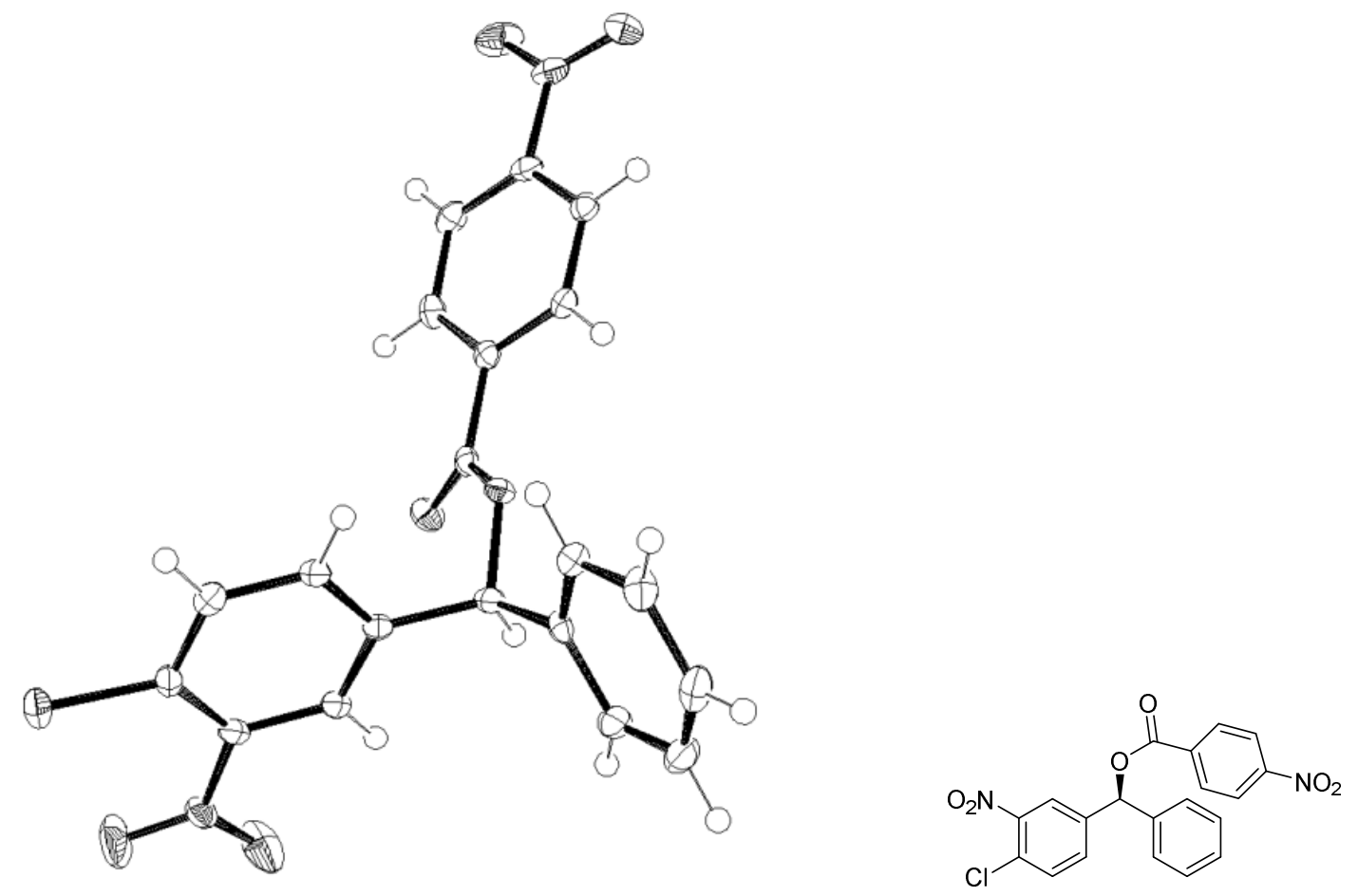

Figure S5. X-ray crystallographic structure of 13g. All hydrogens except those attached to chiral carbons are omitted for clarity.
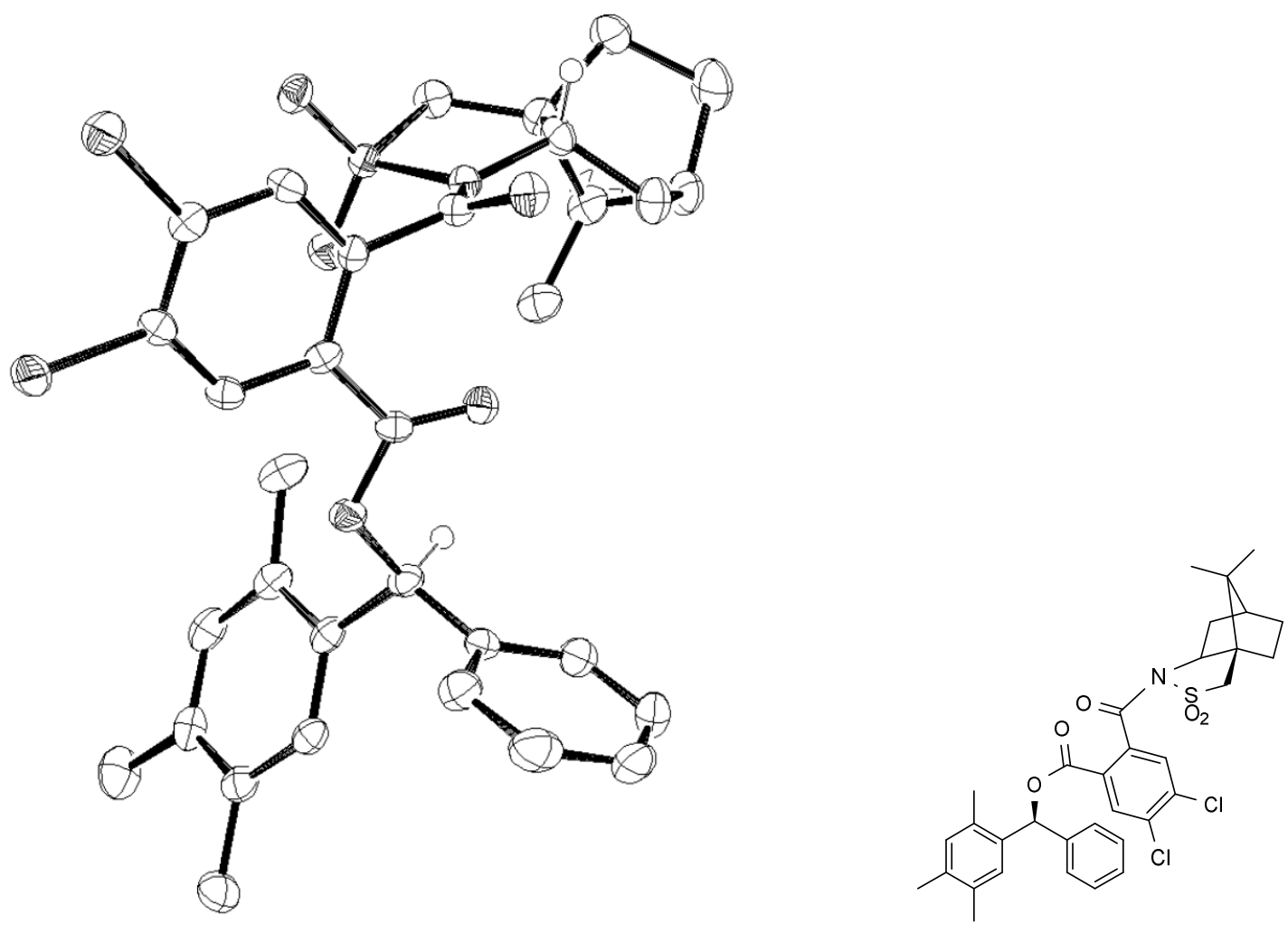
Figure S6. X-ray crystallographic structure of $\mathbf{1 3 j}$. All hydrogens except those attached to chiral carbons are omitted for clarity.
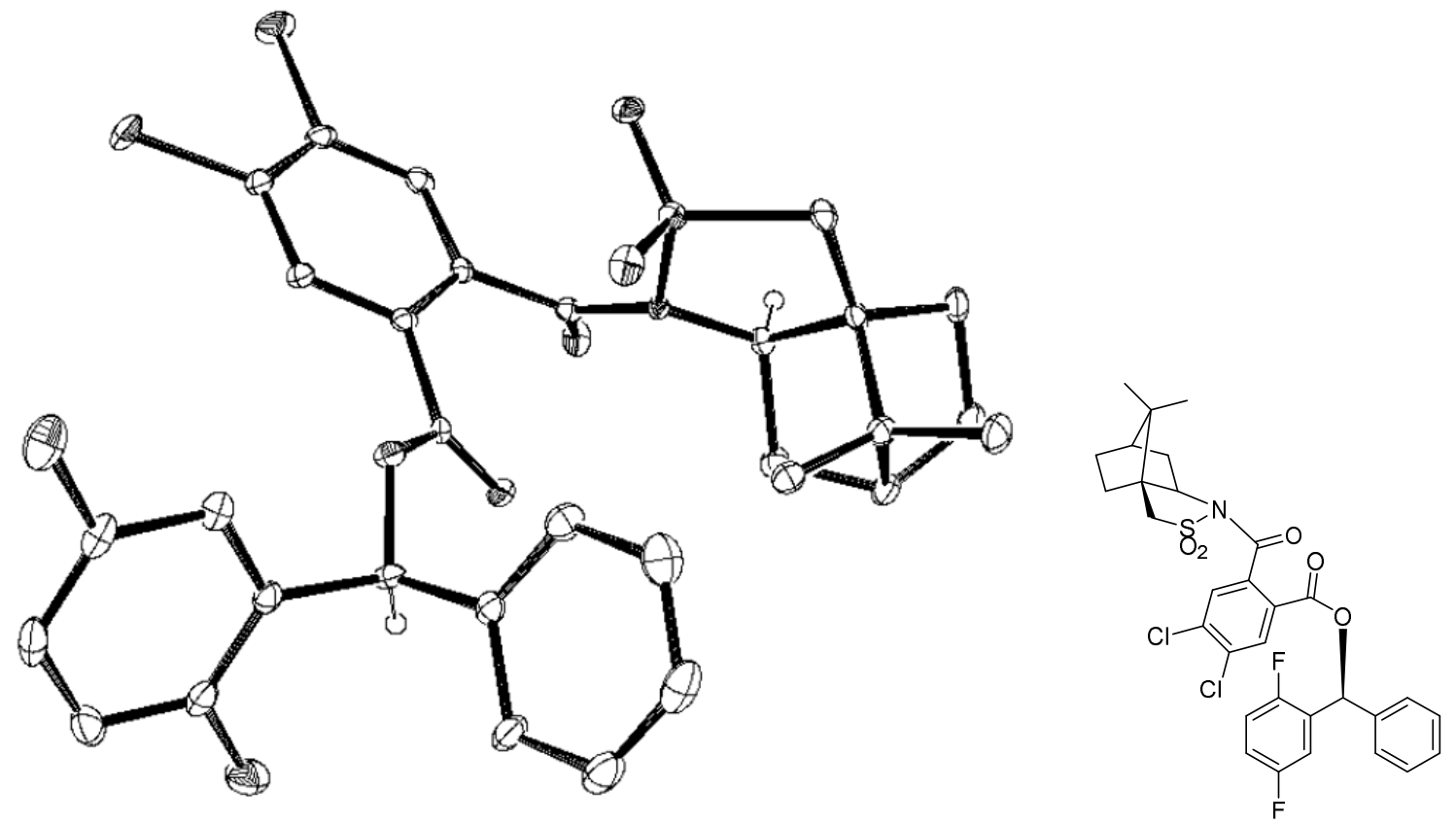

Figure S7. X-ray crystallographic structure of 13k. All hydrogens except those attached to chiral carbons are omitted for clarity.
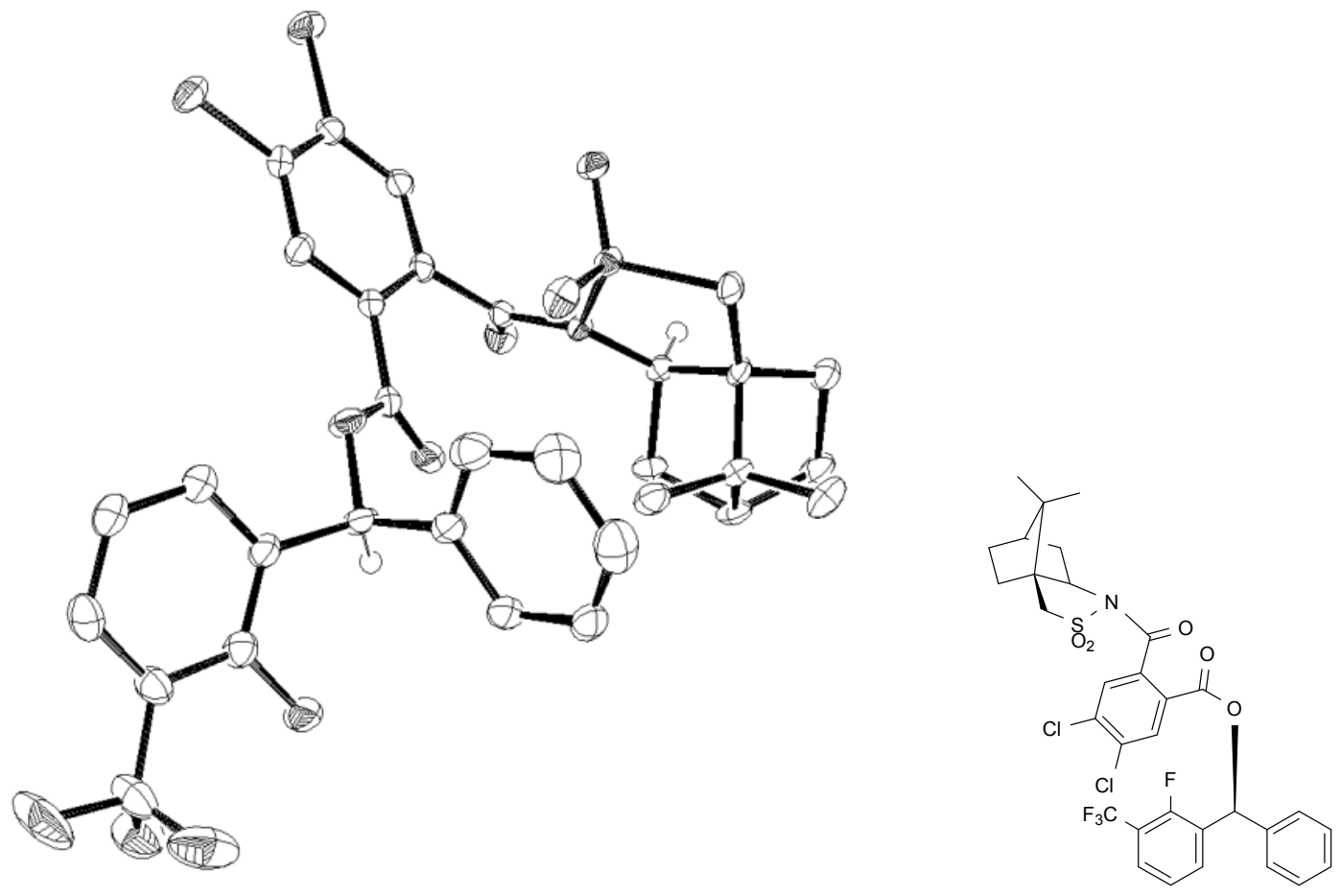
Figure S8. X-ray crystallographic structure of 14c. All hydrogens except those attached to chiral carbons are omitted for clarity.
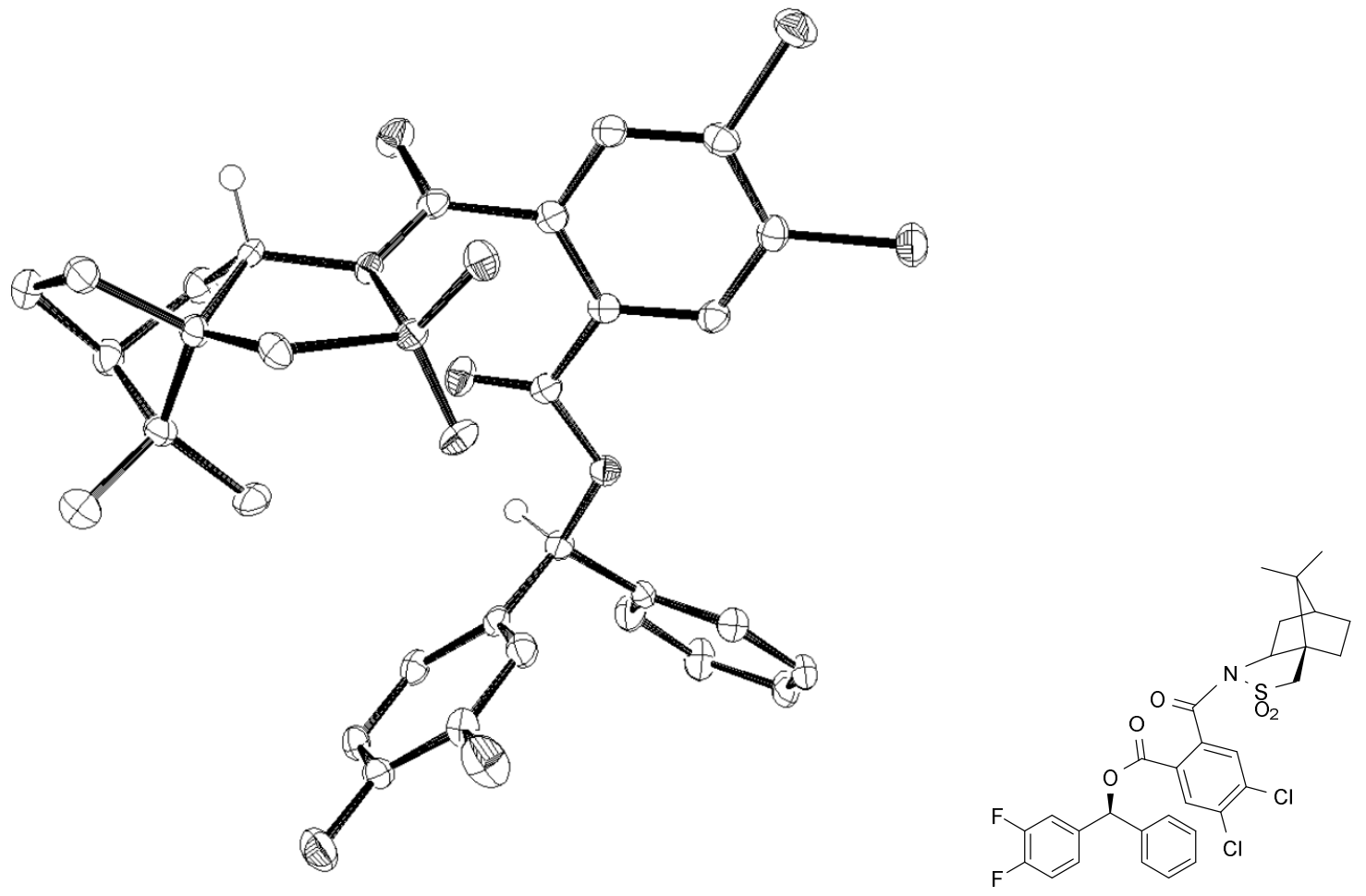

Figure S9. X-ray crystallographic structure of 14f. All hydrogens except those attached to chiral carbons are omitted for clarity.
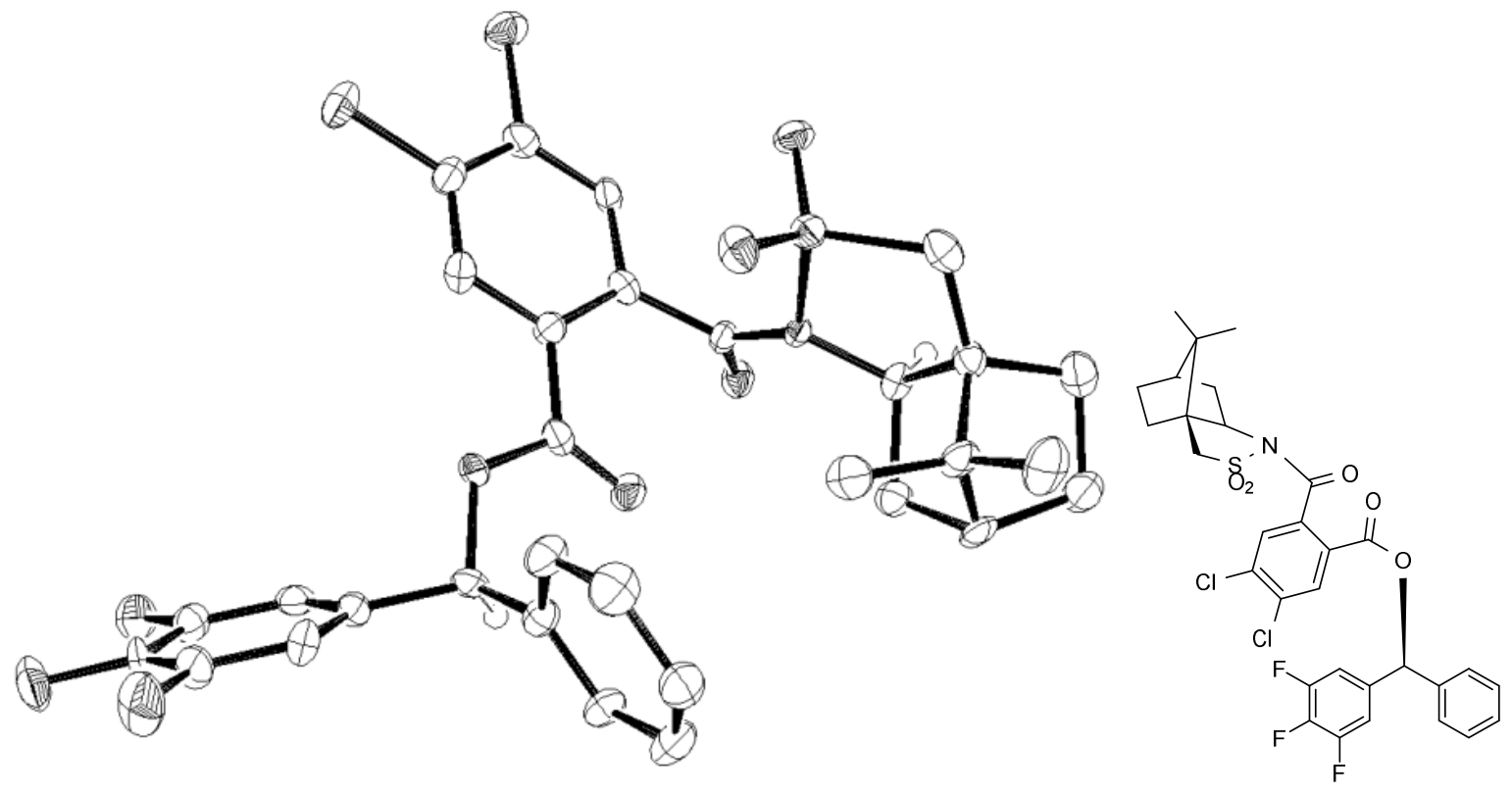
Figure S10. X-ray crystallographic structure of 14n. All hydrogens except those attached to chiral carbons are omitted for clarity.
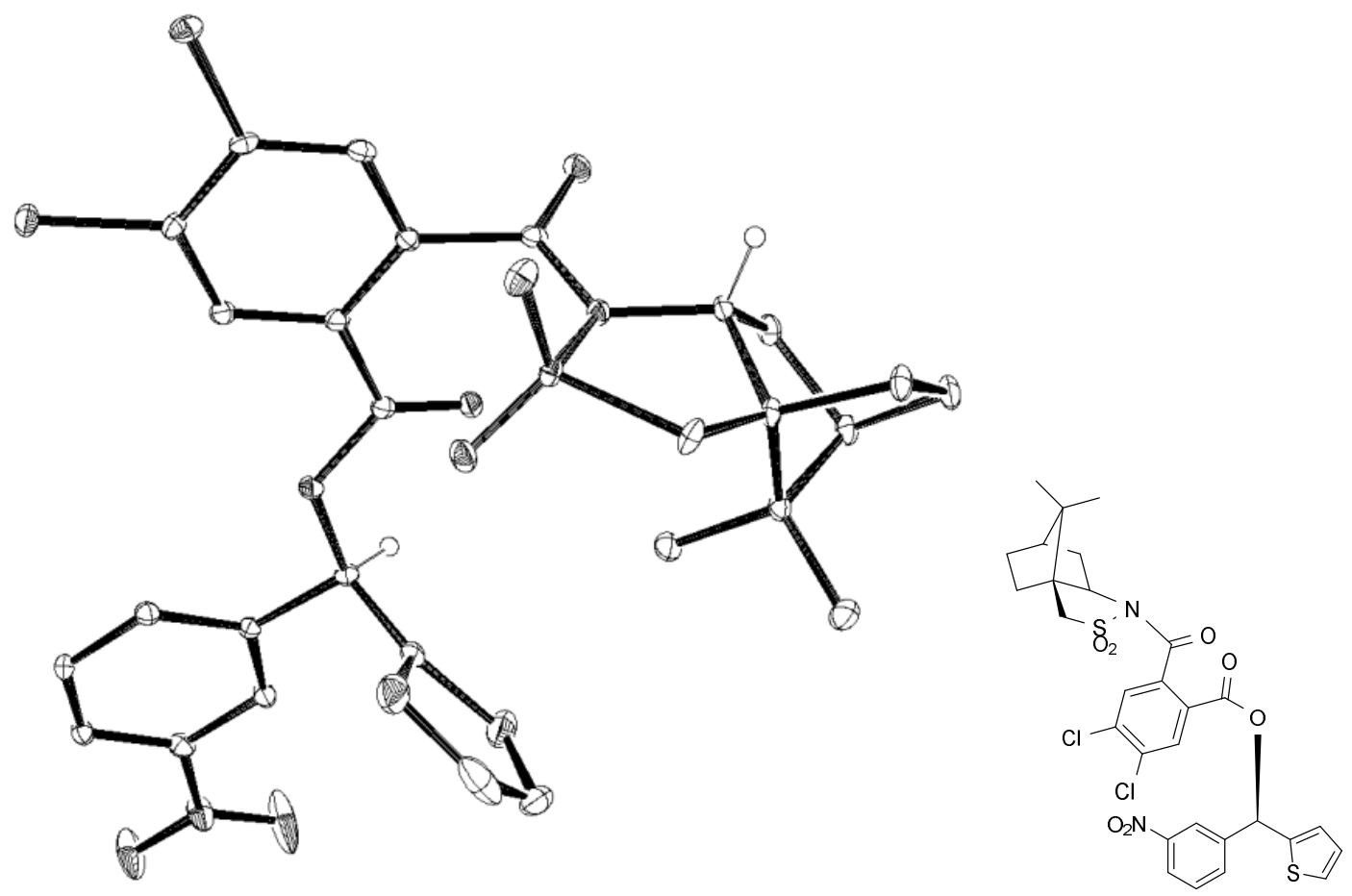

\section{F. References}

(1) Woydziak, Z. R.; Fu, L.; Peterson, B. R. J. Org. Chem. 2012, 77, 473.

(2) Ohkuma, T.; Koizumi, M.; Ikehira, H.; T. Yokozawa, T.; Noyori, R. Org. Lett. 2000, 2, 659.

(3) Morikawa, S.; Michigami, K.; Amii, H. Org. Lett. 2010, 12, 2520.

(4) Uenishi, A.; Nakagawa, Y.; Osumi, H.; Harada, T. Chem. Eur. J. 2013, 19, 4896.

(5) Duan, H.-F.; Xie, J.-H.; Shi, W.-J.; Zhang, Q.; Zhou, Q.-L. Org. Lett. 2006, 8, 1479.

(6) Yamamoto, Y.; Kurihara, K.; Miyaura, N. Angew. Chem. Int. Ed. 2009, 48, 4414.

(7) Kokura, A.; Tanaka, S.; Ikeno, T.; Yamada, T. Org. Lett. 2006, 8, 3025.

(8) Salvi, L.; Kim, J. G.; Walsh, P. J. J. Am. Chem. Soc. 2009, 131, 12483.

(9) Wang, M.-C.; Zhang, Q.-J.; Zhao, W.-X.; Wang, X.-D.; Ding, X.; Jing, T.-T.; Song, M.-P. J. Org. Chem. 2008, 73, 168.

(10) Terada, M.; Moriya, K.; Kanomata, K.; Sorimachi, K. Angew. Chem. Int. Ed. 2011, 52, 12586. 


\section{G. NMR Spectra for Products.}

Phenyl(3,4,5-trifluorophenyl)methanone (10f)

${ }^{1} \mathrm{H}$ NMR $\left(400 \mathrm{MHz}, \mathrm{CDCl}_{3}\right)$

Agilent Technologies

150601-2

$\begin{array}{ll}\text { Sample Name } & \text { 150601-2 } \\ \text { Date collected } & \text { 2015-06-01 }\end{array}$

Pulse sequence PROTON
Solvent cdcl3

Temperature
Spectrometer

Study owner vnmr1
Operator vnmr1

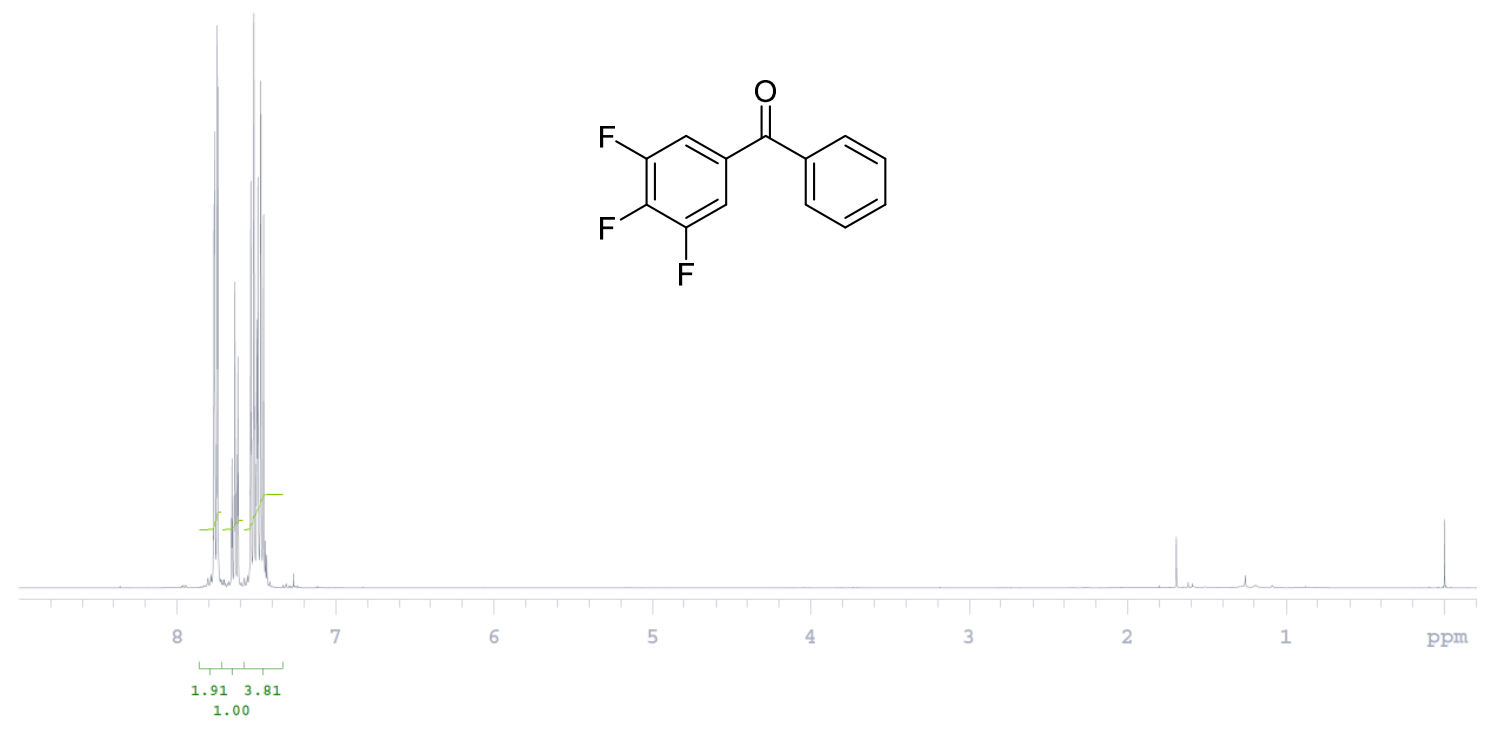

${ }^{13} \mathrm{C}$ NMR (125 MHz, $\left.\mathrm{CDCl}_{3}\right)$
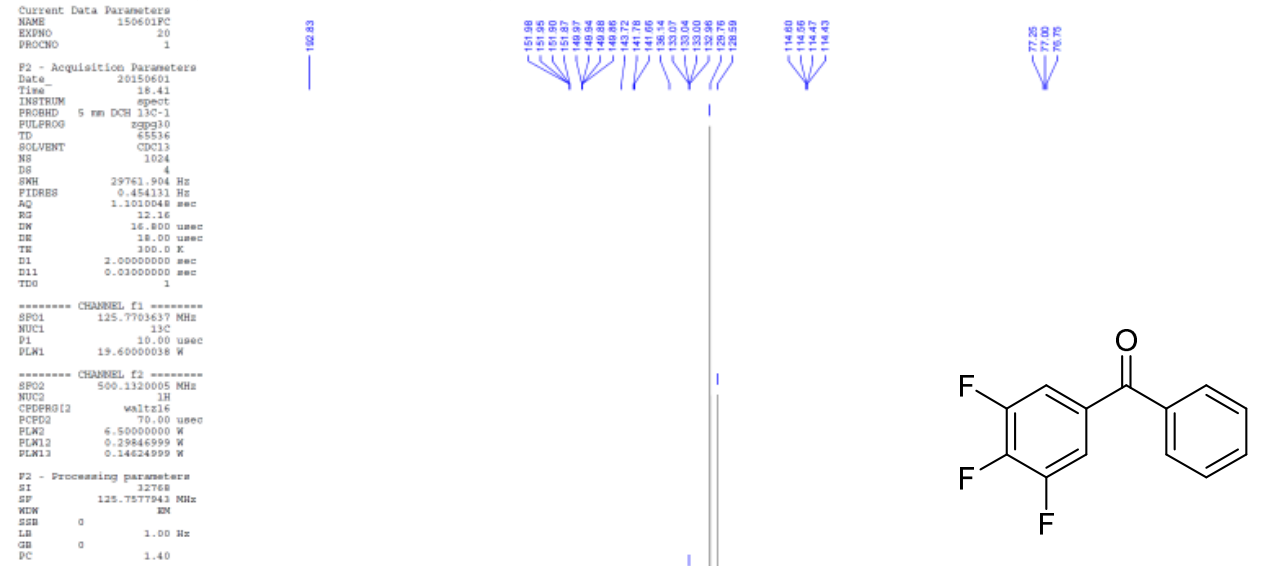


\section{(3,5-Dinitrophenyl)(phenyl)methanone (10g)}

${ }^{1} \mathrm{H}$ NMR (400 MHz, $\left.\mathrm{CDCl}_{3}\right)$

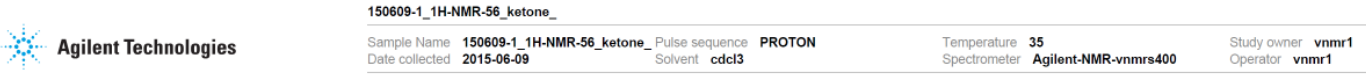

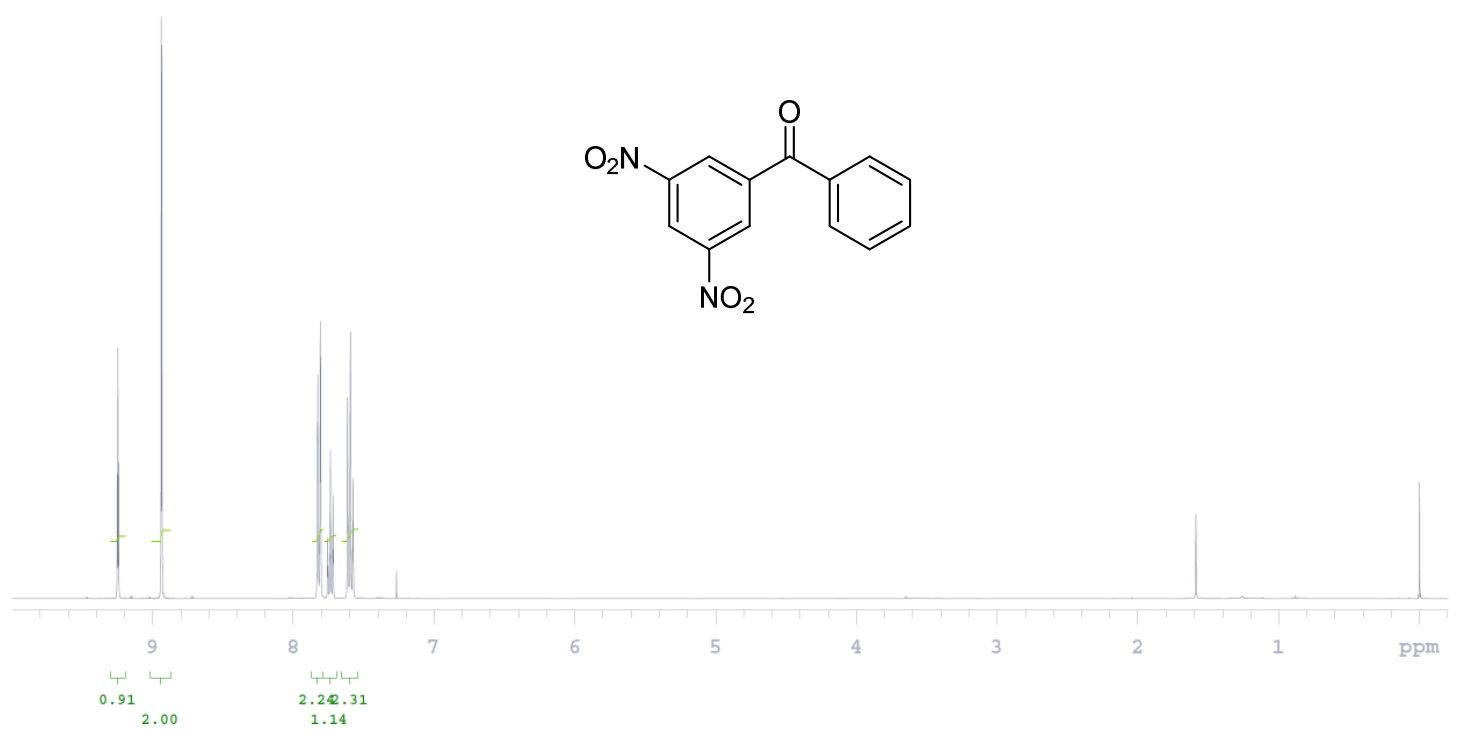

${ }^{13} \mathrm{C}$ NMR $\left(125 \mathrm{MHz}, \mathrm{CDCl}_{3}\right)$
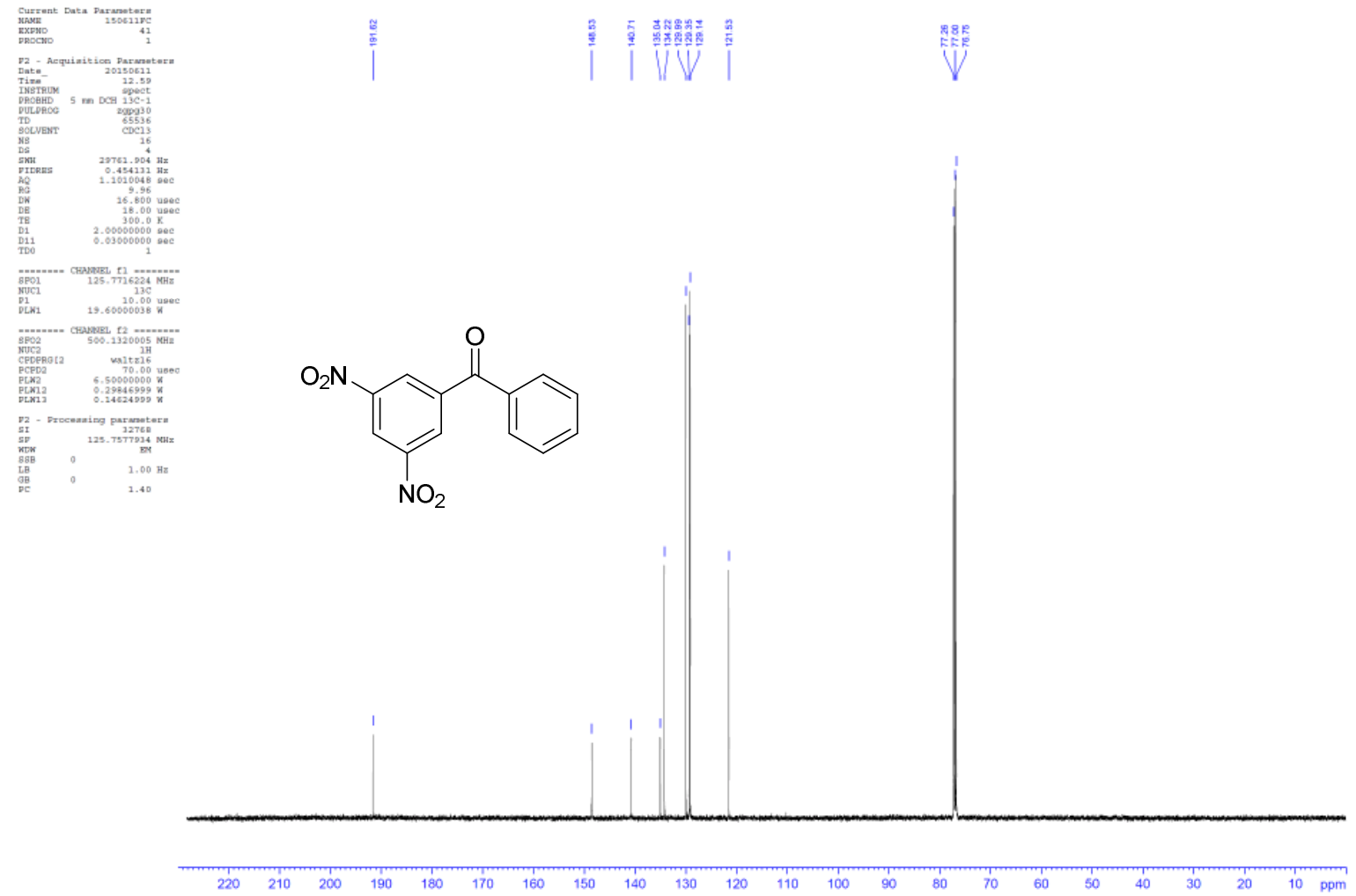


\section{(3,5-Dinitrophenyl)(4-methoxyphenyl)methanone (10I)}

${ }^{1} \mathrm{H}$ NMR $\left(400 \mathrm{MHz}, \mathrm{CDCl}_{3}\right)$

150609-2__H-NMR.62_ketone_

Agilent Technologies

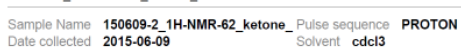

Temperature 35

Agilent-NMR-vnmrs 400

Study oumer vnmm1
Operator vnmer

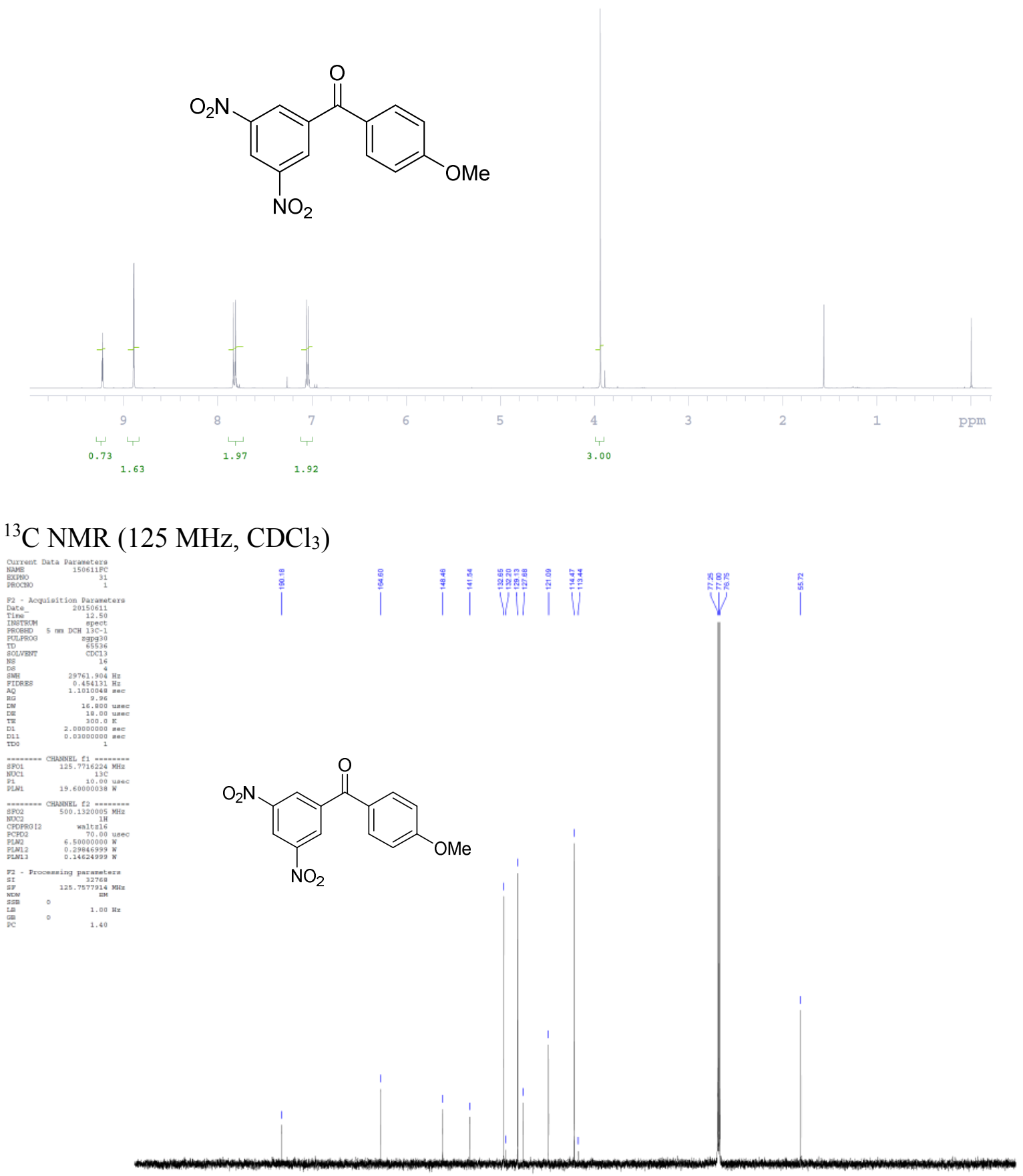

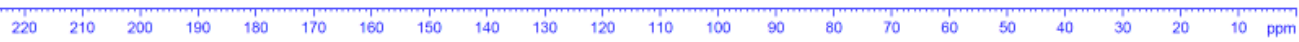




\section{(3-Nitrophenyl)(thiophen-2-yl)methanone (10n)}

${ }^{1} \mathrm{H}$ NMR $\left(400 \mathrm{MHz}, \mathrm{CDCl}_{3}\right)$

Agilent Technologies

150708-1_1H-NMR-64_ketotone_

Sample Name 150708-114-1H-NMR-64_ketone_Pulse sequence PROTON
Date collected 2015-07-08

Temperature 35
Spectrometer

Agilent-NMR-vnmmrs400

Study owner vnmr1
Operator vnmr1<smiles>O=C(c1cccc([N+](=O)[O-])c1)c1cccs1</smiles>
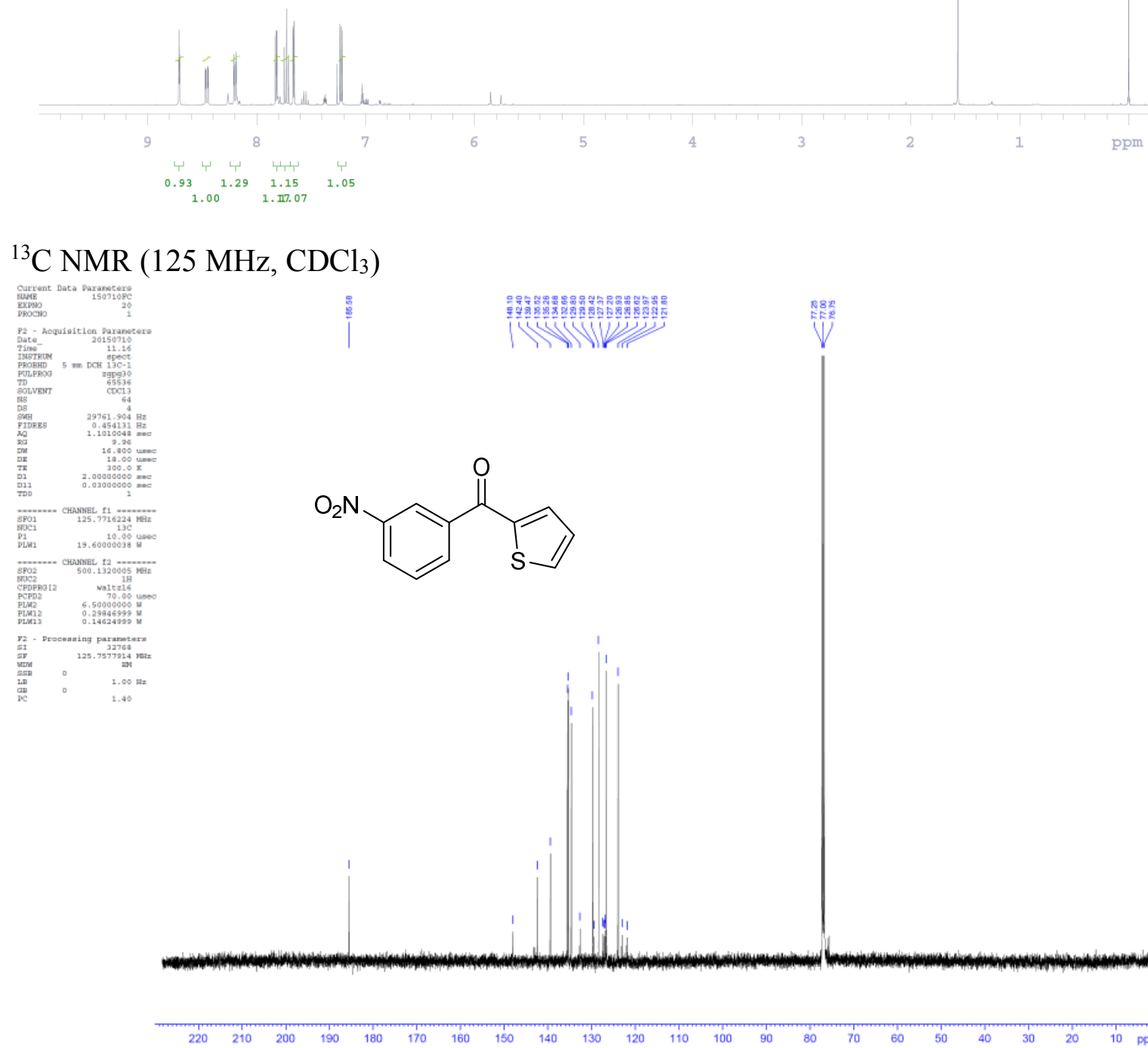


\section{(S)-Phenyl(o-tolyl)methanol (6a)}

${ }^{1} \mathrm{H}$ NMR (400 MHz, $\left.\mathrm{CDCl}_{3}\right)$

\begin{tabular}{|c|c|c|c|c|}
\hline & 150323-2 (1H-NMR-01) & & & \\
\hline jilent Technologies & $\begin{array}{ll}\text { Sample Name } & 150323-2 \text { (1H-NMR-01) } \\
\text { Date collected } & 2015-03-23\end{array}$ & $\begin{array}{l}\text { Pulse sequence PROTON } \\
\text { Solvent cdcl3 }\end{array}$ & $\begin{array}{l}\text { Temperature } 35 \\
\text { Spectrometer } \\
\text { Agilent-NMR-vnmrs } 400\end{array}$ & $\begin{array}{l}\text { Study owner vnmr1 } \\
\text { Operator vnmr1 }\end{array}$ \\
\hline
\end{tabular}

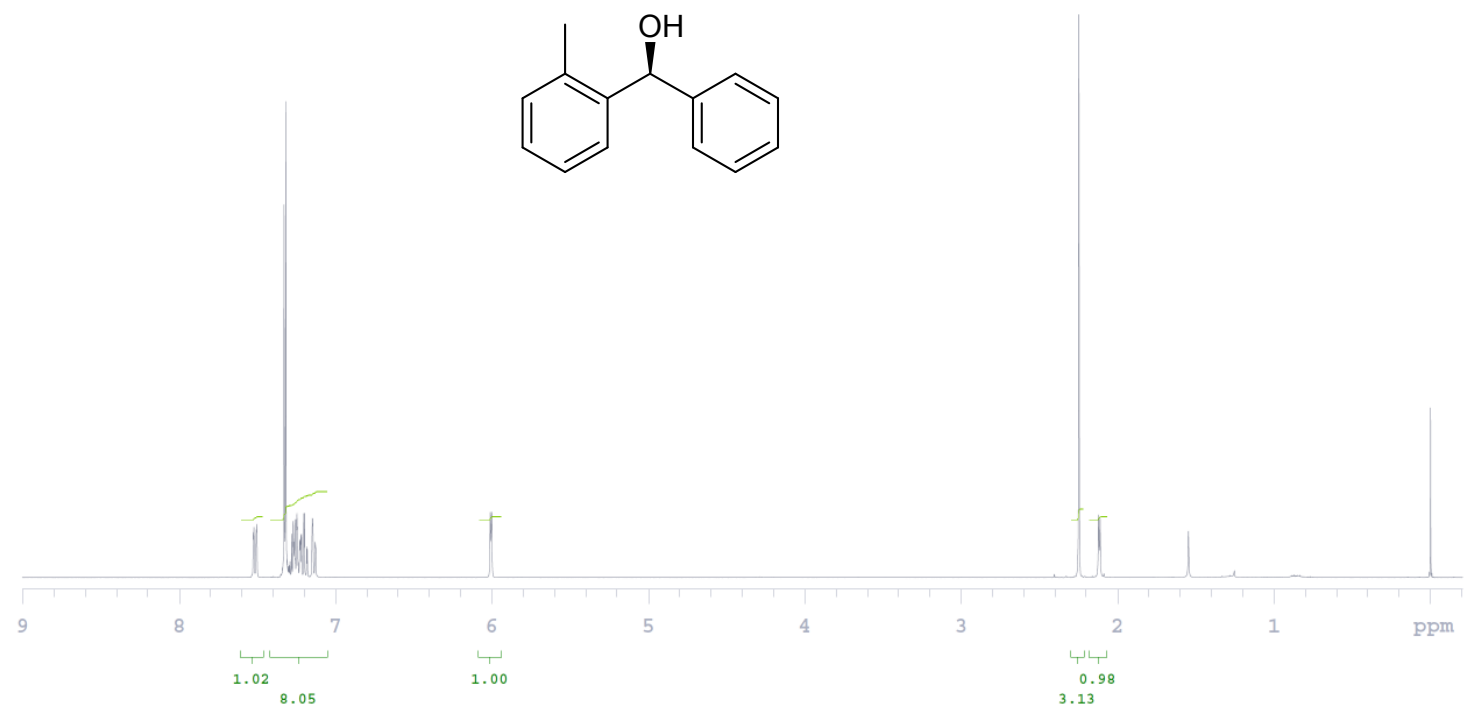

${ }^{13} \mathrm{C}$ NMR $\left(125 \mathrm{MHz}, \mathrm{CDCl}_{3}\right)$
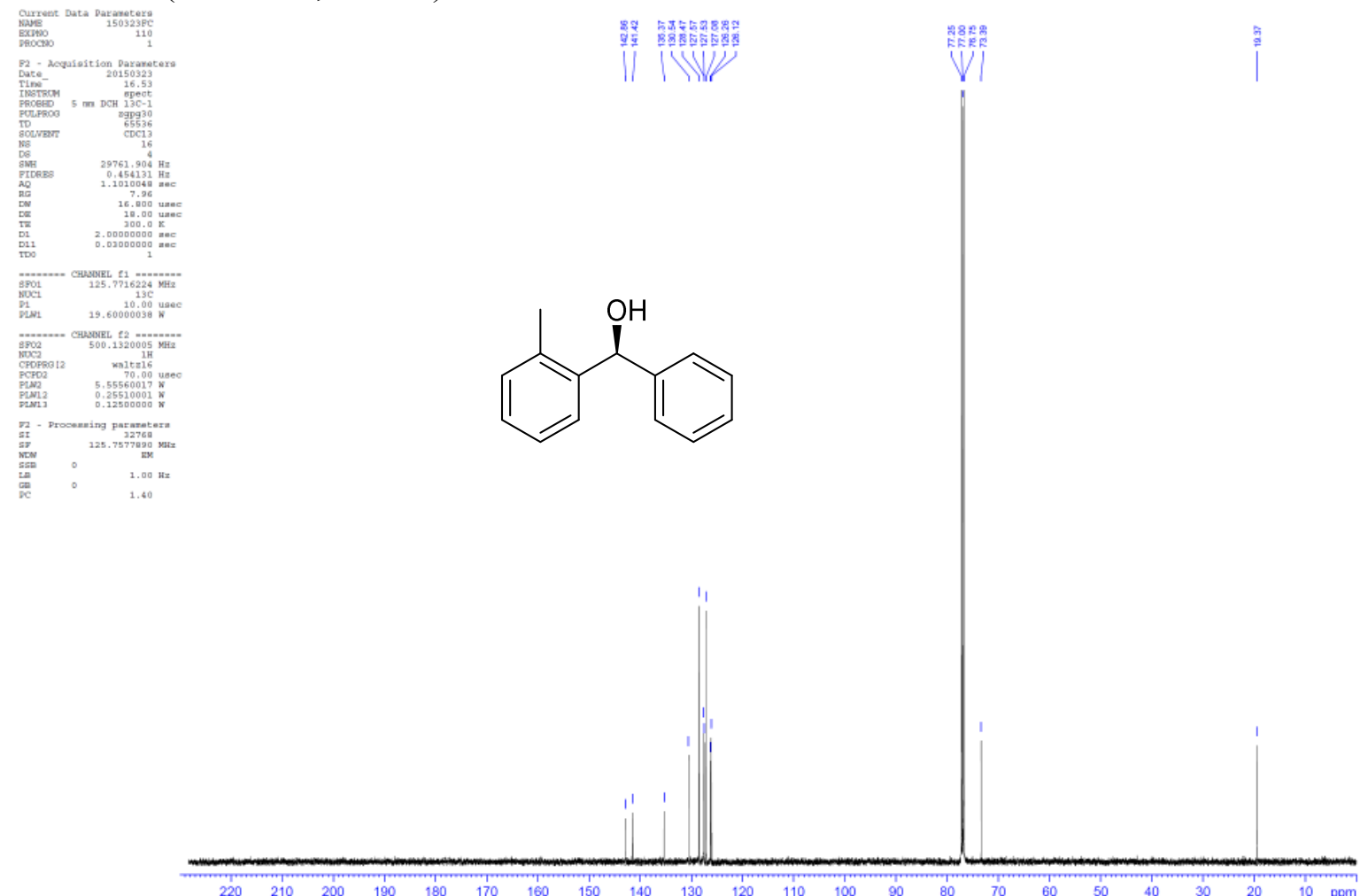


\section{(S)-(2-Chlorophenyl)(phenyl)methanol (6b)}

${ }^{1} \mathrm{H}$ NMR $\left(400 \mathrm{MHz}, \mathrm{CDCl}_{3}\right)$

\begin{tabular}{|c|c|c|c|c|}
\hline & 150323-3 (1H-NMR-02) & & & \\
\hline Agilent Technologies & $\begin{array}{l}\text { Sample Name } 150323-3(1 \mathrm{H}-\mathrm{NMR}-02) \\
\text { Date collected } 2015-03-23\end{array}$ & $\begin{array}{l}\text { Pulse sequence PROTON } \\
\text { Solvent cdcl3 }\end{array}$ & $\begin{array}{l}\text { Temperature } 35 \\
\text { Spectrometer } \\
\text { Agilent-NMR-vnmrs } 400\end{array}$ & $\begin{array}{l}\text { Study owner vnmr1 } \\
\text { Operator vnmr1 }\end{array}$ \\
\hline
\end{tabular}

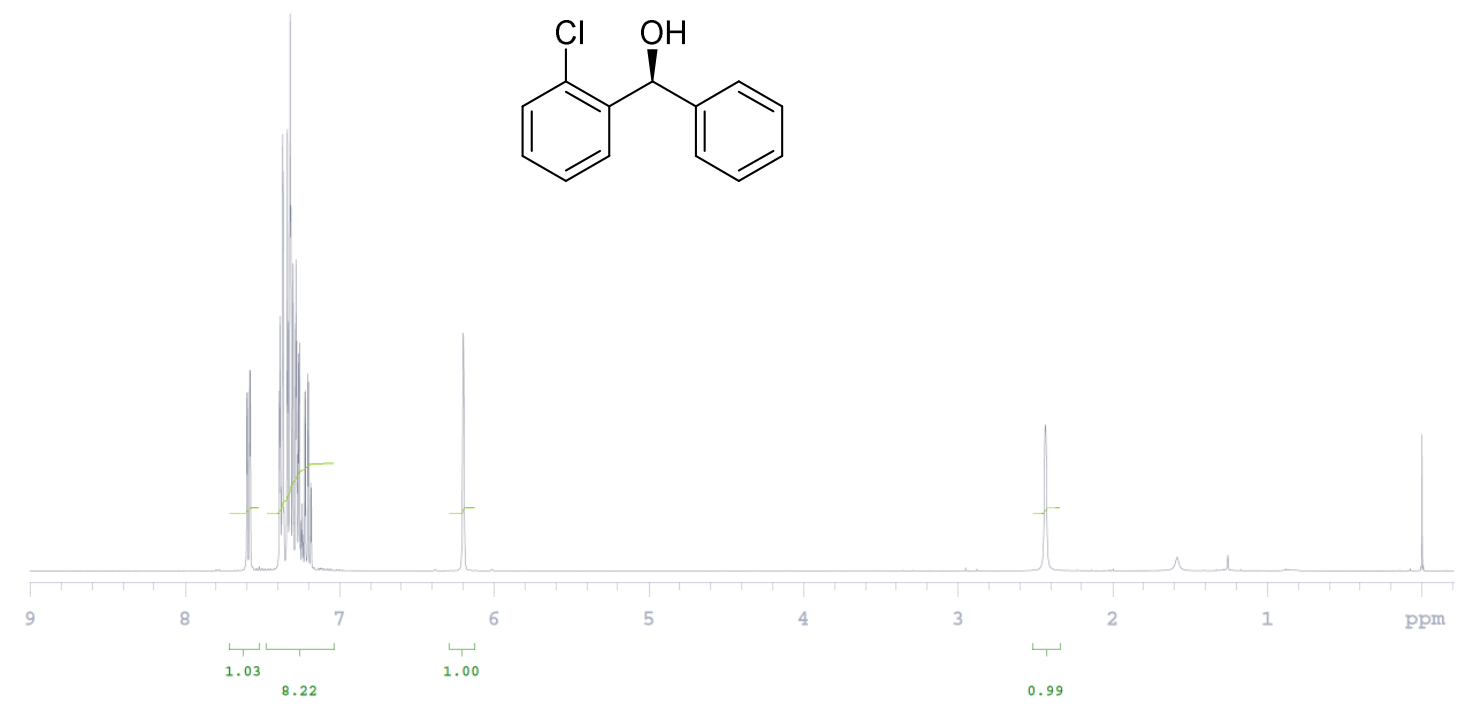

${ }^{13} \mathrm{C} \mathrm{NMR}\left(125 \mathrm{MHz}, \mathrm{CDCl}_{3}\right)$

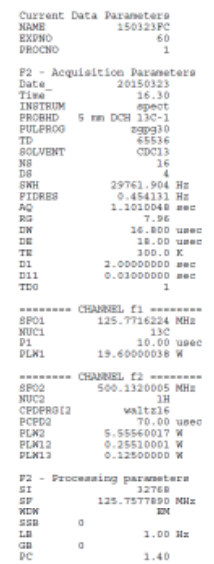<smiles>OC(c1ccccc1)c1ccccc1Cl</smiles>
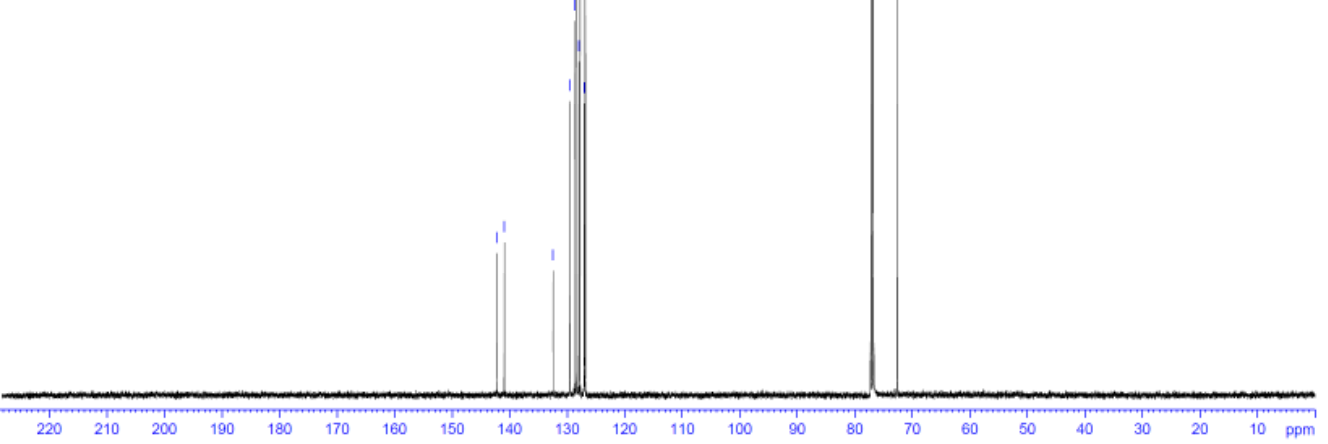


\section{(S)-(2-Bromophenyl)(phenyl)methanol (6c)}

${ }^{1} \mathrm{H}$ NMR $\left(400 \mathrm{MHz}, \mathrm{CDCl}_{3}\right)$

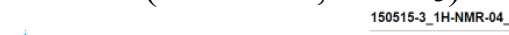

Agilent Technologies

Sample Name 150515-3 11-NMR-04

Pusse sequence PROTON
Solvent cdcl3

Temperature 35

Study owner vnmr
Opoerator vnmer

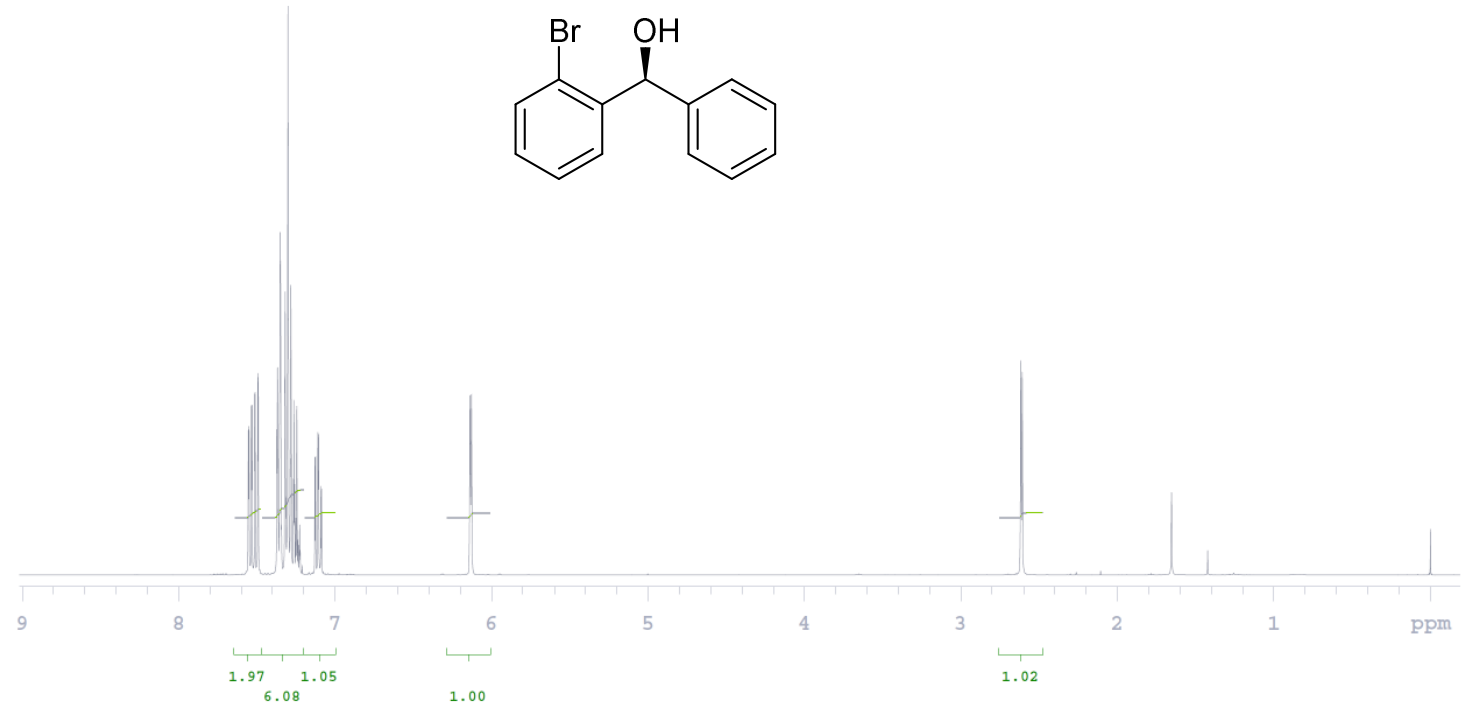

${ }^{13} \mathrm{C}$ NMR $\left(125 \mathrm{MHz}, \mathrm{CDCl}_{3}\right)$

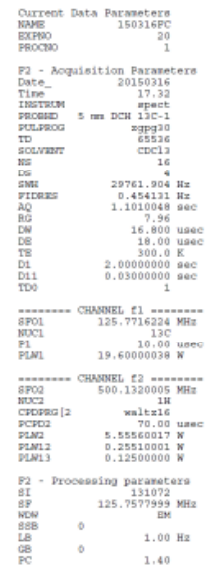

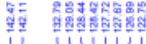

$\checkmark$ IVI

$\sqrt{2}$

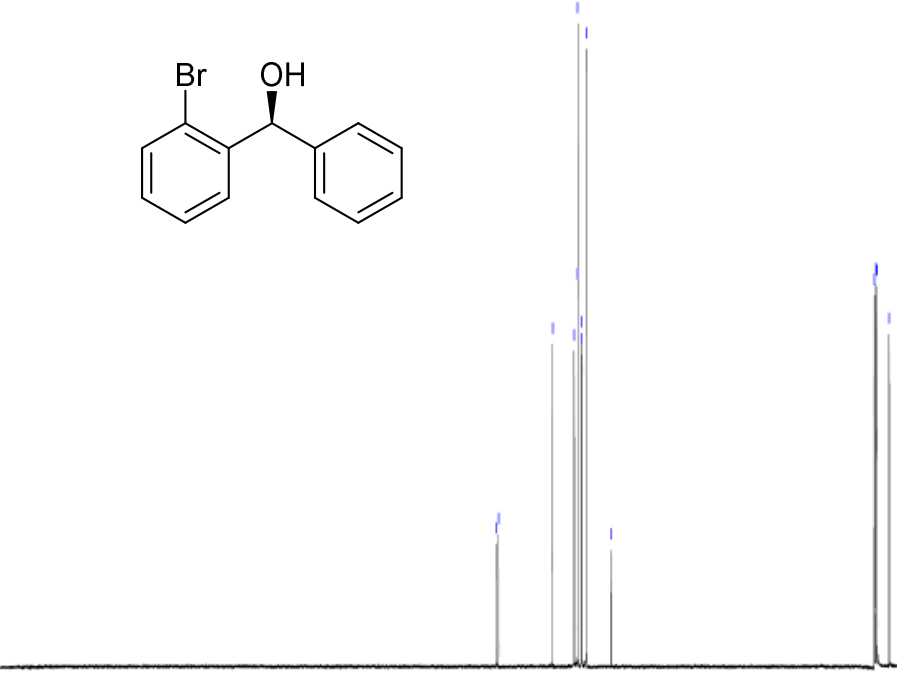

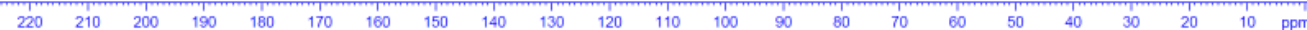




\section{(S)-Phenyl(2-(trifluoromethyl)phenyl)methanol (6d)}

${ }^{1} \mathrm{H}$ NMR $\left(400 \mathrm{MHz}, \mathrm{CDCl}_{3}\right)$

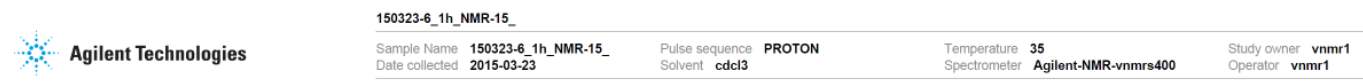

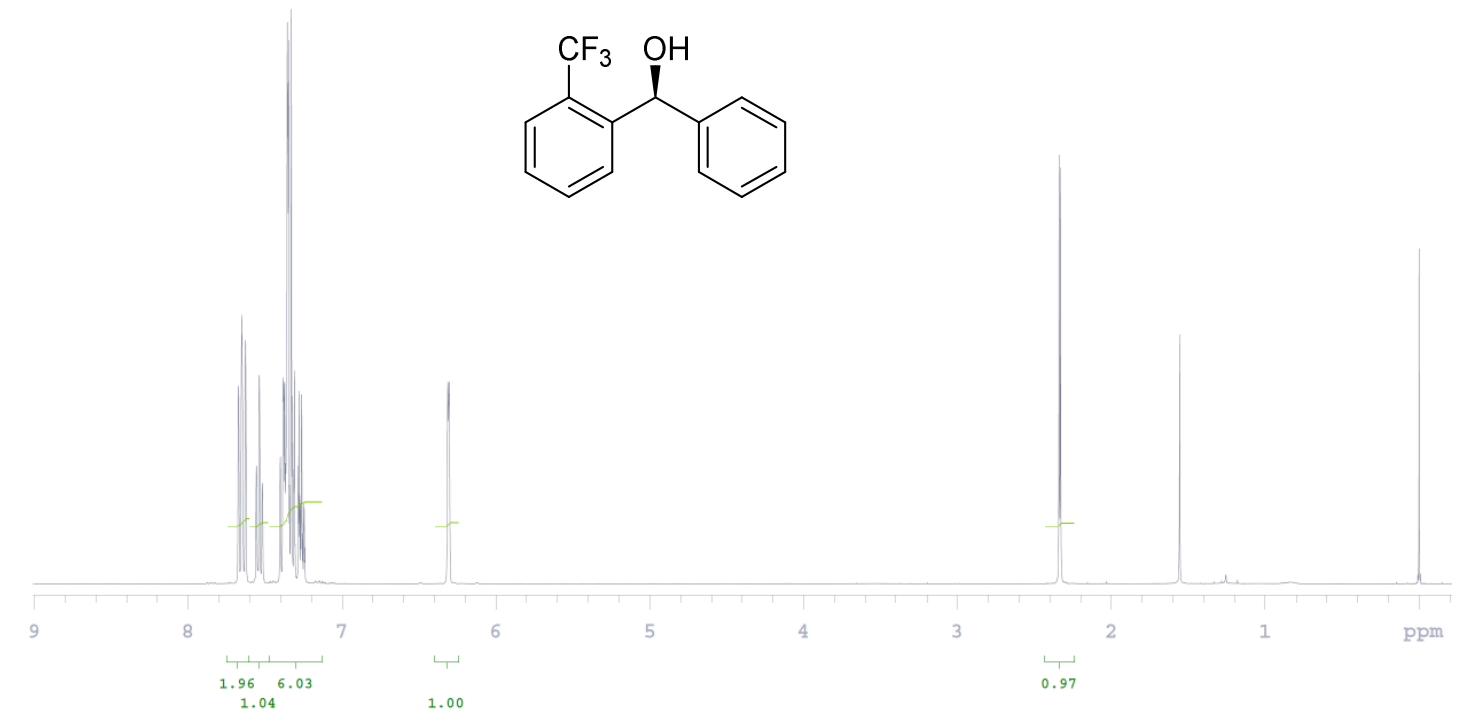

${ }^{13} \mathrm{C}$ NMR $\left(125 \mathrm{MHz}, \mathrm{CDCl}_{3}\right)$
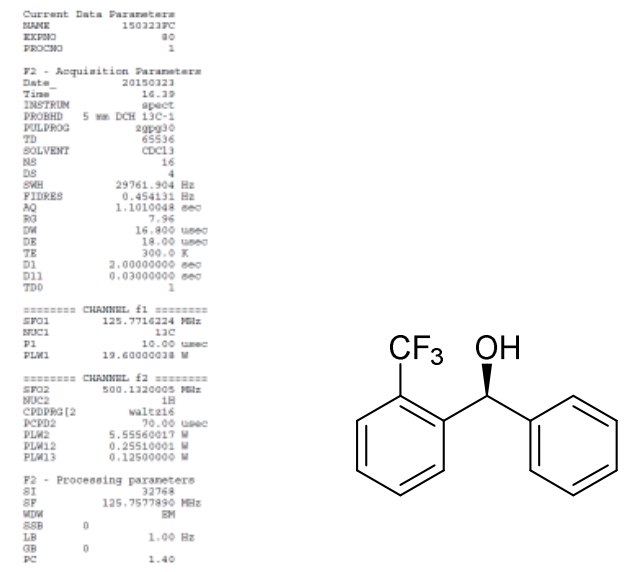

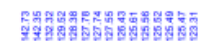

Wwily

$V^{88 n}$

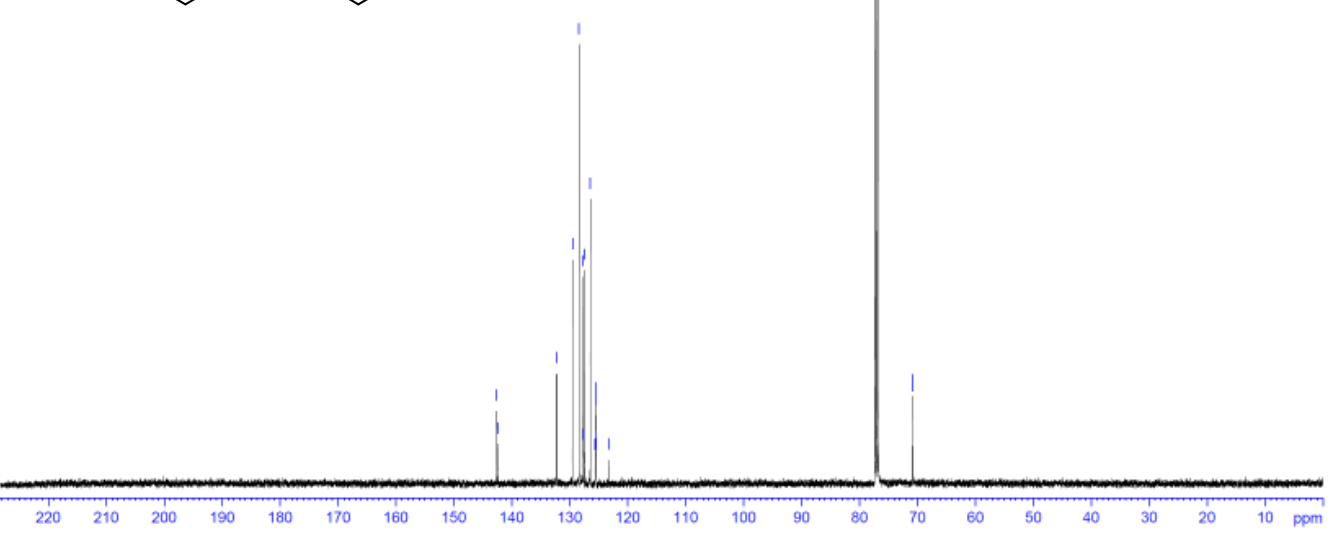


(S)-2-(Hydroxy(phenyl)methyl)phenol (6e)

${ }^{1} \mathrm{H}$ NMR (400 MHz, $\left.\mathrm{CDCl}_{3}\right)$

Agilent Technologies

150610-1_1H-NMR-05

$\begin{array}{ll}\text { Sample Name } & 150610-1 \text { 1 } 1 \text { H-NMR-05 } \\ \text { Date collected } & 2015-06-10\end{array}$

$\begin{array}{ll}\text { Temperature } & \mathbf{3 5} \\ \text { Spectrometer } & \text { Agilent-NMR-vnmrs400 }\end{array}$

Study owner vnmr1
Operator vnmr1

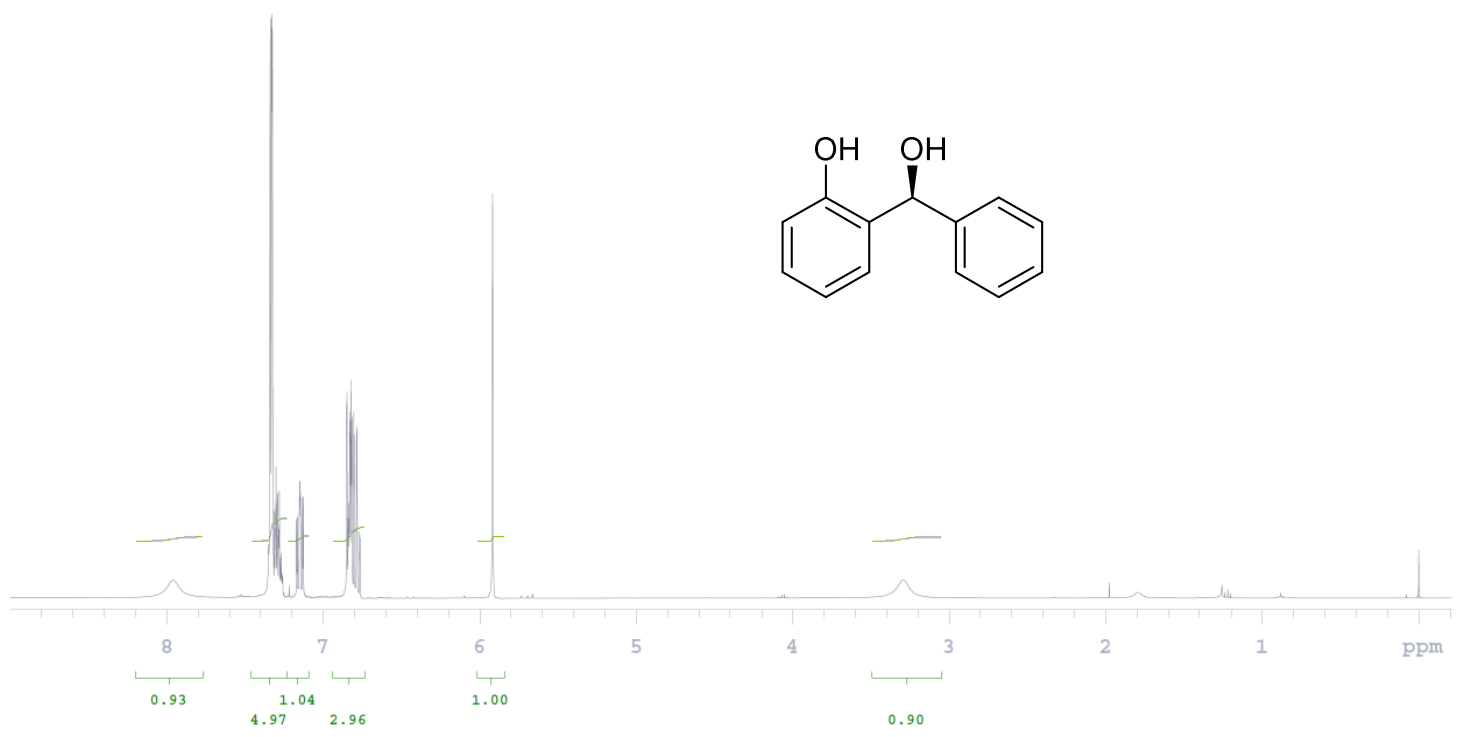

${ }^{13} \mathrm{C}$ NMR (125 MHz, $\left.\mathrm{CDCl}_{3}\right)$

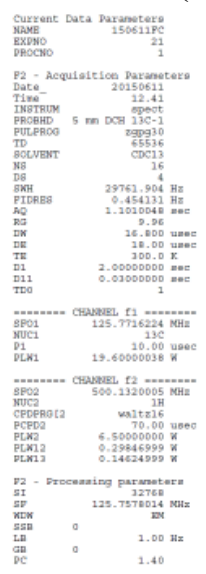

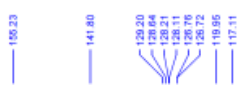

策<smiles>Oc1ccccc1[C@@H](O)c1ccccc1</smiles>

S45 
(S)-(2,4-Dimethylphenyl)(phenyl)methanol (bf)

${ }^{1} \mathrm{H}$ NMR (400 MHz, $\left.\mathrm{CDCl}_{3}\right)$

Agilent Technologies

150323-4 (1H-NMR-03)

$\begin{array}{ll}\text { Sample Name } & \text { 150323-4 (1H-NMR-03) } \\ \text { Date collected } & 2015-03-23\end{array}$

Pulse sequence PROTON
Solvent cdcl3

Temperature 35

$\begin{array}{ll} & \\ \text { Spectrometer } & \text { Agilent-NMR-vnmrs } 400\end{array}$

Study owner nmr

Detected
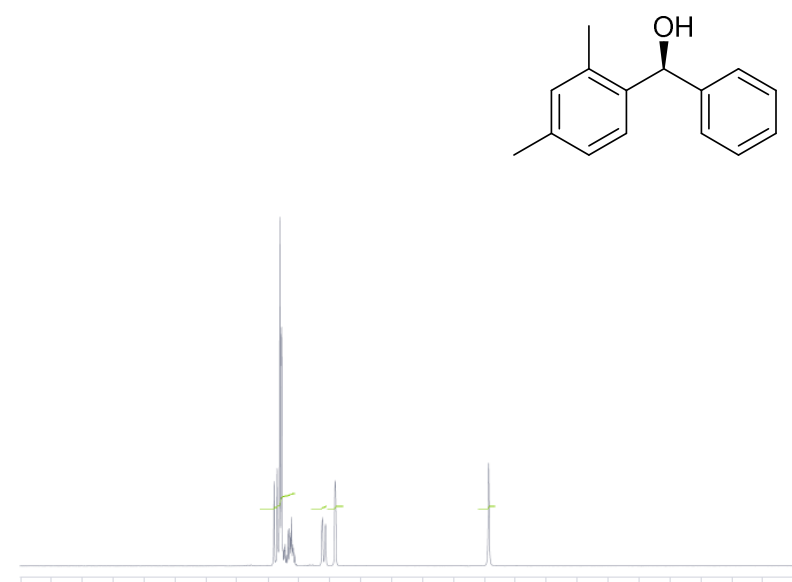

9

8

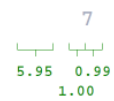

6
4
1.0

5

${ }^{13} \mathrm{C}$ NMR $\left(125 \mathrm{MHz}, \mathrm{CDCl}_{3}\right)$
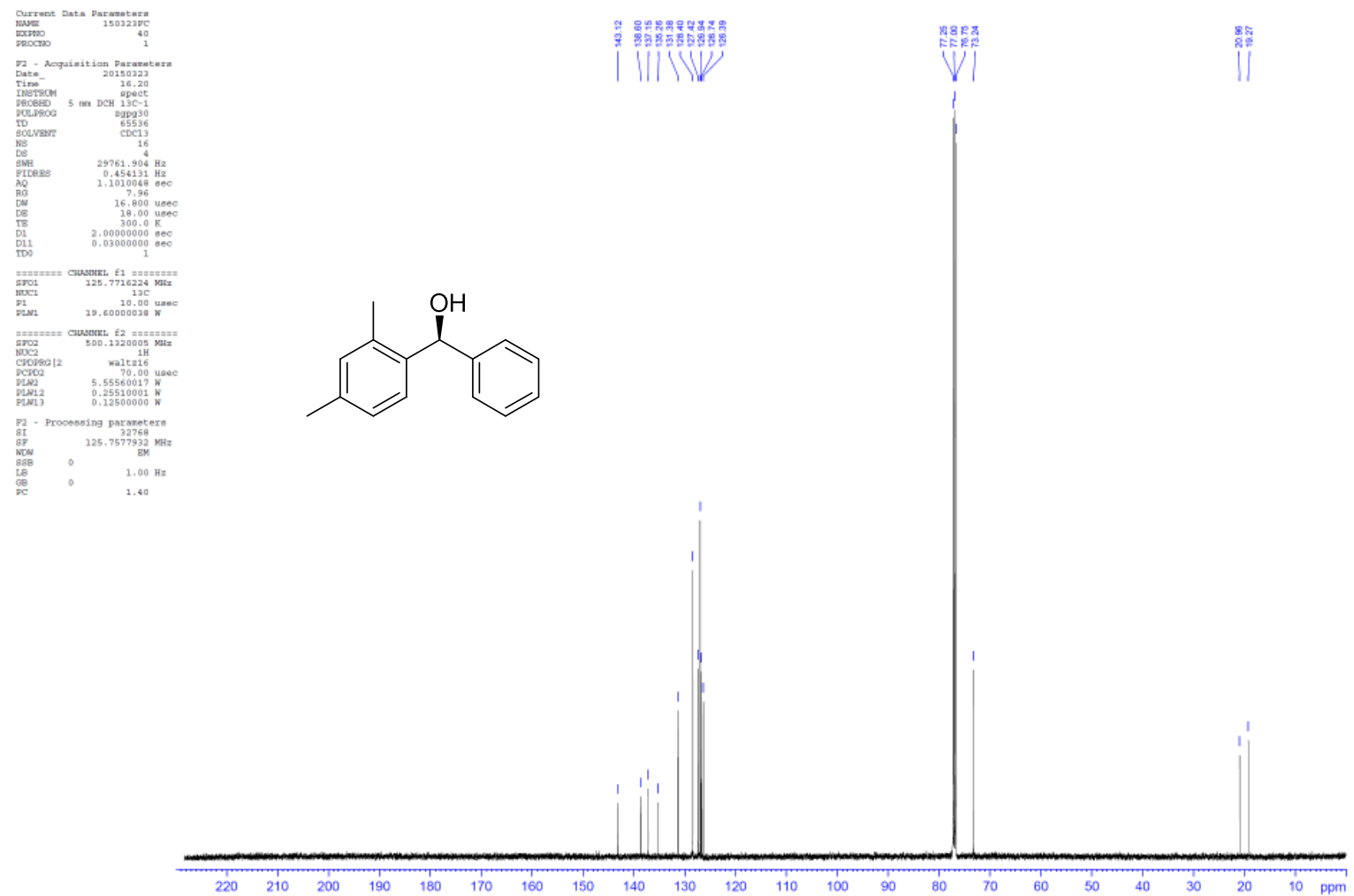

S46 
(S)-Phenyl(2,4,5-trimethylphenyl)methanol (6g)

${ }^{1} \mathrm{H}$ NMR $\left(400 \mathrm{MHz}, \mathrm{CDCl}_{3}\right)$

Agilent Technologies

150401-1_1 1 -NMR-22

$\begin{array}{ll}\text { Sample Name 150401-1_1-1H-NMR-22_ } & \begin{array}{l}\text { Pulse sequence PROTON } \\ \text { Date collected 2015-04-01 }\end{array}\end{array}$

Temperature 35
Spectrometer

Study owner vnmr1
Operator vnmr1

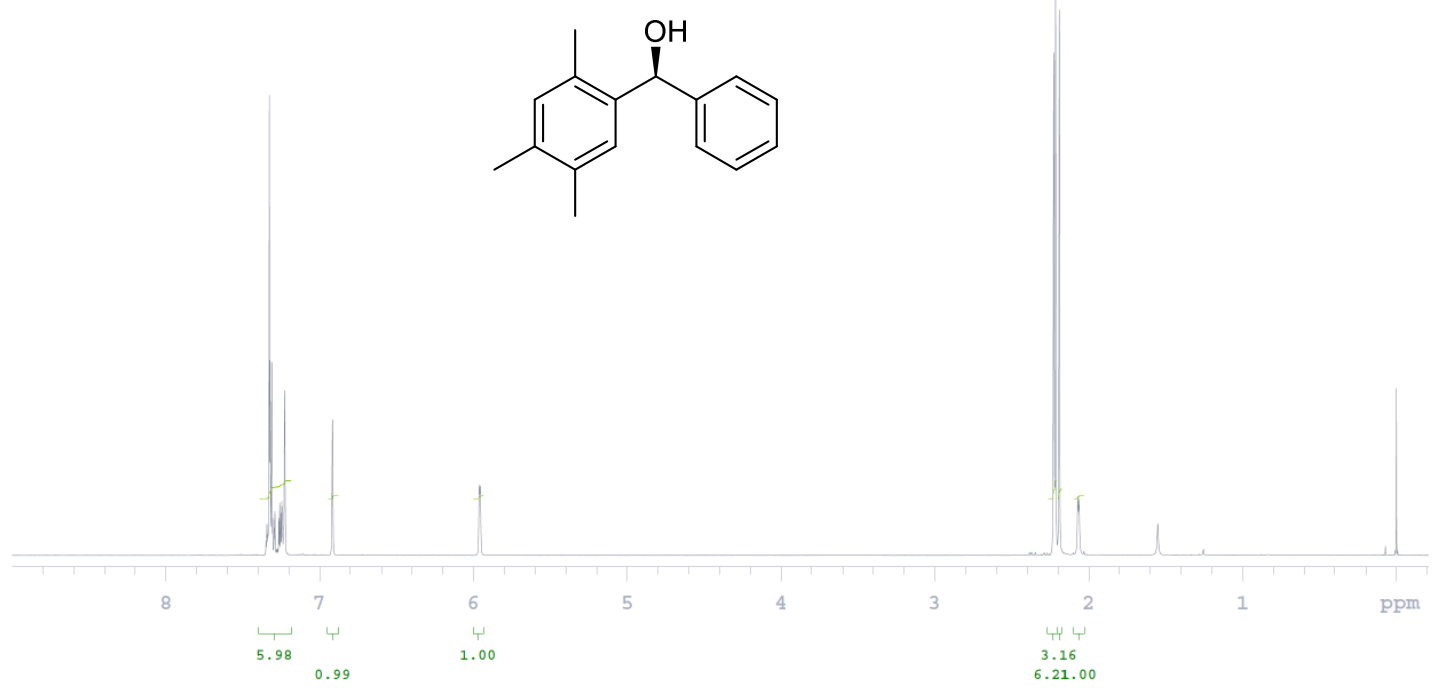

${ }^{13} \mathrm{C}$ NMR $\left(125 \mathrm{MHz}, \mathrm{CDCl}_{3}\right)$
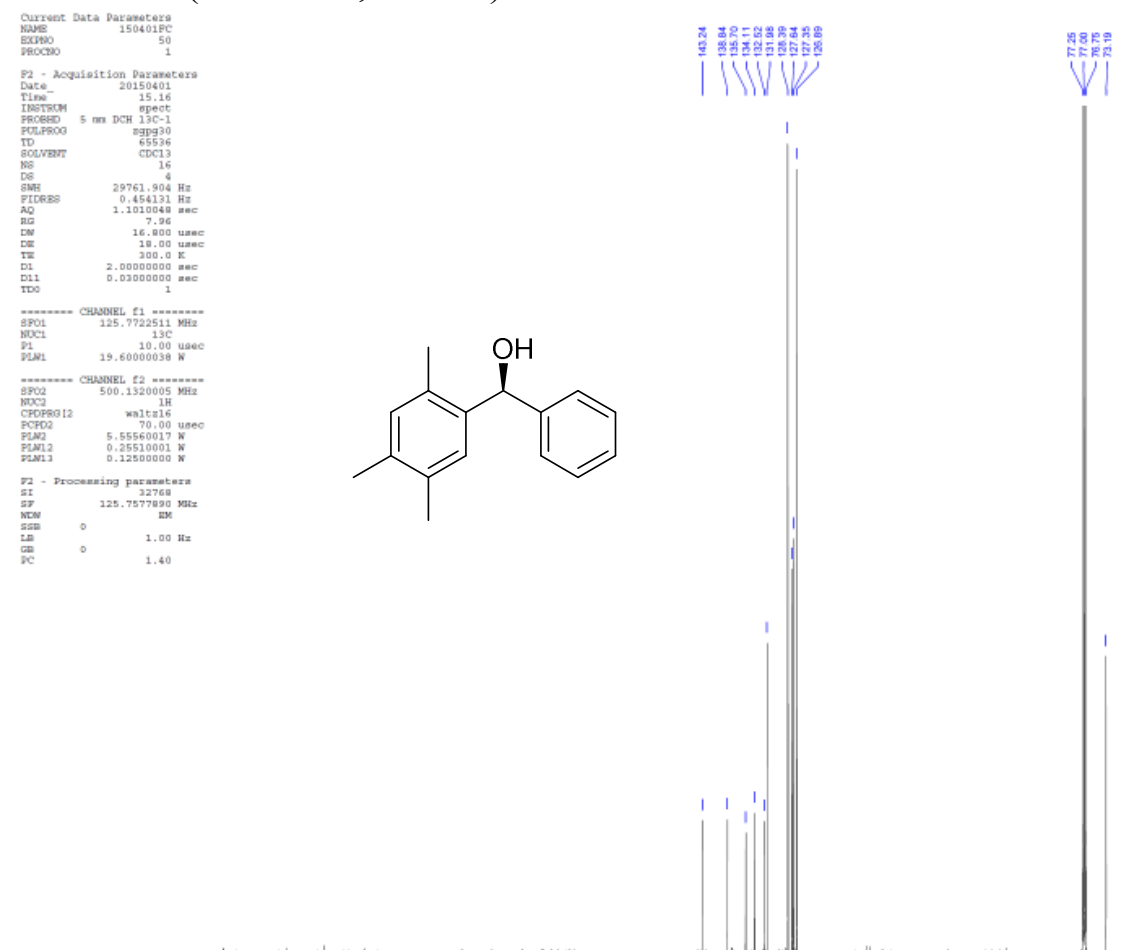

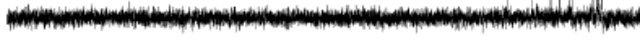

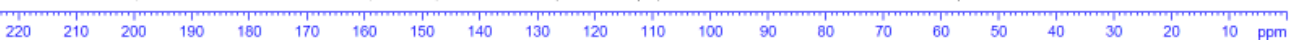




\section{(S)-(2,4-Dichlorophenyl)(phenyl)methanol (6h)}

${ }^{1} \mathrm{H}$ NMR $\left(400 \mathrm{MHz}, \mathrm{CDCl}_{3}\right)$

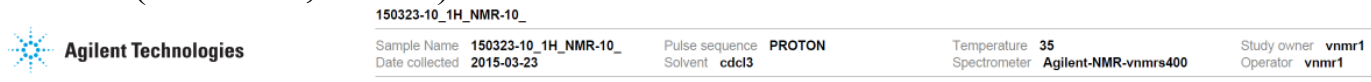

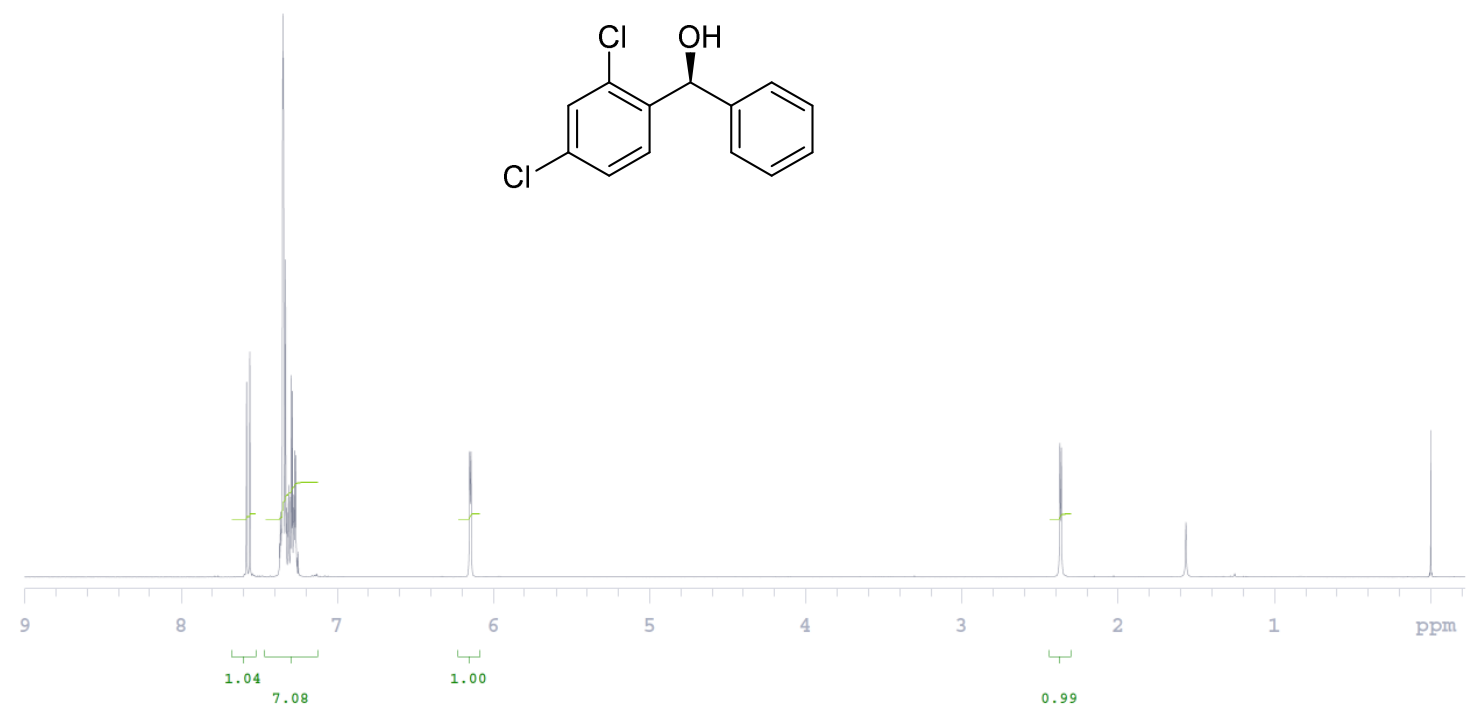

${ }^{13} \mathrm{C}$ NMR $\left(125 \mathrm{MHz}, \mathrm{CDCl}_{3}\right)$
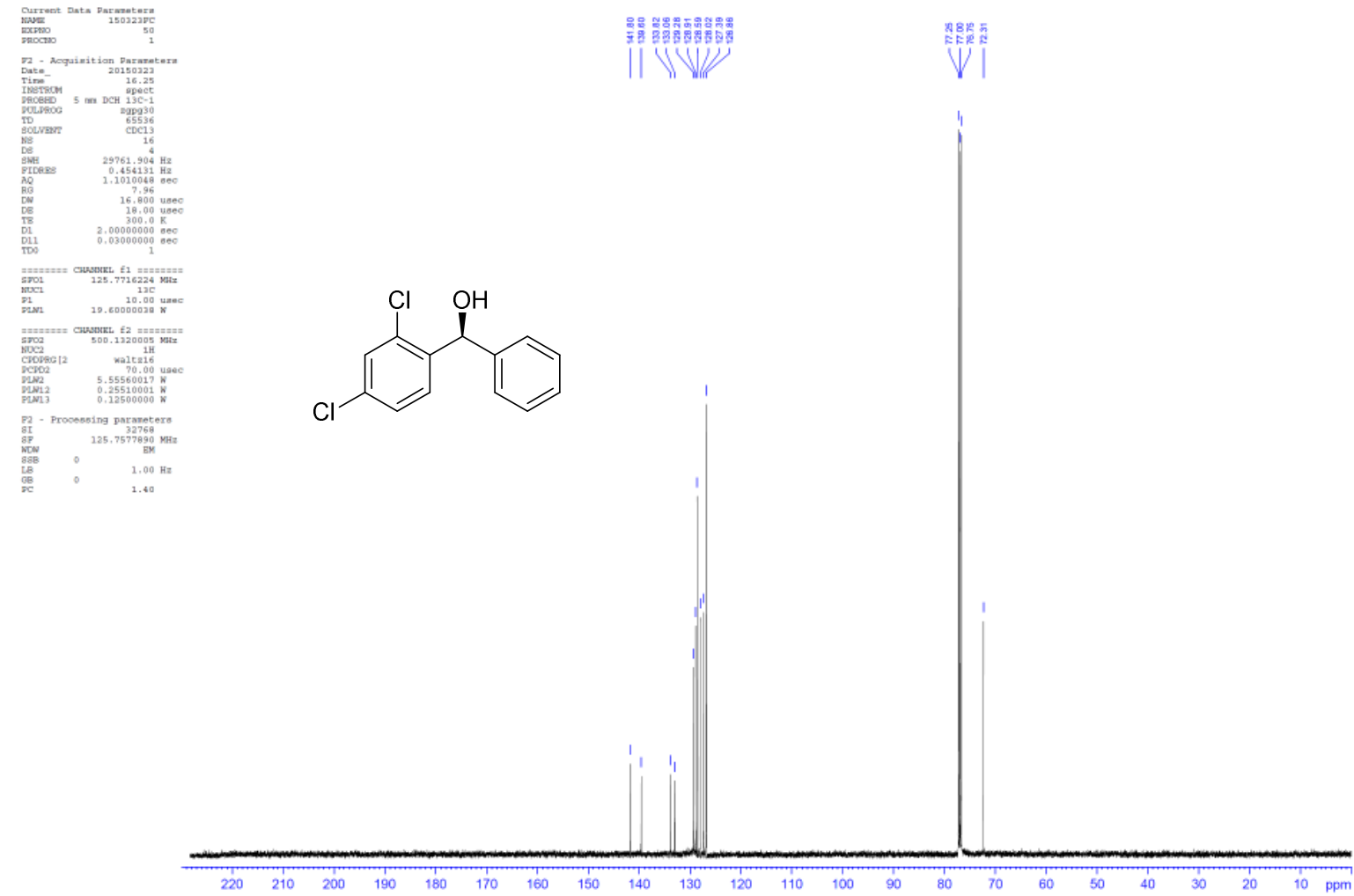
(S)-(2-Chloro-5-nitrophenyl)(phenyl)methanol (6i)

${ }^{1} \mathrm{H}$ NMR $\left(400 \mathrm{MHz}, \mathrm{CDCl}_{3}\right)$

Agilent Technologies

150401-8_1H-NMR-12

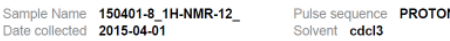

Temperature 35
Spectrometer

gilent-NMR-vnmrs400

Study owner vnmr1
Operator vnmr1

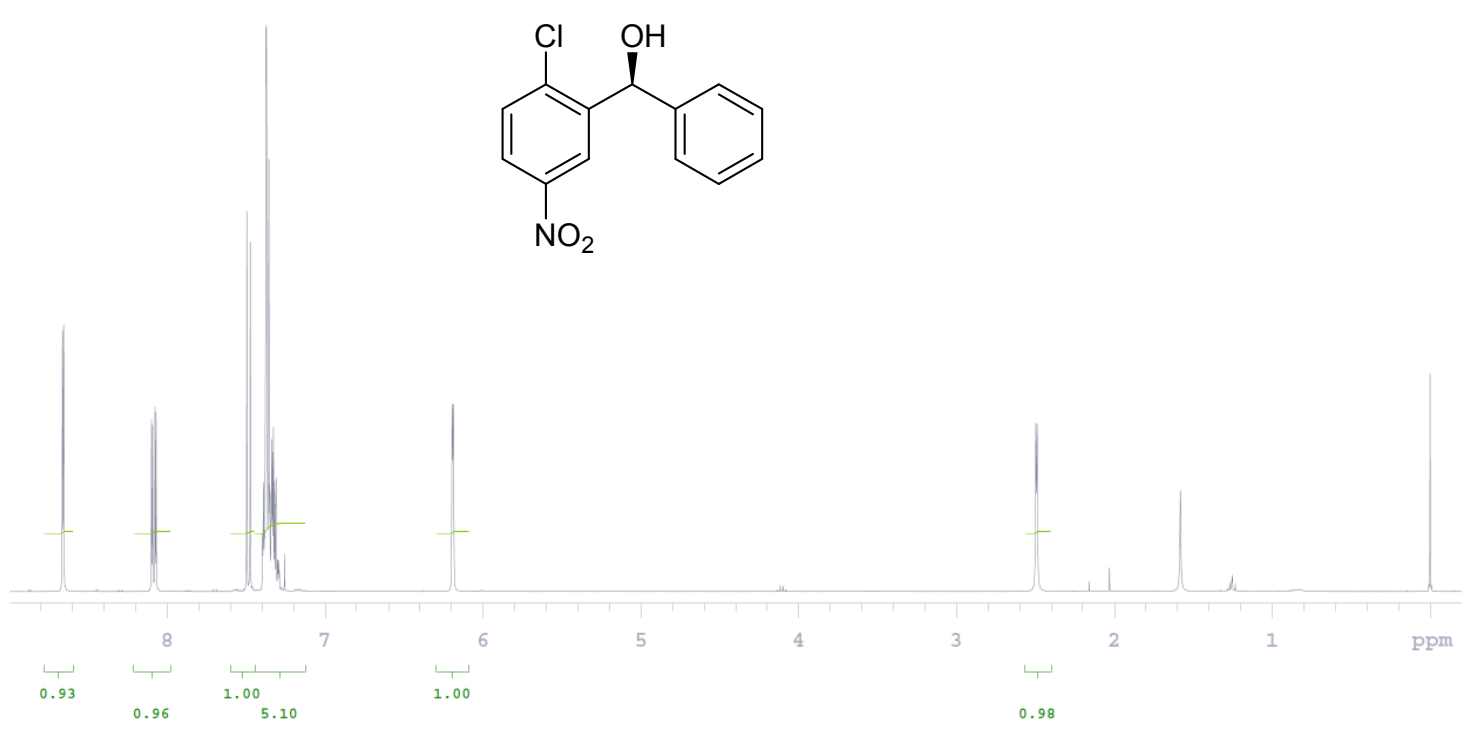

${ }^{13} \mathrm{C}$ NMR $\left(125 \mathrm{MHz}, \mathrm{CDCl}_{3}\right)$
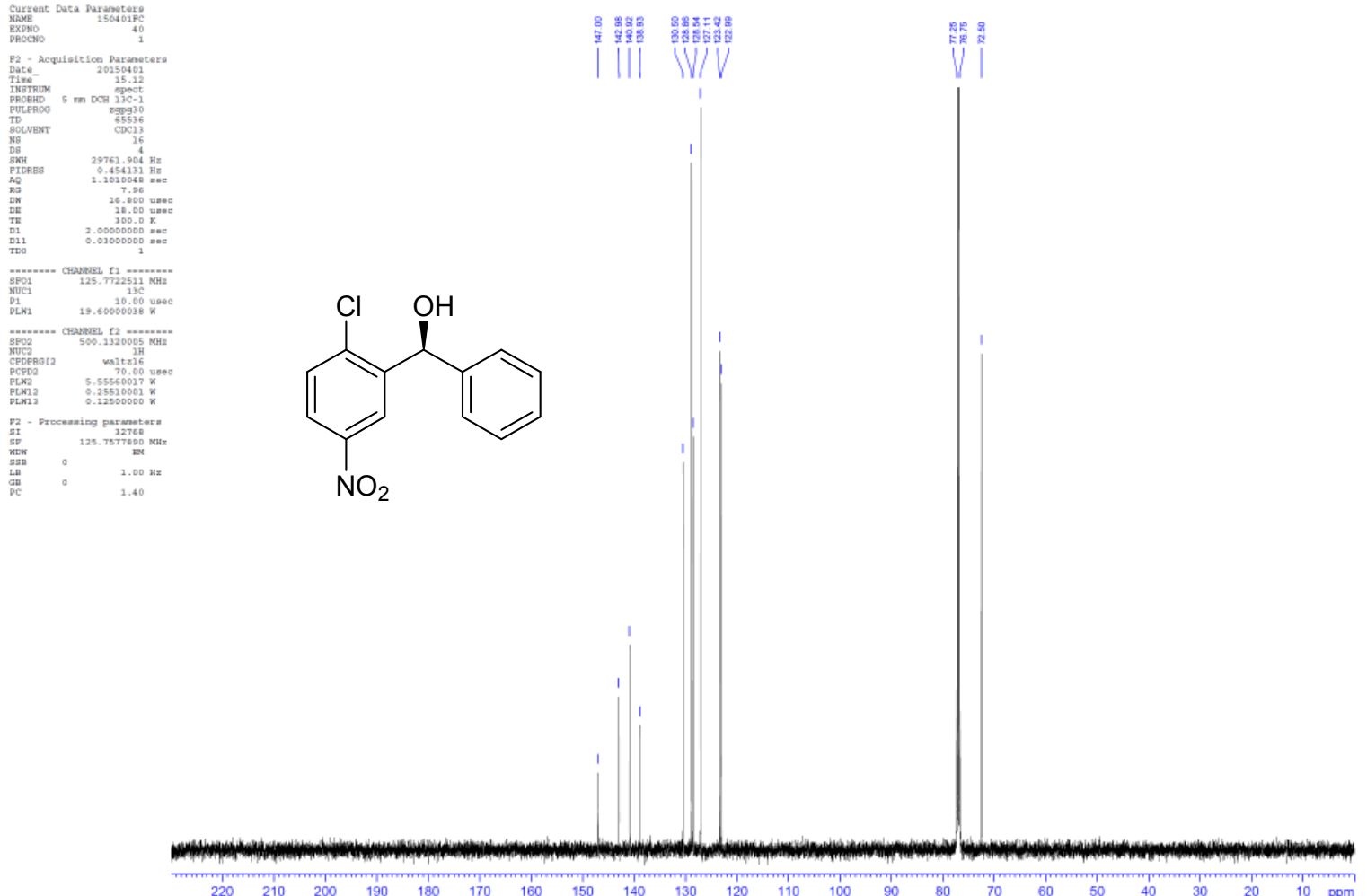


\section{(S)-(2,5-Difluorophenyl)(phenyl)methanol (6j)}

\section{${ }^{1} \mathrm{H}$ NMR $\left(400 \mathrm{MHz}, \mathrm{CDCl}_{3}\right)$}

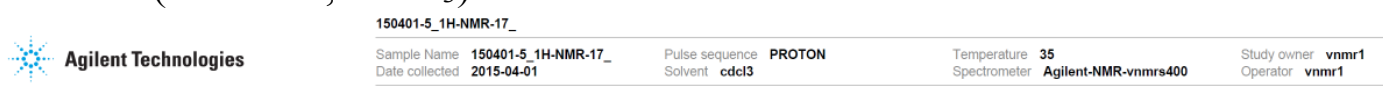

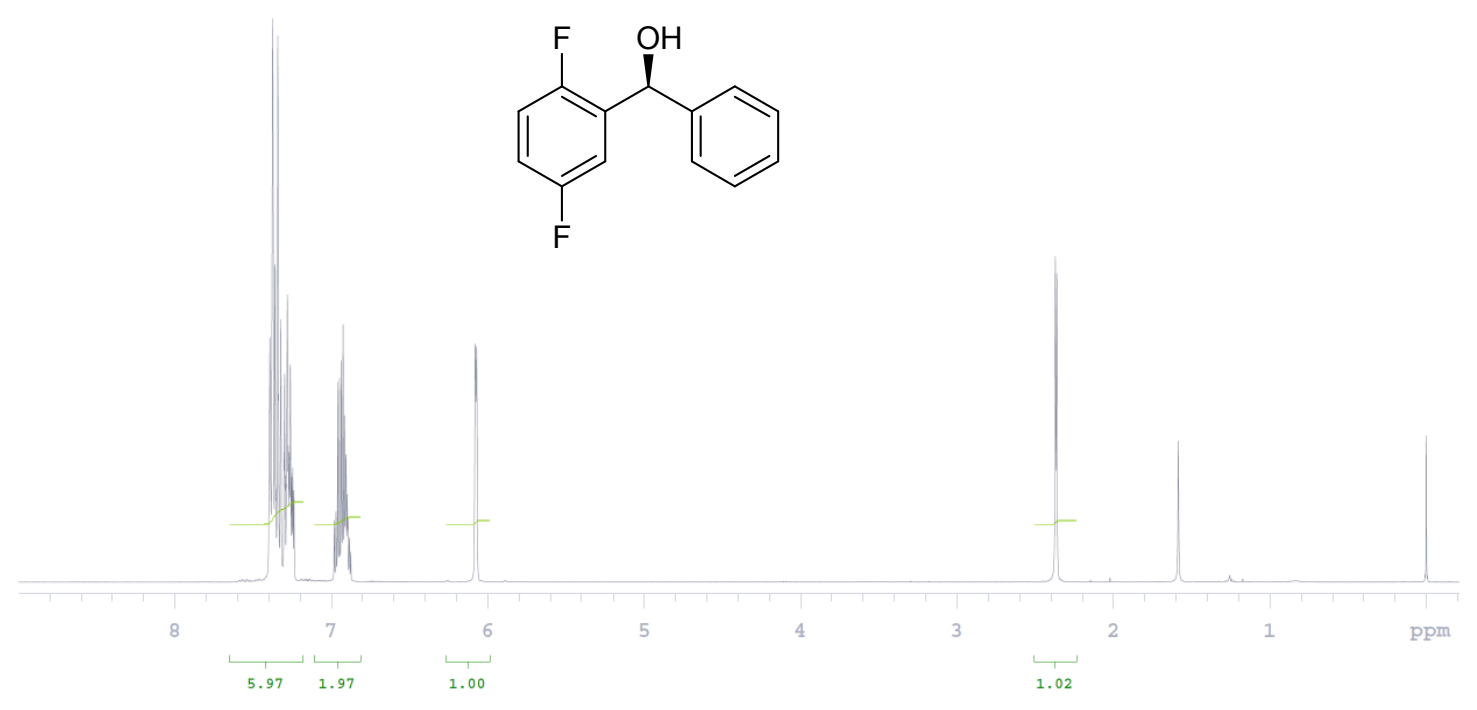

${ }^{13} \mathrm{C}$ NMR $\left(125 \mathrm{MHz}, \mathrm{CDCl}_{3}\right)$
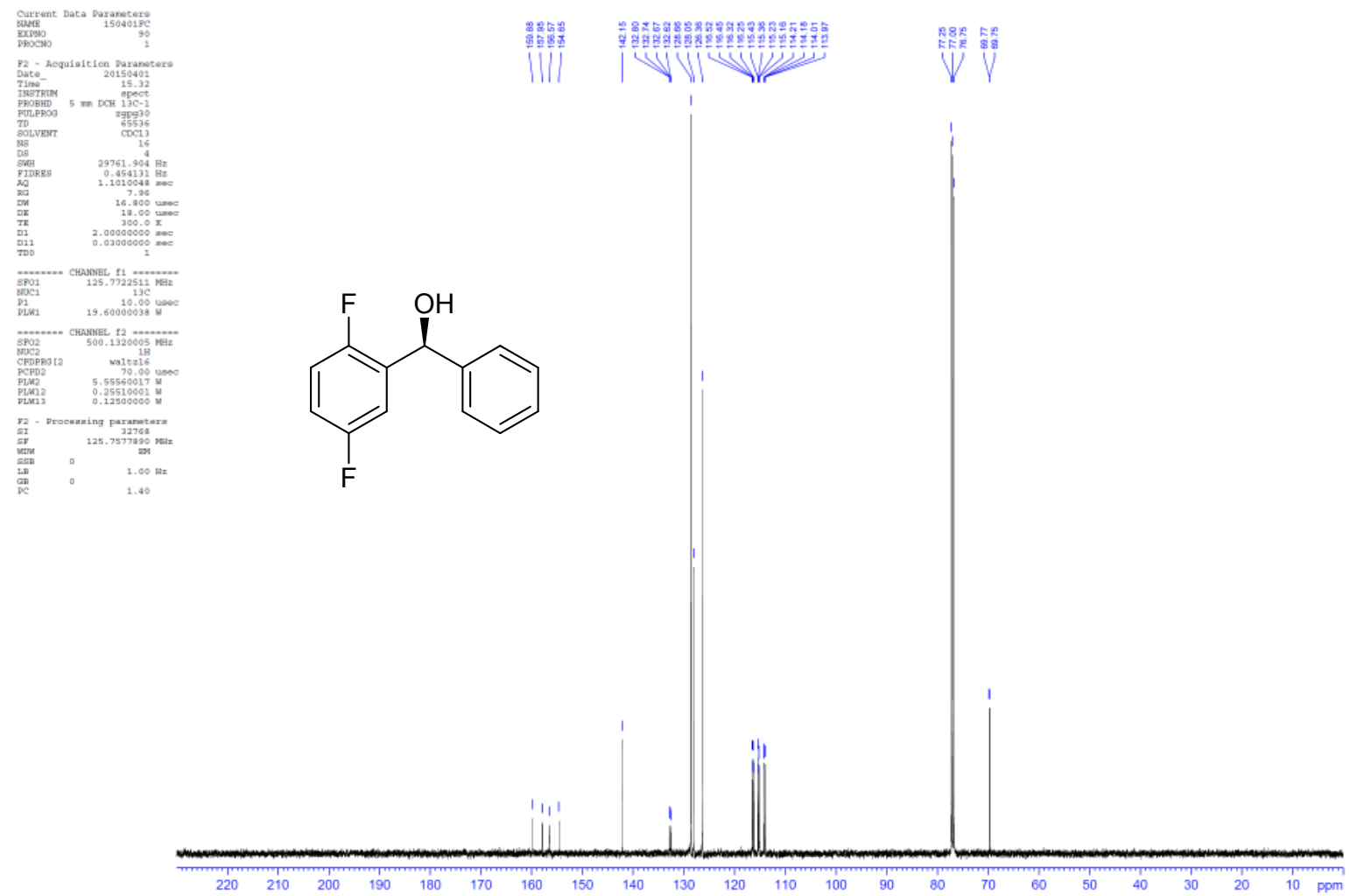


\section{(S)-(2-Fluoro-3-(trifluoromethyl)phenyl)(phenyl)methanol (6k)}

\section{${ }^{1} \mathrm{H}$ NMR $\left(400 \mathrm{MHz}, \mathrm{CDCl}_{3}\right)$}

Agilent Technologies

\section{1-7__ H-NMR-25}

$\begin{array}{ll}\text { Sample Name } 150401-7 \text { 1 } 1 \text { 1H-NMR-25_ } & \text { Pulse sequence PROTON } \\ \text { Datecollected 2015-04-01 } & \text { Solvent cdcl3 }\end{array}$

$\begin{array}{ll}\text { Temperature } & 35 \\ \text { Spectrometer Agilent-NMR-vnmrs400 } & \text { Study owner vnmr1 } \\ \text { Operator vnmr1 }\end{array}$
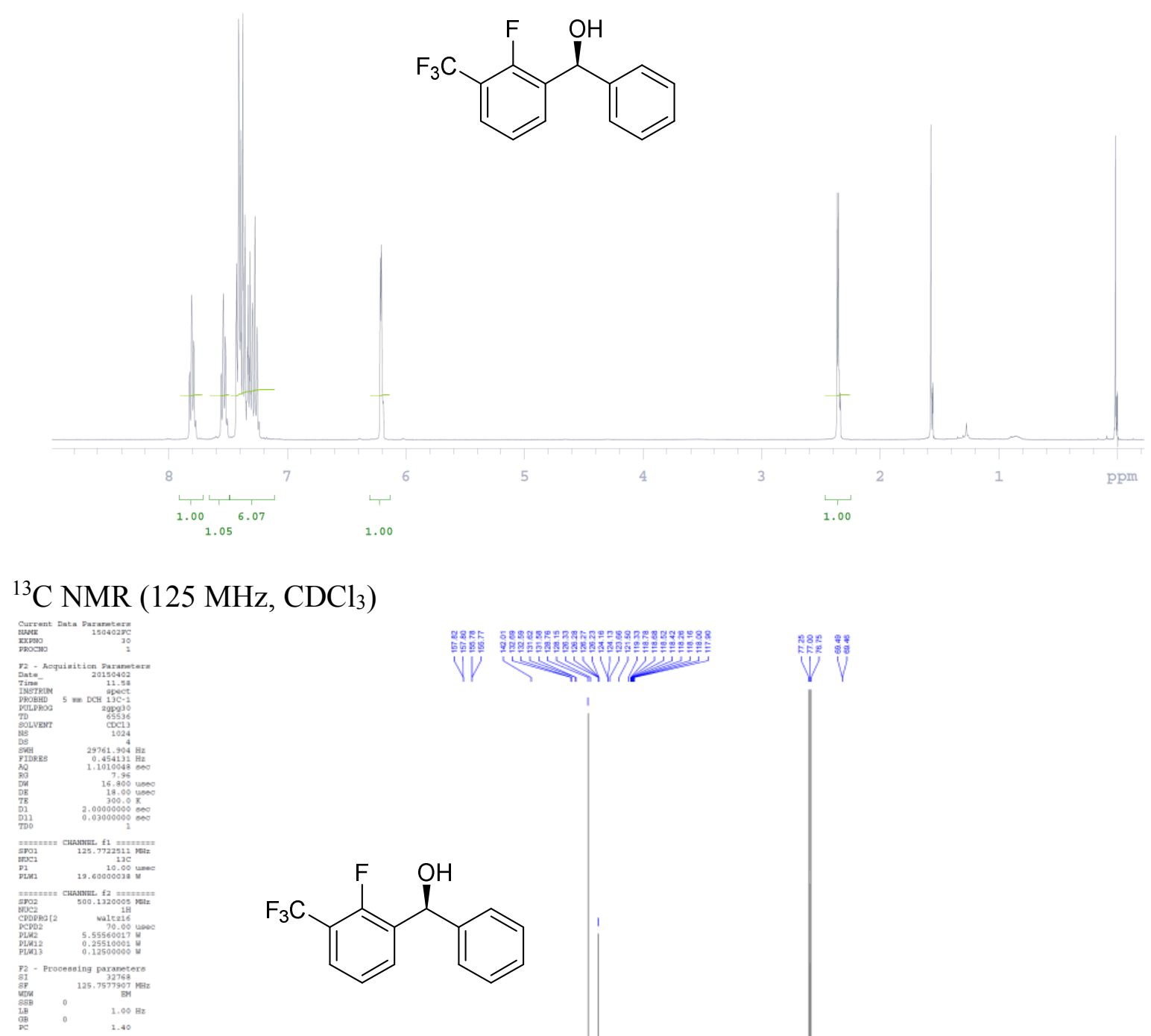

$\left.25 \mathrm{MHz}, \mathrm{CDCl}_{3}\right)$
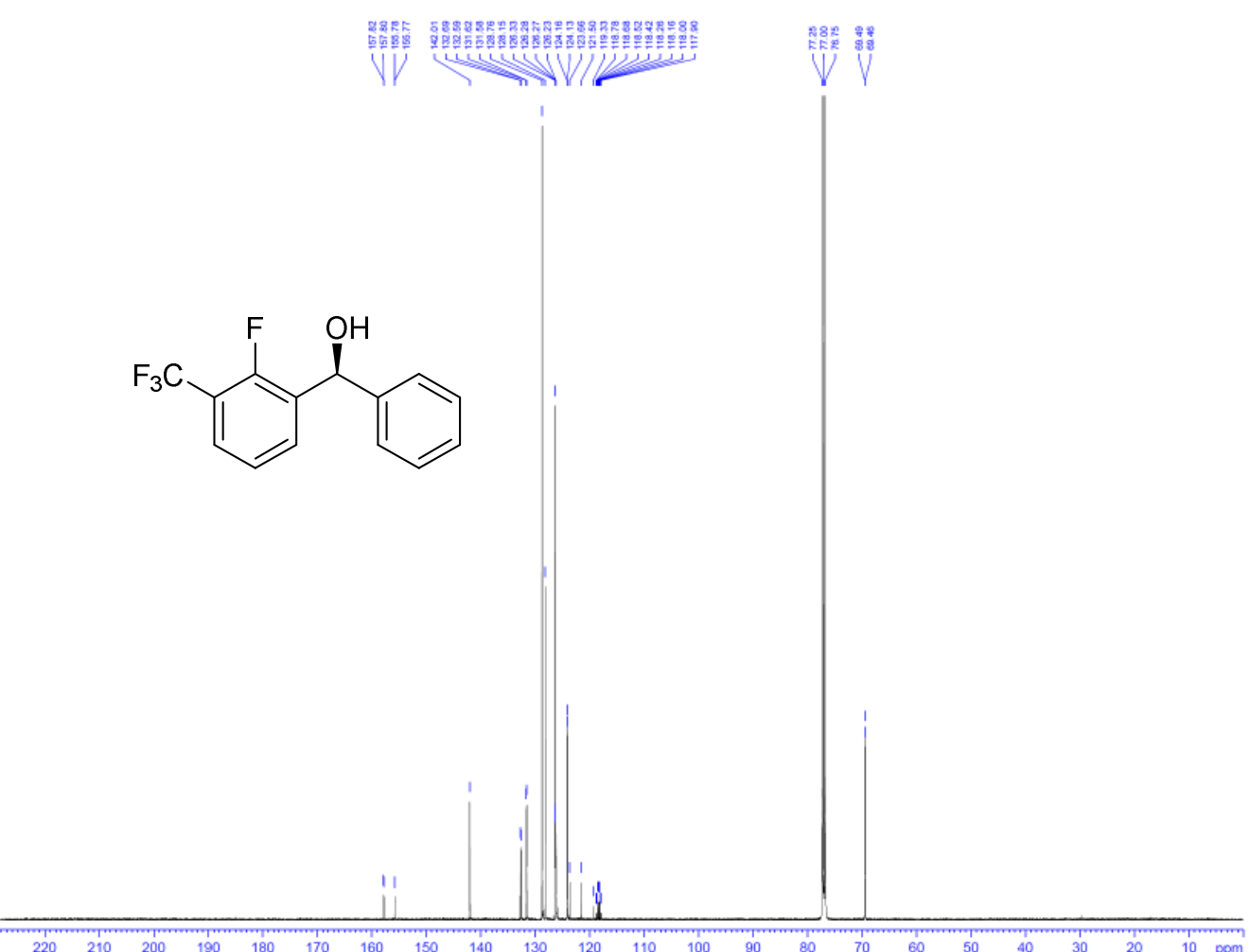


\section{(S)-(Perfluorophenyl)(phenyl)methanol (6l)}

${ }^{1} \mathrm{H}$ NMR $\left(400 \mathrm{MHz}, \mathrm{CDCl}_{3}\right)$

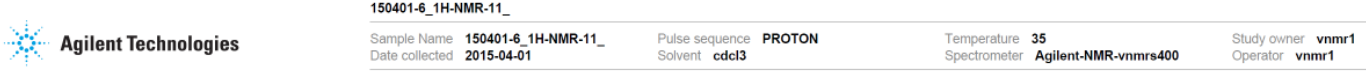
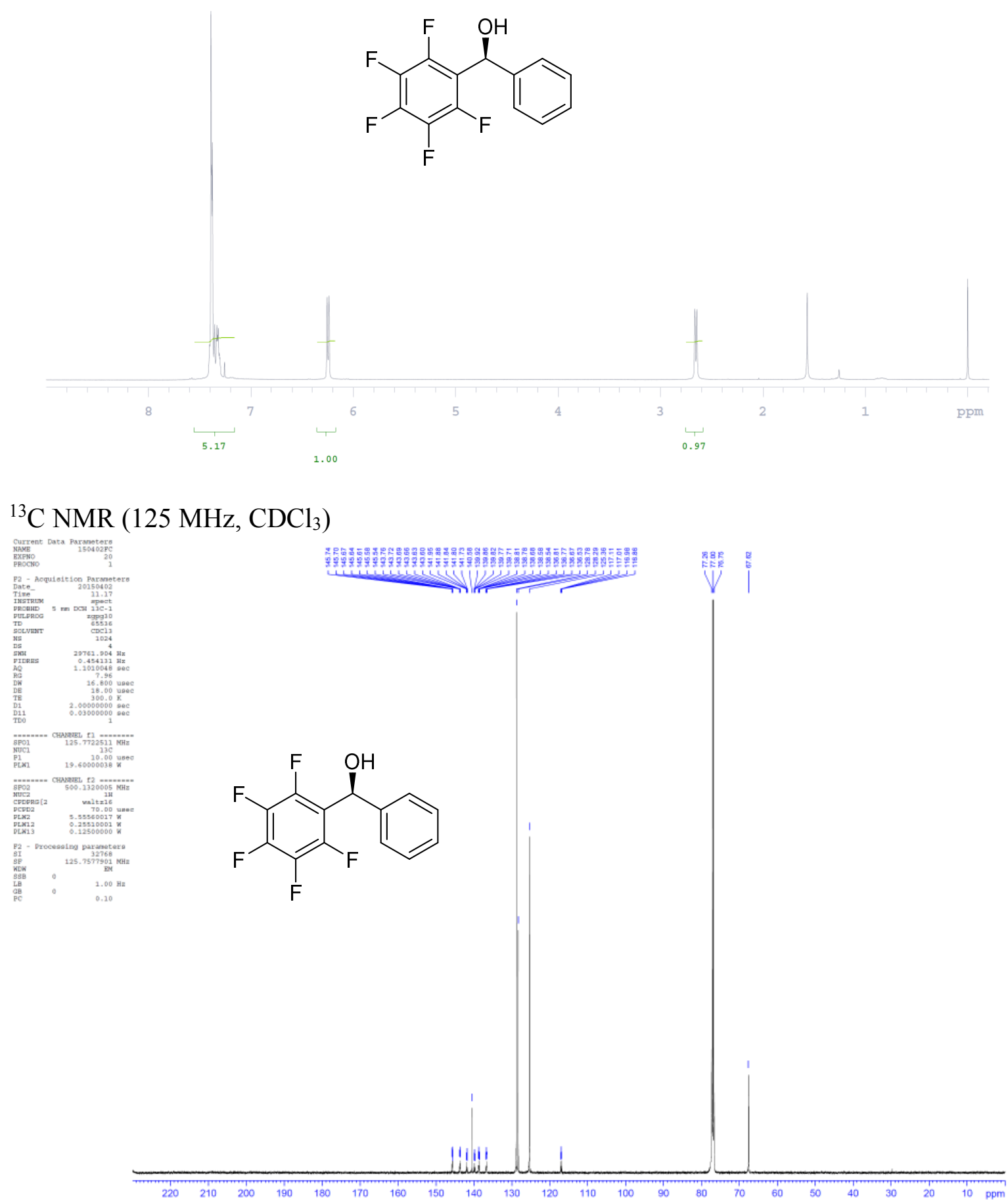


\section{(S)-(2-Chlorophenyl)(4-chlorophenyl)methanol (6m)}

${ }^{1} \mathrm{H}$ NMR $\left(400 \mathrm{MHz}, \mathrm{CDCl}_{3}\right)$

\begin{tabular}{|c|c|c|c|c|}
\hline & 150323-5 (1H-NMR-07) & & & \\
\hline ilent Technologies & $\begin{array}{ll}\text { Sample Name } & 150323-5(1 \text { H-NMR-07) } \\
\text { Date collected } & 2015-03-23\end{array}$ & $\begin{array}{l}\text { Pulse sequence PROTON } \\
\text { Solvent cdcl3 }\end{array}$ & $\begin{array}{l}\text { Temperature } 35 \\
\text { Spectrometer }\end{array}$ & $\begin{array}{l}\text { Study owner vnmr1 } \\
\text { Operator vnmr1 }\end{array}$ \\
\hline
\end{tabular}
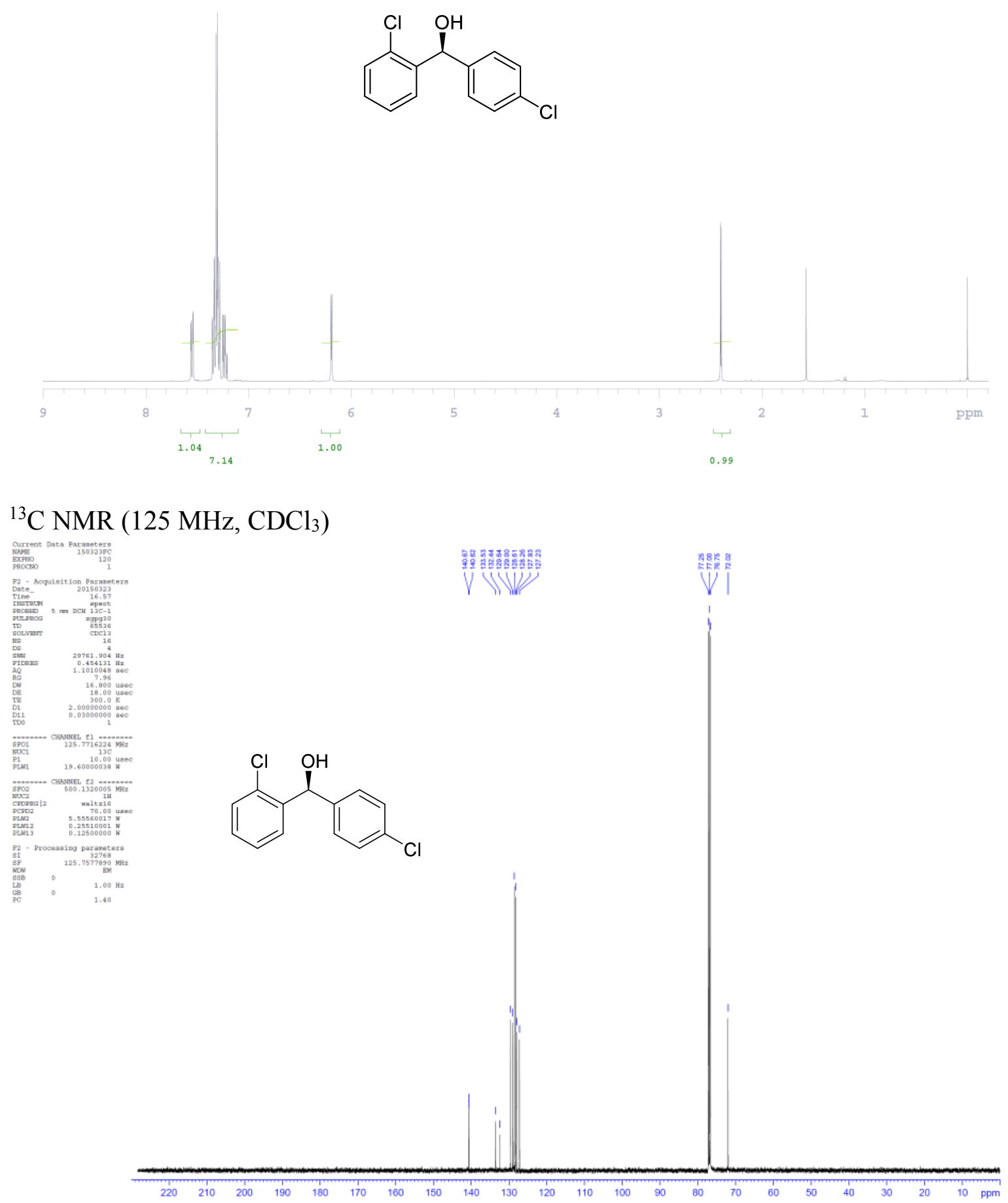
(S)-(2-Chlorophenyl)(4-fluorophenyl)methanol (bn)

${ }^{1} \mathrm{H}$ NMR (400 MHz, $\left.\mathrm{CDCl}_{3}\right)$

Agilent Technologies

150401-9_1H-NMR-09_.

Sample Name 150401-9_1H-NMR-09
Date collected 2015-04-01

Pulse sequence PROTON
Solvent cdcl3

Temperature $\mathbf{3 5}$

$\begin{array}{ll} & \\ \text { Spectrometer } & \text { Agilent-NMR-vnmrs } 400\end{array}$

Study owner nmr
Operator nmr
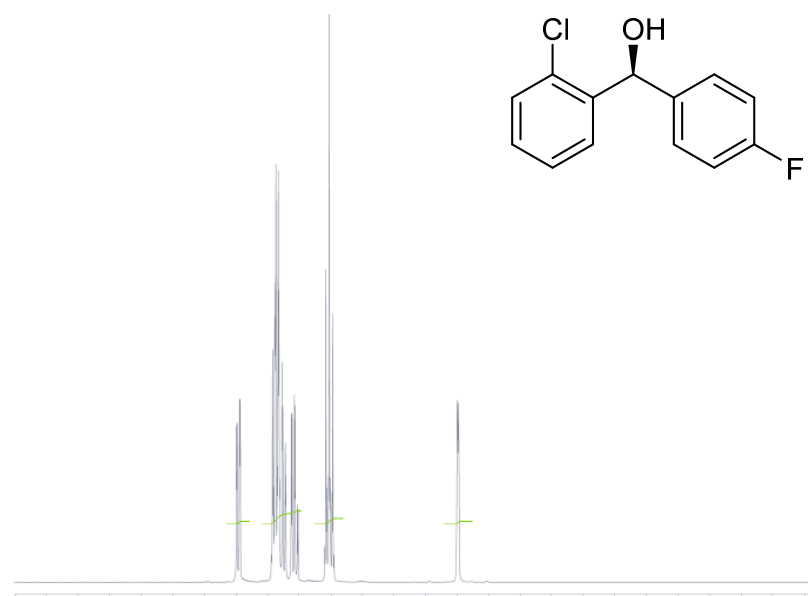

9

8

${ }_{1.02} \underbrace{2.04}_{5.10}$
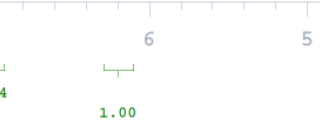

3

2

1

${ }^{13} \mathrm{C}$ NMR $\left(125 \mathrm{MHz}, \mathrm{CDCl}_{3}\right)$

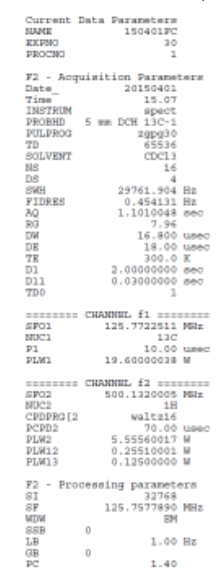

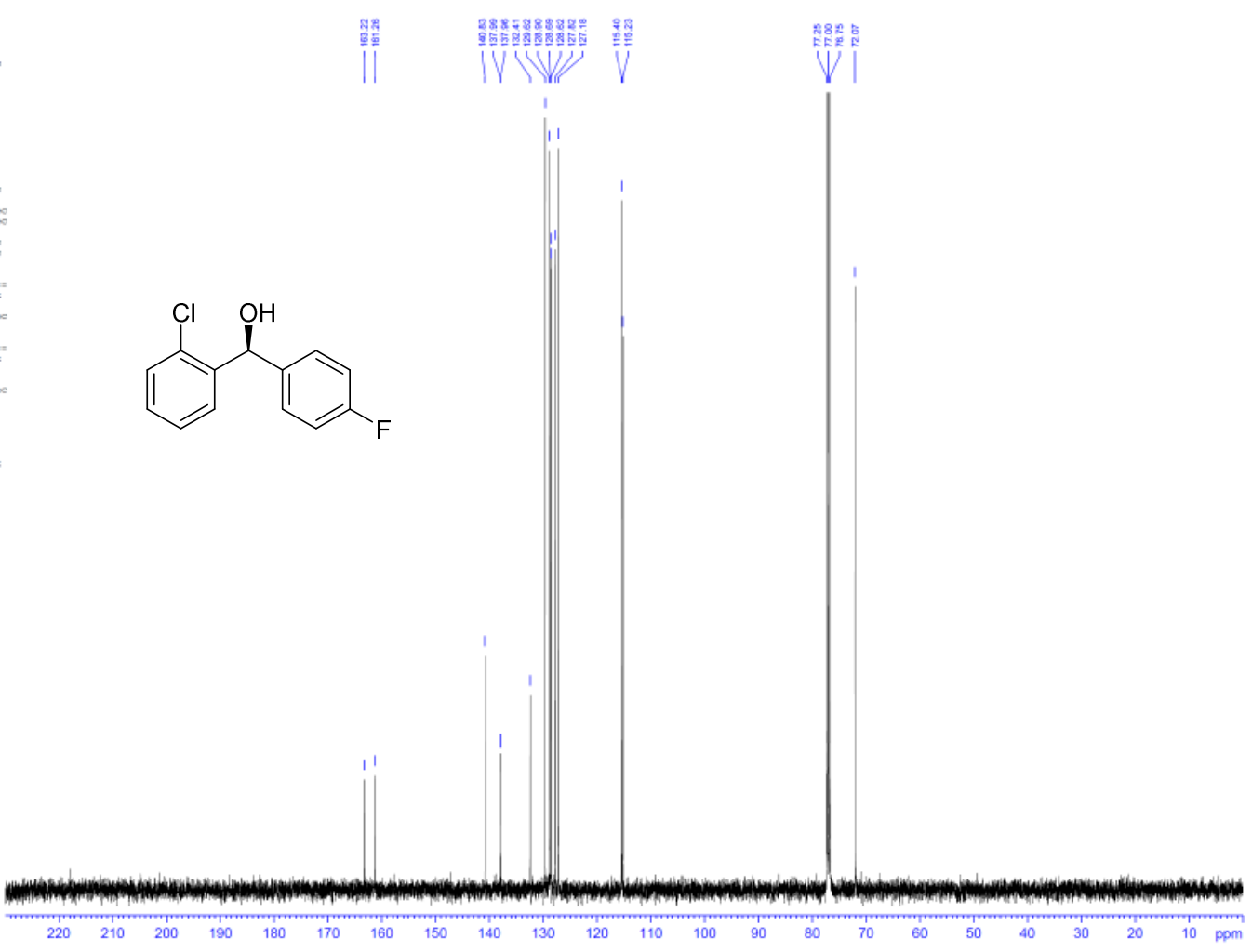

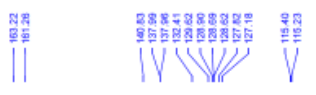

0.99

ppm

S54 
(S)-6-Phenyl-6H-benzo[c]chromene (9)

${ }^{1} \mathrm{H}$ NMR (400 MHz, $\mathrm{CDCl}_{3}$ )

Agilent Technologies

150515-4_1H-NMR-60_

\begin{tabular}{ll} 
Sample Name & 150515-4_1H-NMR-60_ \\
Date collected & 2015-05-15 \\
\hline
\end{tabular}

$\begin{array}{ll}\text { Temperature } & \mathbf{3 5} \\ \text { Spectrometer } & \text { Agilent-NMR-vnmrs400 }\end{array}$

Study owner vnmr1
Operator vnmr1

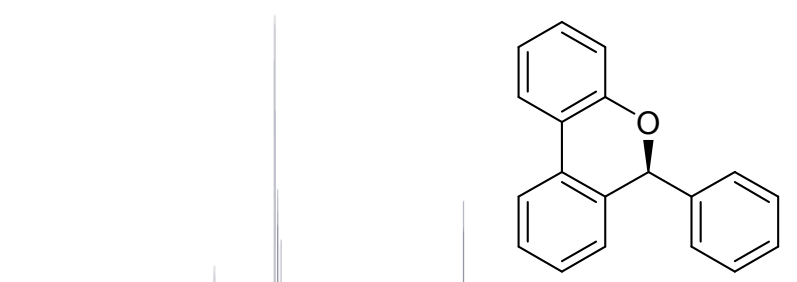

9

8

$\frac{1}{2.30}$

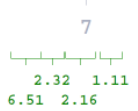

1.00

${ }^{13} \mathrm{C} \mathrm{NMR}\left(125 \mathrm{MHz}, \mathrm{CDCl}_{3}\right)$

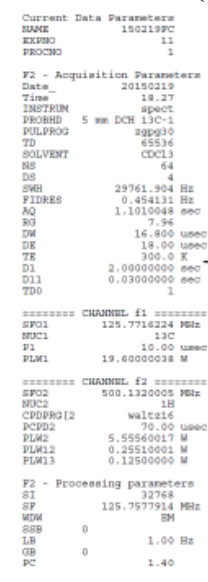

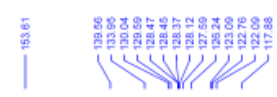

${ }^{3 x^{2}}$

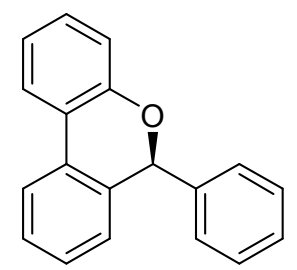
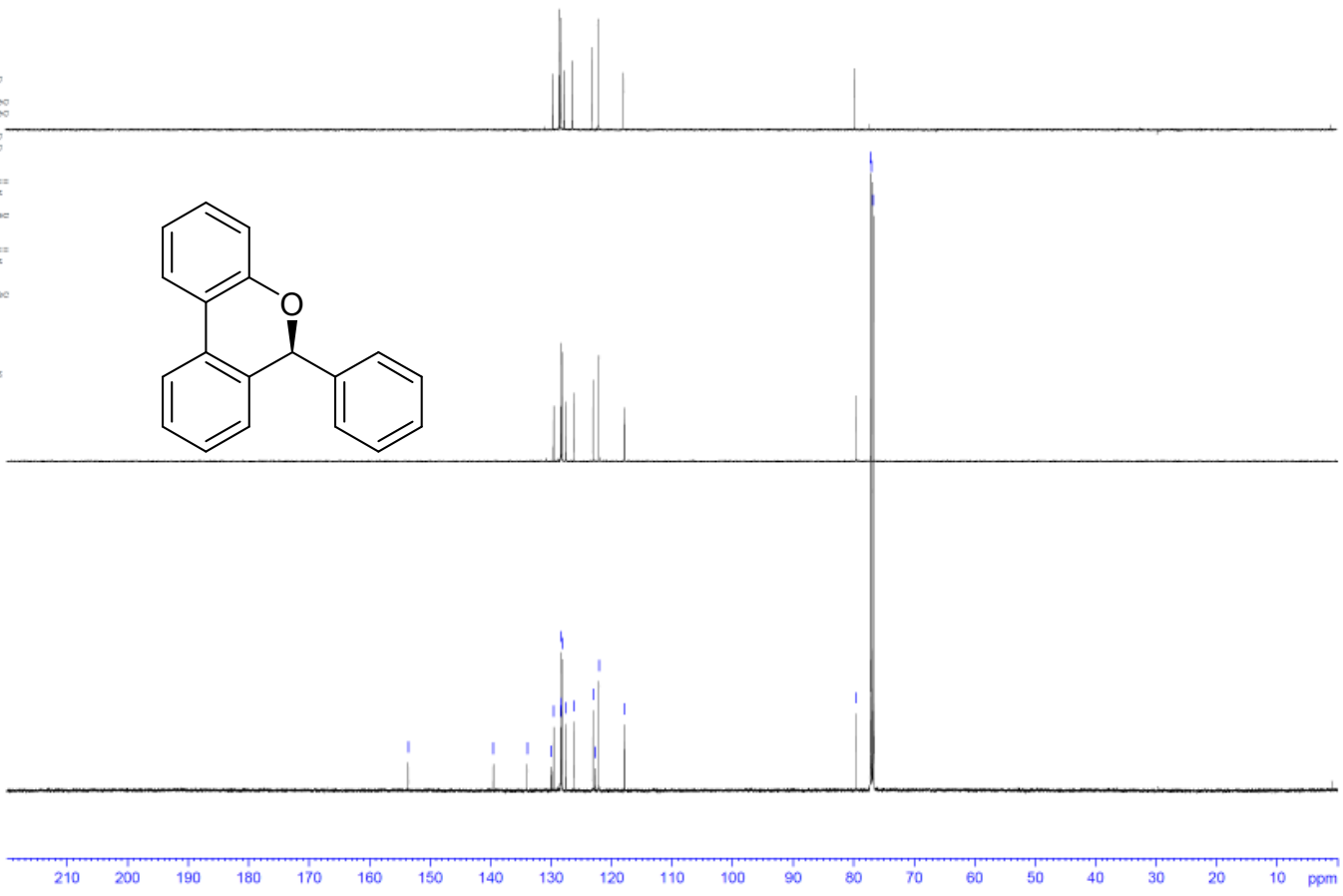

S55 


\section{(S)-(4-Chlorophenyl)(phenyl)methanol (11a)}

${ }^{1} \mathrm{H}$ NMR (400 MHz, $\left.\mathrm{CDCl}_{3}\right)$

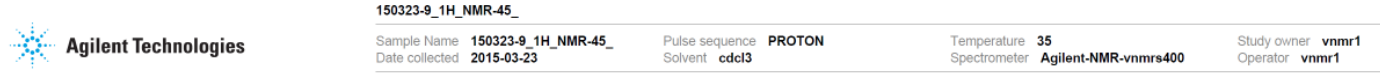
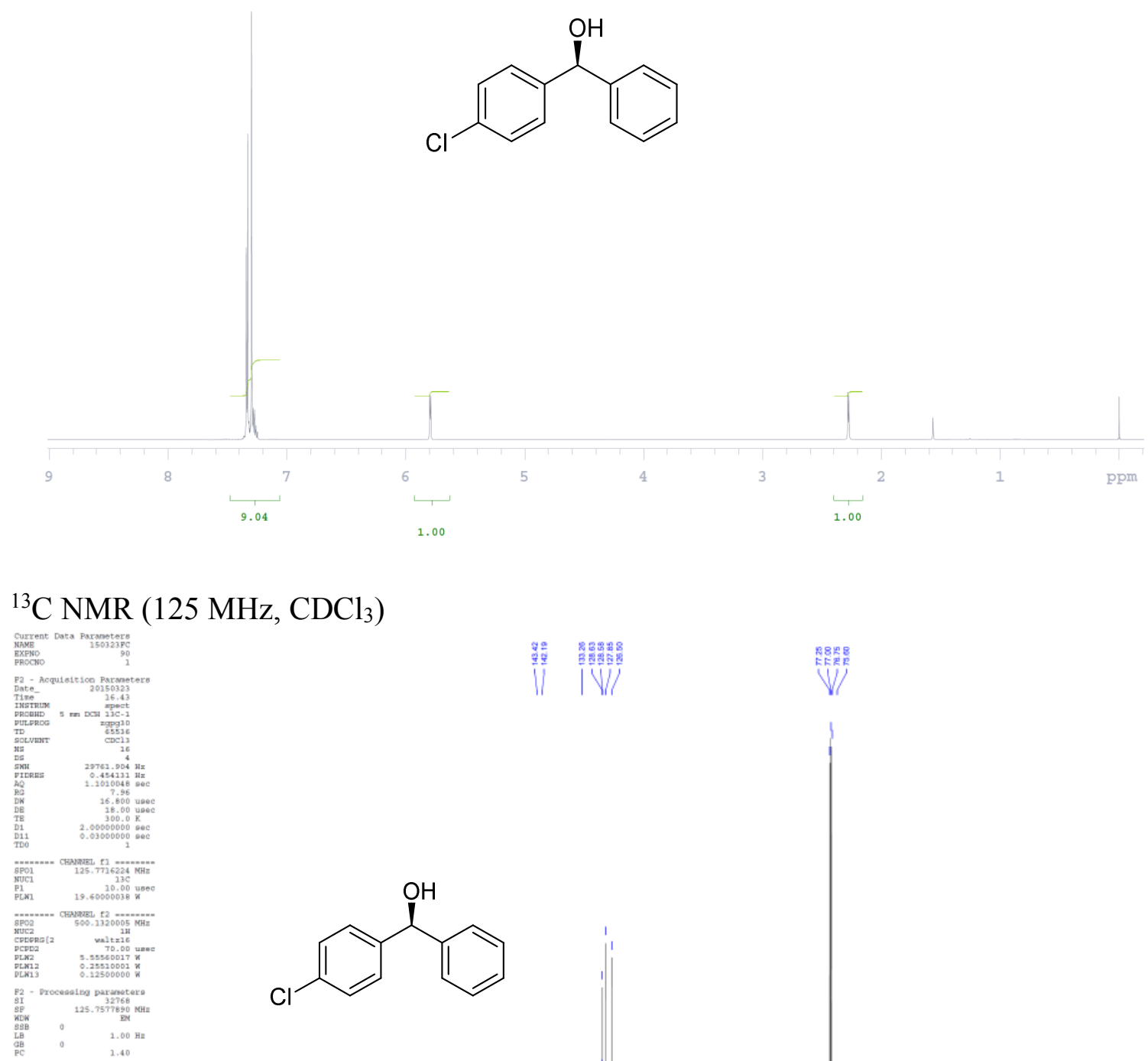

$\left(125 \mathrm{MHz}, \mathrm{CDCl}_{3}\right)$

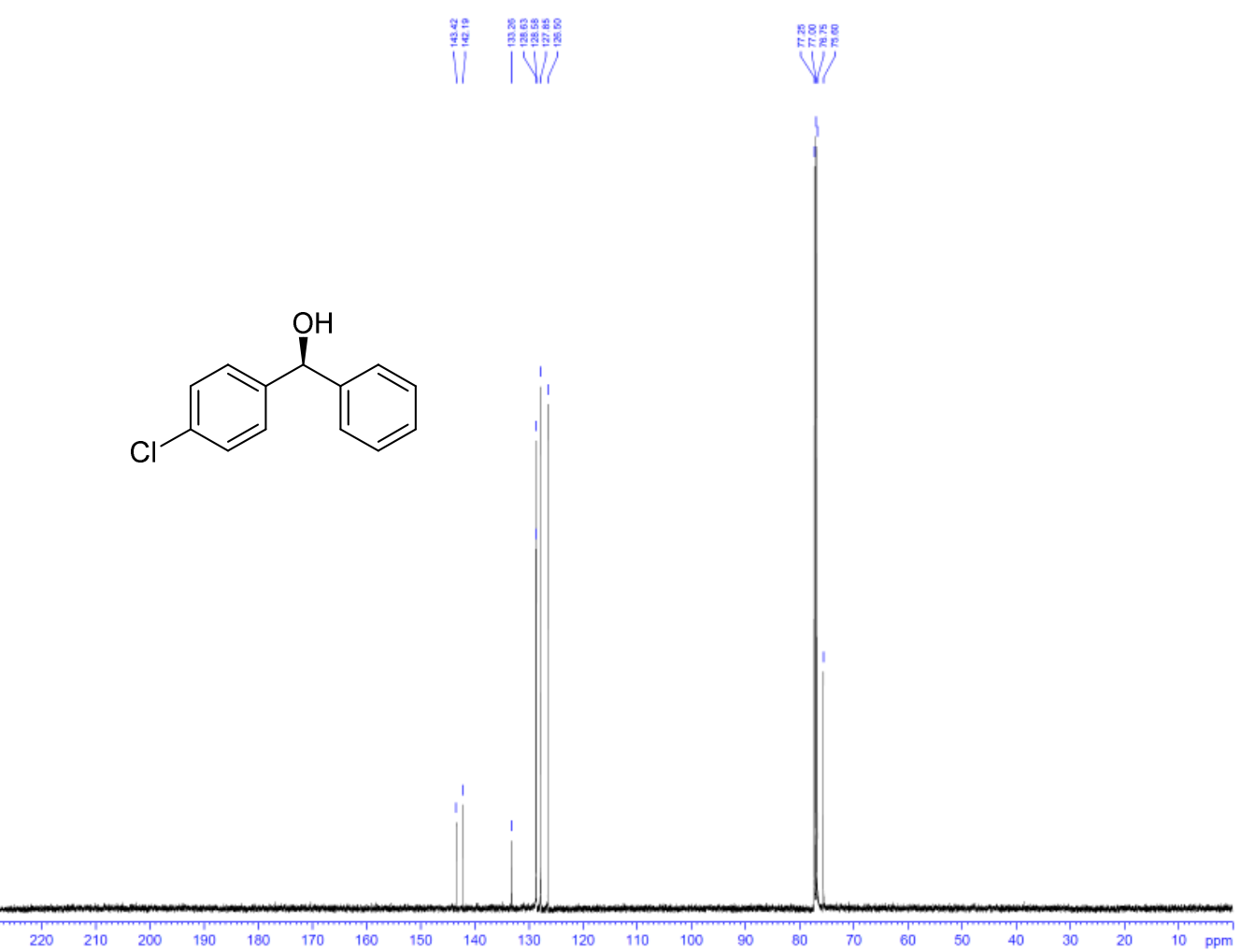


(S)-(3,4-Dichlorophenyl)(phenyl)methanol (11b)

${ }^{1} \mathrm{H}$ NMR (400 MHz, $\left.\mathrm{CDCl}_{3}\right)$

Agilent Technologies

Sample Name $150401-4$-1H-NMR-23
Date collected $2015-04-01$

Pulse sequence PROTON
Solvent cdcl3

Temperature $\mathbf{3 5}$
Spectrometer $\quad$ Agilent-NMR-vnmrs400

Study owner nmr
Operator vnmr1

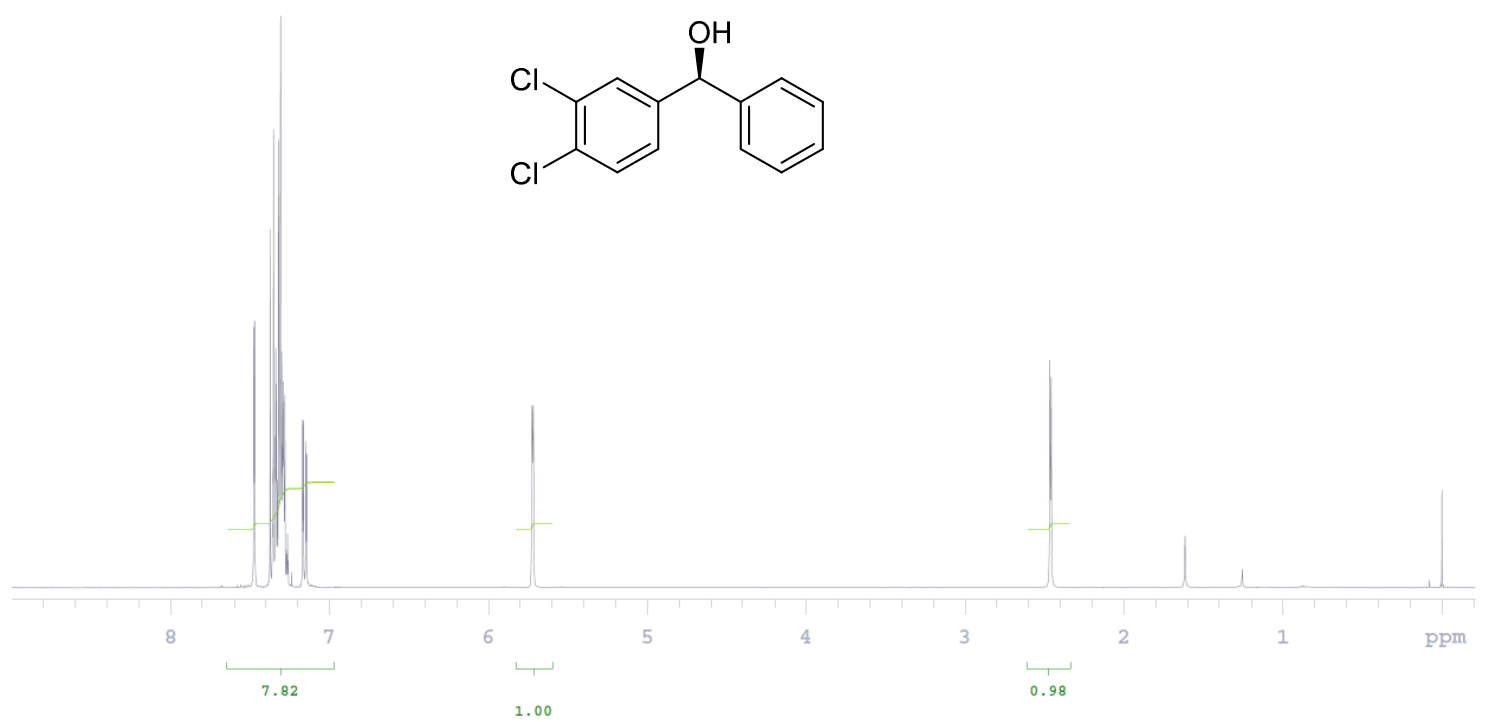

$\left.{ }^{13} \mathrm{C} \mathrm{NMR} \mathrm{(125} \mathrm{MHz,} \mathrm{CDCl}_{3}\right)$

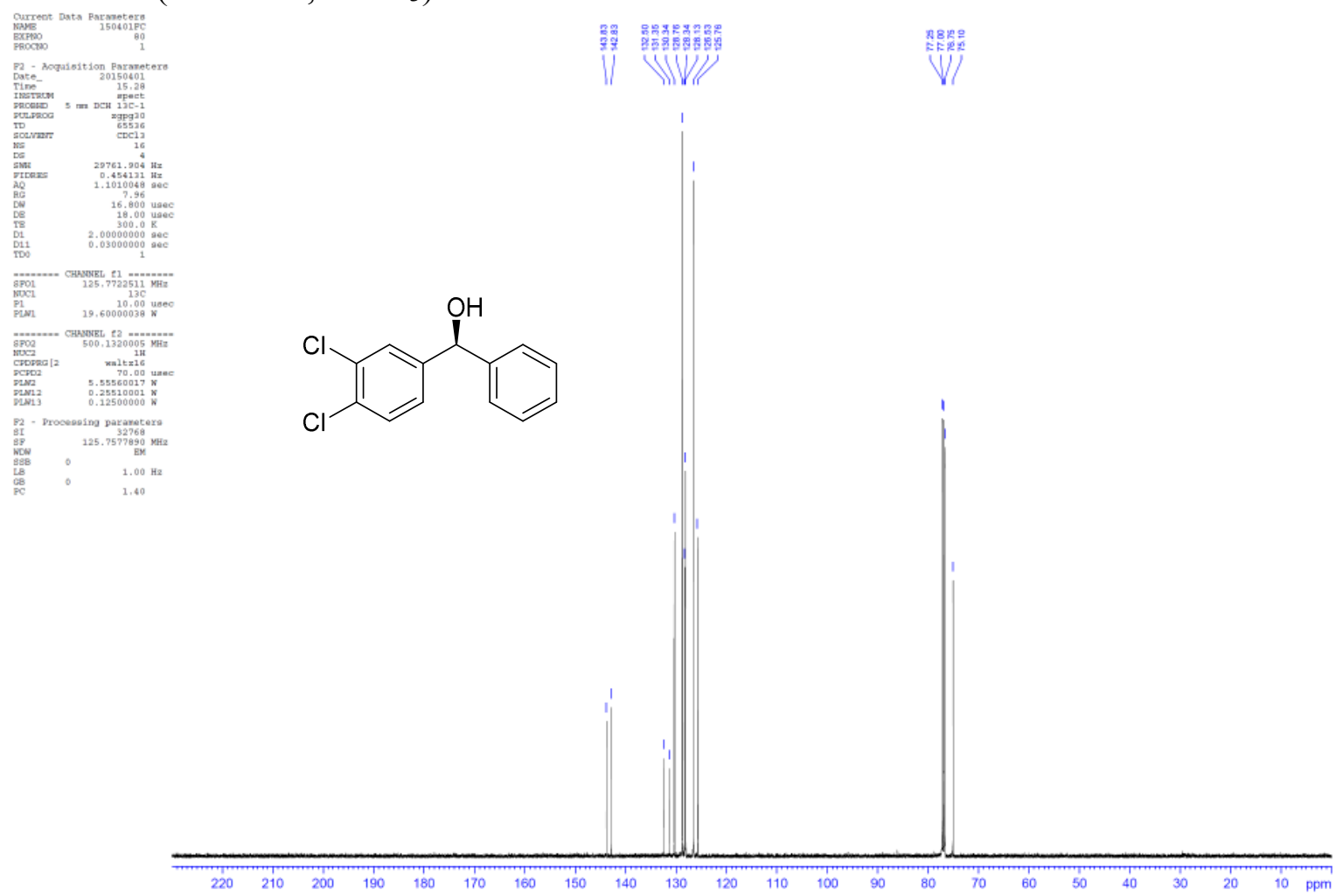

S57 


\section{(S)-(3,4-Difluorophenyl)(phenyl)methanol (11c)}

\section{${ }^{1} \mathrm{H}$ NMR $\left(400 \mathrm{MHz}, \mathrm{CDCl}_{3}\right)$}

Agilent Technologies

150401-2_1H-NMR-13

$\begin{array}{ll}\text { Sample Name 150401-2_1H-NMR-13__ } & \begin{array}{l}\text { Pulse sequence PROTON } \\ \text { Date collected } 2015-04-01\end{array}\end{array}$

Temperature 35

5 Agilent-NMR-vnmrs400

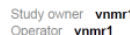
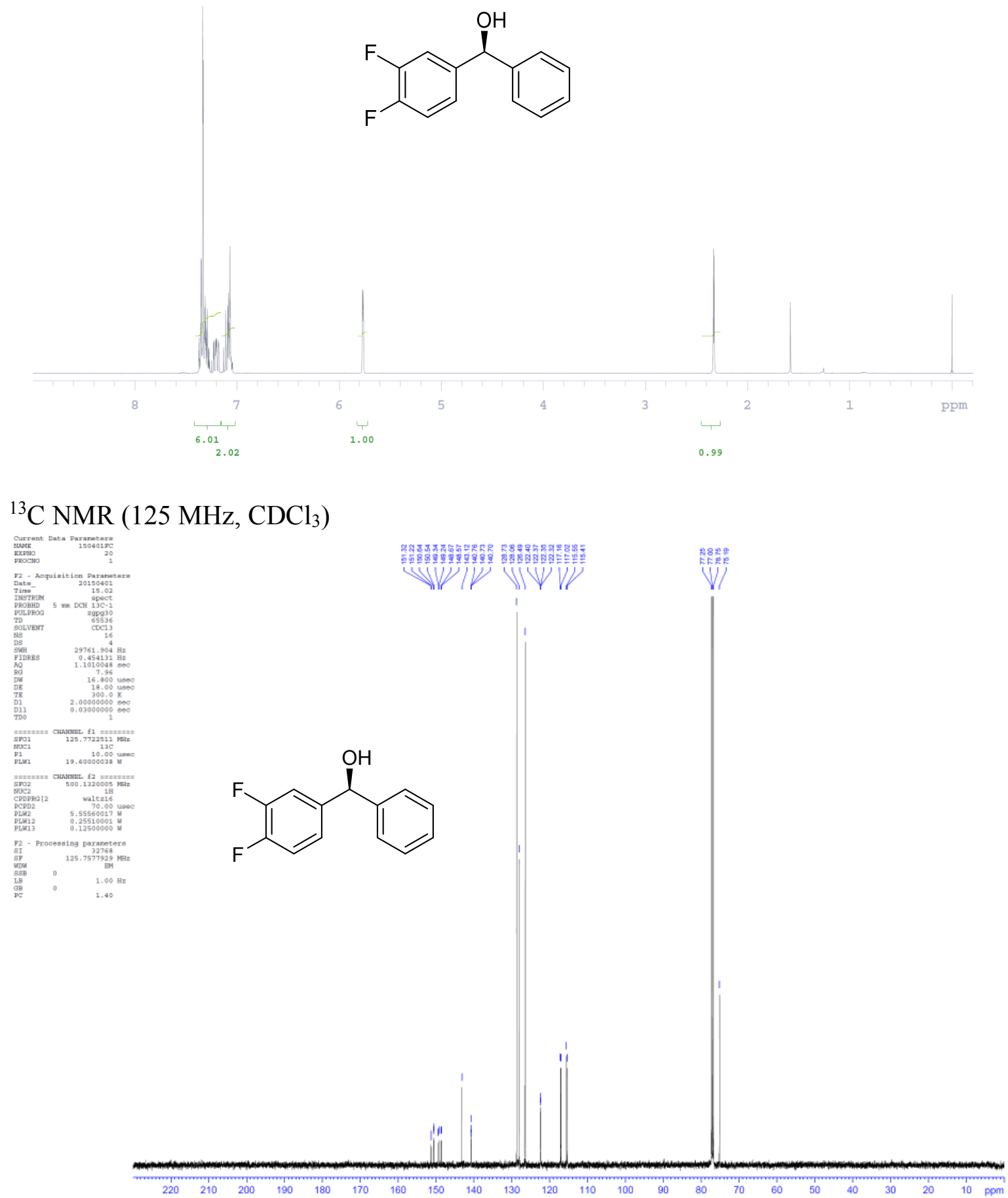

$\left(125 \mathrm{MHz}, \mathrm{CDCl}_{3}\right)$ 


\section{(S)-(4-Nitrophenyl)(phenyl)methanol (11d)}

${ }^{1} \mathrm{H}$ NMR $\left(400 \mathrm{MHz}, \mathrm{CDCl}_{3}\right)$

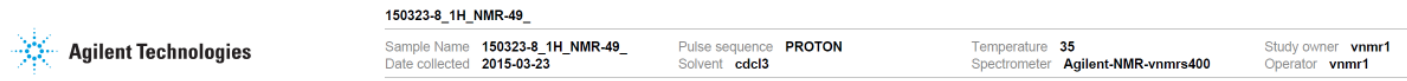

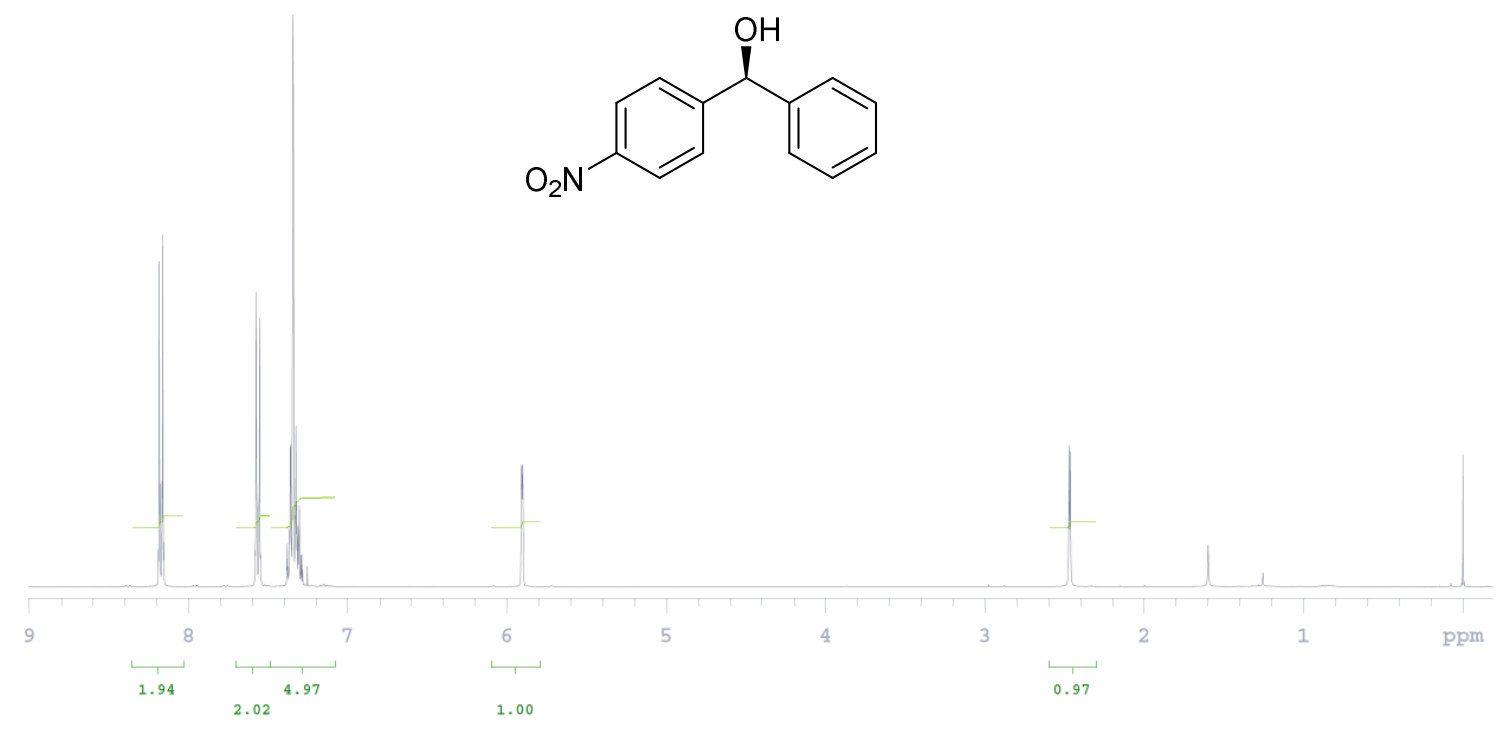

${ }^{13} \mathrm{C}$ NMR $\left(125 \mathrm{MHz}, \mathrm{CDCl}_{3}\right)$
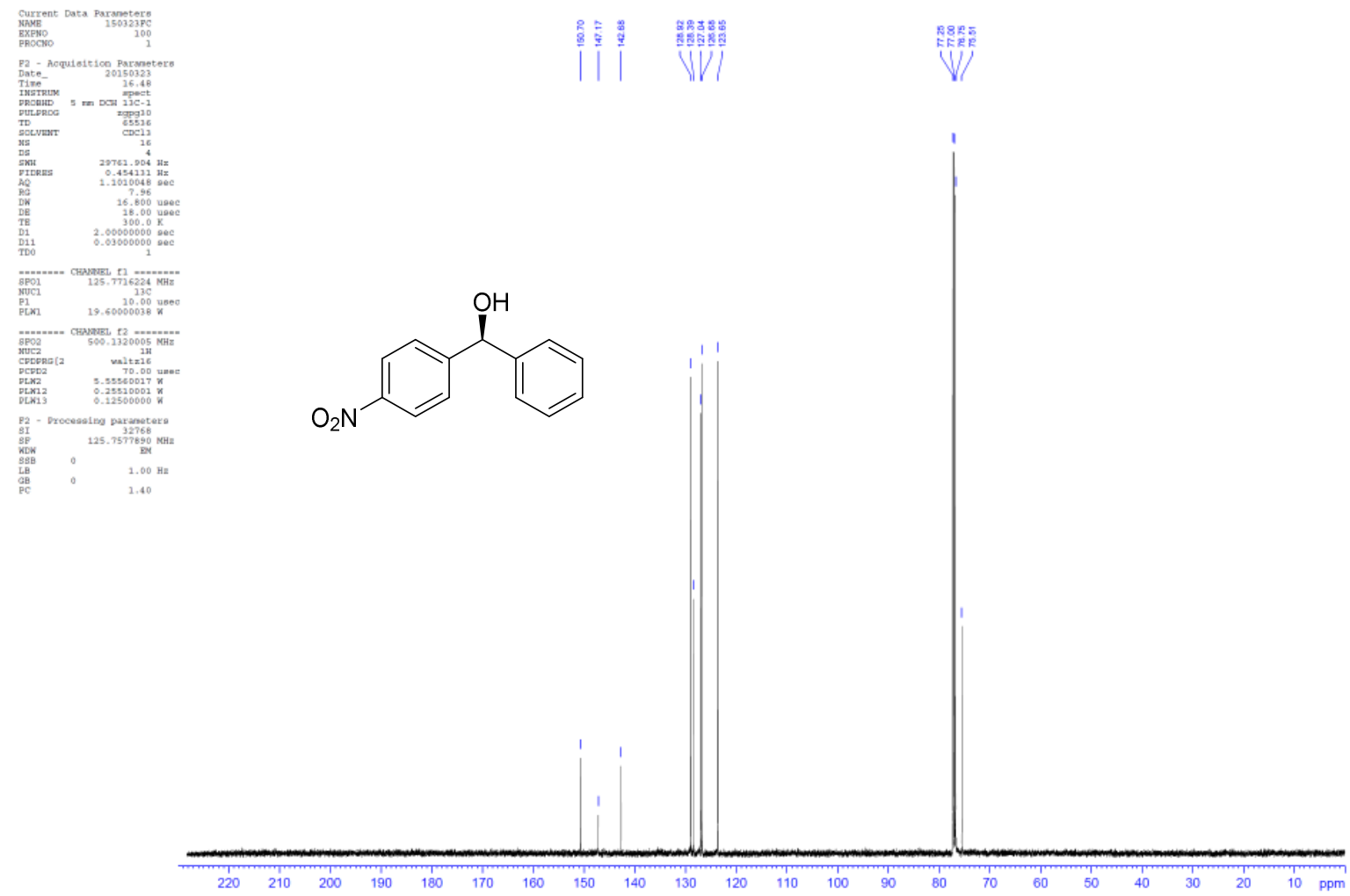


\section{(S)-(4-Chloro-3-nitrophenyl)(phenyl)methanol (11e)}

${ }^{1} \mathrm{H}$ NMR $\left(400 \mathrm{MHz}, \mathrm{CDCl}_{3}\right)$

Agilent Technologies

150323-1 (1H-NMR-24)

Sample Name 150323-1 (1H-NMR-24)

Pulse sequence PROTON
Solvent cdcl3

Temperature 35

Agilent-NMR-vnmrs400

Study owner vnmr1
Operator vnmr1

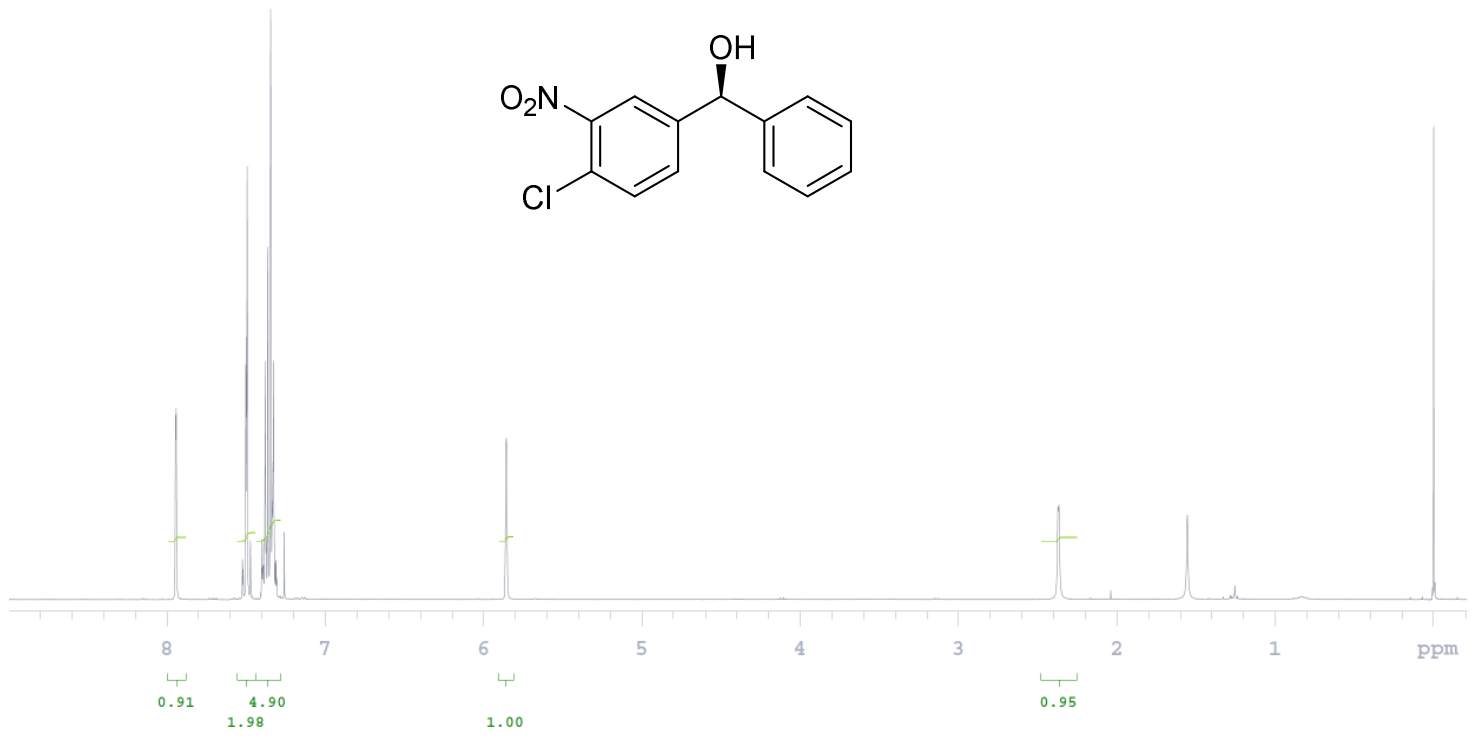

${ }^{13} \mathrm{C}$ NMR $\left(125 \mathrm{MHz}, \mathrm{CDCl}_{3}\right)$
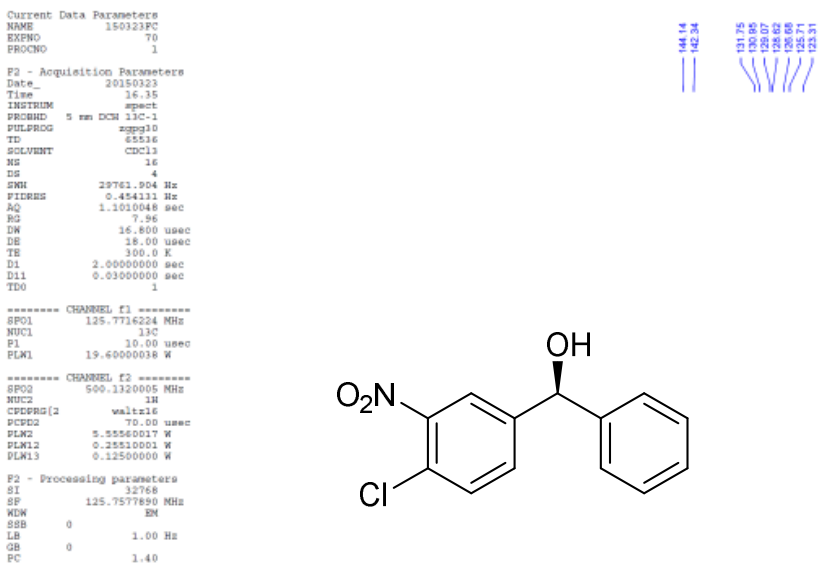

$\sqrt{9828}$

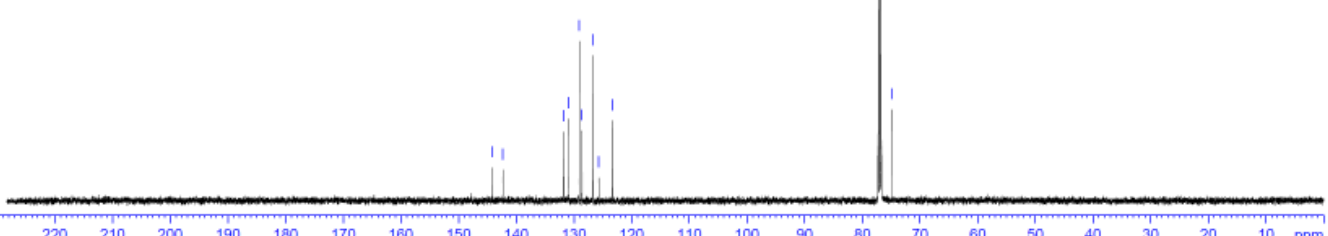




\section{(S)-Phenyl(3,4,5-trifluorophenyl)methanol (11f)}

${ }^{1} \mathrm{H}$ NMR $\left(400 \mathrm{MHz}, \mathrm{CDCl}_{3}\right)$

150604-3_1H-NMR-59

Agilent Technologies

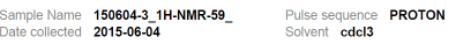

$\begin{array}{ll}\text { Temperature } & 35 \\ \text { Spectrometer Agilent-NMR-vnmrs400 } & \text { Study owner } \text { vnmr }^{2} \\ \text { Operator vnmr1 }\end{array}$

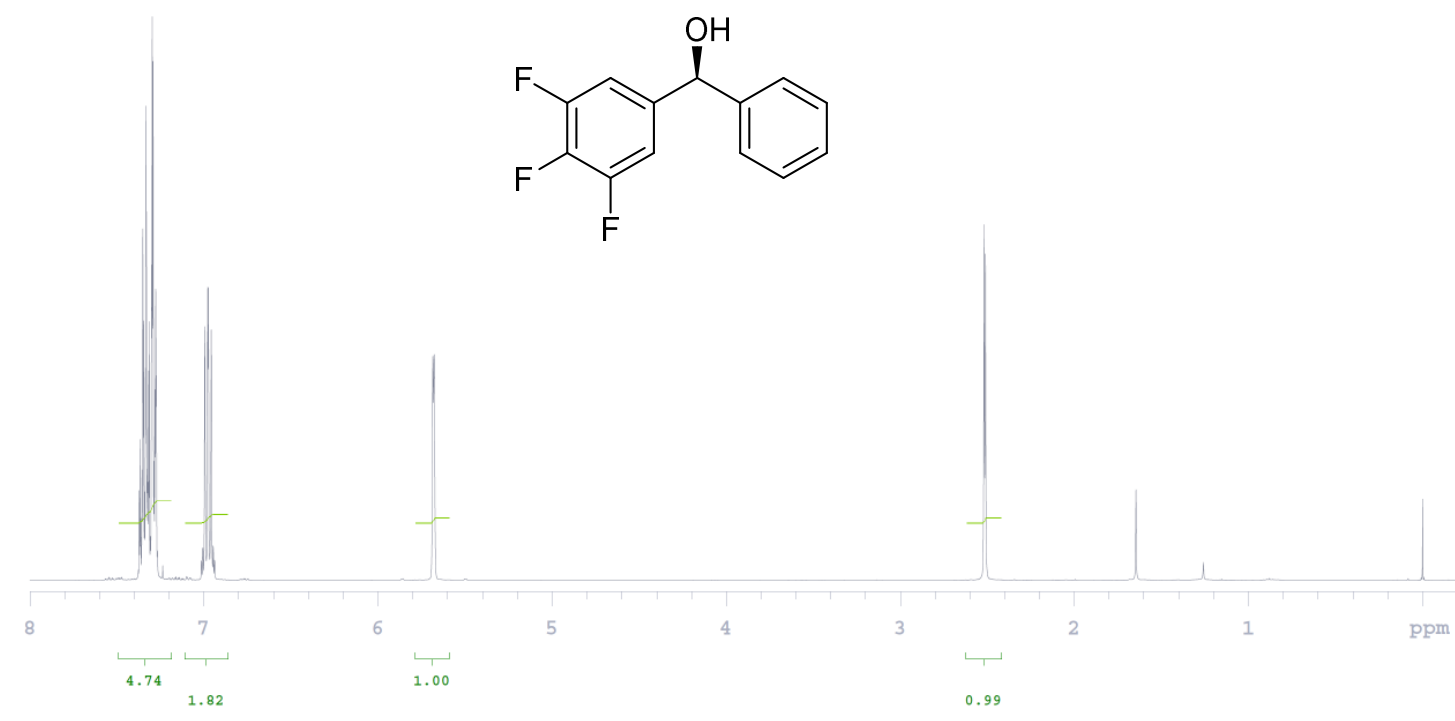

${ }^{13} \mathrm{C}$ NMR $\left(125 \mathrm{MHz}, \mathrm{CDCl}_{3}\right)$
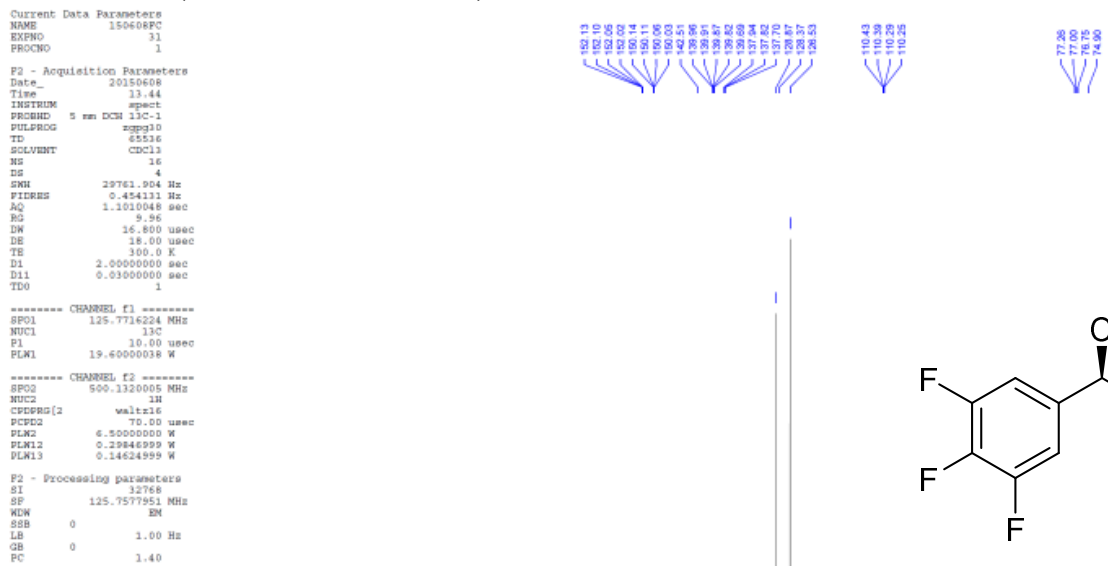<smiles>O[C@H](c1ccccc1)c1cc(F)c(F)c(F)c1</smiles>

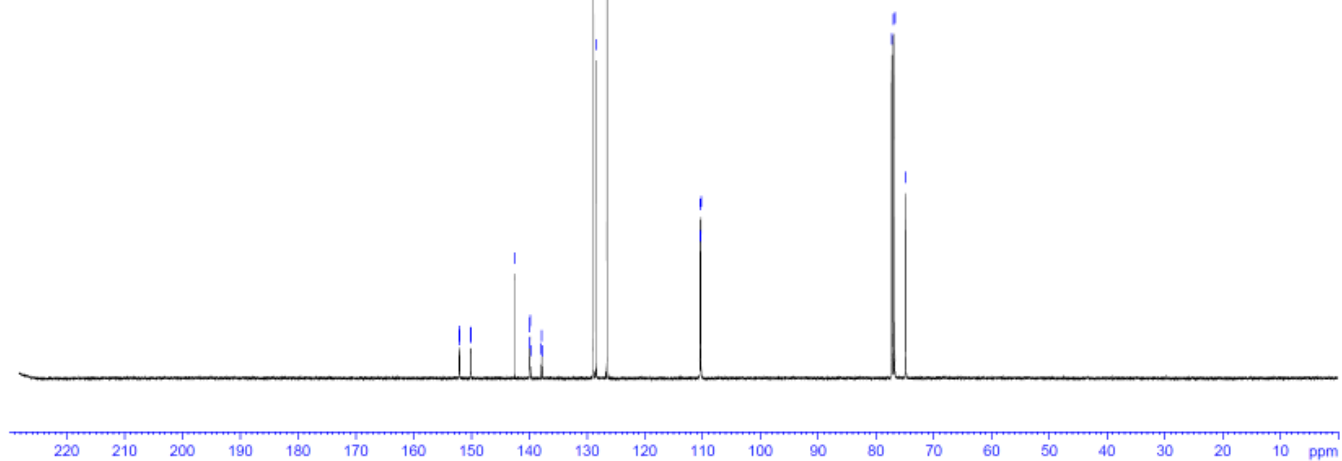




\section{(S)-(3,5-Dinitrophenyl)(phenyl)methanol (11g)}

${ }^{1} \mathrm{H}$ NMR $\left(400 \mathrm{MHz}, \mathrm{CDCl}_{3}\right)$

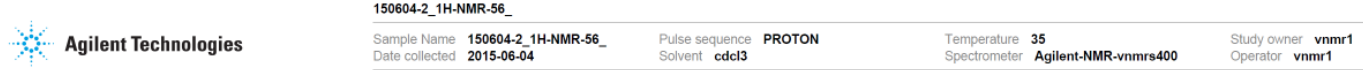

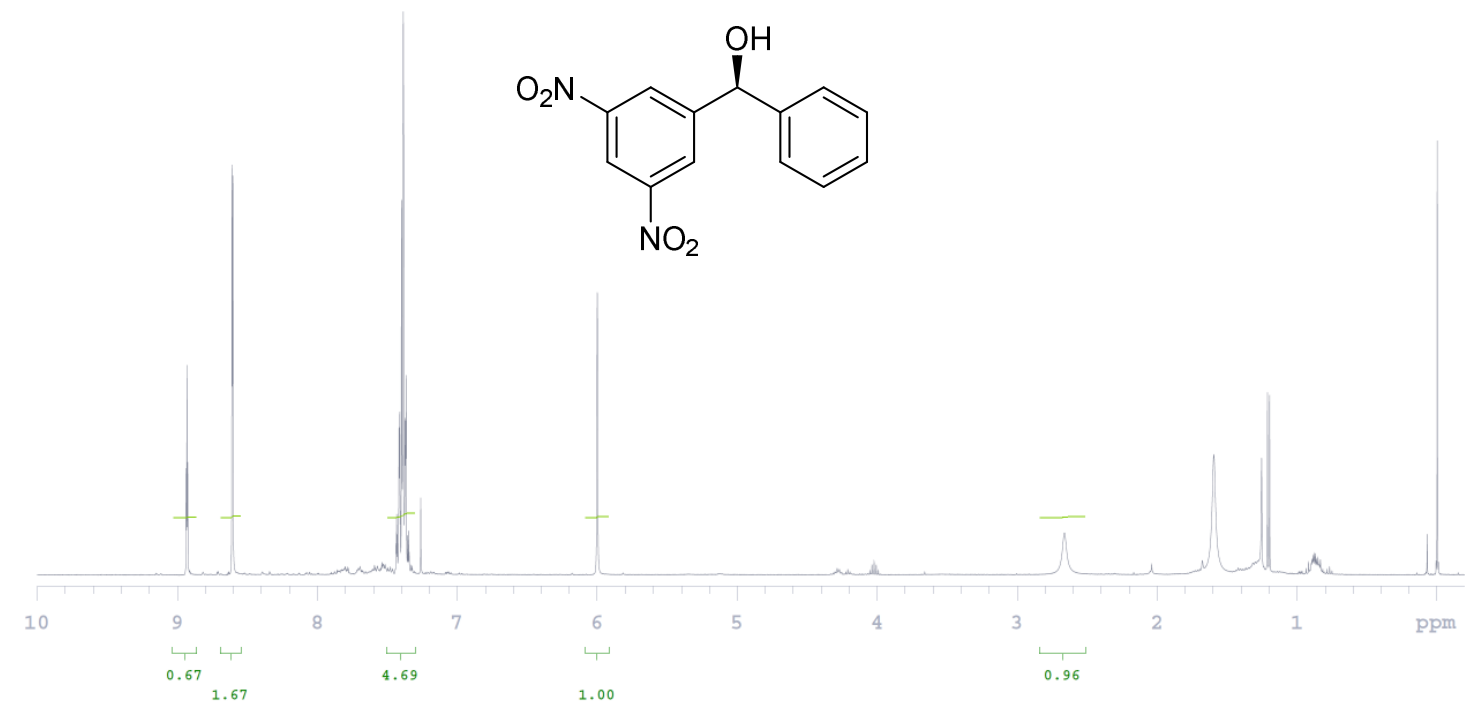

${ }^{13} \mathrm{C}$ NMR $\left(125 \mathrm{MHz}, \mathrm{CDCl}_{3}\right)$
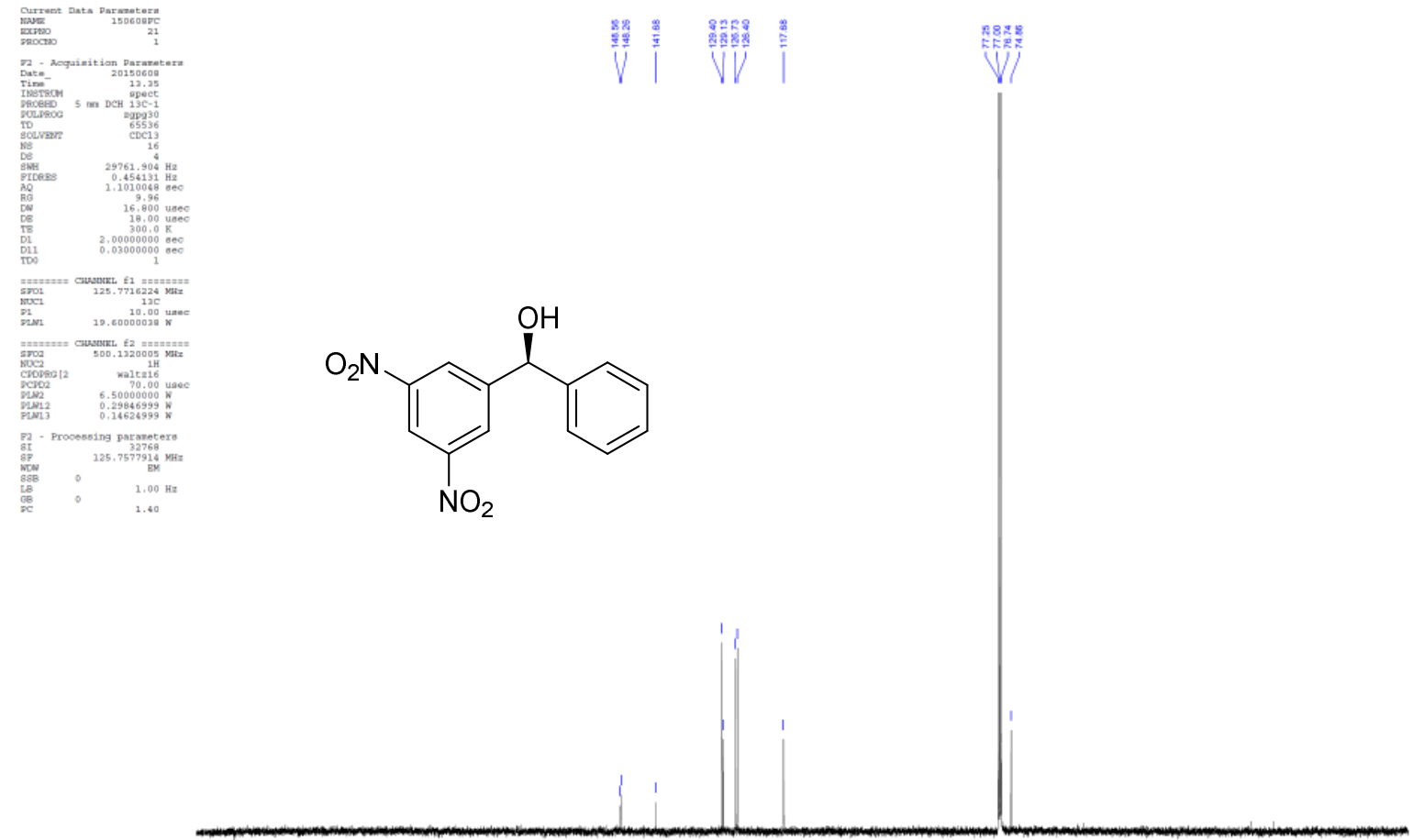

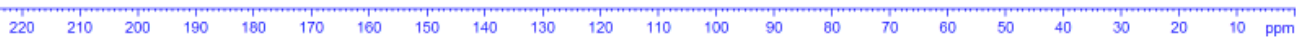


(R)-(4-Methoxyphenyl)(phenyl)methanol (11h)

${ }^{1} \mathrm{H}$ NMR $\left(400 \mathrm{MHz}, \mathrm{CDCl}_{3}\right)$

Agilent Technologies

150401-3_ HH-NMR-43

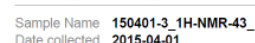

Pulse sequence PROTON
Solvent cdcl3

Temperature 35
Sppectrometer

$\begin{array}{ll}35 & \text { Study owner vnmr1 } \\ \text { Agilent-NMR-vnmrs400 } & \text { Operator vnmr1 }\end{array}$

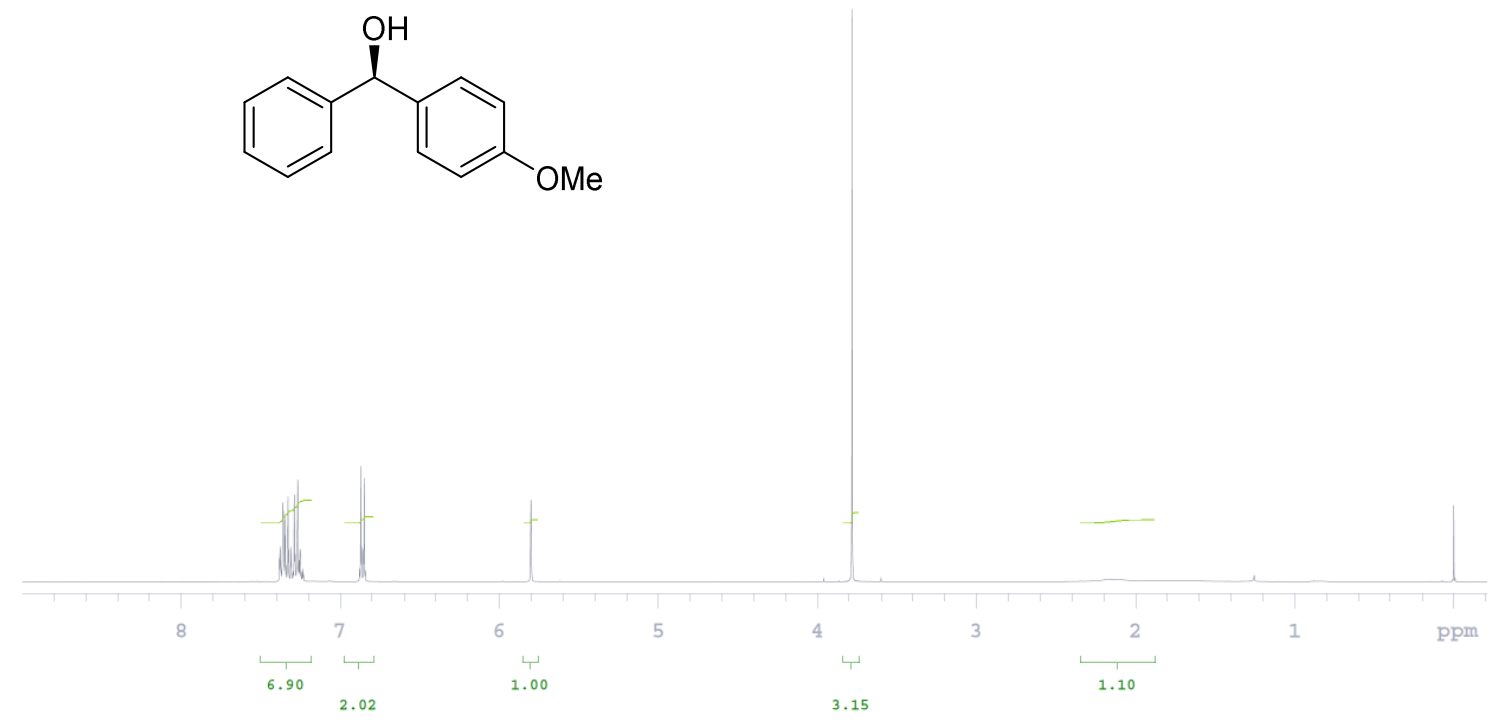

${ }^{13} \mathrm{C}$ NMR $\left(125 \mathrm{MHz}, \mathrm{CDCl}_{3}\right)$
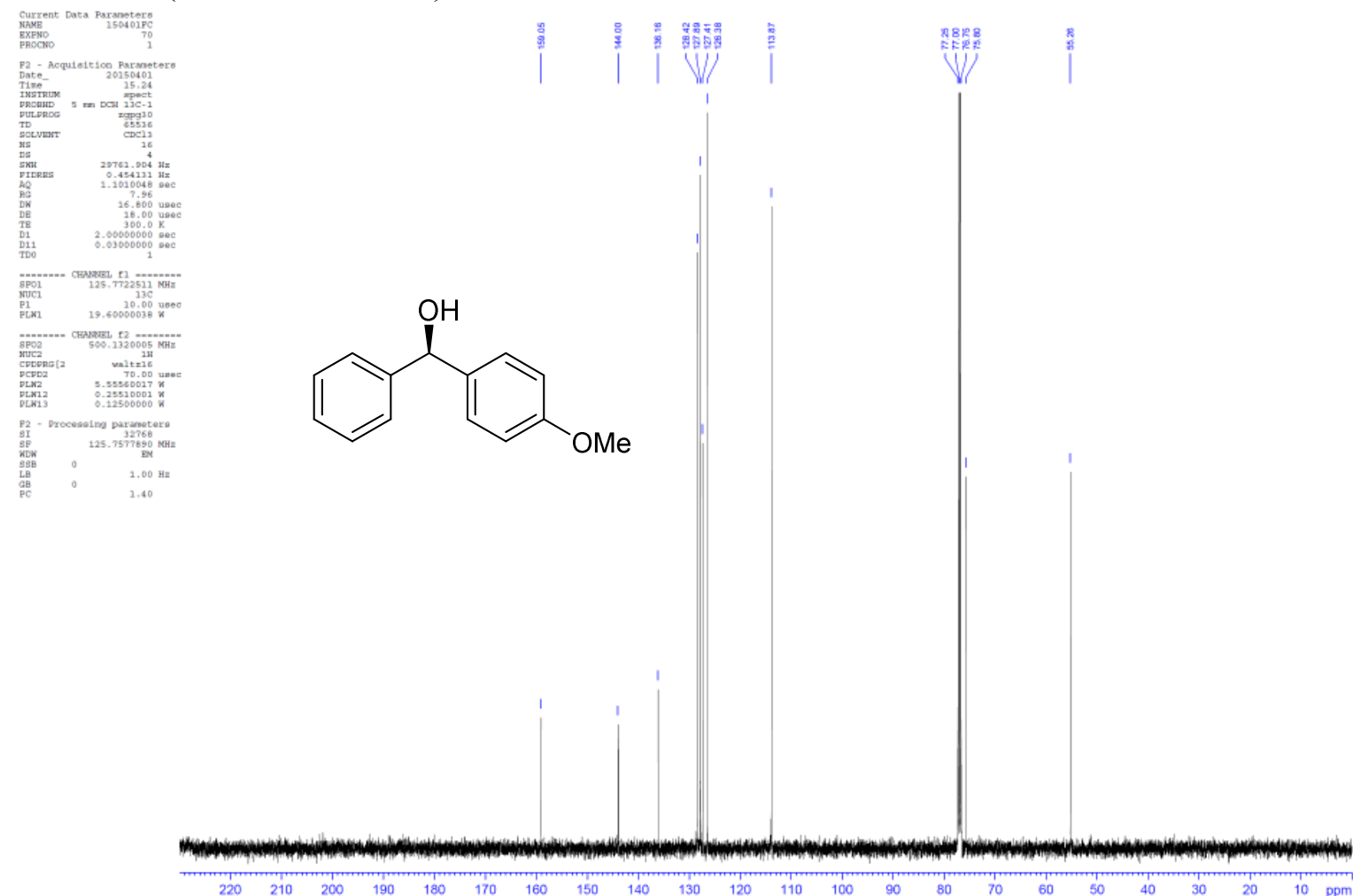


\section{(S)-(4-Chlorophenyl)(4-methoxyphenyl)methanol (11i)}

${ }^{1} \mathrm{H}$ NMR $\left(400 \mathrm{MHz}, \mathrm{CDCl}_{3}\right)$

Agilent Technologies

150323-7__1H_NMR-35_

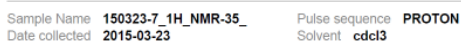

$\begin{array}{ll}\text { Temperature } 35 & \text { Study owner vnmr1 } \\ \text { Spectrometer Agilent-NMR-vnmrs400 } & \text { Operator vnmr1 }\end{array}$

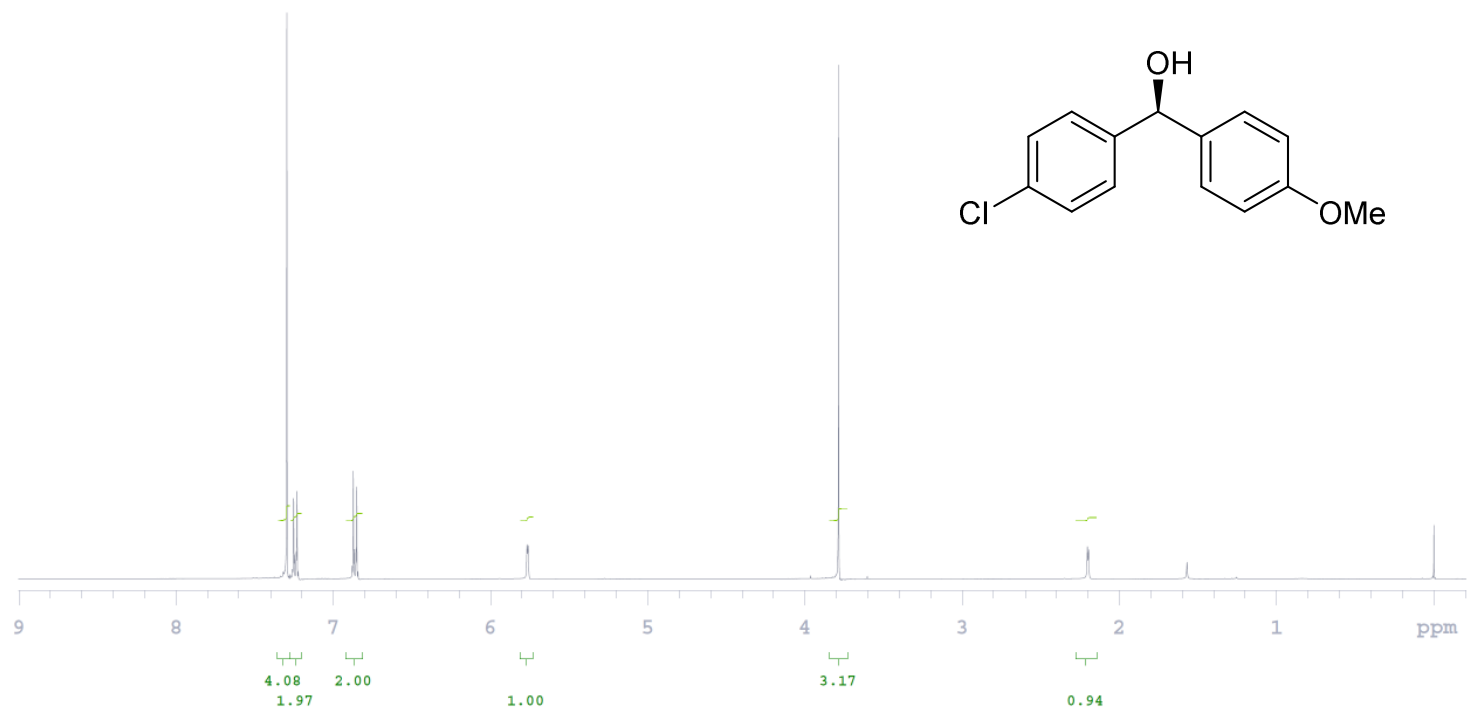

${ }^{13} \mathrm{C}$ NMR $\left(125 \mathrm{MHz}, \mathrm{CDCl}_{3}\right)$
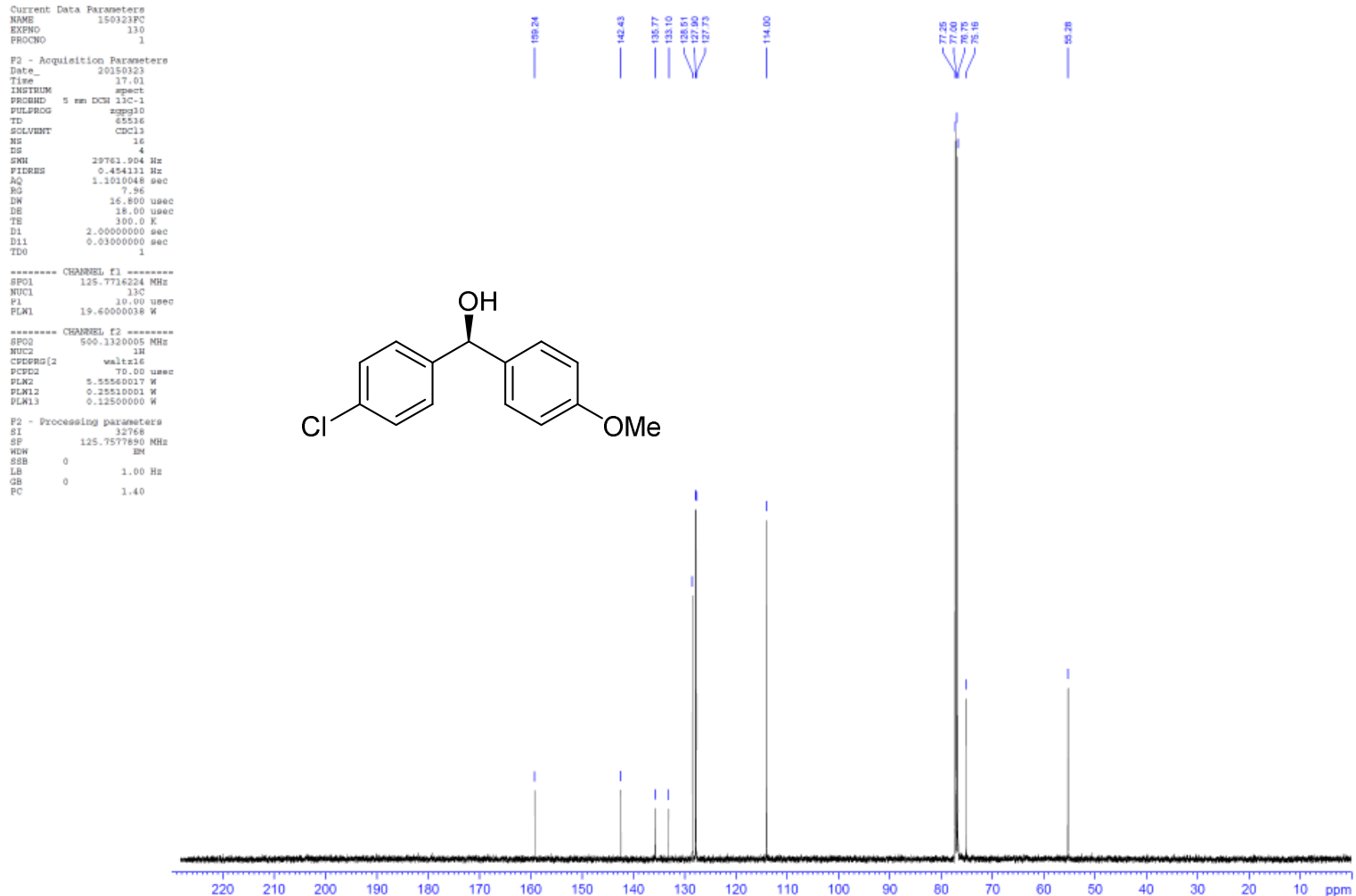


\section{(S)-4-((4-Chlorophenyl)(hydroxy)methyl)phenol (11j)}

${ }^{1} \mathrm{H}$ NMR (400 MHz, CD $\mathrm{CD}_{3} \mathrm{OD}$ )

Agilent Technologies
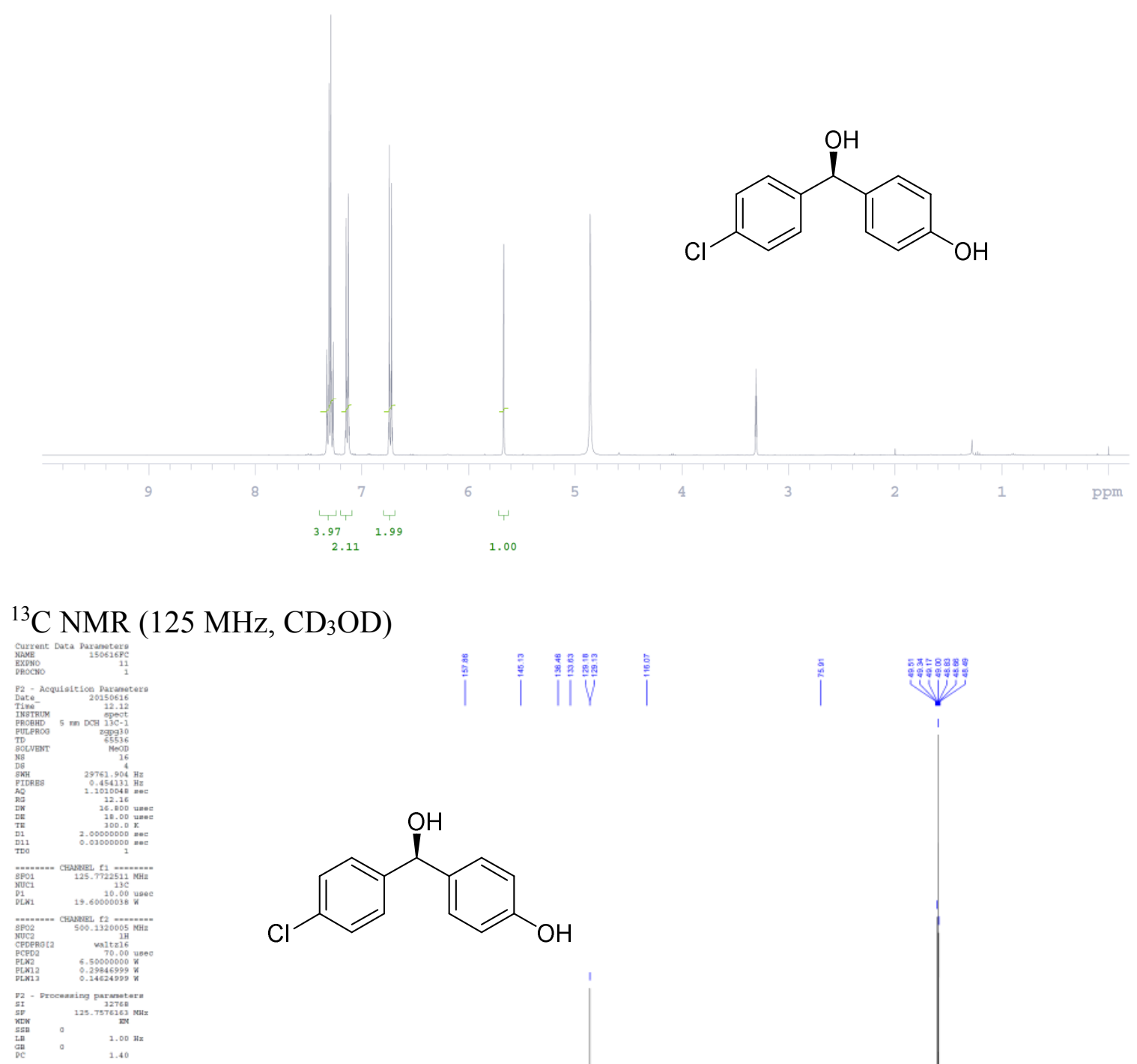<smiles>Oc1ccc([C@@H](O)c2ccc(Cl)cc2)cc1</smiles> 


\section{(R)-(4-Methoxyphenyl)(4-nitrophenyl)methanol (11k)}

${ }^{1} \mathrm{H}$ NMR (400 MHz, $\left.\mathrm{CDCl}_{3}\right)$

Agilent Technologies

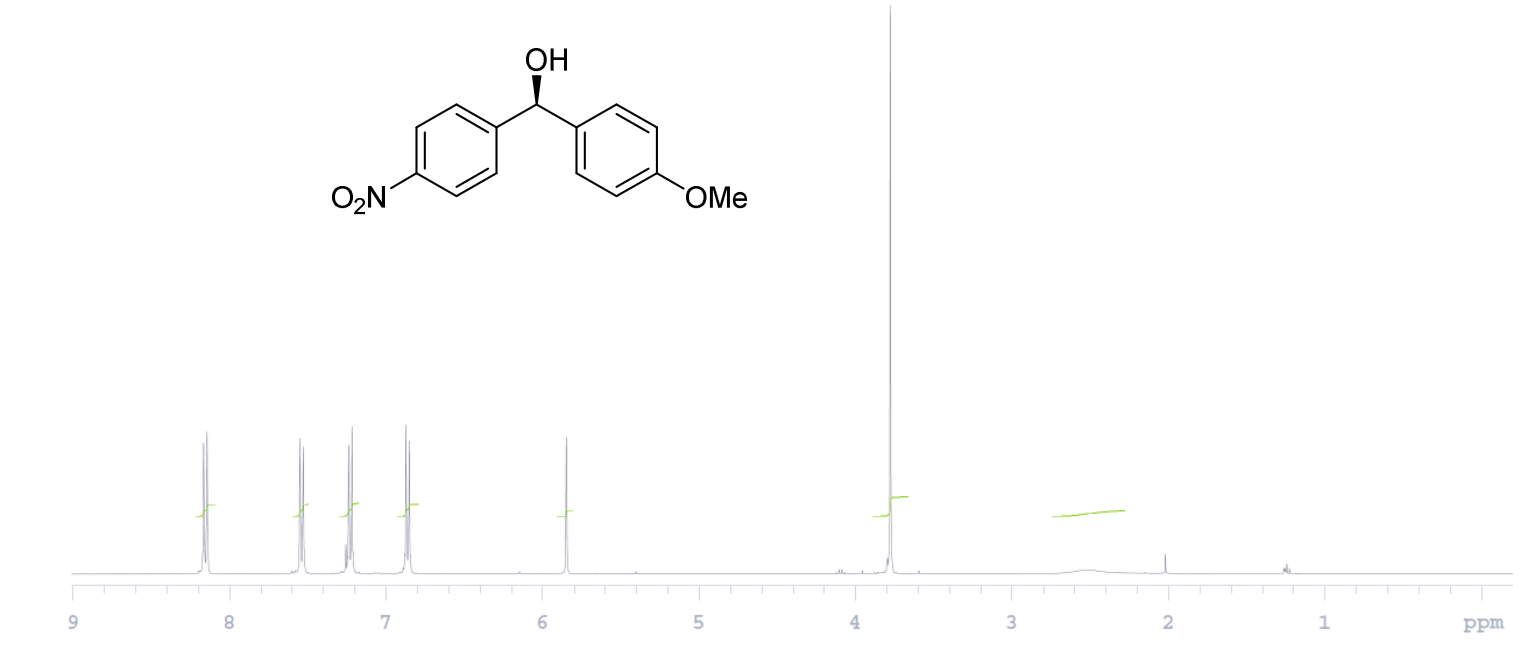

${ }^{13} \mathrm{C}$ NMR $\left(125 \mathrm{MHz}, \mathrm{CDCl}_{3}\right)$
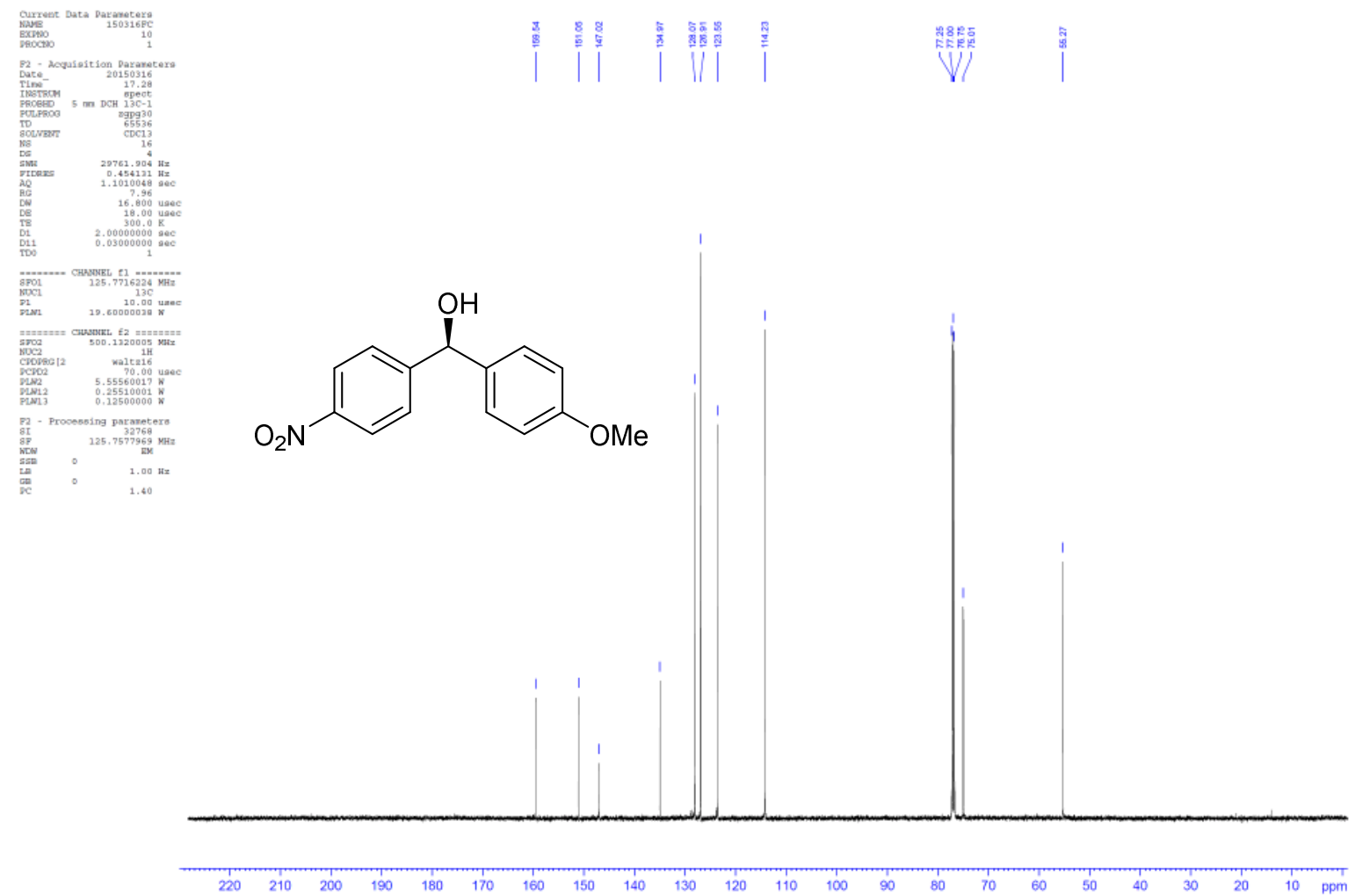


\section{(S)-(3,5-Dinitrophenyl)(4-methoxyphenyl)methanol (111)}

\section{${ }^{1} \mathrm{H}$ NMR $\left(400 \mathrm{MHz}, \mathrm{CDCl}_{3}\right)$}

Agilent Technologies

150616-2_1H-NMR-62

$\begin{array}{ll}\text { Sample Name 150616-2__H-NMR-62_ } & \text { Pulse sequence PROTON } \\ \text { Date collected 2015-06-16 } & \text { Solvent cdd }\end{array}$

Temperature 35

Agilent-NMR-vnmrs400

Study owner vnmr1
Operator vnmr1

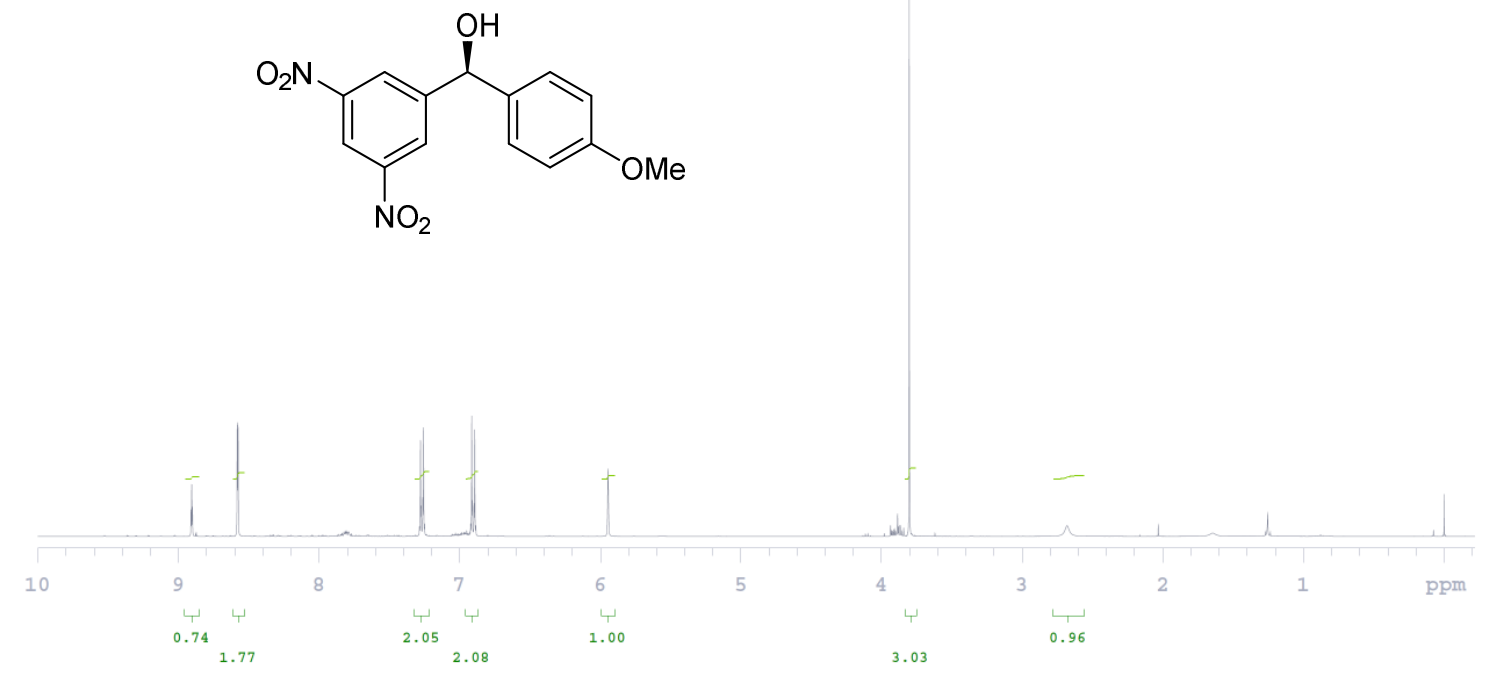

${ }^{13} \mathrm{C} \mathrm{NMR}\left(125 \mathrm{MHz}, \mathrm{CDCl}_{3}\right)$
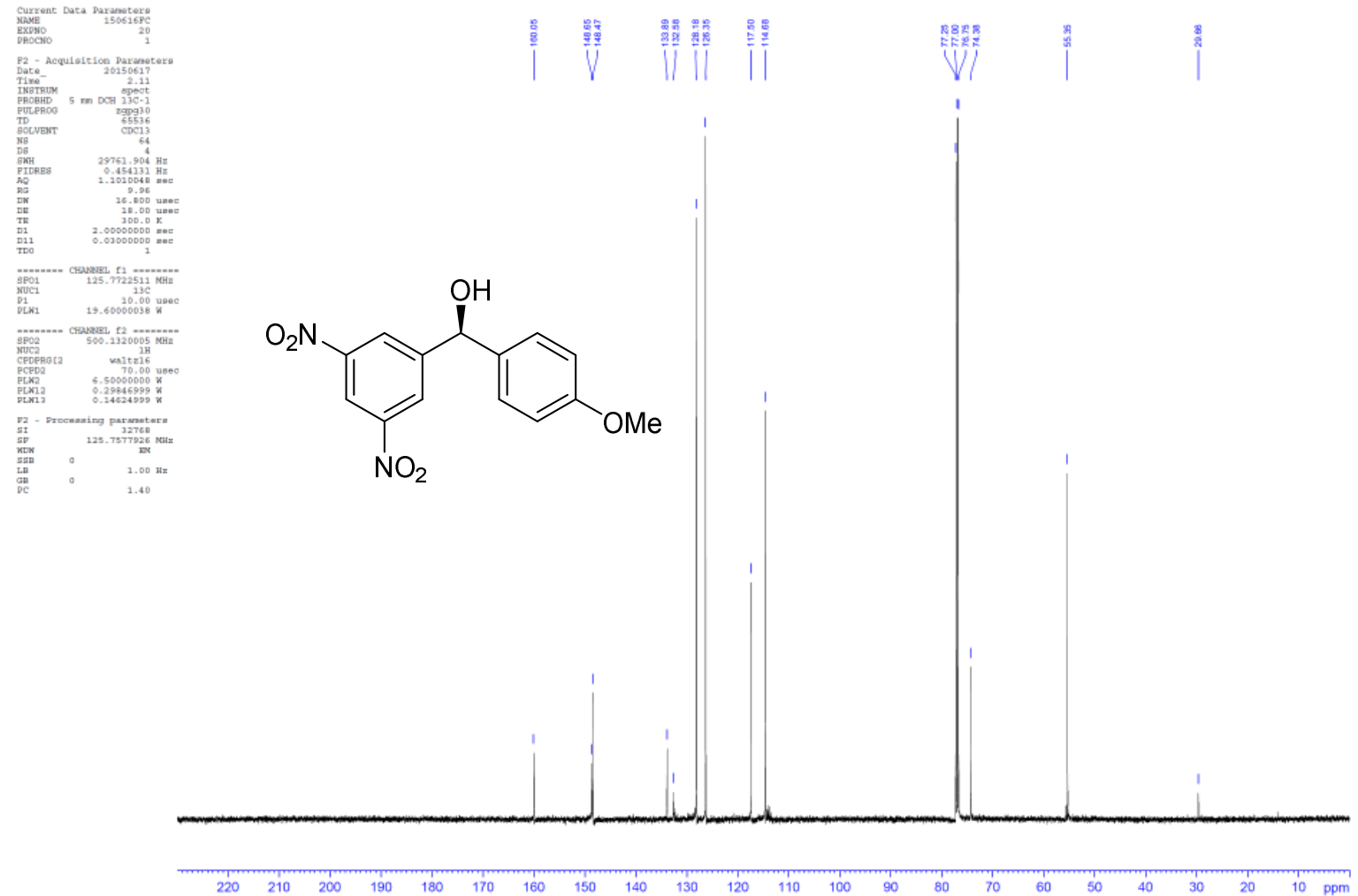


\section{(S)-Phenyl(ferrocenyl)methanol (11 m)}

${ }^{1} \mathrm{H}$ NMR $\left(400 \mathrm{MHz}, \mathrm{CDCl}_{3}\right)$

150401-10_1H-NMR-53

Agilent Technologies

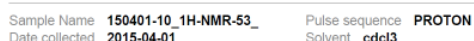

Temperature 35 Study owner vnmr1

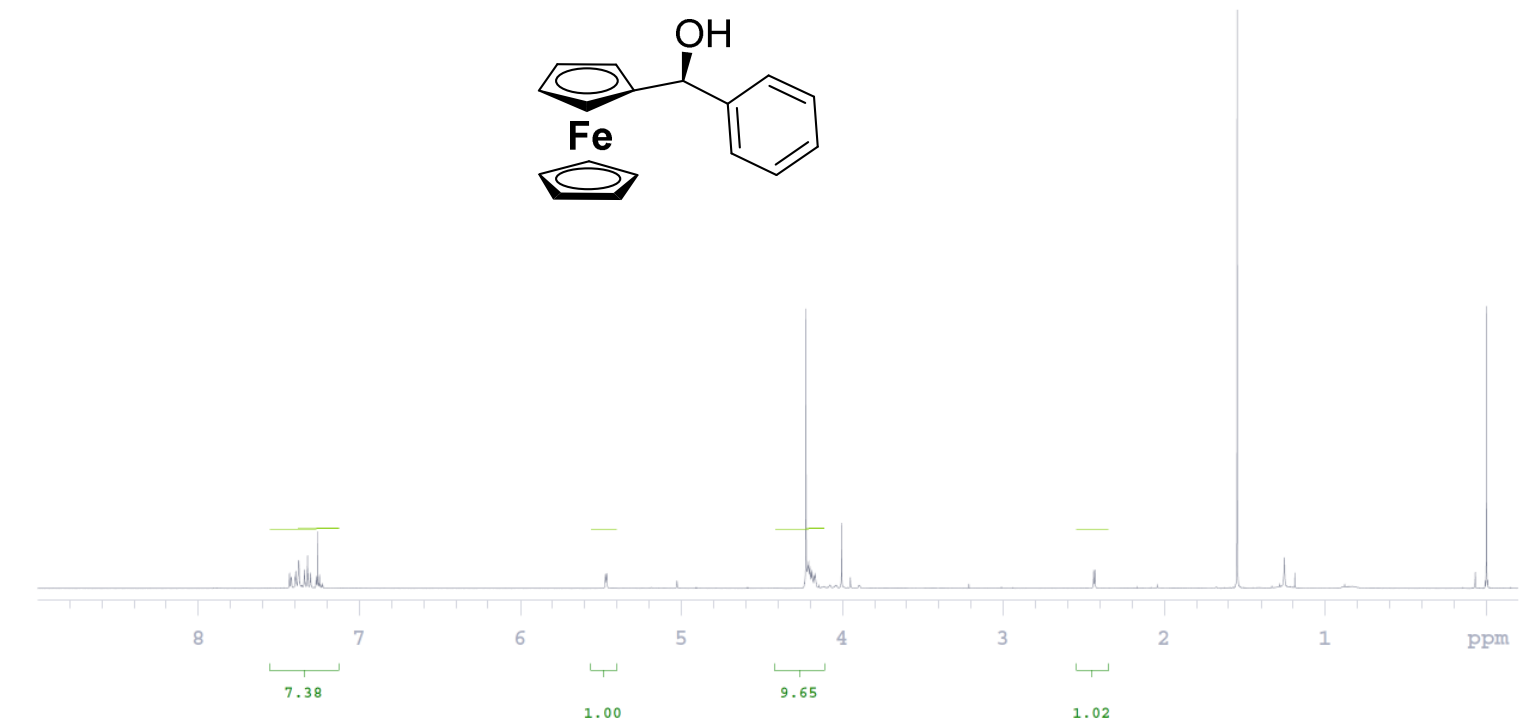

${ }^{13} \mathrm{C} \mathrm{NMR}\left(125 \mathrm{MHz}, \mathrm{CDCl}_{3}\right)$
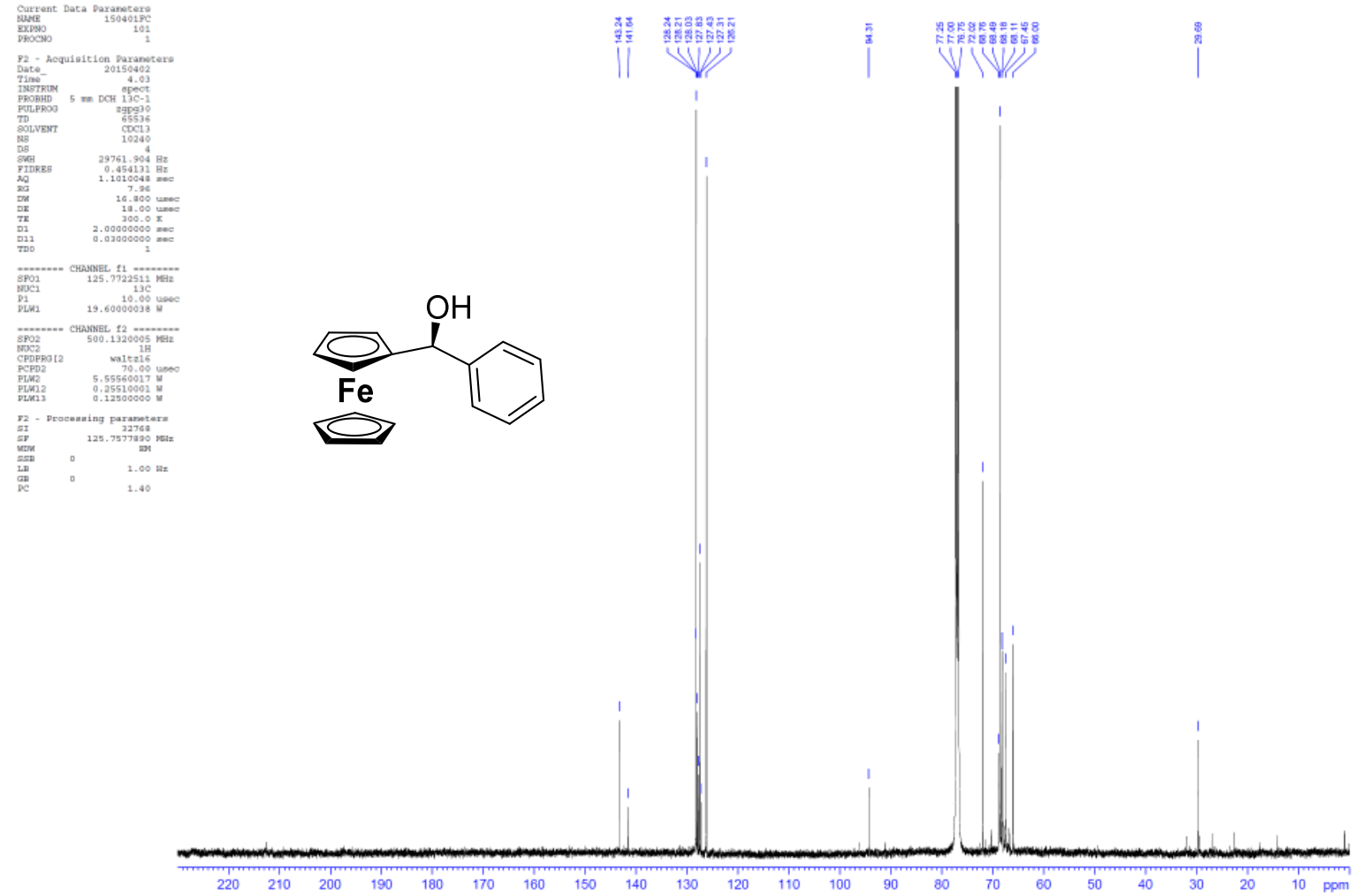


\section{(R)-(3-Nitrophenyl)(thiophen-2-yl)methanol (11n)}

${ }^{1} \mathrm{H}$ NMR $\left(400 \mathrm{MHz}, \mathrm{CDCl}_{3}\right)$

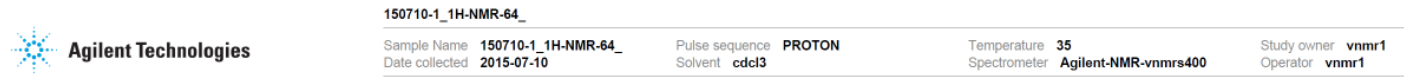

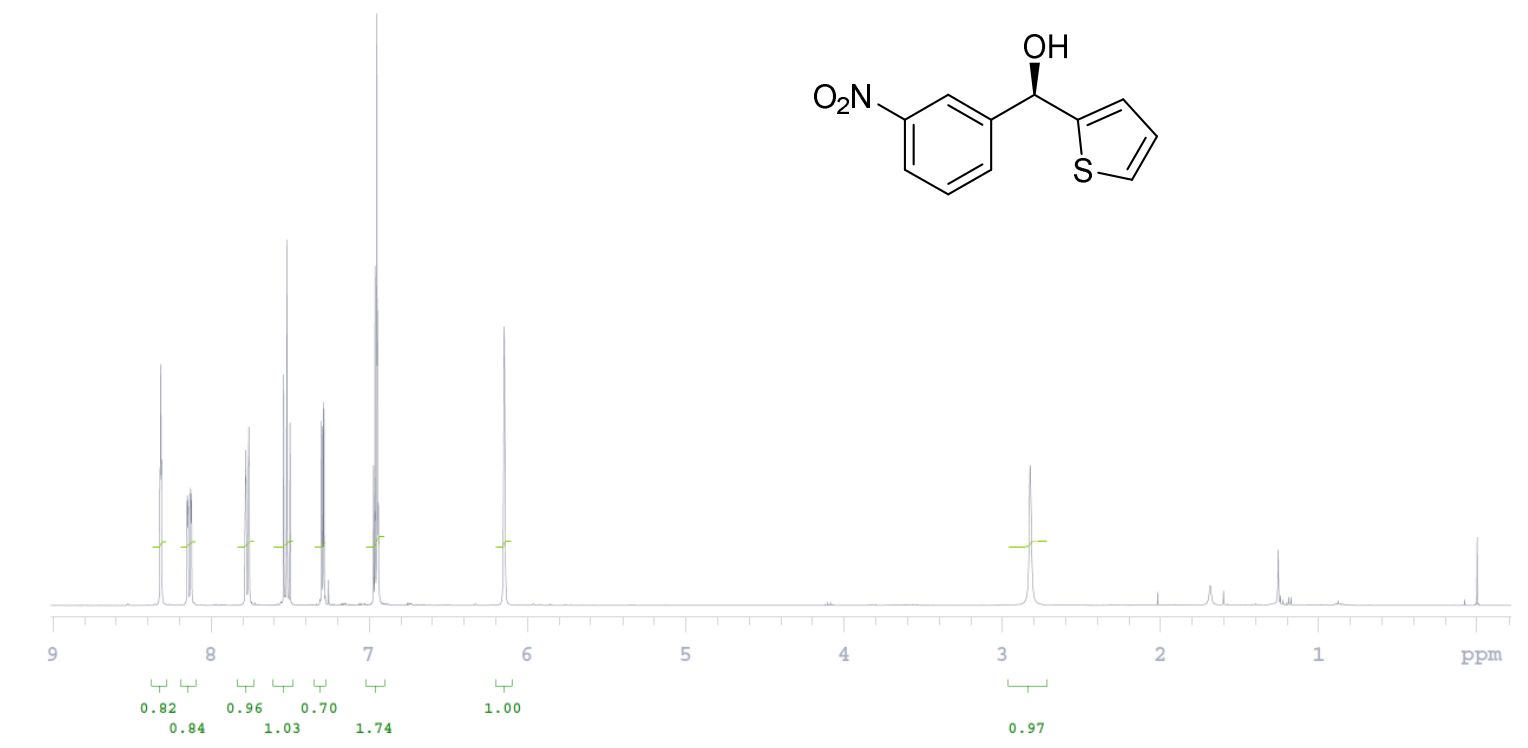

${ }^{13} \mathrm{C}$ NMR $\left(125 \mathrm{MHz}, \mathrm{CDCl}_{3}\right)$
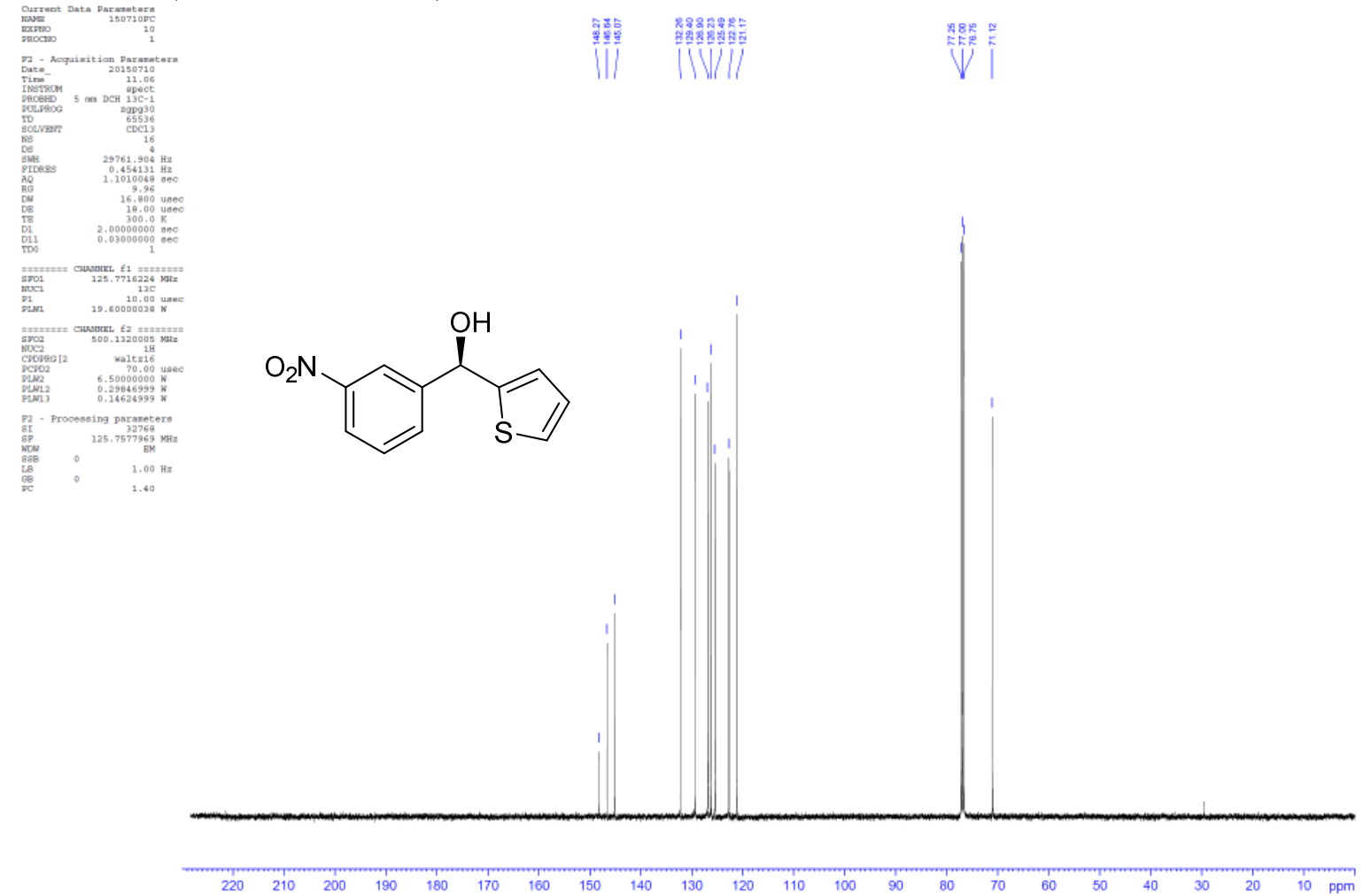


\section{(S)-(2-Chloro-5-nitrophenyl)(phenyl)methyl 4-nitrobenzoate (13i)}

${ }^{1} \mathrm{H}$ NMR (400 MHz, $\left.\mathrm{CDCl}_{3}\right)$

Agilent Technologies

151203-1_1H-NMR-71_

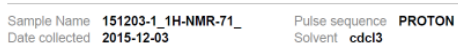

Temperature 25

Study owner vnmr1

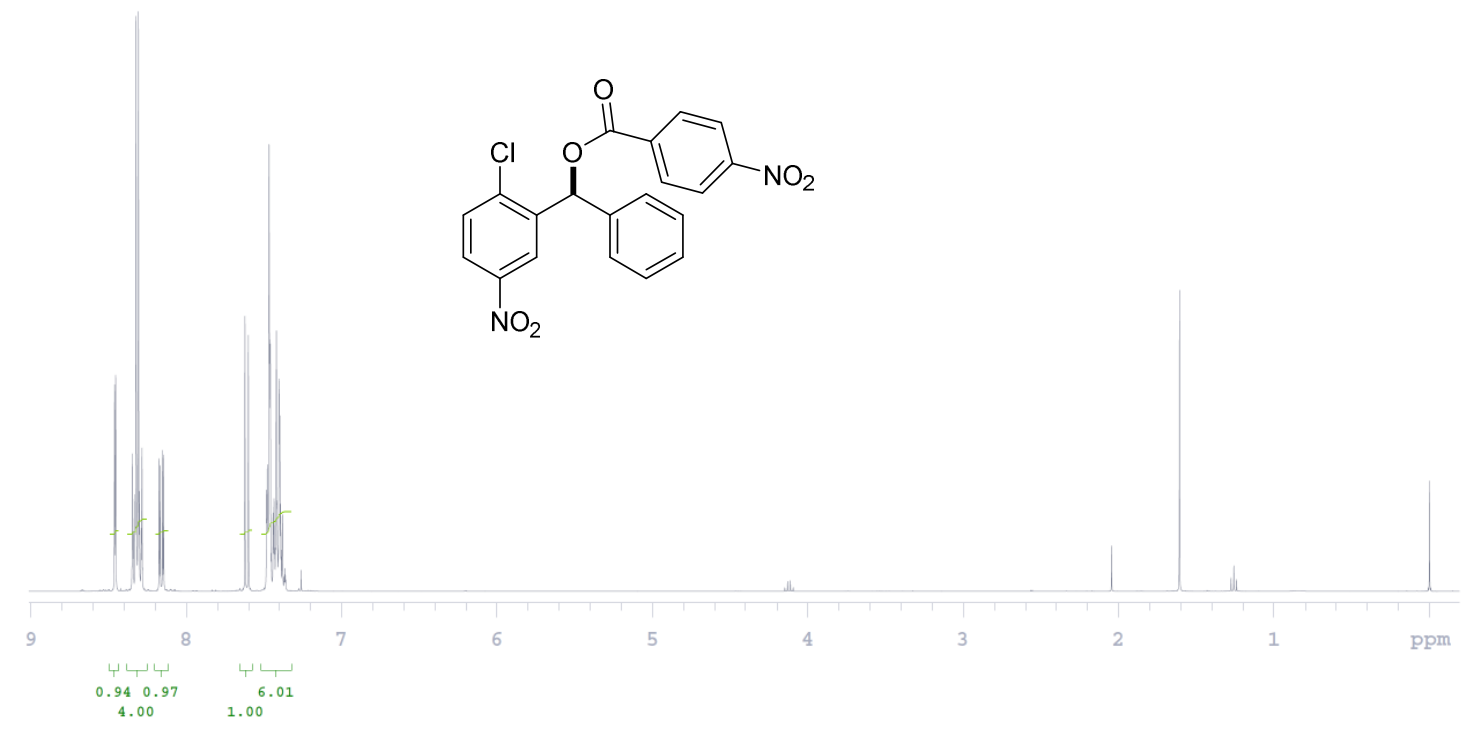

${ }^{13} \mathrm{C} \mathrm{NMR}\left(125 \mathrm{MHz}, \mathrm{CDCl}_{3}\right)$
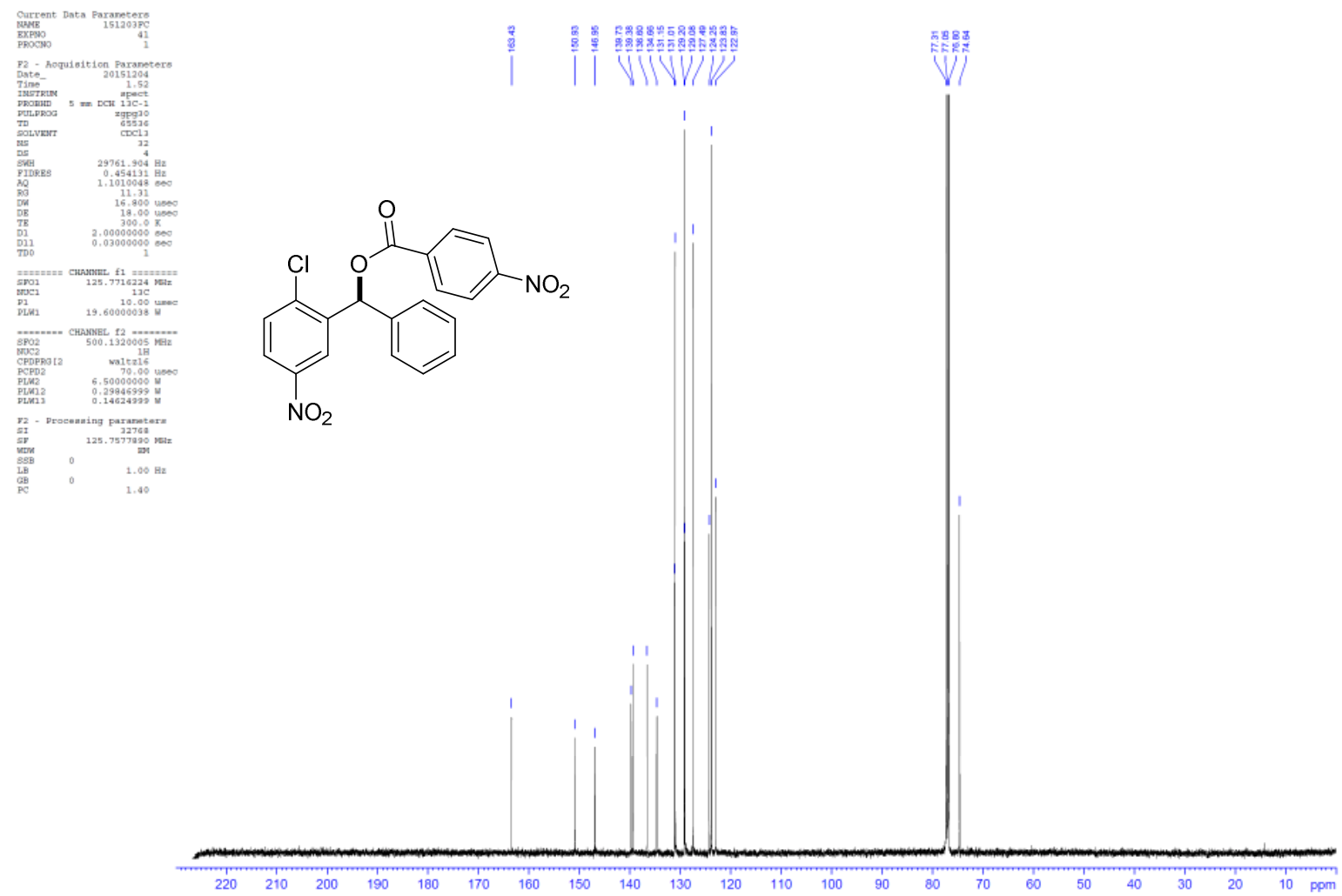


\section{(S)-(3,4-Dichlorophenyl)(phenyl)methyl 4-nitrobenzoate (14b)}

${ }^{1} \mathrm{H}$ NMR $\left(400 \mathrm{MHz}, \mathrm{CDCl}_{3}\right)$

Agilent Technologies

151124-1_1H-NMR-67_

Sample Name 151124-1_1H-NMR-67

Pulse sequence PROTON
Solvent cdcl3

$\begin{array}{ll}\text { Temperature } 25 & \text { Study owner vnmr } \\ \text { Spectrometer Agilent-NMR-vnmms400 } & \text { Operator vnmr1 }\end{array}$

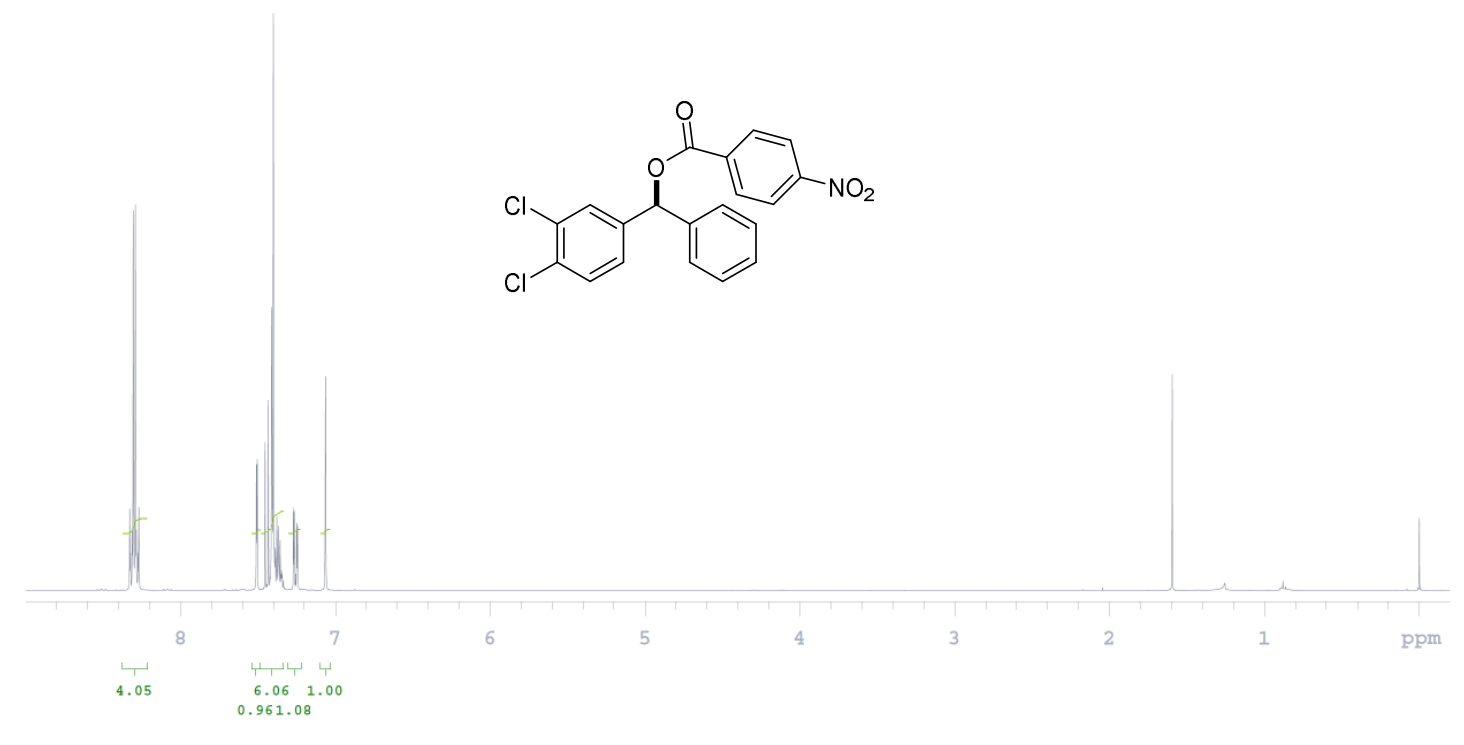

${ }^{13} \mathrm{C}$ NMR $\left(125 \mathrm{MHz}, \mathrm{CDCl}_{3}\right)$
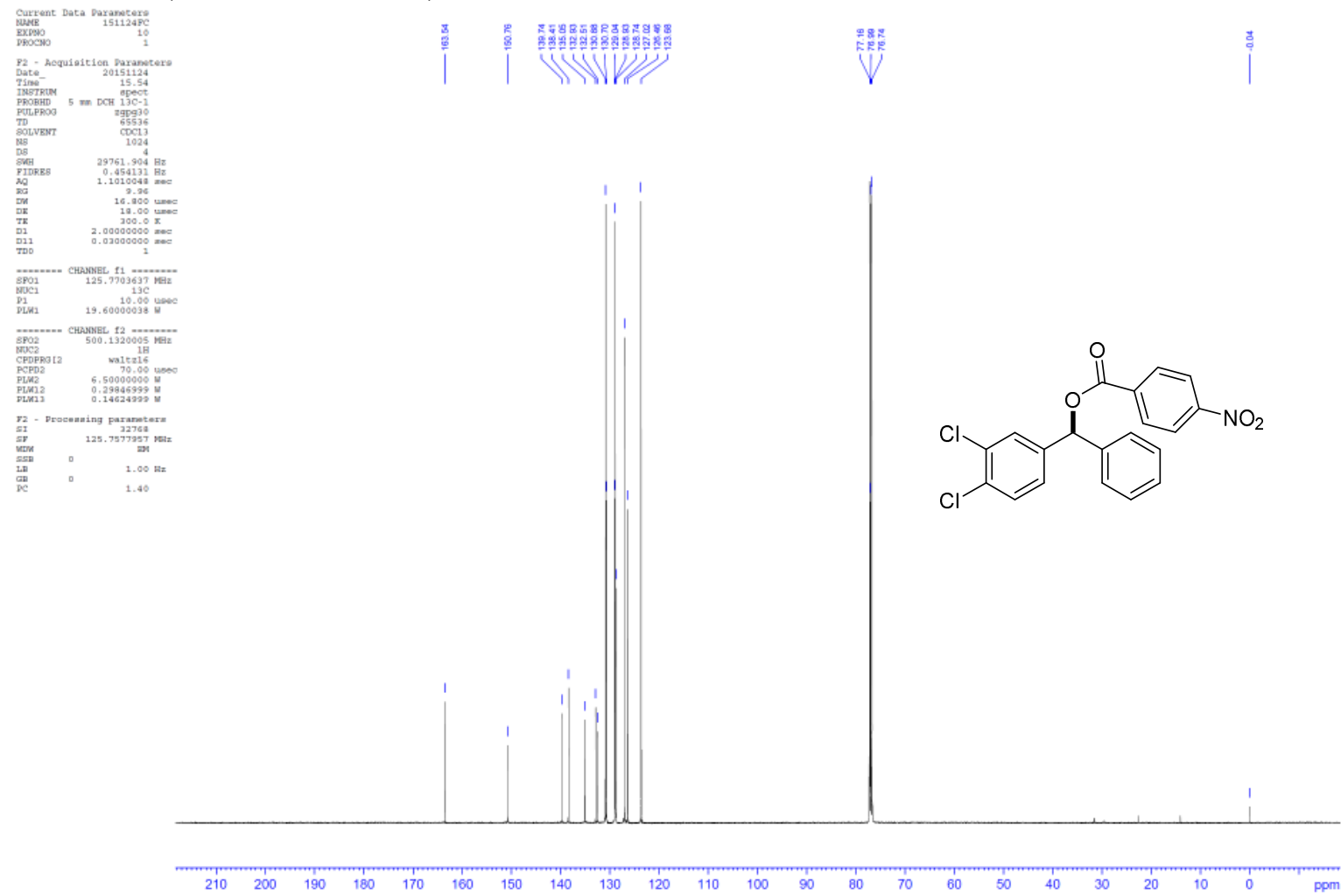
(S)-(4-Chloro-3-nitrophenyl)(phenyl)methyl 4-nitrobenzoate (14e)

${ }^{1} \mathrm{H}$ NMR (400 MHz, $\left.\mathrm{CDCl}_{3}\right)$

Agilent Technologies
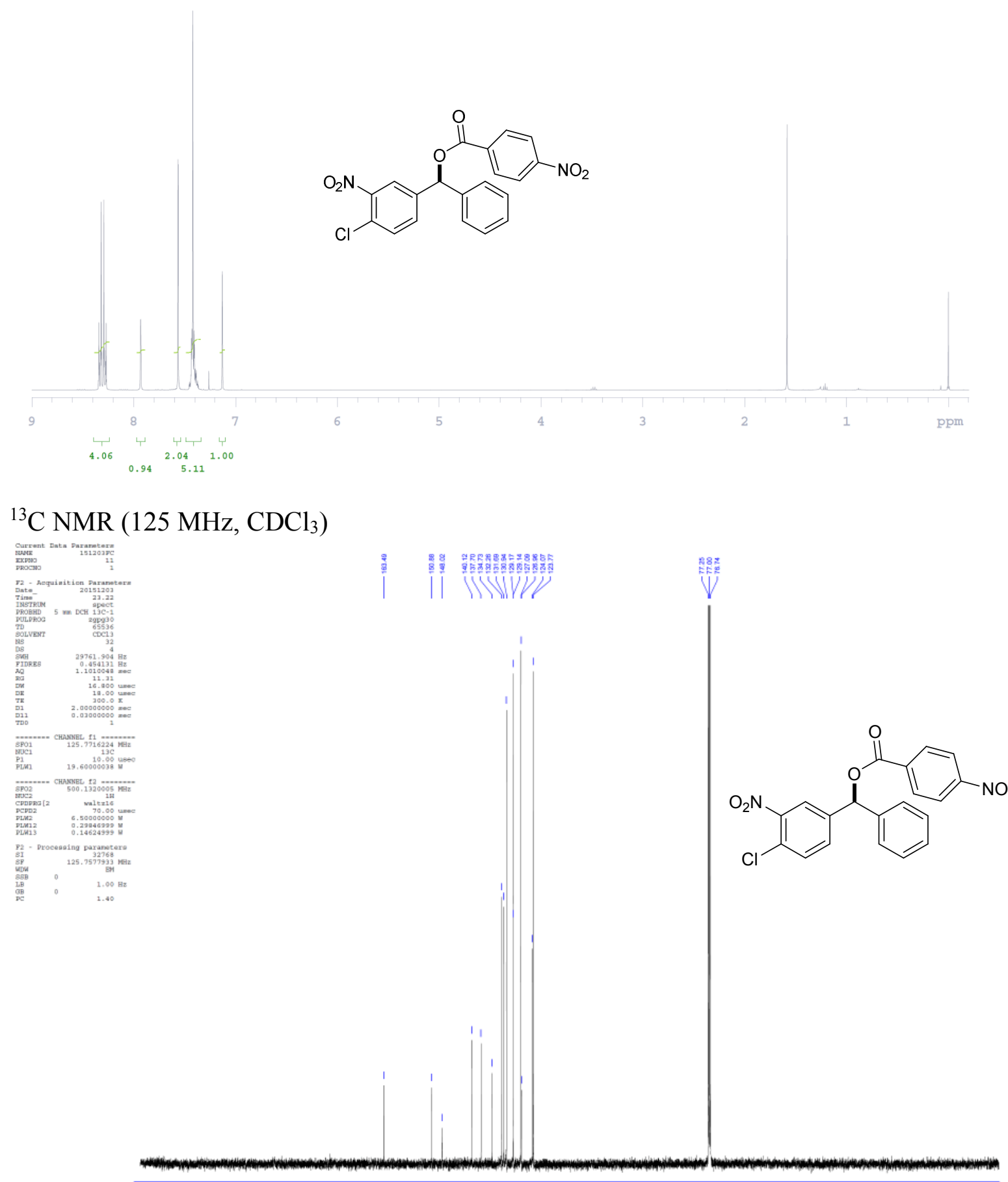
(S)-Phenyl(2,4,5-trimethylphenyl)methyl

4,5-dichloro-2-((3aR,6S)-8,8-dimethyl-2,2-dioxidohexahydro-3H-3a,6-methanobenzo[c]isothiazole-1-car bonyl)benzoate (13g)

${ }^{1} \mathrm{H} \mathrm{NMR}\left(500 \mathrm{MHz}, \mathrm{CDCl}_{3}\right)$

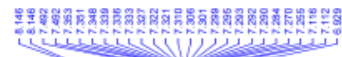

1

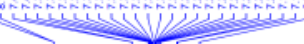
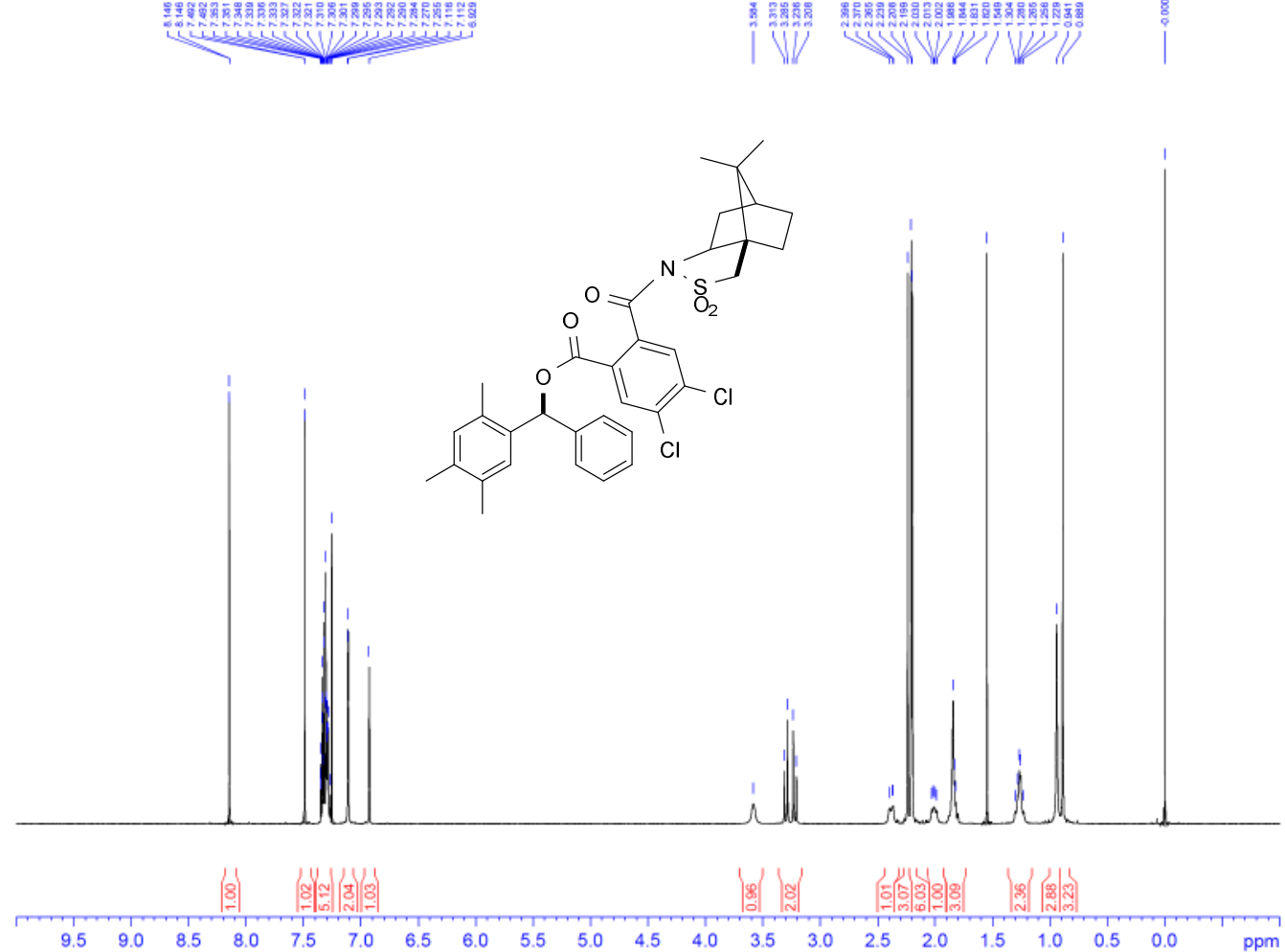

${ }^{13} \mathrm{C}$ NMR $\left(125 \mathrm{MHz}, \mathrm{CDCl}_{3}\right)$
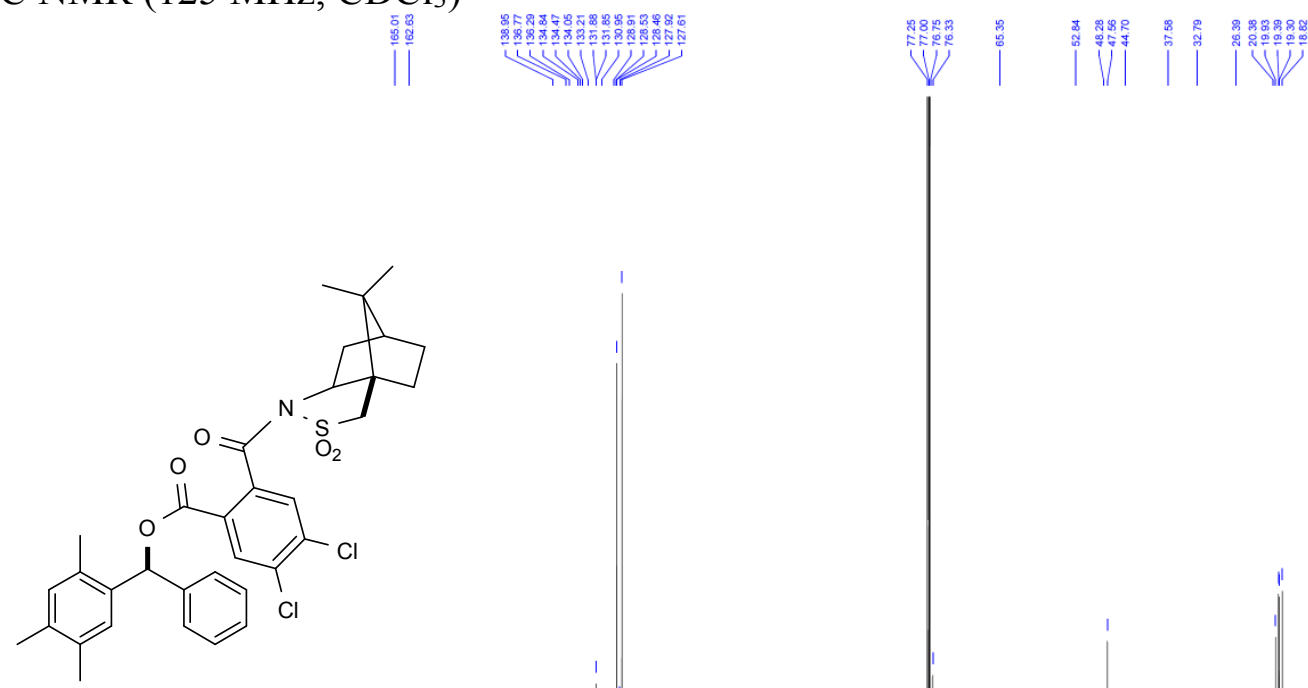
(S)-(2,5-Difluorophenyl)(phenyl)methyl

4,5-dichloro-2-((3aS,6R)-8,8-dimethyl-2,2-dioxidohexahydro-3H-3a,6-methanobenzo[c]isothiazole-1-car bonyl)benzoate (13j)

${ }^{1} \mathrm{H} \mathrm{NMR}\left(500 \mathrm{MHz}, \mathrm{CDCl}_{3}\right)$

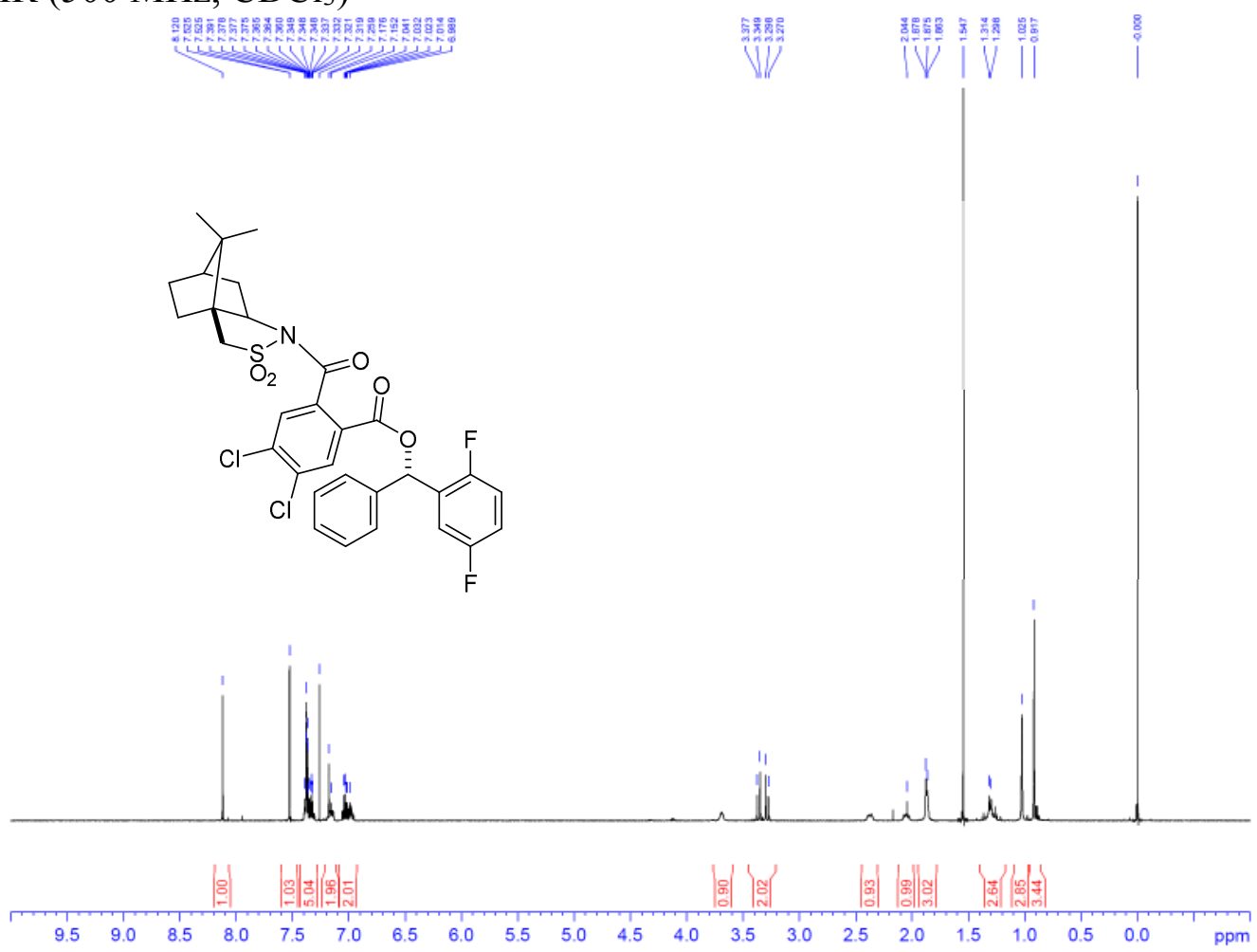

${ }^{13} \mathrm{C} \mathrm{NMR}\left(125 \mathrm{MHz}, \mathrm{CDCl}_{3}\right)$
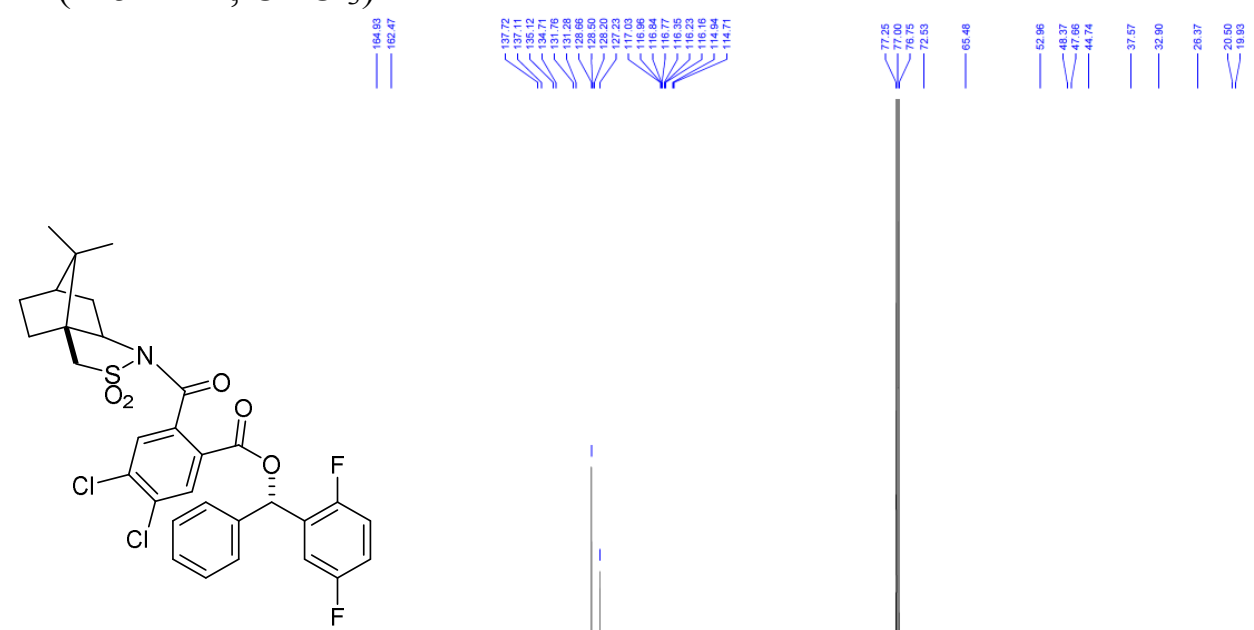
(S)-(2-Fluoro-3-(trifluoromethyl)phenyl)(phenyl)methyl 4,5-dichloro-2-((3aS,6R)-8,8-dimethyl-2,2-dioxidohexahydro-3H-3a,6-methanobenzo[c]isothiazole-1-car bonyl)benzoate (13k)

${ }^{1} \mathrm{H} \mathrm{NMR}\left(500 \mathrm{MHz}, \mathrm{CDCl}_{3}\right)$
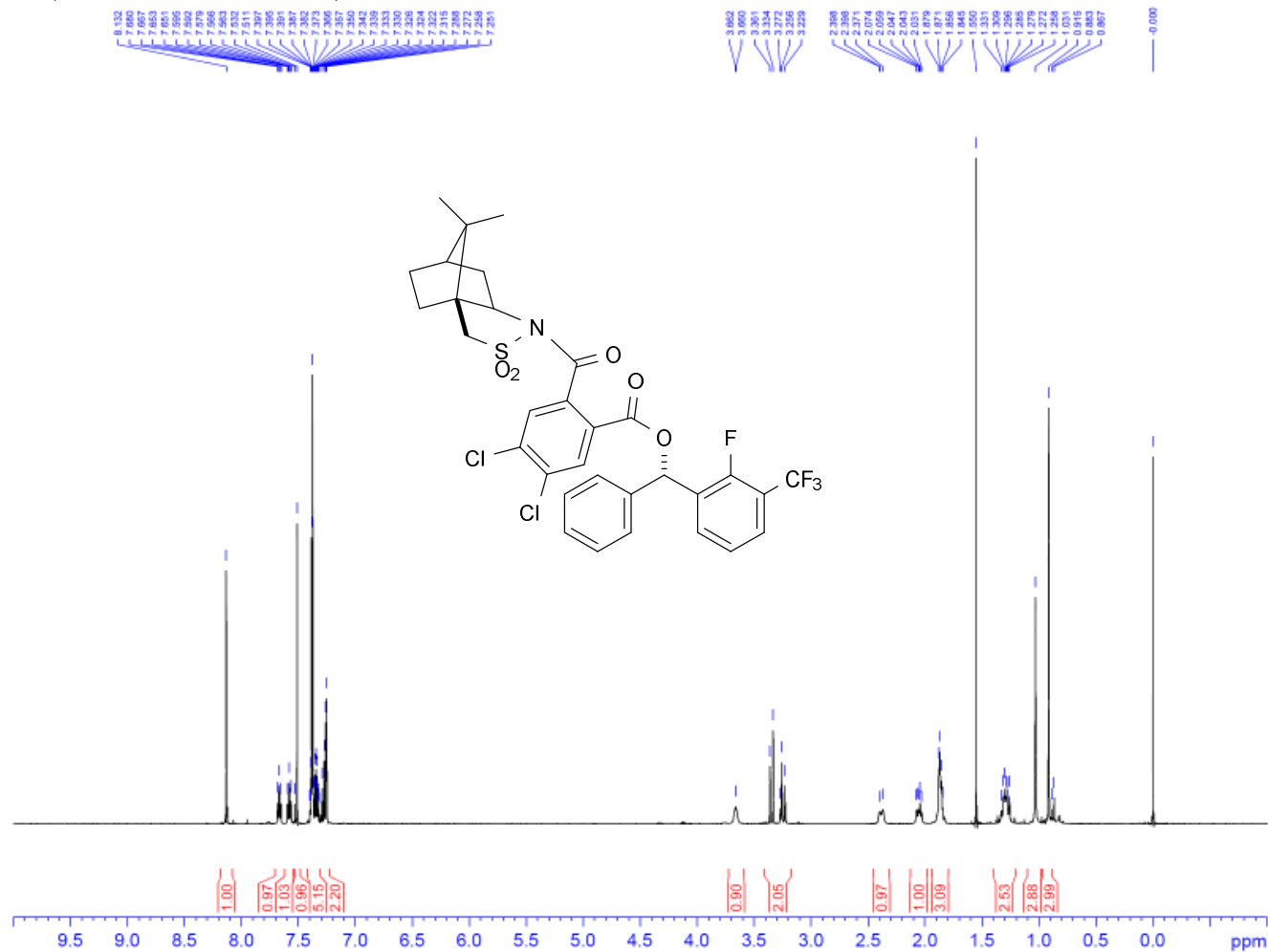

${ }^{13} \mathrm{C} \mathrm{NMR}\left(125 \mathrm{MHz}, \mathrm{CDCl}_{3}\right)$
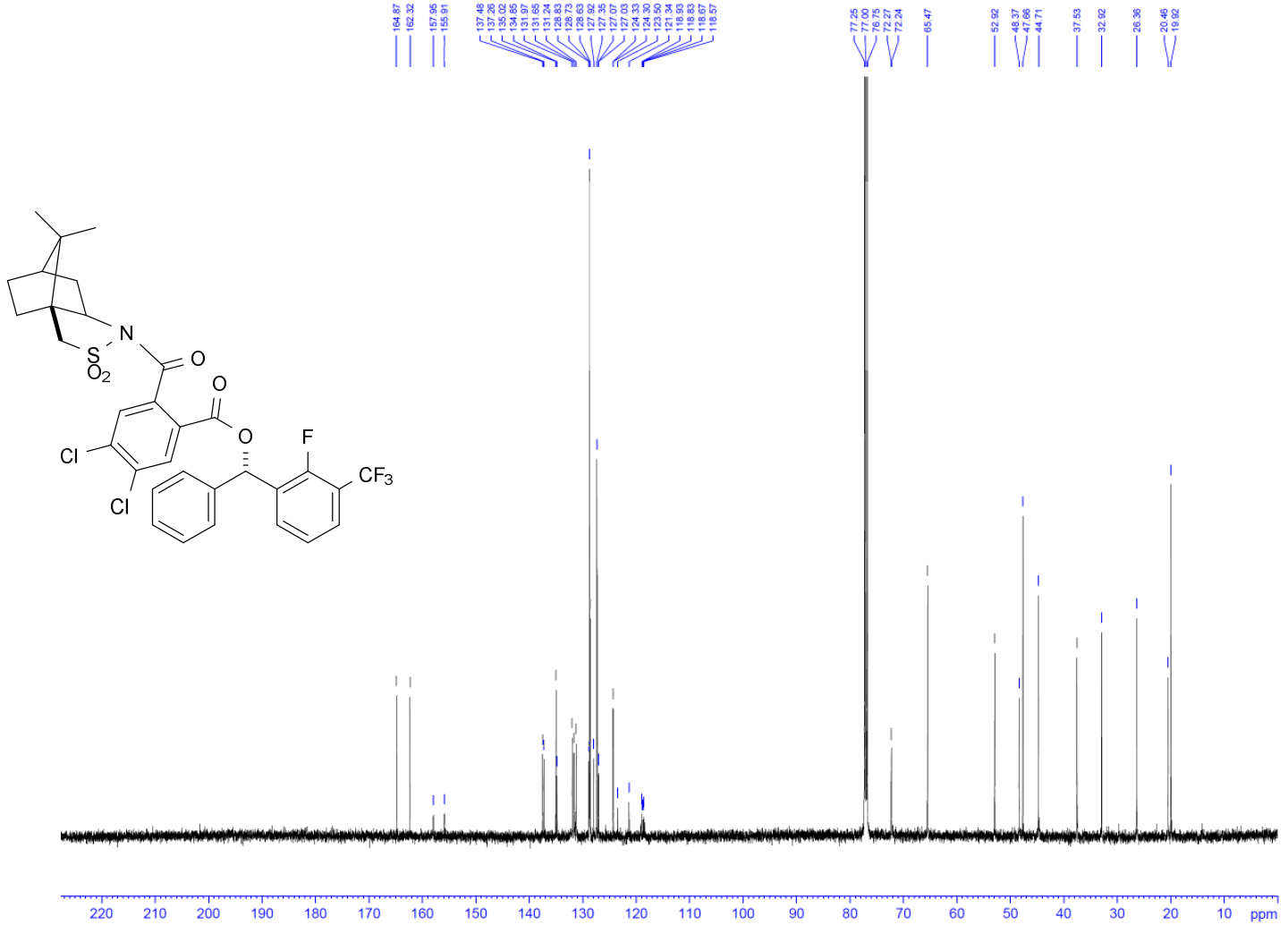
(S)-(3,4-Difluorophenyl)(phenyl)methyl

4,5-dichloro-2-((3aR,6S)-8,8-dimethyl-2,2-dioxidohexahydro-3H-3a,6-methanobenzo[c]isothiazole-1-car bonyl)benzoate (14c)

${ }^{1} \mathrm{H} \mathrm{NMR}\left(500 \mathrm{MHz}, \mathrm{CDCl}_{3}\right)$

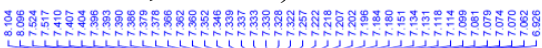
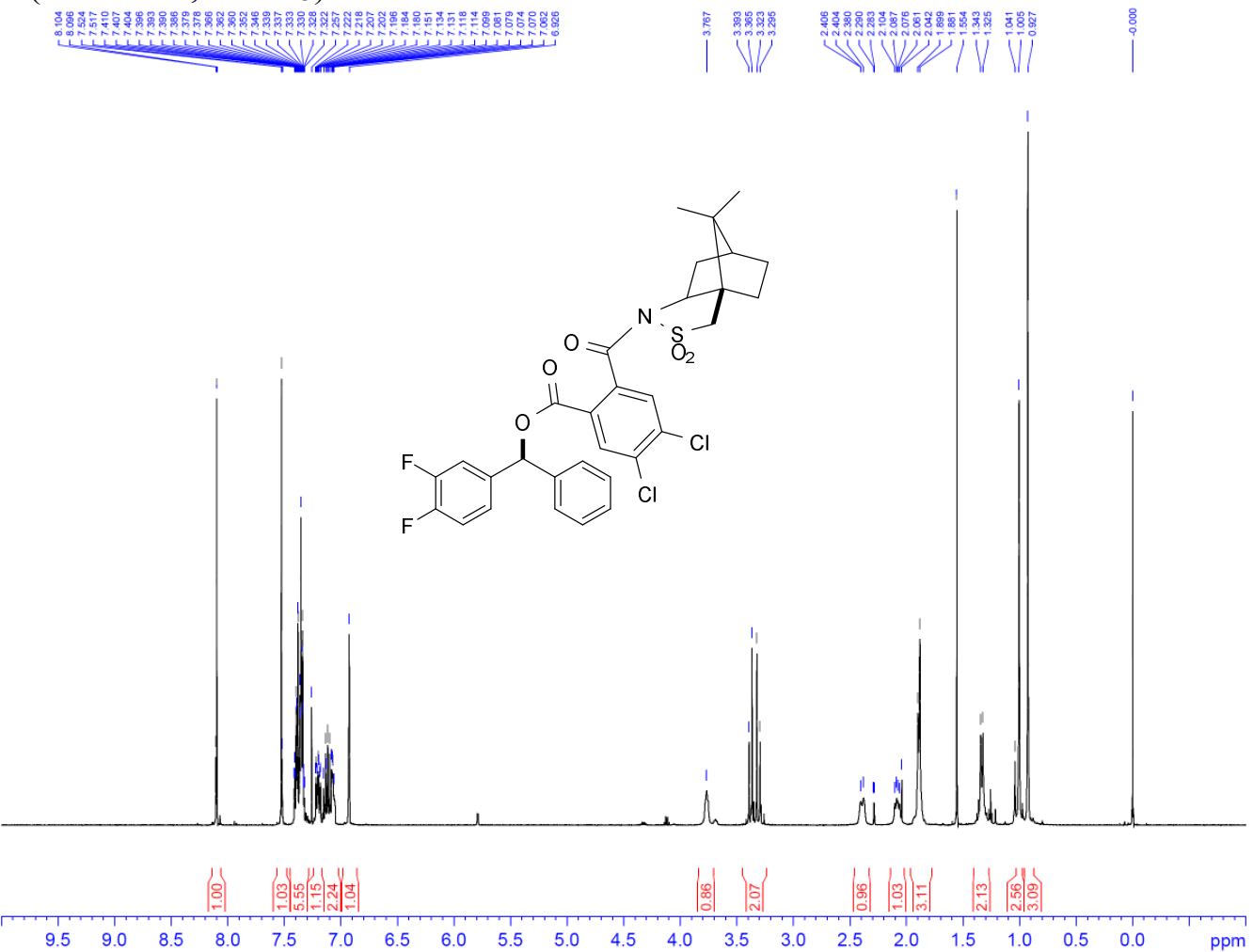

${ }^{13} \mathrm{C} \mathrm{NMR}\left(125 \mathrm{MHz}, \mathrm{CDCl}_{3}\right)$

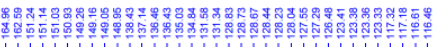

W. W W WIIIIIIII

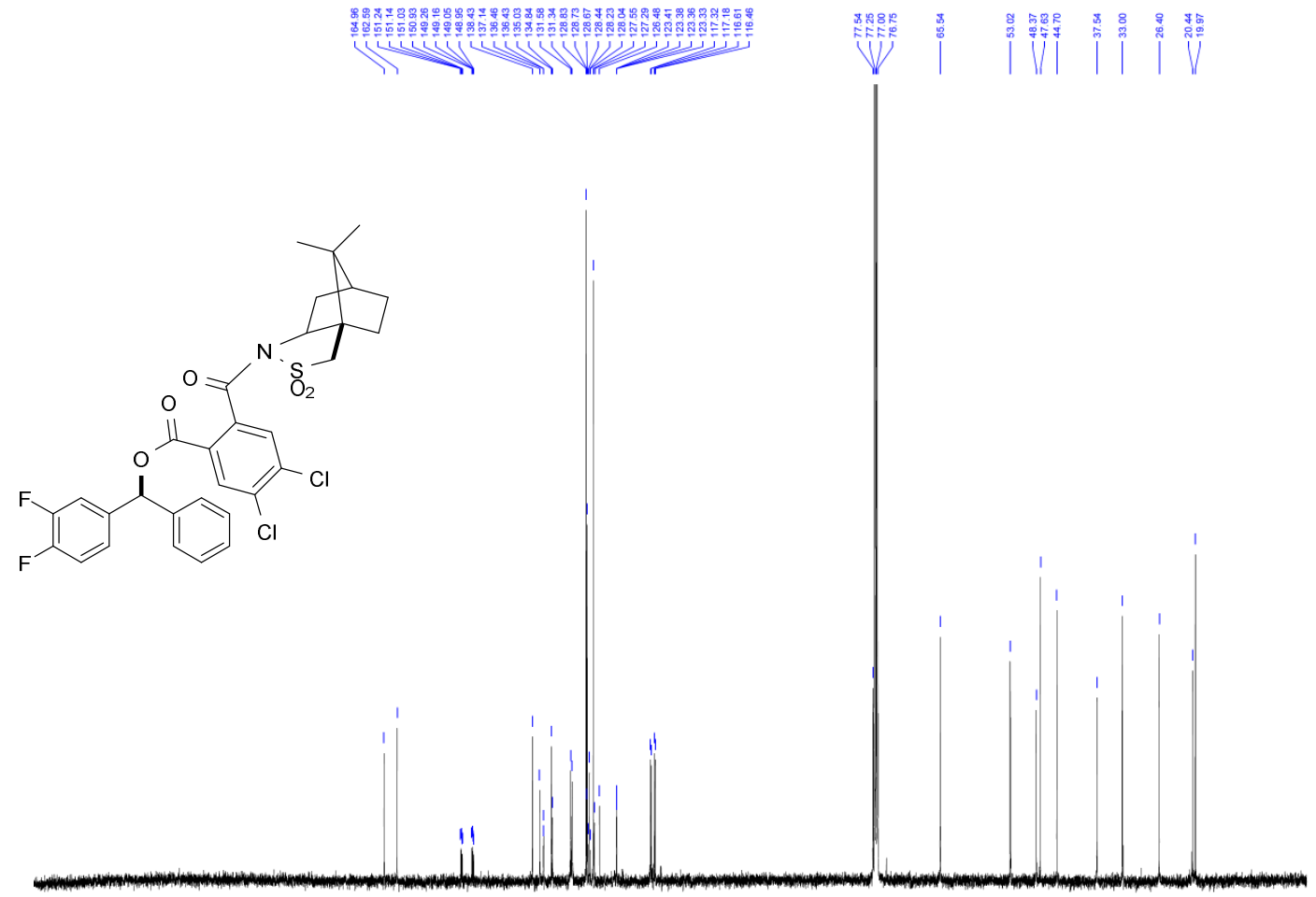


(S)-Phenyl(3,4,5-trifluorophenyl)methyl

4,5-dichloro-2-((3aS,6R)-8,8-dimethyl-2,2-dioxidohexahydro-3H-3a,6-methanobenzo[c]isothiazole-1-car bonyl)benzoate (14f)

${ }^{1} \mathrm{H} \mathrm{NMR}\left(500 \mathrm{MHz}, \mathrm{CDCl}_{3}\right)$
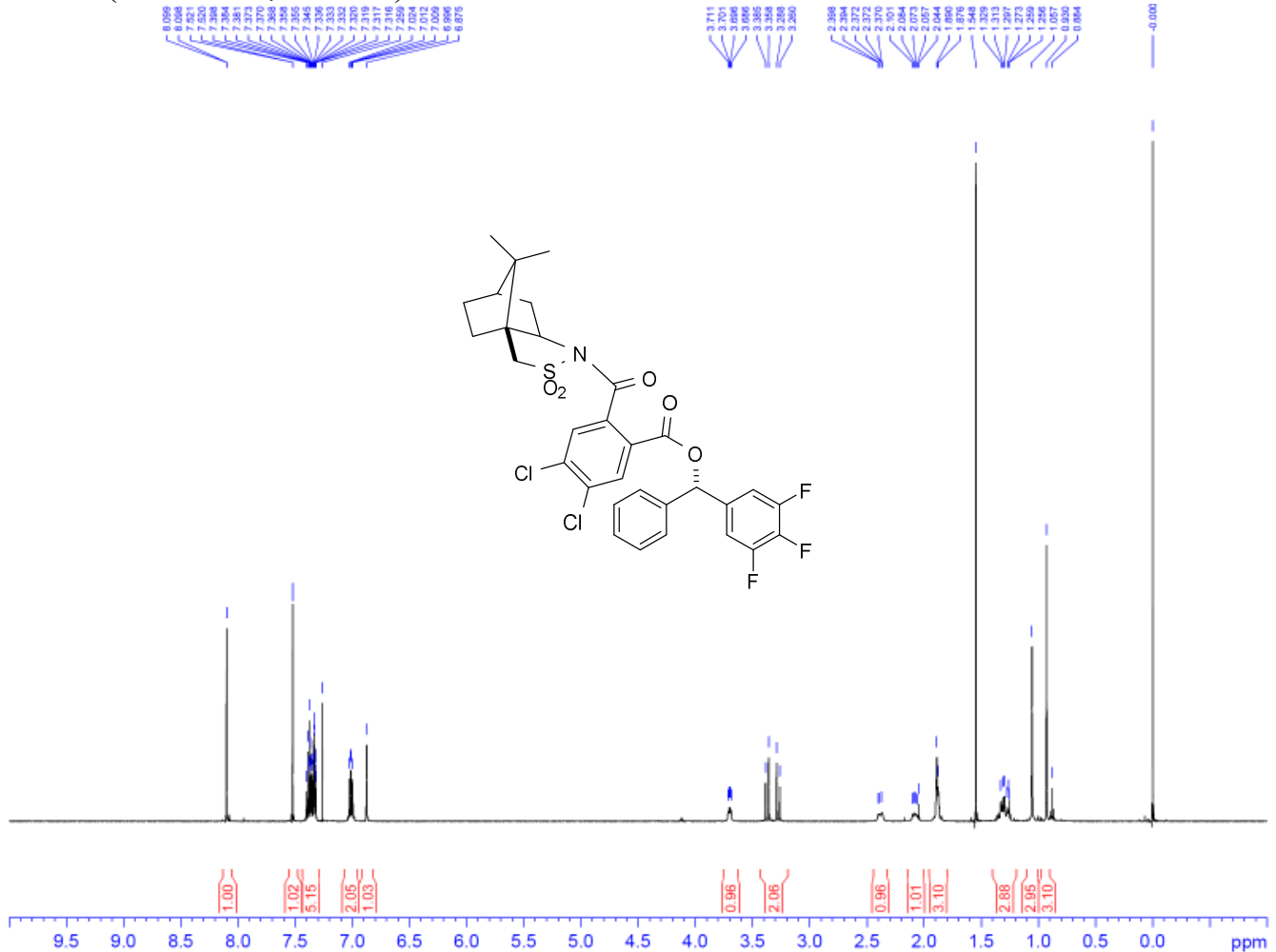

${ }^{13} \mathrm{C} \mathrm{NMR}\left(125 \mathrm{MHz}, \mathrm{CDCl}_{3}\right)$
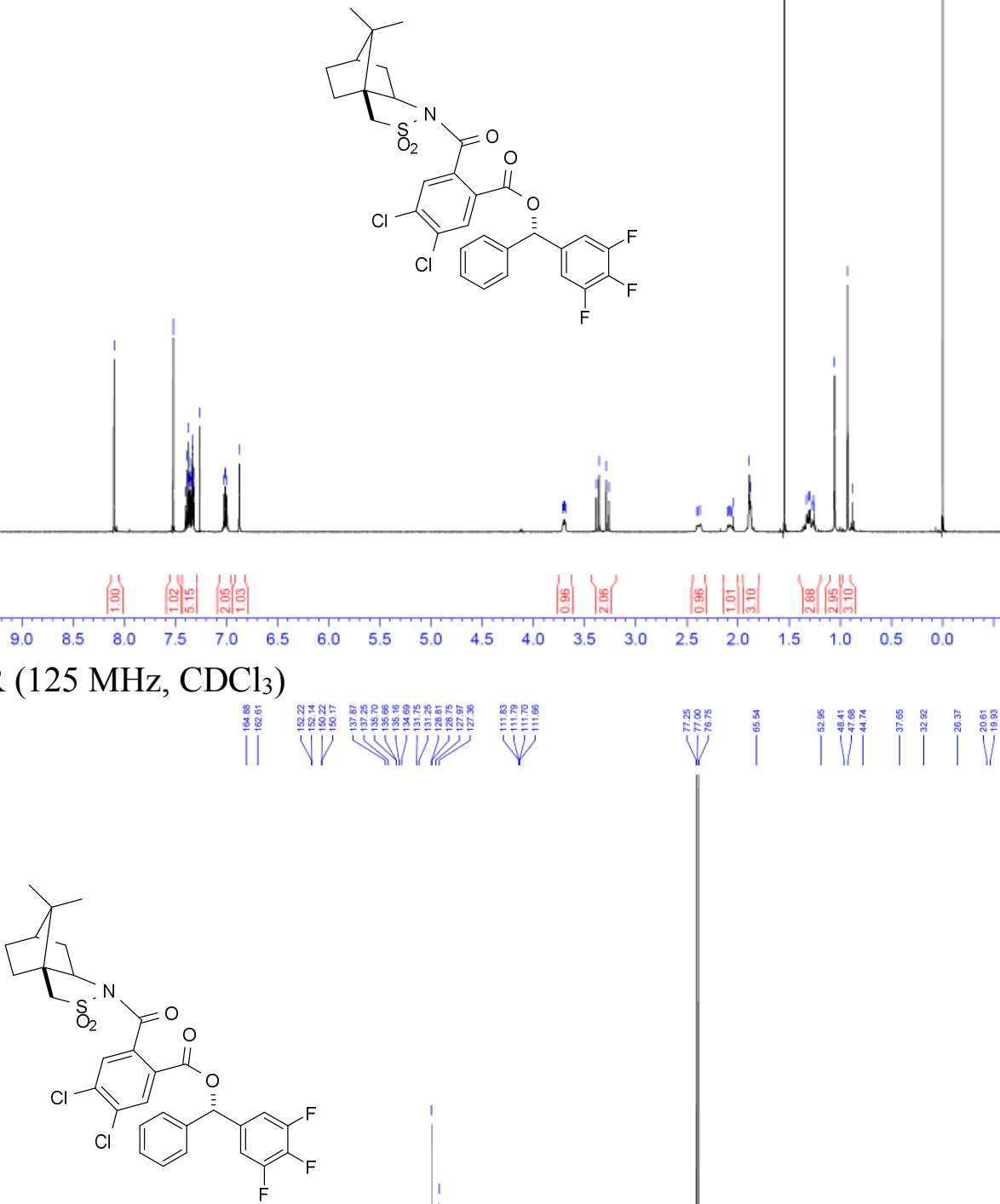
(R)-(3-Ditrophenyl)(thiophen-2-yl)methyl

4,5-dichloro-2-((3aS,6R)-8,8-dimethyl-2,2-dioxidohexahydro-3H-3a,6-methanobenzo[c]isothiazole-1-car bonyl)benzoate (14n)

${ }^{1} \mathrm{H}$ NMR $\left(500 \mathrm{MHz}, \mathrm{CDCl}_{3}\right)$

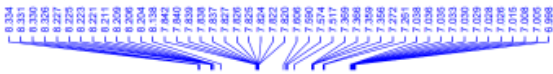
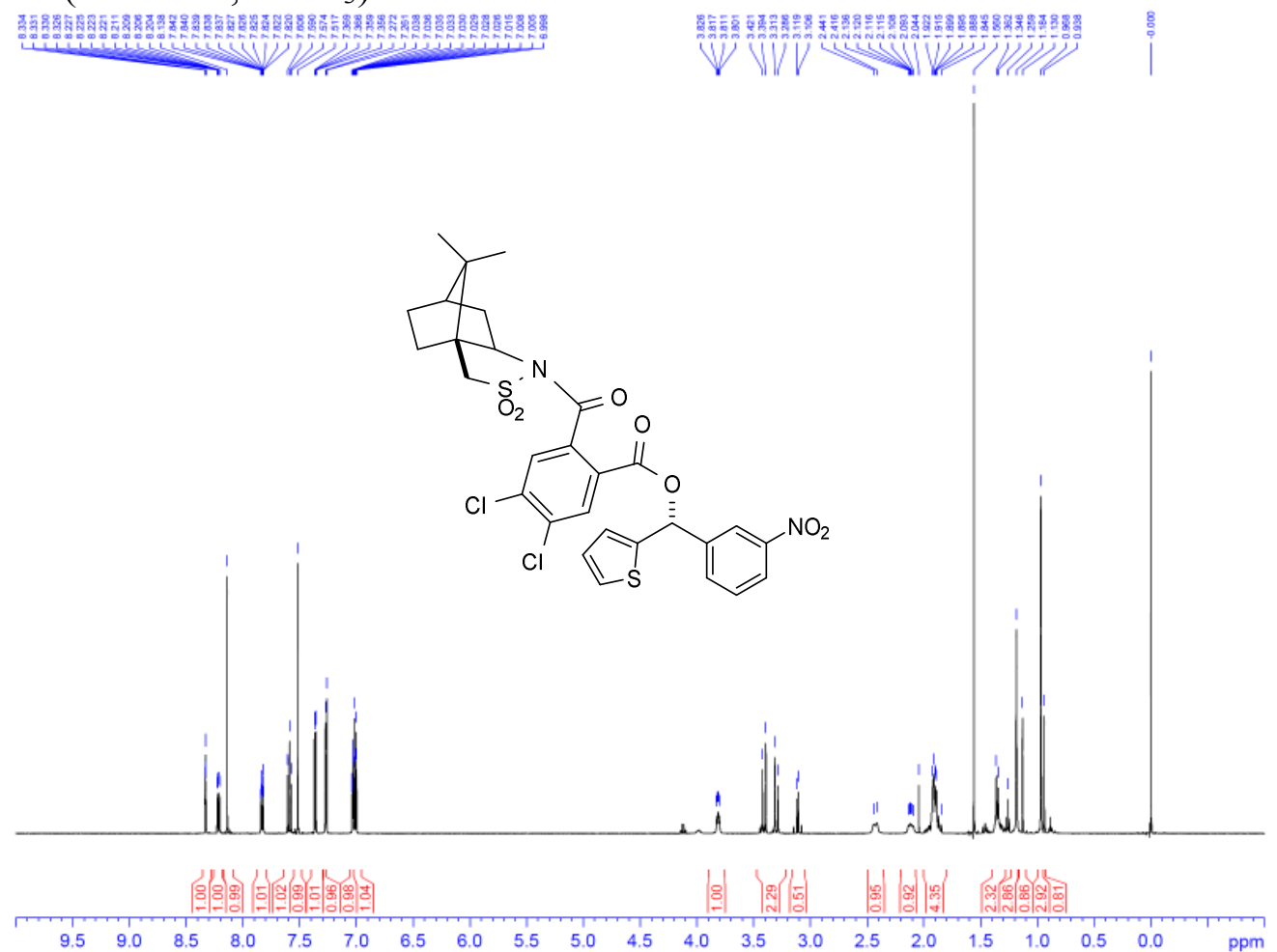

${ }^{13} \mathrm{C} \mathrm{NMR}\left(125 \mathrm{MHz}, \mathrm{CDCl}_{3}\right)$

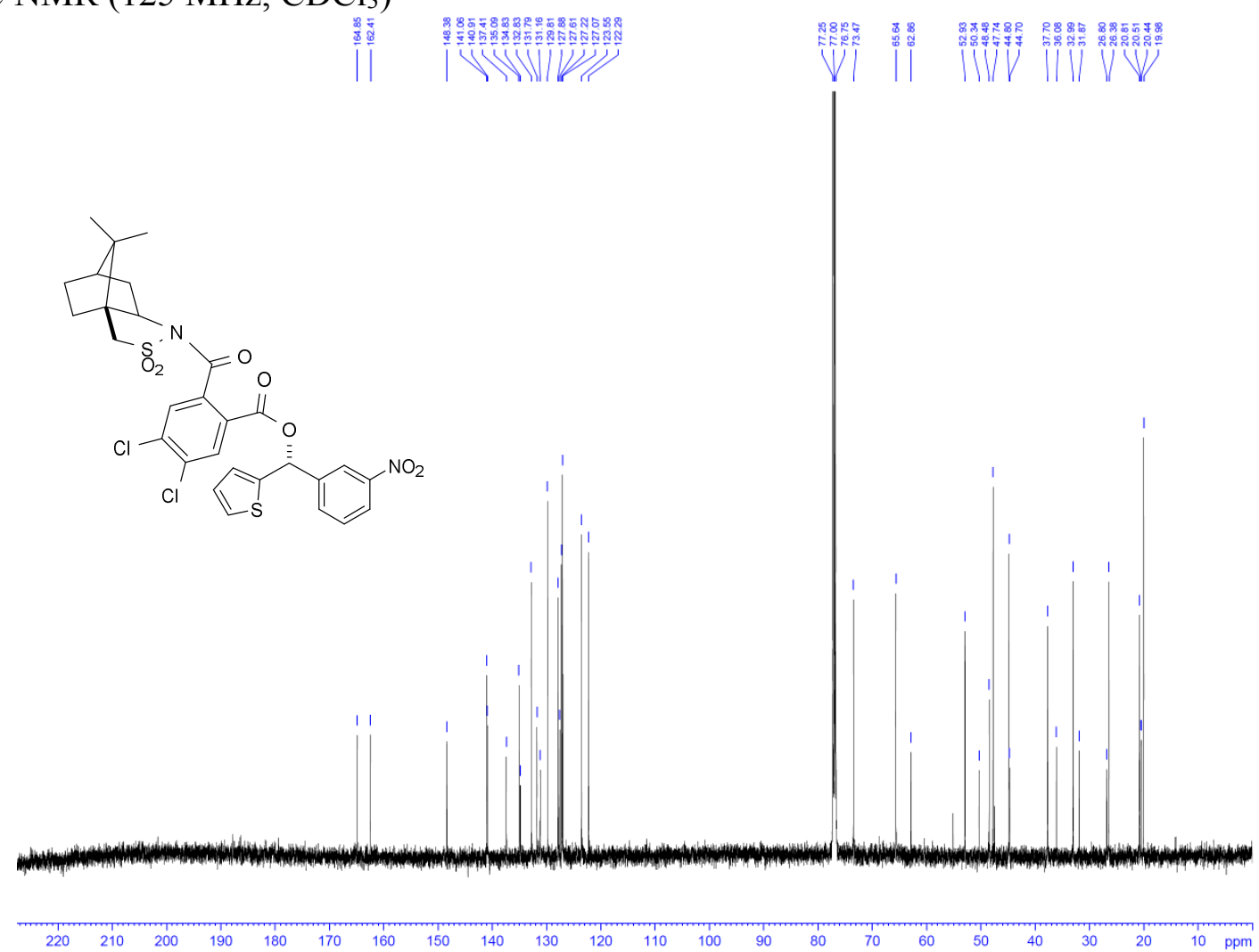


H. HR-MS Spectra for Products.

\section{(3,5-Dinitrophenyl)(phenyl)methanone (10g)}

(FI)

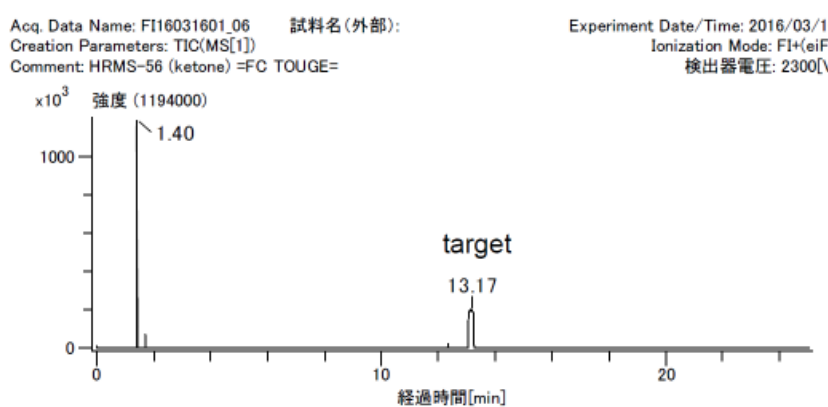

Standard for calibration: AT STD MIX

MS
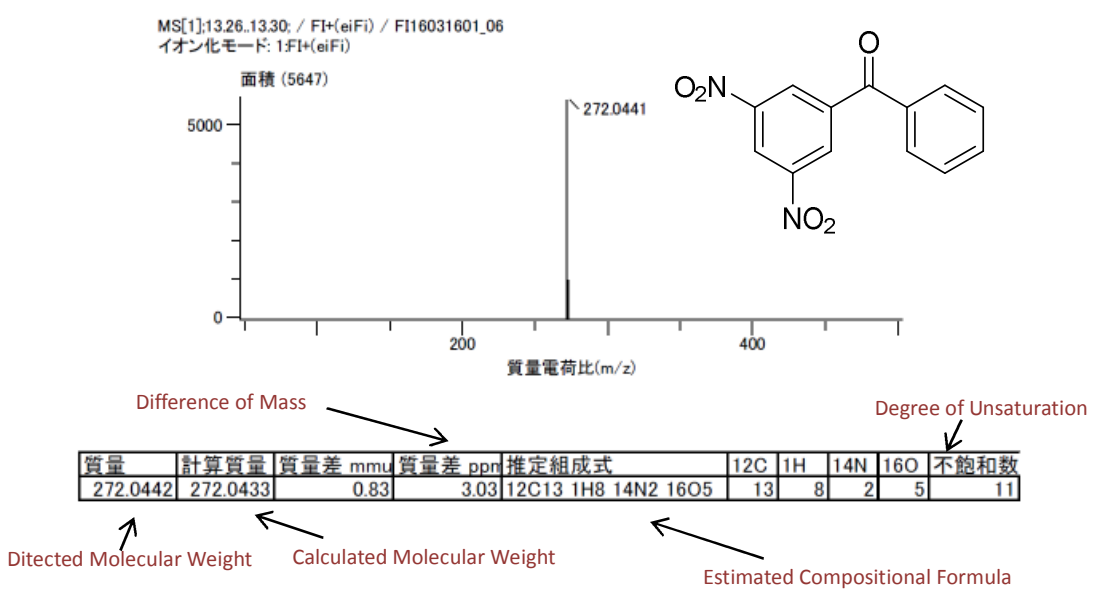

Phenyl(3,4,5-trifluorophenyl)methanone (10f) (FI)

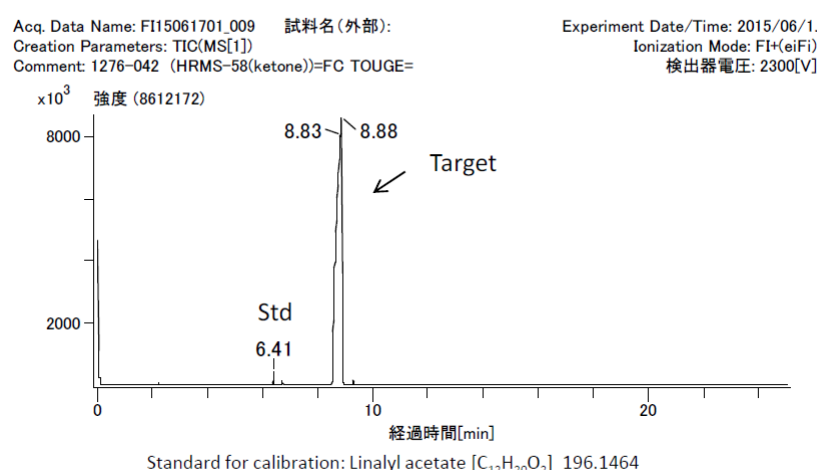

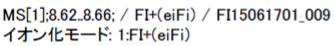

$\times 10^{3}$ 面積 $(1859113)$

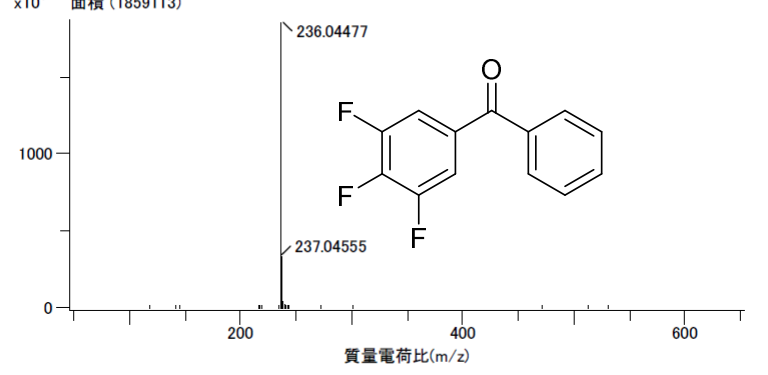

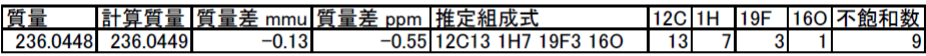

[Condition]

Column: Rxi-5ms $30 \mathrm{~m} \times 0.25 \mathrm{~mm}$ i.d., $0.25 \mu \mathrm{m}$

Instrument: GC:Agilent7890, MS:JMS-T100GCV

Oven: $60(1 \mathrm{~min}){ }^{\circ} \mathrm{C}-300^{\circ} \mathrm{C} @ 20^{\circ} \mathrm{C} / \mathrm{min}$

onization: $\mathrm{Fl}$

MS Range: $m / z$ 50-650

Inj. Vol.: Sample $0.5 \mu \mathrm{L}+$ STD 


\section{(3,5-Dinitrophenyl)(4-methoxyphenyl)methanone (101)}

(FI)

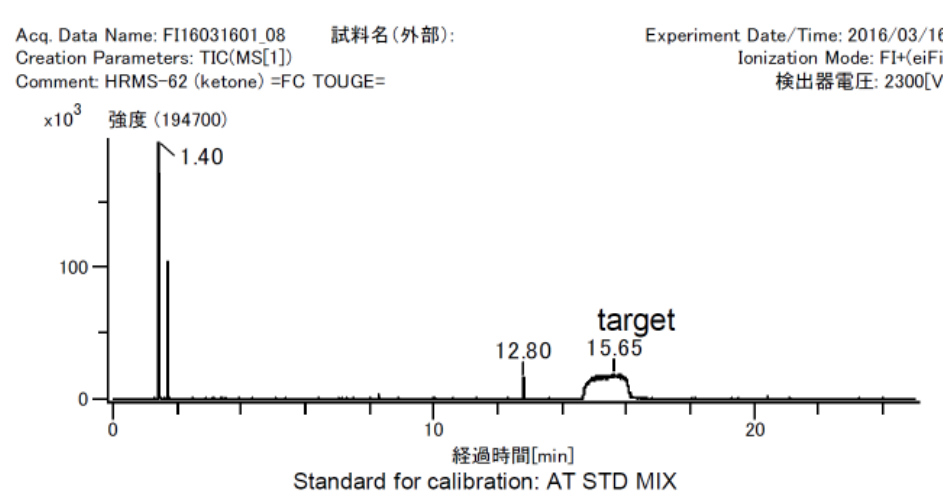

MS
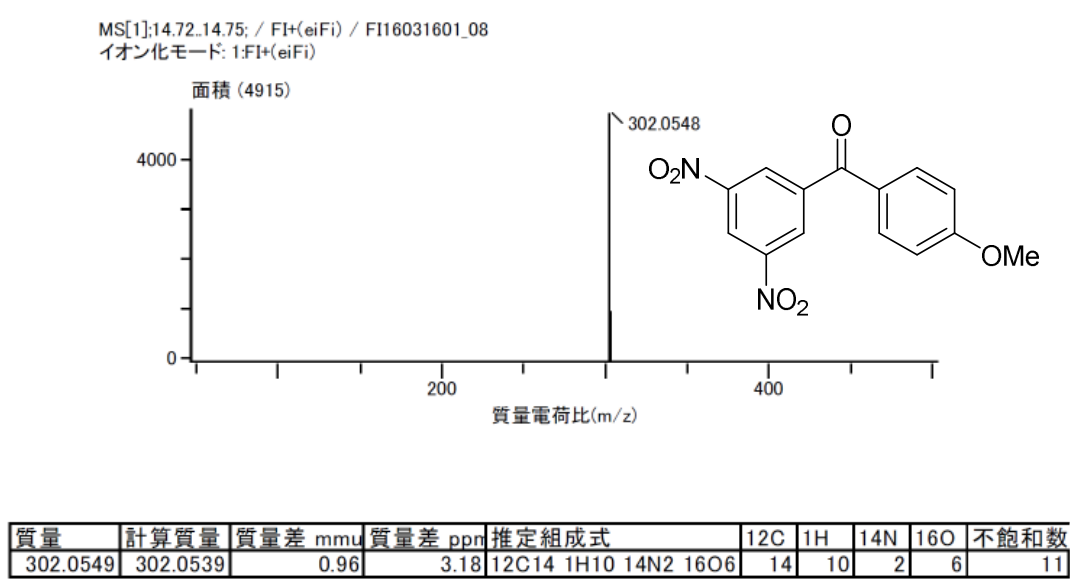

\section{(3,5-Dinitrophenyl)(thiophen-2-yl)methanone (10n)}

(FI)

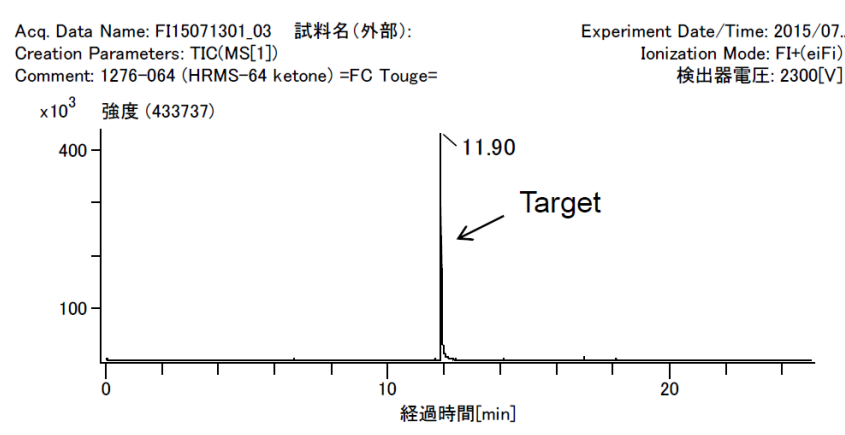

Standard for calibration: Linalyl acetate $\left[\mathrm{C}_{12} \mathrm{H}_{20} \mathrm{O}_{2}\right] 196.1464$

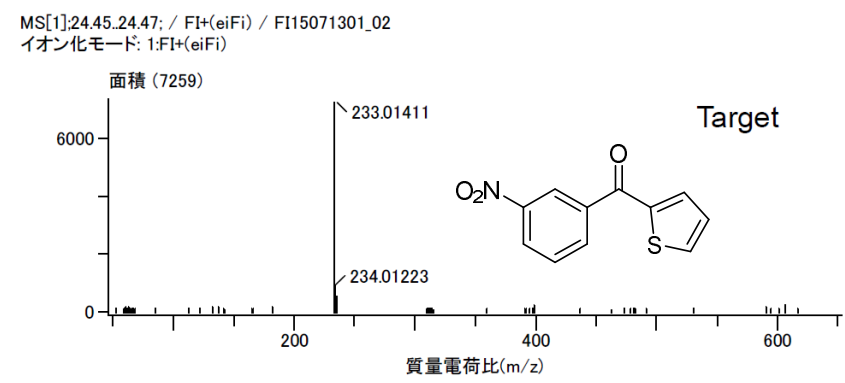

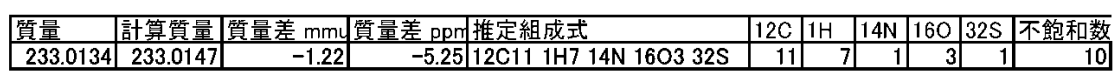

[Condition]

Column: Rxi-5ms $30 \mathrm{~m} \times 0.25 \mathrm{~mm}$ i.d, $0.25 \mu \mathrm{m}$

Instrument: GC:Agilent7890, MS:JMS-T100GCV

Oven: $60(1 \mathrm{~min}){ }^{\circ} \mathrm{C}-280^{\circ} \mathrm{C} @ 8^{\circ} \mathrm{C} / \mathrm{min}$

onization: $\mathrm{FI}$

MS Range: $m / 2$ 50-650

Inj. Vol.: Sample $2 \mu L+$ STD 


\section{(S)-(2'-Fluoro-[1,1'-biphenyl]-2-yl)(phenyl)methanol (8)}

(FI)

Acq. Data Name: F115061701_006 試料名(外部): Creation Parameters: TIC(MS[1])
Comment: $1261-162$ (HRMS-59)=FC TOUGE=

$\times 10^{3}$ 强度 (4688330)

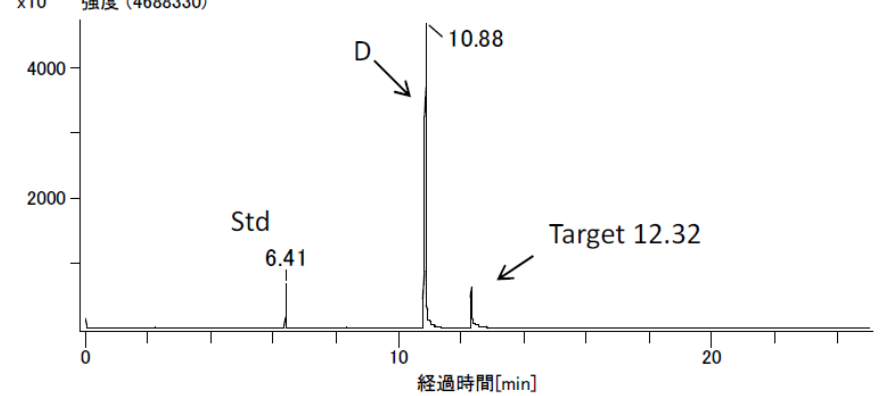

Standard for calibration: Linalyl acetate $\left[\mathrm{C}_{12} \mathrm{H}_{20} \mathrm{O}_{2}\right] 196.1464$ MS[1];12.31..12.36; / FI+(eiFi) / FI15061701_006 イオン化モード: 1:FI+(eiFi)

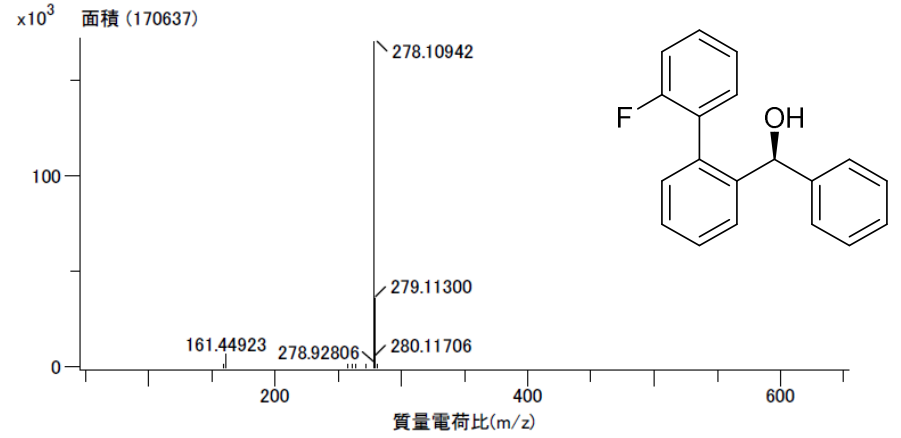

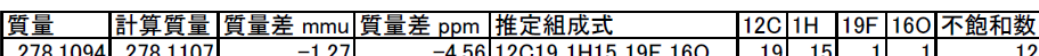

\section{(S)-6-Phenyl-6H-benzo[c]chromene (9)}

(APCI)

$\begin{array}{ll}\text { Instrument : LCMS-IT-TOF } & \text { Flow rate : } 0.2 \mathrm{ml} / \mathrm{min} \\ \text { Ionization : APCI Infusion } & \text { MS range : } 150-1000\end{array}$

Solvent: $\mathrm{CH}_{2} \mathrm{Cl}_{2} \quad$ Data file : T15020902_002.Icd
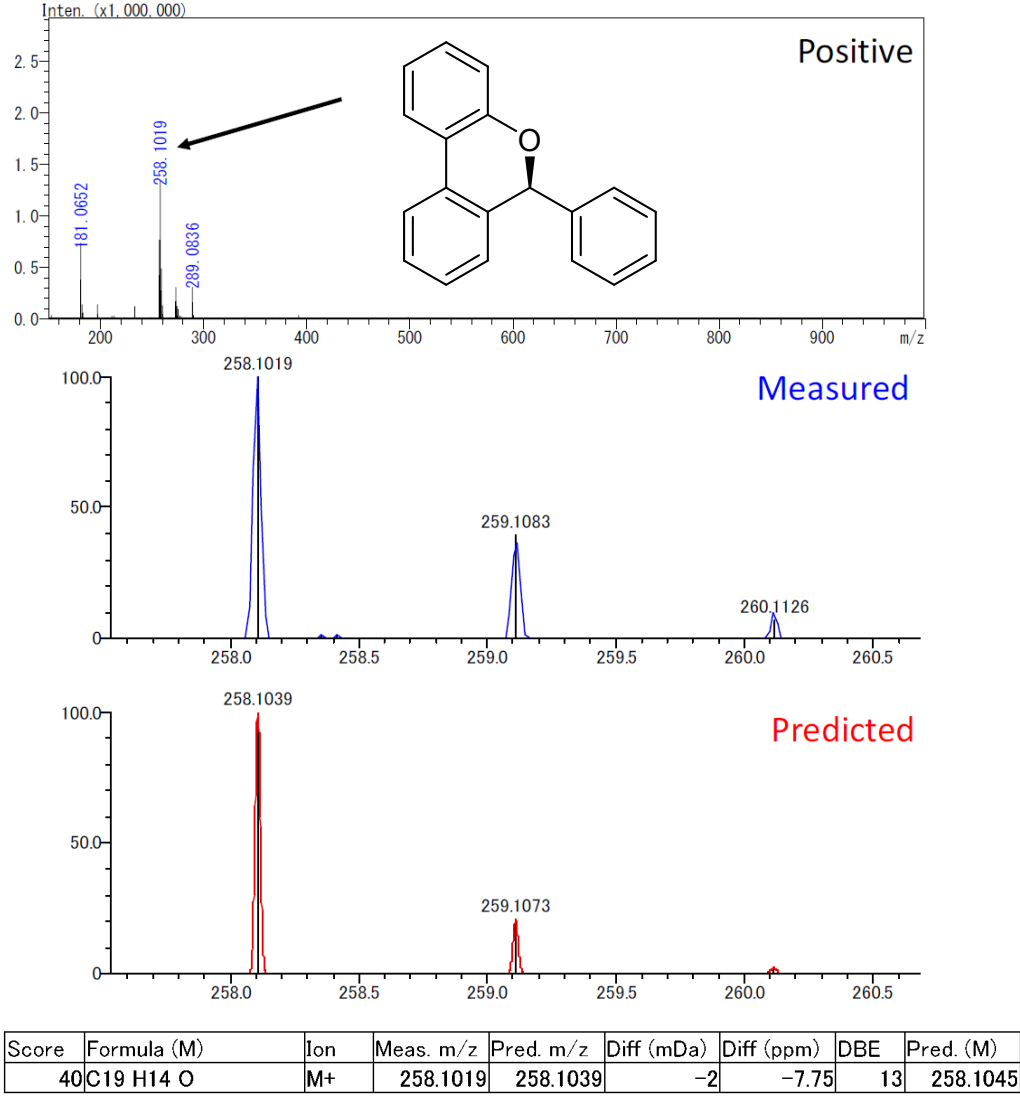

$\mathrm{C}: 0-200, \mathrm{H}: 0-200,0: 0-3$ 


\section{(S)-Phenyl(o-tolyl)methanol (6a)}

(FI)

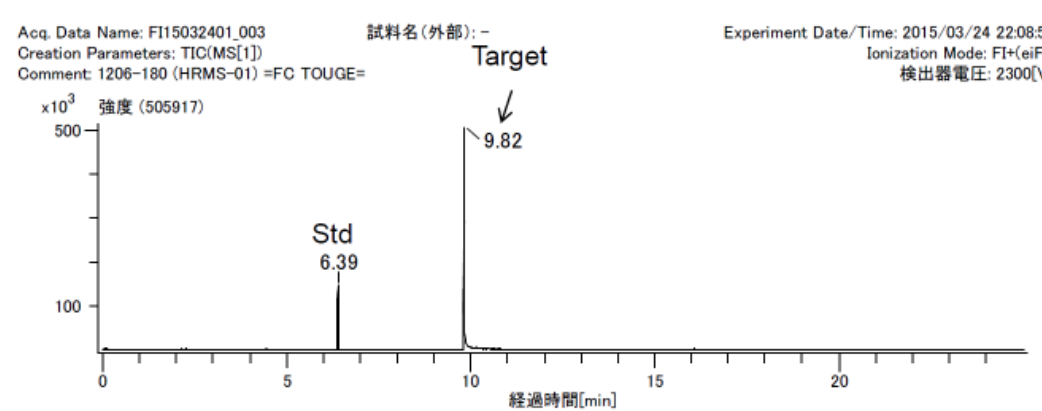

Standard for calibration: Linalyl acetate $\left[\mathrm{C}_{12} \mathrm{H}_{20} \mathrm{O}_{2}\right] 196.1464$

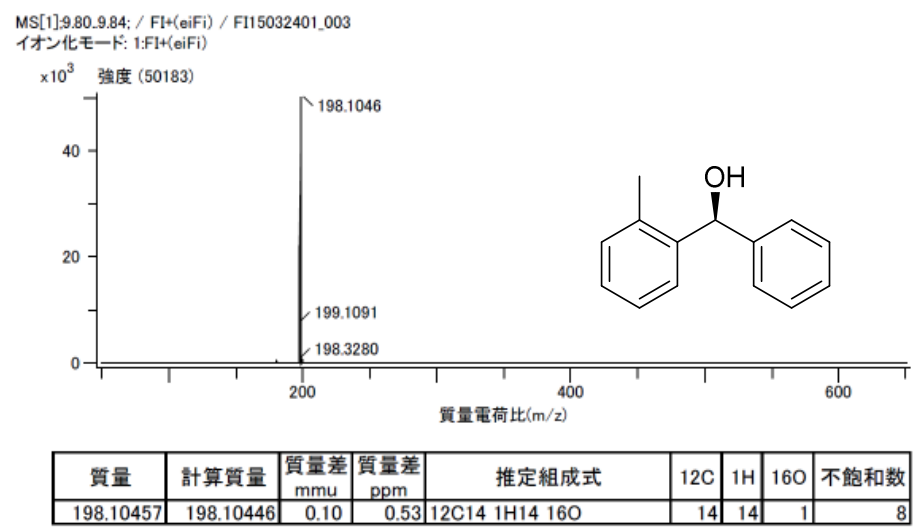

\section{[Condition]}

Column: Rxi-5ms $30 \mathrm{~m} \times 0.25 \mathrm{~mm}$ i.d., $0.25 \mu \mathrm{m}$

Instrument: GC:Agilent7890, MS:JMS-T100GCV

Oven: $60(1 \mathrm{~min}){ }^{\circ} \mathrm{C}-300^{\circ} \mathrm{C} @ 20^{\circ} \mathrm{C} / \mathrm{min}$

Ionization: $\mathrm{Fl}$

MS Range: $m / z$ 50-650

Inj. Vol.: Sample $0.5 \mu \mathrm{L}+$ STD

\section{(S)-(2-Chlorophenyl)(phenyl)methanol (6b)}

(FI)

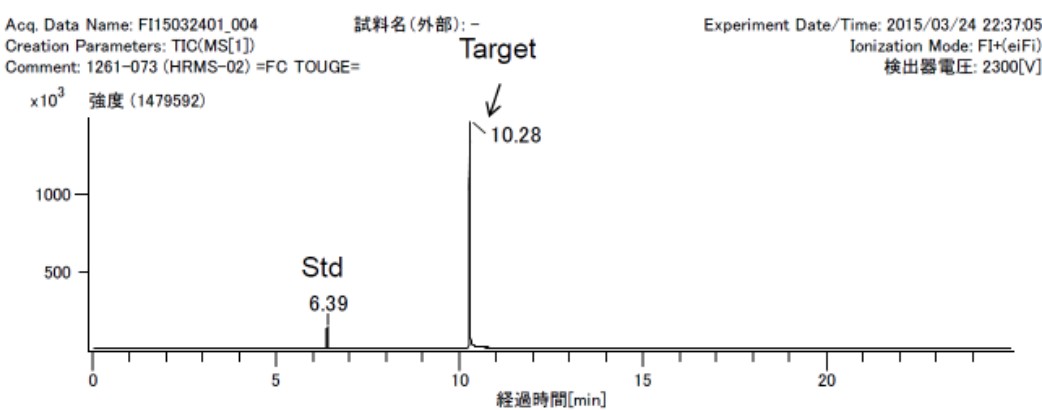

Standard for calibration: Linalyl acetate $\left[\mathrm{C}_{12} \mathrm{H}_{20} \mathrm{O}_{2}\right] 196.1464$

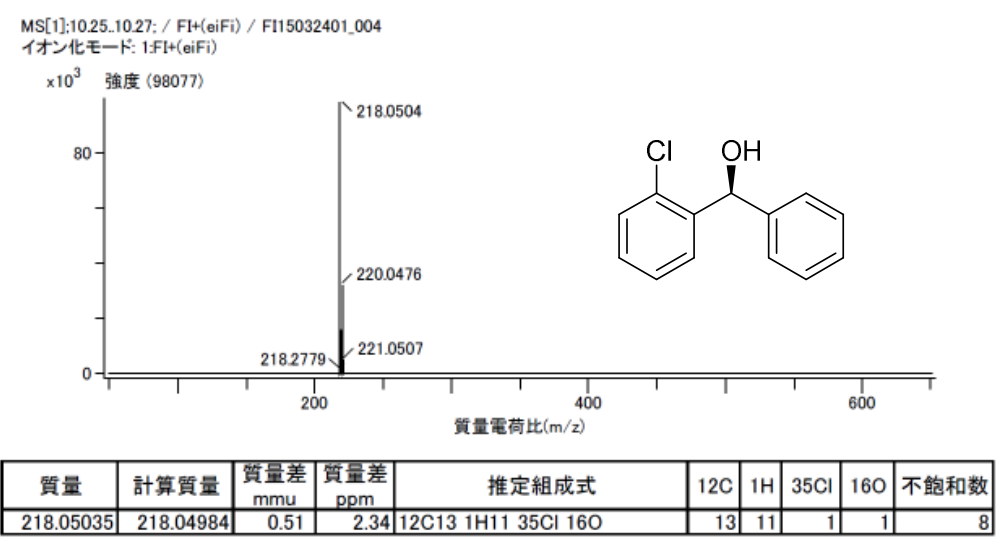

[Condition]

Column: Rxi-5ms $30 \mathrm{~m} \times 0.25 \mathrm{~mm}$ i.d., $0.25 \mu \mathrm{m}$

Instrument: GC:Agilent7890, MS:JMS-T100GCV

Oven: $60(1 \mathrm{~min}){ }^{\circ} \mathrm{C}-300^{\circ} \mathrm{C} @ 20^{\circ} \mathrm{C} / \mathrm{min}$

onization: $F$

MS Range: $m / z 50-650$

Inj. Vol.: Sample $0.5 \mu \mathrm{L}+$ STD 


\section{(S)-(2-Bromophenyl)(phenyl)methanol (6c)}

(FD)

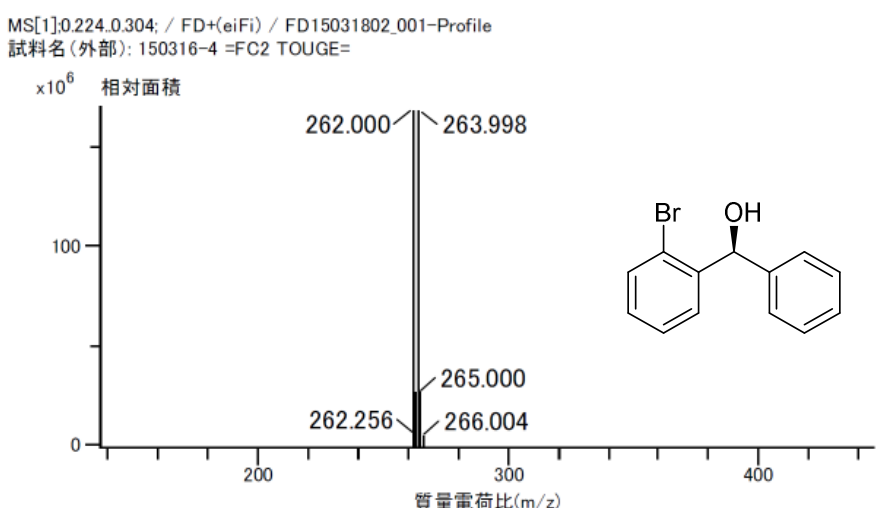

\begin{tabular}{|l|r|r|r|l|r|r|r|r|r|}
\hline 質量 & 計算質量 & $\begin{array}{c}\text { 質量差 } \\
\mathrm{mmu}\end{array}$ & $\begin{array}{c}\text { 質量差 } \\
\mathrm{ppm}\end{array}$ & 推定組成式 & $12 \mathrm{C}$ & $1 \mathrm{H}$ & $79 \mathrm{Br}$ & 160 & 不飽和数 \\
\hline 261.9996 & 261.9993 & 0.29 & 1.1 & $12 \mathrm{C} 131 \mathrm{H} 1179 \mathrm{Br} 16 \mathrm{O}$ & 13 & 11 & 1 & 1 & 8 \\
\hline
\end{tabular}

External standard : hexafluorobenzene

\section{(S)-Phenyl(2-(trifluoromethyl)phenyl)methanol (6d)}

(FI)

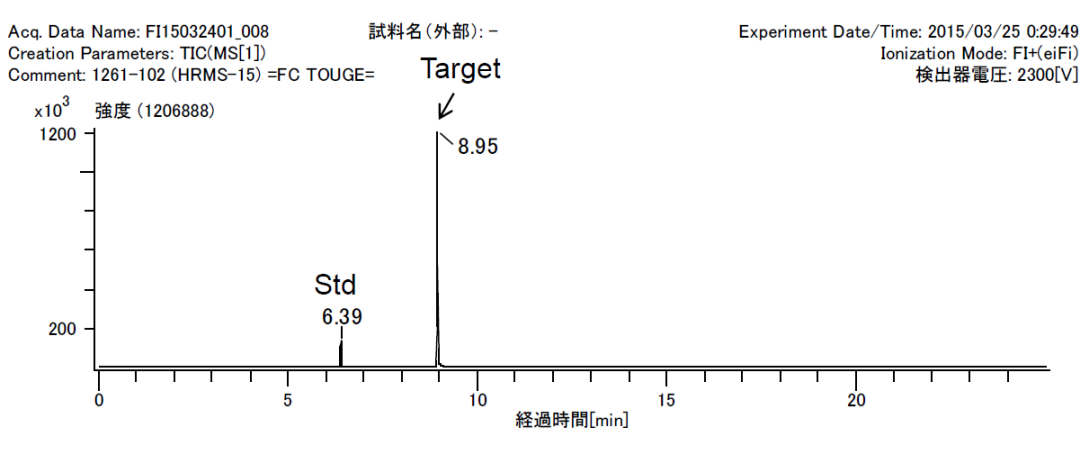

Standard for calibration: Linalyl acetate $\left[\mathrm{C}_{12} \mathrm{H}_{20} \mathrm{O}_{2}\right] 196.1464$

MS[1]:8.92.8.94: / FI+(eiFi) / FI1 5032401__008

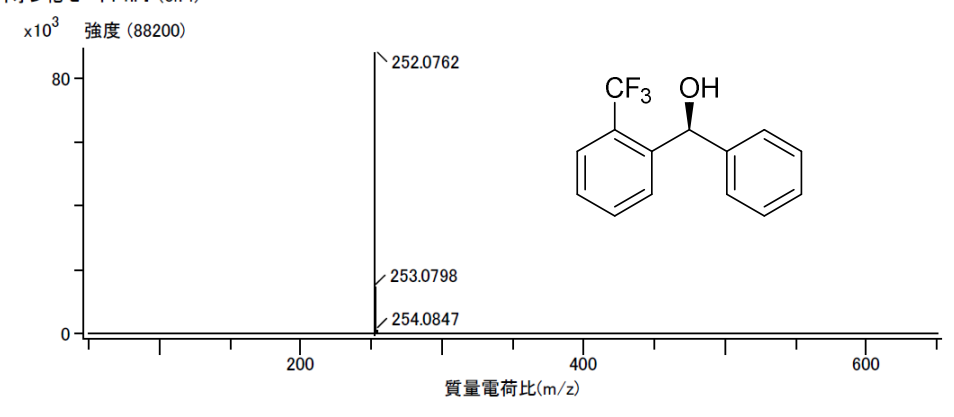

\begin{tabular}{|c|c|c|c|c|r|r|r|r|r|}
\hline 質量 & 計算質量 & $\begin{array}{c}\text { 質量差 } \\
\text { mmu }\end{array}$ & $\begin{array}{c}\text { 摬量差 } \\
\text { ppm }\end{array}$ & 推定組成式 & $12 \mathrm{C}$ & $1 \mathrm{H}$ & $19 \mathrm{~F}$ & 160 & 不飽和数 \\
\hline 252.07615 & 252.07620 & -0.05 & -0.18 & $12 \mathrm{C} 141 \mathrm{H} 11119 \mathrm{~F} 316 \mathrm{O}$ & 14 & 11 & 3 & 1 & 8 \\
\hline
\end{tabular}

[Condition]

Column: Rxi-5ms $30 \mathrm{~m} \times 0.25 \mathrm{~mm}$ i.d. $0.25 \mu \mathrm{m}$

Instrument: GC:Agilent7890, MS:JMS-T100GCV

Oven: $60(1 \mathrm{~min}){ }^{\circ} \mathrm{C}-300^{\circ} \mathrm{C} @ 20^{\circ} \mathrm{C} / \mathrm{min}$

Ionization: $\mathrm{FI}$

0-650

Inj. Vol.: Sample $0.5 \mu \mathrm{L}+$ STD 


\section{(S)-2-(Hydroxy(phenyl)methyl)phenol (6e)}

(ESI)
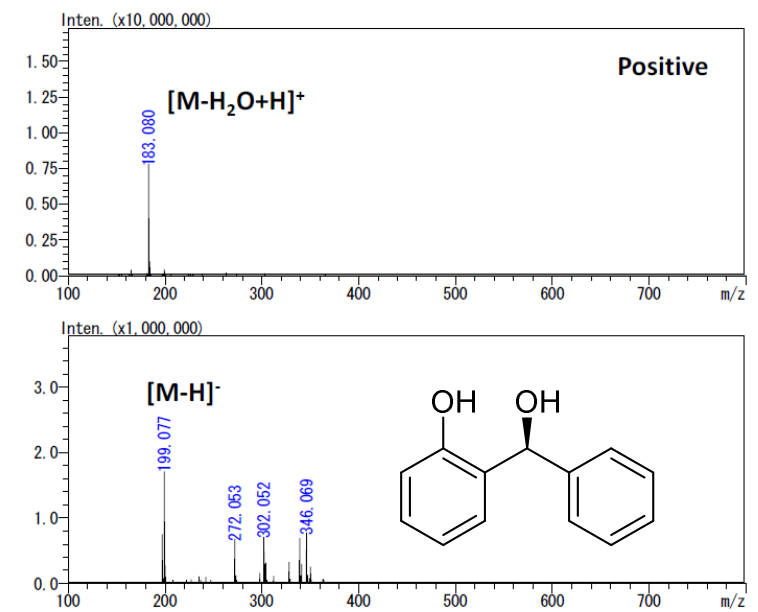

$\left[\mathrm{M}-\mathrm{H}_{2} \mathrm{O}+\mathrm{H}\right]^{+}$

\begin{tabular}{|l|l|l|r|r|r|r|r|r|}
\hline Score & Formula $(\mathrm{M})$ & Ion & Meas. $\mathrm{m} / \mathrm{z}$ & Pred. $\mathrm{m} / \mathrm{z}$ & Diff $(\mathrm{mDa})$ & Diff (ppm) & DBE & Pred. (M) \\
\hline 100 & $\mathrm{C} 13 \mathrm{H} 10 \mathrm{O}$ & {$[\mathrm{M}+\mathrm{H}]+$} & 183.0803 & 183.0804 & -0.1 & -0.55 & 9 & 182.0732 \\
\hline
\end{tabular} $[\mathrm{M}-\mathrm{H}]^{-}$

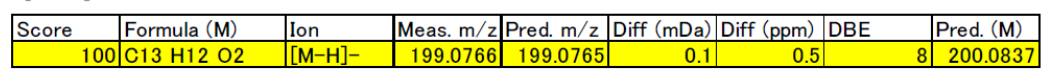

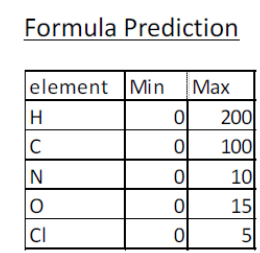

Analytical conditions

Instrument : LCMS-IT-TOF

lonization : ESI Infusion (positive \& negative)

Flow rate : $0.2 \mathrm{~mL} / \mathrm{min}$

$B: A C N$

Eluent $\quad A:$ water

data file : $\quad 15061702012$

\section{(S)-(2,4-Dimethylphenyl)(phenyl)methanol (6f)}

(FI)

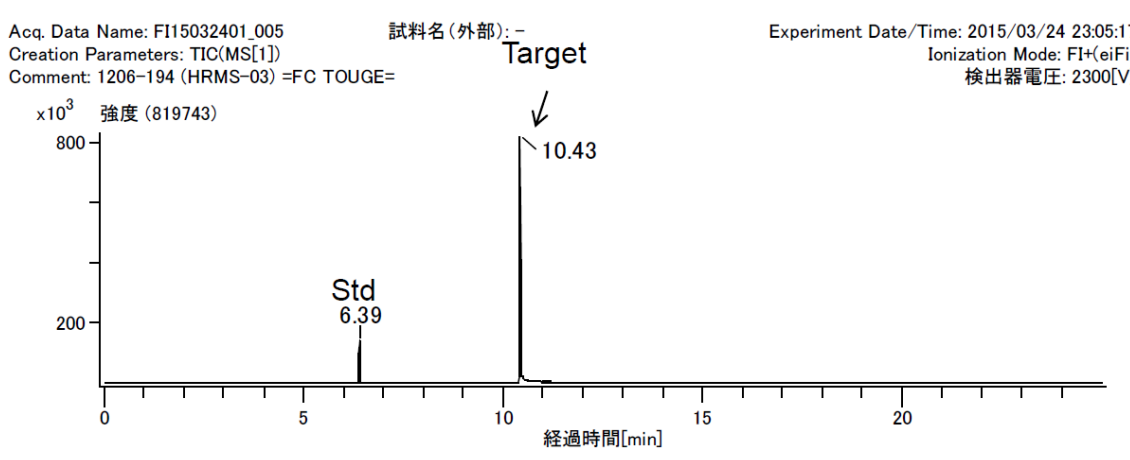

Standard for calibration: Linalyl acetate $\left[\mathrm{C}_{12} \mathrm{H}_{20} \mathrm{O}_{2}\right] 196.1464$

MS[1];10.40.10.41; / FI+(eiFi) / FI15032401_005

イオン化モード: 1:FI+(eiFi)

$\times 10^{3}$ 強度 (19967)

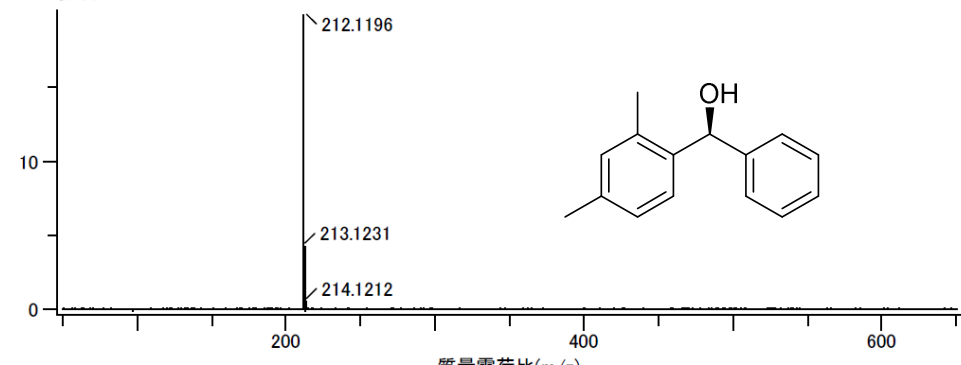

質量電荷比 $(m / z)$

\begin{tabular}{|c|c|c|c|c|r|r|r|r|}
\hline 質量 & 計算質量 & $\begin{array}{c}\text { 質量差 } \\
\text { mmu }\end{array}$ & $\begin{array}{c}\text { 質量差美 } \\
\text { ppm }\end{array}$ & 推定組成式 & $12 \mathrm{C}$ & $1 \mathrm{H}$ & 160 & 不飽和数 \\
\hline 212.11955 & 212.12011 & -0.56 & -2.64 & $12 \mathrm{C} 151 \mathrm{H} 1616 \mathrm{O}$ & 15 & 16 & 1 & 8 \\
\hline
\end{tabular}

[Condition]

Column: Rxi- $5 \mathrm{~ms} 30 \mathrm{~m} \times 0.25 \mathrm{~mm}$ i.d., $0.25 \mu \mathrm{m}$

Instrument: GC:Agilent7890, MS:JMS-T100GCV

Oven: $60(1 \mathrm{~min}){ }^{\circ} \mathrm{C}-300^{\circ} \mathrm{C} @ 20^{\circ} \mathrm{C} / \mathrm{min}$

lonization: $\mathrm{FI}$

MS Range: $m / z$ 50-650

Inj. Vol.: Sample $0.5 \mu \mathrm{L}+$ STD 


\section{(S)-Phenyl(2,4,5-trimethylphenyl)methanol (6g)}

(FI)

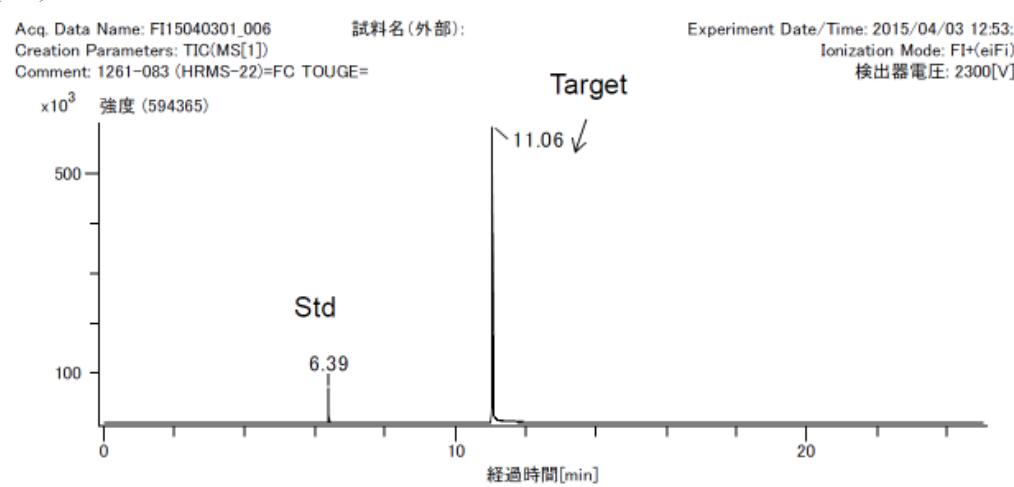

Standard for calibration: Linalyl acetate $\left[\mathrm{C}_{12} \mathrm{H}_{20} \mathrm{O}_{2}\right] 196.1464$ MS[1]:11.05.11.06: / Fl+(eiFi) / F115040301_006

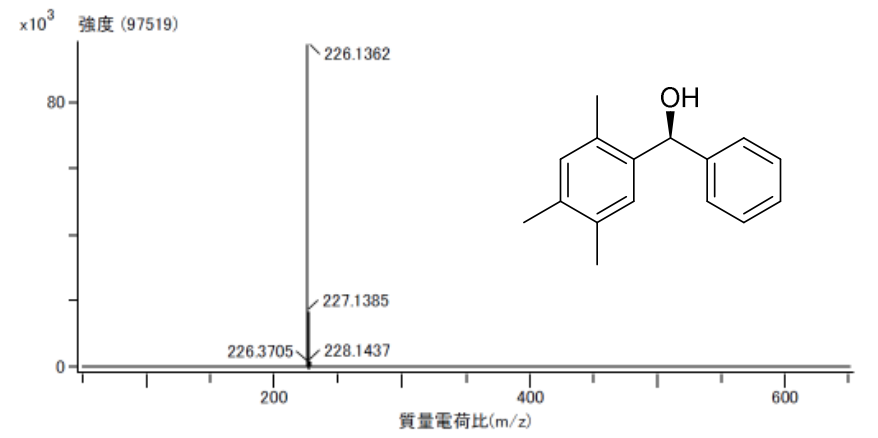

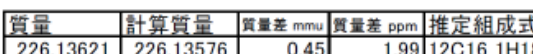

\begin{tabular}{|l|l|l|l|}
\hline $12 \mathrm{C}$ & $1 \mathrm{H}$ & 160 & 不飽和数 \\
\hline & 16 & 18 & 1
\end{tabular}

[Condition]

Column: Rxi-5ms $30 \mathrm{~m} \times 0.25 \mathrm{~mm}$ i.d., $0.25 \mathrm{~m}$

Instrument: GC:Agilent7890, MS:JMS-T100GCV

Oven: $60(1 \mathrm{~min}){ }^{\circ} \mathrm{C}-300^{\circ} \mathrm{C} @ 20^{\circ} \mathrm{C} / \mathrm{min}$

Ionization: $F$

Inj. Vol.: Sample $0.5 \square L+$ STD

\section{(S)-(2,4-Dichlorophenyl)(phenyl)methanol (6h)}

(FI)

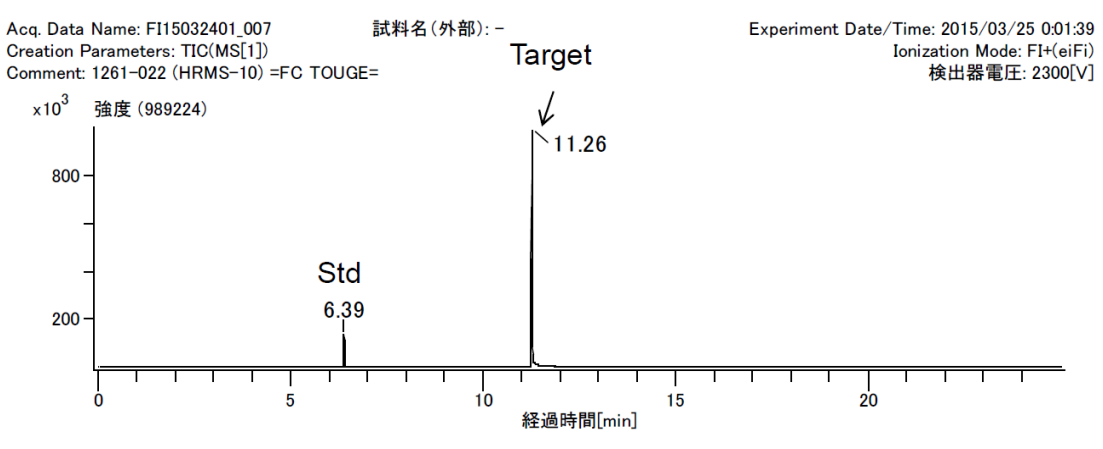

Standard for calibration: Linalyl acetate $\left[\mathrm{C}_{12} \mathrm{H}_{20} \mathrm{O}_{2}\right] 196.1464$

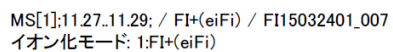

$\times 10^{3}$ 強度 (40351)

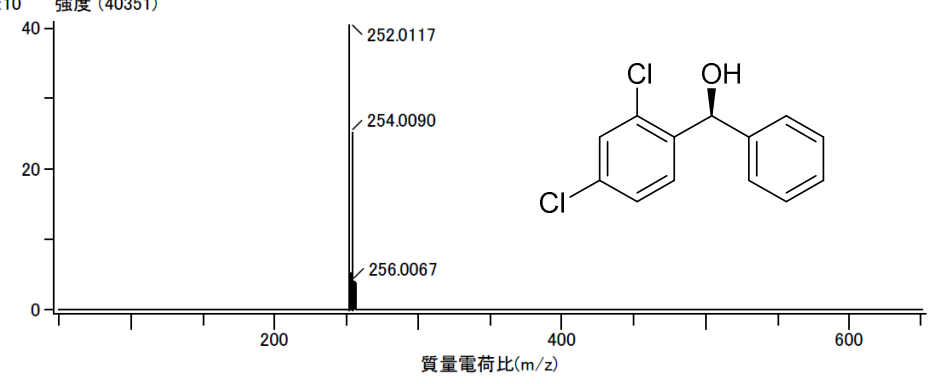

\begin{tabular}{|c|c|c|c|c|r|r|r|r|r|}
\hline 質量 & 計算質量 & $\begin{array}{c}\text { 皆量差質量差 } \\
\text { mmu }\end{array}$ & ppm & 推定組成式 & $12 \mathrm{C}$ & $1 \mathrm{H}$ & $35 \mathrm{Cl}$ & 160 & 不飽和数 \\
\hline 252.01169 & 252.01087 & 0.82 & 3.25 & $12 \mathrm{C} 131 \mathrm{H} 1035 \mathrm{C} 1216 \mathrm{O}$ & 13 & 10 & 2 & 1 & 8 \\
\hline
\end{tabular}

[Condition]

Column: Rxi-5ms $30 \mathrm{~m} \times 0.25 \mathrm{~mm}$ i.d., $0.25 \mu \mathrm{m}$

Instrument: GC:Agilent7890, MS:JMS-T100GCV

Oven: $60(1 \mathrm{~min}){ }^{\circ} \mathrm{C}-300^{\circ} \mathrm{C} @ 20^{\circ} \mathrm{C} / \mathrm{min}$

onization: $\mathrm{Fl}$

Inj. Vol.: Sample $0.5 \mu \mathrm{L}+$ STD 


\section{(S)-(2-Chloro-5-nitrophenyl)(phenyl)methanol (6i)}

(FI)

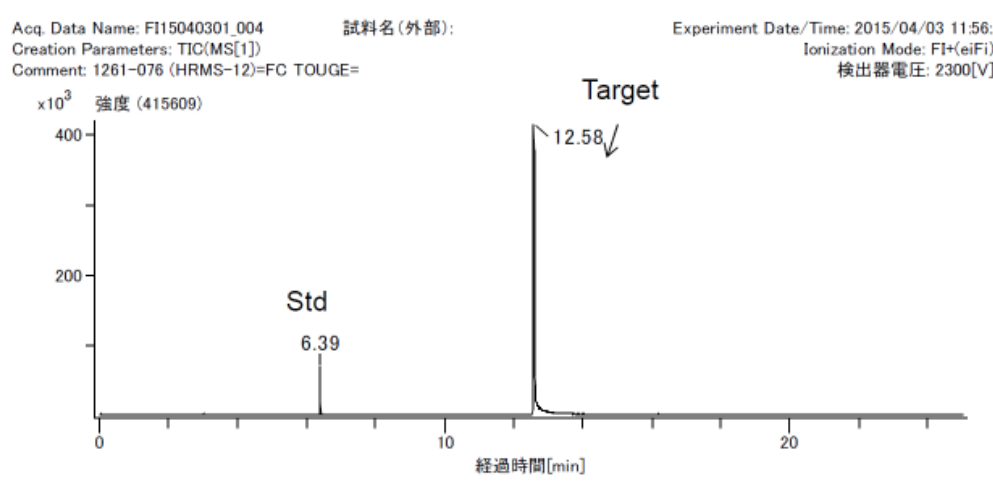

Standard for calibration: Linalyl acetate $\left[\mathrm{C}_{12} \mathrm{H}_{20} \mathrm{O}_{2}\right] 196.1464$

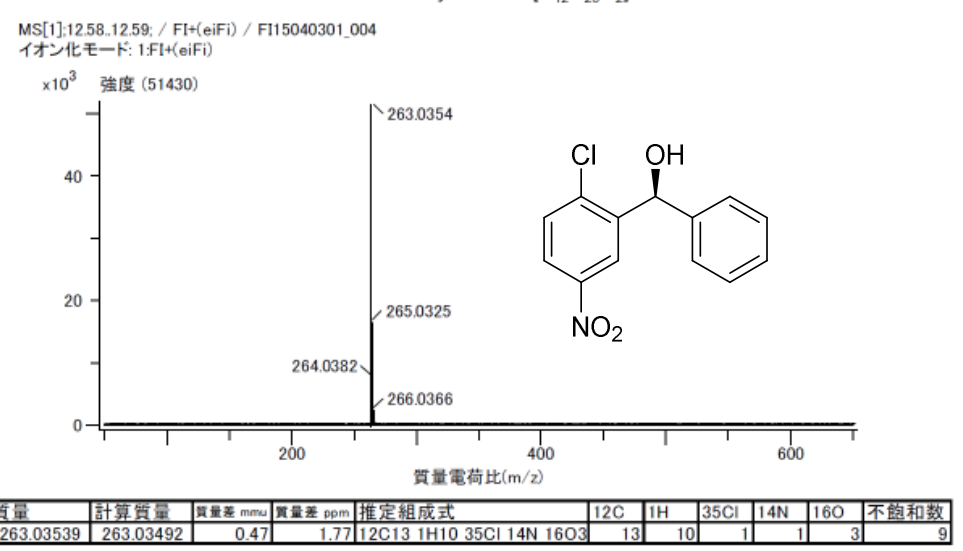

[Condition]

Column: Rxi- $5 \mathrm{~ms} 30 \mathrm{~m} \times 0.25 \mathrm{~mm}$ i.d., $0.25-\mathrm{m}$

Instrument: GC:Agilent7890, MS:JMS-T100GCV

Oven: $60(1 \mathrm{~min}){ }^{\circ} \mathrm{C}-300^{\circ} \mathrm{C} @ 20^{\circ} \mathrm{C} / \mathrm{min}$

lonization: $\mathrm{Fl}$

MS Range: $m / z$ 50-650

Inj. Vol.: Sample $0.5 \square L+S T D$

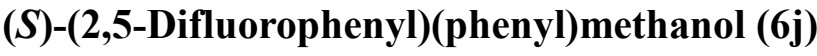

(FI)

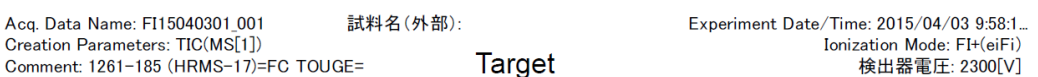

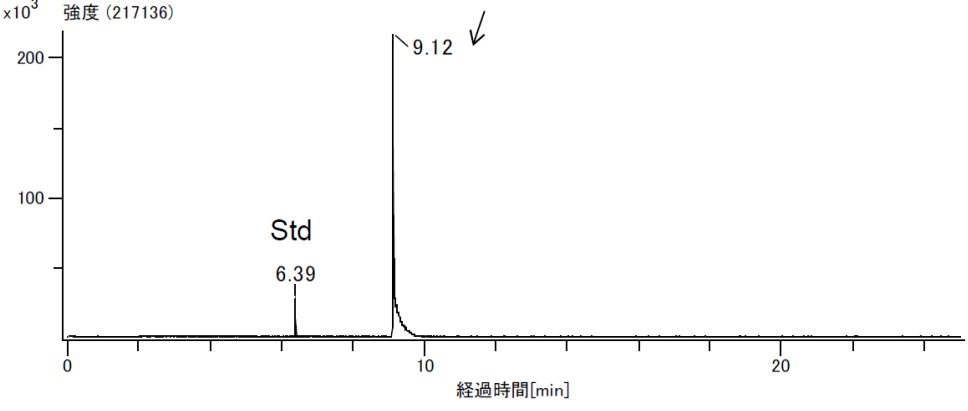

Standard for calibration: Linalyl acetate $\left[\mathrm{C}_{12} \mathrm{H}_{20} \mathrm{O}_{2}\right] 196.1464$

MS[1]:9.09.9.09:/ FI+(eiFi) / F115040301_001

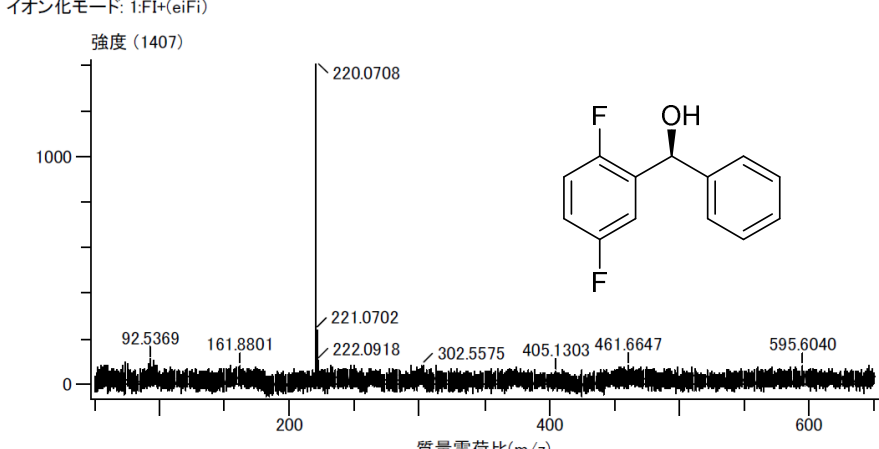

質量電荷比 $(m / z)$

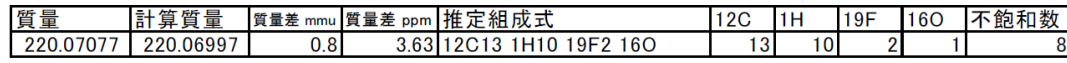

[Condition]

Column: Rxi-5ms $30 \mathrm{~m} \times 0.25 \mathrm{~mm}$ i.d., $0.25 \square \mathrm{m}$

Instrument: GC:Agilent7890, MS:JMS-T100GCV

Oven: 60(1min) ${ }^{\circ} \mathrm{C}-300^{\circ} \mathrm{C} @ 20^{\circ} \mathrm{C} / \mathrm{min}$

lonization: $\mathrm{FI}$

MS Range: $m / z$ 50-650

Inj. Vol.: Sample 0.5 _L + STD 
(S)-(2-Fluoro-3-(trifluoromethyl)phenyl)(phenyl)methanol (6k)

$(\mathrm{FI})$

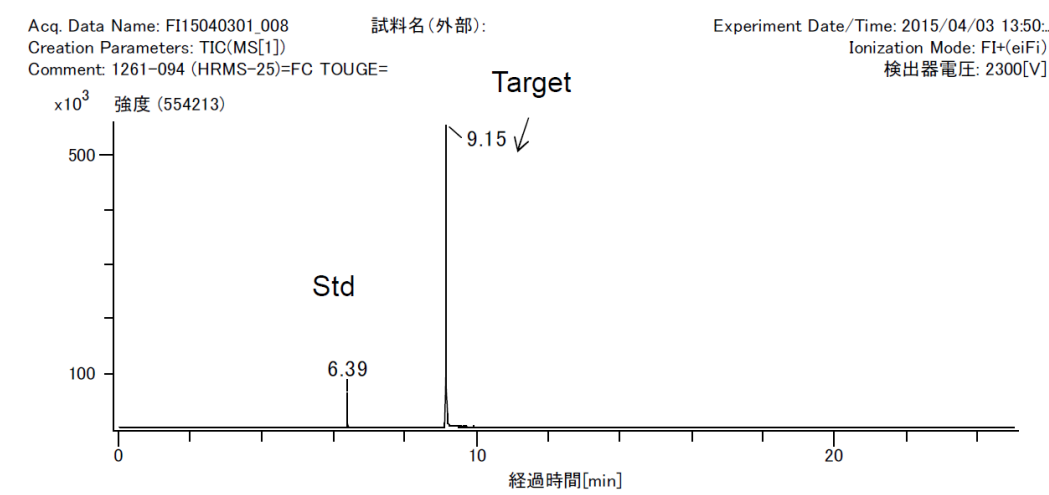

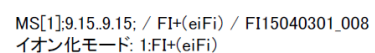

1オコ一า

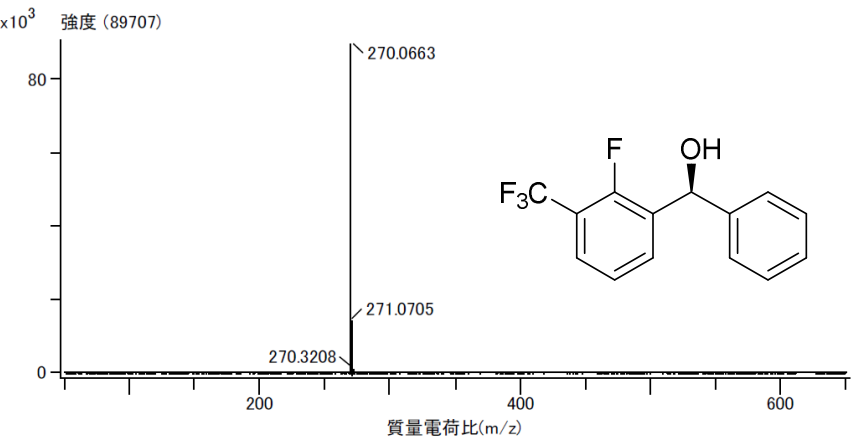

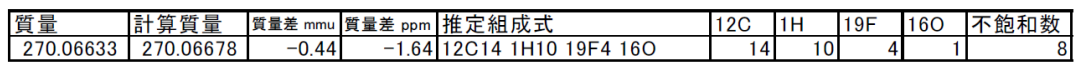

[Condition]

Column: Rxi-5ms $30 \mathrm{~m} \times 0.25 \mathrm{~mm}$ i.d., $0.25 \beth \mathrm{m}$

Instrument: GC:Agilent7890, MS:JMS-T100GCV

Oven: $60(1 \mathrm{~min}){ }^{\circ} \mathrm{C}-300^{\circ} \mathrm{C} @ 20^{\circ} \mathrm{C} / \mathrm{min}$

onization: $F$

MS Range: $m / z$ 50-650

Inj. Vol.: Sample $0.5 \square L+S T D$

\section{(S)-(Perfluorophenyl)(phenyl)methanol (61)}

(FI)

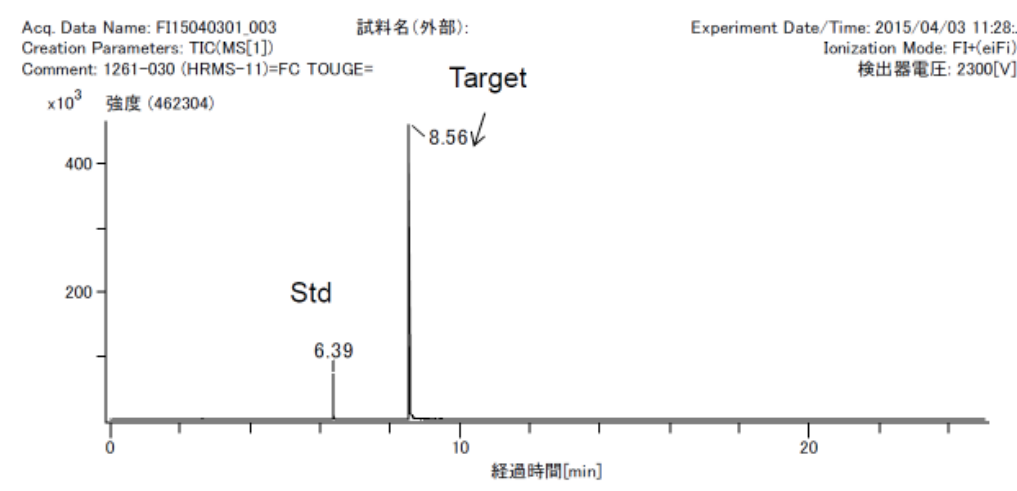

Standard for calibration: Linalyl acetate $\left[\mathrm{C}_{12} \mathrm{H}_{20} \mathrm{O}_{2}\right] 196.1464$

MS[1] 8.56.8.56: / F1+(eiFi) / F115040301_003

(10 $10^{3}$

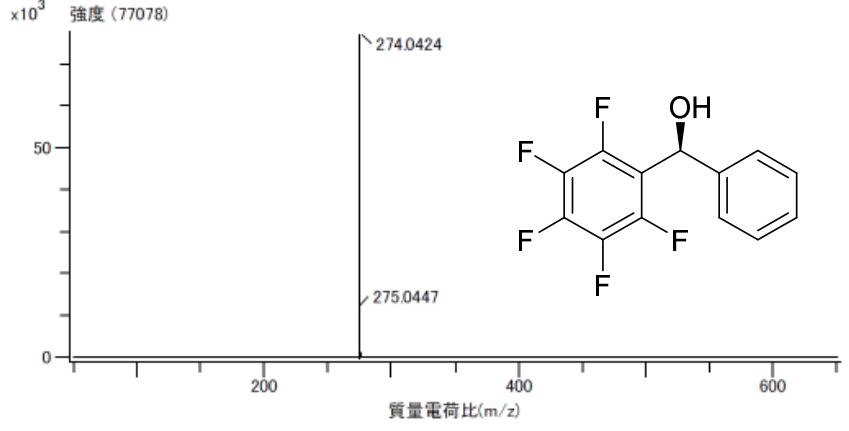

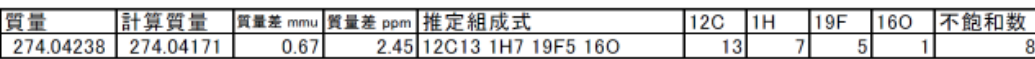

[Condition]

Column: Rxi-5ms $30 \mathrm{~m} \times 0.25 \mathrm{~mm}$ i.d., $0.25 \square \mathrm{m}$

Instrument: GC:Agilent7890, MS:JMS-T100GCV

Oven: $60(1 \mathrm{~min}){ }^{\circ} \mathrm{C}-300^{\circ} \mathrm{C} @ 20^{\circ} \mathrm{C} / \mathrm{min}$

Ionization: $\mathrm{F}$

MS Range: $m / 2$ 50-650

Inj. Vol.: Sample 0.5 $\square$ L STD 


\section{(S)-(2-Chlorophenyl)(4-chlorophenyl)methanol (6m)}

(FI)

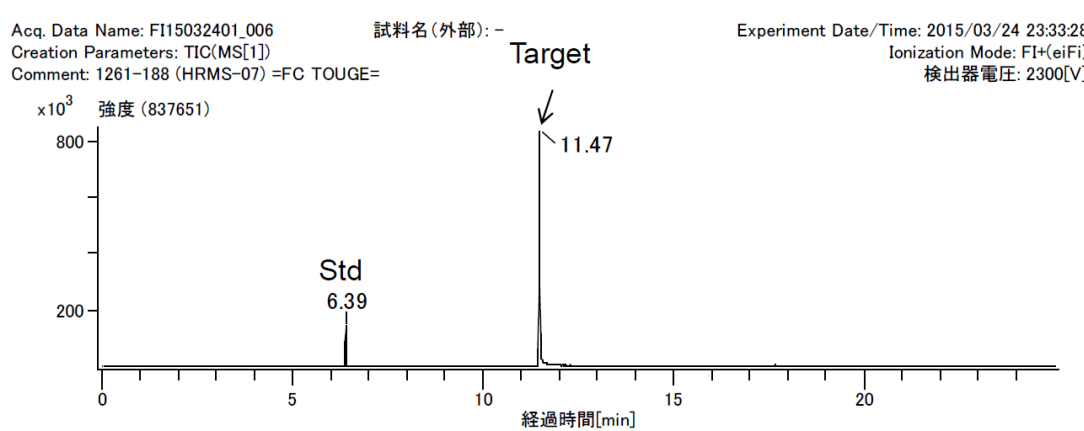

Standard for calibration: Linalyl acetate $\left[\mathrm{C}_{12} \mathrm{H}_{20} \mathrm{O}_{2}\right] 196.1464$

MS[1]:11.46.11.48; / FI+(eiFi) / F115032401_006

偖:

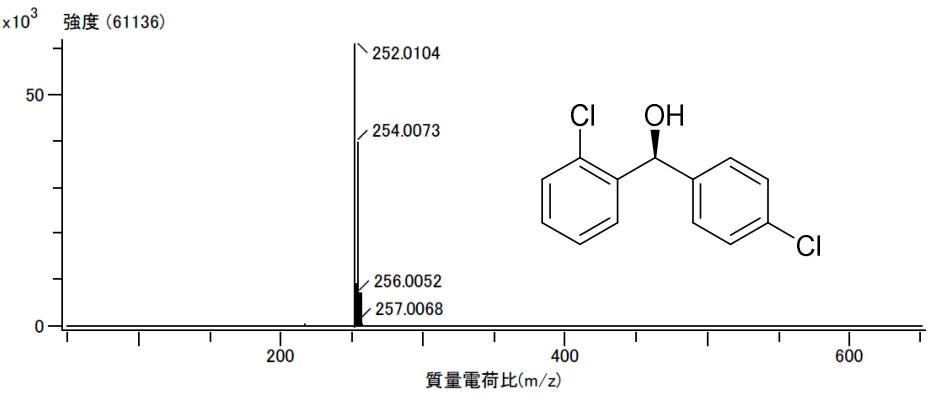

質量 計算質量 質量差 質量差

\begin{tabular}{c|c|c} 
筫量差 & $\begin{array}{c}\text { 筫量差 } \\
\text { mmu } \\
\text { ppm }\end{array}$ 推定組成式 \\
\hline
\end{tabular}

\begin{tabular}{l|l|l|l|l|}
$12 \mathrm{C}$ & $1 \mathrm{H}$ & $35 \mathrm{Cl}$ & 160 & 不飽和数 \\
\hline
\end{tabular}

\section{(S)-(2-Chlorophenyl)(4-fluorophenyl)methanol (6n)}

(FI)

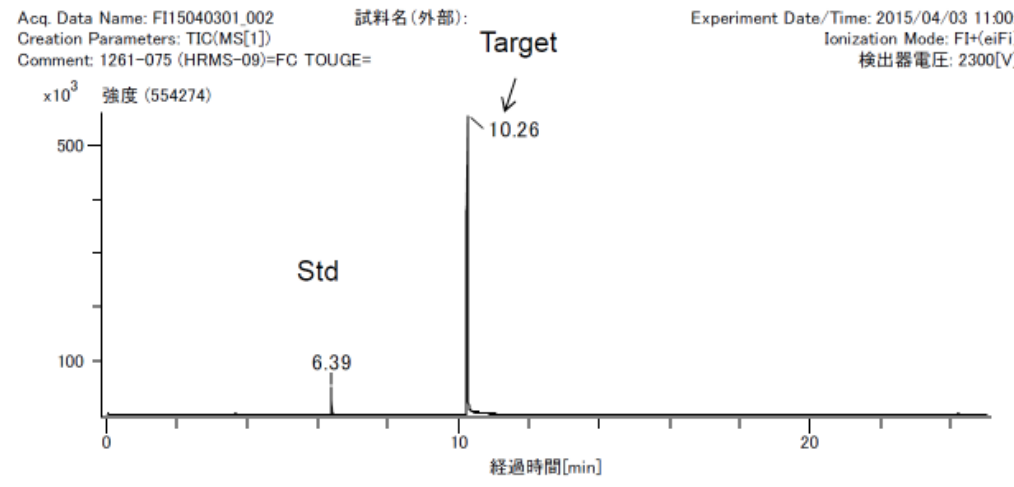

Standard for calibration: Linalyl acetate $\left[\mathrm{C}_{12} \mathrm{H}_{20} \mathrm{O}_{2}\right] 196.1464$

MS[1]:10.23.10.24: FI+(eiFi) / F115040301_002

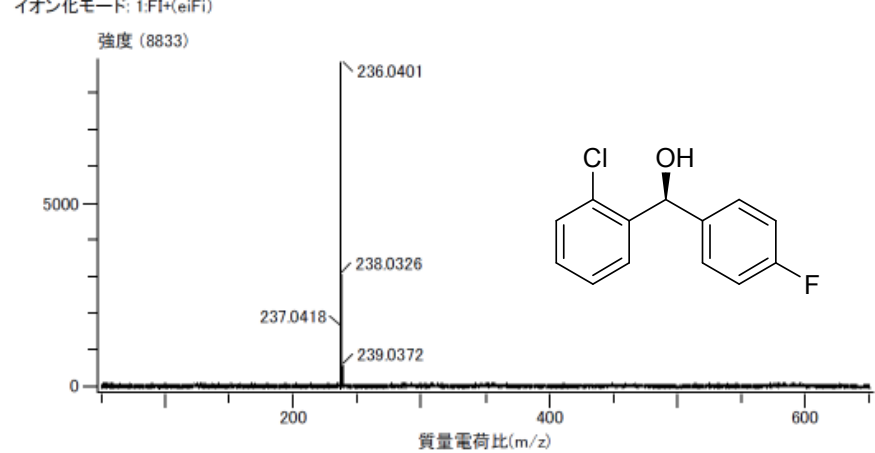

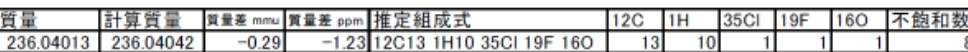

[Condition]

Column: Rxi-5ms $30 \mathrm{~m} \times 0.25 \mathrm{~mm}$ i.d., $0.25 \mathrm{~m}$

Instrument: GC:Agilent7890, MS:JMS-T100GCV

Oven: $60(1 \mathrm{~min}){ }^{\circ} \mathrm{C}-300^{\circ} \mathrm{C} @ 20^{\circ} \mathrm{C} / \mathrm{min}$

Ionization: $\mathrm{F}$

MS Range: $m / z$ 50-650

Inj. Vol.: Sample 0.5 $\square \mathrm{L}+\mathrm{STD}$ 


\section{(S)-(4-Chlorophenyl)(phenyl)methanol (11a)}

(FI)

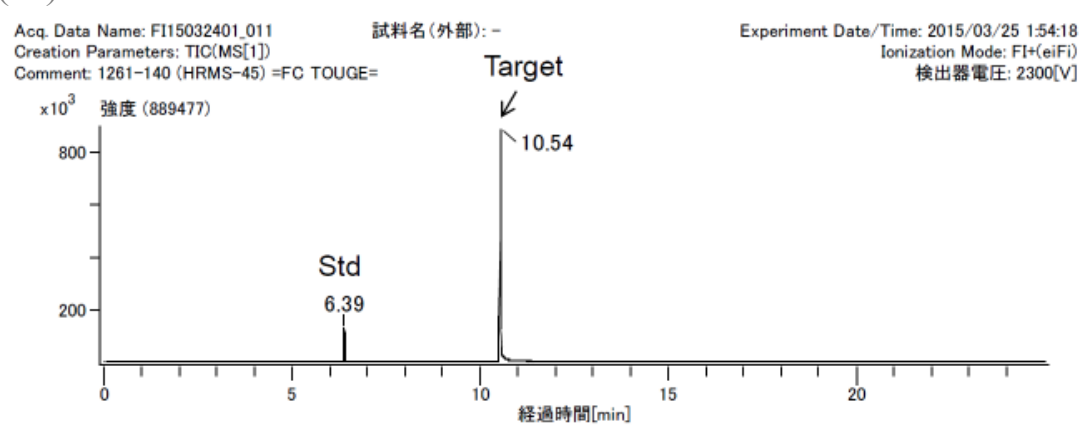

Standard for calibration: Linalyl acetate $\left[\mathrm{C}_{12} \mathrm{H}_{20} \mathrm{O}_{2}\right] 196.1464$

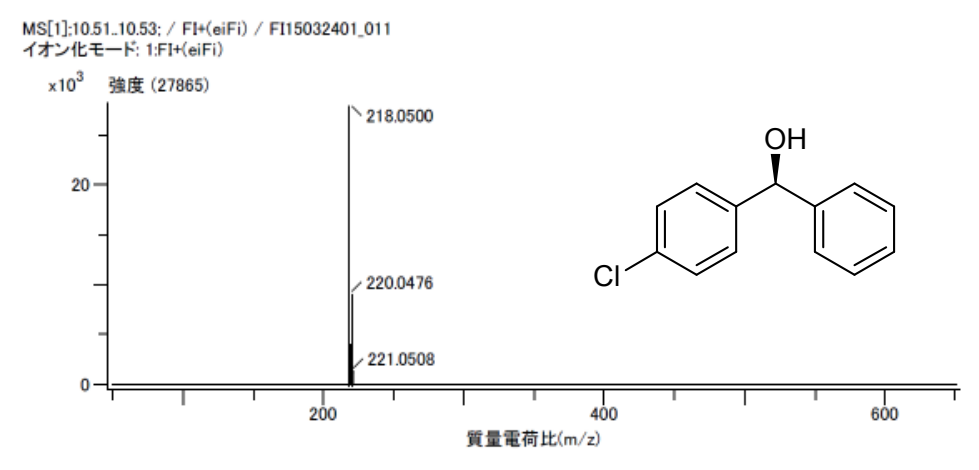

\begin{tabular}{|c|c|c|c|c|r|r|r|r|r|}
\hline 質量 & 計算質量 & $\begin{array}{c}\text { 質量差買量差 } \\
\text { mmu }\end{array}$ & 推定組成式 & $12 \mathrm{C}$ & $1 \mathrm{H}$ & $35 \mathrm{CI}$ & 160 & 不飽和数 \\
\hline 218.05004 & 218.04984 & 0.20 & 0.90 & $12 \mathrm{C} 131 \mathrm{H} 1135 \mathrm{Cl} 16 \mathrm{O}$ & 13 & 11 & 1 & 1 & 8 \\
\hline
\end{tabular}

[Condition]

Column: Rxi-5ms $30 \mathrm{~m}$ x $0.25 \mathrm{~mm}$ i.d., $0.25 \mu \mathrm{m}$

Instrument: GC:Agilent7890, MS:JMS-T100GCV

Oven: $60(1 \mathrm{~min}){ }^{\circ} \mathrm{C}-300^{\circ} \mathrm{C} @ 20^{\circ} \mathrm{C} / \mathrm{min}$

MS Range: $m / z$ 50-650

Inj. Vol.: Sample $0.5 \mu L+$ STD 


\section{(S)-(3,4-Dichlorophenyl)(phenyl)methanol (11b)}

(FI)

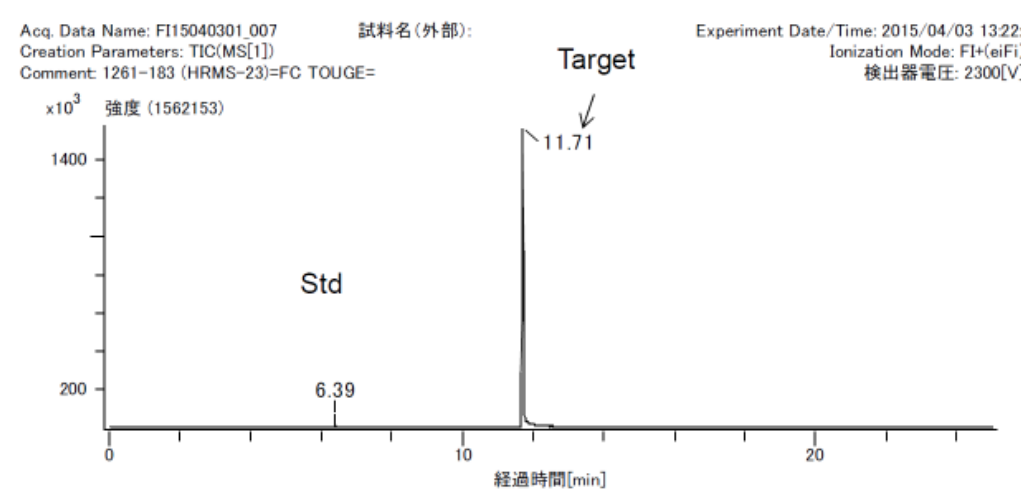

Standard for calibration: Linalyl acetate $\left[\mathrm{C}_{12} \mathrm{H}_{20} \mathrm{O}_{2}\right] 196.1464$ MS[1]:11.72-11.72: / Fl+(eiFi) / F115040301_007$$
\times 10^{3} \quad \text { 强度 }(90664
$$
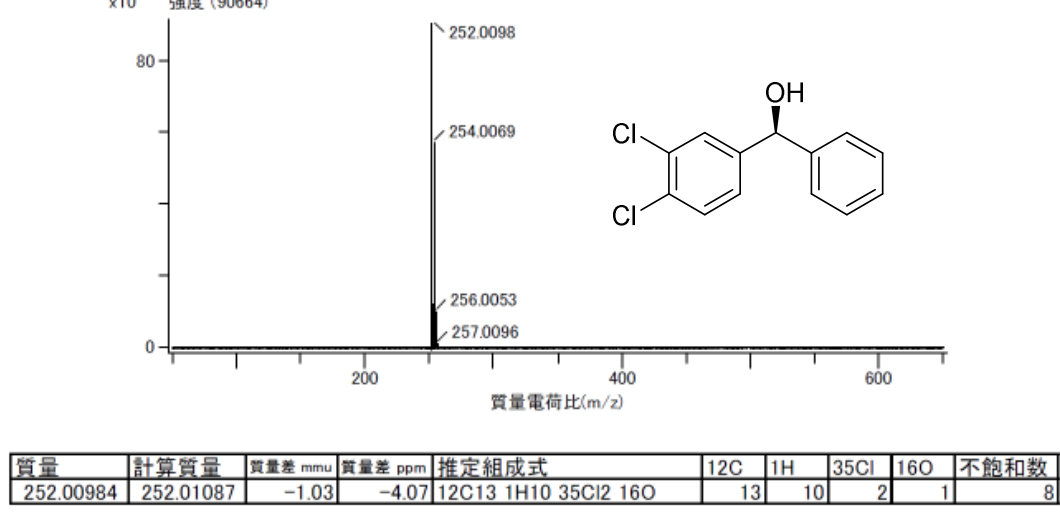

[Condition]

Column: Rxi-5ms $30 \mathrm{~m} \times 0.25 \mathrm{~mm}$ i.d., $0.25 \_\mathrm{m}$

Instrument: GC:Agilent7890, MS:JMS-T100GCV

Oven: $60(1 \mathrm{~min}){ }^{\circ} \mathrm{C}-300^{\circ} \mathrm{C} @ 20^{\circ} \mathrm{C} / \mathrm{min}$

Ionization: $\mathrm{Fl}$

MS Range: $m / 2$ 50-650

Inj. Vol.: Sample 0.5 $\square \mathrm{L}+\mathrm{STD}$

\section{(S)-(3,4-Difluorophenyl)(phenyl)methanol (11c)}

(FI)

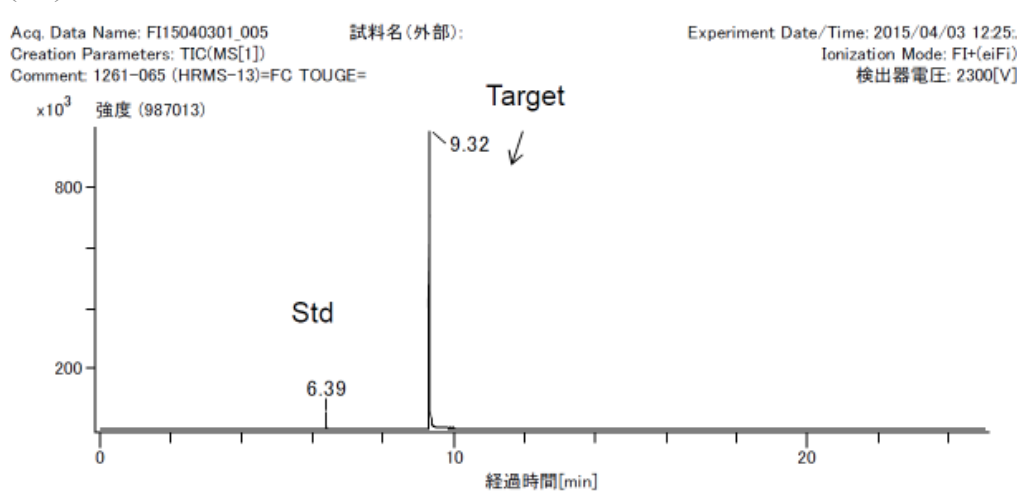

Standard for calibration: Linalyl acetate $\left[\mathrm{C}_{12} \mathrm{H}_{20} \mathrm{O}_{2}\right] 196.1464$

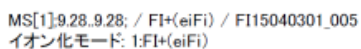

$$
\begin{array}{r}
\text { イオン化モート゚: 1:Fl+(eiFi) } \\
\text { 強度 (2956) }
\end{array}
$$

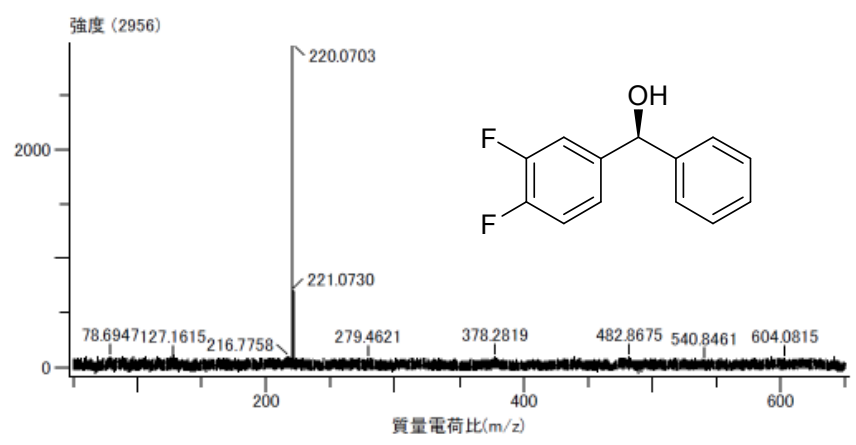

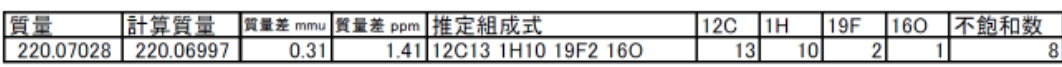

[Condition]

Column: Rxi-5ms $30 \mathrm{~m} \times 0.25 \mathrm{~mm}$ i.d., $0.25 \_\mathrm{m}$

Instrument: GC:Agilent7890, MS:JMS-T100GCV

Oven: $60(1 \mathrm{~min}){ }^{\circ} \mathrm{C}-300^{\circ} \mathrm{C} @ 20^{\circ} \mathrm{C} / \mathrm{min}$

Ionization: $\mathrm{FI}$

MS Range: $m / z$ 50-650

Inj. Vol.: Sample 0.5 $\square$ L + STD 


\section{(S)-(4-Nitrophenyl)(phenyl)methanol (11d)}

(FI)

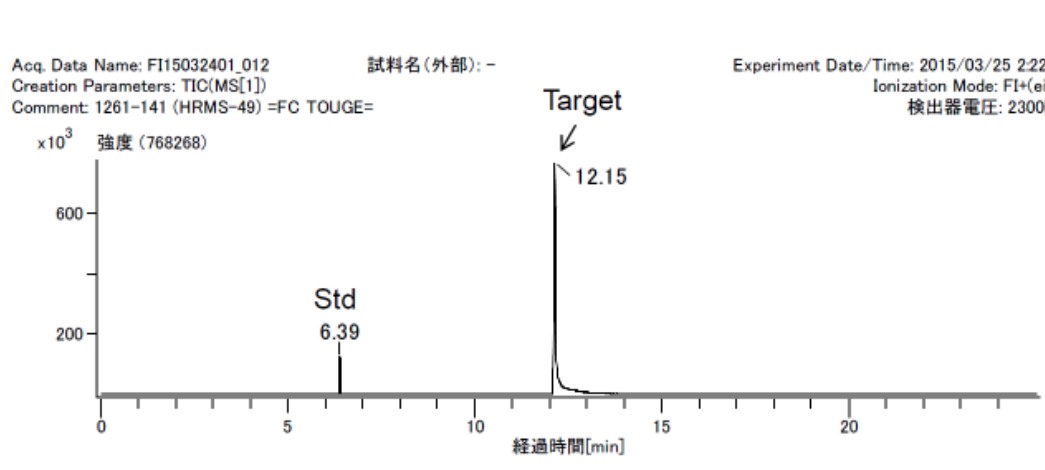

Standard for calibration: Linalyl acetate $\left[\mathrm{C}_{12} \mathrm{H}_{20} \mathrm{O}_{2}\right] 196.1464$

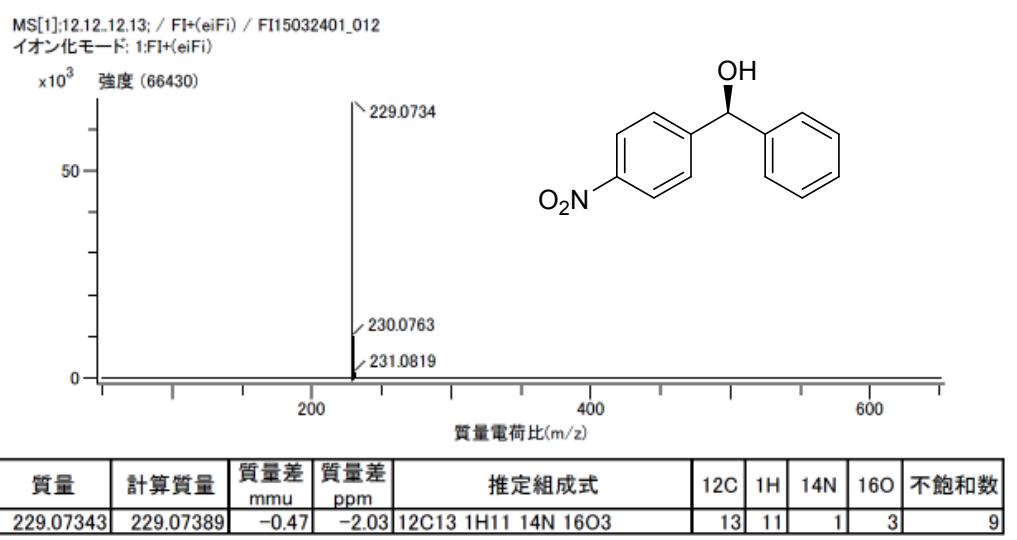

[Condition]

Column: Rxi-5ms $30 \mathrm{~m} \times 0.25 \mathrm{~mm}$ i.d., $0.25 \mu \mathrm{m}$

Instrument: GC:Agilent7890, MS:JMS-T100GCV

Oven: $60(1 \mathrm{~min}){ }^{\circ} \mathrm{C}-300^{\circ} \mathrm{C} @ 20^{\circ} \mathrm{C} / \mathrm{min}$

Ionization: $\mathrm{F}$

MS Range: $m / 250-650$

Inj. Vol.: Sample $0.5 \mu \mathrm{L}+$ STD

\section{(S)-(4-Chloro-3-nitrophenyl)(phenyl)methanol (11e)}

(FI)

Acq. Data Name: F115032401009 試料名(外部): -

Creation Parameters: TIC(MS[1])

$10^{3}$ 胎度 (122748)

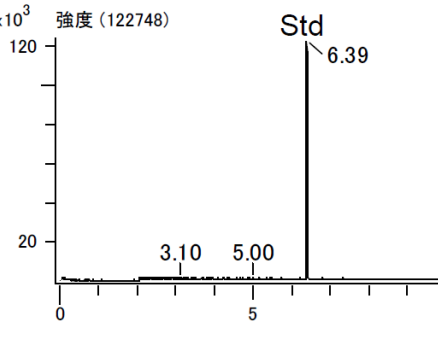

Target

Experiment Date/Time: 2015/03/25 0:57:59 模出器電王: $2300[\mathrm{~V}$ ]

Standard for calibration: Linalyl acetate $\left[\mathrm{C}_{12} \mathrm{H}_{20} \mathrm{O}_{2}\right] 196.1464$

MS[1]:12.75. 12.84; / FI+(eiFi) / FI15032401_009

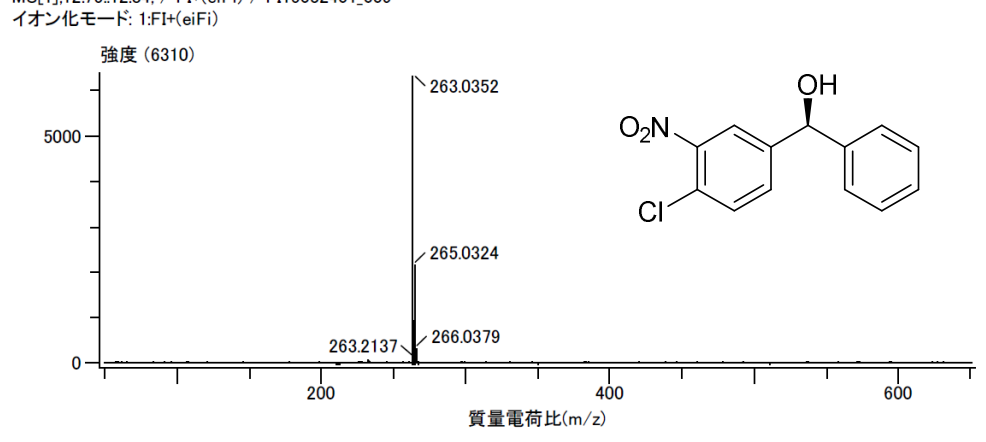

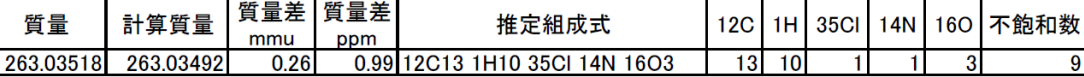

[Condition]

Column: Rxi-5ms $30 \mathrm{~m} \times 0.25 \mathrm{~mm}$ id. $0.25 \mu \mathrm{m}$

Instrument: GC:Agi

Oven: $60(1 \mathrm{~min}){ }^{\circ} \mathrm{C}-300^{\circ} \mathrm{C} @ 20^{\circ} \mathrm{C} / \mathrm{min}$

$@ 20^{\circ} \mathrm{C} / \mathrm{min}$

MS Rang: $m / z$

$250-650$

Inj. Vol.: Sample $0.5 \mu \mathrm{L}+\mathrm{STD}$ 
(S)-Phenyl(3,4,5-trifluorophenyl)methanol (11f)

(FI)

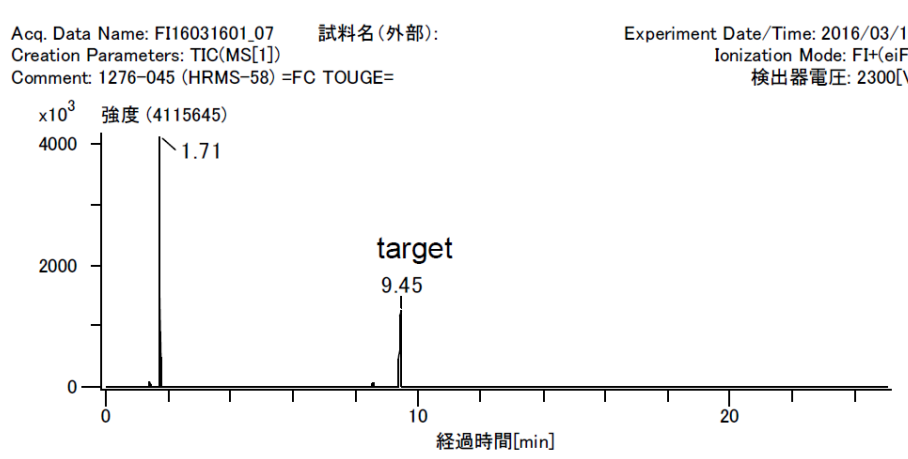

Standard for calibration: AT STD MIX

MS

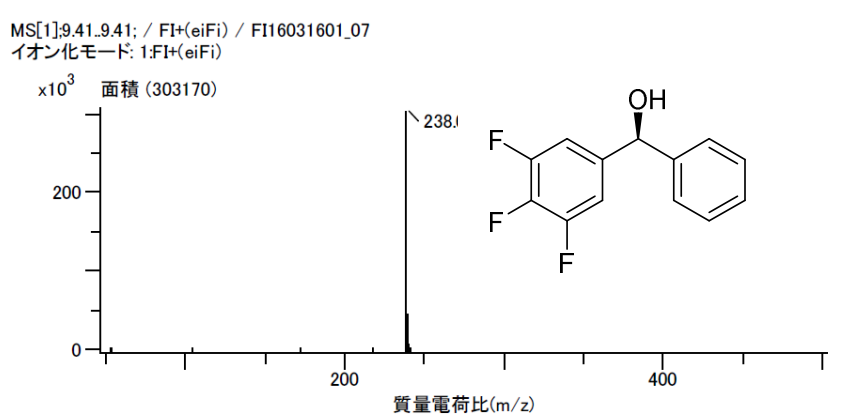

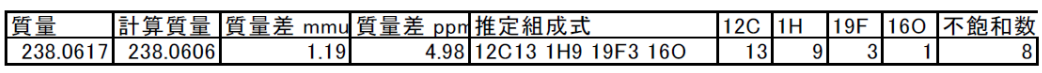

\section{(S)-(3,5-Dinitrophenyl)(phenyl)methanol (11g)}

(FI)
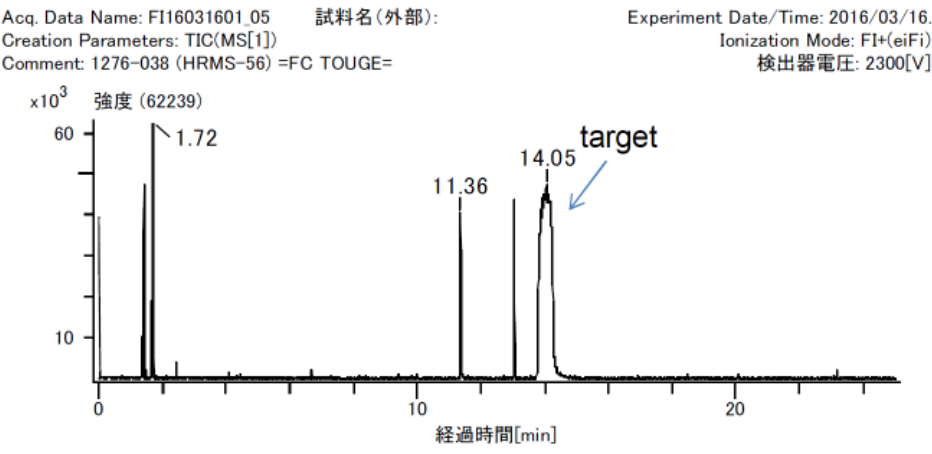

Standard for calibration: AT STD MIX

MS

MS[1]:13.88.14.26: / FI+(eiFi) / F16031601_05

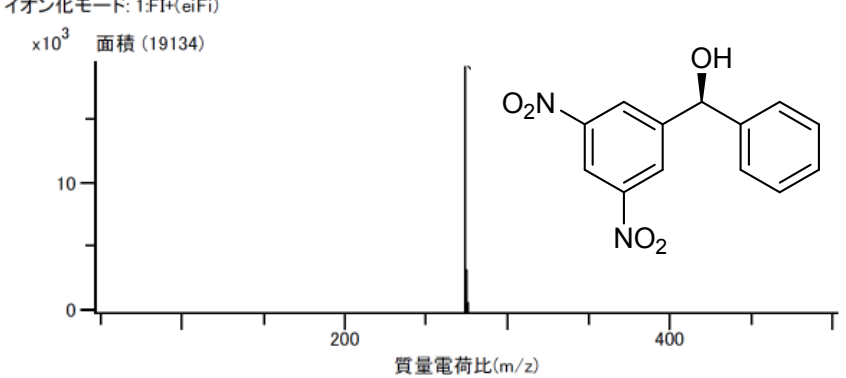

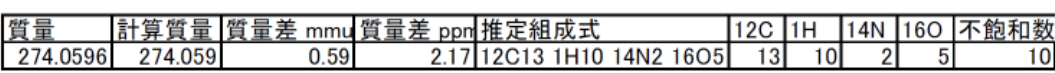




\section{(R)-(4-Methoxyphenyl)(phenyl)methanol (11h)}

(FI)

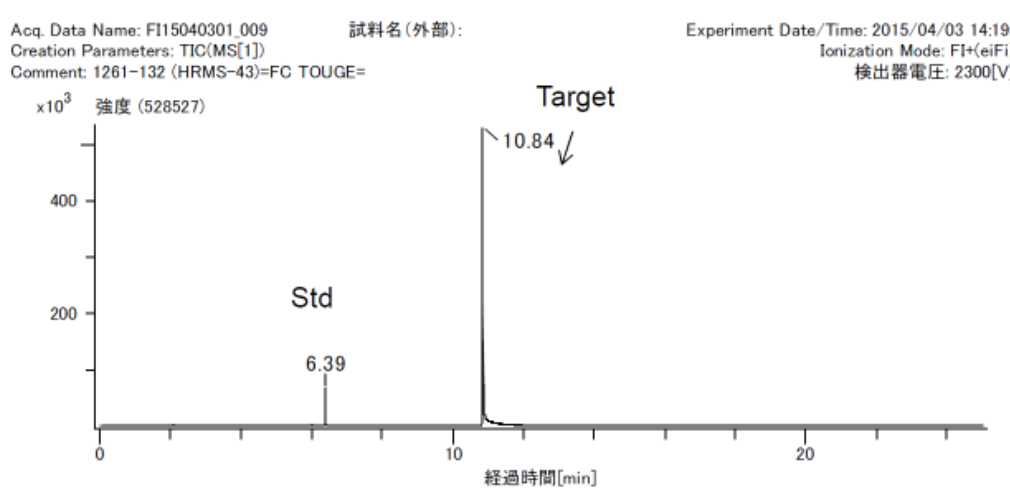

Standard for calibration: Linalyl acetate $\left[\mathrm{C}_{12} \mathrm{H}_{20} \mathrm{O}_{2}\right] 196.1464$

MS[1]:10.84.10.84: $/ \mathrm{FI}+($ (eifi) / F115040301_009

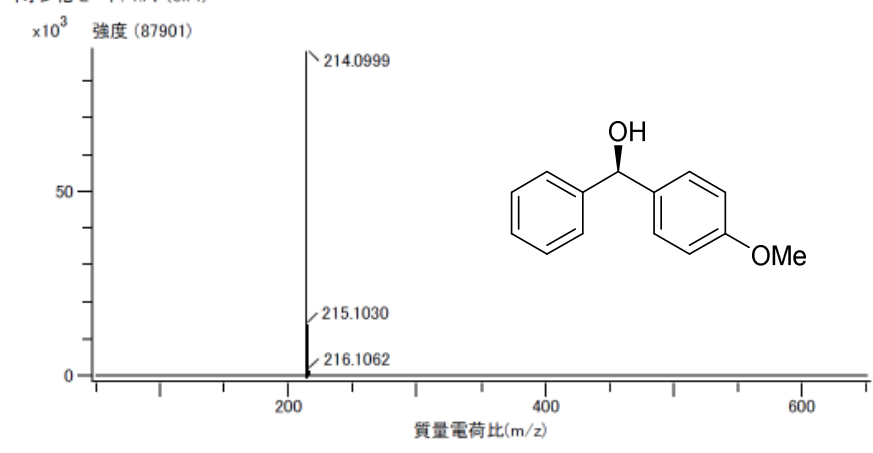

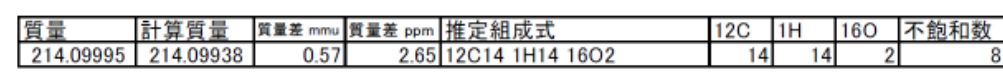

[Condition]

Column: Rxi-5ms $30 \mathrm{~m} \times 0.25 \mathrm{~mm}$ i.d., $0.25-\mathrm{m}$

Instrument: GC:Agilent7890, MS:JMS-T100GCV

Oven: $60(1 \mathrm{~min}){ }^{\circ} \mathrm{C}-300^{\circ} \mathrm{C} @ 20^{\circ} \mathrm{C} / \mathrm{min}$

Ionization: $\mathrm{FI}$

MS Range: $m / 250-650$

Inj. Vol.: Sample $0.5 \square L+S T D$

\section{(S)-(4-Chlorophenyl)(4-methoxyphenyl)methanol (11i)}

\section{(FI)}

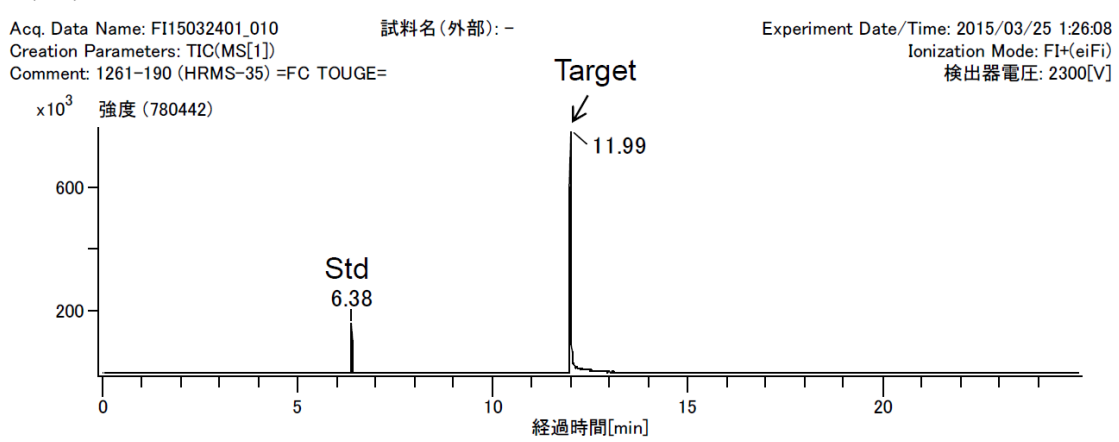

Standard for calibration: Linalyl acetate $\left[\mathrm{C}_{12} \mathrm{H}_{20} \mathrm{O}_{2}\right] 196.1464$

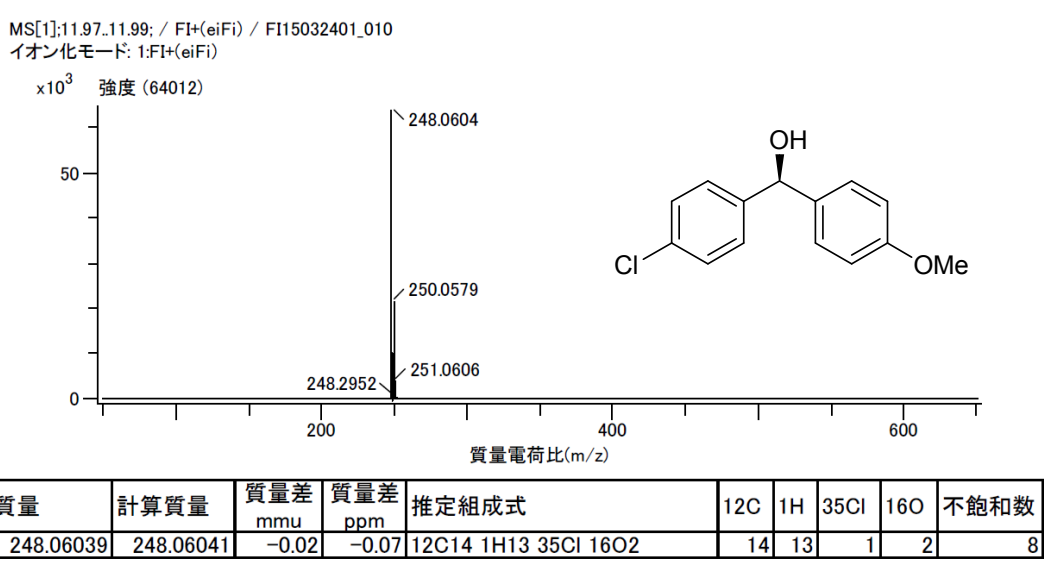

[Condition]

Column: Rxi- $5 \mathrm{~ms} 30 \mathrm{~m} \times 0.25 \mathrm{~mm}$ i.d., $0.25 \mu \mathrm{m}$

Instrument: GC:Agilent7890, MS:JMS-T100GCV

Oven: 60(1min) ${ }^{\circ} \mathrm{C}-300^{\circ} \mathrm{C} @ 20^{\circ} \mathrm{C} / \mathrm{min}$

Ionization: $\mathrm{FI}$

MS Range: $m / z$ 50-650

Inj. Vol.: Sample $0.5 \mu \mathrm{L}+$ STD 
(S)-4-((4-Chlorophenyl)(hydroxy)methyl)phenol (11j) (FI)

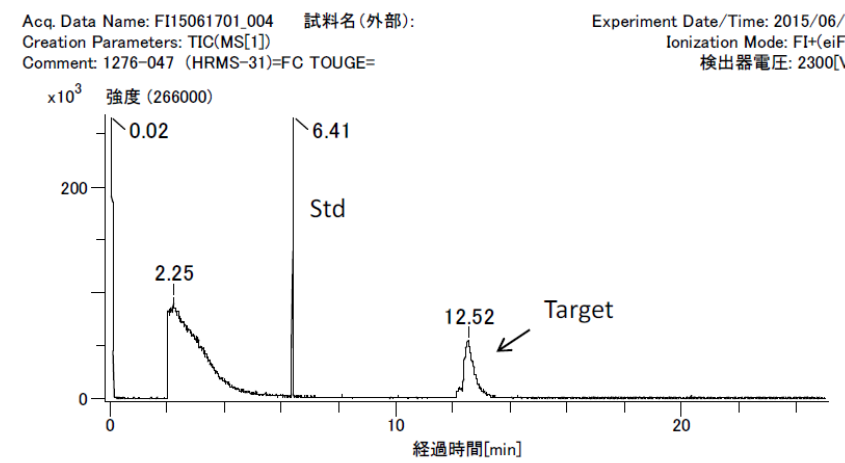

Standard for calibration: Linalyl acetate $\left[\mathrm{C}_{12} \mathrm{H}_{20} \mathrm{O}_{2}\right] 196.1464$

MS[1]:12.39.12.73; / FI+(eiFi) / FI15061701_004

$$
\times 10^{3} \text { 面積 }(14465)
$$
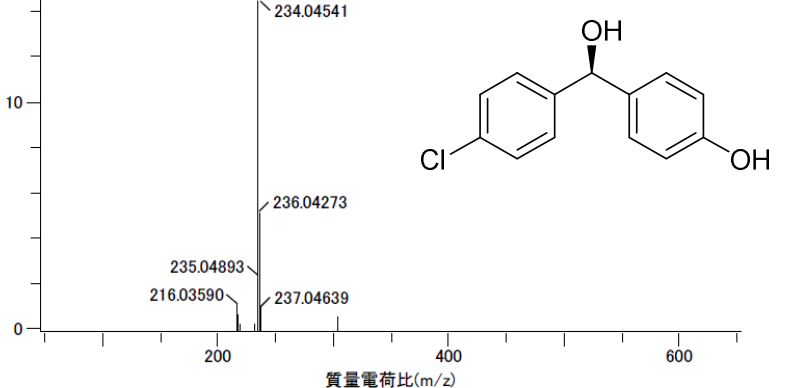

筫量電荷比 $(\mathrm{m} / \mathrm{z})$

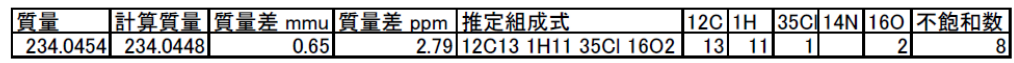

[Condition]

Column: Rxi- $5 \mathrm{~ms} 30 \mathrm{~m} \times 0.25 \mathrm{~mm}$ i.d., $0.25 \mu \mathrm{m}$

Instrument: GC:Agilent7890, MS:JMS-T100GCV

Oven: $60(1 \mathrm{~min}){ }^{\circ} \mathrm{C}-300^{\circ} \mathrm{C} @ 20^{\circ} \mathrm{C} / \mathrm{min}$

Ionization: $\mathrm{Fl}$

MS Range: $m / z$ 50-650

Inj. Vol.: Sample $0.5 \mu \mathrm{L}+$ STD

\section{(R)-(4-Methoxyphenyl)(4-nitrophenyl)methanol (11k)}

(FI)
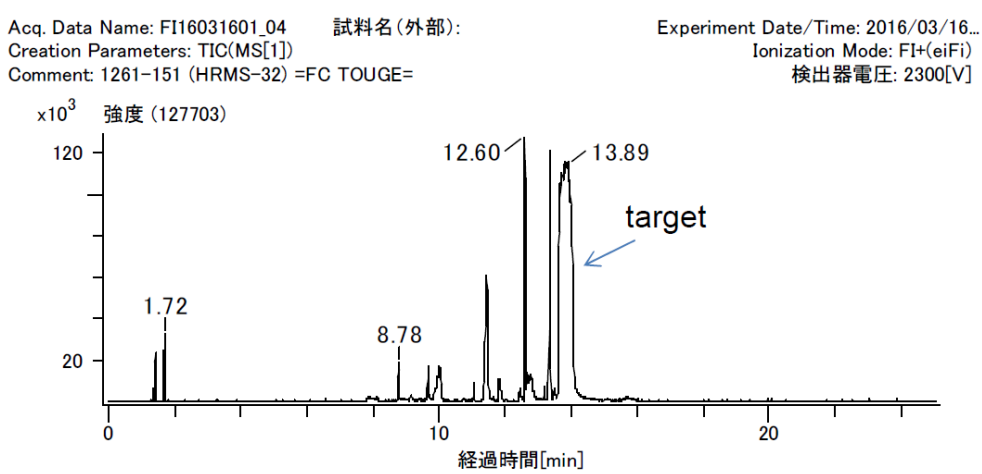

Standard for calibration: AT STD MIX

MS

MS[1];13.66..14.02; / FI+(eiFi) / FI16031601_04

イオン化モード: 1:FI+(eiFi)

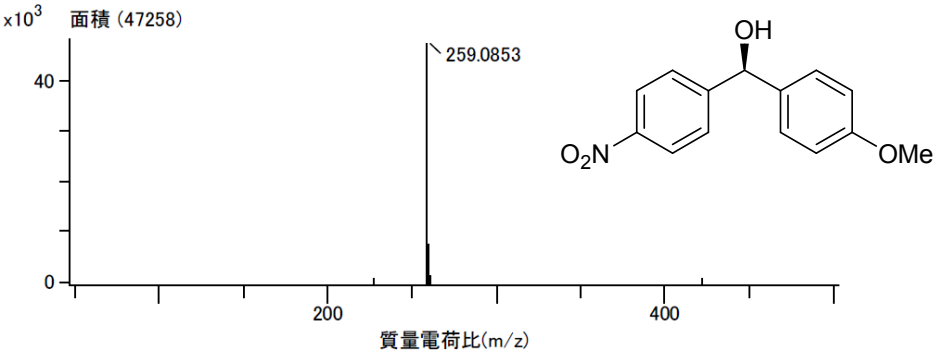

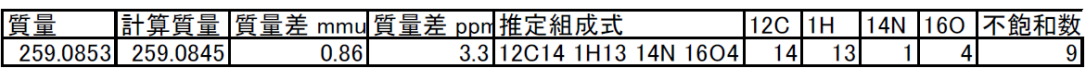


(S)-(3,5-Dinitrophenyl)(4-methoxyphenyl)methanol (11I) (ESI)
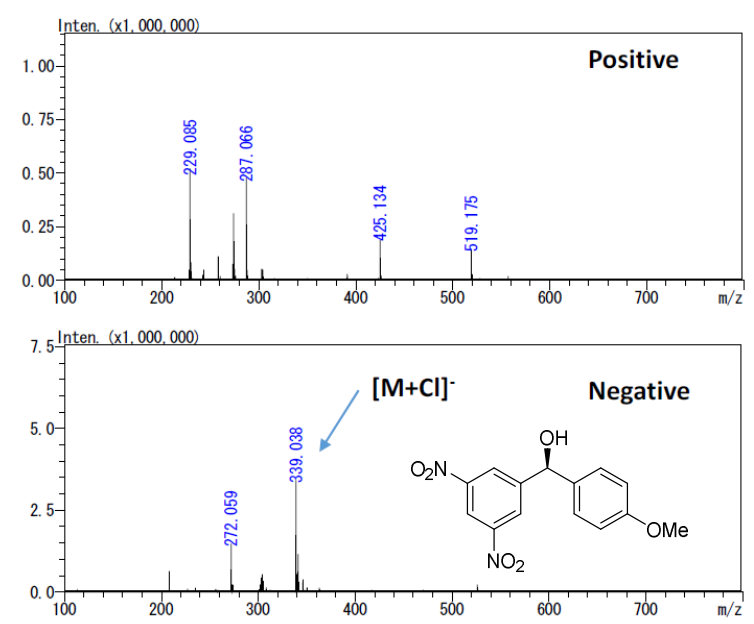

$[\mathrm{M}+\mathrm{Cl}]$

\begin{tabular}{|c|c|c|c|c|c|c|c|c|}
\hline Score & Formula (M) & Ion & Meas. $\mathrm{m} / 2$ & Pred. $\mathrm{m} / \mathrm{z}$ & Diff (mDa) & Diff (ppm) & DBE & Pred. (M) \\
\hline 60.2 & $\mathrm{C} 14 \mathrm{H} 12 \mathrm{~N} 2 \mathrm{O6}$ & {$[\mathrm{M}+\mathrm{Cl}]-$} & 339.0382 & 339.0389 & -0.7 & -2.06 & 10 & 304.0695 \\
\hline
\end{tabular}

Formula Prediction

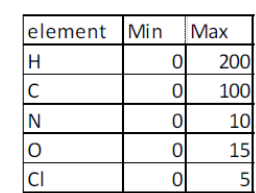

\section{Analytical conditions}

Instrument : LCMS-IT-TOF

Inization ESI Infusion (positive \& negative)

Flow rate : $0.2 \mathrm{~mL} / \mathrm{min}$

Eluent $\quad A$ : water $B: A C N$

data file : $\quad 15061702015$

\section{(S)-Phenyl(ferrocenyl)methanol (11 m)}

(ESI)
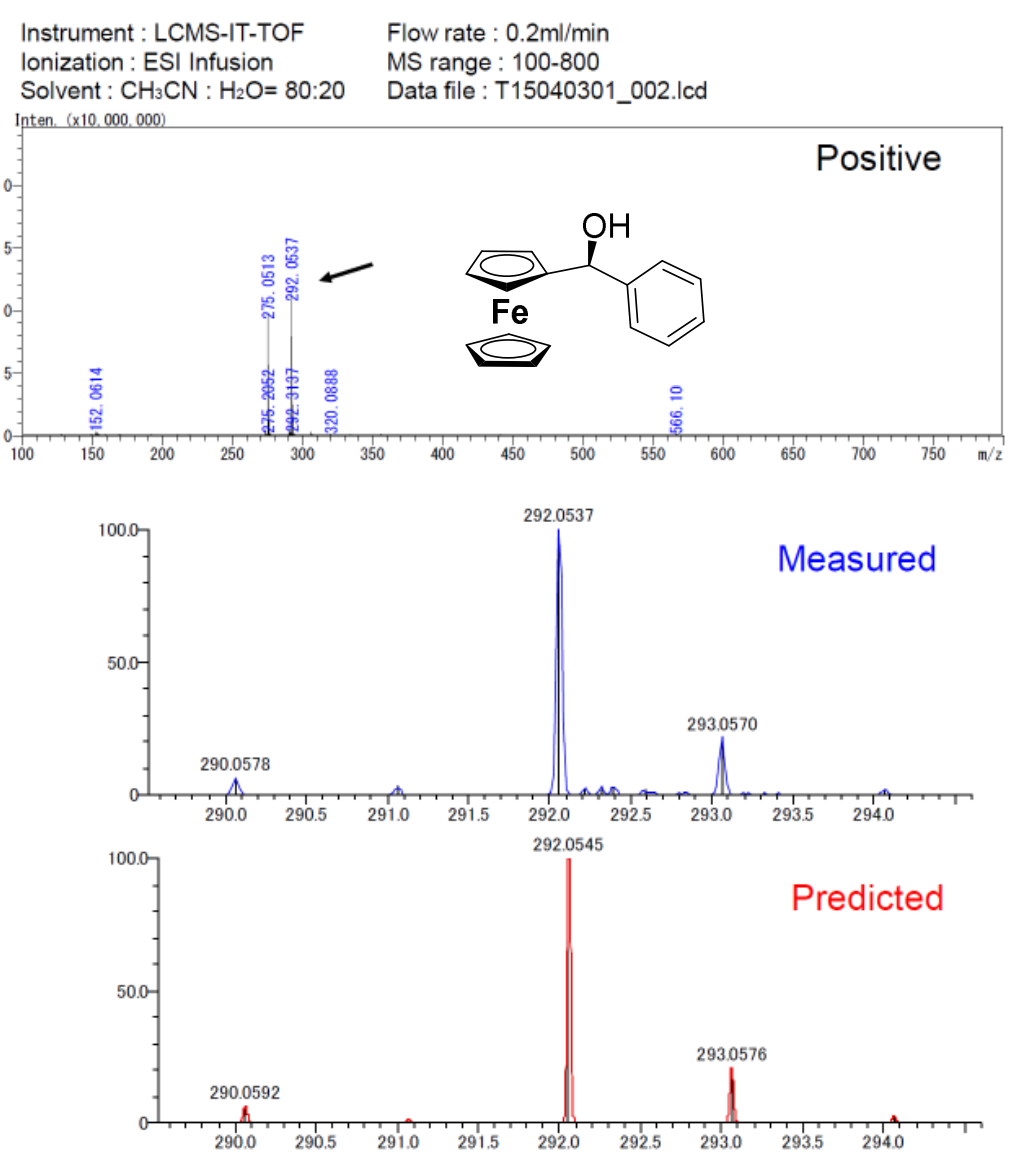

Formula (M) \begin{tabular}{|l|l|l|l|l|l|l|}
\hline Ion & Meas. $\mathrm{m} / \mathrm{z}$ & Pred. $\mathrm{m} / \mathrm{z}$ & Diff $(\mathrm{mDa})$ & Diff $(\mathrm{ppm})$ & DBE & Pred. (M) \\
\hline
\end{tabular} 


\section{(R)-(3-Nitrophenyl)(thiophen-2-yl)methanol (11n)}

(FI)

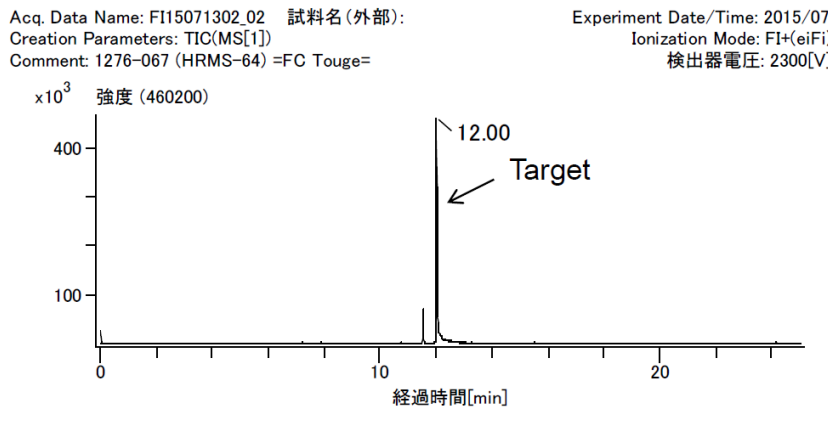

Standard for calibration: Linalyl acetate $\left[\mathrm{C}_{12} \mathrm{H}_{20} \mathrm{O}_{2}\right] 196.1464$ MS[1]:24.63.24.69; / FI+(eiFi) / FI15071302_.01

$$
\text { ×10 }
$$

$\times 10^{3}$ 面積 (124561)

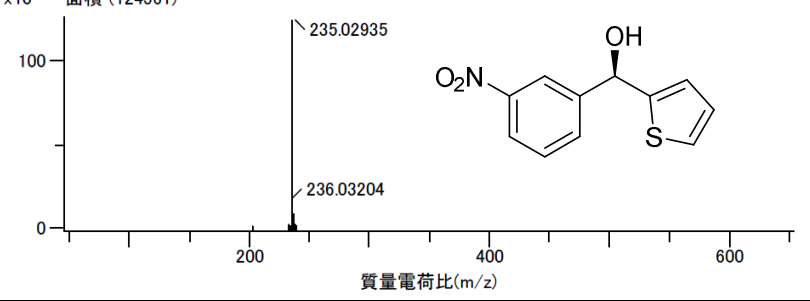

質量電荷比 $(\mathrm{m} / \mathrm{z})$

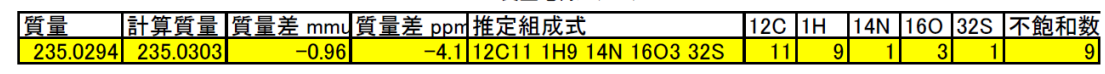

[Condition]

Column: Rxi-5ms $30 \mathrm{~m} \times 0.25 \mathrm{~mm}$ i.d., $0.25 \mu \mathrm{m}$

Instrument: GC:Agilent7890, MS:JMS-T100GCV

Oven: $60(1 \mathrm{~min}){ }^{\circ} \mathrm{C}-280^{\circ} \mathrm{C} @ 8^{\circ} \mathrm{C} / \mathrm{min}$

Ionization: $\mathrm{F}$

MS Range: $m / z$ 50-650

Inj. Vol.: Sample $2 \mu \mathrm{L}+$ STD
(S)-(2-Chloro-5-nitrophenyl)(phenyl)methyl 4-nitrobenzoate (13i)

\section{(APCI)}
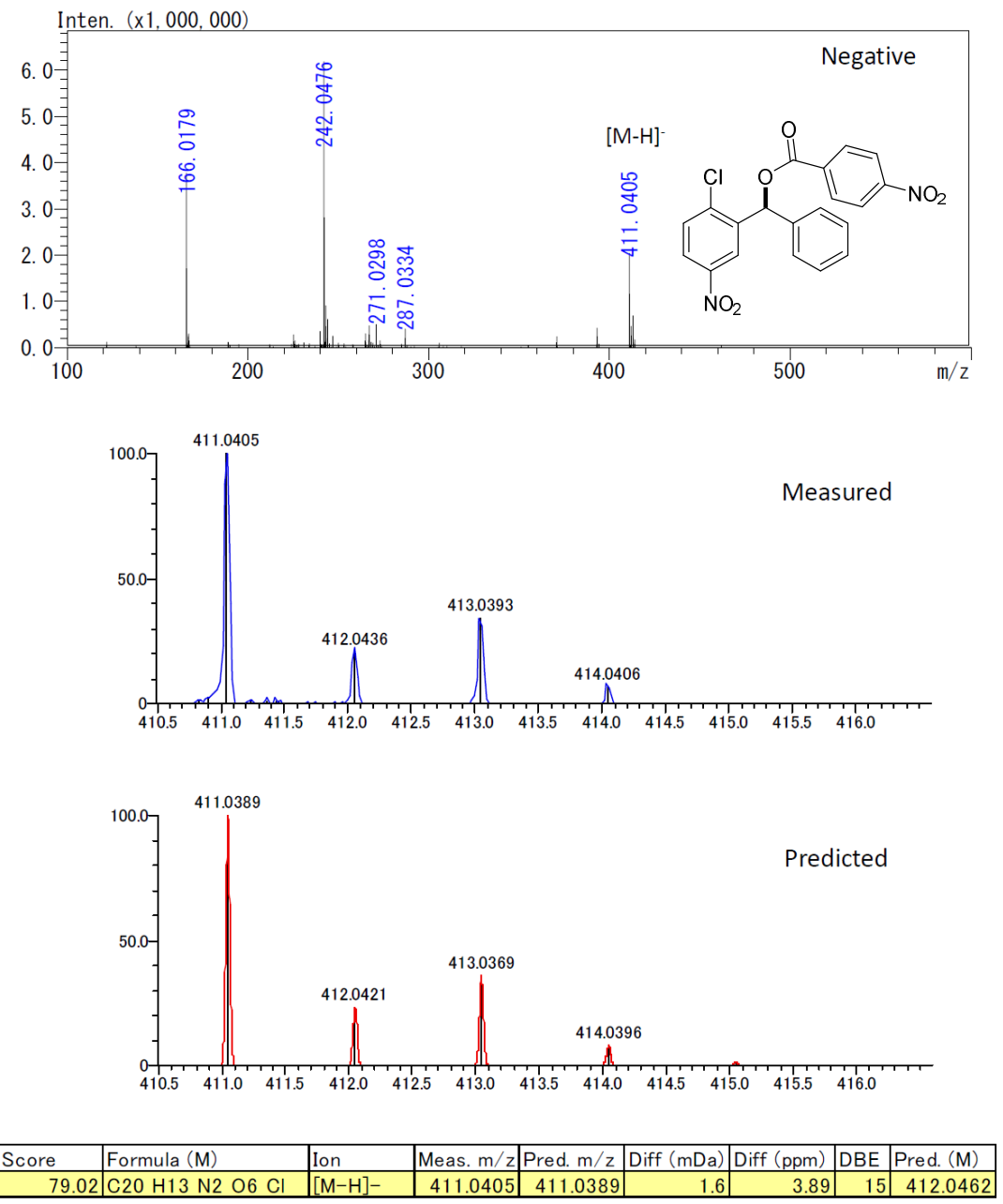

Elements for formula prediction <H: 0-200, C: 0-100, 0: 0-10, N:0-10, F:0-5, Cl:0-1 > Data file 15121701 005 
(S)-(3,4-Dichlorophenyl)(phenyl)methyl 4-nitrobenzoate (14b)

(APCI)
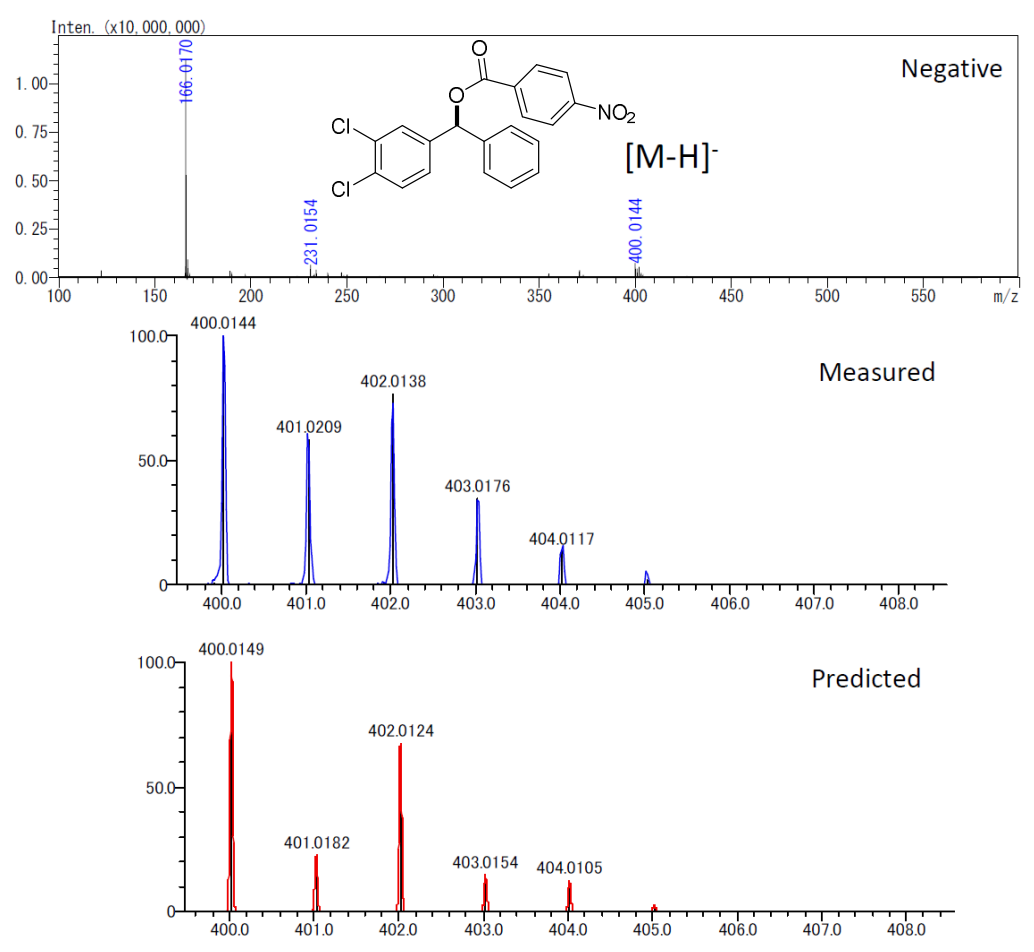

Score $\quad$ Formula $(\mathrm{M}$

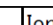

Meas. $\mathrm{m} / \mathrm{z}$ Pred. $\mathrm{m} / \mathrm{z}$ Diff (mDa) Diff (ppm) DBE

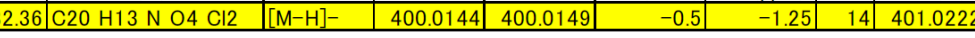

Elements for formula prediction < H: 0-200, C: 0-100, 0: 0-10, N:0-10, Cl:0-5>
(S)-(4-Chloro-3-nitrophenyl)(phenyl)methyl 4-nitrobenzoate (14e)

(APCI)

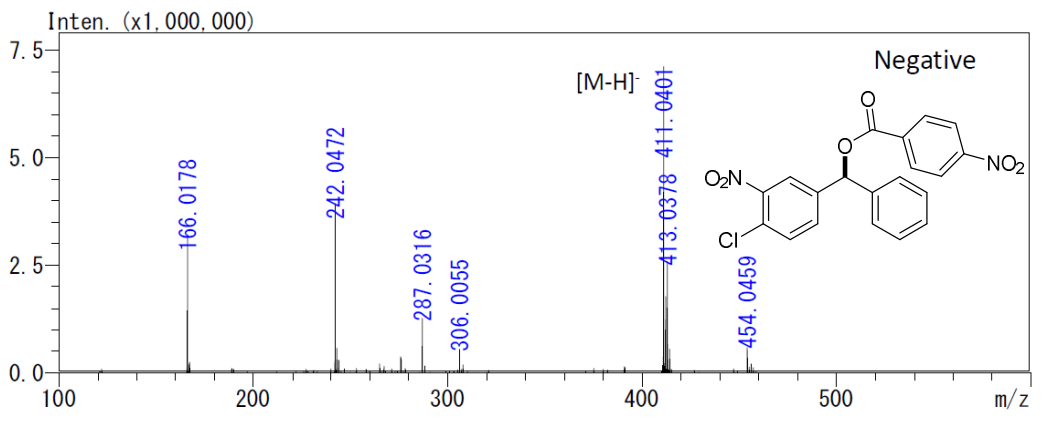

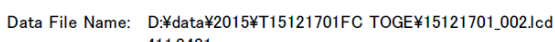
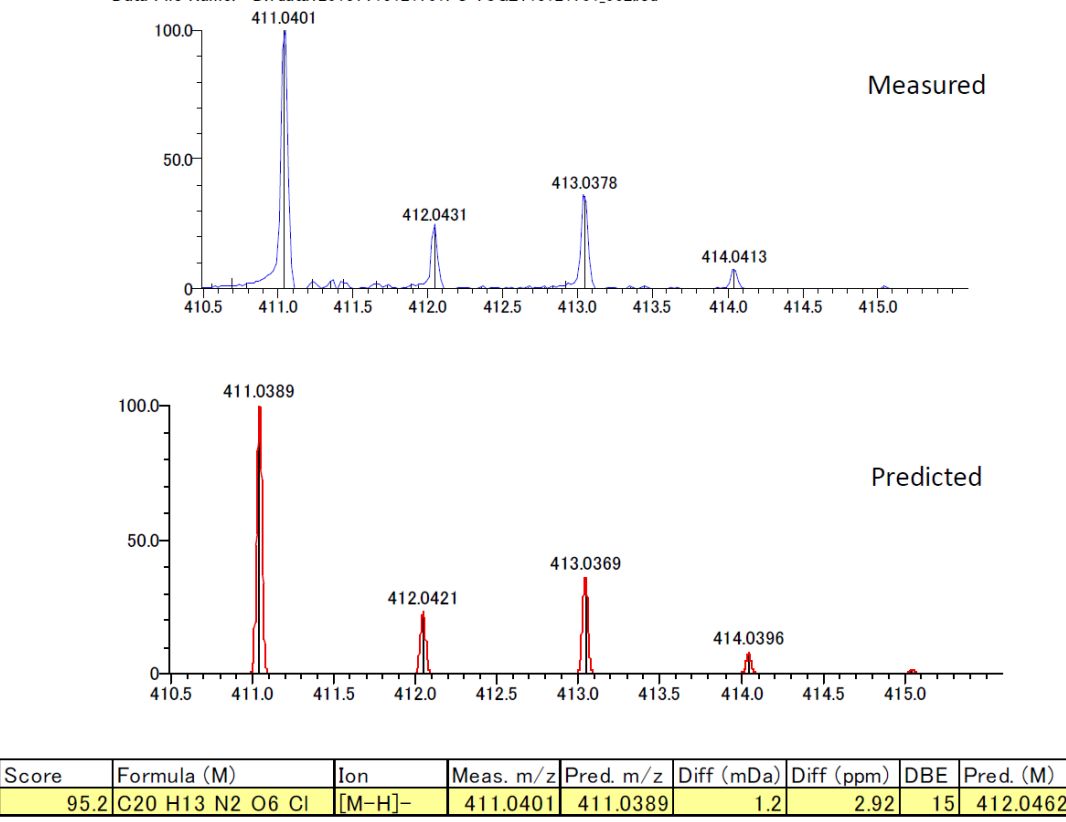

Elements for formula prediction < H: 0-200, C: 0-100, O: 0-10, N:0-10, F:0-5, Cl:0-1> 
(S)-Phenyl(2,4,5-trimethylphenyl)methyl

4,5-dichloro-2-((3aR,6S)-8,8-dimethyl-2,2-dioxidohexahydro-3H-

3a,6-methanobenzo[c]isothiazole-1-carbonyl)benzoate (13g) (ESI)
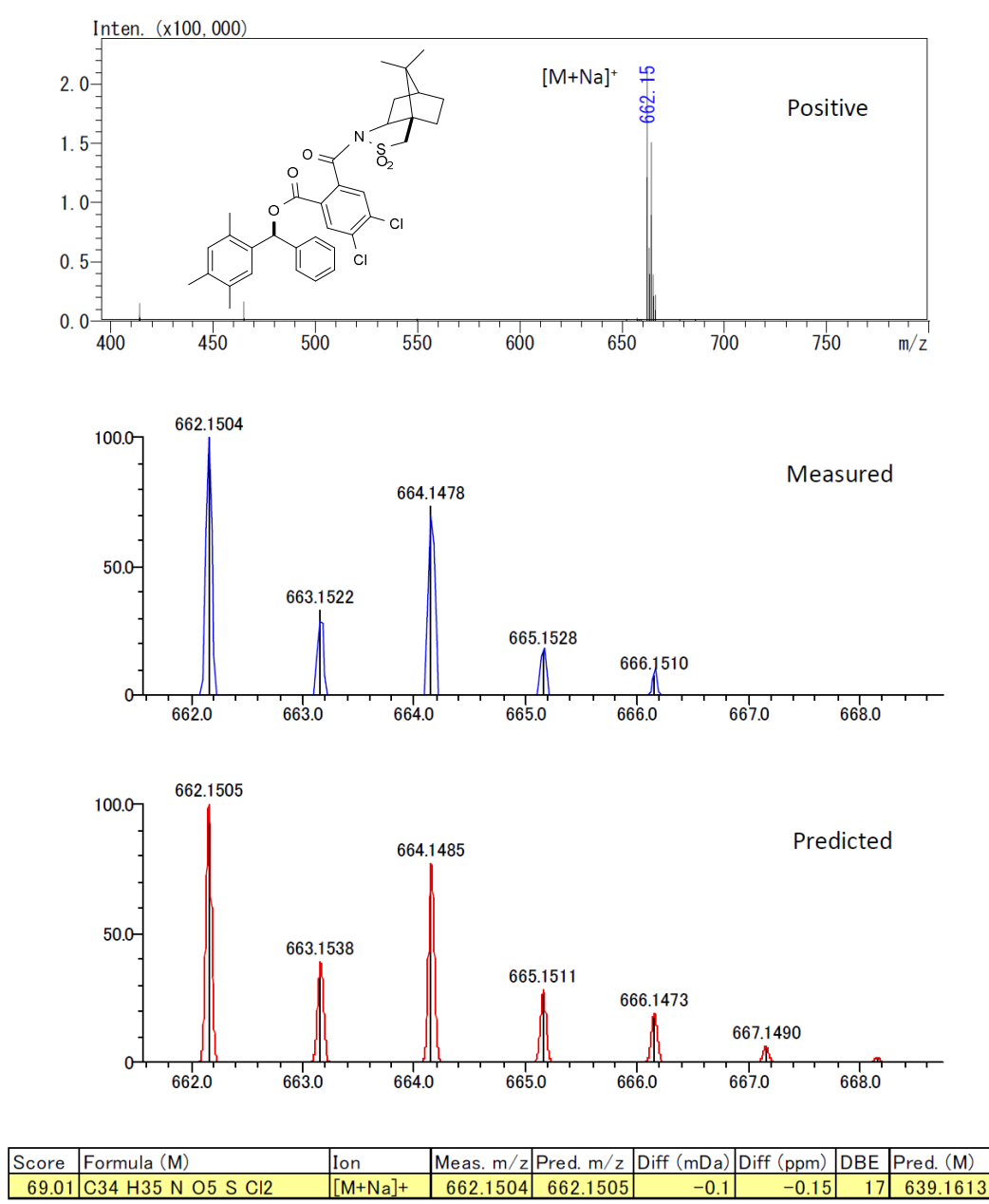

Elements for formula prediction < $\mathrm{H}: 0-200, \mathrm{C}: 30-500,0: 0-5, \mathrm{~N}: 0-5, \mathrm{~F}: 0-5, \mathrm{~S}: 0-3, \mathrm{Cl}: 0-3>$
(S)-(2,5-Difluorophenyl)(phenyl)methyl

4,5-dichloro-2-((3aS,6R)-8,8-dimethyl-2,2-dioxidohexahydro-3H-

3a,6-methanobenzo[c]isothiazole-1-carbonyl)benzoate (13j)

(ESI)
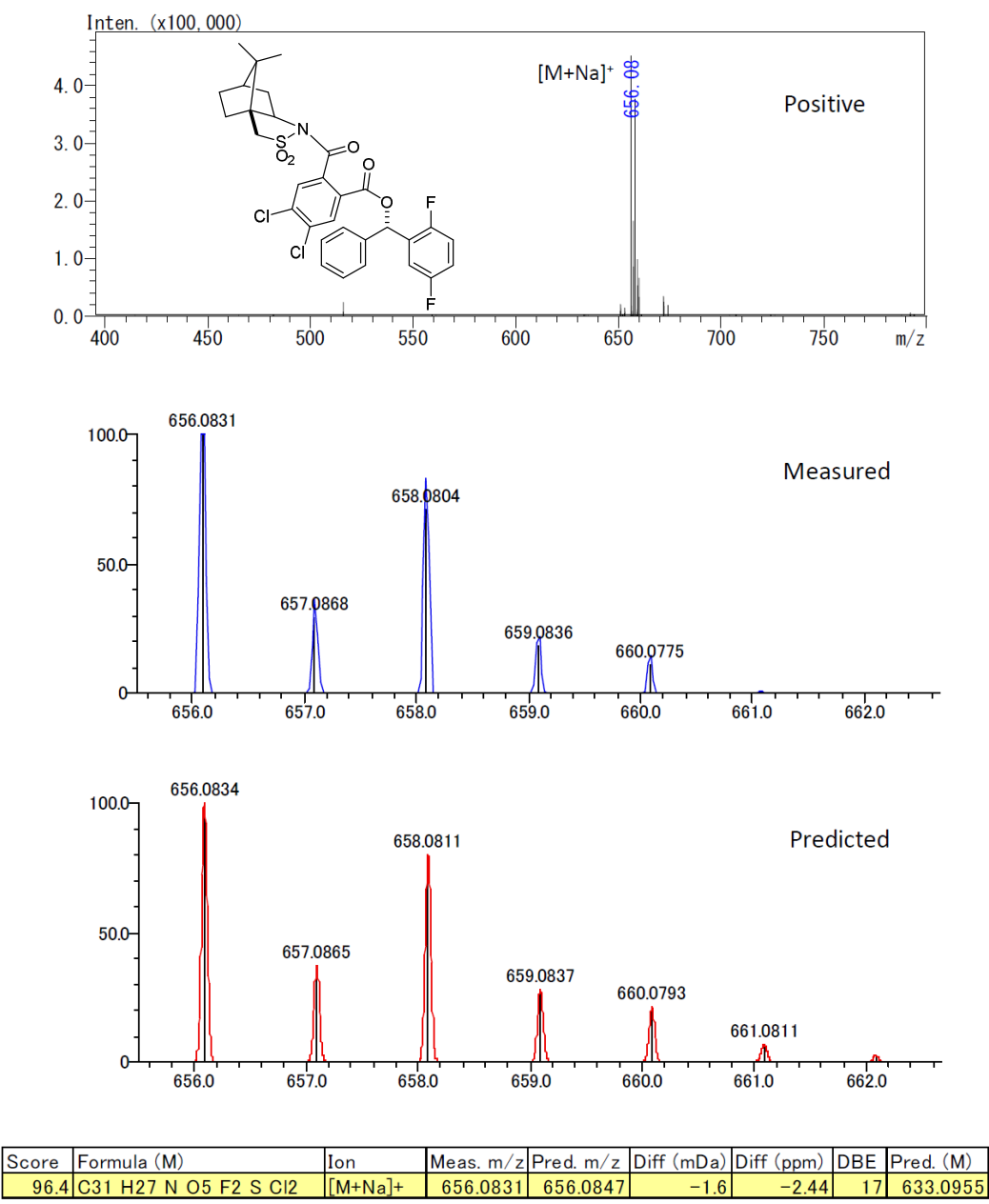

Elements for formula prediction < H: 0-200, C: 30-500, 0: 0-5, N: 0-5, F: 0-5, S: 0-3, Cl: 0-3 > 
(S)-(2-Fluoro-3-(trifluoromethyl)phenyl)(phenyl)methyl 4,5-dichloro-2-((3aS,6R)-8,8-dimethyl-2,2-dioxidohexahydro-3H$3 a, 6$-methanobenzo $[c]$ isothiazole-1-carbonyl)benzoate $(13 \mathrm{k})$ (ESI)
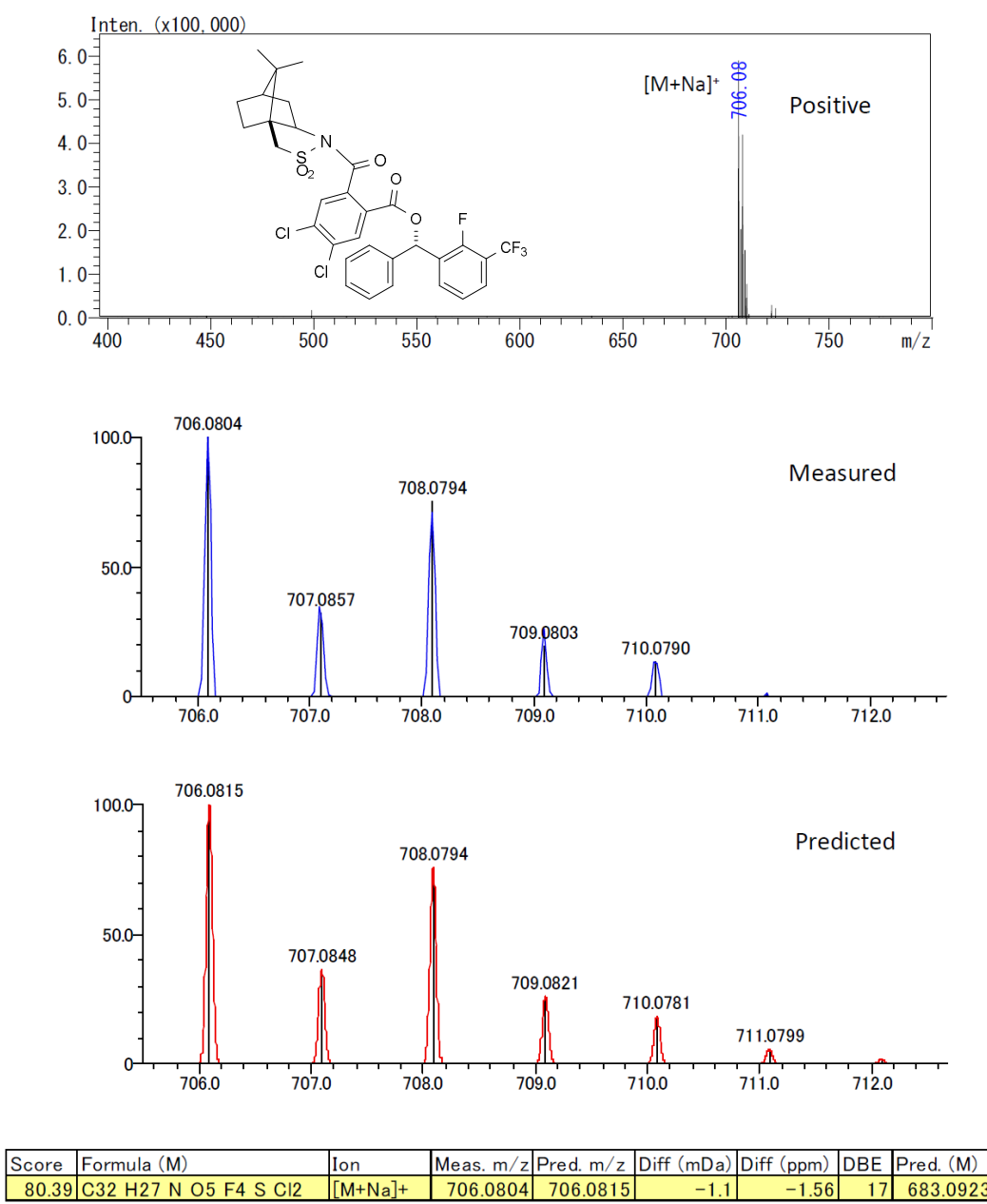

Elements for formula prediction $<\mathrm{H}: 0-200, \mathrm{C}: 30-500,0: 0-5, \mathrm{~N}: 0-5, \mathrm{~F}: 0-5, \mathrm{~S}: 0-3, \mathrm{Cl}: 0-3>$
(S)-(3,4-Difluorophenyl)(phenyl)methyl

4,5-dichloro-2-((3aR,6S)-8,8-dimethyl-2,2-dioxidohexahydro-3H3a,6-methanobenzo[c]isothiazole-1-carbonyl)benzoate (14c)

(ESI)
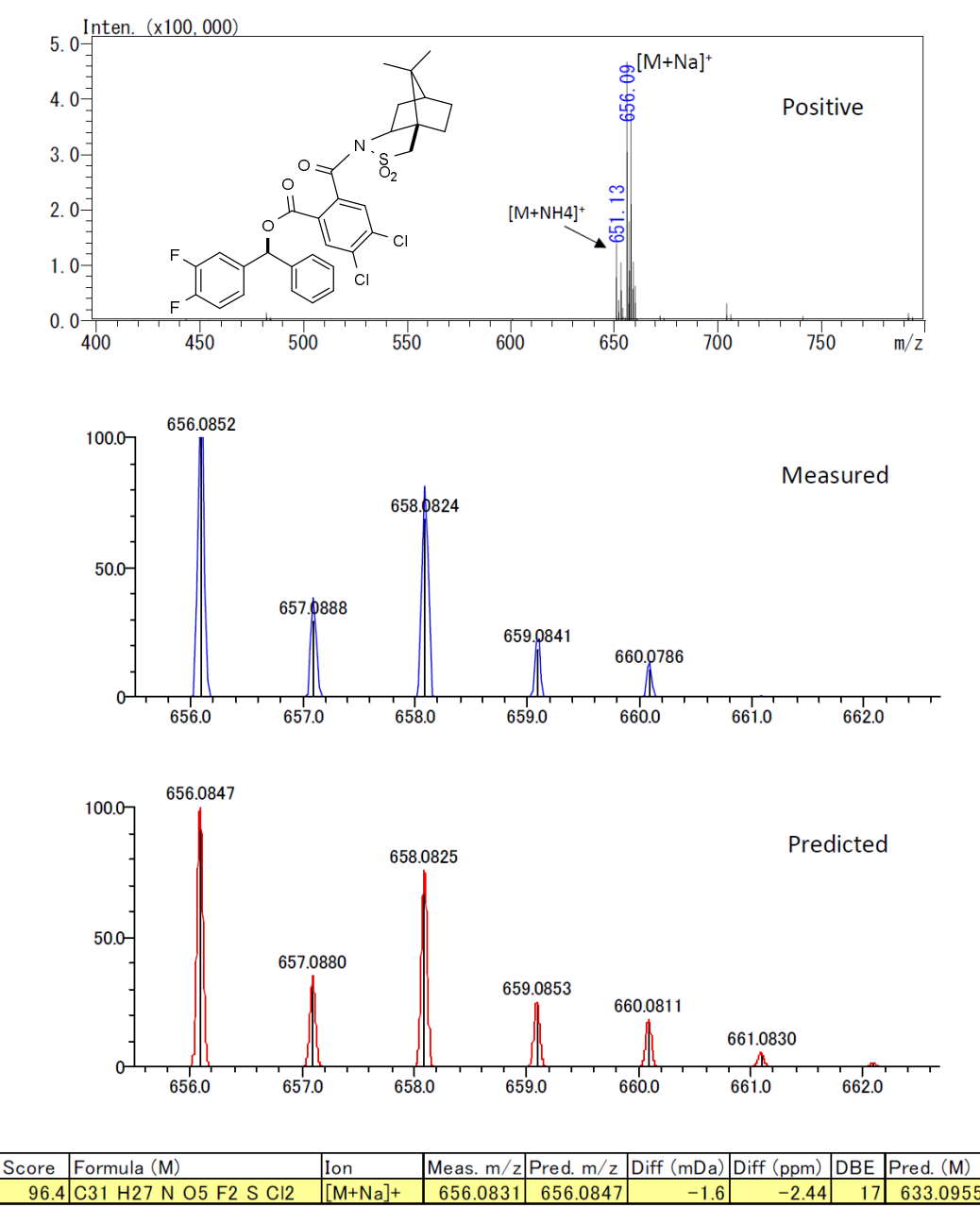

Elements for formula prediction < H: 0-200, C: 30-500, O: 0-5, N: 0-5, F: 0-5, S: 0-3, Cl: 0-3> 
(S)-Phenyl(3,4,5-trifluorophenyl)methyl

4,5-dichloro-2-((3aS,6R)-8,8-dimethyl-2,2-dioxidohexahydro-3H-

3a,6-methanobenzo[c]isothiazole-1-carbonyl)benzoate (14f)

(ESI)
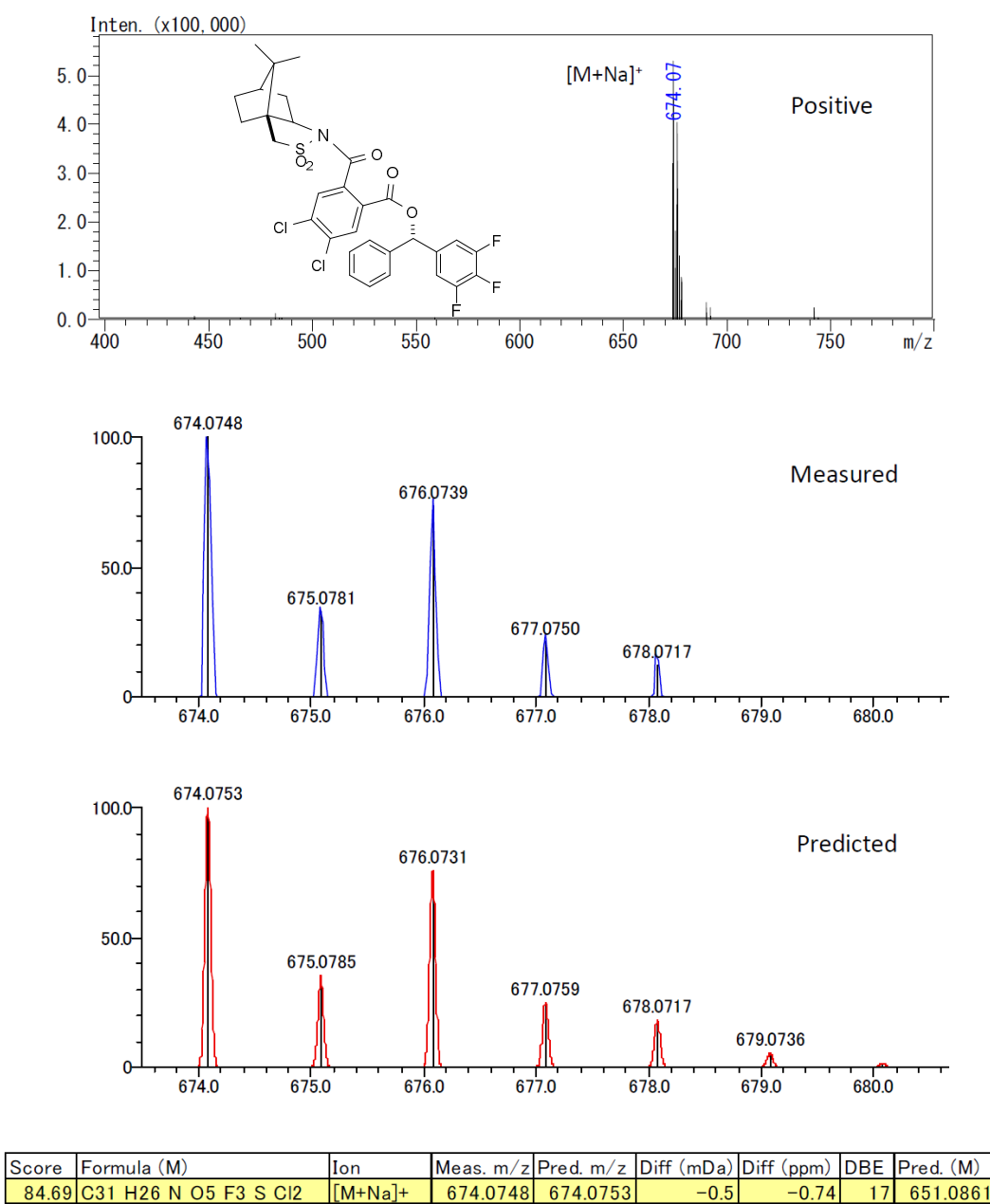

Elements for formula prediction $<\mathrm{H}: 0-200, \mathrm{C}: 30-500,0: 0-5, \mathrm{~N}: 0-5, \mathrm{~F}: 0-5, \mathrm{~S}: 0-3, \mathrm{Cl}: 0-3>$
(R)-(3-Nitrophenyl)(thiophen-2-yl)methyl

4,5-dichloro-2-((3aS,6R)-8,8-dimethyl-2,2-dioxidohexahydro-3H$3 a, 6$-methanobenzo[c]isothiazole-1-carbonyl)benzoate (14n)

$(\mathrm{ESI})$
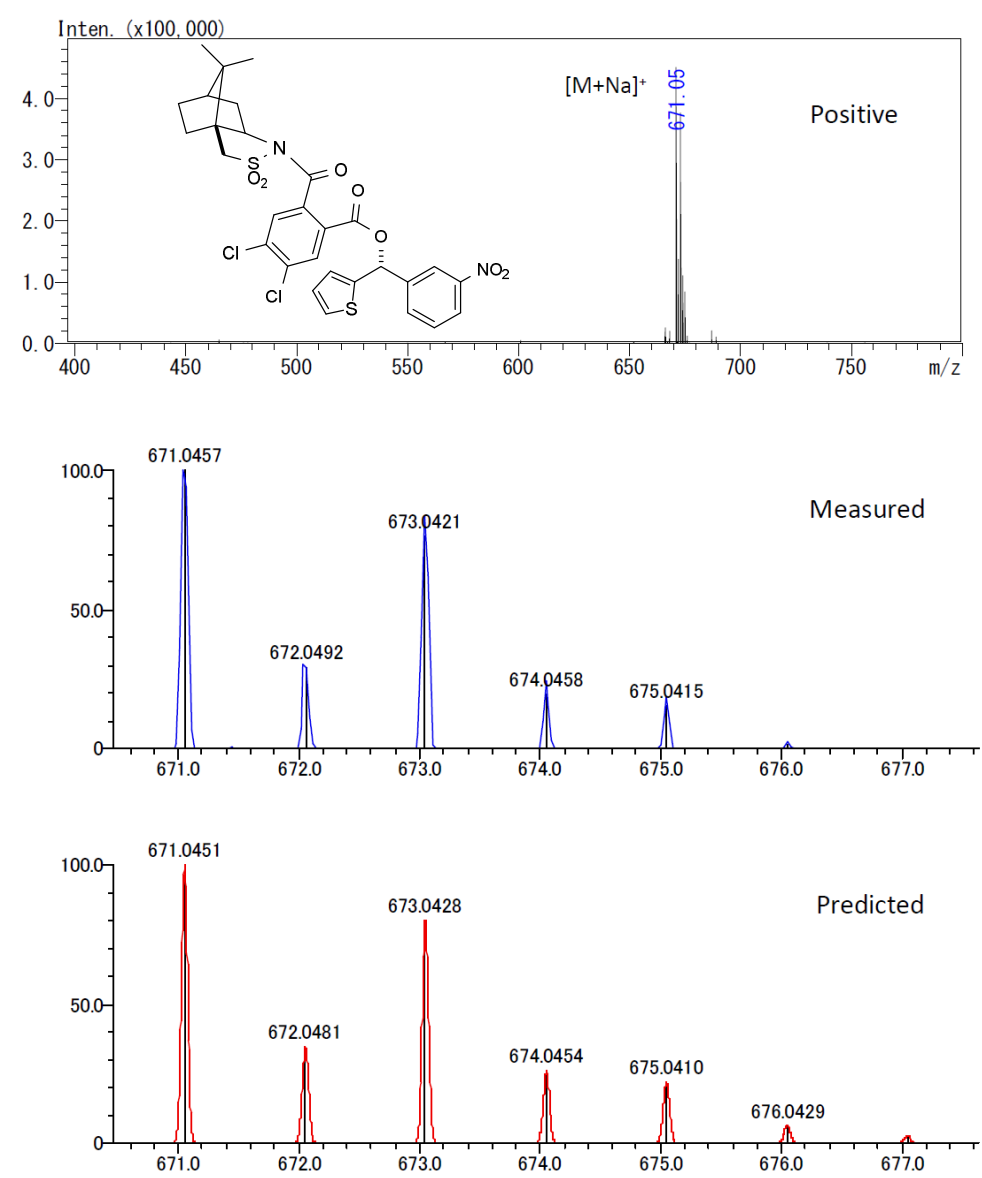

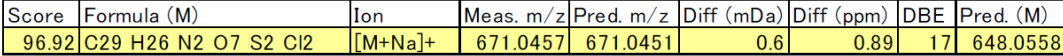

Elements for formula prediction < H: 0-200, C: 25-500, O: 0-10, N: 0-5, F: 0-5, S: 0-3, Cl: 0-3 > 


\section{Chiral HPLC Charts for Recduction Products}

Phenyl(o-tolyl)methanol (6a)

(OD-H, Hex/2-PrOH=97/3, F=1.0 mL/min, 220 nm, 30deg.C)

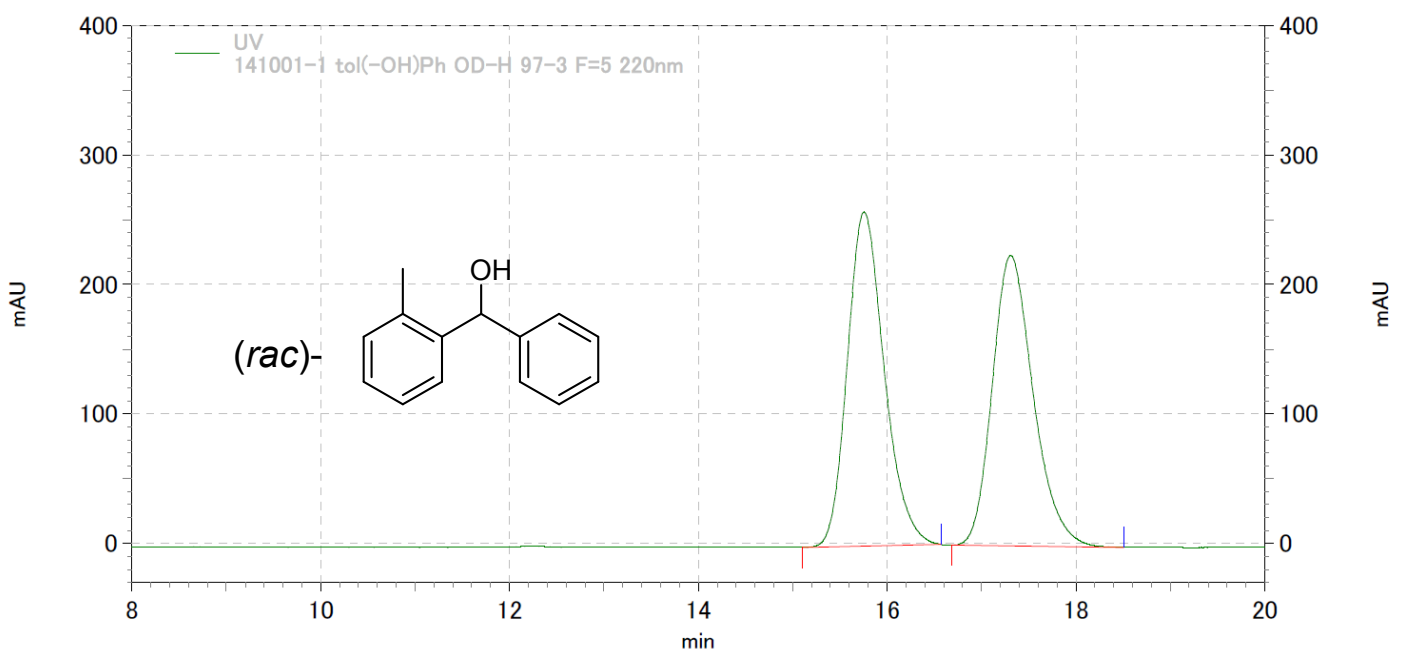

141001-1 tol (-OH) Ph OD-H 97-3 F=5 220nm

\begin{tabular}{|c|c|c|c|c|c|}
\hline $\begin{array}{l}\text { UVV結果 } \\
\text { Pk } \\
\text { No. }\end{array}$ & R. T. & Area & Area\% & B. C. & Symmetry \\
\hline $\begin{array}{l}1 \\
2\end{array}$ & $\begin{array}{l}15.757 \\
17.310\end{array}$ & $\begin{array}{l}28043333 \\
27079357\end{array}$ & $\begin{array}{l}50.874 \\
49.126\end{array}$ & $\begin{array}{l}\text { MM } \\
\text { MM }\end{array}$ & $\begin{array}{l}0.00000 \\
0.00000\end{array}$ \\
\hline$r-8$ & & 55122690 & 100.000 & & \\
\hline
\end{tabular}

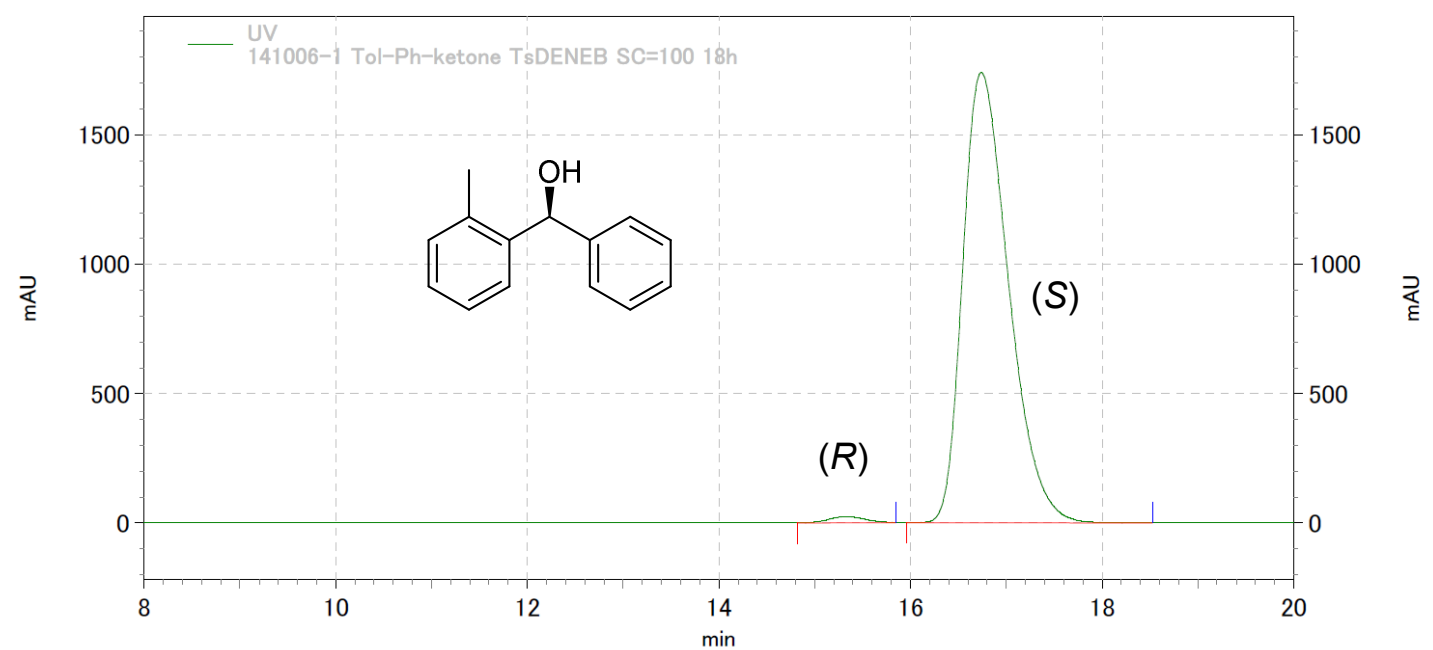

141006-1 Tol-Ph-ketone TsDENEB SC=100 18h

\begin{tabular}{|c|c|c|c|c|c|}
\hline $\begin{array}{l}\text { UV 結身 } \\
\text { Pk } \\
\text { No. }\end{array}$ & R. T. & Area & Area\% & B. C. & Symmetry \\
\hline $\begin{array}{l}1 \\
2\end{array}$ & $\begin{array}{l}15.330 \\
16.740\end{array}$ & $\begin{array}{r}2550291 \\
231459589\end{array}$ & $\begin{array}{r}1.090 \\
98.910\end{array}$ & $\begin{array}{l}\text { MM } \\
\text { MM }\end{array}$ & $\begin{array}{l}0.00000 \\
0.00000\end{array}$ \\
\hline $\begin{array}{r}1-8 \\
\|\end{array}$ & & 234009880 & 100.000 & & \\
\hline
\end{tabular}




\section{(2-Chlorophenyl)(phenyl)methanol (6b)}

(OD-H, Hex/2-PrOH=97/3, F=1.0 mL/min, 220 nm, 30deg.C)

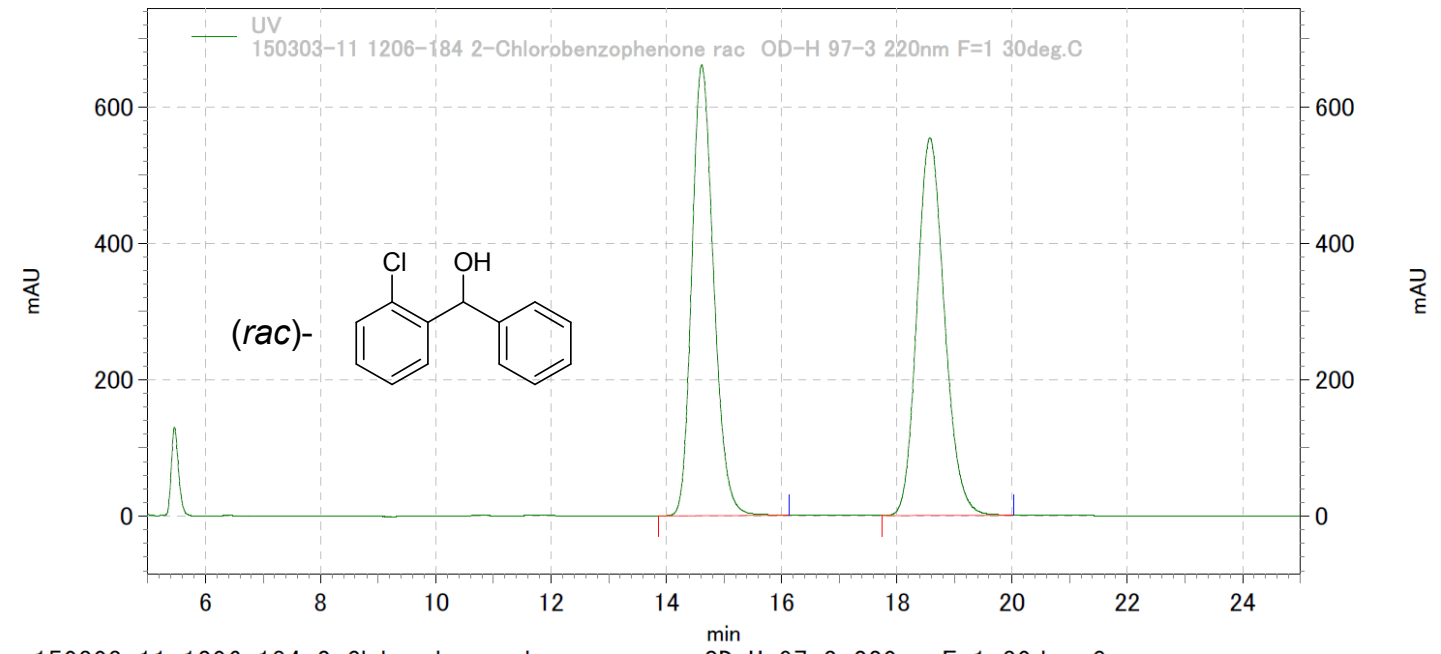

150303-11 1206-184 2-Chlorobenzophenone rac OD-H 97-3 220nm F=1 30deg. C

\begin{tabular}{rlllll}
$\begin{aligned} \text { UV } \\
\text { Pk } \\
\text { No. }\end{aligned}$ & R. T. & Area & Area\% & B. C. & Symmetry \\
\hline 1 & 14.613 & 69594118 & 48.740 & MM & 0.00000 \\
2 & 18.573 & 73193394 & 51.260 & MM & 0.00000
\end{tabular}

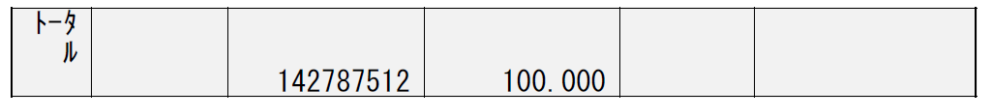

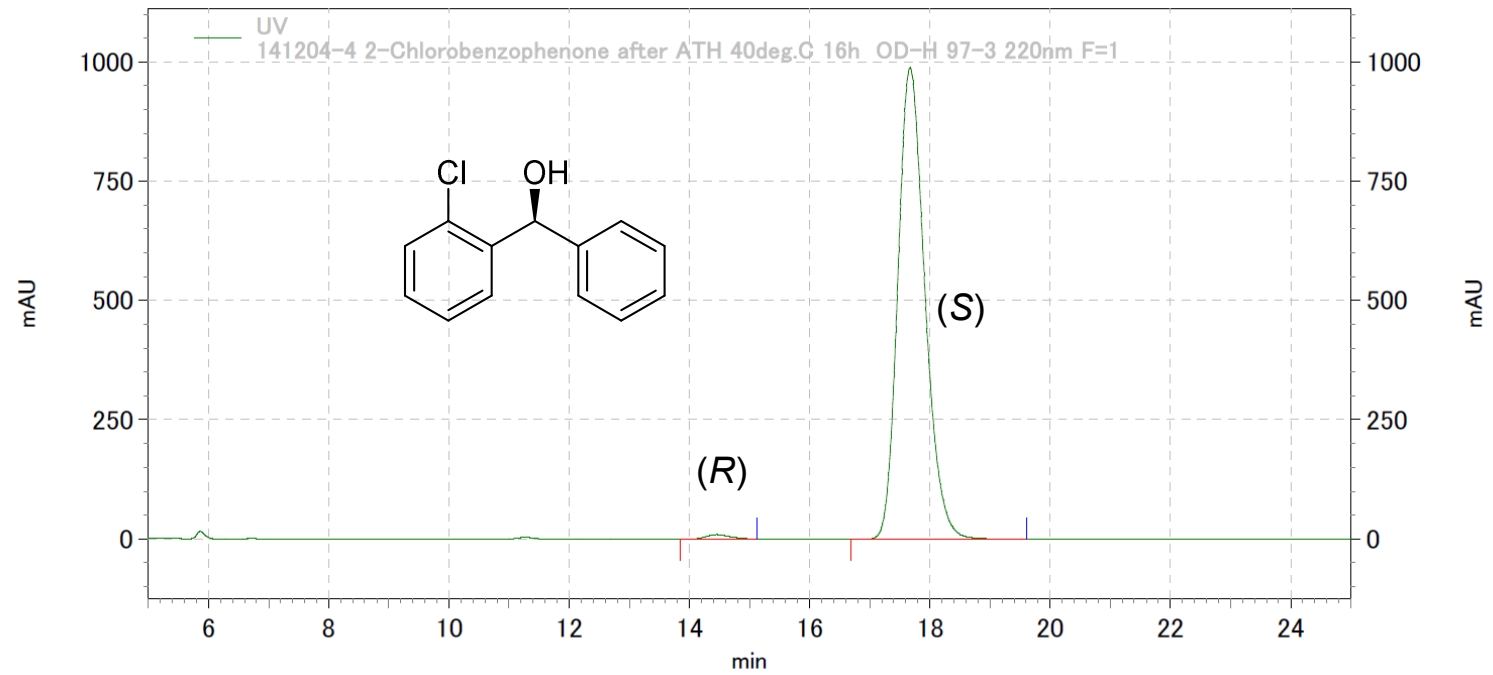

141204-4 2-Chlorobenzophenone after ATH 40deg. C 16h OD-H 97-3 220nm F=1

\begin{tabular}{|c|c|c|c|c|c|}
\hline $\begin{array}{l}\text { UVV結身 } \\
\text { Pk } \\
\text { No. }\end{array}$ & R. T. & Area & Area\% & B. C. & Symmetry \\
\hline $\begin{array}{l}1 \\
2\end{array}$ & $\begin{array}{l}14.457 \\
17.670\end{array}$ & $\begin{array}{r}1011814 \\
123305091\end{array}$ & $\begin{array}{r}0.814 \\
99.186\end{array}$ & $\begin{array}{l}M M \\
M M\end{array}$ & $\begin{array}{l}0.00000 \\
0.00000\end{array}$ \\
\hline $\begin{array}{r}r-夕 \\
\|\end{array}$ & & 124316905 & 100.000 & & \\
\hline
\end{tabular}




\section{(2-Bromophenyl)(phenyl)methanol (6c)}

(OD-H, Hex/2-PrOH=97/3, F=1.0 mL/min, $220 \mathrm{~nm}, 30$ deg.C)

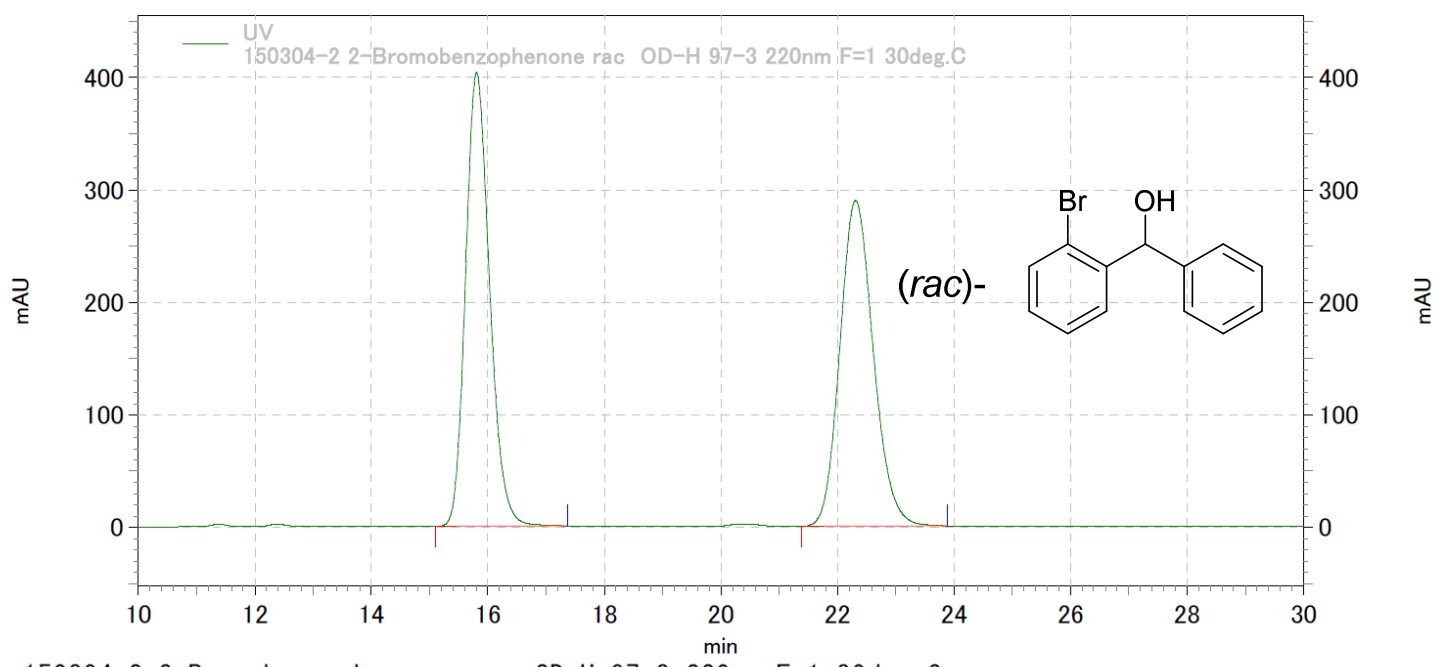

150304-2 2-Bromobenzophenone rac OD-H 97-3 220nm F=1 30deg. C

\begin{tabular}{|c|c|c|c|c|c|}
\hline $\begin{array}{l}\text { UV結身 } \\
\text { Pk } \\
\text { No. } \\
\end{array}$ & R. T. & Area & Area\% & B. C. & Symmetry \\
\hline $\begin{array}{l}1 \\
2\end{array}$ & $\begin{array}{l}15.807 \\
\text { 22. } 313\end{array}$ & $\begin{array}{l}46072109 \\
46055179\end{array}$ & $\begin{array}{l}50.009 \\
49.991\end{array}$ & $\begin{array}{l}\text { MM } \\
\text { MM }\end{array}$ & $\begin{array}{l}0.00000 \\
0.00000\end{array}$ \\
\hline $\begin{array}{r}r-8 \\
\mu\end{array}$ & & 92127288 & 100.000 & & \\
\hline
\end{tabular}

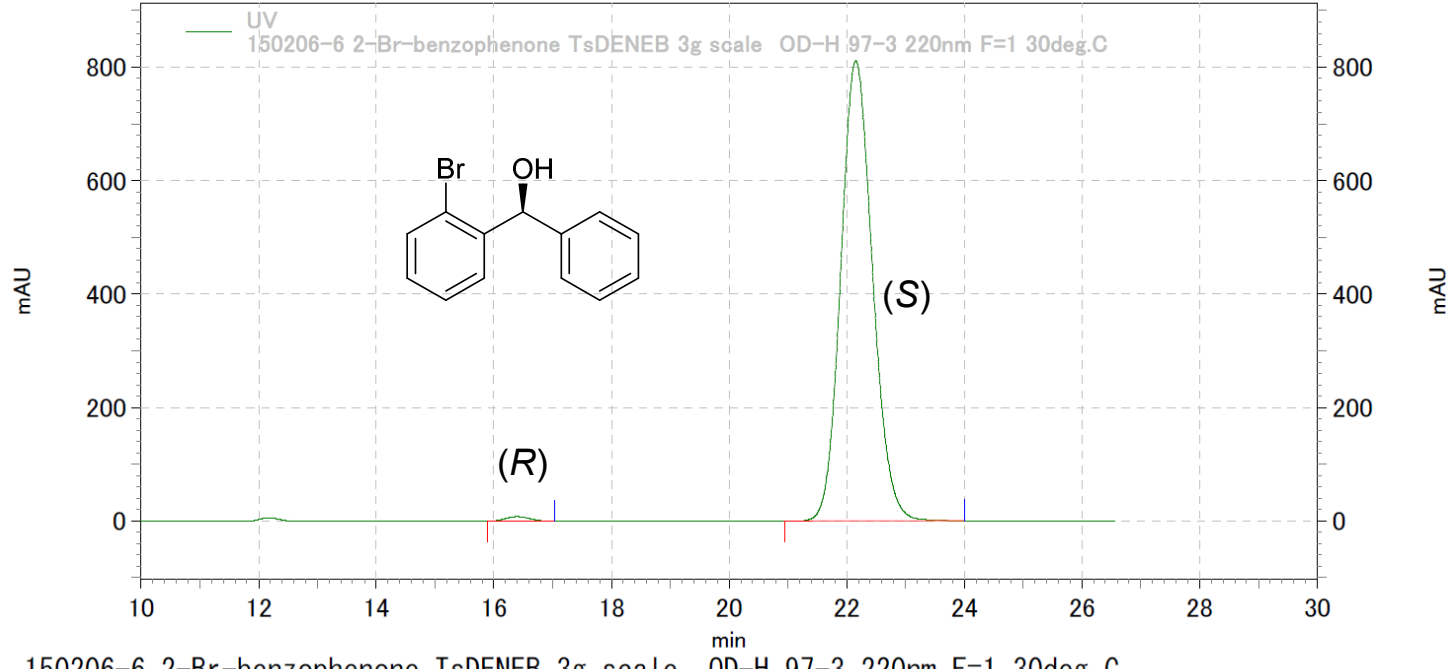

150206-6 2-Br-benzophenone TsDENEB 3g scale OD-H 97-3 220nm F=1 30deg. C

\begin{tabular}{|c|c|c|c|c|c|}
\hline $\begin{array}{l}\text { UV結身 } \\
\text { Pk } \\
\text { No. }\end{array}$ & R. T. & Area & Area\% & B. C. & Symmetry \\
\hline $\begin{array}{l}1 \\
2\end{array}$ & $\begin{array}{l}16.397 \\
22.153\end{array}$ & $\begin{array}{r}872753 \\
121643499\end{array}$ & $\begin{array}{r}0.712 \\
99.288\end{array}$ & $\begin{array}{l}\text { MM } \\
\text { MM }\end{array}$ & $\begin{array}{l}0.00000 \\
0.00000\end{array}$ \\
\hline $\begin{array}{r}r-夕 \\
ル\end{array}$ & & 122516252 & 100.000 & & \\
\hline
\end{tabular}




\section{Phenyl(2-(trifluoromethyl)phenyl)methanol (6d)}

(OD-H, Hex/2-PrOH=97/3, F=1.0 mL/min, 220 nm, 30deg.C)

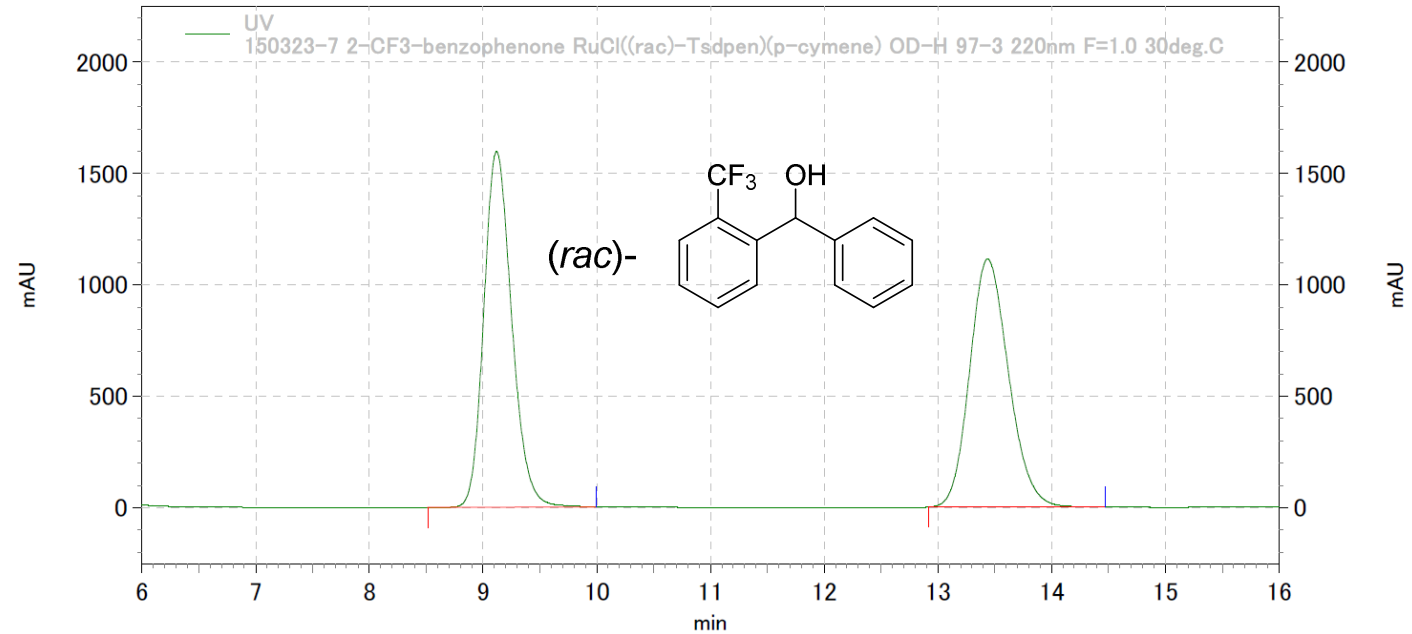

150323-7 2-CF3-benzophenone RuCl ( (rac)-Tsdpen) (p-cymene) OD-H 97-3 220nm F=1. 0 30deg. C

\begin{tabular}{|c|c|c|c|c|c|}
\hline $\begin{array}{l}\text { UV 結身 } \\
\text { Pk } \\
\text { No }\end{array}$ & R. T. & Area & Area\% & B. C. & Symmetry \\
\hline $\begin{array}{l}1 \\
2\end{array}$ & $\begin{array}{r}9.120 \\
13.437\end{array}$ & $\begin{array}{l}108878070 \\
105852591\end{array}$ & $\begin{array}{l}50.704 \\
49.296\end{array}$ & $\begin{array}{l}\mathrm{MM} \\
\mathrm{HH}\end{array}$ & $\begin{array}{l}0.00000 \\
0.00000\end{array}$ \\
\hline $\begin{array}{r}r-8 \\
\|\end{array}$ & & 214730661 & 100.000 & & \\
\hline
\end{tabular}

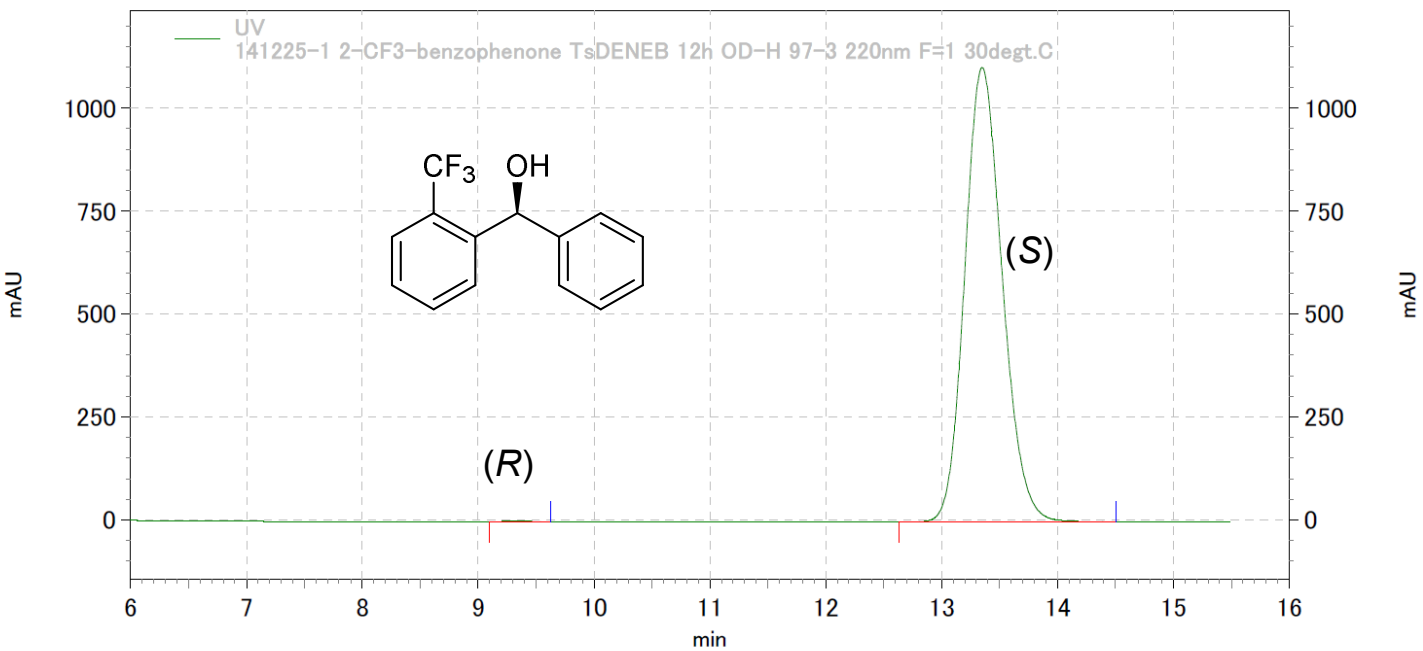

141225-1 2-CF3-benzophenone TsDENEB 12h OD-H 97-3 220nm F=1 30degt. C

\begin{tabular}{|c|c|c|c|c|c|}
\hline $\begin{array}{l}\text { UV 結果 } \\
\text { Pk } \\
\text { No. }\end{array}$ & R. T. & Area & Area\% & B. C. & Symmetry \\
\hline $\begin{array}{l}1 \\
2\end{array}$ & $\begin{array}{r}9.333 \\
13.350\end{array}$ & $\begin{array}{r}150603 \\
99286210\end{array}$ & $\begin{array}{r}0.151 \\
99.849\end{array}$ & $\begin{array}{l}\mathrm{MM} \\
\mathrm{MM}\end{array}$ & $\begin{array}{l}0.00000 \\
0.00000\end{array}$ \\
\hline $\begin{array}{ll}r-8 \\
\pi\end{array}$ & & 99436813 & 100.000 & & \\
\hline
\end{tabular}




\section{2-(Hydroxy(phenyl)methyl)phenol (6e)}

(OD-H, Hex/2-PrOH=80/20, F=1.0 mL/min, 220 nm, 30deg.C)

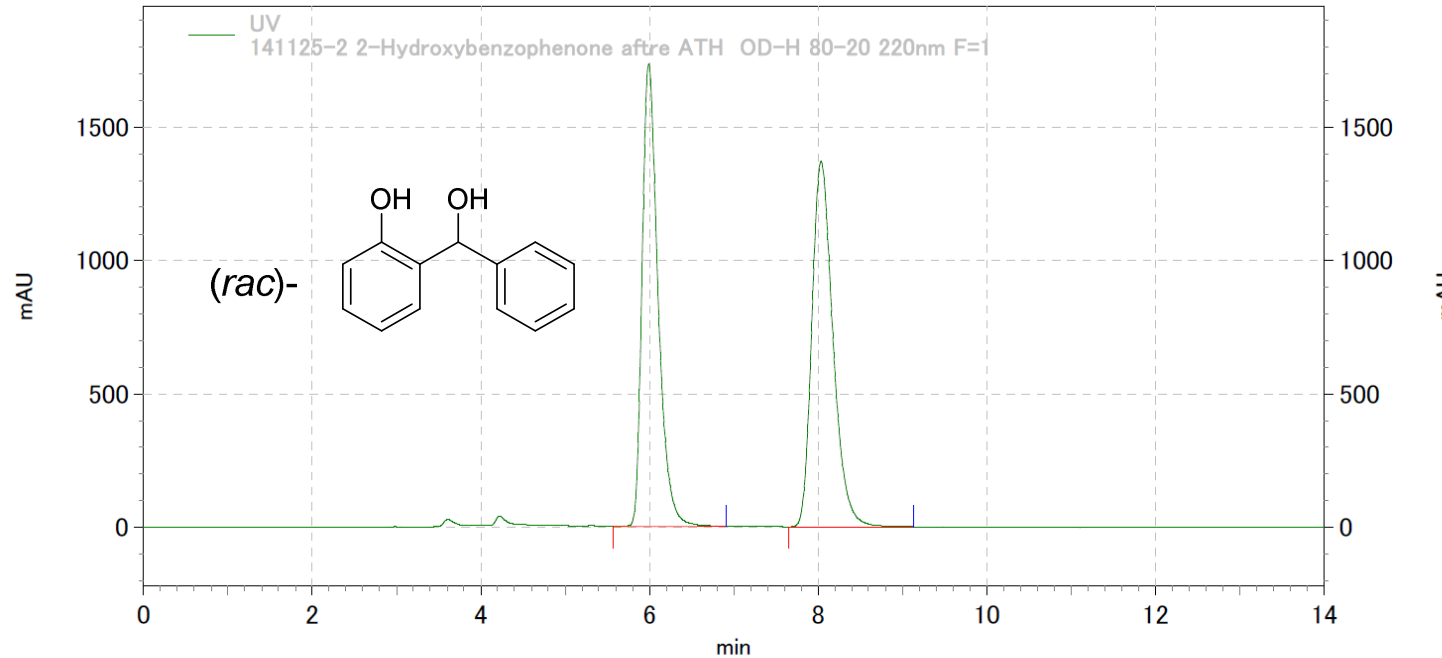

141125-2 2-Hydroxybenzophenone aftre ATH OD-H 80-20 220nm F=1

\begin{tabular}{|c|c|c|c|c|c|}
\hline $\begin{array}{l}\text { UV結果 } \\
\text { Pk } \\
\text { No. }\end{array}$ & R. T. & Area & Area\% & B. C. & Symmetry \\
\hline $\begin{array}{l}1 \\
2\end{array}$ & $\begin{array}{l}5.990 \\
8.037\end{array}$ & $\begin{array}{l}91465330 \\
95329171\end{array}$ & $\begin{array}{l}48.966 \\
51.034\end{array}$ & $\begin{array}{l}\text { MM } \\
\text { MM }\end{array}$ & $\begin{array}{l}0.00000 \\
0.00000\end{array}$ \\
\hline $\begin{array}{r}r-夕 \\
\mu\end{array}$ & & 186794501 & 100.000 & & \\
\hline
\end{tabular}

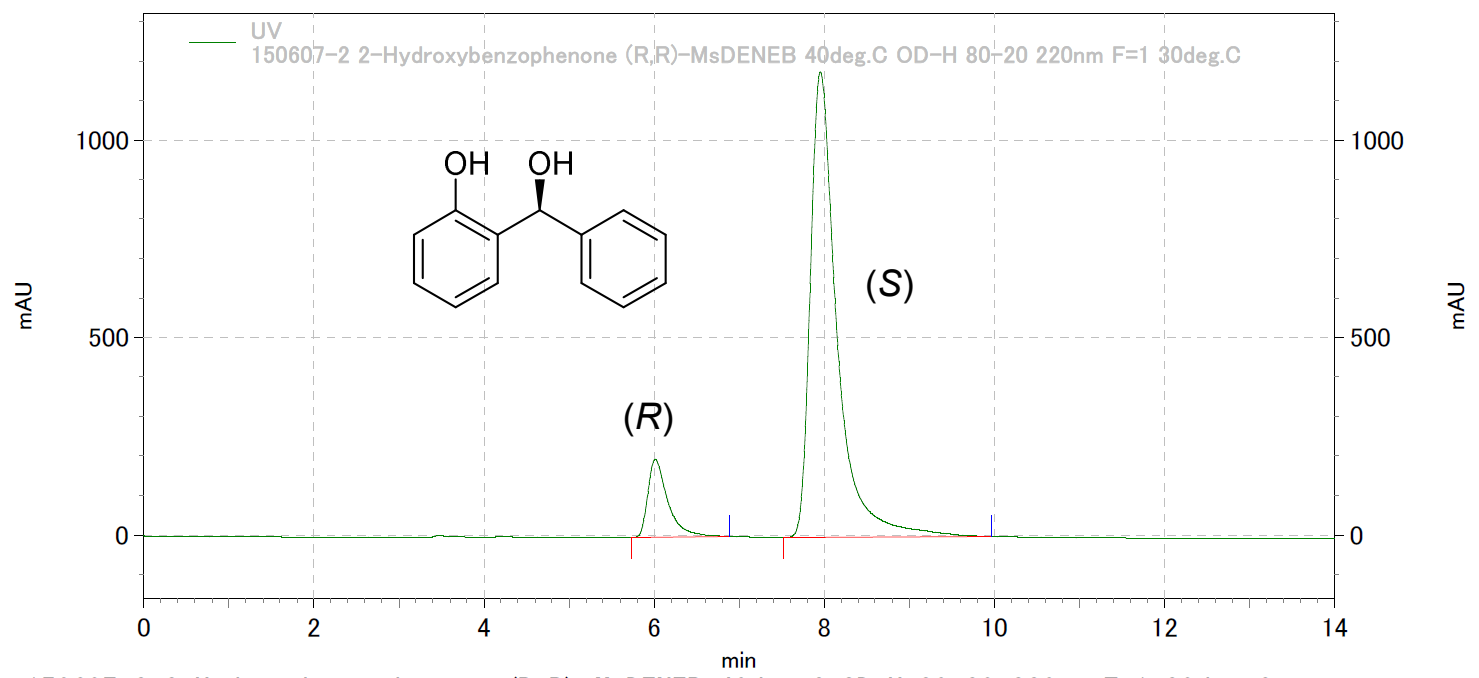

150607-2 2-Hydroxybenzophenone (R, R)-MsDENEB 40deg. C OD-H 80-20 220nm F=1 30deg. C

\begin{tabular}{|c|c|c|c|c|c|}
\hline $\begin{array}{l}\text { UV結果 } \\
\text { Pk } \\
\text { No. }\end{array}$ & R. T. & Area & Area\% & B. C. & Symmetry \\
\hline $\begin{array}{l}1 \\
2\end{array}$ & $\begin{array}{l}6.013 \\
7.957\end{array}$ & $\begin{array}{r}12959189 \\
102929586\end{array}$ & $\begin{array}{l}11.182 \\
88.818\end{array}$ & $\begin{array}{l}\overline{M M} \\
\mathrm{MM}\end{array}$ & $\begin{array}{l}0.00000 \\
0.00000\end{array}$ \\
\hline $\begin{array}{r}r-夕 \\
\mu\end{array}$ & & 115888775 & 100.000 & & \\
\hline
\end{tabular}




\section{(2,4-Dimethylphenyl)(phenyl)methanol (6f)}

(OD-H, Hex/2-PrOH=97/3, F=1.0 mL/min, $220 \mathrm{~nm}$, 30deg.C)

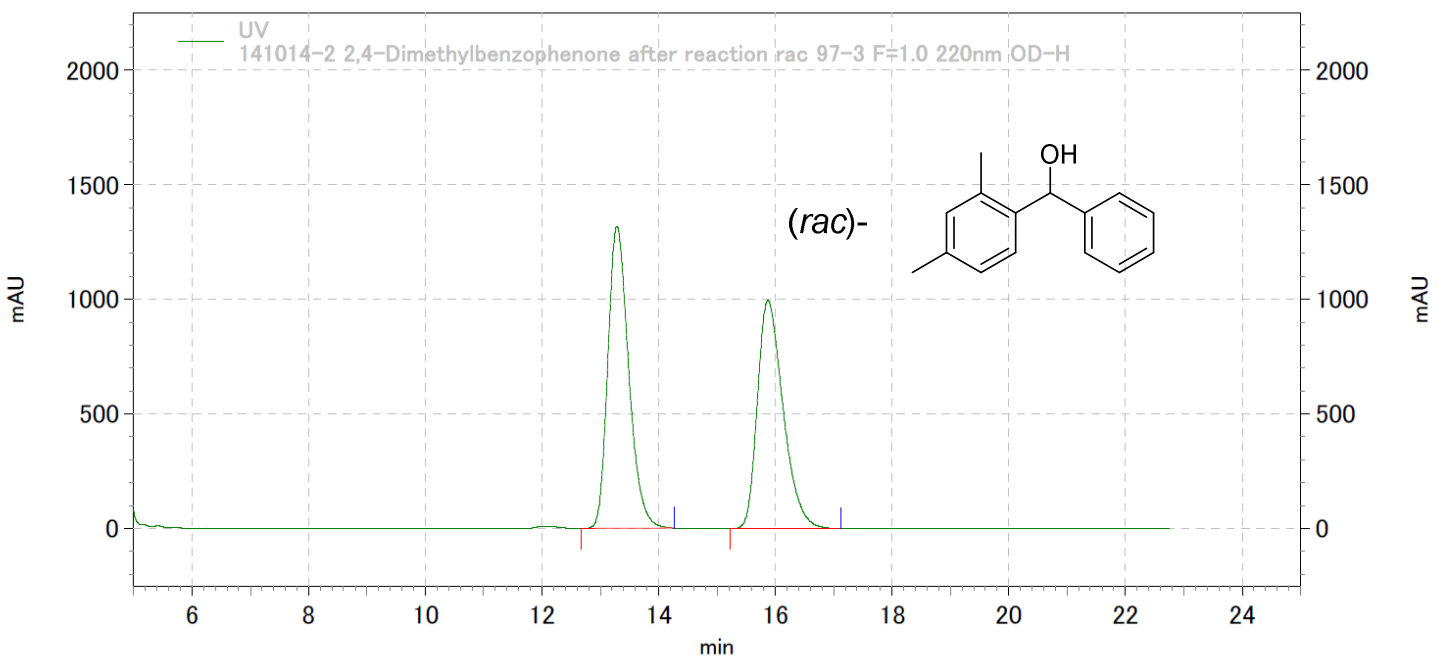

141014-2 2, 4-Dimethylbenzophenone after reaction rac 97-3 F=1.0 220nm OD-H

\begin{tabular}{|c|c|c|c|c|c|}
\hline $\begin{array}{l}\text { UV結果 } \\
\text { Pk } \\
\text { No. }\end{array}$ & R. T. & Area & Area\% & B. C. & Symmetry \\
\hline $\begin{array}{l}1 \\
2\end{array}$ & $\begin{array}{l}13.287 \\
15.873\end{array}$ & $\begin{array}{l}130482635 \\
121480975\end{array}$ & $\begin{array}{l}51.786 \\
48.214\end{array}$ & $\begin{array}{l}\mathrm{MM} \\
\mathrm{MM}\end{array}$ & $\begin{array}{l}0.00000 \\
0.00000\end{array}$ \\
\hline $\begin{array}{r}r-夕 \\
ル\end{array}$ & & 251963610 & 100.000 & & \\
\hline
\end{tabular}

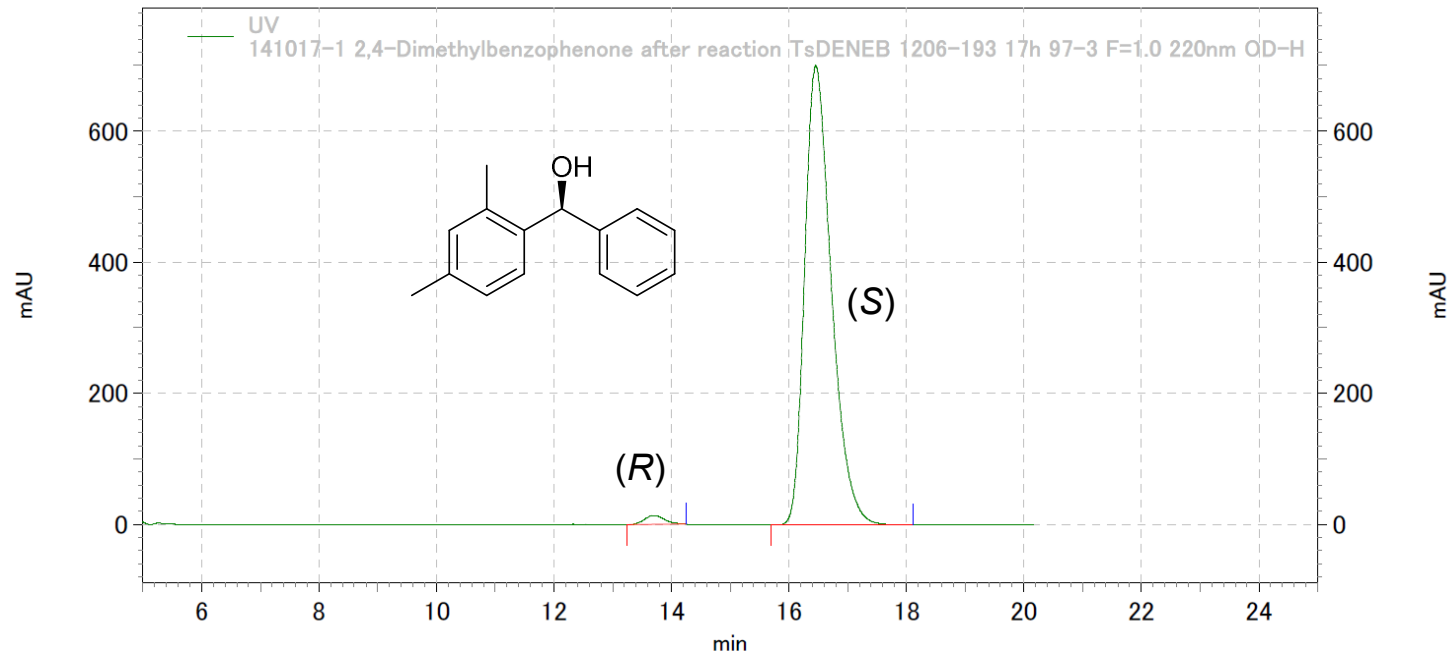

141017-1 2, 4-Dimethylbenzophenone after reaction TsDENEB 1206-193 17h 97-3 F=1.0 220nm $\mathrm{OD}-\mathrm{H}$

\begin{tabular}{|c|c|c|c|c|c|}
\hline $\begin{array}{l}\text { UV結身 } \\
\text { Pk } \\
\text { No. }\end{array}$ & R. T. & Area & Area\% & B. C. & Symmetry \\
\hline $\begin{array}{l}1 \\
2\end{array}$ & $\begin{array}{l}13.700 \\
16.457\end{array}$ & $\begin{array}{r}1284051 \\
88523368\end{array}$ & $\begin{array}{r}1.430 \\
98.570\end{array}$ & $\begin{array}{l}\text { MM } \\
\text { MM }\end{array}$ & $\begin{array}{l}0.00000 \\
0.00000\end{array}$ \\
\hline $\begin{array}{r}r-夕 \\
\mu\end{array}$ & & 89807419 & 100.000 & & \\
\hline
\end{tabular}




\section{Phenyl(2,4,5-trimethylphenyl)methanol (6g)}

(OD-H, Hex/2-PrOH=97/3, F=1.0 mL/min, $220 \mathrm{~nm}, 30$ deg.C)

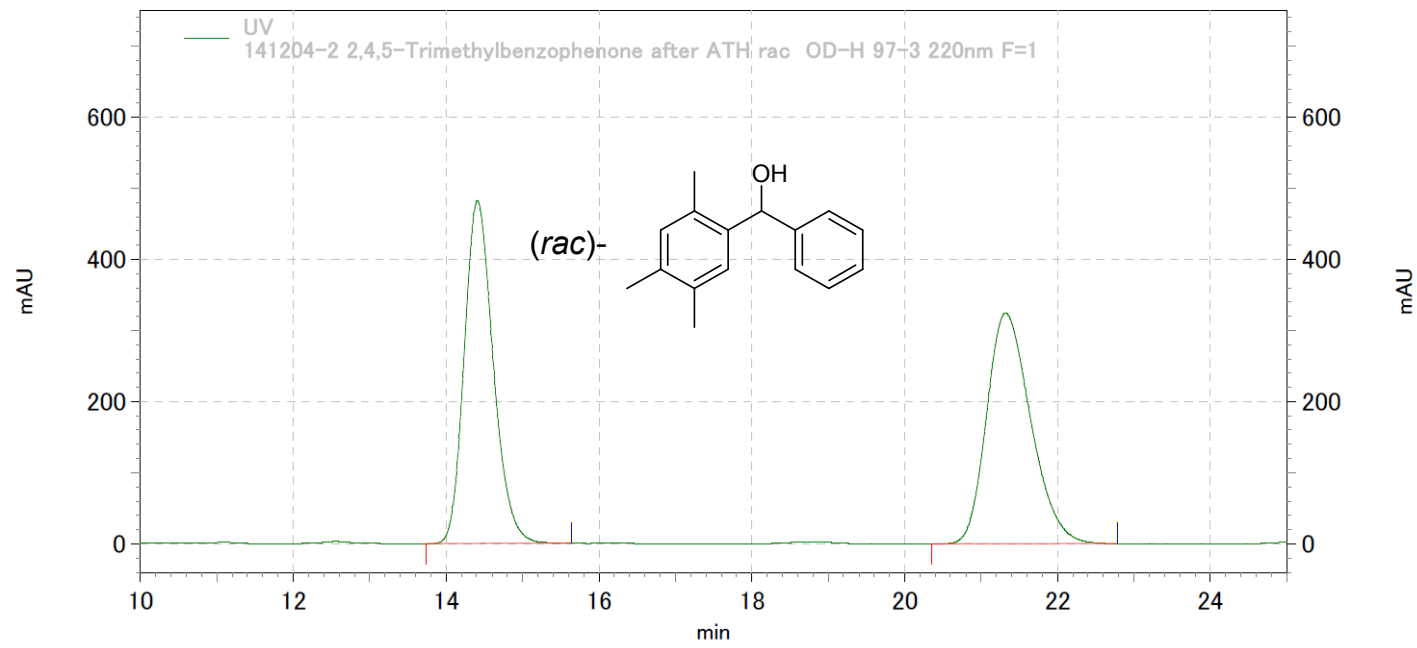

141204-2 2, 4, 5-Trimethylbenzophenone after ATH rac 0D-H 97-3 220nm F=1

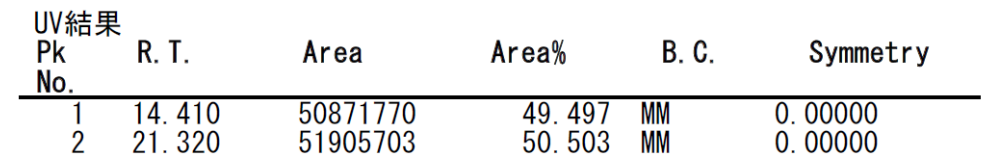

\begin{tabular}{|r|r|r|r|r|r|}
\hline $\begin{array}{r}\text { r-夕 } \\
\text { ル }\end{array}$ & & 102777473 & 100.000 & & \\
\hline
\end{tabular}

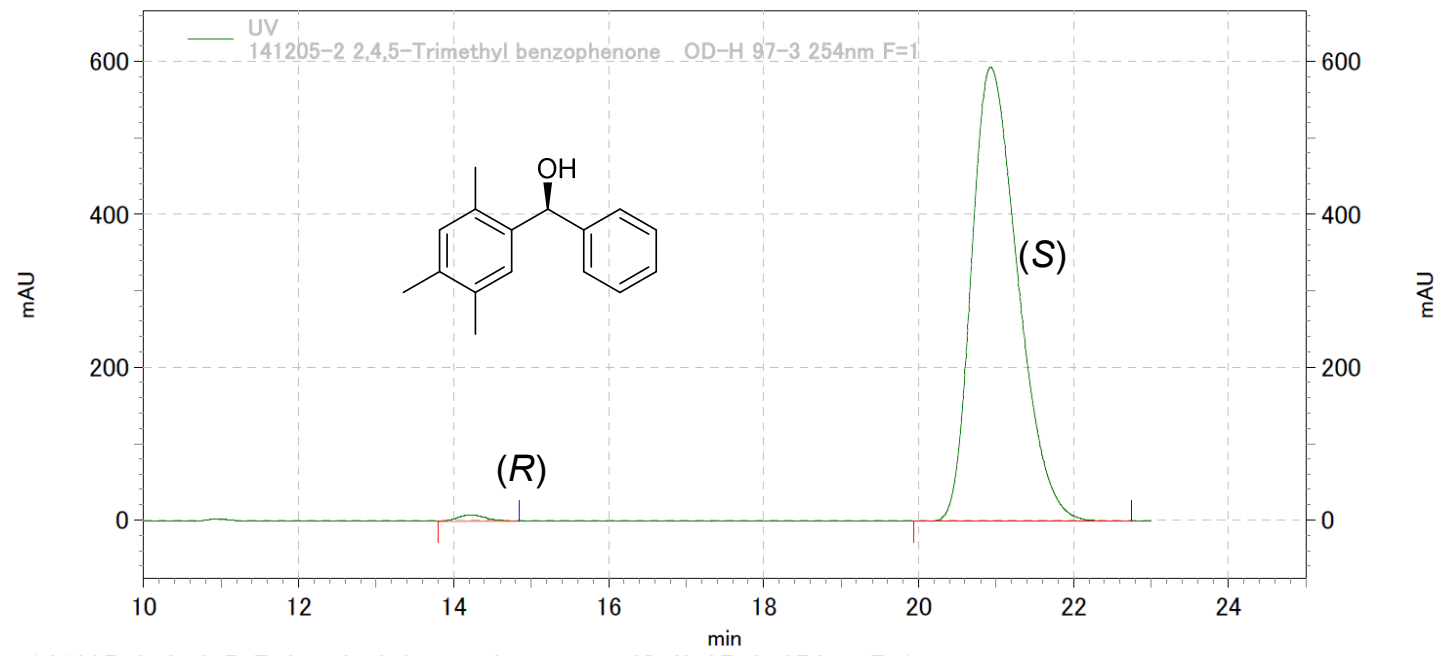

141205-2 2, 4,5-Trimethy| benzophenone OD-H 97-3 254nm F=1

\begin{tabular}{|c|c|c|c|c|c|}
\hline $\begin{array}{l}\text { UV結果 } \\
\text { Pk } \\
\text { No. }\end{array}$ & R. T. & Area & Area\% & B. C. & Symmetry \\
\hline $\begin{array}{l}1 \\
2\end{array}$ & $\begin{array}{l}14.223 \\
20.930\end{array}$ & $\begin{array}{r}845344 \\
98515005\end{array}$ & $\begin{array}{r}0.851 \\
99.149\end{array}$ & $\begin{array}{l}\text { MM } \\
\text { MM }\end{array}$ & $\begin{array}{l}0.00000 \\
0.00000\end{array}$ \\
\hline $\begin{array}{r}1-夕 夕 \\
\|\end{array}$ & & 99360349 & 100.000 & & \\
\hline
\end{tabular}




\section{(2,4-Dichlorophenyl)(phenyl)methanol (6h)}

(OD-H, Hex/2-PrOH=97/3, F=1.0 mL/min, $220 \mathrm{~nm}, 30 \mathrm{deg} . \mathrm{C}$ )

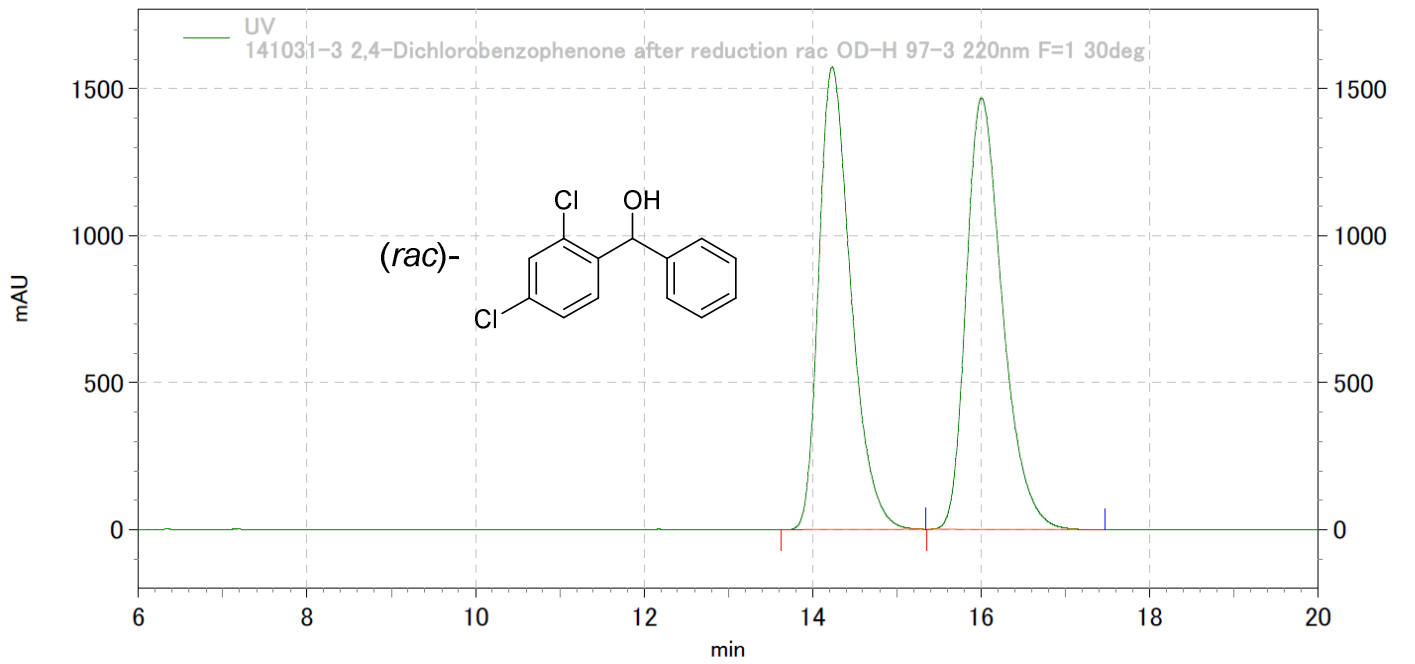

141031-3 2, 4-Dichlorobenzophenone after reduction rac 0D-H 97-3 220nm $F=130 \mathrm{deg}$

\begin{tabular}{|c|c|c|c|c|c|}
\hline $\begin{array}{l}\text { UV結果 } \\
\text { Pk } \\
\text { No. }\end{array}$ & R. T. & Area & Area\% & B. C. & Symmetry \\
\hline $\begin{array}{l}1 \\
2\end{array}$ & $\begin{array}{l}14.233 \\
16.007\end{array}$ & $\begin{array}{l}167070017 \\
175783402\end{array}$ & $\begin{array}{l}48.729 \\
51.271\end{array}$ & $\begin{array}{l}\text { MM } \\
M M\end{array}$ & $\begin{array}{l}0.00000 \\
0.00000\end{array}$ \\
\hline $\begin{array}{r}1-8 \\
\|\end{array}$ & & 342853419 & 100.000 & & \\
\hline
\end{tabular}

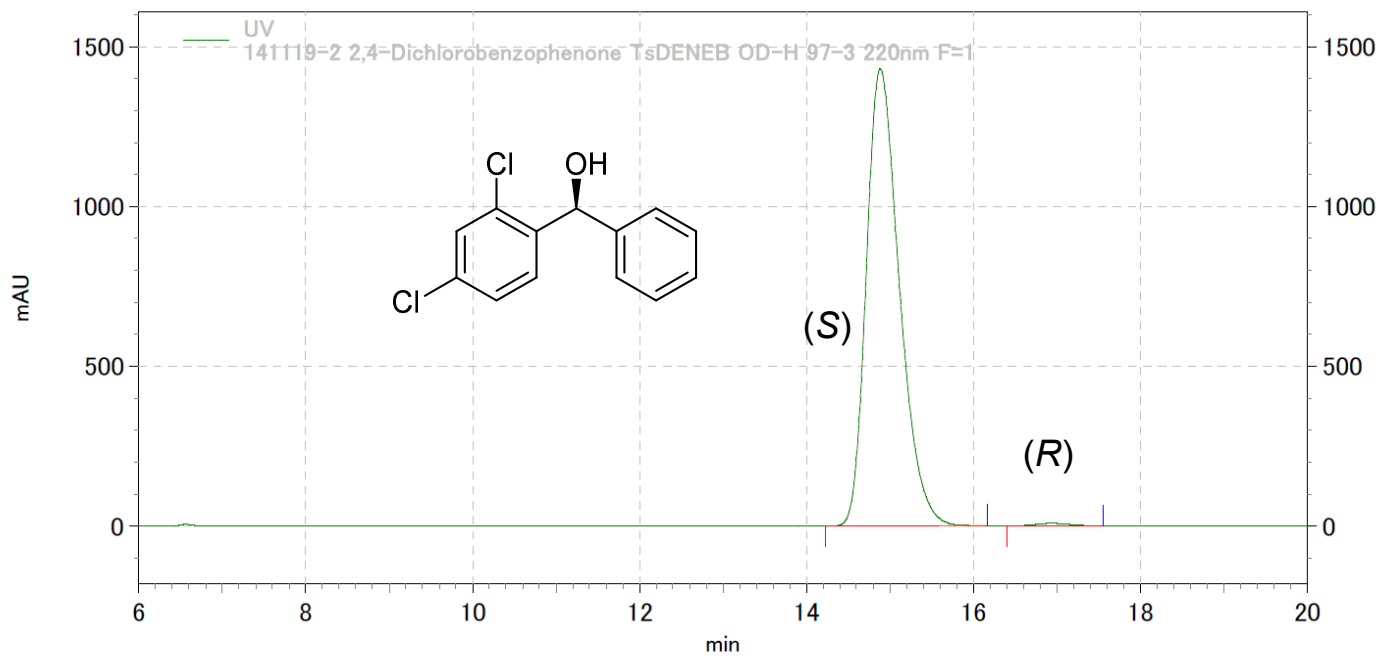

141119-2 2, 4-Dichlorobenzophenone TsDENEB OD-H 97-3 220nm F=1

\begin{tabular}{|c|c|c|c|c|c|}
\hline $\begin{array}{l}\text { UVV結果 } \\
\text { Pk } \\
\text { No. }\end{array}$ & R. T. & Area & Area\% & B. C. & Symmetry \\
\hline $\begin{array}{l}1 \\
2\end{array}$ & $\begin{array}{l}14.883 \\
16.940\end{array}$ & $\begin{array}{r}157259637 \\
1010406\end{array}$ & $\begin{array}{r}99.362 \\
0.638\end{array}$ & $\begin{array}{l}\text { MM } \\
\text { MM }\end{array}$ & $\begin{array}{l}0.00000 \\
0.00000\end{array}$ \\
\hline $\begin{array}{r}1-8 \\
\|\end{array}$ & & 158270043 & 100.000 & & \\
\hline
\end{tabular}




\section{(2-Chloro-5-nitrophenyl)(phenyl)methanol (6i)}

(OD-H, Hex/2-PrOH=97/3, F=1.0 mL/min, 220 nm, 30deg.C)

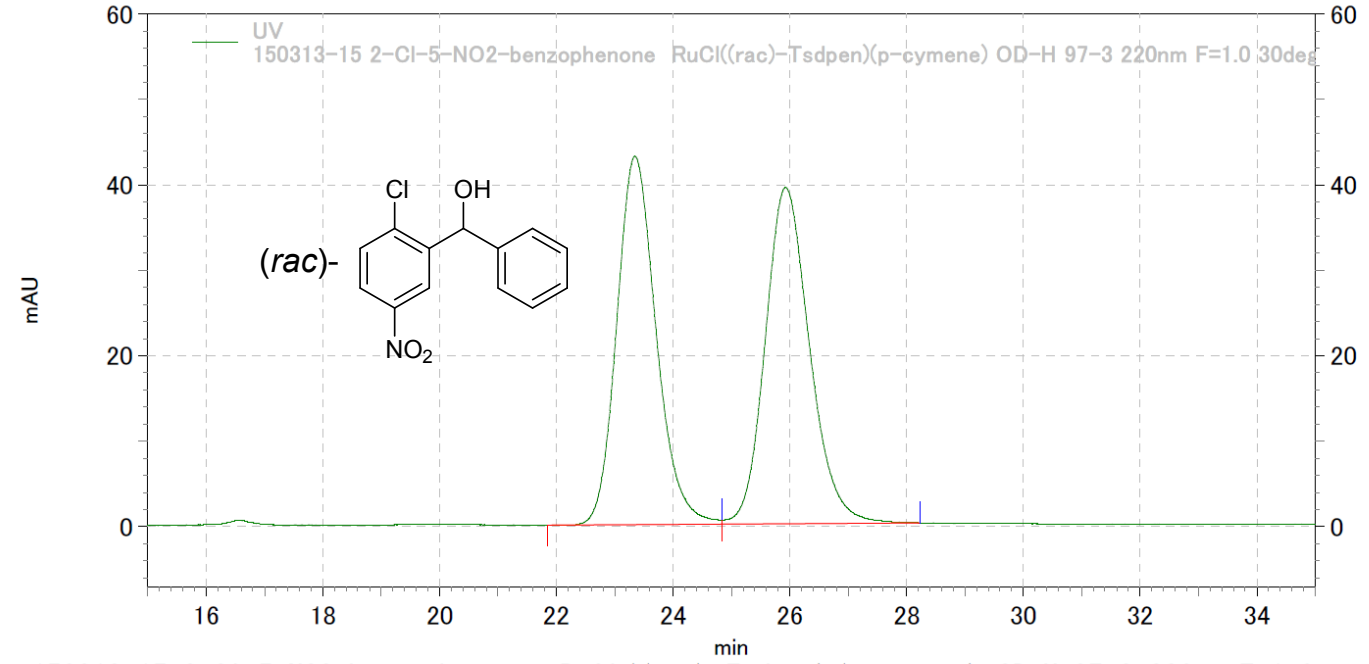

150313-15 2-Cl-5-N02-benzophenone RuCI ( (rac)-Tsdpen) (p-cymene) OD-H 97-3 220nm F=1.0 30deg. C

\begin{tabular}{|c|c|c|c|c|c|}
\hline $\begin{array}{l}\text { UV結果 } \\
\text { Pk } \\
\text { No. }\end{array}$ & R. T. & Area & Area\% & B. C. & Symmetry \\
\hline $\begin{array}{l}1 \\
2\end{array}$ & $\begin{array}{l}23.347 \\
25.930\end{array}$ & $\begin{array}{l}8075289 \\
8309276\end{array}$ & $\begin{array}{l}49.286 \\
50.714\end{array}$ & $\begin{array}{l}M x \\
x M\end{array}$ & $\begin{array}{l}0.00000 \\
0.00000\end{array}$ \\
\hline $\begin{array}{r}r-夕 \\
ル\end{array}$ & & 16384565 & 100.000 & & \\
\hline
\end{tabular}

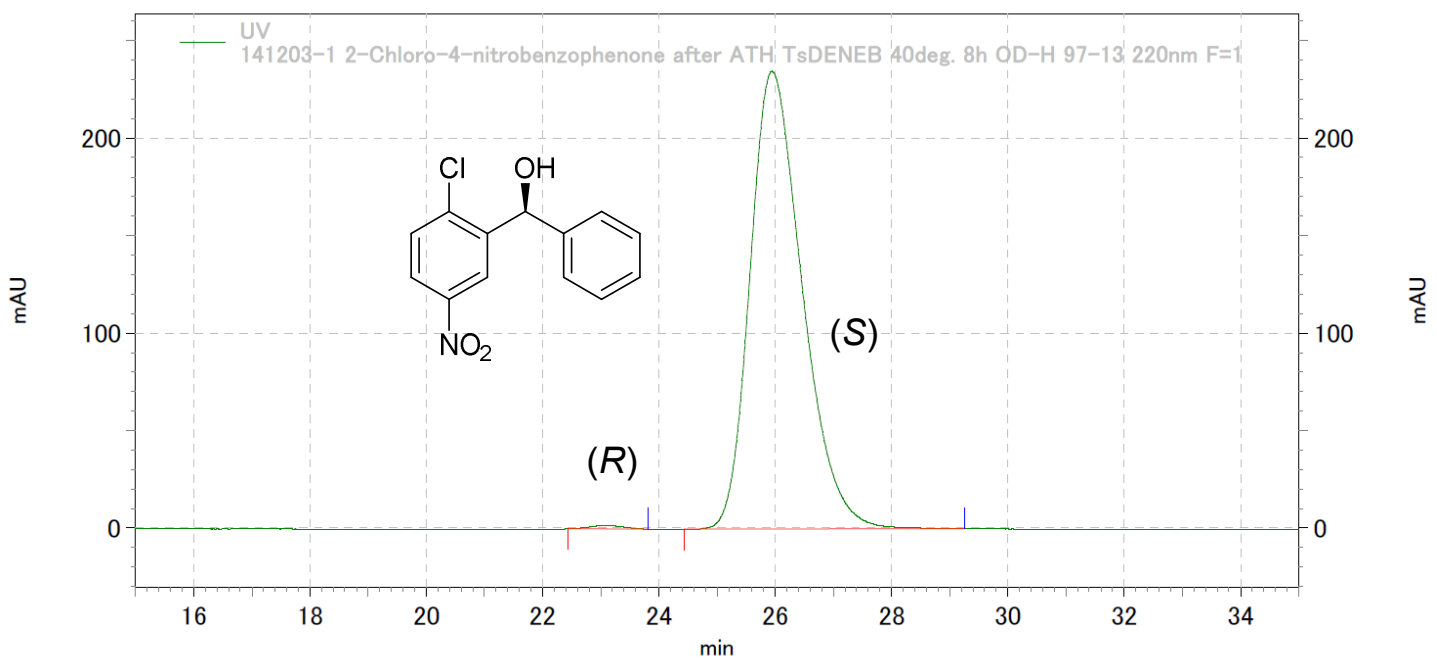

141203-1 2-Chloro-4-nitrobenzophenone after ATH TsDENEB 40deg. 8h 0D-H 97-13 220nm F=1

\begin{tabular}{|c|c|c|c|c|c|}
\hline $\begin{array}{l}\text { UV 結 } \\
\text { Pk } \\
\text { No. }\end{array}$ & R. T. & Area & Area\% & B. C. & Symmetry \\
\hline $\begin{array}{l}1 \\
2\end{array}$ & $\begin{array}{l}23.100 \\
25.947\end{array}$ & $\begin{array}{r}273912 \\
58518116\end{array}$ & $\begin{array}{r}0.466 \\
99.534\end{array}$ & $\begin{array}{l}\text { MM } \\
\text { MM }\end{array}$ & $\begin{array}{l}0.00000 \\
0.00000\end{array}$ \\
\hline $\begin{array}{r}r-夕 \\
\|\end{array}$ & & 58792028 & 100.000 & & \\
\hline
\end{tabular}




\section{(2,5-Difluorophenyl)(phenyl)methanol (6j)}

(OJ-H, Hex/2-PrOH=97/3, F=1.0 mL/min, 220 nm, 30deg.C)

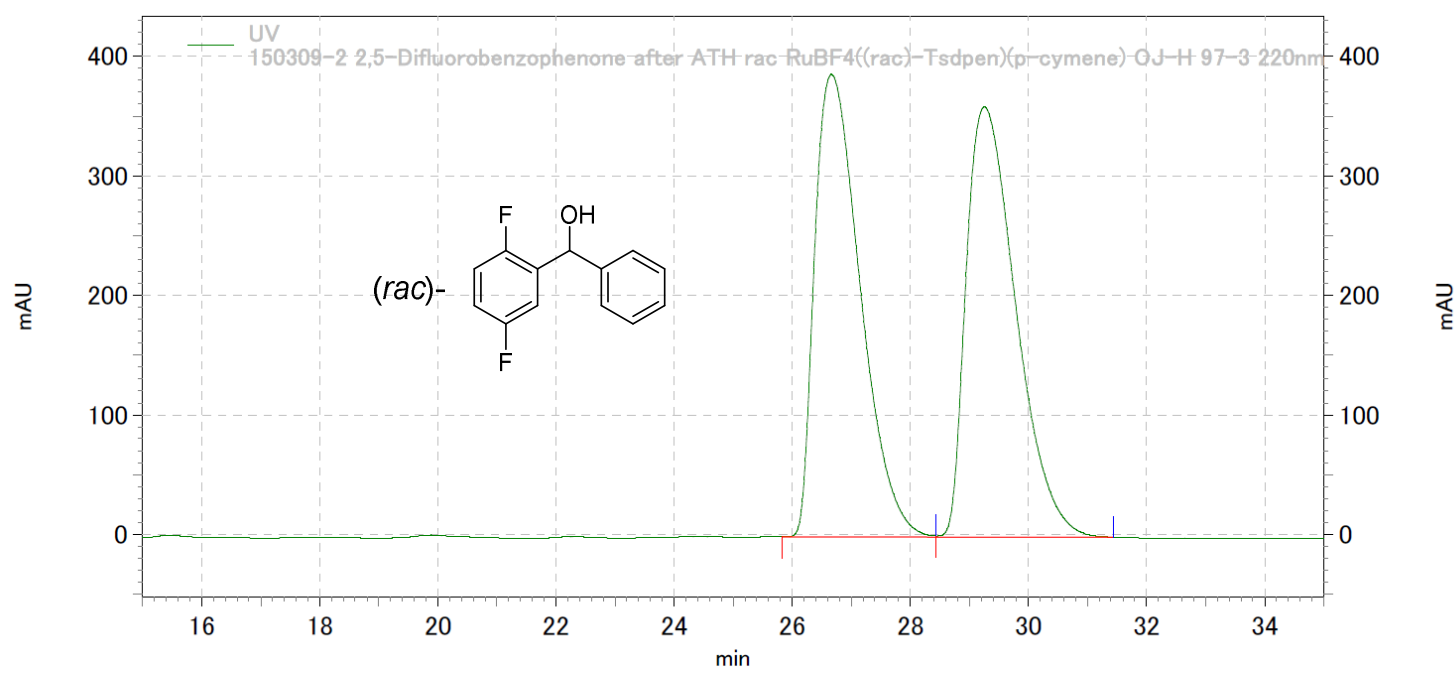

150309-2 2,5-Difluorobenzophenone after ATH rac RuBF4 ( (rac)-Tsdpen) (p-cymene) 0J-H 97-3 $220 \mathrm{~nm} F=1.0$ 30deg. C

\begin{tabular}{|c|c|c|c|c|c|}
\hline $\begin{array}{l}\text { UV 結 } \\
\text { Pk } \\
\text { No. } \\
\end{array}$ & R. T. & Area & Area\% & B. C. & Symmetry \\
\hline $\begin{array}{l}1 \\
2\end{array}$ & $\begin{array}{l}26.667 \\
29.257\end{array}$ & $\begin{array}{l}83197685 \\
84242879\end{array}$ & $\begin{array}{l}49.688 \\
50.312\end{array}$ & $\begin{array}{l}M x \\
x M\end{array}$ & $\begin{array}{l}0.00000 \\
0.00000\end{array}$ \\
\hline $\begin{array}{r}r-g \\
\pi\end{array}$ & & 167440564 & 100.000 & & \\
\hline
\end{tabular}

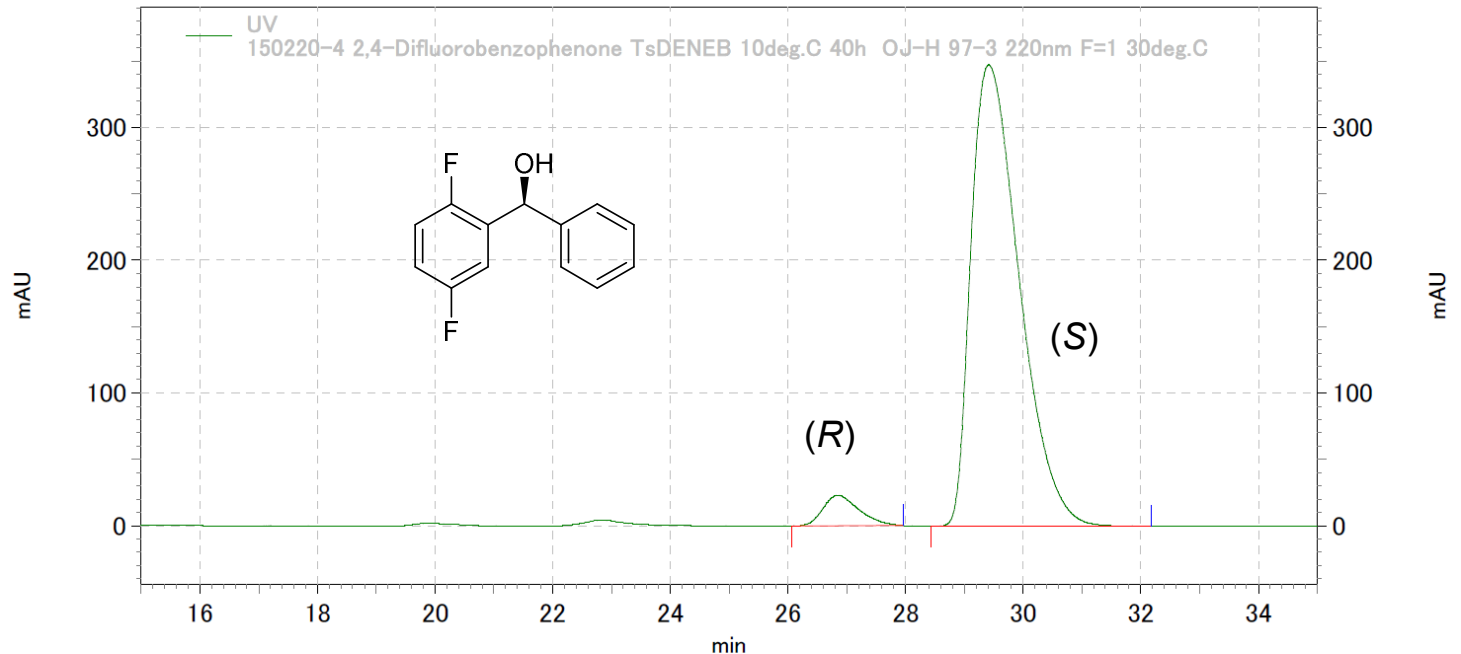

150220-4 2, 4-Difluorobenzophenone TsDENEB 10deg. C 40h 0J-H 97-3 220nm F=1 30deg. C

\begin{tabular}{|c|c|c|c|c|c|}
\hline $\begin{array}{l}\text { UV結果果 } \\
\mathrm{Pk} \\
\mathrm{No}\end{array}$ & R. T. & Area & Area\% & B. C. & Symmetry \\
\hline $\begin{array}{l}1 \\
2\end{array}$ & $\begin{array}{l}26.847 \\
29.417\end{array}$ & $\begin{array}{r}3903986 \\
79279779\end{array}$ & $\begin{array}{r}4.693 \\
95.307\end{array}$ & $\begin{array}{l}\text { MM } \\
\text { MM }\end{array}$ & $\begin{array}{l}0.00000 \\
0.00000\end{array}$ \\
\hline $\begin{array}{r}r-夕 \\
\|\end{array}$ & & 83183765 & 100.000 & & \\
\hline
\end{tabular}




\section{(2-Fluoro-3-(trifluoromethyl)phenyl)(phenyl)methanol (6k)}

(OD-H, Hex/2-PrOH=97/3, F=1.0 mL/min, $220 \mathrm{~nm}$, 30deg.C)

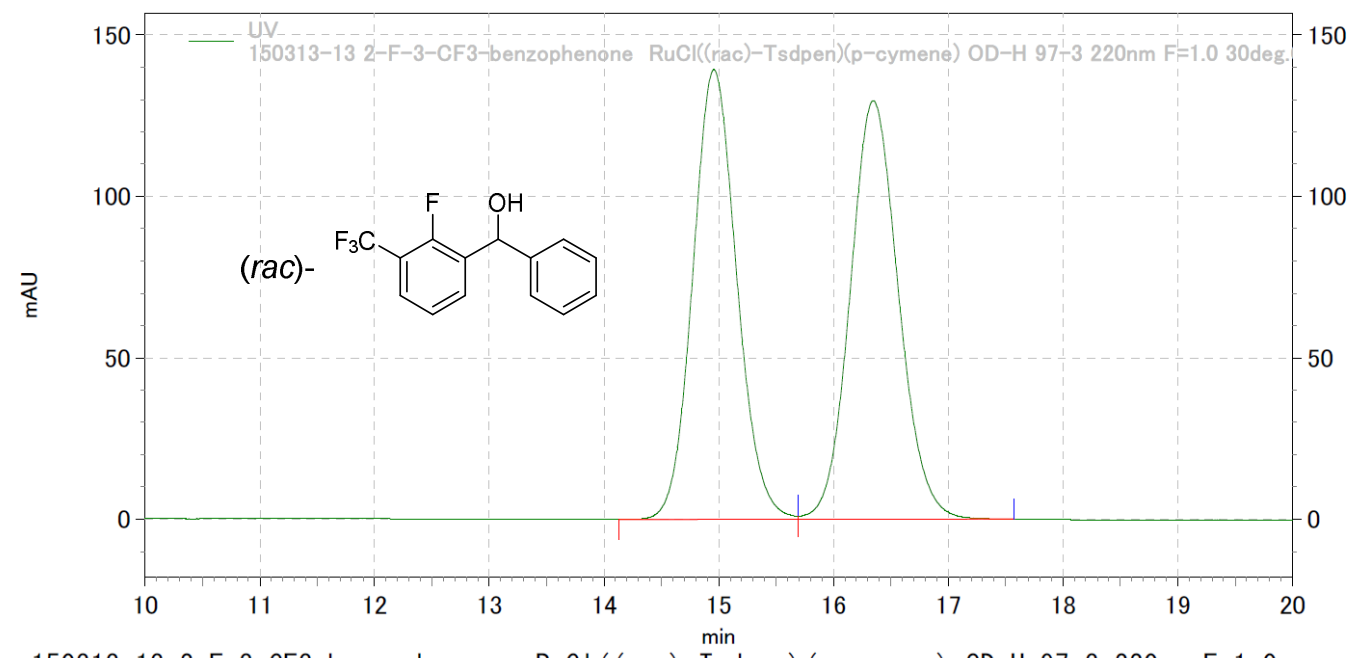

150313-13 2-F-3-CF3-benzophenone RuCl ( ( rac)-Tsdpen) (p-cymene) OD-H 97-3 220nm F=1.0 30deg. C

\begin{tabular}{|c|c|c|c|c|c|}
\hline $\begin{array}{l}\text { UV結果 } \\
\text { Pk } \\
\text { No. }\end{array}$ & R. T. & Area & Area\% & B. C. & Symmetry \\
\hline $\begin{array}{l}1 \\
2\end{array}$ & $\begin{array}{l}14.957 \\
16.347\end{array}$ & $\begin{array}{l}14849901 \\
15224921\end{array}$ & $\begin{array}{l}49.377 \\
50.623\end{array}$ & $\begin{array}{l}\overline{M x} \\
x M\end{array}$ & $\begin{array}{l}0.00000 \\
0.00000\end{array}$ \\
\hline $\begin{array}{r}r-夕 \\
\mu\end{array}$ & & 30074822 & 100.000 & & \\
\hline
\end{tabular}

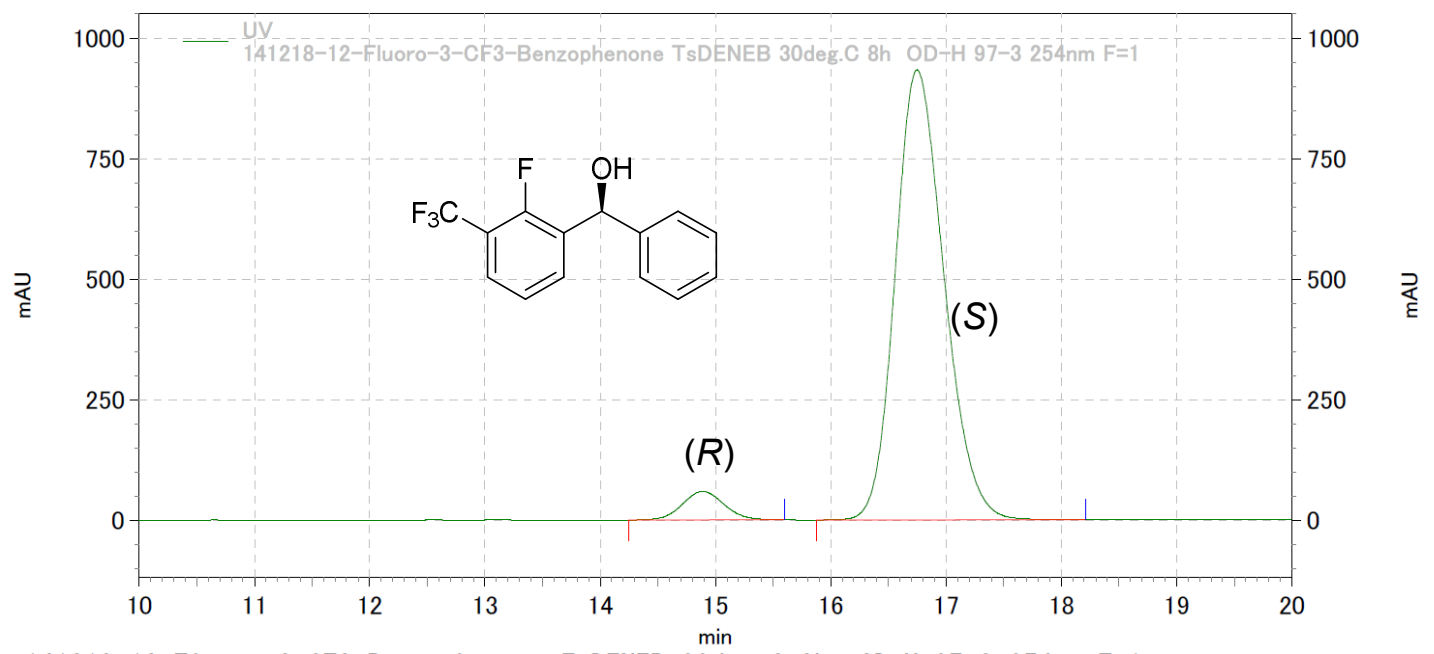

141218-12-Fluoro-3-CF3-Benzophenone TsDENEB 30deg. C 8h OD-H 97-3 254nm F=1

\begin{tabular}{|c|c|c|c|c|c|}
\hline $\begin{array}{l}\text { UV結身 } \\
\text { Pk } \\
\text { No. }\end{array}$ & R. T. & Area & Area\% & B. C. & Symmetry \\
\hline $\begin{array}{l}1 \\
2\end{array}$ & $\begin{array}{l}14.890 \\
16.750\end{array}$ & $\begin{array}{r}5914496 \\
109810110\end{array}$ & $\begin{array}{r}5.111 \\
94.889\end{array}$ & $\begin{array}{l}\mathrm{MM} \\
\mathrm{MM}\end{array}$ & $\begin{array}{l}0.00000 \\
0.00000\end{array}$ \\
\hline $\begin{array}{r}r-3 \\
\|\end{array}$ & & 115724606 & 100.000 & & \\
\hline
\end{tabular}




\section{(Perfluorophenyl)(phenyl)methanol (61)}

(OD-H, Hex/2-PrOH=97/3, F=1.0 mL/min, 220 nm, 30deg.C)

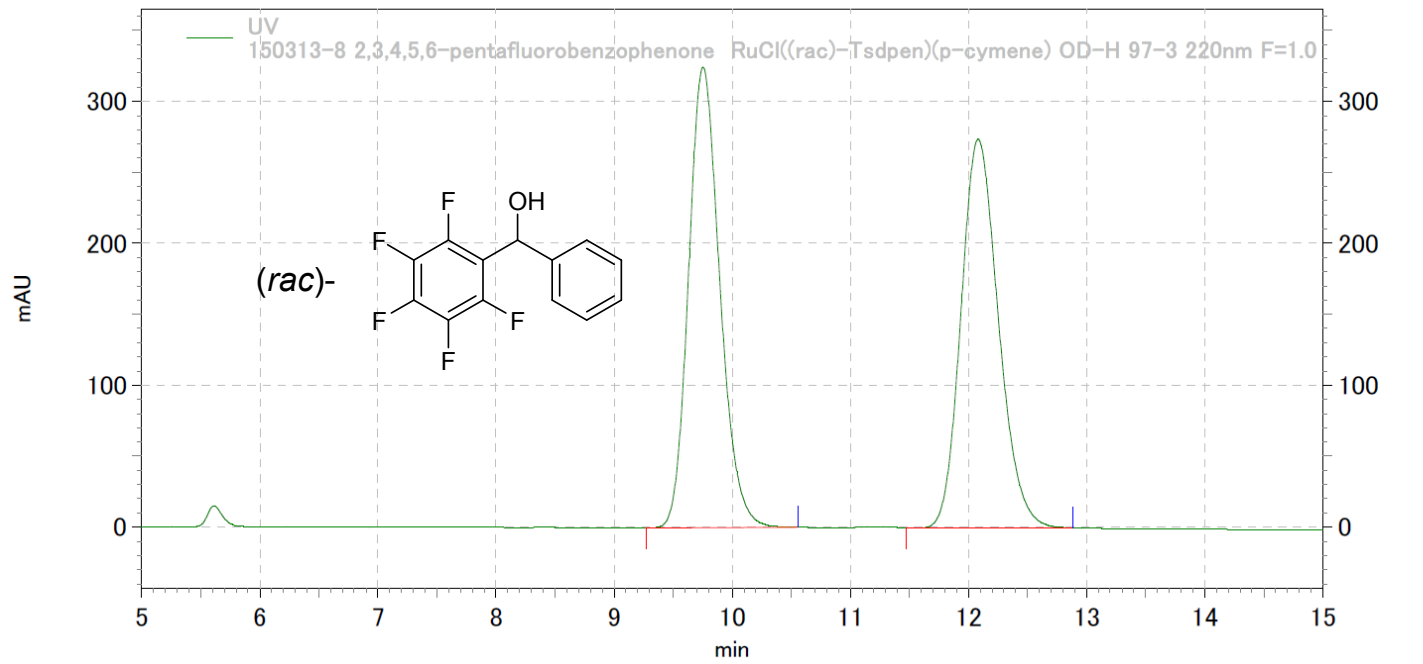

150313-8 2, 3, 4, 5, 6-pentafluorobenzophenone RuCl ((rac)-Tsdpen) (p-cymene) 0D-H 97-3 $220 \mathrm{~nm} \mathrm{~F}=1.0$ 30deg. C

\begin{tabular}{|c|c|c|c|c|c|}
\hline $\begin{array}{l}\text { UV結 } \\
\text { Pk } \\
\text { No. }\end{array}$ & R. T. & Area & Area\% & B. C. & Symmetry \\
\hline $\begin{array}{l}1 \\
2\end{array}$ & $\begin{array}{r}9.753 \\
12.083\end{array}$ & $\begin{array}{l}23887183 \\
24147367\end{array}$ & $\begin{array}{l}49.729 \\
50.271\end{array}$ & $\begin{array}{l}\mathrm{MM} \\
\mathrm{HH}\end{array}$ & $\begin{array}{l}0.00000 \\
0.00000\end{array}$ \\
\hline $\begin{array}{r}r-夕 \\
\mu\end{array}$ & & 48034550 & 100.000 & & \\
\hline
\end{tabular}

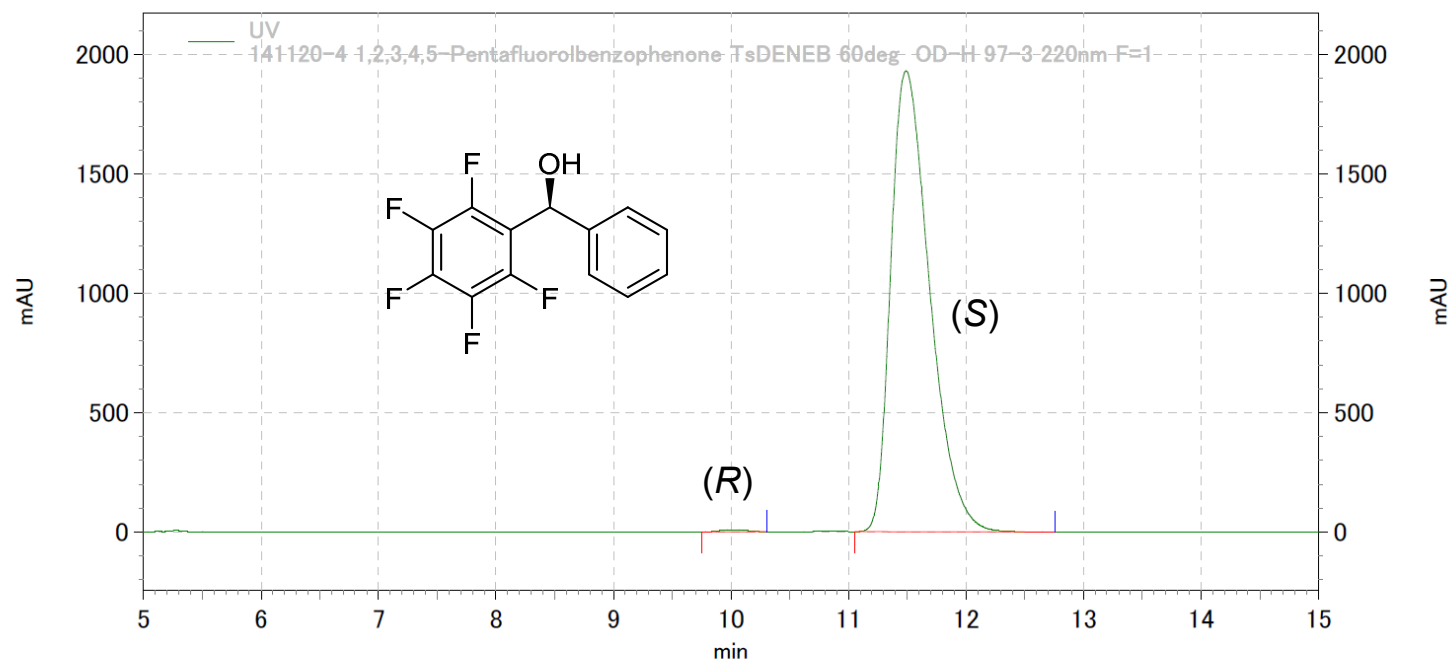

141120-4 1, 2, 3, 4,5-Pentafluorolbenzophenone TsDENEB 60deg OD-H 97-3 220nm F=1

\begin{tabular}{|c|c|c|c|c|c|}
\hline $\begin{array}{l}\text { UV結果 } \\
\mathrm{Pk} \\
\text { No. }\end{array}$ & R. T. & Area & Area\% & B. C. & Symmetry \\
\hline $\begin{array}{l}1 \\
2\end{array}$ & $\begin{array}{l}10.017 \\
11.490\end{array}$ & $\begin{array}{r}547578 \\
177594665\end{array}$ & $\begin{array}{r}0.307 \\
99.693\end{array}$ & $\begin{array}{l}\mathrm{MM} \\
\mathrm{MM}\end{array}$ & $\begin{array}{l}0.00000 \\
0.00000\end{array}$ \\
\hline $\begin{array}{r}r-夕 \\
\mu\end{array}$ & & 178142243 & 100.000 & & \\
\hline
\end{tabular}




\section{(2-Chlorophenyl)(4-chlorophenyl)methanol (6m)}

(OD-H, Hex/2-PrOH=97/3, F=1.0 mL/min, 220 nm, 30deg.C)

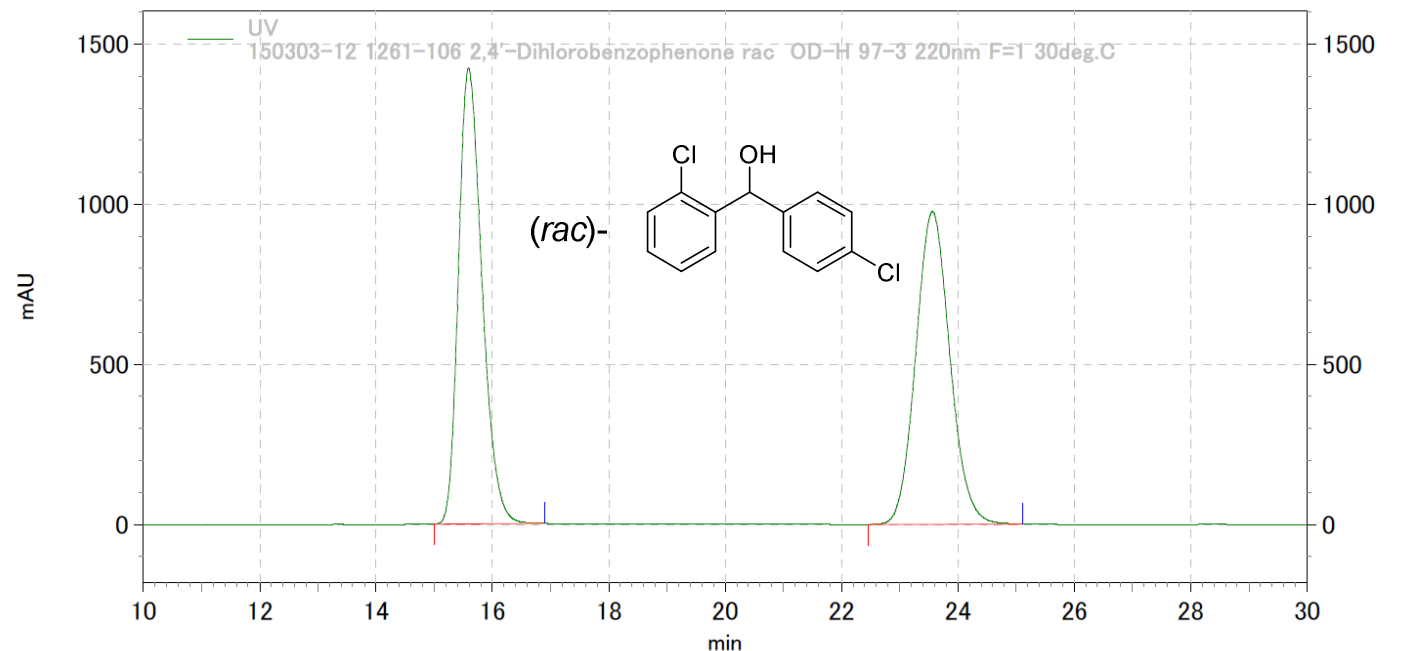

150303-12 1261-106 2, 4' -Dihlorobenzophenone rac OD-H 97-3 220nm F=1 30deg. C

\begin{tabular}{|c|c|c|c|c|c|}
\hline $\begin{array}{l}\text { UV結果 } \\
\text { Pk } \\
\text { No. }\end{array}$ & R. T. & Area & Area\% & B. C. & Symmetry \\
\hline $\begin{array}{l}1 \\
2\end{array}$ & $\begin{array}{l}15.590 \\
23.563\end{array}$ & $\begin{array}{l}160344873 \\
159071049\end{array}$ & $\begin{array}{l}50.199 \\
49.801\end{array}$ & $\begin{array}{l}\text { MM } \\
\text { MM }\end{array}$ & $\begin{array}{l}0.00000 \\
0.00000\end{array}$ \\
\hline $\begin{array}{r}\text { ト-タ } \\
ル\end{array}$ & & 319415922 & 100.000 & & \\
\hline
\end{tabular}

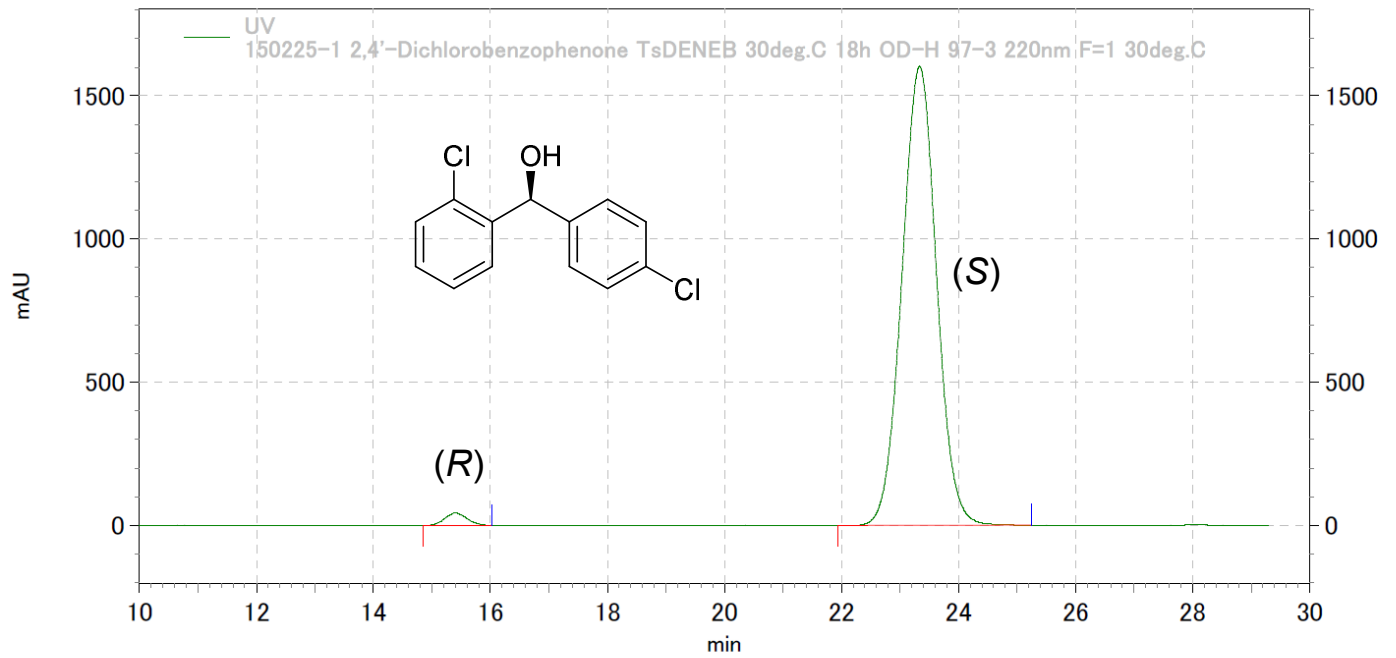

150225-1 2, 4' -Dichlorobenzophenone TsDENEB 30deg. C 18h 0D-H 97-3 220nm F=1 30deg. C

\begin{tabular}{|c|c|c|c|c|c|}
\hline $\begin{array}{l}\text { UV結身 } \\
\text { Pk } \\
\text { No. }\end{array}$ & R. T. & Area & Area\% & B. C. & Symmetry \\
\hline $\begin{array}{l}1 \\
2\end{array}$ & $\begin{array}{l}15.400 \\
23.333\end{array}$ & $\begin{array}{r}4601430 \\
263561425\end{array}$ & $\begin{array}{r}1.716 \\
98.284\end{array}$ & $\begin{array}{l}\text { MM } \\
\text { MM }\end{array}$ & $\begin{array}{l}0.00000 \\
0.00000\end{array}$ \\
\hline $\begin{array}{r}r-y \\
\|\end{array}$ & & 268162855 & 100.000 & & \\
\hline
\end{tabular}




\section{(2-Chlorophenyl)(4-fluorophenyl)methanol (6n)}

(OD-H, Hex/2-PrOH=97/3, F=1.0 mL/min, 220 nm, 30deg.C)

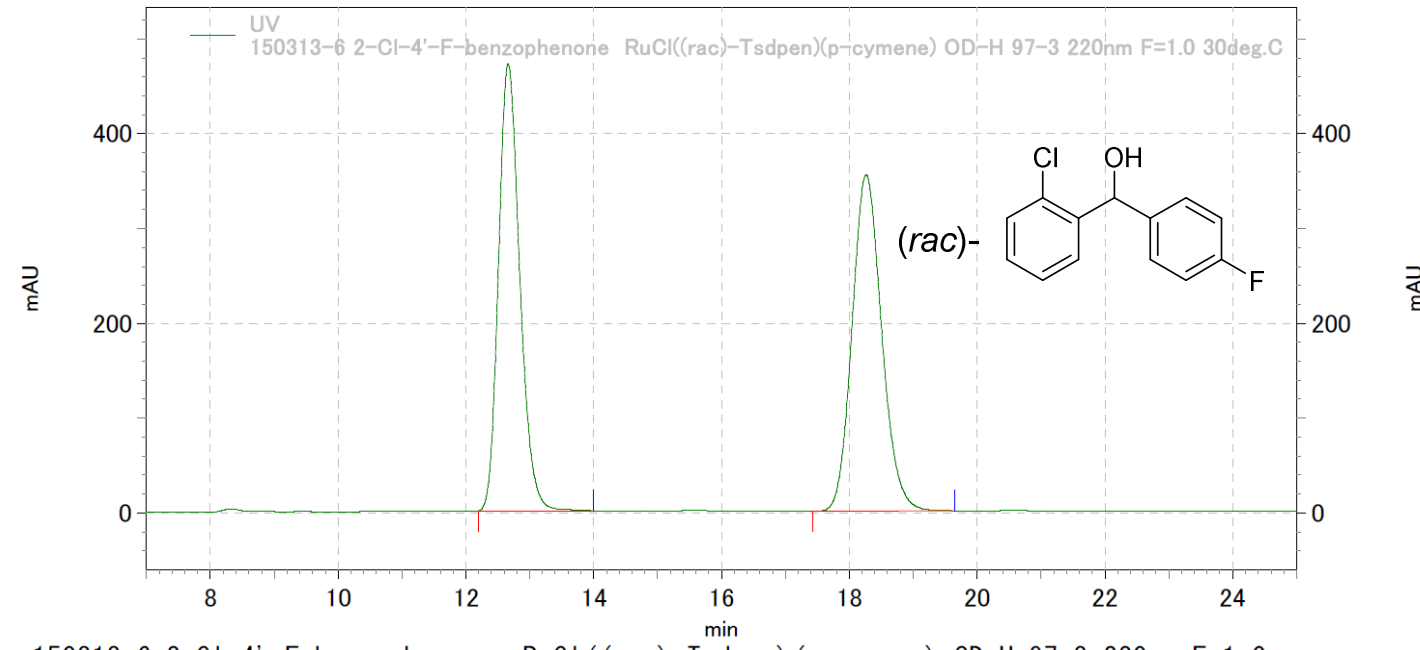

150313-6 2-Cl-4' -F-benzophenone RuCl ( (rac)-Tsdpen) (p-cymene) 0D-H 97-3 220nm F=1.0 30deg. C

\begin{tabular}{|c|c|c|c|c|c|}
\hline $\begin{array}{l}\text { UV結身 } \\
\text { Pk } \\
\text { No. }\end{array}$ & R. T. & Area & Area\% & B. C. & Symmetry \\
\hline $\begin{array}{l}1 \\
2\end{array}$ & $\begin{array}{l}12.660 \\
18.263\end{array}$ & $\begin{array}{l}43554474 \\
45673358\end{array}$ & $\begin{array}{l}48.813 \\
51.187\end{array}$ & $\begin{array}{l}\mathrm{HH} \\
\mathrm{MM}\end{array}$ & $\begin{array}{l}0.00000 \\
0.00000\end{array}$ \\
\hline $\begin{array}{r}r-\not k \\
\|\end{array}$ & & 89227832 & 100.000 & & \\
\hline
\end{tabular}

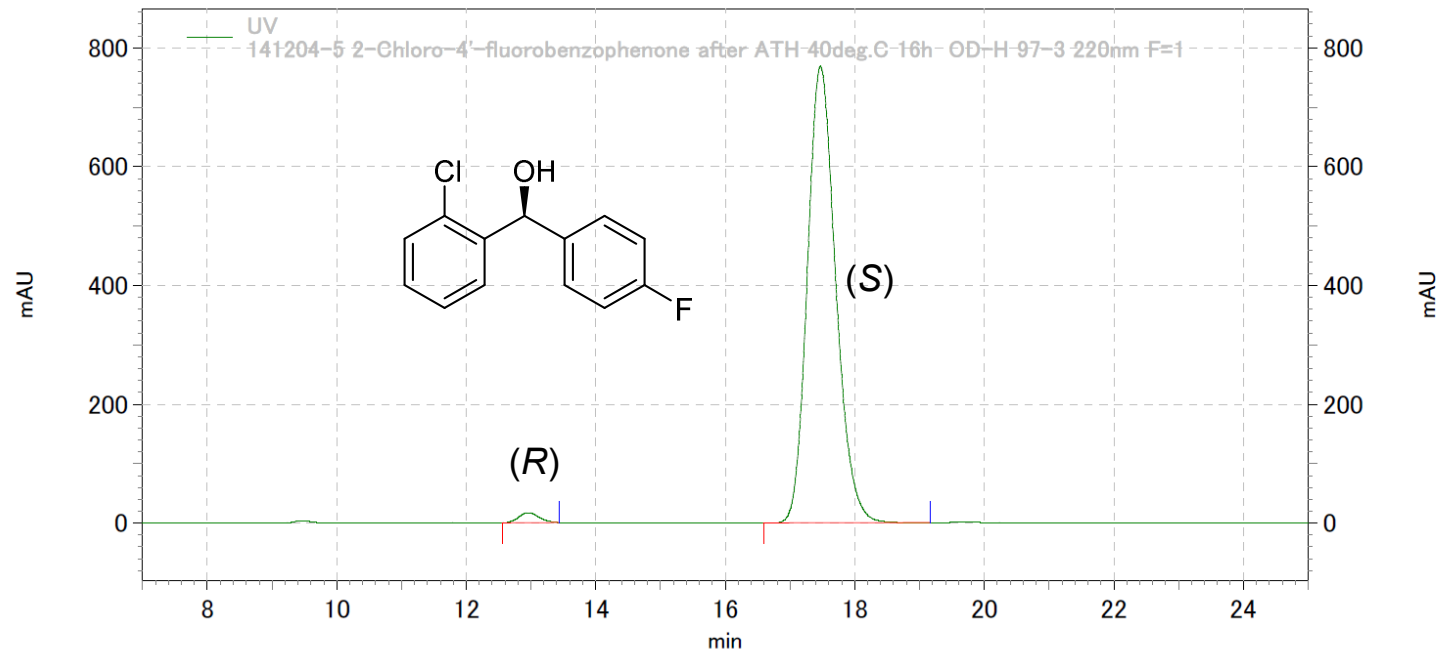

141204-5 2-Chloro-4'-fluorobenzophenone after ATH 40deg. C 16h OD-H 97-3 220nm F=1

\begin{tabular}{|c|c|c|c|c|c|}
\hline $\begin{array}{l}\text { UV結果 } \\
\text { Pk } \\
\text { No. }\end{array}$ & R. T. & Area & Area\% & B. C. & Symmetry \\
\hline $\begin{array}{l}1 \\
2\end{array}$ & $\begin{array}{l}12.957 \\
17.470\end{array}$ & $\begin{array}{r}1431178 \\
93438465\end{array}$ & $\begin{array}{r}1.509 \\
98.491\end{array}$ & $\begin{array}{l}\text { MM } \\
\text { MM }\end{array}$ & $\begin{array}{l}0.00000 \\
0.00000\end{array}$ \\
\hline $\begin{array}{r}r-夕 \\
ル\end{array}$ & & 94869643 & 100.000 & & \\
\hline
\end{tabular}




\section{(2'-Fluoro-[1,1'-biphenyl]-2-yl)(phenyl)methanol (8)}

(OD-H, Hex/2-PrOH=97/3, F=1.0 mL/min, $220 \mathrm{~nm}$, 30deg.C)

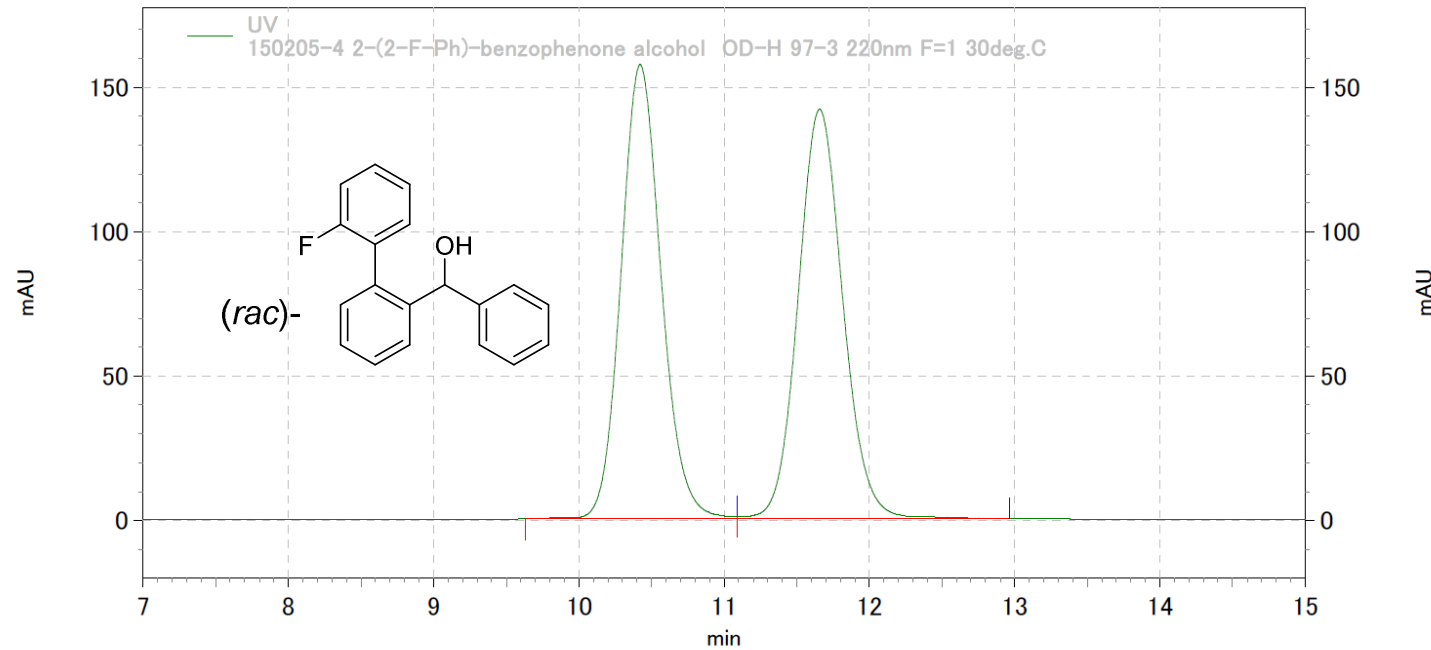

150205-4 2-(2-F-Ph)-benzophenone alcohol OD-H 97-3 220nm F=1 30deg. C

\begin{tabular}{rl|l|l|l|l|}
$\begin{array}{l}\text { UV 結果 } \\
\text { Pk } \\
\text { No. }\end{array}$ & R. T. & Area & Area\% & B. C. & Symmetry \\
\hline 1 & 10.423 & 12100169 & 49.881 & Mx & 0.00000 \\
2 & 11.657 & 12157999 & 50.119 & XM & 0.00000 \\
\begin{tabular}{|r|l|l|l|l|}
\hline ト-夕 \\
ル
\end{tabular} & & & & & \\
\hline
\end{tabular}

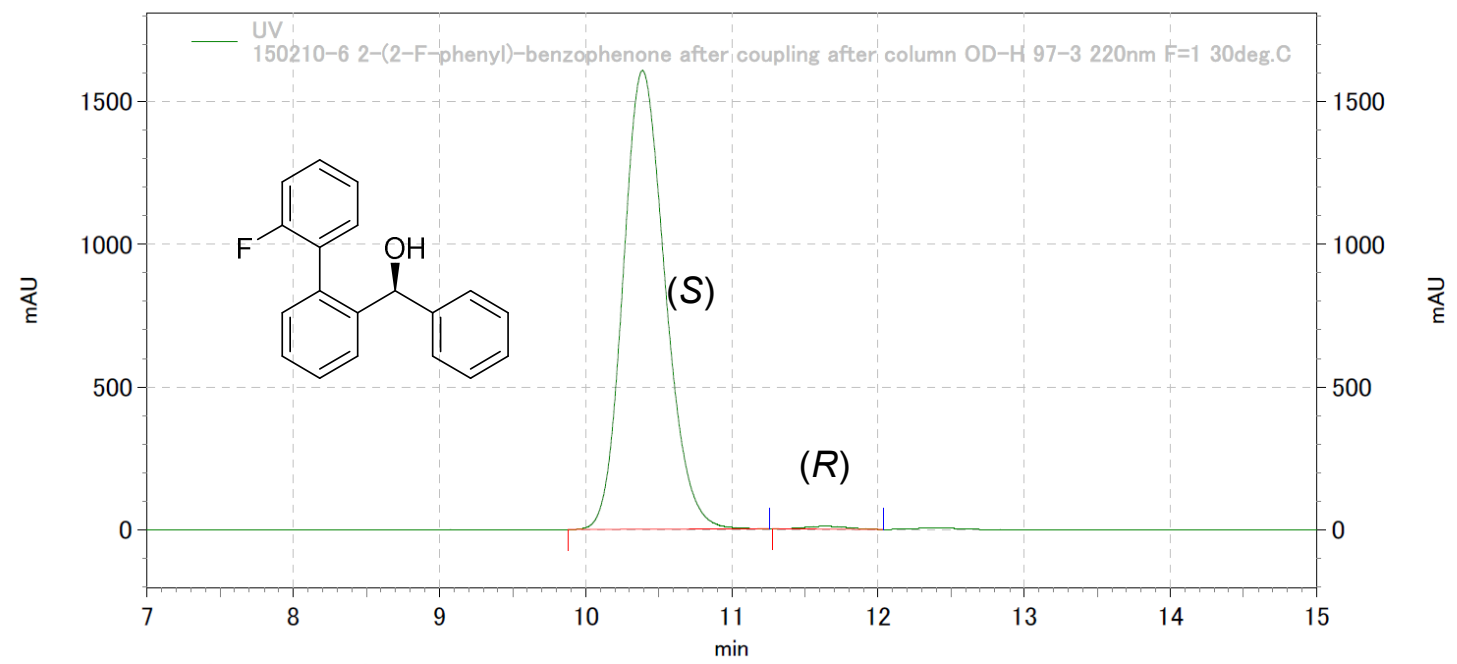

150210-6 2-(2-F-phenyl)-benzophenone after coupling after column OD-H 97-3 220nm F=1 30deg. C

\begin{tabular}{|c|c|c|c|c|c|}
\hline $\begin{array}{l}\text { UV結果 } \\
\text { Pk } \\
\text { No. }\end{array}$ & R. T. & Area & Area\% & B. C. & Symmetry \\
\hline $\begin{array}{l}1 \\
2\end{array}$ & $\begin{array}{l}10.390 \\
11.640\end{array}$ & $\begin{array}{r}128283334 \\
781437\end{array}$ & $\begin{array}{r}99.395 \\
0.605\end{array}$ & $\begin{array}{l}\text { MM } \\
\text { MM }\end{array}$ & $\begin{array}{l}0.00000 \\
0.00000\end{array}$ \\
\hline $\begin{array}{r}r-タ \\
ル\end{array}$ & & 129064771 & 100.000 & & \\
\hline
\end{tabular}




\section{(S)-6-Phenyl-6H-benzo[c]chromene (9)}

(OJ-H, Hex/2-PrOH=98/2, F=1.0 mL/min, 220 mm, 30deg.C)

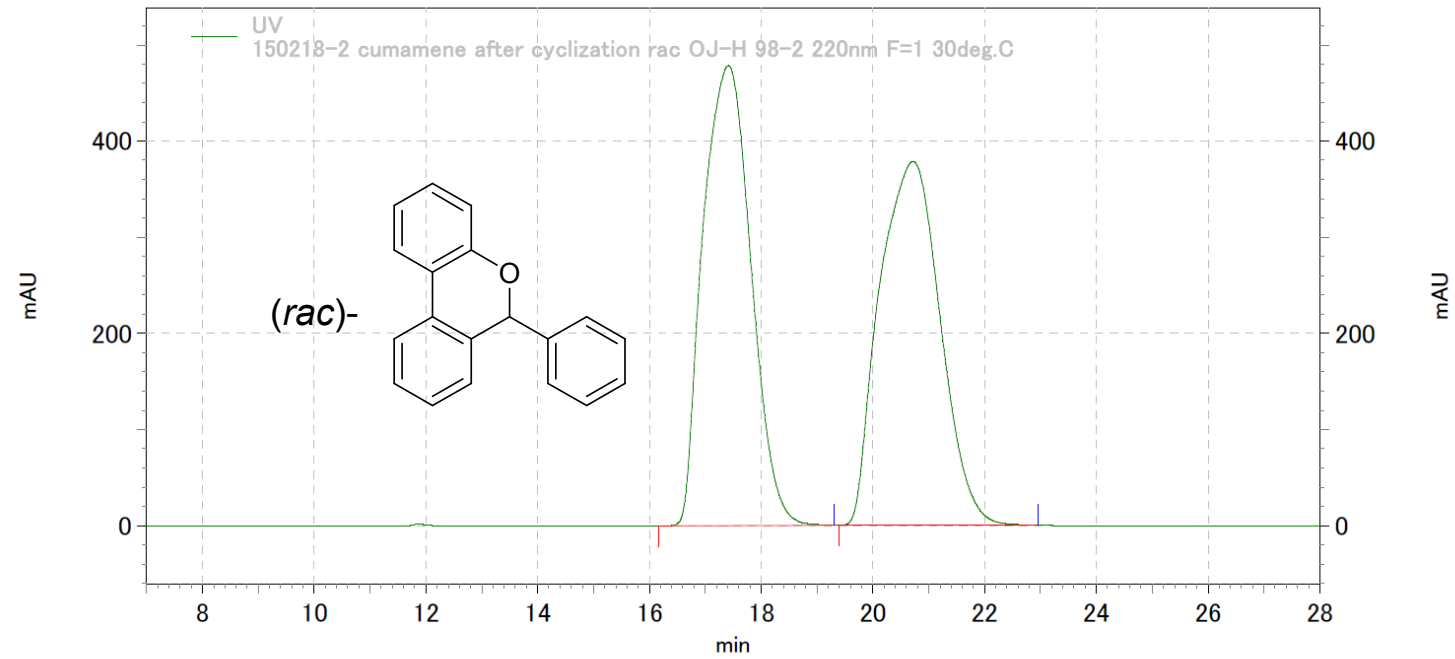

150218-2 cumamene after cyclization rac 0J-H 98-2 220nm F=1 30deg. C

\begin{tabular}{|c|c|c|c|c|c|}
\hline $\begin{array}{l}\text { UV } \\
\text { Pk } \\
\text { No. }\end{array}$ & R. T. & Area & Area\% & B. C. & Symmetry \\
\hline $\begin{array}{l}1 \\
2\end{array}$ & $\begin{array}{l}17.413 \\
20.717\end{array}$ & $\begin{array}{l}114307131 \\
114491224\end{array}$ & $\begin{array}{l}49.960 \\
50.040\end{array}$ & $\begin{array}{l}\text { MM } \\
\text { MM }\end{array}$ & $\begin{array}{l}0.00000 \\
0.00000\end{array}$ \\
\hline
\end{tabular}

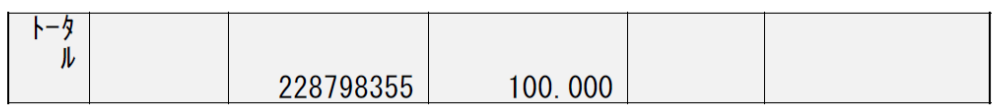

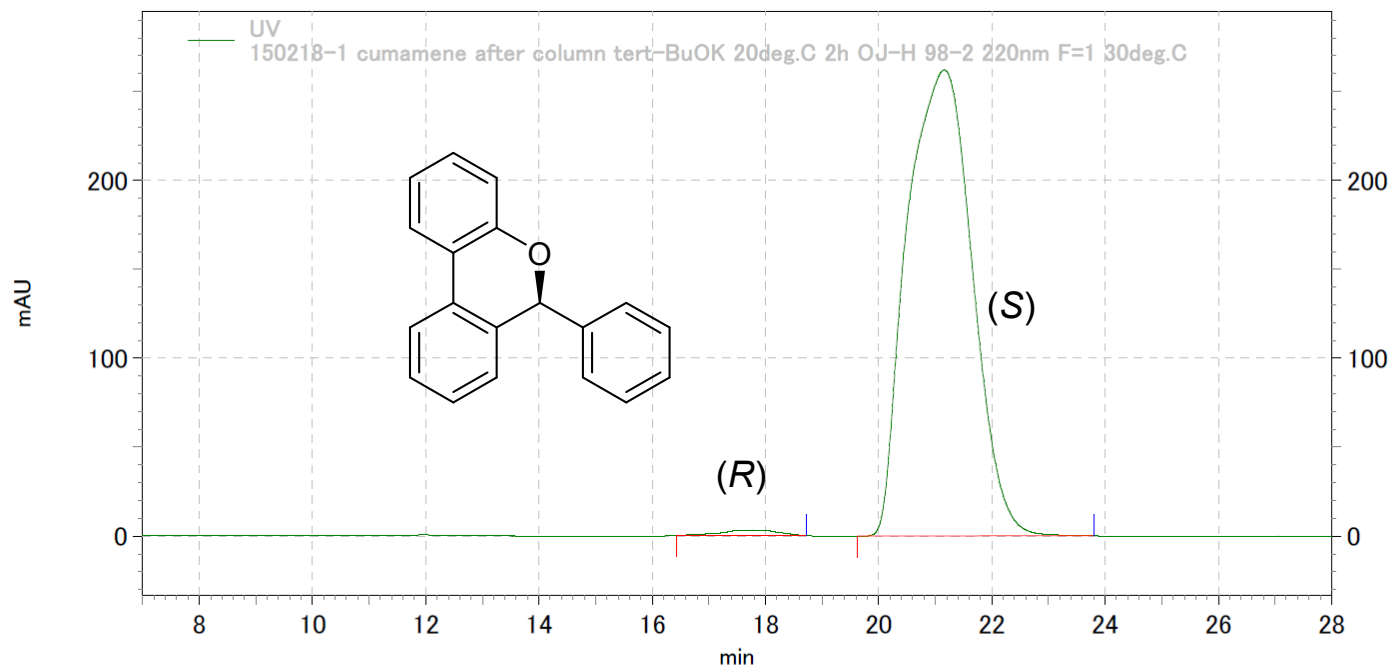

150218-1 cumamene after column tert-Bu0K 20deg. C 2h 0J-H 98-2 220nm F=1 30deg. C

\begin{tabular}{|c|c|c|c|c|c|}
\hline $\begin{array}{l}\text { UV } \\
\text { Pk } \\
\text { No. }\end{array}$ & R. T. & Area & Area\% & B. C. & Symmetry \\
\hline $\begin{array}{l}1 \\
2\end{array}$ & $\begin{array}{l}17.670 \\
21.160\end{array}$ & $\begin{array}{r}865531 \\
83874454\end{array}$ & $\begin{array}{r}1.021 \\
98.979\end{array}$ & $\begin{array}{l}\mathrm{MM} \\
\mathrm{MM}\end{array}$ & $\begin{array}{l}0.00000 \\
0.00000\end{array}$ \\
\hline $\begin{array}{r}r-8 \\
\pi\end{array}$ & & 84739985 & 100.000 & & \\
\hline
\end{tabular}




\section{(4-Chlorophenyl)(phenyl)methanol (11a)}

(AD-H, Hex/2-PrOH=97/3, F=1.0 mL/min, 220 nm, 30deg.C)

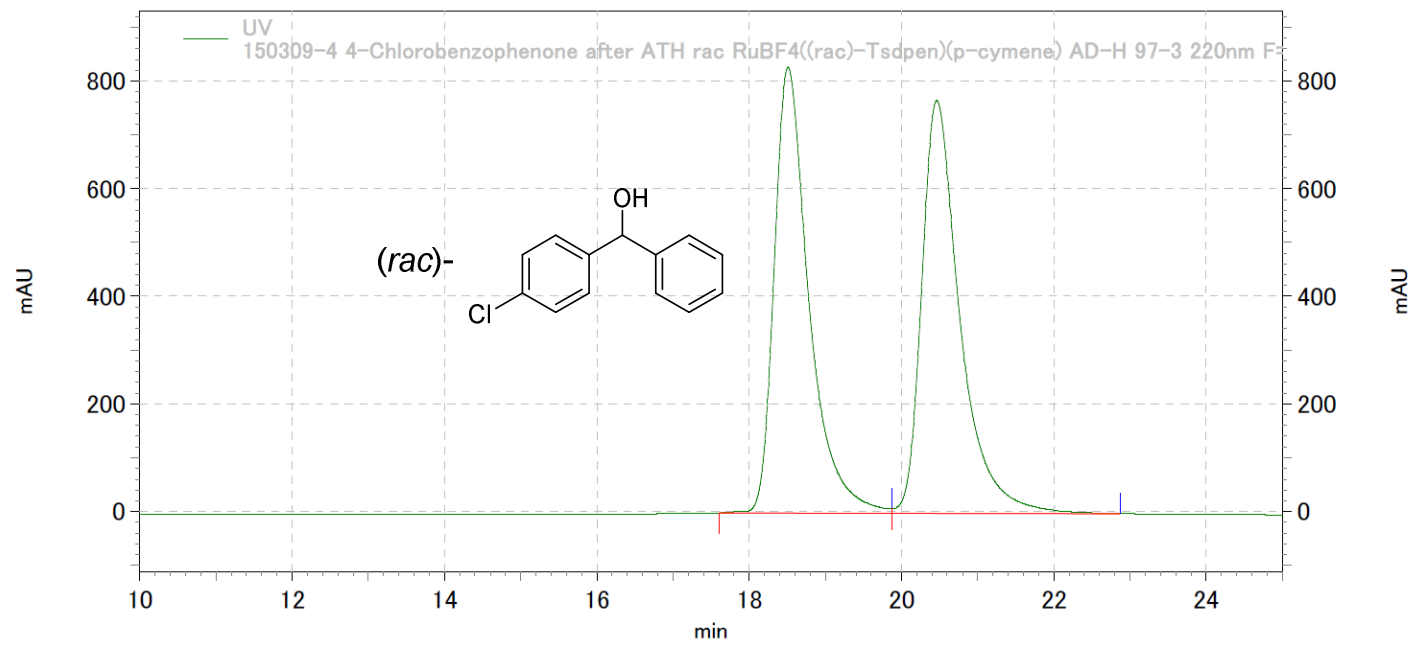

150309-4 4-Chlorobenzophenone after ATH rac RuBF4 ( $(\mathrm{rac})-T$ sdpen) ( $p$-cymene) AD-H 97-3 $220 \mathrm{~nm} F=1.0$ 30deg. C

\begin{tabular}{|c|c|c|c|c|c|}
\hline $\begin{array}{l}\text { UV結身 } \\
\text { Pk } \\
\text { No. }\end{array}$ & R. T. & Area & Area\% & B. C. & Symmetry \\
\hline $\begin{array}{l}1 \\
2\end{array}$ & $\begin{array}{l}18.510 \\
20.463\end{array}$ & $\begin{array}{l}105197605 \\
107192046\end{array}$ & $\begin{array}{l}49.530 \\
50.470\end{array}$ & $\begin{array}{l}\mathrm{MX} \\
\mathrm{XM}\end{array}$ & $\begin{array}{l}0.00000 \\
0.00000\end{array}$ \\
\hline $\begin{array}{r}r-夕 \\
ル\end{array}$ & & 212389651 & 100.000 & & \\
\hline
\end{tabular}

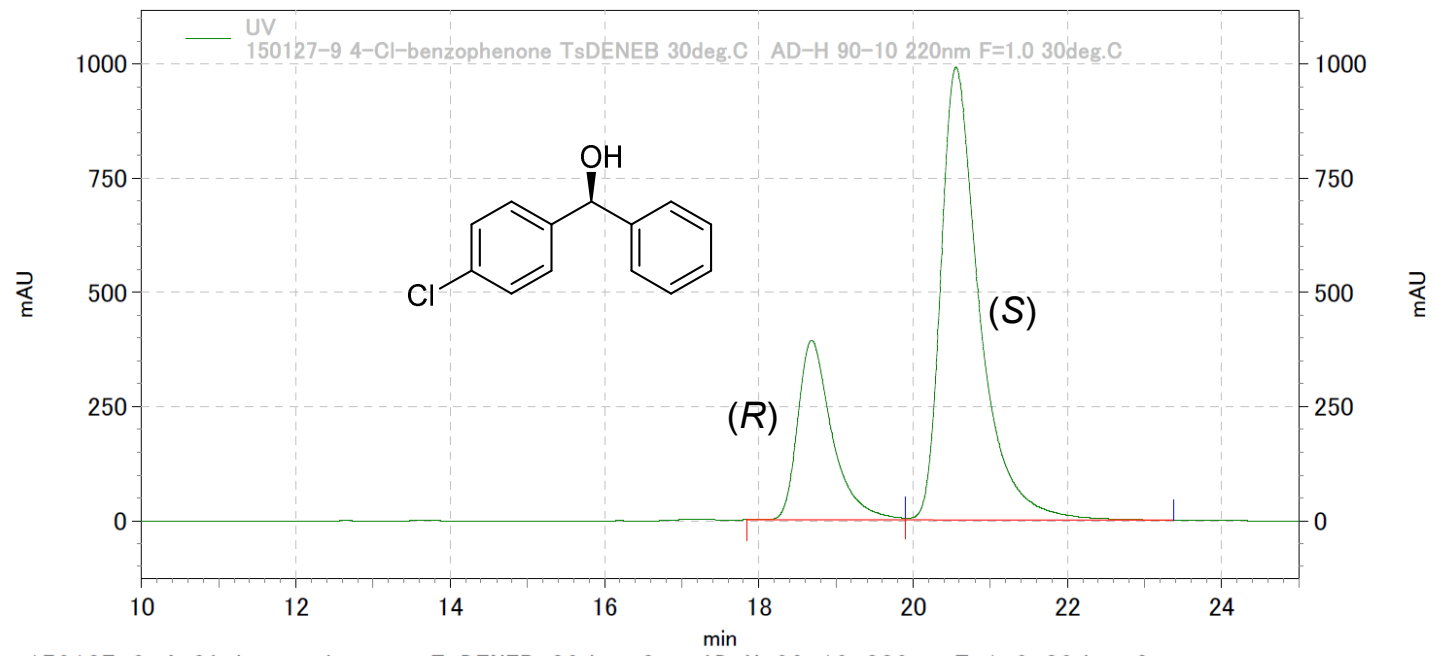

150127-9 4-Cl-benzophenone TsDENEB 30deg. C AD-H 90-10 220nm $F=1.0$ 30deg. C

\begin{tabular}{|c|c|c|c|c|c|}
\hline $\begin{array}{l}\text { UV結身 } \\
\text { Pk } \\
\text { No. }\end{array}$ & R. T. & Area & Area\% & B. C. & Symmetry \\
\hline $\begin{array}{l}1 \\
2\end{array}$ & $\begin{array}{l}18.683 \\
20.553\end{array}$ & $\begin{array}{r}48591555 \\
137493721\end{array}$ & $\begin{array}{l}26.113 \\
73.887\end{array}$ & $\begin{array}{l}\mathrm{Mx} \\
\mathrm{XM}\end{array}$ & $\begin{array}{l}0.00000 \\
0.00000\end{array}$ \\
\hline $\begin{array}{r}r-夕 \\
ル\end{array}$ & & 186085276 & 100.000 & & \\
\hline
\end{tabular}




\section{(3,4-Dichlorophenyl)(phenyl)methanol (11b)}

(OJ-H, Hex/2-PrOH=90/10, F=1.0 mL/min, $220 \mathrm{~nm}$, 30deg.C)

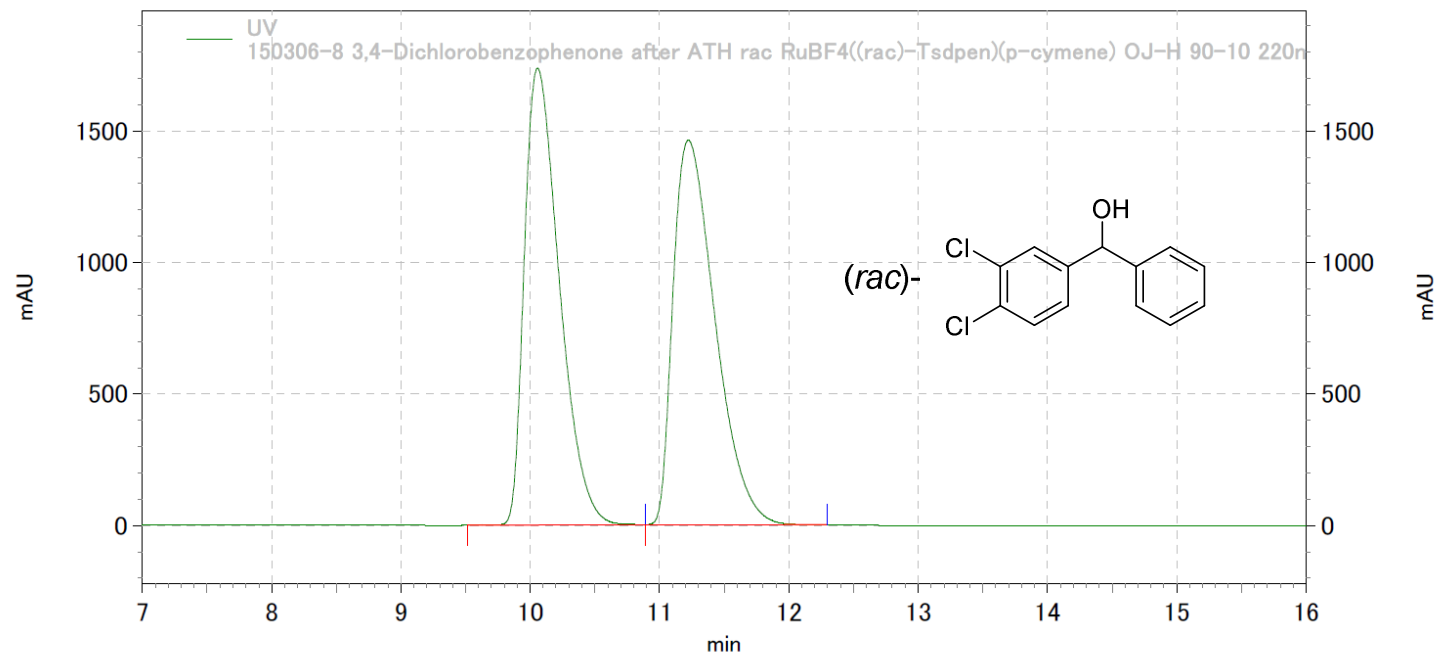

150306-8 3, 4-Dichlorobenzophenone after ATH rac RuBF4 ( (rac)-Tsdpen) (p-cymene) OJ-H 90-10 220nm $F=1.0$ 30deg. C

\begin{tabular}{rrrrrr}
$\begin{array}{l}\text { UV 結果 } \\
\text { Pk }\end{array}$ & R. T. & Area & Area\% & B. C. & Symmetry \\
No. & & & & & \\
\hline 1 & 10.057 & 130889329 & 49.743 & Mx & 0.00000 \\
2 & 11.223 & 132243961 & 50.257 & xM & 0.00000
\end{tabular}

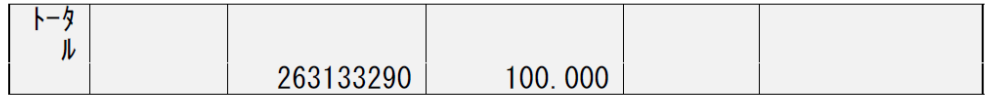

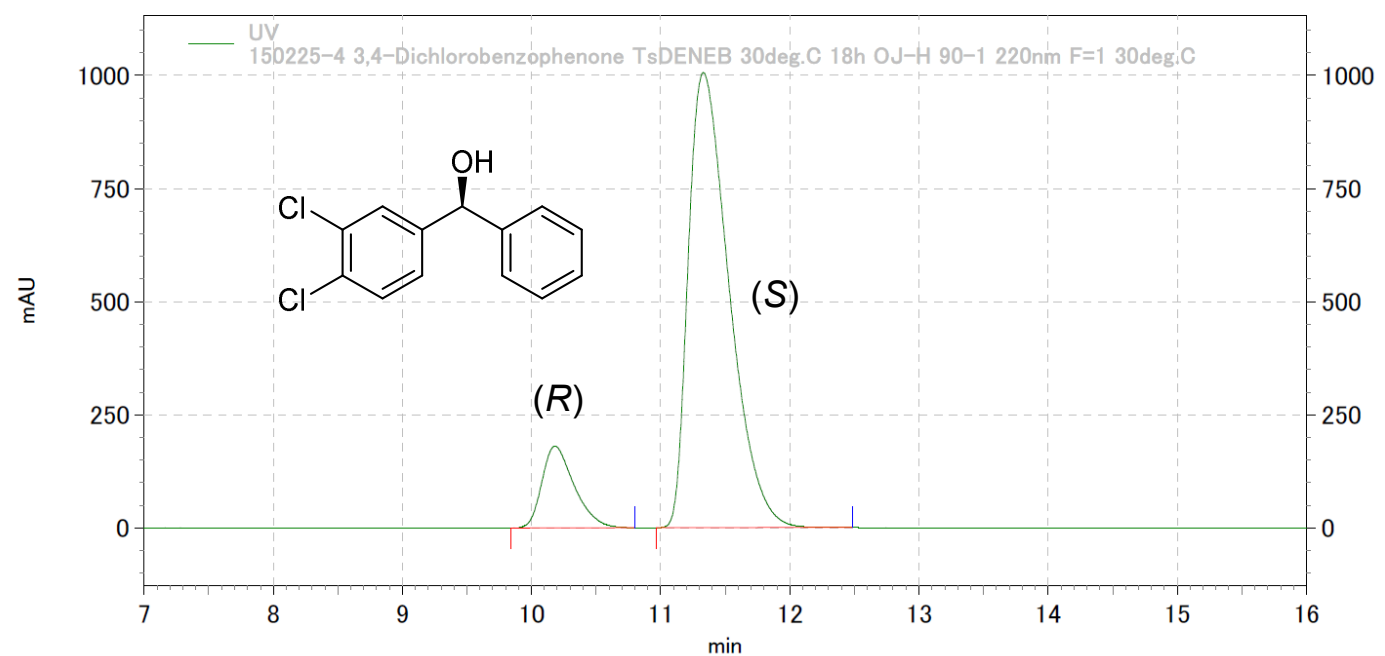

150225-4 3,4-Dichlorobenzophenone TsDENEB 30deg. C 18h 0J-H 90-1 220nm F=1 30deg. C

\begin{tabular}{|c|c|c|c|c|c|}
\hline $\begin{array}{l}\text { UV結茅 } \\
\text { Pk } \\
\text { No. }\end{array}$ & R. T. & Area & Area\% & B. C. & Symmetry \\
\hline $\begin{array}{l}1 \\
2\end{array}$ & $\begin{array}{l}10.180 \\
11.330\end{array}$ & $\begin{array}{l}12486120 \\
88195771\end{array}$ & $\begin{array}{l}\text { 12. } 402 \\
87.598\end{array}$ & $\begin{array}{l}\text { MM } \\
\text { MM }\end{array}$ & $\begin{array}{l}0.00000 \\
0.00000\end{array}$ \\
\hline $\begin{array}{r}r-8 \\
ル\end{array}$ & & 100681891 & 100.000 & & \\
\hline
\end{tabular}




\section{(S)-(3,4-Difluorophenyl)(phenyl)methanol (11c)}

(OD-H, Hex/2-PrOH=97/3, F=1.0 mL/min, $220 \mathrm{~nm}, 30$ deg.C)

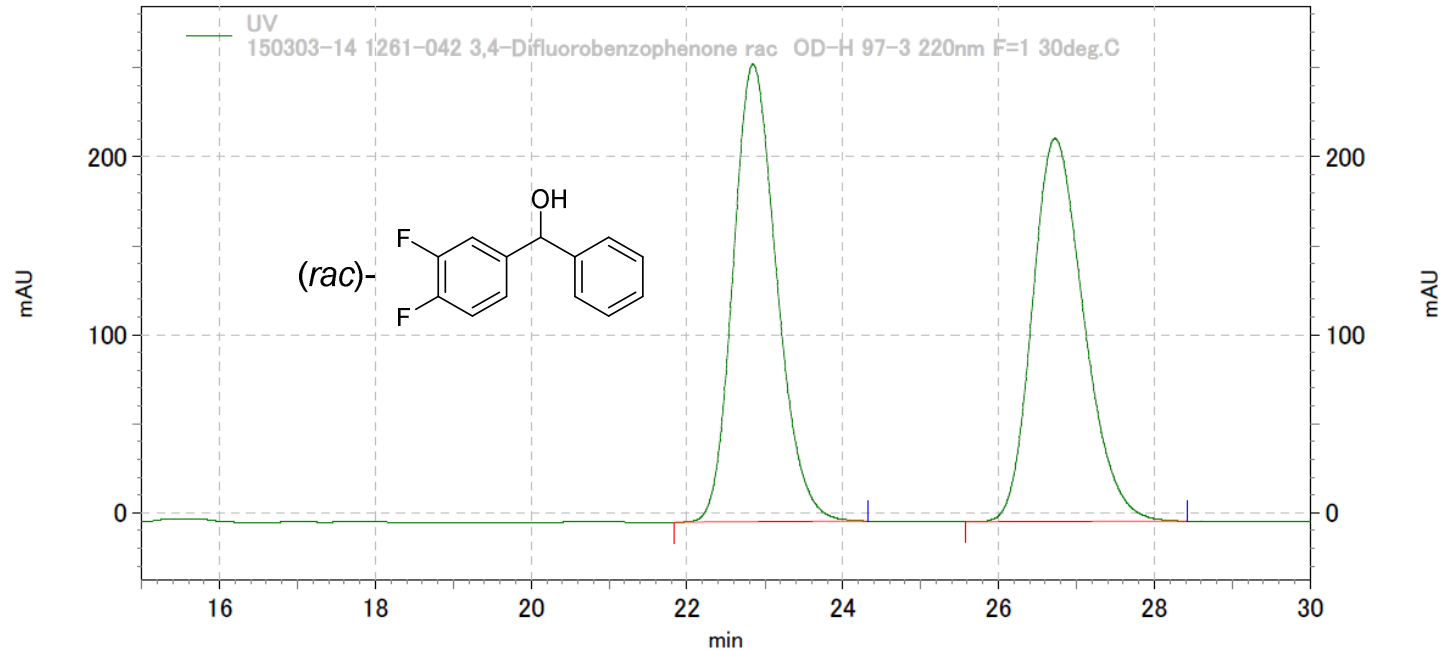

150303-14 1261-042 3, 4-Difluorobenzophenone rac OD-H 97-3 220nm F=1 30deg. C

\begin{tabular}{|c|c|c|c|c|c|}
\hline $\begin{array}{l}\text { UV結果 } \\
\text { Pk } \\
\text { No. }\end{array}$ & R. T. & Area & Area\% & B. C. & Symmetry \\
\hline 1 & $\begin{array}{l}22.847 \\
26.727\end{array}$ & $\begin{array}{l}39999463 \\
39610863\end{array}$ & $\begin{array}{l}50.244 \\
49.756\end{array}$ & $\begin{array}{l}\text { MM } \\
\text { MM }\end{array}$ & $\begin{array}{l}0.00000 \\
0.00000\end{array}$ \\
\hline $\begin{array}{r}r-3 \\
\|\end{array}$ & & 79610326 & 100.000 & & \\
\hline
\end{tabular}

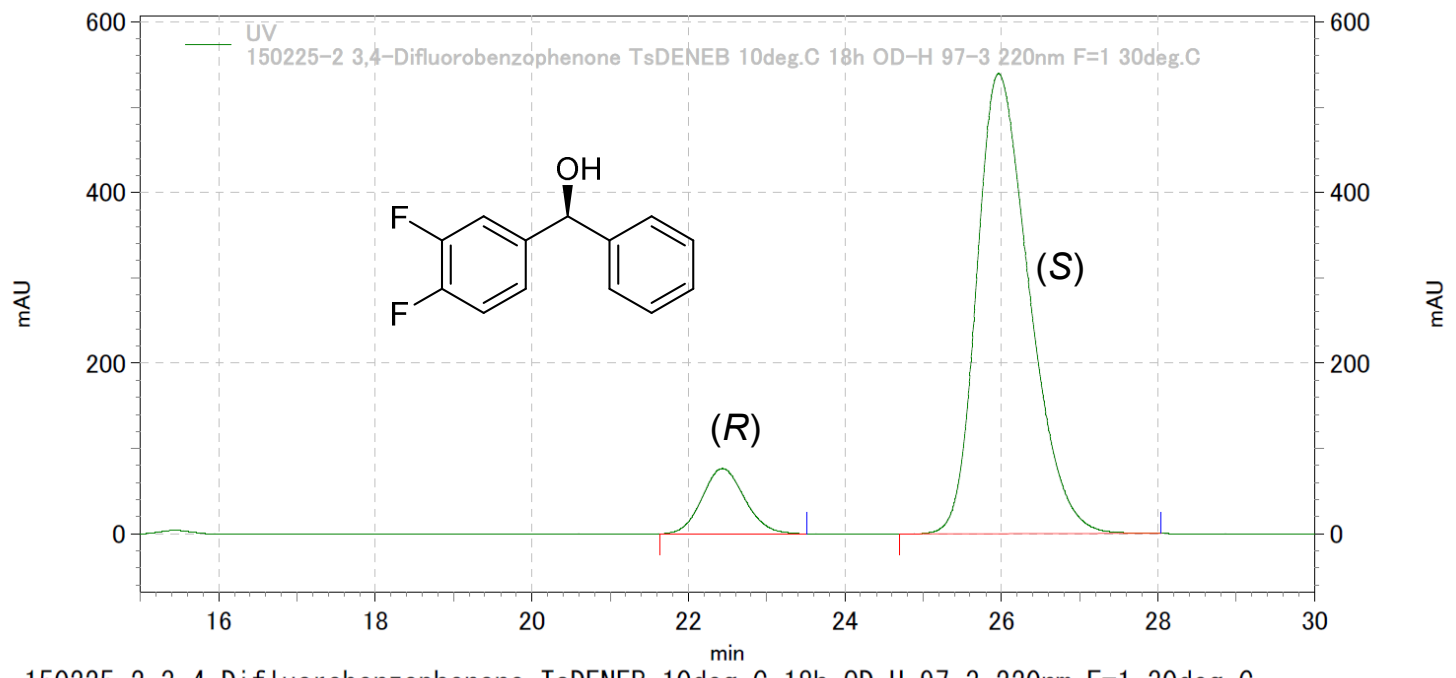

150225-2 3,4-Difluorobenzophenone TsDENEB 10deg. C 18h OD-H 97-3 220nm F=1 30deg. C

\begin{tabular}{|c|c|c|c|c|c|}
\hline $\begin{array}{l}\text { UV 結果 } \\
\text { Pk } \\
\text { No. }\end{array}$ & R. T. & Area & Area\% & B. C. & Symmetry \\
\hline $\begin{array}{l}1 \\
2\end{array}$ & $\begin{array}{l}22.433 \\
25.963\end{array}$ & $\begin{array}{r}11529214 \\
100276414\end{array}$ & $\begin{array}{l}10.312 \\
89.688\end{array}$ & $\begin{array}{l}\text { MM } \\
\text { MM }\end{array}$ & $\begin{array}{l}0.00000 \\
0.00000\end{array}$ \\
\hline $\begin{array}{r}\text { r-夕 } \\
\pi\end{array}$ & & 111805628 & 100.000 & & \\
\hline
\end{tabular}




\section{(4-Nitrophenyl)(phenyl)methanol (11d)}

(AD-H, Hex/2-PrOH=90/10, F=1.0 mL/min, 220 nm, 30deg.C)

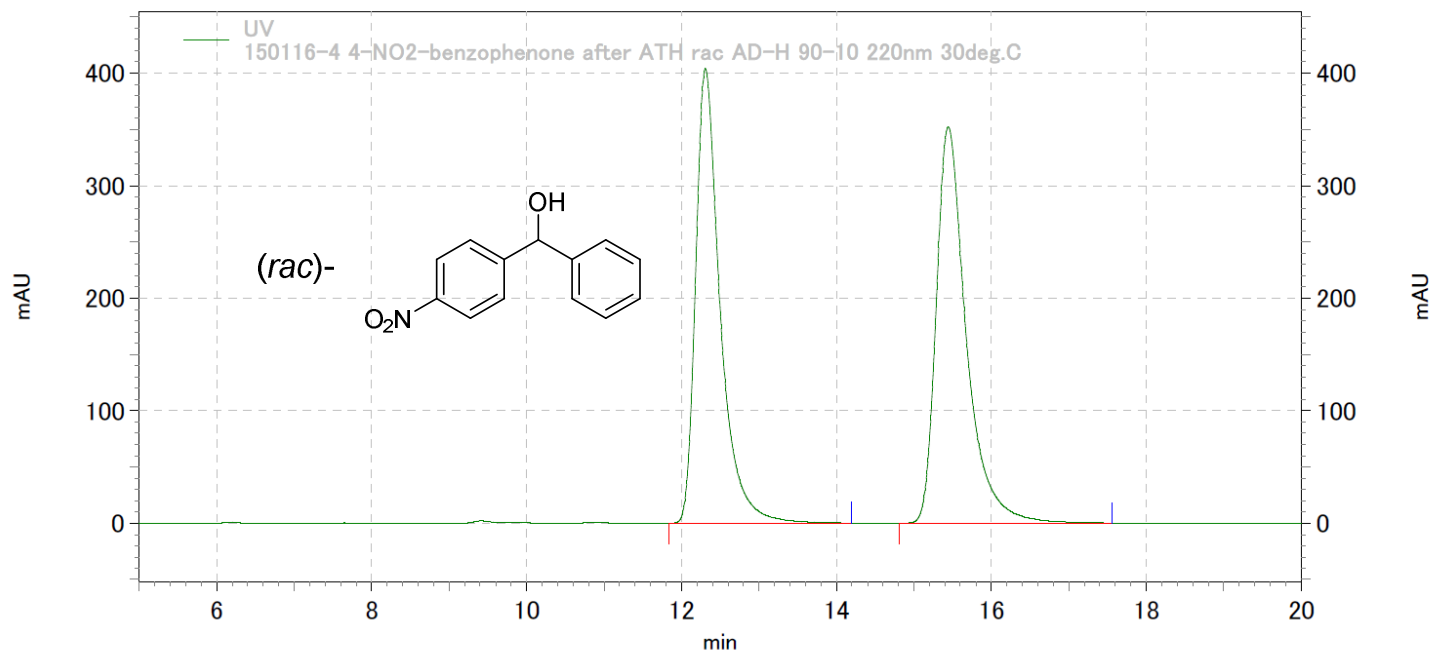

150116-4 4-N02-benzophenone after ATH rac AD-H 90-10 220nm 30deg. C

\begin{tabular}{|c|c|c|c|c|c|}
\hline $\begin{array}{l}\text { UV結果 } \\
\text { Pk } \\
\text { No. }\end{array}$ & R. T. & Area & Area\% & B. C. & Symmetry \\
\hline $\begin{array}{l}1 \\
2\end{array}$ & $\begin{array}{l}12.310 \\
15.443\end{array}$ & $\begin{array}{l}35592019 \\
38027201\end{array}$ & $\begin{array}{l}48.346 \\
51.654\end{array}$ & $\begin{array}{l}\mathrm{MM} \\
\mathrm{MM}\end{array}$ & $\begin{array}{l}0.00000 \\
0.00000\end{array}$ \\
\hline $\begin{array}{r}r-夕 \\
\mu\end{array}$ & & 73619220 & 100. 000 & & \\
\hline
\end{tabular}

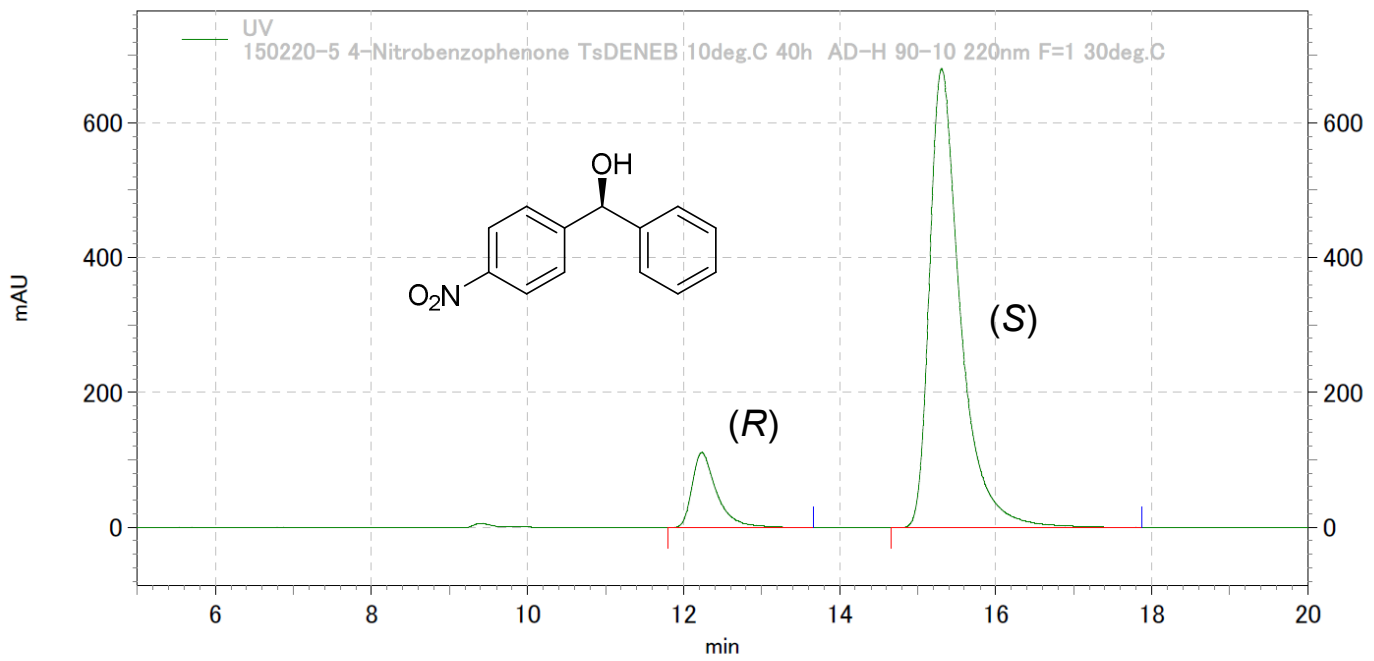

150220-5 4-Nitrobenzophenone TsDENEB 10deg. C 40h AD-H 90-10 220nm F=1 30deg. C

\begin{tabular}{|c|c|c|c|c|c|}
\hline $\begin{array}{l}\text { UV結身 } \\
\mathrm{Pk} \\
\text { No. }\end{array}$ & R. T. & Area & Area\% & B. C. & Symmetry \\
\hline $\begin{array}{l}1 \\
2\end{array}$ & $\begin{array}{l}12.233 \\
15.307\end{array}$ & $\begin{array}{r}9739803 \\
74800392\end{array}$ & $\begin{array}{l}11.521 \\
88.479\end{array}$ & $\begin{array}{l}\mathrm{MM} \\
\mathrm{MM}\end{array}$ & $\begin{array}{l}0.00000 \\
0.00000\end{array}$ \\
\hline $\begin{array}{r}r-y \\
\mu\end{array}$ & & 84540195 & 100.000 & & \\
\hline
\end{tabular}




\section{(4-Chloro-3-nitrophenyl)(phenyl)methanol (11e)}

(OJ-H, Hex/2-PrOH=90/10, F=1.0 mL/min, 220 nm, 30deg.C)

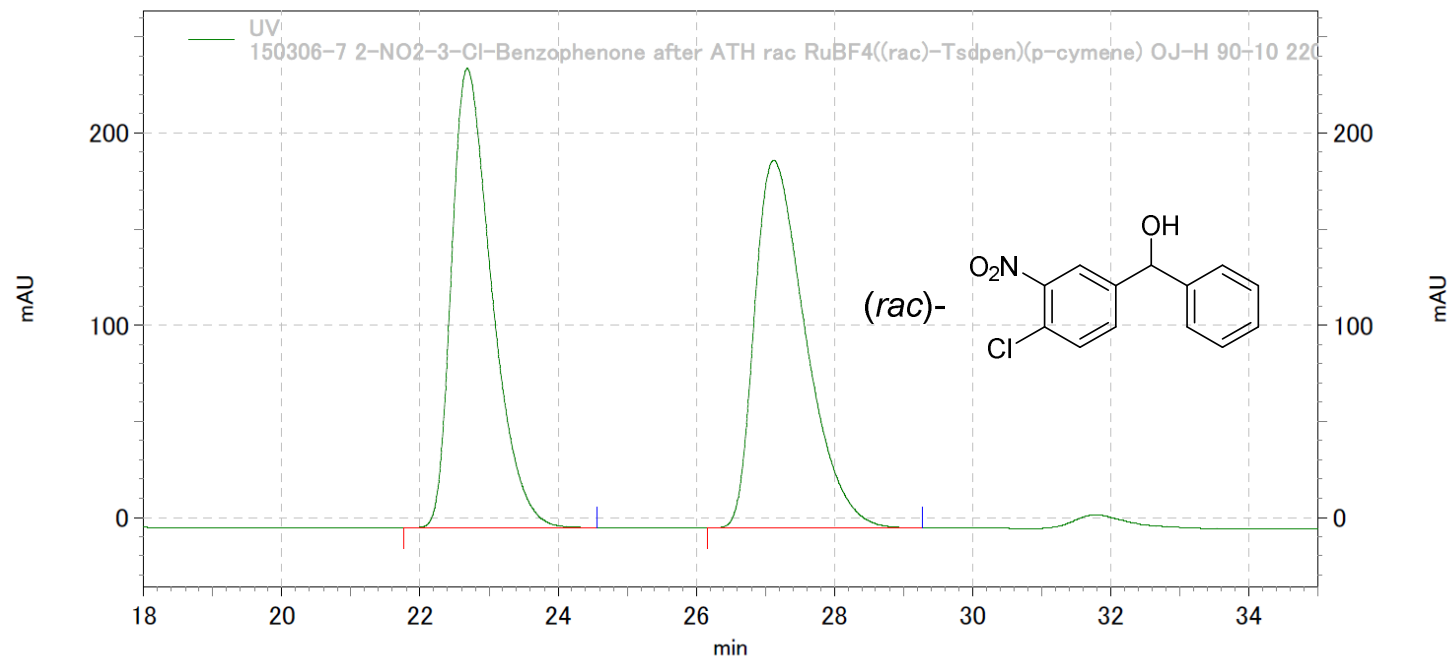

150306-7 2-N02-3-Cl-Benzophenone after ATH rac RuBF4 ( (rac)-Tsdpen) (p-cymene) 0J-H 90-10 $220 \mathrm{~nm} F=1.0$ 30deg. C

\begin{tabular}{|c|c|c|c|c|c|}
\hline $\begin{array}{l}\text { UV結身 } \\
\text { Pk } \\
\text { No. }\end{array}$ & R. T. & Area & Area\% & B. C. & Symmetry \\
\hline $\begin{array}{l}1 \\
2\end{array}$ & $\begin{array}{l}22.683 \\
27.123\end{array}$ & $\begin{array}{l}38647877 \\
39427863\end{array}$ & $\begin{array}{l}49.500 \\
50.500\end{array}$ & $\begin{array}{l}\mathrm{MM} \\
\mathrm{MM}\end{array}$ & $\begin{array}{l}0.00000 \\
0.00000\end{array}$ \\
\hline $\begin{array}{r}1-8 \\
\mu\end{array}$ & & 78075740 & 100.000 & & \\
\hline
\end{tabular}

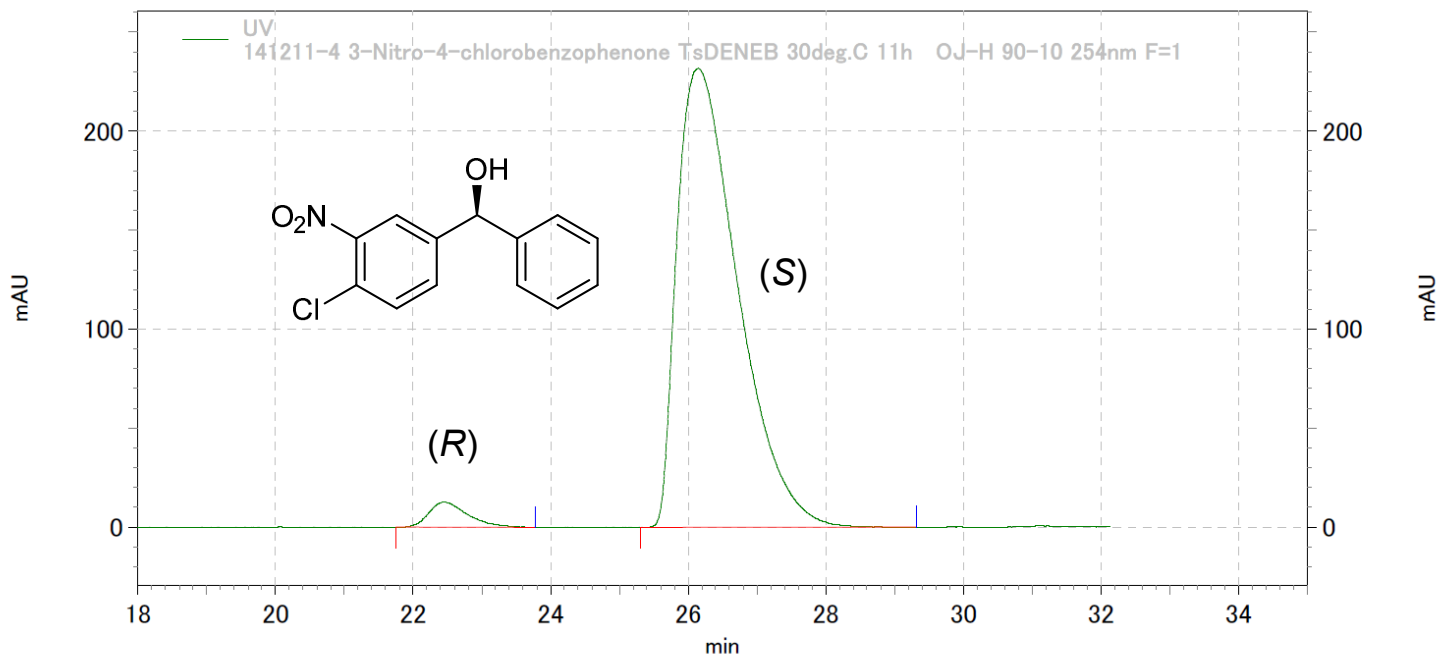

141211-4 3-Nitro-4-chlorobenzophenone TsDENEB 30deg. C 11h 0J-H 90-10 254nm F=1

\begin{tabular}{|c|c|c|c|c|c|}
\hline $\begin{array}{l}\text { UV結果 } \\
\text { Pk } \\
\text { No. }\end{array}$ & R. T. & Area & Area\% & B. C. & Symmetry \\
\hline $\begin{array}{l}1 \\
2\end{array}$ & $\begin{array}{l}22.453 \\
26.147\end{array}$ & $\begin{array}{r}1982425 \\
56908186\end{array}$ & $\begin{array}{r}3.366 \\
96.634\end{array}$ & $\begin{array}{l}\mathrm{MM} \\
\mathrm{MM}\end{array}$ & $\begin{array}{l}0.00000 \\
0.00000\end{array}$ \\
\hline $\begin{array}{r}r-夕 \\
ル\end{array}$ & & 58890611 & 100.000 & & \\
\hline
\end{tabular}




\section{Phenyl(3,4,5-trifluorophenyl)methanol (11f)}

(OD-H, Hex/2-PrOH=95/5, F=1.0 mL/min, 220 nm, 30deg.C)

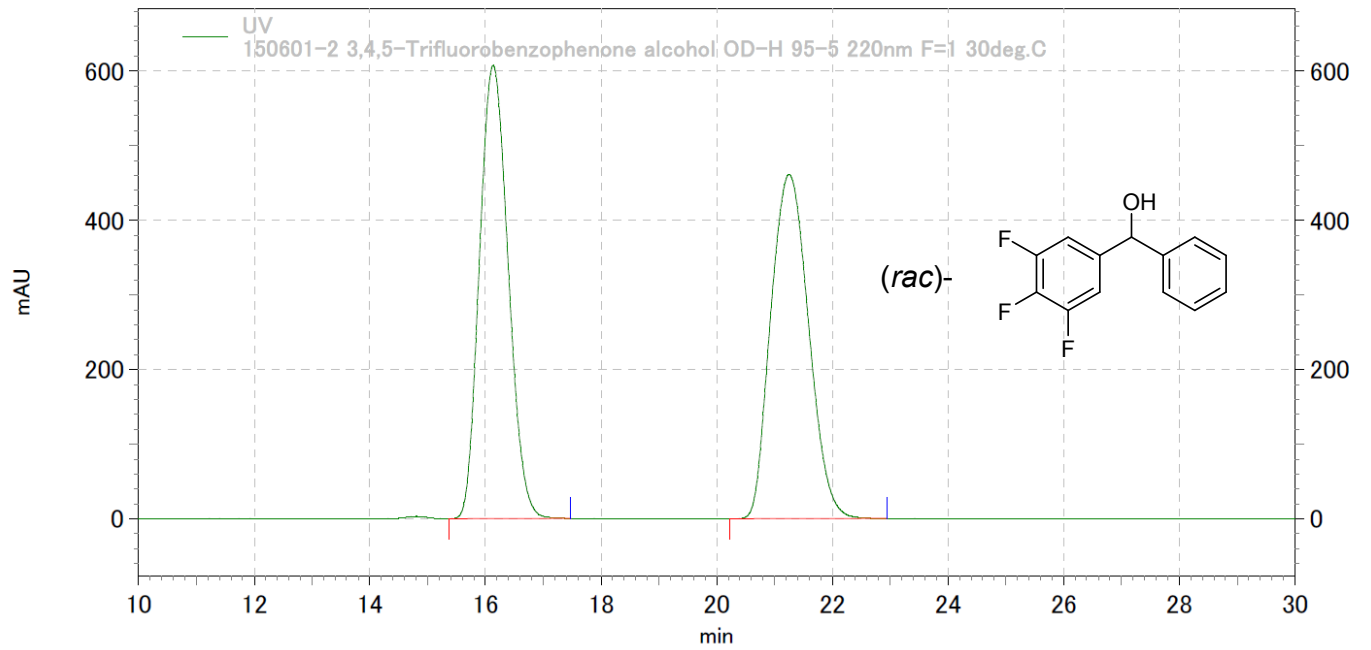

150601-2 3,4,5-Trifluorobenzophenone alcohol OD-H 95-5 220nm F=1 30deg. C

\begin{tabular}{|c|c|c|c|c|c|}
\hline $\begin{array}{l}\text { UV結果 } \\
\text { Pk } \\
\text { No. }\end{array}$ & R. T. & Area & Area\% & B. C. & Symmetry \\
\hline $\begin{array}{l}1 \\
2\end{array}$ & $\begin{array}{l}16.133 \\
21.253\end{array}$ & $\begin{array}{l}83683265 \\
83920056\end{array}$ & $\begin{array}{l}49.929 \\
50.071\end{array}$ & $\begin{array}{l}\text { MM } \\
\text { MM }\end{array}$ & $\begin{array}{l}0.00000 \\
0.00000\end{array}$ \\
\hline $\begin{array}{r}r-8 \\
\|\end{array}$ & & 167603321 & 100.000 & & \\
\hline
\end{tabular}

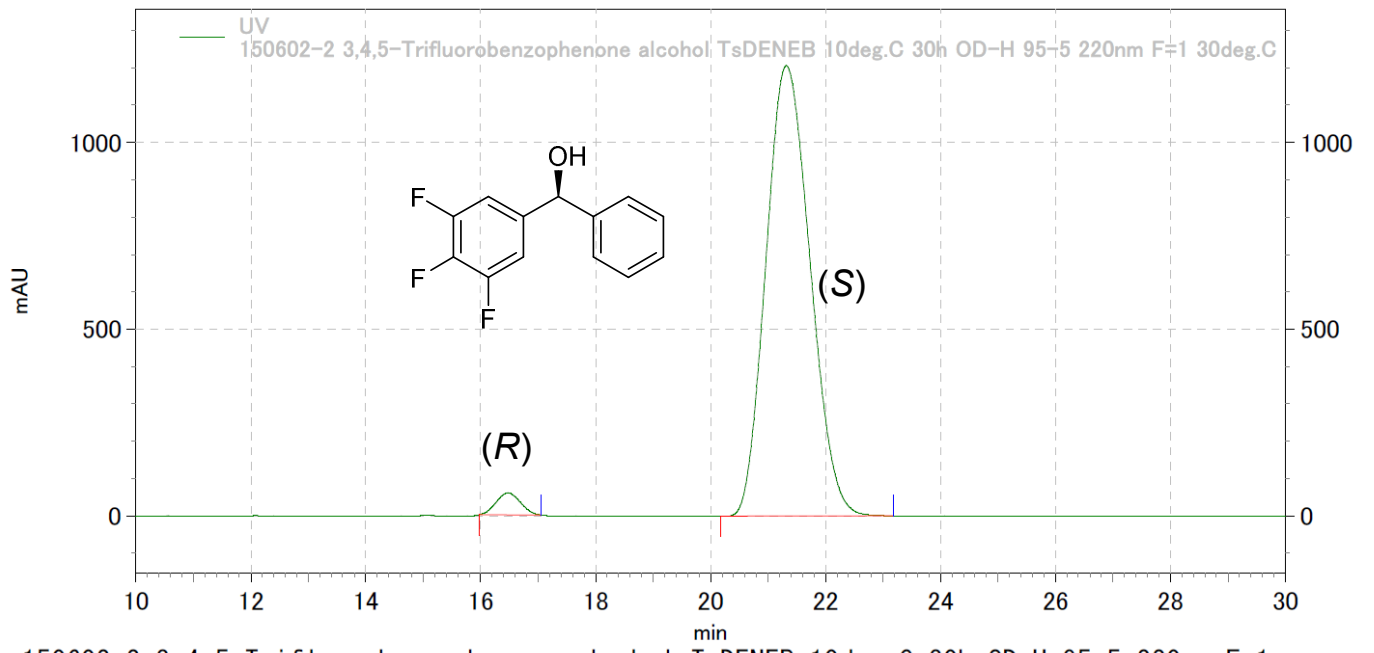

150602-2 3, 4,5-Trifluorobenzophenone alcohol TsDENEB 10deg. C 30h OD-H 95-5 220nm F=1 30deg. C

\begin{tabular}{|c|c|c|c|c|c|}
\hline $\begin{array}{l}\text { UV結果 } \\
\text { Pk } \\
\text { No. }\end{array}$ & R. T. & Area & Area\% & B. C. & Symmetry \\
\hline $\begin{array}{l}1 \\
2\end{array}$ & $\begin{array}{l}16.473 \\
21.317\end{array}$ & $\begin{array}{r}6931930 \\
257392737\end{array}$ & $\begin{array}{r}2.623 \\
97.377\end{array}$ & $\begin{array}{l}\overline{M M} \\
\mathrm{MM}\end{array}$ & $\begin{array}{l}0.00000 \\
0.00000\end{array}$ \\
\hline $\begin{array}{r}r-夕 \\
\mu\end{array}$ & & 264324667 & 100.000 & & \\
\hline
\end{tabular}




\section{(3,5-Dinitrophenyl)(phenyl)methanol (11g)}

(AS-H, Hex/2-PrOH=90/10, F=1.0 mL/min, $220 \mathrm{~nm}$, 30deg.C)

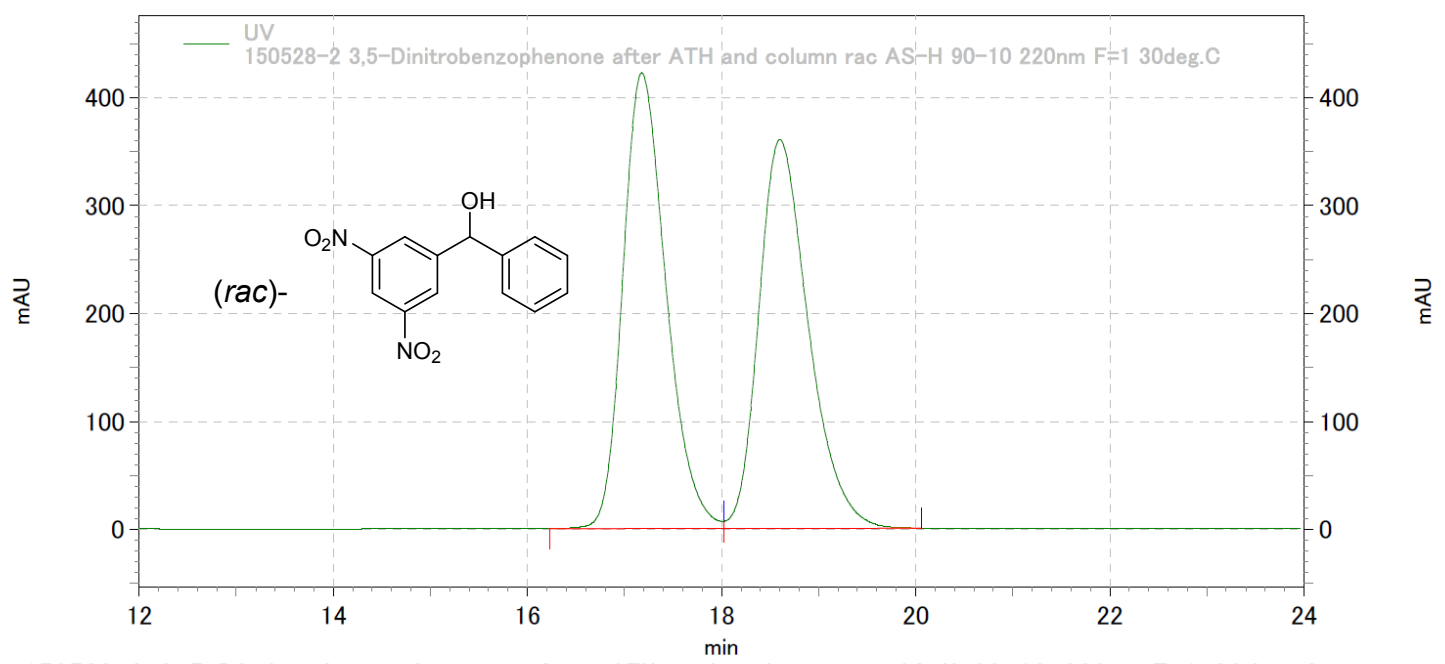

150528-2 3,5-Dinitrobenzophenone after ATH and column rac AS-H 90-10 220nm F=1 30deg. C

\begin{tabular}{rlllll}
$\begin{array}{l}\text { UV 結果 } \\
\text { Pk }\end{array}$ & R. T. & Area & Area\% & B. C. & Symmetry \\
No. & & & & & \\
\hline 1 & 17.180 & 52230953 & 50.484 & $M x$ & 0.00000 \\
2 & 18.600 & 51229853 & 49.516 & xM & 0.00000
\end{tabular}

\begin{tabular}{|r|r|r|r|r|r|}
\hline $\begin{array}{r}\text { r-夕夕 } \\
\|\end{array}$ & & 103460806 & 100.000 & & \\
\hline
\end{tabular}

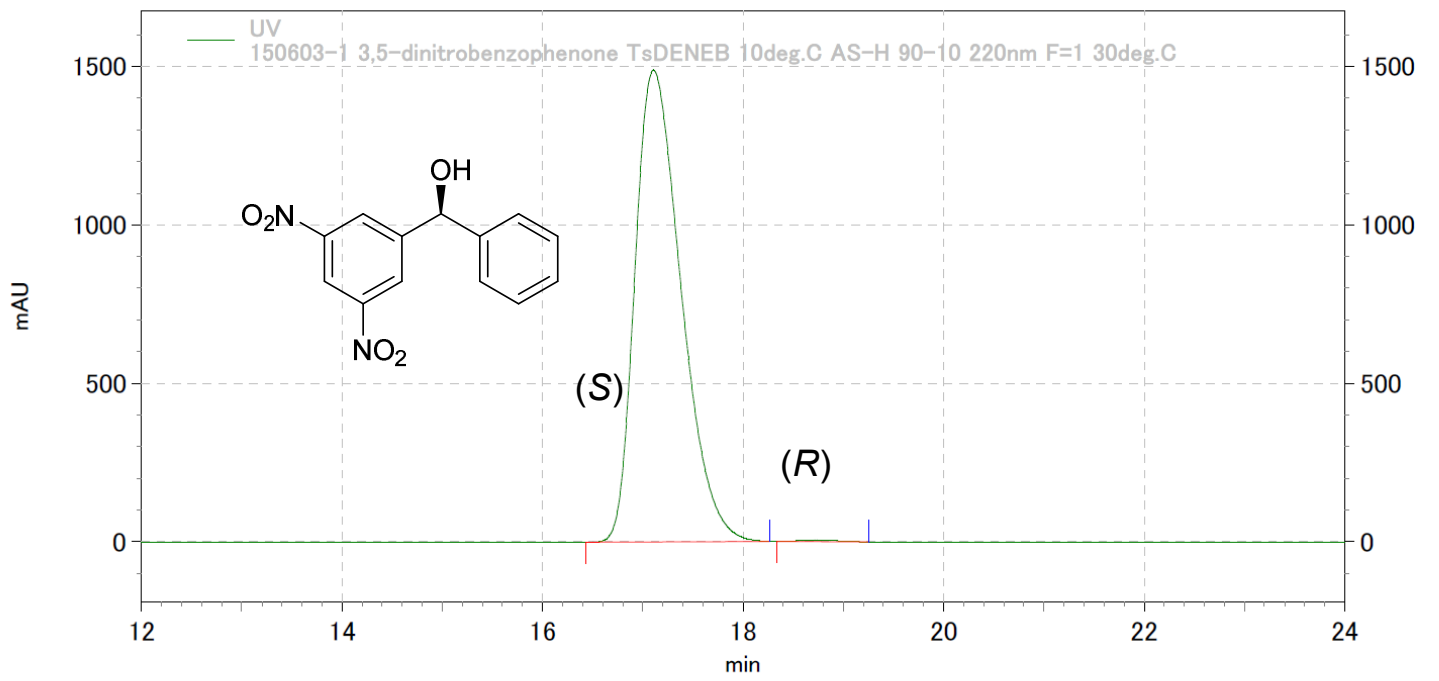

150603-1 3,5-dinitrobenzophenone TsDENEB 10deg. C AS-H 90-10 220nm F=1 30deg. C

\begin{tabular}{|c|c|c|c|c|c|}
\hline $\begin{array}{l}\text { UV 結 } \\
\text { Pk } \\
\text { No. }\end{array}$ & R. T. & Area & Area\% & B. C. & Symmetry \\
\hline $\begin{array}{l}1 \\
2\end{array}$ & $\begin{array}{l}17.107 \\
18.727\end{array}$ & $\begin{array}{r}190193858 \\
509586\end{array}$ & $\begin{array}{r}99.733 \\
0.267\end{array}$ & $\begin{array}{l}\mathrm{MM} \\
\mathrm{MM}\end{array}$ & $\begin{array}{l}0.00000 \\
0.00000\end{array}$ \\
\hline $\begin{array}{r}r-夕 \\
\mu\end{array}$ & & 190703444 & 100.000 & & \\
\hline
\end{tabular}




\section{(4-Methoxyphenyl)(phenyl)methanol (11h)}

(AD-H, Hex/2-PrOH=90/10, F=0.5 mL/min, $220 \mathrm{~nm}, 30$ deg.C)

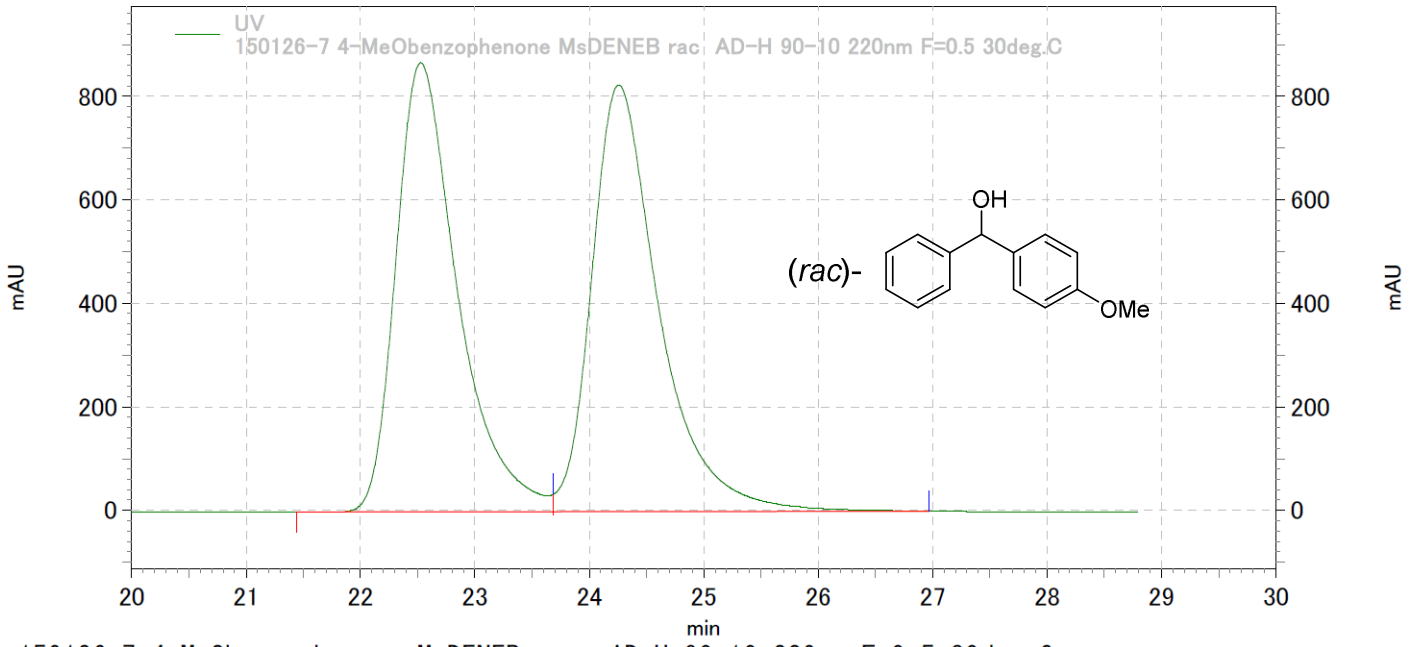

150126-7 4-MeObenzophenone MsDENEB rac AD-H 90-10 220nm $F=0.5$ 30deg. $C$

\begin{tabular}{rrrrrl}
$\begin{array}{l}\text { UV 結果 } \\
\text { Pk }\end{array}$ & R. T. & Area & Area\% & B. C. & Symmetry \\
No. & & & & & \\
\hline 1 & 22.527 & 130126209 & 49.210 & Mx & 0.00000 \\
2 & 24.257 & 134306685 & 50.790 & xM & 0.00000
\end{tabular}
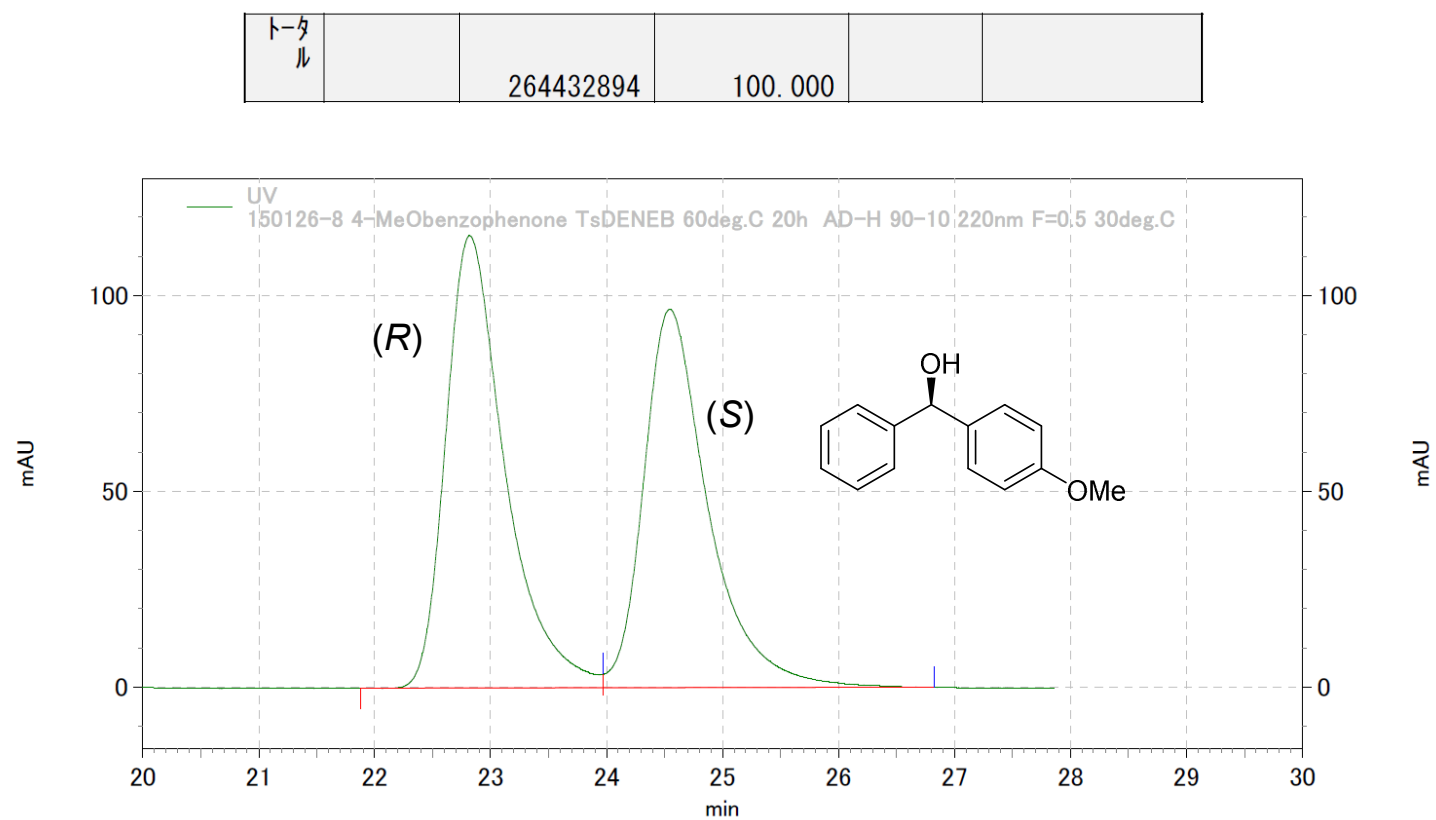

150126-8 4-MeObenzophenone TsDENEB 60deg. C 20h AD-H 90-10 220nm F=0. 5 30deg. C

\begin{tabular}{|c|c|c|c|c|c|}
\hline $\begin{array}{l}\text { UV結 } \operatorname{y} \\
\text { Pk } \\
\text { No. }\end{array}$ & R. T. & Area & Area\% & B. C. & Symmetry \\
\hline $\begin{array}{l}1 \\
2\end{array}$ & $\begin{array}{l}22.820 \\
24.547\end{array}$ & $\begin{array}{l}16421737 \\
14970708\end{array}$ & $\begin{array}{l}52.311 \\
47.689\end{array}$ & $\begin{array}{l}\overline{M x} \\
\mathrm{XM}\end{array}$ & $\begin{array}{l}0.00000 \\
0.00000\end{array}$ \\
\hline $\begin{array}{r}r-夕 \\
\mu\end{array}$ & & 31392445 & 100.000 & & \\
\hline
\end{tabular}




\section{(4-Chlorophenyl)(4-methoxyphenyl)methanol (11i)}

(OD-H, Hex/2-PrOH=95/5, F=0.5 mL/min, $220 \mathrm{~nm}$, 30deg.C)

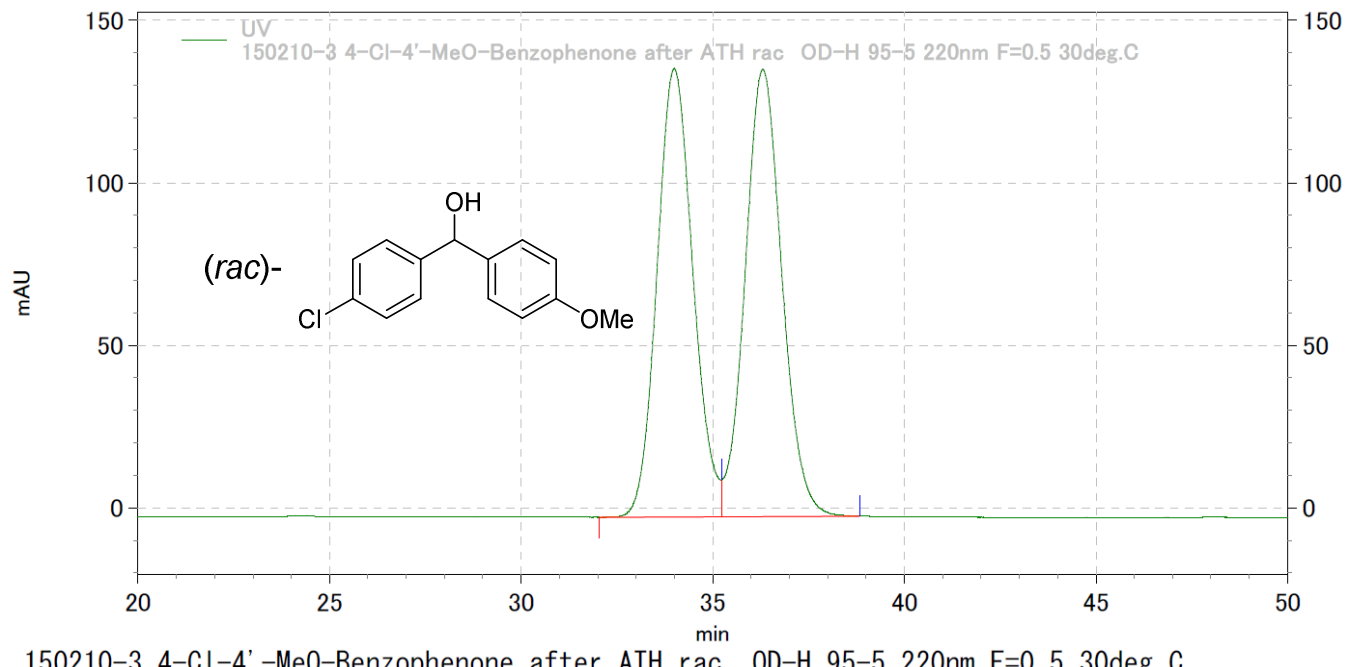

150210-3 4-CI-4'-Me0-Benzophenone after ATH rac 0D-H 95-5 220nm F=0. 5 30deg. C

\begin{tabular}{|c|c|c|c|c|c|}
\hline $\begin{array}{l}\text { UV結果 } \\
\text { Pk } \\
\text { No. }\end{array}$ & R. T. & Area & Area\% & B. C. & Symmetry \\
\hline $\begin{array}{l}1 \\
2\end{array}$ & $\begin{array}{l}33.993 \\
36.307\end{array}$ & $\begin{array}{l}55915402 \\
36869927\end{array}$ & $\begin{array}{l}49.344 \\
50.656\end{array}$ & $\begin{array}{l}\mathrm{Mx} \\
\mathrm{XM}\end{array}$ & $\begin{array}{l}0.00000 \\
0.00000\end{array}$ \\
\hline $\begin{array}{r}r-y \\
ル\end{array}$ & & 72785329 & 100.000 & & \\
\hline
\end{tabular}

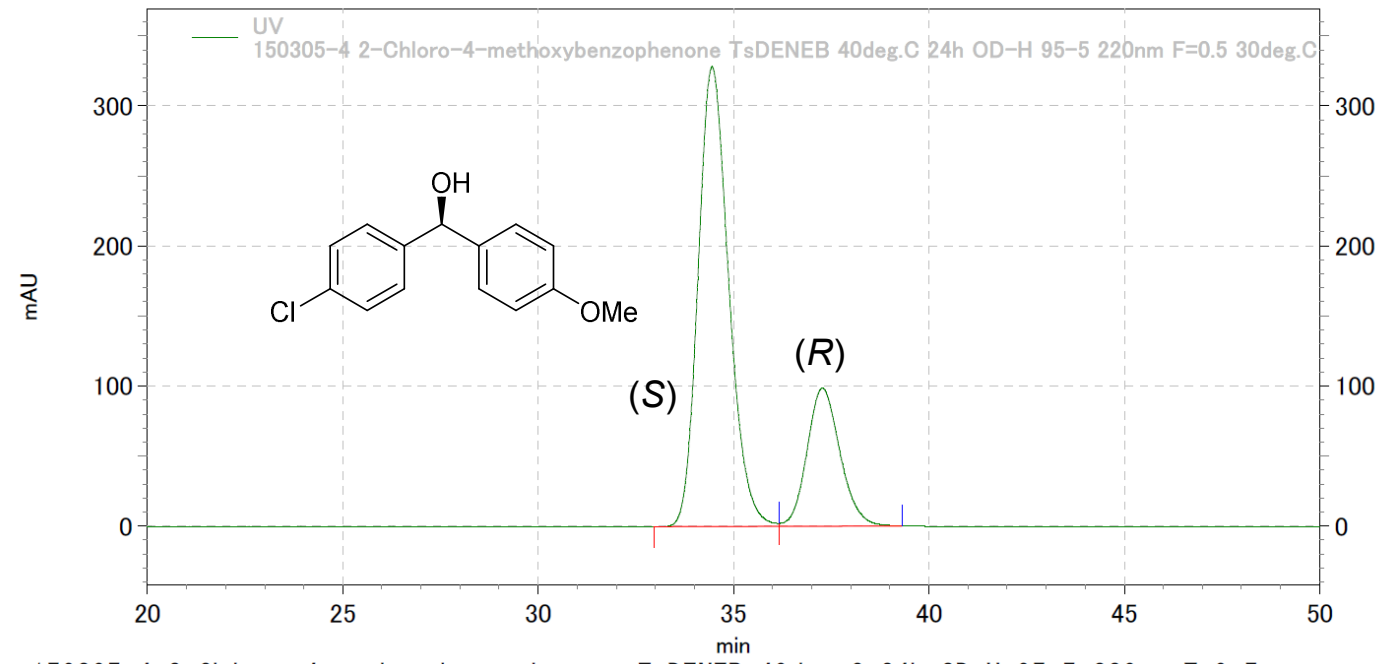

150305-4 2-Chloro-4-methoxybenzophenone TsDENEB 40deg. C 24h 0D-H 95-5 220nm F=0.5 30deg. C

\begin{tabular}{|c|c|c|c|c|c|}
\hline $\begin{array}{l}\text { UV結身 } \\
\text { Pk } \\
\text { No. }\end{array}$ & R. T. & Area & Area\% & B. C. & Symmetry \\
\hline $\begin{array}{l}1 \\
2\end{array}$ & $\begin{array}{l}34.453 \\
37.277\end{array}$ & $\begin{array}{l}71486123 \\
23102869\end{array}$ & $\begin{array}{l}75.576 \\
24.424\end{array}$ & $\begin{array}{l}\overline{M x} \\
\mathrm{XM}\end{array}$ & $\begin{array}{l}0.00000 \\
0.00000\end{array}$ \\
\hline $\begin{array}{r}r-y \\
\mu\end{array}$ & & 94588992 & 100.000 & & \\
\hline
\end{tabular}




\section{4-((4-Chlorophenyl)(hydroxy)methyl)phenol (11j)}

(OD-H, Hex/2-PrOH=90/10, F=1.0 mL/min, 220 nm, 30deg.C)

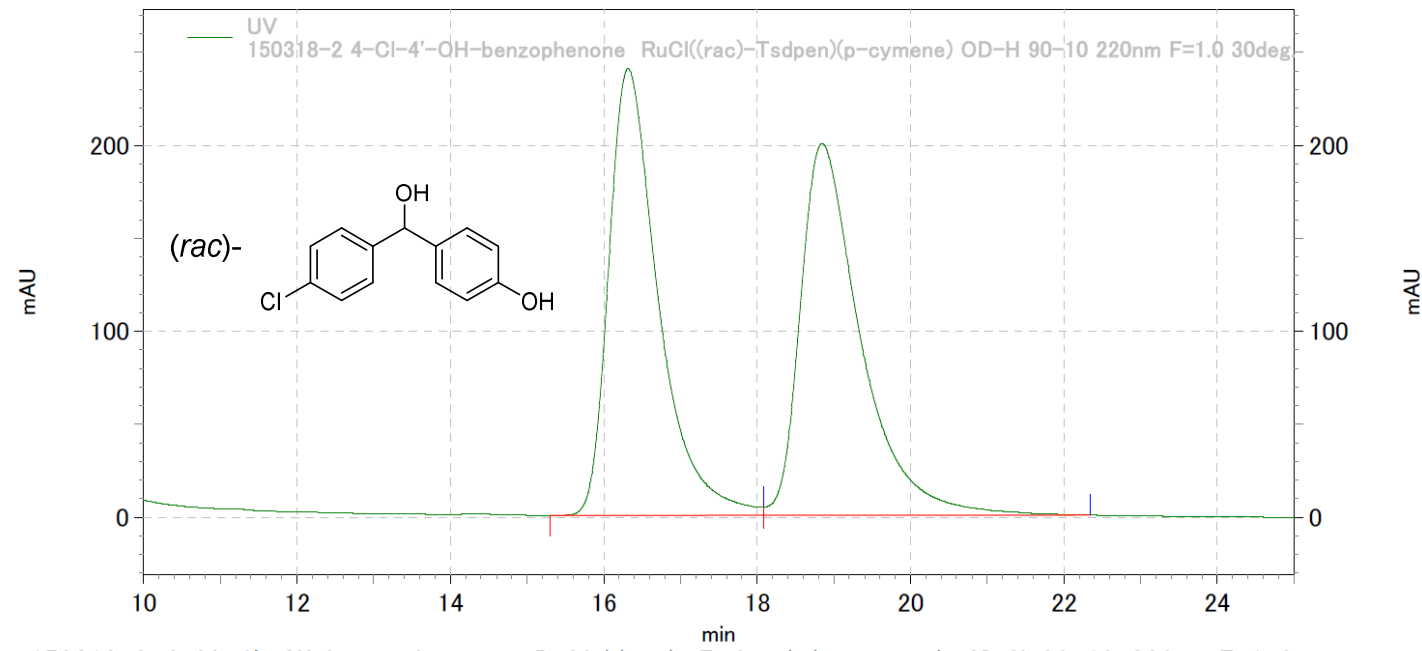

150318-2 4-Cl-4' -OH-benzophenone RuCl ( (r ac)-Tsdpen) (p-cymene) OD-H 90-10 220nm F=1.0 30deg. C

\begin{tabular}{|c|c|c|c|c|c|}
\hline $\begin{array}{l}\text { UV結果 } \\
\text { Pk } \\
\text { No. }\end{array}$ & R. T. & Area & Area\% & B. C. & Symmetry \\
\hline $\begin{array}{l}1 \\
2\end{array}$ & $\begin{array}{l}16.317 \\
18.847\end{array}$ & $\begin{array}{l}43230491 \\
43691450\end{array}$ & $\begin{array}{l}49.735 \\
50.265\end{array}$ & $\begin{array}{l}\mathrm{Mx} \\
\mathrm{XM}\end{array}$ & $\begin{array}{l}0.00000 \\
0.00000\end{array}$ \\
\hline $\begin{array}{r}r-夕 \\
\mu\end{array}$ & & 86921941 & 100.000 & & \\
\hline
\end{tabular}

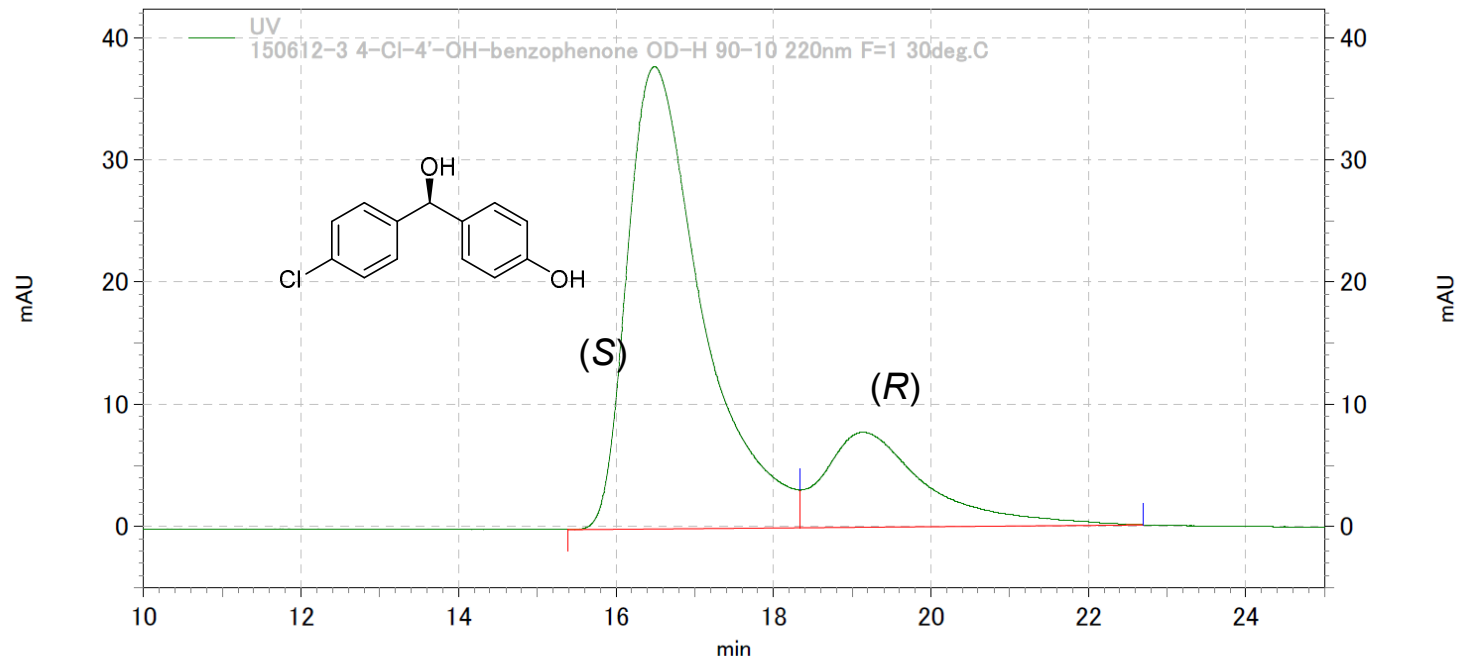

150612-3 4-Cl-4' -OH-benzophenone OD-H 90-10 220nm F=1 30deg. C

\begin{tabular}{|c|c|c|c|c|c|}
\hline $\begin{array}{l}\text { UV結果 } \\
\text { Pk } \\
\text { No. }\end{array}$ & R. T. & Area & Area\% & B. C. & Symmetry \\
\hline $\begin{array}{l}1 \\
2\end{array}$ & $\begin{array}{l}16.487 \\
19.140\end{array}$ & $\begin{array}{r}10022067 \\
2803561\end{array}$ & $\begin{array}{l}78.141 \\
21.859\end{array}$ & $\begin{array}{l}M x \\
X M\end{array}$ & $\begin{array}{l}0.00000 \\
0.00000\end{array}$ \\
\hline $\begin{array}{r}r-夕 \\
\pi\end{array}$ & & 12825628 & 100.000 & & \\
\hline
\end{tabular}




\section{(4-Methoxyphenyl)(4-nitrophenyl)methanol (11k)}

(AD-H, Hex/2-PrOH=90/10, F=1.0 mL/min, 220 nm, 30deg.C)

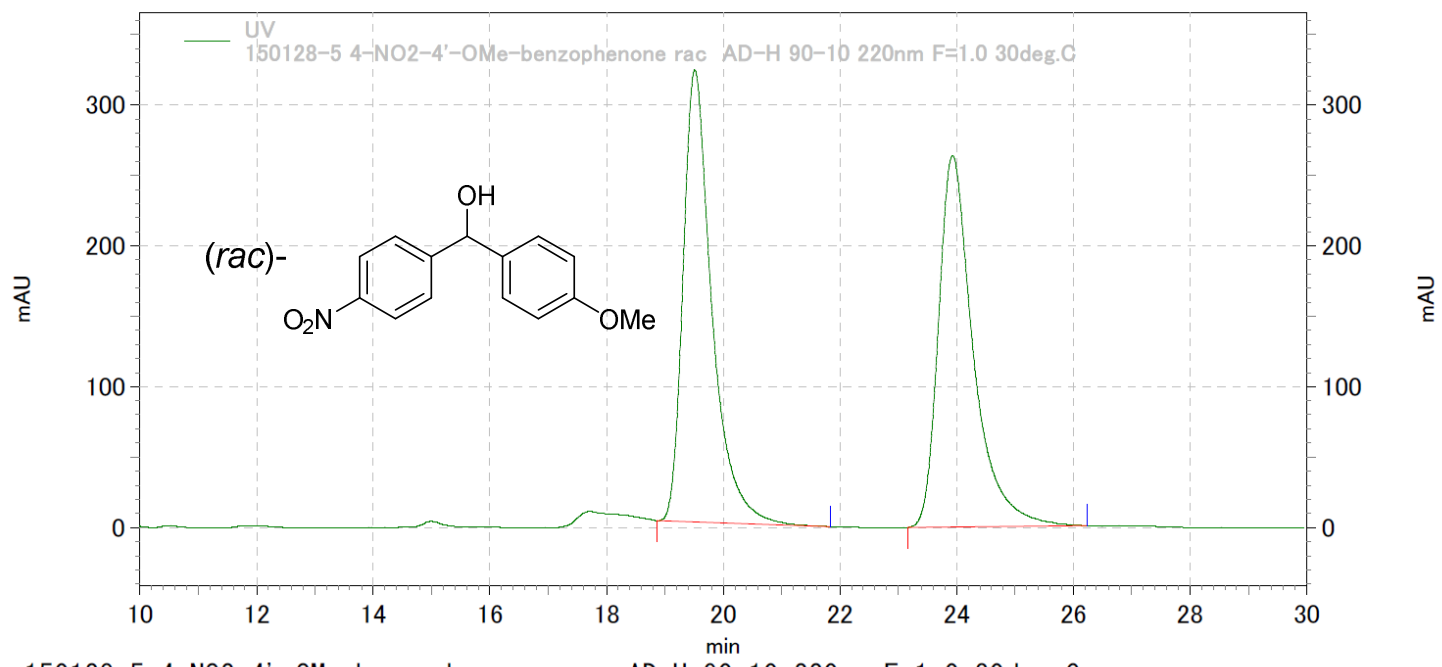

150128-5 4-N02-4' -OMe-benzophenone rac AD-H 90-10 220nm F=1. 0 30deg. C

\begin{tabular}{|c|c|c|c|c|c|}
\hline $\begin{array}{l}\text { UV結身 } \\
\text { Pk } \\
\text { No. }\end{array}$ & R. T. & Area & Area\% & B. C. & Symmetry \\
\hline $\begin{array}{l}1 \\
2\end{array}$ & $\begin{array}{l}\text { 19. } 513 \\
23.933\end{array}$ & $\begin{array}{l}43949905 \\
43734601\end{array}$ & $\begin{array}{l}50.123 \\
49.877\end{array}$ & $\begin{array}{l}\mathrm{MM} \\
\mathrm{MM}\end{array}$ & $\begin{array}{l}0.00000 \\
0.00000\end{array}$ \\
\hline $\begin{array}{r}r-夕 \\
\|\end{array}$ & & 87684506 & 100.000 & & \\
\hline
\end{tabular}

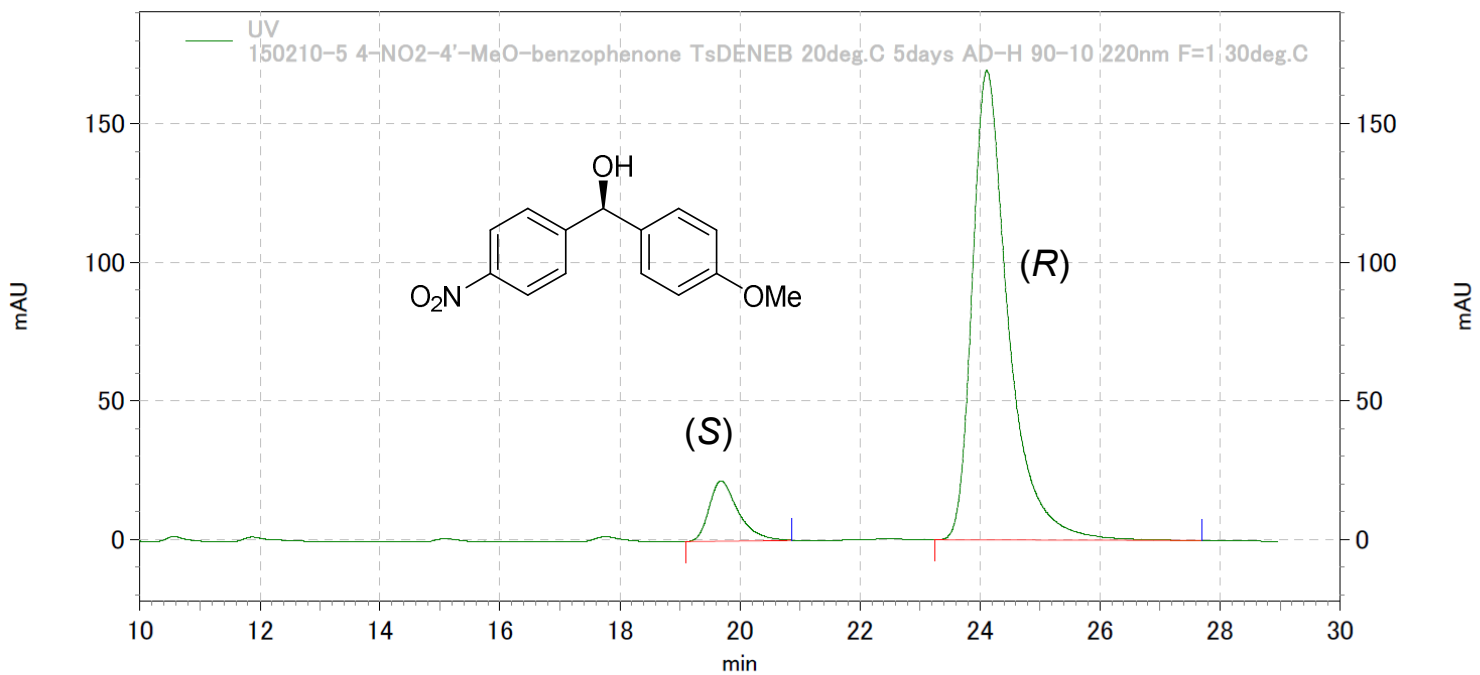

150210-5 4-N02-4' -Me0-benzophenone TsDENEB 20deg. C 5days AD-H 90-10 220nm F=1 30deg. C

\begin{tabular}{|c|c|c|c|c|c|}
\hline $\begin{array}{l}\text { UV 結果 } \\
\text { Pk } \\
\text { No. }\end{array}$ & R. T. & Area & Area\% & B. C. & Symmetry \\
\hline $\begin{array}{l}1 \\
2\end{array}$ & $\begin{array}{l}19.683 \\
24.117\end{array}$ & $\begin{array}{r}2904526 \\
28238814\end{array}$ & $\begin{array}{r}9.326 \\
90.674\end{array}$ & $\begin{array}{l}\text { MM } \\
\text { MM }\end{array}$ & $\begin{array}{l}0.00000 \\
0.00000\end{array}$ \\
\hline $\begin{array}{r}r-y \\
\pi\end{array}$ & & 31143340 & 100.000 & & \\
\hline
\end{tabular}




\section{(3,5-Dinitrophenyl)(4-methoxyphenyl)methanol (11I)}

(OJ-H, Hex/2-PrOH=85/15, F=1.0 mL/min, $220 \mathrm{~nm}$, 30deg.C)

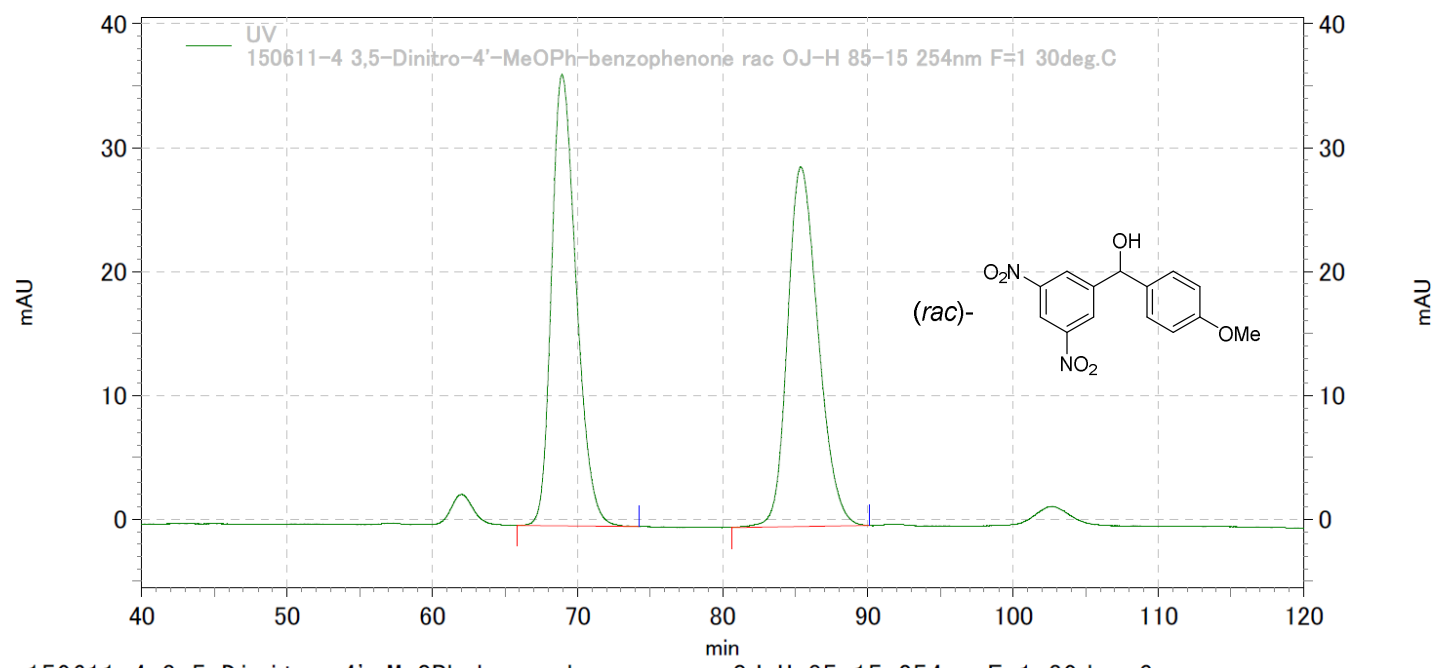

150611-4 3, 5-Dinitro-4' -MeOPh-benzophenone rac 0J-H 85-15 254nm F=1 30deg. C

\begin{tabular}{rlllll}
$\begin{array}{llll}\text { UV } \\
\text { Pk }\end{array}$ & R. T. & Area & Area\% & B. C. & Symmetry \\
No. & & & & & \\
\hline 1 & 68.940 & 17220260 & 50.271 & MM & 0.00000 \\
2 & 85.370 & 17034703 & 49.729 & MM & 0.00000
\end{tabular}

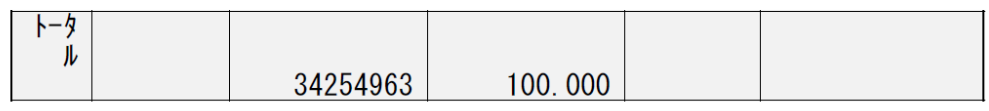

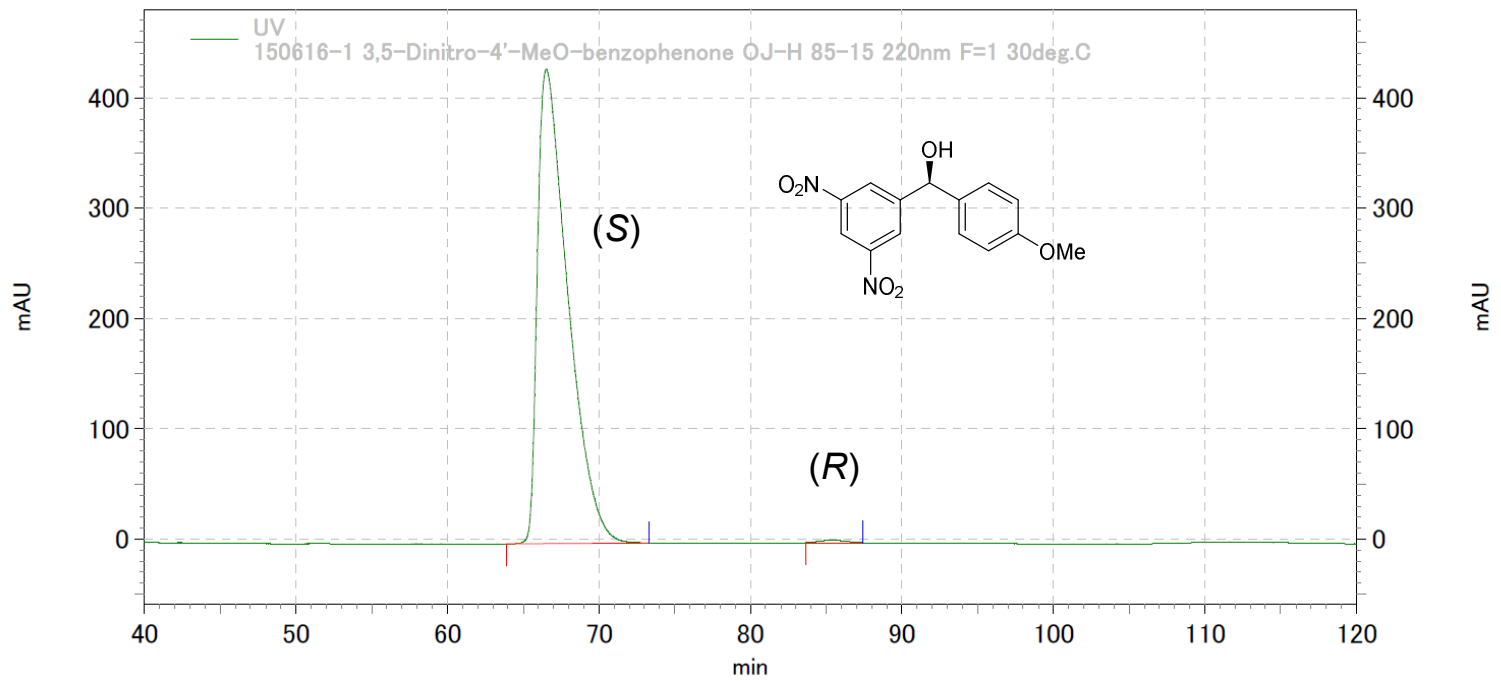

150616-1 3, 5-Dinitro-4' -Me0-benzophenone 0J-H 85-15 220nm F=1 30deg. C

\begin{tabular}{|c|c|c|c|c|c|}
\hline $\begin{array}{l}\text { UV 結 } \\
\text { Pk } \\
\text { No. }\end{array}$ & R. T. & Area & Area\% & B. C. & Symmetry \\
\hline $\begin{array}{l}1 \\
2\end{array}$ & $\begin{array}{l}66.527 \\
85.413\end{array}$ & $\begin{array}{r}240063042 \\
1179485\end{array}$ & $\begin{array}{r}99.511 \\
0.489\end{array}$ & $\begin{array}{l}M M \\
M M\end{array}$ & $\begin{array}{l}0.00000 \\
0.00000\end{array}$ \\
\hline $\begin{array}{r}r-夕 \\
\|\end{array}$ & & 241242527 & 100.000 & & \\
\hline
\end{tabular}




\section{Phenyl(ferrocenyl)methanol (11 m)}

(AS-H, Hex/2-PrOH=95/5, F=1.0 mL/min, 220 nm, 30deg.C)

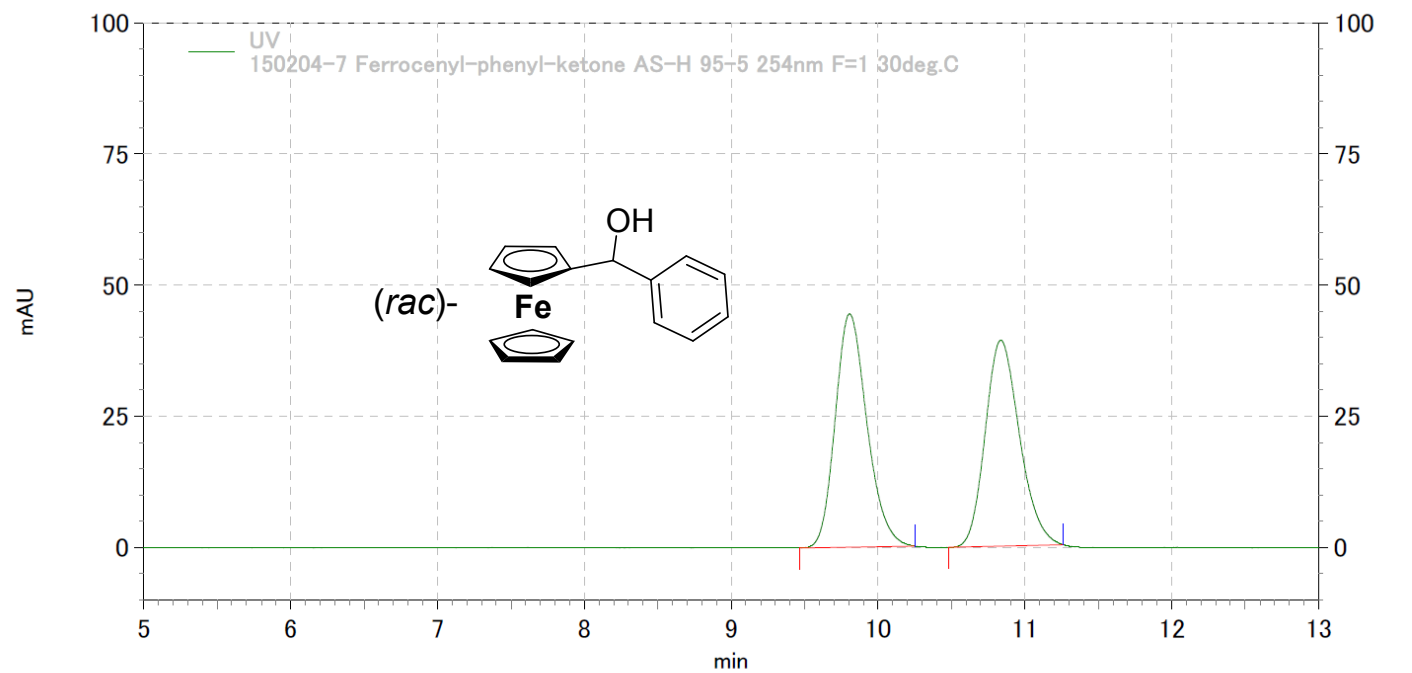

150204-7 Ferrocenyl-phenyl-ketone AS-H 95-5 254nm F=1 30deg. C

\begin{tabular}{|c|c|c|c|c|c|}
\hline $\begin{array}{l}\text { UV結身 } \\
\text { Pk } \\
\text { No. }\end{array}$ & R. T. & Area & Area\% & B. $\mathrm{C}$. & Symmetry \\
\hline $\begin{array}{l}1 \\
2\end{array}$ & $\begin{array}{r}9.807 \\
10.837\end{array}$ & $\begin{array}{l}2672390 \\
2551811\end{array}$ & $\begin{array}{l}51.154 \\
48.846\end{array}$ & $\begin{array}{l}\text { MM } \\
\text { MM }\end{array}$ & $\begin{array}{l}0.00000 \\
0.00000\end{array}$ \\
\hline $\begin{array}{r}r-g \\
\mu\end{array}$ & & 5224201 & 100.000 & & \\
\hline
\end{tabular}

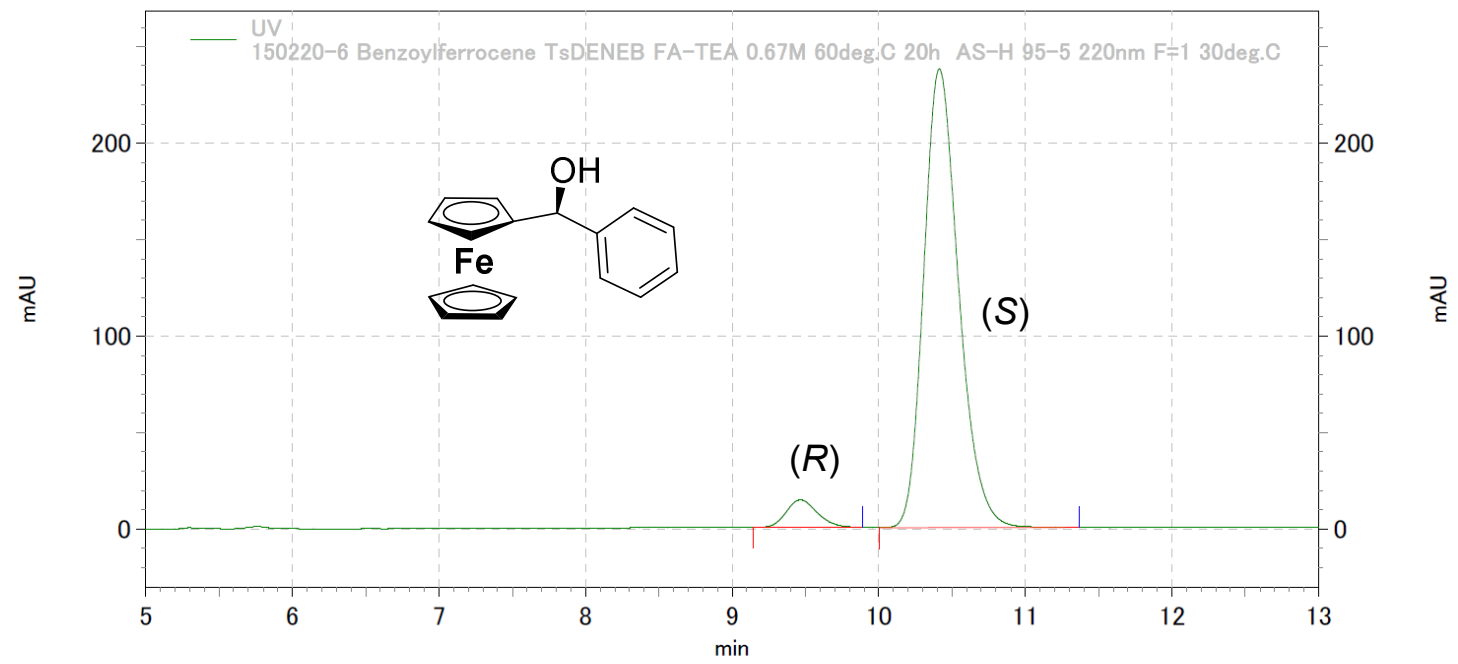

150220-6 Benzoylferrocene TsDENEB FA-TEA 0.67M 60deg. C 20h AS-H 95-5 220nm F=1 30deg. C

\begin{tabular}{|c|c|c|c|c|c|}
\hline $\begin{array}{l}\text { UV 結 } \\
\text { Pk } \\
\text { No. }\end{array}$ & R. T. & Area & Area\% & B. $\mathrm{C}$ & Symmetry \\
\hline $\begin{array}{l}1 \\
2\end{array}$ & $\begin{array}{r}9.467 \\
10.413\end{array}$ & $\begin{array}{r}850689 \\
15707397\end{array}$ & $\begin{array}{r}5.138 \\
94.862\end{array}$ & $\begin{array}{l}\mathrm{MM} \\
\mathrm{MM}\end{array}$ & $\begin{array}{l}0.00000 \\
0.00000\end{array}$ \\
\hline $\begin{array}{r}r-夕 \\
ル\end{array}$ & & 16558086 & 100.000 & & \\
\hline
\end{tabular}




\section{(3-Nitrophenyl)(thiophen-2-yl)methanol (11n)}

(OJ-H, Hex/2-PrOH=90/10, F=1.0 mL/min, 220 nm, 30deg.C)

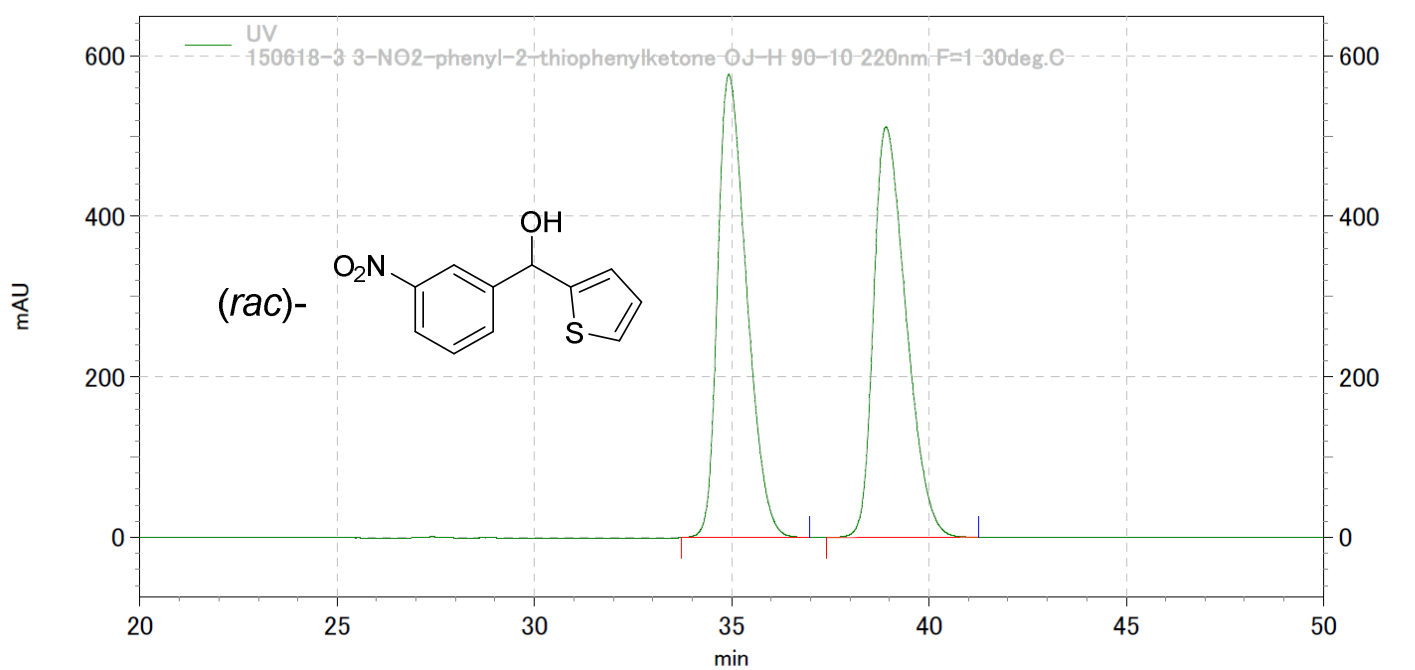

150618-3 3-N02-phenyl-2-thiophenylketone 0J-H 90-10 220nm F=1 30deg. C

\begin{tabular}{|c|c|c|c|c|c|}
\hline $\begin{array}{l}\text { UV 結身 } \\
\text { Pk } \\
\text { No. }\end{array}$ & R. T. & Area & Area\% & B. C. & Symmetry \\
\hline $\begin{array}{l}1 \\
2\end{array}$ & $\begin{array}{l}34.927 \\
38.913\end{array}$ & $\begin{array}{l}117310664 \\
117393200\end{array}$ & $\begin{array}{l}49.982 \\
50.018\end{array}$ & $\begin{array}{l}\text { MM } \\
\text { MM }\end{array}$ & $\begin{array}{l}0.00000 \\
0.00000\end{array}$ \\
\hline $\begin{array}{r}r-夕 \\
\|\end{array}$ & & 234703864 & 100.000 & & \\
\hline
\end{tabular}

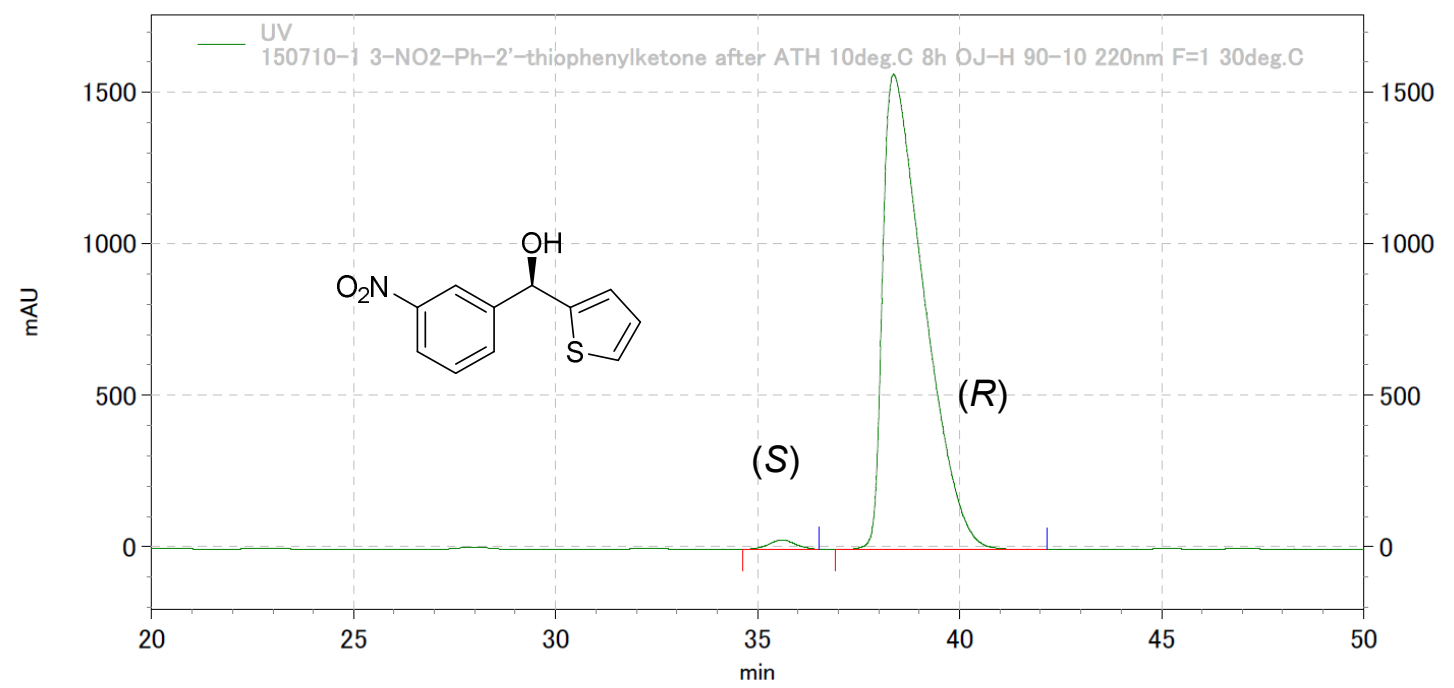

150710-1 3-N02-Ph-2' -thiophenylketone after ATH 10deg. C 8h 0J-H 90-10 220nm F=1 30deg. C

\begin{tabular}{|c|c|c|c|c|c|}
\hline $\begin{array}{l}\text { UV結果 } \\
\mathrm{Pk}\end{array}$ & R. T. & Area & Area\% & B. C. & Symmetry \\
\hline $\begin{array}{l}1 \\
2\end{array}$ & $\begin{array}{l}35.603 \\
38.370\end{array}$ & $\begin{array}{r}5311190 \\
445444406\end{array}$ & $\begin{array}{r}1.178 \\
98.822\end{array}$ & $\begin{array}{l}\mathrm{MM} \\
\mathrm{MM}\end{array}$ & $\begin{array}{l}0.00000 \\
0.00000\end{array}$ \\
\hline $\begin{array}{r}r-夕 \\
M\end{array}$ & & 450755596 & 100.000 & & \\
\hline
\end{tabular}

